\title{
Identifizierung eines Flattermechanismus an einem superkritischen Profil unter laminarer Strömung
}

\author{
Dissertation \\ zur Erlangung des mathematisch-naturwissenschaftlichen Doktorgrades \\ „Doctor rerum naturalium“ \\ der Georg-August-Universität Göttingen \\ im Promotionsprogramm ProPhys \\ der Georg-August University School of Science (GAUSS)
}

vorgelegt von

Marc Braune

aus Schmallenberg-Bödefeld

Göttingen, 2021 


\section{Betreuungsausschuss}

\section{Prof. Dr. Andreas Dillmann}

Institut für Aerodynamik und Strömungstechnik, Deutsches Zentrum für Luft- und Raumfahrt e. V. (DLR) Institut für Dynamik komplexer Systeme, Georg-August-Universität Göttingen

\section{Prof. Dr. Martin Rein}

Institut für Aerodynamik und Strömungstechnik, Deutsches Zentrum für Luft- und Raumfahrt e. V. (DLR) Institut für Dynamik komplexer Systeme, Georg-August-Universität Göttingen

\section{Mitglieder der Prüfungskommission}

\section{Referent: Prof. Dr. Andreas Dillmann}

Institut für Aerodynamik und Strömungstechnik, Deutsches Zentrum für Luft- und Raumfahrt e. V. (DLR) Institut für Dynamik komplexer Systeme, Georg-August-Universität Göttingen

\section{Korreferent: Prof. Dr. Martin Rein}

Institut für Aerodynamik und Strömungstechnik, Deutsches Zentrum für Luft- und Raumfahrt e. V. (DLR) Institut für Dynamik komplexer Systeme, Georg-August-Universität Göttingen

\section{Weitere Mitglieder der Prüfungskommission}

\section{Prof. Dr. Stefan Dreizler}

Institut für Astrophysik, Georg-August-Universität Göttingen

\section{Prof. Dr. Wolfgang Glatzel}

Institut für Astrophysik, Georg-August-Universität Göttingen

\section{Prof. Dr. Stefan Klumpp}

Institut für Dynamik komplexer Systeme, Georg-August-Universität Göttingen

\section{Prof. Dr. Ulrich Parlitz}

Max-Planck-Institut für Dynamik und Selbstorganisation (MPIDS)

Institut für Dynamik komplexer Systeme, Georg-August-Universität Göttingen

Tag der mündlichen Prüfung: 02.06.2021 


\section{Danksagung}

If I have seen further than others,

it is by standing upon the shoulders of giants.

\section{ISAAC NEWTON (1643-1727)}

In Anlehnung an das Zitat ist es ein Name, der auf dieser Arbeit steht, aber eine Vielzahl von Menschen, die dies ermöglicht haben. Jedem von ihnen gebührt mein tiefster Dank. So mögen sich auch diejenigen in gleichem Maße angesprochen fühlen, die nicht namentlich genannt werden, mich aber auf meinem Wege begleitet und unterstützt haben, mich auch zu Boden warfen, mir wieder auf halfen, mich geprägt haben.

So gilt an erster Stelle mein Dank dem Institut für Aeroelastik des Deutschen Zentrums für Luft- und Raumfahrt e. V., dem gesamten Team und insbesondere seinem Direktor Herrn Prof. Dr.-Ing. Lorenz Tichy für die Möglichkeit und die Mittel, diese Arbeit anfertigen und abschließen zu können. Großer Dank gilt ebenso meinem Abteilungsleiter Herrn Dr. rer. nat. Holger Mai für die Unterstützung, den Rückhalt, das Vertrauen und den mir gewährten Freiraum.

Herrn Prof. Dr. rer. nat. Dr.-Ing. habil. Andreas Dillmann sowie Herrn Prof. Dr. rer. nat. Martin Rein danke ich herzlichst für die Betreuung der Arbeit und die Übernahme des Referats und Korreferats. Weiterhin sei ihnen dafür gedankt, dass sie mich für die Strömungsmechanik und Aerodynamik begeistert und hierin ausgebildet haben, bei Fragen und Problemen mir immer hilfsbereit mit Rat und Tat zur Seite standen. Auch den weiteren Mitgliedern der Prüfungskommission Herrn Prof. Dr. rer. nat. Stefan Dreizler, Herrn Prof. Dr. rer. nat. Wolfgang Glatzel, Herrn Prof. Dr. rer. nat. Stefan Klumpp und Herrn Prof. Dr. rer. nat. Ulrich Parlitz sei vielmals gedankt.

Erfolgreiche Experimente sind nie die Leistung eines Einzelnen. Mein größter Dank gilt daher dem gesamten Team der Arbeitsgruppe Aeroelastische Windkanalexperimente. Insbesondere Frau Anne Hebler bin ich für ihre Unterstützung während der Vorbereitung und Durchführung der Windkanalversuche sowie für ihre Vorarbeiten sehr verbunden. Danke auch an die Herren Heiko Böhlken, Thomas Büte, Holger Ernst und Johannes Nuhn für die technische Unterstützung und die vielen unterhaltsamen Stunden im Windkanal und darüber hinaus. Ein großes Dankeschön an Herrn Christian Stieg für die technische Unterstützung und Vorbereitung des Windkanalmodells sowie Herrn Carsten Fuchs für die durchgeführten Schönheitskorrekturen. Herrn Dr. rer. nat. Stefan Koch und Herrn 
Sebastian Weiss sei für die Bereitstellung der Heißfilm-Anemometrie-Messtechnik und ihrer Unterstützung während der Messungen gedankt. Auch dem gesamten Team vom DNW-TWG gilt mein Dank, hier insbesondere Herrn Dr. rer. nat. Andreas Benkel für die Projektleitung und den Herren Sven Fiedler, Andreas Grimme und Karsten Steiner für die Umsetzung und Geduld bei speziellen, den Wind betreffenden Wünschen.

Danke an meinen Gruppenleiter Herrn Dr.-Ing. Nils van Hinsberg für seine Unterstützung bei den Experimenten, für die vielen Hilfestellungen, Anregungen und Ratschläge im Rahmen meiner wissenschaftlichen Tätigkeit, Korrekturen und Anmerkungen in diesem und weiteren Manuskripten sowie für die vielen Gespräche zu frühen Stunden. Gleichermaßen möchte ich mich bei Herrn Dr.-Ing. Jan Schwochow und Herrn Dr.-Ing. Fritz Kießling dafür bedanken, dass sie mich an ihrem Wissen stets teilhaben ließen. Bei einer Vielzahl an Fragen hatten sie immer ein offenes Ohr und bei Problemen zumeist die Lösung oder die passende Veröffentlichung in der Tasche. Auch für die Durchsicht des Manuskripts mitsamt den vielen hilfreichen Anmerkungen und der Hilfe bei diversen Literaturquellen bin ich überaus dankbar.

Ferner sei einem gewissen Flugschüler für sehr viele Höhenmeter, aber auch für die Vielzahl von unterhaltsamen und gleichsam lehrreichen Gesprächen und Ratschlägen gedankt. Die hieraus geschöpfte Motivation und das Durchhaltevermögen haben entscheidend zum Gelingen dieser Arbeit beigetragen.

Der größte Dank aber gilt meinem Bürokollegen und wissenschaftlichem Mentor Herrn Dr. rer. nat. Günter Schewe. Ohne ihn wäre diese Arbeit weder mit Inhalt gefüllt, noch wäre sie jemals zu einem Abschluss gekommen. Danke für die unzähligen Stunden an Gesprächen, die vielen Diskussionen, die zahlreichen Ratschläge und Weisheiten, die aufmunternden aber auch kritischen Worte sowie die Weitergabe von über Jahrzehnte gesammeltem Wissen und Erfahrungen. Auch für die vielen Stunden der Durchsicht und Korrektur des Manuskripts bin ich ihm sehr verbunden.

$\mathrm{Zu}$ guter Letzt möchte ich mich bei den Menschen bedanken, die meinem Leben Inhalt und mir Rückhalt geben, Freud und Leid mit mir teilen und es mir erst ermöglicht haben, dass ich diese Worte an dieser Stelle niederschreiben darf. Aus tiefstem Herzen Danke

an Dirk, Hilke und Gerrit, an meine Eltern, an meine Schwester, an meine Liebste. 


\section{Kurzfassung}

Die transsonische Umströmung von elastischen Körpern kann zu komplexen, nichtlinearen aeroelastischen Wechselwirkungen führen. Bisher kaum untersucht sind dabei aeroelastische Systeme, bei denen die laminar-turbulente Grenzschichttransition eine zentrale Rolle für die stationäre und instationäre Aerodynamik darstellt. Ein Zusammenspiel dieser Komponenten führt an dem superkritischen CAST 10-2 Flügelprofil zu einem neuartigen Flattermechanismus, welcher in der vorliegenden Arbeit untersucht und identifiziert wird. Hierzu wurden transsonische Windkanalexperimente mit zwangserregten Nick- und selbsterregten Flatterschwingungen durchgeführt. Die Untersuchungen fanden schwerpunktmäßig bei einer freien Grenzschichttransition in einem Mach-Zahl-Bereich von $0,73 \lesssim \mathrm{Ma}_{\infty} \lesssim 0,75$ bei einer Reynolds-Zahl von $\operatorname{Re}_{\infty} \approx 2 \cdot 10^{6}$ statt.

Der neuartige Flattermechanismus wird auf Mach-Zahlen und Anstellwinkel eingegrenzt, die mit dem oberen Übergangsbereich der Laminardelle des CAST 10-2 Flügelprofils zusammenfallen. Das aeroelastische Systemverhalten tritt als subkritische Hopf-Bifurkation in Erscheinung. Der Flattermechanismus wird als Transitionsflattern identifiziert. Eine freie Grenzschichttransition stellt hierfür eine notwendige Bedingung dar. Ferner führt der neue Flattertyp zu einer Vergrößerung des instabilen Bereiches, einer Ausdehnung des Transonic Dips. Für Strömungsparameter, bei denen das Transitionsflattern auftritt, zeigen zwangserregte Nickschwingungen, dass ein Energietransfer von der Strömung in die Struktur stattfindet. Dieser geht mit einer Phasenvoreilung des Nickmomentes einher und tritt bei einer turbulenten Grenzschicht nicht auf. Eine im Rahmen dieser Arbeit entwickelte Auswertungsmethodik ermöglicht dabei erstmalig auch die Auflösung der nichtlinearen Dynamik der Position der laminar-turbulenten Grenzschichttransition mitsamt der Stoß-Grenzschicht-Interaktion. Aerodynamische Grenzzyklusschwingungen, die bei festen Anstellwinkeln im oberen Übergangsbereich der Laminardelle auftreten, können so auf eine Oszillation der Transitionslage zurückgeführt werden. Ferner zeigt sich eine Phasenvoreilung der Transitionsbewegung gegenüber der Bewegung des Verdichtungsstoßes und eine damit einhergehende Steuerung der Stoßlage. Die Existenz eines, vom Flattern zu unterscheidenden Transitionsbuffets und dessen Interaktion mit dem aeroelastischen System können gezeigt werden. Der Übergang von einem aerodynamischen in einen aeroelastischen Grenzzyklus werden experimentell aufgelöst. Diese Beobachtungen unterstützen die Hypothese, dass eine Instationarität des laminar-turbulenten Grenzschichtumschlags ursächlich zum neuartigen Flattermechanismus führt.

Die Ergebnisse der Arbeit zeigen, dass aus bzw. mit der laminar-turbulenten Grenzschichttransition neuartige aerodynamische und aeroelastische Instabilitäten resultieren. Hieraus leitet sich die Notwendigkeit ab, die laminar-turbulente Grenzschichttransition in einer vollständigen aeroelastischen Systembewertung mit zu berücksichtigen. 


\section{Abstract}

The transonic flow around elastic bodies can lead to complex, nonlinear aeroelastic interactions. So far, hardly any aeroelastic systems have been investigated in which the laminar-turbulent boundary layer transition plays a central role for the steady and unsteady aerodynamics. An interaction of these components leads to a novel flutter mechanism at the supercritical CAST 10-2 airfoil, which is investigated and identified in the present work. For this purpose, transonic wind tunnel experiments with forced pitch and self-excited flutter oscillations were performed. The investigations focused on a free boundary layer transition in a Mach number range of $0.73 \lesssim \mathrm{Ma}_{\infty} \lesssim 0.75$ at a Reynolds number of $\operatorname{Re}_{\infty} \approx 2 \cdot 10^{6}$.

The novel flutter mechanism is confined to Mach numbers and angles of attack that coincide with the upper transition region of the laminar drag bucket of the CAST 10-2 airfoil. The aeroelastic system behavior appears as a subcritical Hopf bifurcation. The flutter mechanism is identified as transition-flutter. A free boundary layer transition represents a necessary condition for this. Furthermore, the new type of flutter leads to a larger unstable region, hence an extension of the transonic dip. For flow parameters at which transition flutter occurs, forced pitch oscillations show that there is a transfer of energy from the flow to the structure. This is associated with a phase lead of the pitching moment and does not occur for a turbulent boundary layer. An evaluation methodology developed within the framework of this study also enables the resolution of the nonlinear dynamics of the laminar-turbulent boundary layer transition position together with the shock-boundary layer interaction for the first time. Aerodynamic limit cycle oscillations that occur at fixed angles of attack in the upper transition region of the drag bucket, can thus be attributed to an oscillation of the transition position. Furthermore, a phase lead of the transition movement with respect to the motion of the compression shock and an associated control of the shock position is shown. The existence of a transition-buffet, to be distinguished from flutter, and its interaction with the aeroelastic system can be shown. The crossover from an aerodynamic to an aeroelastic limit cycle is resolved experimentally. These observations support the hypothesis that an unsteadiness of the laminar-turbulent boundary layer transition causally leads to the novel flutter mechanism.

The results of this work demonstrate that novel aerodynamic and aeroelastic instabilities result from or in combination with the laminar-turbulent boundary layer transition. This requires the inclusion of the laminar-turbulent boundary layer transition in a complete aeroelastic system assessment. 


\section{Inhaltsverzeichnis}

$\begin{array}{ll}\text { 1. Einleitung } & 1\end{array}$

1.1. Motivation und Zielsetzung der Arbeit . . . . . . . . . . . . 2

1.2. Stand der Forschung . . . . . . . . . . . . . . . . . 5

1.3. Gliederung und wesentliche Ergebnisse der Arbeit . . . . . . . . . . 8

2. Physikalische Grundlagen $\quad 11$

2.1. Laminar-turbulente Grenzschichttransition . . . . . . . . . . . . . 11

2.1.1. Natürlicher Transitionsprozess und Primäre Stabilitätstheorie . . 13

2.1.2. Einfluss des Druckgradienten und Laminarerhaltung . . . . . . . 17

2.1.3. Stoß-Grenzschicht-Interaktion . . . . . . . . . . . . . 20

2.1.4. Wandschubspannungsverlauf und Definition der Transition . . . 22

2.2. Physikalische Aspekte der Aeroelastik . . . . . . . . . . . . . . 24

2.2.1. Grundzüge der instationären Aerodynamik . . . . . . . . . . 25

2.2.2. Flattern . . . . . . . . . . . . . . . 30

2.2.3. Aeroelastische Instabilitäten unter transsonischer Strömung . . . 33

3. Experimenteller Aufbau $\quad 37$

3.1. Transsonischer Windkanal . . . . . . . . . . . . . . . . 37

3.2. Versuchsstände . . . . . . . . . . . . . . . . . 38

3.2.1. Nickversuchsstand für zwangserregte Profilschwingungen . . . . . 38

3.2.2. Flatterversuchstand für selbsterregte Profilschwingungen . . . . . 39

3.3. Laminarflügelprofil CAST $10-2 \ldots \ldots$. . . . . . . . . . . . . 42

3.4. Messtechnik und Sensorik . . . . . . . . . . . . . . . . . . . 44

3.4.1. Drucksensoren . . . . . . . . . . . . . . . . 45

3.4.2. Beschleunigungssensoren . . . . . . . . . . . . . . 46

3.4.3. Heißfilm-Anemometrie . . . . . . . . . . . . . . 46

3.4.4. Lasertriangulatoren . . . . . . . . . . . . . . . . . . 47

3.4.5. Infrarotthermographie . . . . . . . . . . . . . . 48 48

3.4.6. Piezoelektrische Waage . . . . . . . . . . . . . . 48

3.4.7. Datenerfassung und Aufbereitung. . . . . . . . . . . . 49 
4. Datenanalyse und Methodik 51

4.1. Datenanalyse im Frequenzbereich . . . . . . . . . . . . . . . . . . . 51

4.1.1. Fourier-Reihe und Fourier-Transformation . . . . . . . . . . 51

4.1.2. Korrelationsfunktionen und Leistungsdichtespektren . . . . . . . 52

4.1.3. Übertragungsfunktion . . . . . . . . . . . . . . . . . . . . . 53

4.1.4. Integration und Differentiation im Frequenzbereich . . . . . . . . 54

4.2. Berechnung der aerodynamischen Beiwerte . . . . . . . . . 55

4.2.1. Aerodynamische Beiwerte und stationäre Derivativa . . . . . . . 55

4.2.2. Aerodynamischer Energie- und Leistungseintrag . . . . . . . . 56

4.2.3. Instationäre aerodynamische Beiwerte . . . . . . . . . . 58

4.3. Identifikation der Grenzschichttransition . . . . . . . . . . . . . . . . 58

4.3.1. Quasiwandschubspannung . . . . . . . . . . . . . . . 59

4.3.2. Identifikation der Grenzschichtzustände . . . . . . . . . . . 60

4.3.3. Datenverarbeitung und Transitionsdetektion . . . . . . . . . 61

5. Aerodynamisches Verhalten bei statischen Anstellwinkeln 65

5.1. Verhalten der aerodynamischen Kräfte . . . . . . . . . . . . . 65

5.2. Verhalten der Druckverteilung . . . . . . . . . . . . . . . . . . 70

5.3. Verhalten der Grenzschicht . . . . . . . . . . . . . . . . . 74

5.4. Instationäre Effekte bei statischen Anstellwinkeln . . . . . . . . . . . . 77

5.5. Zusammenfassung der stationären Aerodynamik . . . . . . . . . . . . . . 81

6. Aerodynamisches Verhalten bei zwangserregten Nickschwingungen 83

6.1. Instationäre Derivativa der aerodynamischen Beiwerte . . . . . . . . . . 83

6.2. Energiebetrachtung . . . . . . . . . . . . . . . . 88

6.3. Instationäres Druckverhalten . . . . . . . . . . . . . . . . . . 93

6.4. Instationäres Transitionsverhalten . . . . . . . . . . . . . . . . . 98

6.5. Zusammenfassung der instationären Aerodynamik . . . . . . . . . . . . 104

$\begin{array}{ll}\text { 7. Aeroelastisches Verhalten } & 107\end{array}$

7.1. Aeroelastische Konfigurationen . . . . . . . . . . . . . . . . . . . . 107

7.2. Aeroelastische Stabilität der 2-DoF Konfiguration . . . . . . . . . . . . . 109

7.3. Aeroelastische Stabilität der 1-DoF Konfiguration . . . . . . . . . . . . . 113

7.3.1. Vergleichbarkeit zur 2-DoF Konfiguration . . . . . . . . . . . . 115

7.3.2. Einfluss der Mach-Zahl . . . . . . . . . . . . . . . . . . 117

7.3.3. Einfluss des Anstellwinkels . . . . . . . . . . . . . . . . . . 118

7.4. Grenzzyklusschwingung der 1-DoF Konfiguration . . . . . . . . . . . . 120

7.4.1. Verhalten von Stoß und Transition . . . . . . . . . . . . . . . . 121

7.4.2. Energie- und Leistungsbetrachtung . . . . . . . . . . . . . . . . 125

7.5. Zusammenfassung des aeroelastischen Verhaltens . . . . . . . . . . . . . 129 
$\begin{array}{ll}\text { 8. Diskussion und Ausblick } & 131\end{array}$

8.1. Diskussion . . . . . . . . . . . . . . . . . . . . . . . . . . . 132

8.2. Ausblick . . . . . . . . . . . . . . . . . 137

$\begin{array}{ll}\text { Literaturverzeichnis } & 141\end{array}$

$\begin{array}{ll}\text { Nomenklatur } & 168\end{array}$

$\begin{array}{ll}\text { Abbildungsverzeichnis } & 174\end{array}$

$\begin{array}{ll}\text { Tabellenverzeichnis } & 175\end{array}$

$\begin{array}{ll}\text { Anhang } & 177\end{array}$

$\begin{array}{lr}\text { A. Ergänzungen zu Kapitel } 2 & 179\end{array}$

A.1. Stabilitätsrechnung mit variierendem Pohlhausen-Parameter . . . . . . . 179

A.2. Kinematische Strömungsbedingung . . . . . . . . . . . . . . . . . . 180

A.3. Zur Lösung der Integralgleichung Gl. (2.2.8) . . . . . . . . . . . . . . . 181

A.4. Berechnung der instationären Luftkräfte . . . . . . . . . . . . . . . 183

$\begin{array}{ll}\text { B. Ergänzungen zu Kapitel } 3 & 187\end{array}$

B.1. Berechnung von Strömungsparametern und Diskussion von Messfehlern 187

B.2. Federkonstanten und generalisierte Massen des FVS . . . . . . . . . . . 191

B.2.1. Federkonstanten der Rechteck-Parallelfedern . . . . . . . . . . . 192

B.2.2. Generalisierte Massen der Rechteck-Parallelfedern . . . . . . . . 193

B.2.3. Federkonstanten der Torsionsfedern . . . . . . . . . . . . . . . . 194

B.2.4. Generalisierte Massen des Torsionsfedersystems . . . . . . . . . . 195

B.3. Vermessung der Profilgeometrie . . . . . . . . . . . . . . . . . . 196

B.4. Messungenauigkeiten der Sensorik . . . . . . . . . . . . . . . . . . 199

B.4.1. Messfehler der Drucksensoren . . . . . . . . . . . . . . . . . . . . 199

B.4.2. Messfehler des Anstellwinkels bei Flatterversuchen . . . . . . . . 201

B.4.3. Messfehler des Anstellwinkels bei Nickversuchen . . . . . . . . . 203

B.4.4. Messfehler der Schlagbewegung . . . . . . . . . . . . . . . . . . 204

$\begin{array}{ll}\text { C. Ergänzungen zu Kapitel } 4 & 207\end{array}$

C.1. Diskrete Fourier-Transformation _. . . . . . . . . . . . . . . . 207

C.2. Welch-Methode . . . . . . . . . . . . . . . . . . . . . 209

C.3. Schätzung der Übertragungsfunktion . . . . . . . . . . . . . . . . . . 210

C.4. Interpolation der Profilkontur und Druckverteilung . . . . . . . . . . . . 210

C.5. Diskretisierte Berechnung der aerodynamischen Kräfte . . . . . . . . . . 212

C.6. Rekonstruktion der Modellbewegung . . . . . . . . . . . . . . . . . . 213

C.7. Diskretisierte Berechnung des Energieeintrags . . . . . . . . . . . . . . . 214 
C.8. Vorzeichenkonvention bei Phasenverschiebungen . . . . . . . . . . . . . . 214

$\begin{array}{ll}\text { D. Ergänzungen zu Kapitel } 5 & 217\end{array}$

D.1. Zu 5.4: Instationäre Effekte bei statischen Anstellwinkeln . . . . . . . . 217

$\begin{array}{ll}\text { E. Ergänzungen zu Kapitel } 6 & 227\end{array}$

E.1. Zu 6.1: Instationäre Derivativa der aerodynamischen Beiwerte . . . . . . 227

E.2. Zu 6.2: Energiebetrachtung . . . . . . . . . . . . . . . . . . 230

E.3. Zu 6.3: Instationäres Druckverhalten . . . . . . . . . . . . . . . . . 231

E.4. Zu 6.4: Instationäres Transitionsverhalten . . . . . . . . . . . . . . . 237

$\begin{array}{ll}\text { F. Ergänzungen zu Kapitel } 7 & 243\end{array}$

F.1. Dämpfungen der aeroelastischen Konfigurationen . . . . . . . . . . . . 243

F.2. Schwingungsmoden der aeroelastischen Konfigurationen . . . . . . . . 246

F.3. Windkanalresonanzen . . . . . . . . . . . . . . . . . . . . . 248

F.4. Zu 7.3.3: Einfluss des Anstellwinkels . . . . . . . . . . . . . . . . . . 249

F.5. Zu 7.4.1: Verhalten von Stoß und Transition . . . . . . . . . . . . . . . 250

F.6. Zu 7.4.2: Energie- und Leistungsbetrachtung . . . . . . . . . . . . . . . . 251 


\section{Einleitung}

Grenzschichten und die damit verbundenen physikalischen Vorgänge sind für die Strömungsmechanik von essentieller Bedeutung. Sie entwickeln sich an der Oberfläche umströmter Körper und bilden einen der „Grundpfeiler der modernen Strömungsforschung" [1, S. XXII]. Der Übergang von einer laminaren zu einer turbulenten Grenzschicht, die laminar-turbulente Grenzschichttransition, ist dabei einer der fundamentalsten Vorgänge der Strömungsmechanik.

In einer transsonischen Strömung kommt es zu einer Interaktion von Verdichtungsstößen mit der Grenzschicht. Die Auswirkungen der Stoß-Grenzschicht-Interaktionen hängen maßgeblich vom Zustand der Grenzschicht ab [2]. Bei einer laminaren bzw. transitionellen Grenzschicht führt eine solche Wechselwirkung nahezu immer zu einer Ablösung [3]. Diese kann sich entweder wieder an den Körper anlegen und eine Ablöseblase bilden oder zu einem vollständigen aerodynamischen Strömungsabriss führen [4].

Die drei aerodynamischen Komponenten: Grenzschicht samt Grenzschichttransition, Verdichtungsstöße und Ablösungen sind dabei untereinander verknüpft und beeinflussen sich gegenseitig. Solche transsonischen Strömungsfelder sind inhärent nichtlinear [5-7] und zumeist auch instationär. Sowohl die Lage und Stärke von Verdichtungsstößen, die Position und Ausdehnung des laminar-turbulenten Grenzschichtumschlags sowie die Topologie von Ablösestrukturen können sich dynamisch verändern oder eine eigene Dynamik entwickeln. Dies wirkt sich auf die aerodynamischen Kräfte aus, die auf einen umströmten Körper wirken, wenn er sich in einem solchen komplexen Strömungsfeld befindet. Schematisch ist dies in Abb. 1.1 (links) am Beispiel eines Flügelprofils veranschaulicht.
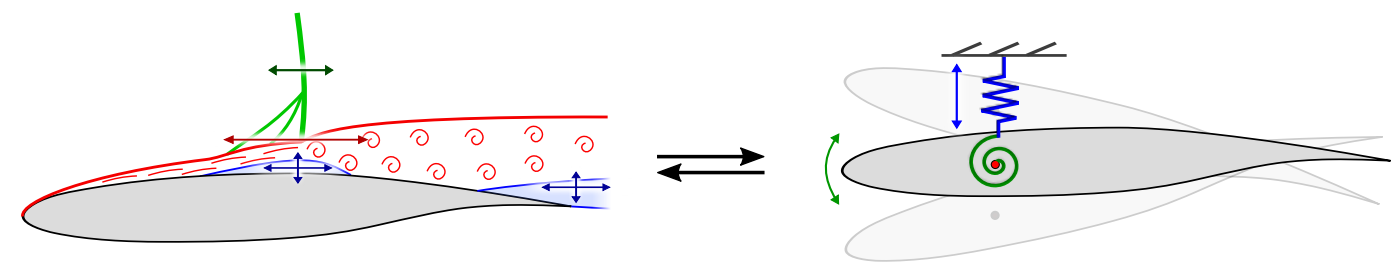

Abbildung 1.1.: Schematische Darstellung der Wechselwirkung eines transsonischen Strömungsfeldes (links) samt den drei Komponenten Verdichtungsstoß (grün), Ablösung (blau) und Grenzschicht (rot), mit einer elastischen Struktur (rechts) am Beispiel eines Flügelprofils. 
Umströmte Körper sind zumeist elastisch, wie in Abb. 1.1 (rechts) dargestellt. Statische und dynamische Deformationen bewirken eine Änderung des Strömungsfeldes, welches wiederum mit geänderten aerodynamischen Kräften auf die Struktur einwirkt. Derartige aeroelastische Wechselwirkungen können zu einem Energietransfer aus der Strömung in die Struktur führen. Selbsterregte, als Flattern bezeichnete Schwingungen können auftreten und zu „destruktiven strukturellen Instabilitäten mit katastrophalen Folgen führen“ [8, S.399]. In transsonischer Strömung sind solche aeroelastischen Instabilitäten infolge des Transonic Dips kritischer [9], da die aeroelastische Stabilitätsgrenze herabgesetzt ist.

Ein Zusammenspiel all dieser Komponenten tritt bei Flügelprofilen in transsonischer Strömung auf, wo eine laminare bzw. transitionelle Grenzschicht eine essentielle Rolle spielt. Laminare Grenzschichten weisen einen erheblich kleineren Reibungsbeiwert auf als turbulente Grenzschichten [1, S.160]. Dieser Aspekt wird bei Laminarprofilen ausgenutzt, um einen möglichst geringen Profilwiderstand zu erzielen. Eine spezielle Formgebung dieser Flügelprofile erhöht den Abstand zwischen Staupunkt und Druckminimum [10], woraus ein ausgedehnter Bereich mit einem negativen Druckgradienten resultiert. Dieser begrenzt das Wachstum von Störungen [11] und bewirkt eine Stabilisierung der laminaren Grenzschicht. Die laminar-turbulente Grenzschichttransition wird verzögert. Unter spezifischen Randbedingungen, insbesondere für passende Anstellwinkelbereiche und Reynolds-Zahlen werden so lange laminare Laufängen und damit geringere Profilwiderstände erzielt [1, S.453 f.]. Jedoch beschränkt sich der geringe Widerstand auf einen ausgewiesenen Bereich, der im Polarendiagramm als charakteristische Laminardelle ersichtlich ist. Gerade in den Übergängen der Laminardelle kann es zu ausgeprägten Bewegungen des laminar-turbulenten Grenzschichtumschlags kommen [12-14]. Diese können wie zuvor erläutert zu einer Änderung des gesamten Strömungsfeldes führen und aeroelastische Instabilitäten sowie unerwartete Flattermechanismen zur Folge haben.

Auch wenn die allgemeinen aerodynamischen Aspekte von Laminarprofilen gut verstanden sind [15], so sind aeroelastische Auswirkungen und die instationäre Aerodynamik einer laminaren bzw. transitionellen Strömung kaum erforscht [16]. Insbesondere bei transsonischer Strömung ist das physikalische Verständnis der Phänomene lückenhaft, obwohl es für die Bewertung möglicher Instabilitäten von essentieller Bedeutung ist.

\subsection{Motivation und Zielsetzung der Arbeit}

Ausgangspunkt der vorliegenden Arbeit sind Windkanalexperimente [12,13, 17-19] und begleitende numerische Studien [20-23] zum Einfluss der laminar-turbulenten Grenzschichttransition auf das aeroelastische Verhalten eines superkritischen CAST 10-2 Laminarprofils. Untersuchungen fanden bei subsonischen sowie transsonischen Strömungen, 
Reynolds-Zahlen von $\operatorname{Re}_{\infty} \approx 2 \cdot 10^{6}$ und bei freier und fixierter Grenzschichttransition statt. Der zugrunde liegende Transitionsmechanismus ist vorwiegend einer TollmienSchlichting-Instabilität zuzuordnen. Bei freier Grenzschichttransition wurden ausgeprägte Nichtlinearitäten in der stationären und instationären Aerodynamik nachgewiesen, lokalisiert bei Anstellwinkeln im oberen Übergangsbereich der auftretenden Laminardelle. In einem ersten Flatterversuch wurde ferner die aeroelastische Stabilität des CAST 10-2 Laminarprofils mit den zwei Starrkörper-Freiheitsgraden Schlag und Drehung direkt untersucht [24, 25]. Im Vergleich zu einer turbulenten Grenzschicht traten aeroelastische Instabilitäten bei freier Grenzschichttransition bereits bei kleineren Mach-Zahlen und Ruhedrücken auf. Eine Verschiebung des Transonic Dips wurde auch numerisch beobachtet $[20,22,23]$. Darüber hinaus wurde im Experiment unter spezifischen Randbedingungen und einer laminaren bzw. transitionellen Grenzschichtströmung ein unerwarteter und neuartiger Flattermechanismus an diesem Profil beobachtet.
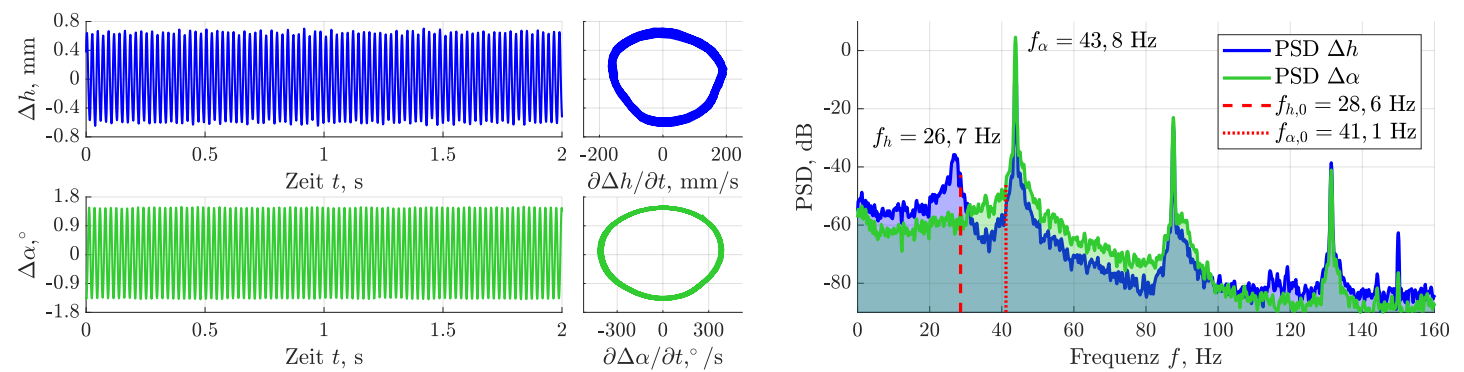

(a) Grenzzyklusschwingung des Laminarprofils bei $\mathrm{Ma}_{\infty}=0,75, p_{0}=50 \mathrm{kPa}$ und $\bar{\alpha}=0,8^{\circ}$

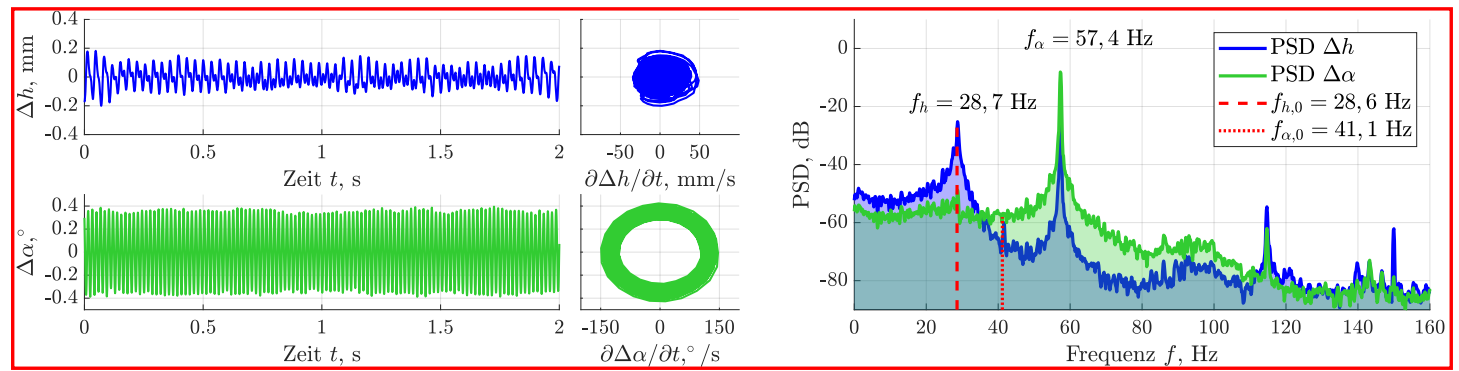

(b) Grenzzyklusschwingung des Laminarprofils bei $\mathrm{Ma}_{\infty}=0,75, p_{0}=50 \mathrm{kPa}$ und $\bar{\alpha}=0,0^{\circ}$

Abbildung 1.2.: Beispielhafte Flatterfälle, welche im Rahmen des ersten Flatterversuchs mit dem CAST 10-2 Laminarfügel [24] bei freier Grenzschichttransition und mittleren Anstellwinkeln von $\bar{\alpha}=0,8^{\circ}$ (a) und $\bar{\alpha}=0,0^{\circ}$ (b) beobachtet wurden.

Abb. 1.2 zeigt die Zeitreihen, Phasenraumdarstellungen und Leistungsdichtespektren der instationären Schlag- und Drehbewegung $\Delta h$ und $\Delta \alpha$ des Laminarflügelmodells für zwei beispielhafte Flatterfälle. Der in Abb. 1.2(a) dargestellte Fall korrespondiert mit einem mittleren Anstellwinkel von $\bar{\alpha}=0,8^{\circ}$. Es treten Grenzzyklusschwingungen des Schlag- und Dreh-Freiheitsgrades mit einer, in der Nähe der Dreh-Eigenfrequenz $f_{\alpha, 0}$ liegenden Flatterfrequenz von $f_{\alpha}=43,8 \mathrm{~Hz}$ auf. Das durch die Drehung und deren Eigenfrequenz dominierte Flatterverhalten weist Analogien zu früheren Flatterversuchen 
am superkritischen NLR 7301 Profil und den dort beobachteten, zumeist jedoch schlagdominierten Flatterfällen auf [26-28]. Die Flatterfrequenzen lagen in diesen Fällen in der Nähe der Schlag-Eigenfrequenz $f_{h, 0}$.

Ein abweichendes Verhalten zeigt hingegen der in Abb. 1.2(b) dargestellte und rot umrandete Flatterfall, welcher bei $\bar{\alpha}=0,0^{\circ}$ im oberen Übergangsbereich der Laminardelle beobachtet wurde. Das Flatterverhalten tritt annähernd als ein Ein-Freiheitsgrad-Flattern der Drehung in Erscheinung, wobei Grenzzyklusschwingungen mit im Vergleich zu Abb. 1.2(a) deutlich geringeren Amplituden auftreten. Die Flatterfrequenz liegt mit $f_{\alpha}=57,4 \mathrm{~Hz}$ deutlich oberhalb von $f_{\alpha, 0}$, entspricht jedoch der doppelten SchlagEigenfrequenz. Hieraus ergab sich die Hypothese, dass höhere Harmonische des SchlagFreiheitsgrades an dem Flattermechanismus beteiligt sind und dieser im Zusammenhang mit einer instationären Bewegung der Grenzschichttransition steht.

Aus diesem Flatterfall entstand die Motivation zur vorliegenden Arbeit. Zielsetzung ist es, auf Basis experimenteller Untersuchungen eine genauere Charakterisierung und Identifizierung des angesprochenen Flattermechanismus durchzuführen. Im Detail ergeben sich folgende Punkte, welche im Rahmen der Arbeit untersucht werden:

- Untersuchung der stationären und instationären Aerodynamik bei Randbedingungen, unter denen der neuartige Flatterfall beobachtet wurde. Ausgehend von den Beobachtungen des ersten Flatterversuches [24] leitet sich ein relevanter MachZahl-Bereich von $0,73 \lesssim \mathrm{Ma}_{\infty} \lesssim 0,75$ ab. Schwerpunktmäßig schließt dies eine Untersuchung der stationären und instationären aerodynamischen Kräfte sowie eine Identifizierung des Verhaltens der Grenzschichttransition und der Druckverteilung bei laminarer bzw. transitioneller Grenzschichtströmung ein.

- Genauere Abgrenzung und Charakterisierung des neuartigen Flatterverhaltens im Transonic Dip des CAST 10-2 Laminarprofils und Klärung des Einflusses von Mach-Zahl Ma $\mathrm{\infty}_{\infty}$ und mittlerem Anstellwinkel $\bar{\alpha}$.

- Abklärung des Einflusses des Schlag-Starrkörper-Freiheitsgrades und einer möglichen Beteiligung von höheren Harmonischen an dem Flattermechanismus.

- Identifizierung der instationären Aerodynamik während der aeroelastischen Instabilität, insbesondere Auflösung des Verhaltens der laminar-turbulenten Grenzschichttransition und der Stoß-Grenzschicht-Interaktion.

- Abklärung der Rolle der laminar-turbulenten Grenzschichttransition, insbesondere deren Einfluss auf den Flattermechanismus und die aeroelastische Stabilität. 


\subsection{Stand der Forschung}

Strömungs-Struktur-Wechselwirkungen im Zusammenhang mit laminaren bzw. transitionellen Grenzschichtströmungen wurden bislang in nur wenigen Untersuchungen thematisiert. Zumeist traten während Windkanalversuchen spontane Schwingungen an Flügelstrukturen auf, die nur bei laminaren Grenzschichten beobachtet wurden.

VAN DE VOOREN und Bergh [29] berichten von spontanen Oszillationen eines torsionselastisch gelagerten Flügelprofils während eines subsonischen Windkanalversuches bei geringen Reynolds-Zahlen $\operatorname{Re}_{\infty} \approx 2 \cdot 10^{5}$. Es traten bei laminarer Grenzschichtströmung Schwingungen mit kleinen Amplituden und Frequenzen im Bereich $60 \mathrm{~Hz}$ bis $300 \mathrm{~Hz}$ auf, die bei einer turbulenten Grenzschicht nicht beobachtet wurden. Die Instabilitäten wurden auf einen selbsterregten Rückkopplungs-Mechanismus zwischen der Strukturschwingung und hierdurch angeregten Instabilitäten der laminaren Grenzschicht zurückgeführt.

POIREL ET AL. [30-33] (siehe auch [34-38]) untersuchten experimentell sowie numerisch das dynamische Verhalten eines elastisch gelagerten NACA 0012 Profils bei transitionellen Reynolds-Zahlen ${ }^{1}$. Aeroelastische Grenzzyklusschwingungen wurden für einen Reynolds-Zahl-Bereich von $5 \cdot 10^{4} \lesssim \operatorname{Re}_{\infty} \lesssim 1,5 \cdot 10^{5}$ beobachtet. Diese wurden auf eine negative aerodynamische Dämpfung infolge von Ablösungen der laminaren Grenzschicht zurückgeführt. Bei einer Fixierung der Transition oder bei einem zu großen Turbulenzgrad verschwand die, als laminares Ablöseflattern (engl.: laminar separation flutter) [31] bezeichnete aeroelastische Instabilität. BARNES und VISBAL [39-43] führten hierzu implizite Large-Eddy-Simulationen (ILES) mit einer Strömungs-Struktur-Kopplung bei $7,7 \cdot 10^{4} \lesssim \operatorname{Re}_{\infty} \lesssim 2 \cdot 10^{5}$ durch. Diese bestätigten eine negative aerodynamische Dämpfung durch eine laminare Ablöseblase. Insgesamt wurden die Grenzzyklusschwingungen auf die laminar-turbulente Grenzschichttransition und deren instationäres Verhalten, insbesondere die zeitliche Verzögerung zwischen Modellbewegung, Transition und Antwort des übrigen Strömungsfeldes, zurückgeführt. Ferner wurde für Reynolds-Zahlen von $\operatorname{Re}_{\infty}=1,5 \cdot 10^{5}$ und $\operatorname{Re}_{\infty}=2 \cdot 10^{5}$ ein subkritisches Bifurkationsverhalten beobachtet. Mithilfe von Plasma-Aktuatoren konnte die Transition und somit das laminare Ablöseflattern kontrolliert und vollständig unterdrückt werden [44].

Bei größeren Reynolds-Zahlen von $\operatorname{Re}_{\infty} \approx 1 \cdot 10^{6}$ und transsonischen Strömungen beobachteten HouwINK ET AL. [45] eine zusätzliche aeroelastische Instabilität an einem superkritischen 3D-Flügelmodell im Rahmen eines Windkanalversuches. Bei Ruhedrücken von $p_{0}=45 \mathrm{kPa}$ fiel die Dämpfung der Flügelbiegung auf Null, was mit einer EinFreiheitsgrad-Schwingung des Modells in Verbindung gebracht wurde. Diese wurde auf eine variable Position der laminar-turbulenten Grenzschichttransition und deren Einfluss

\footnotetext{
${ }^{1}$ Reynolds-Zahlen bei denen ein substantieller Anteil einer laminaren Strömung vorliegt.
} 
auf die instationären aerodynamischen Kräfte zurückgeführt. Die Transitionsfixierung verlor bei den geringen Ruhedrücken ihre Wirkung. HouwINK ET AL. [45] merkten an, dass „diese Instabilität nicht aufgetreten wäre, wenn der Transitionsstreifen bei allen Strömungsbedingungen wirksam gewesen wäre“.

Motiviert durch ein ungewöhnliches Flatterverhalten eines Raumfahrzeug-Flügel-Konzepts [46], führte ERICKSON [47] transsonische Flatterversuche an einem 3D-Flügelmodell mit geringer Streckung durch. Mit einer Transitionsfixierung traten Grenzzyklusschwingungen in einem schmalen Mach-Zahl-Bereich auf, die durch die erste Modell-Eigenmode und deren Frequenz dominiert wurden. Bei freier Grenzschichttransition setzte Flattern erst bei leicht höheren Mach-Zahlen ein, jedoch mit einer vermeintlichen Instabilität der zweiten Eigenmode. In beiden Fällen wurden die Flattermechanismen auf eine instationäre Stoß-Grenzschicht-Interaktion und Ablösung zurückgeführt, die jedoch durch die verschiedenen Grenzschichtzustände zu verschiedenen Flatterfällen führte.

Ebenfalls in transsonischer Strömung beobachteten MABEY ET AL. [48] Schwingungen der ersten Biegemode eines 3D-Flügelmodells. Nahe der Flügel-Vorderkante beeinflussten MABEy ET AL. den Grenzschichtzustand durch Ausblasen von Luft in die Grenzschicht. Dies ermöglichte eine Steuerung der Grenzschichtzustände. Maximale Schwingungsamplituden traten bei einer Reynolds-Zahl von $\operatorname{Re}_{\infty} \approx 6 \cdot 10^{6}$ bzw. einer transitionellen Grenzschicht (mäßiges Ausblasen) auf. Bei einer laminaren Grenzschicht (kein Ausblasen) waren die Amplituden geringer. Mit fixierter Transition (maximales Ausblasen) traten keine aeroelastischen Instabilitäten auf. Diese wurden auf eine oszillatorische Bewegung der Grenzschicht-Transition und eine damit verbundene Reduzierung der aerodynamischen Dämpfung zurückgeführt. Eine quasi-stationäre Überlegung hierzu [48] wurde durch ERICSSON [49] kritisiert. ERICSSOn [50] führte eine Kopplung von Strukturbewegung und laminar-turbulenter Grenzschichttransition auf den Einfluss des Druckgradienten und Verzögerungseffekte in instationärer Strömung zurück. Als wichtigsten Faktor benannte ERICSSON jedoch den Einfluss von bewegten Wänden (vgl. auch [51]). ERICSSON's Begründungen [50] wurden wiederum von MABEY und AsHill [52] als „mangelhaft" beanstandet. Eine abschließende Klärung der Sachverhalte blieb aus.

Erste transsonische Flattermessungen an einem 2D-Modell mit einem superkritischen NLR 7301 Profil und den zwei Freiheitsgraden Schlag und Drehung führten ScHEwE und Deyhle [53] bei Reynolds-Zahlen von $\operatorname{Re}_{\infty} \approx 2 \cdot 10^{6}$ durch. Zielsetzung dieser Messungen war hauptsächlich die Untersuchung von nichtlinearen Effekten. Bei freier Grenzschichttransition wurden im Bereich des Minimums des Transonic Dips verschiedene Arten von Flatterschwingungen mit unerwartet kleinen Amplituden beobachtet. U. a. traten Grenzzyklusschwingungen der Drehung auf. Eine vergleichbare Instabilität wurde in einer zweiten Messung auch bei einer fixierten Transition gefunden, jedoch 
unter anderen Randbedingungen [26]. Bei einem weiteren Flatterversuch mit nur einem Starrkörper-Drehfreiheitsgrad [54] wurden Grenzzyklusschwingungen wider Erwarten nur bei einer fixierten Transition beobachtet. Mit freier Transition traten hingegen keine Instabilitäten auf. Schwingungen der Position der Grenzschicht wurden daher als Ursache für die Grenzyklusschwingungen ausgeschlossen [54]. In einem Folgeexperiment [26] mit den zwei Freiheitsgraden Schlag und Drehung wurde wiederum bei freier Transition eine Grenzzyklusschwingung beobachtet. Dieser Flatterfall „MP77“ wurde international als Benchmark-Test für numerische Simulationen genutzt. Jedoch zeigte sich, dass die berechneten Grenzzyklusamplituden deutlich zu groß waren. Die Rechnungen wurden mit einer fixierten Transitionslage durchgeführt. So ist als Ursache dieser Abweichung zu vermuten, dass man sich des Einflusses der laminar-turbulenten Grenzschichttransition bzgl. aeroelastischer Instabilitäten noch nicht bewusst war.

Eine erste systematische Untersuchung zum Einfluss laminarer Grenzschichtströmungen bzw. des laminar-turbulenten Grenzschichtumschlags auf aeroelastische Instabilitäten ist weitestgehend auf eine langfristige Strategie des Instituts für Aeroelastik am Deutschen Zentrum für Luft- und Raumfahrt e.V. (DLR) [16] zurückzuführen. In diesem Rahmen beobachteten Mai und Hebler [12] sowie Hebler et Al. [13] (siehe auch [17-19]) in Windkanalversuchen ausgeprägte Nichtlinearitäten in der stationären und instationären Aerodynamik eines superkritischen CAST 10-2 Laminarprofils für $\operatorname{Re}_{\infty} \approx 2 \cdot 10^{6}$. Die Nichtlinearitäten konzentrierten sich auf Anstellwinkel im oberen Übergangsbereich der Laminardelle und lagen nur bei freier Grenzschichttransition vor. Fenrs [20] sowie Fehrs ET AL. [21,23] konnten die Ergebnisse mit Reynolds-gemittelten Navier-Stokes-Rechnungen (engl.: Reynolds Averaged Navier-Stokes, RANS) und einem $\gamma-\operatorname{Re}_{\Theta}$ Transitionsmodell qualitativ nachbilden. Die instationären Rechnungen zeigten für $\mathrm{Ma}_{\infty}=0,72$ und $\mathrm{Ma}_{\infty}=0,74$ aerodynamische Resonanzen bei Anstellwinkeln inmitten der Übergangsregion der Laminardelle [23]. Für $\mathrm{Ma}_{\infty}=0,73$ trat eine Instationarität der Transitionsposition auf. Die numerischen Ergebnisse wiesen hier auf eine aerodynamische Schwingung hin, es stellte sich jedoch kein stabiles Strömungsfeld ein. Rechnungen mit einem neu entwickelten $\gamma$-Transitionsmodell $[55,56]$ zeigten später auch aerodynamische Resonanzen bei $\mathrm{Ma}_{\infty}=0,73$ [57]. Im Experiment deutete sich eine aerodynamische Resonanz hingegen bei $\mathrm{Ma}_{\infty}=0,75$ an [13]. Für die übrigen Mach-Zahlen lagen keine experimentellen Ergebnisse vor.

Fehrs Et AL. [21,23] beobachteten ferner eine Phasenvoreilung des Nickmoments gegenüber der Drehbewegung des Flügelprofils, was auf ein mögliches Ein-FreiheitsgradFlattern hinweist. Die Voreilung trat bei freier Grenzschichttransition und $\mathrm{Ma}_{\infty}=0,72$ auf, jedoch für mittlere Anstellwinkel außerhalb des Übergangsbereiches der Laminardelle. Eine Voreilung des Nickmoments wurde im Experiment bei ähnlichen Anstellwinkeln für 
$\mathrm{Ma}_{\infty}=0,75$ beobachtet [13]. Für Anstellwinkel im Übergangsbereich der Laminardelle lag jedoch eine Phasennacheilung vor.

Nach numerischen Vorbereitungen [58] führte HEBLER [24] des Weiteren eine direkte Untersuchung der aeroelastischen Stabilität des CAST 10-2 Laminarflügels durch. Im Rahmen eines Flatterversuches mit den Starrkörper-Freiheitsgraden Schlag und Drehung bestimmte Hebler die Flattergrenzen bei freier und fixierter Transition. Die Strömungsbedingungen waren mit den vorherigen Versuchen [12,13,17-19] vergleichbar. Es wurde eine Verschiebung des Transonic Dips hin zu kleineren Mach-Zahlen und Ruhedrücken nachgewiesen, wenn eine laminare bzw. transitionelle Grenzschichtströmung vorliegt. Eine solche Verschiebung trat auch in numerischen Rechnungen von FeHrs [20], FEHRS ET AL. [23] und van Roolj und Wegner [22] auf. Ferner beobachtete Hebler in Abhängigkeit vom mittleren Anstellwinkel und dem Grenzschichtzustand verschiedene Typen von Flatterfällen. Dabei trat bei einer freien Grenzschichttransition der in 1.1 dargestellte und in der vorliegenden Arbeit thematisierte Flatterfall erstmalig in Erscheinung.

Ergebnisse, die im Rahmen der vorliegenden Arbeit entstanden und veröffentlicht wurden [14,59-63], sind jeweils in den folgenden Kapiteln zitiert und hier nicht gesondert aufgeführt. Es sei jedoch noch erwähnt, dass jüngste Resultate von ILES-Rechnungen durch Barnes und Visbal [64,65], die durch Ergebnisse [59,62,63] dieser Arbeit motiviert wurden, das komplexe Stoß-Grenzschichtverhalten des CAST 10-2 Profils qualitativ gut wiedergeben konnten. Die Rechnungen zeigten bei zur vorliegenden Arbeit ähnlichen Randbedingungen eine aerodynamische Resonanz sowie ausgeprägte Schwingungen der laminar-turbulenten Grenzschichttransition. Ferner wurde ein positiver Energieeintrag nachgewiesen, der auf eine aeroelastische Instabilität hindeutet. Bei einer Fixierung der Transition mithilfe von Plasma-Aktuatoren verschwanden die Effekte [65].

\subsection{Gliederung und wesentliche Ergebnisse der Arbeit}

Die vorliegende Arbeit befasst sich mit der experimentellen Untersuchung der stationären und instationären Aerodynamik sowie des aeroelastischen Verhaltens eines superkritischen CAST 10-2 Laminarflügelprofils bei Reynolds-Zahlen von $\operatorname{Re} \approx 2 \cdot 10^{6}$ unter transsonischer Strömung. Hierfür werden Windkanaluntersuchungen mit zwangserregten Nickschwingungen und selbsterregten Flatterschwingungen durchgeführt.

Kapitel 2 beginnt mit den physikalischen Grundlagen, die für das Verständnis der Arbeit wichtig sind. So werden die in der Einleitung 1 angesprochenen Zusammenhänge näher erläutert, insbesondere die laminar-turbulente Grenzschichttransition und Grundlagen der Aeroelastik. 
In Kapitel 3 sind die Komponenten des experimentellen Aufbaus wie der Windkanal, die Versuchsstände und die eingesetzten Messtechniken beschrieben. Ferner sind Details zum superkritischen CAST 10-2 Laminarflügelprofil aufgeführt.

In Kapitel 4 sind die Methodiken beschrieben, die zur Auswertung und Analyse der Messergebnisse eingesetzt werden. Unter anderem wurde im Rahmen dieser Arbeit eine Auswertungsmethodik entwickelt, die es erstmalig erlaubt, den laminar-turbulenten Grenzschichtumschlag in instationären, transsonischen Strömungen mithilfe der HeißfilmAnemometrie genauer zu untersuchen. Die Dynamik der Position der Grenzschichttransition kann hiermit in einem bisher nicht vorhandenen Umfang aufgelöst und einer näheren Analyse zugeführt werden.

Kapitel 5 befasst sich dann mit den experimentellen Ergebnissen der stationären Aerodynamik des Laminarprofils (Messungen bei statischen Anstellwinkeln). Durch den Einsatz eines neuen Versuchsstands wurden Messungen erstmalig bei Mach-Zahlen von $\mathrm{Ma}_{\infty}=0,73$ und $\mathrm{Ma}_{\infty}=0,74$ durchgeführt. In vorherigen Untersuchungen $[12,13]$ war dies wegen unkontrollierbaren Modellschwingungen nicht möglich. So wurden erste Hinweise einer Instationarität der Lage der laminar-turbulenten Grenzschichttransition im Übergangsbereich der Laminardelle identifiziert. In diesem Bereich wurden ferner erstmalig aerodynamische Grenzzyklusschwingungen experimentell nachgewiesen. Numerische Rechnungen [23] deuteten dies bereits an. Die selbsterregten Schwingungen der Aerodynamik wurden im Wesentlichen auf Oszillationen der Wandschubspannung bzw. auf Bewegungen der Grenzschichttransition zurückgeführt.

Kapitel 6 umfasst die Auswertung von zwangserregten Nickschwingungen des Laminarflügelprofils. Schwerpunktmäßig wird die instationäre Aerodynamik bei einer Variation der Schwingungsfrequenz analysiert und im Hinblick auf aeroelastische Instabilitäten diskutiert. Auch hier wurden Messungen erstmalig bei $\mathrm{Ma}_{\infty}=0,73$ und $\mathrm{Ma}_{\infty}=0,74$ durchgeführt. Als ein wesentliches Ergebnis wurden in Abhängigkeit der Mach-Zahl, des mittleren Anstellwinkels und der Schwingungsfrequenz Bereiche identifiziert, in denen die Strömung Energie in die Struktur transferiert. Diese Bereiche hängen mit dem Übergangsbereich der Laminardelle sowie einer freien Grenzschichttransition zusammen und lassen einen direkten Rückschluss auf aeroelastische Instabilitäten zu. Das instationäre Verhalten der Druckverteilung und erstmalig auch das des Grenzschichtumschlags wurde aufgelöst. Es zeigte sich eine Voreilung der Transitionbewegung zur Bewegung des Verdichtungsstoßes. Die Transition wurde als notwendige Bedingung für das in 1.1 angesprochene Flatterverhalten identifiziert. Ferner wurden aerodynamische Resonanzen und sich hieran anschließende Bereiche gefunden, in denen der Strömung Energie zugeführt wird. Auch hier zeigte sich ein Zusammenhang zur freien Grenzschichttransition. 
In Kapitel 7 ist abschließend das aeroelastische Verhalten des Laminarprofils unter selbsterregten Flatterschwingungen dargelegt und diskutiert. Es wurde herausgearbeitet, dass der in 1.1 angesprochene Flatterfall sich weitestgehend auf Randbedingungen innerhalb des Übergangsbereiches der Laminardelle konzentriert. Das aeroelastische Systemverhalten lässt sich in Abhängigkeit der Mach-Zahl sowie des mittleren Anstellwinkels durch eine subkritische Bifurkation beschreiben. Eine freie Grenzschichttransition führt insgesamt nicht nur zu einer Verschiebung des Transonic Dips, wie in [20,22-24] beobachtet, sondern der Dip wird durch das unerwartete Flatterverhalten zusätzlich erweitert. Auf Basis der experimentellen Ergebnisse kann dieser Flattermechanismus als „Transitionsflattern" charakterisiert werden. Eine freie Grenzschichttransition wird als notwendige Bedingung hierfür bestätigt. Ferner konnte eine Beteiligung von höheren Harmonischen des Schlag-Freiheitsgrades ausgeschlossen werden. Abschließend wurde die instationäre Aerodynamik des Laminarprofils während des Übergangs von einem aerodynamischen in einen aeroelastischen Grenzzyklus analysiert. Hierbei gelang erstmalig die experimentelle Auflösung der zeitlichen Bewegung der Lage des laminar-turbulenten Grenzschichtumschlags sowie der auftretenden Stoß-Grenzschicht-Interaktion. Es zeigte sich erneut eine Voreilung der Transitions- zur Stoßbewegung sowie eine Steuerung der Stoßposition durch die Transition. Die in diesem Rahmen beobachteten Instationaritäten des Strömungsfeldes deuten dabei auf die Existenz eines „Transitionsbuffets“ hin. Dies wirft die Hypothese auf, dass eine freie Grenzschichttransition nicht nur notwendig für das „Transitionsflattern“ ist, sondern eine Instationarität der Position des laminar-turbulenten Grenzschichtumschlags auch die Ursache für das „Transitionsflattern“ darstellt.

Die Ergebnisse der Arbeit werden in Kapitel 8 nochmals zusammenfassend diskutiert und offene Fragen angeführt. Ein Ausblick enthält Anregungen für weiterführende Untersuchungen und einen experimentellen Lösungsansatz zur möglichen Klärung der zuvor angeführten Hypothese.

Die Arbeit wird durch einen umfangreichen Anhang ergänzt. Dieser beinhaltet weitere Informationen und Abbildungen. Darüber hinaus sind Berechnungen und Voruntersuchungen ausführlich dokumentiert, um nachfolgenden Generationen einen direkten Zugang zu den eingesetzten Verfahren und Methodiken zu ermöglichen. 


\section{Physikalische Grundlagen}

Das folgende Kapitel liefert eine Übersicht über die physikalischen Grundlagen und Konzepte, welche mit der Thematik der vorliegenden Arbeit in Verbindung stehen. Für eine detailliertere Darstellung einzelner Teilgebiete sei auf die im Folgenden genannte Literatur verwiesen.

Das Kapitel gliedert sich in drei Teile. Zunächst wird die Grenzschichttransition unter Berücksichtigung der für die Arbeit relevanten Aspekte (natürliche Transition, Druckgradient und Laminarerhaltung, Stoß-Grenzschicht-Interaktion) behandelt. Ein weiterer Teil umfasst theoretische Informationen zur Wandschubspannung, die im Zusammenhang mit der Messtechnik (vgl. 3.4) und Datenanalyse (vgl. 4.3) stehen, und diskutiert die in der vorliegenden Arbeit verwendete Definition der Transition. Daran anschließend werden die aeroelastischen Aspekte, wie Gründzuge der instationären Aerodynamik, Flattern sowie aeroelastische Instabilitäten unter transonischer Strömung thematisiert.

\subsection{Laminar-turbulente Grenzschichttransition}

Mit der Einführung des Grenzschichtkonzepts [66] Anfang des 20. Jahrhunderts wurde gezeigt, dass sich bei geringer Viskosität (großen Reynolds-Zahlen) der Reibungseinfluss eines umströmten Körpers auf eine wandnahe Grenzschicht beschränkt. Innerhalb dieser besteht ein Geschwindigkeitsgradient, entsprechend einem Übergang der Strömungsgeschwindigkeit von null an der Wand (Haftbedingung) hin zur Geschwindigkeit der Außenströmung. Grenzschichtströmungen können eine laminare oder eine turbulente Struktur aufweisen, wobei Erstere infolge einer beginnenden Instabilität des Strömungsfeldes [67-70] im Rahmen einer laminar-turbulenten Grenzschichttransition in Letztere übergeht. Diese Instabilitäten sind wiederum das Resultat externer Störungen, worauf die Grenzschicht als hochkomplexer Schwinger mit nichtlinearer Charakteristik reagiert [69-73].

In Abb. 2.1 ist der Prozess der laminar-turbulenten Grenzschichttransition vereinfacht dargestellt. Es wird schnell ersichtlich, dass die physikalischen Transitions-Mechanismen 
vielzählig sind und von äußeren Randbedingungen, wie Art der Strömung und Eigenschaften der Störungen abhängen [74]. Eine wesentliche Rolle spielt dabei die Rezeptivität [69,71-73, 75-78]. Diese beschreibt die Interaktion der Grenzschicht mit den externen Störungen und bestimmt deren Anfangsbedingungen innerhalb der laminaren Grenzschicht, insbesondere deren Amplituden und spektralen Eigenschaften [68, 69, 78]. Die Rezeptivität ist somit maßgeblich entscheidend für die Transitions-Mechanismen.

Diese teilen sich im Wesentlichen in drei Gruppen auf. Bei schwachen externen Störungen verläuft die Transition gemäß dem Pfad A (roter Verlauf in Abb. 2.1), was anfangs durch die lineare Stabilitätstheorie der Primärmoden (vgl. 2.1.1) beschrieben wird [69, 78]. Diese natürliche Transition tritt unter realen Flugbedingungen sowie bei turbulenzarmen Windkanälen auf und stellt den für die vorliegende Arbeit bedeutendsten Mechanismus dar. Eine nähere Beschreibung erfolgt im nachfolgenden Abschnitt. Bei sehr großen ansteigendes Störungslevel / Anwachsen der Störamplitude

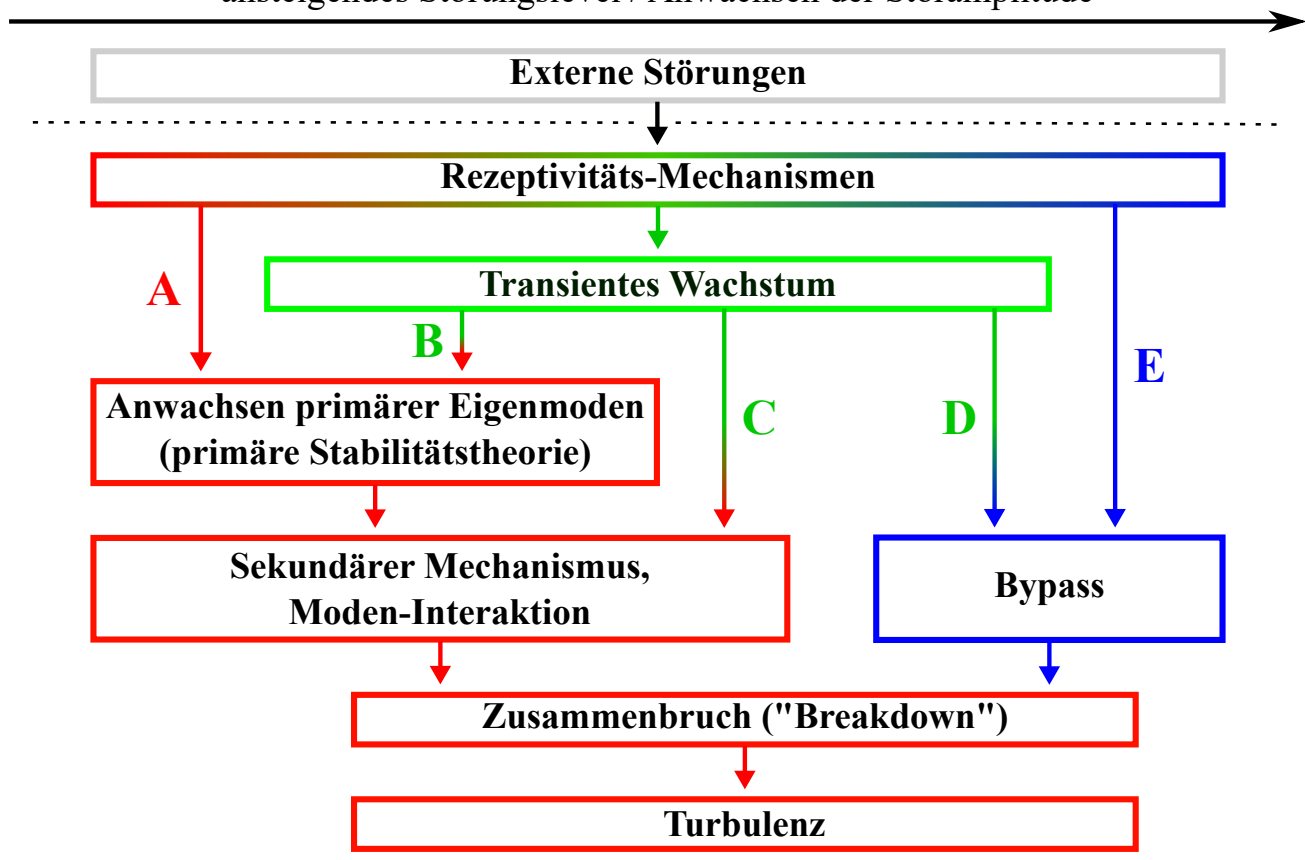

Abbildung 2.1.: Schematische Darstellung des laminar-turbulenten Transitionsprozesses und der unterschiedlichen Mechanismen A bis E, verändert nach [70,78,79].

Störungsamplituden wird das lineare Anwachsen von Primärmoden „übersprungen“ $[69,78]$ und es kommt zu einem direkten, nichtlinearen Zusammenbruch der laminaren Strömung [74]. Diese Bypass-Transition korrespondiert mit dem Pfad E (blauer Verlauf in Abb. 2.1). Einen Übergang von Typ A zu Typ E bilden die Transitions-Mechanismen infolge des transienten Wachstums (grüne Verläufe in Abb. 2.1). Das transiente Wachstum führt zu einer größeren Anfangsamplitude der Eigenmoden bzw. deren Wachstums infolge einer Kopplung und Interaktion von modalen und nichtmodalen Störungen (Pfad B) [70]. Bei Pfad C fehlt das Wachstum von Eigenmoden [70,80] und es kommt direkt zur Anregung von sekundären oder subkritischen Instabilitäten [69,78]. Transientes Wachstum 
vom Typ D führt zu einem vollständig ausgebildeten Störungsspektrum (ähnlich dem eines turbulenten Spektrums) [70,80], was eine Bypass-Transition zur Folge hat [69,78].

\subsubsection{Natürlicher Transitionsprozess und Primäre Stabilitätstheorie}

Wie bereits zuvor erwähnt ist der nach Pfad A (siehe roter Verlauf in Abb. 2.1) verlaufende natürliche Transitionsprozess (auch ToLLMIEN-SCHLichting-Übergang) von zentraler Bedeutung. Dies begründet sich zum einen darin, dass die natürliche Transition für Tragflügel (ohne Pfeilung) im Unterschall dominiert [1, S.485]. Zum anderem existiert eine weitestgehend analytische und im Folgenden skizzierte Beschreibung der primär einsetzenden Instabilitäten, woraus sich direkt Rückschlüsse auf die in 2.1.2 beschriebenen Aspekte der Laminarflügeltechnologie ableiten.

Abb. 2.2 stellt die laminar-turbulente Transition für eine zweidimensionale Grenzschichtströmung am Beispiel der ebenen Platte dar. Der natürliche Transitionsprozess teilt sich

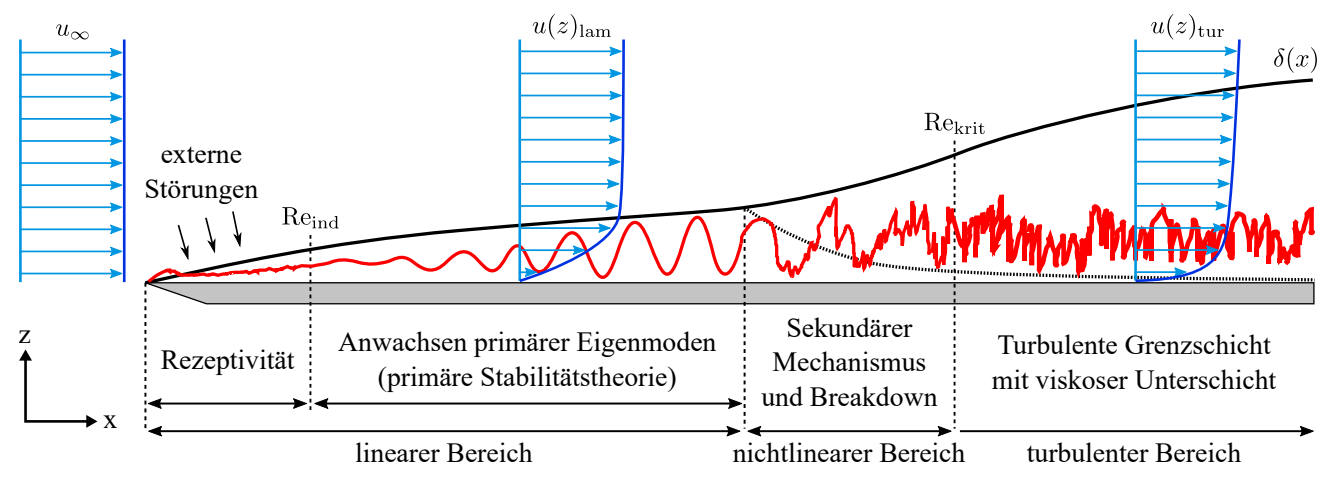

Abbildung 2.2.: Schematische Darstellung der laminar-turbulenten Grenzschichttransition an einer ebenen Platte, verändert nach $[1,74]$. Das Koordinatensystem orientiert sich an dem Windkanal-Koordinatensystem (vgl. 3.1), wie es in der vorliegenden Arbeit verwendet wird.

in einen linearen und einen nichtlinearen Abschnitt auf, welche die bereits in Pfad A in Abb. 2.1 dargestellten drei Hauptaspekte des Transitions-Vorgangs: Rezeptivität, lineare Stabilität und nichtlinearer Zusammenbruch hin zur Turbulenz beinhalten [74]. Wie bereits eingangs erwähnt interagieren externe Störungen mit der laminaren Grenzschicht über einen Rezeptivitäts-Mechanismus, was als Transformation der externe Störungen zu Eigenschwingungen der Grenzschicht verstanden werden kann [74]. Dieses umfasst unter anderem auch eine Art Anpassungsprozess der externen Stör-Wellenzahlen hinzu passenden Wellenzahlen der primären Instabilitäten [69,78], entsprechend der Bereitstellung der Anfangsbedingungen [68,69,78] der primären Eigenmoden.

Sind die primären Instabilitäten generiert, stellt sich die Frage ob diese angefacht oder gedämpft werden. Dies kann mithilfe der primären Stabilitätstheorie (für eine ausführliche Darstellung siehe [1, S.423 ff.] und [79,81]) weitestgehend analytisch beantwortet 
werden. Die grundlegende Idee dabei ist es, die ungestörte stationäre Grenzschichtströmung (Grundströmung) mit den Geschwindigkeitskomponenten $U, V$ und $W$ sowie dem Druck $P$ mit kleinen Störungen $u^{\prime}, v^{\prime}, w^{\prime}$ und $p^{\prime}$ additiv zu überlagern und diese hinsichtlich ihrer Stabilität zu untersuchen [68]. Zur vereinfachten Darstellung sei hierfür eine zweidimensionale inkompressible Grundströmung, überlagert mit einer ebenfalls zweidimensionalen Störungsbewegung angenommen. Des Weiteren wird eine Parallelströmungsannahme ${ }^{1}$ getroffen, womit die Geschwindigkeitskomponente $U$ nur von $z$ abhängt und für die übrigen Komponenten $V=W=0$ gilt. Aus der Superposition der Geschwindigkeitskomponenten und des Drucks folgt somit

$$
u=U(z)+u^{\prime}(x, z, t), \quad v=v^{\prime}(x, z, t), \quad w=0 \quad \text { und } \quad p=P(x, z)+p^{\prime}(x, z, t) .
$$

Es wird vorausgesetzt, dass Gl. (2.1.1) und auch die Grundströmung selbst eine Lösung der NAvieR-Stokes Gleichungen darstellen. Einsetzen von Gl. (2.1.1) in diese führt somit auf einen vereinfachten Satz an (linearen) partiellen Differentialgleichungen für die Störungen $u^{\prime}, v^{\prime}$ und $p^{\prime}$ gemäß

$$
\begin{aligned}
\frac{\partial u^{\prime}}{\partial x}+\frac{\partial v^{\prime}}{\partial z} & =0 \\
\frac{\partial u^{\prime}}{\partial t}+U \frac{\partial u^{\prime}}{\partial x}+v^{\prime} \frac{\mathrm{d} U}{\mathrm{~d} z} & =-\frac{1}{\rho} \frac{\partial p^{\prime}}{\partial x}+\nu \Delta u^{\prime} \\
\frac{\partial v^{\prime}}{\partial t}+U \frac{\partial v^{\prime}}{\partial x} & =-\frac{1}{\rho} \frac{\partial p^{\prime}}{\partial z}+\nu \Delta v^{\prime}
\end{aligned}
$$

mit dem Laplace-Operator $\Delta=\frac{\partial^{2}}{\partial x^{2}}+\frac{\partial^{2}}{\partial z^{2}}$ in zwei Dimensionen. In Gl. (2.1.3) und (2.1.4) wurden dabei alle quadratischen Terme der Störungen vernachlässigt, da die Störungen als klein gegenüber der Grundströmung angesehen werden.

Es wird nunmehr angenommen, dass die Lösungen dieses spezifischen Anfangs- bzw. Randwertproblems als Summe von Partialstörungen bzw. Normalmoden darstellbar sind, welche auf ihr Dämpfungsverhalten hin untersucht werden [79]. Für sich genommen ist eine solche Mode eine in Strömungsrichtung fortschreitende Welle (auch ToLLMiENSchlichting-Welle oder kurz TS-Welle), für deren Stromfunktion $\psi$ der Ansatz

$$
\psi(x, z, t)=\varphi(z) \cdot \exp [\mathrm{i}(k x-\omega t)]
$$

gemacht wird. Dabei ist $k \in \mathbb{R}$ die Wellenzahl und $\lambda=\frac{2 \pi}{k}$ die Wellenlänge der Störung. $\omega \in \mathbb{C}$ ist hingegen komplex, wobei $\operatorname{Re}[\omega]=\omega_{r}$ die Kreisfrequenz der Welle beschreibt

\footnotetext{
${ }^{1}$ Die Parallelströmungsannahme ist für eine Grenzschichtströmung näherungsweise erfüllt [1, S.424] bzw. entspricht einer ersten Approximation [79]. Der Einfluss von nicht-parallelen Effekten bzgl. der Stabilität von 2D-Störungen wird jedoch kontrovers diskutiert [74] und ist $u$. a. vom Druckgradienten abhängig [72]. Die Strömungsannahme erhält jedoch die essentiellen physikalischen Aspekte [81].
} 
und $\operatorname{Im}[\omega]=\omega_{i}$ als Dämpfung aufgefasst werden $\operatorname{kann}^{2} . \varphi(z)$ ist die Amplitudenfunktion der Störung. Darüber hinaus folgt aus $c=\frac{\omega}{k} \in \mathbb{C}$ die Wellengeschwindigkeit $\operatorname{Re}[c]=c_{r}$. $\operatorname{Im}[c]=c_{i}$ sagt wiederum etwas über die Dämpfung der Störung aus. Für $\omega_{i}<0$ bzw. $c_{i}<0$ ist die Welle gedämpft (stabile Strömung), wohingegen $\omega_{i}>0$ bzw. $c_{i}>0$ einer Anfachung der Welle entspricht (Instabilität der Strömung). Die Stromfunktion nach Gl. (2.1.5) ist nun gemäß

$$
\begin{aligned}
& u^{\prime}=\frac{\partial \psi}{\partial z}=\frac{\mathrm{d} \varphi}{\mathrm{d} z} \cdot \exp [\mathrm{i}(k x-\omega t)], \\
& v^{\prime}=-\frac{\partial \psi}{\partial x}=-\mathrm{i} k \varphi(z) \cdot \exp [\mathrm{i}(k x-\omega t)],
\end{aligned}
$$

mit den Störgeschwindigkeiten verbunden. Setzt man diese in Gl. (2.1.3) und (2.1.4) ein, so folgt nach einigen Umformungen die ORR-SOMMERFELD-Gleichung

$$
(U-c)\left(\frac{\mathrm{d}^{2} \varphi}{\mathrm{d} z^{2}}-k^{2} \varphi\right)-\frac{\mathrm{d}^{2} U}{\mathrm{~d} z^{2}} \varphi=-\frac{\mathrm{i}}{k \operatorname{Re}_{\delta}}\left(\frac{\mathrm{d}^{4} \varphi}{\mathrm{d} z^{4}}-2 k^{2} \frac{\mathrm{d}^{2} \varphi}{\mathrm{d} z^{2}}+k^{4} \varphi\right),
$$

hier dargestellt in der Literatur zumeist gebräuchlichen Form mit entdimensionalisierten Größen ${ }^{3}$. Hierbei handelt es sich um eine gewöhnliche Differentialgleichung 4. Ordnung der Störamplitude $\varphi(z)$. Die anfängliche Frage nach der Stabilität der laminaren Grenzschicht ist somit ein Eigenwertproblem mit der Eigenfunktion $\varphi(z)$ und dem Eigenwert $c$. Als Randbedingungen werden zumeist die Haftbedingung sowie ein Verschwinden der Störungen in der Außenströmung gefordert (für $z=0$ und $z=\infty: u^{\prime}=v^{\prime}=\varphi=\frac{\mathrm{d} \varphi}{\mathrm{d} z}=0$ ).

Die Stabilitätsanalyse sieht nun vor, dass zu einem gegebenen Geschwindigkeitsprofil der Grund- bzw. Laminarströmung $U(z)$ sowie zu einer vorgegebenen Wellenlänge $\lambda$ der Störung (bzw. der spezifischen Reynolds-Zahl $\operatorname{Re}_{\delta}$ und der Wellenzahl $k$ ) eine Eigenfunktion $\varphi(z)$ und ein Eigenwert $c$ zu Gl. (2.1.8) bestimmt wird. Aus der grafischen Auftragung von $c_{i}$ mit dem zugehörigen Wertepaar $\left(\operatorname{Re}_{\delta}, k\right)$ folgt die Indifferenzkurve für das gegebene Strömungsprofil $U(z)$. Dies ist in Abb. 2.3 für zwei unterschiedliche Geschwindigkeitsprofile qualitativ dargestellt. Die Wertepaare $\left(\operatorname{Re}_{\delta}, k\right)$ für welche $c_{i}=0$ gilt, bilden die Indifferenzkurve. Diese trennt den stabilen Bereich (gedämpfte Störungen, $c_{i}<0$ ) von dem instabilen Bereich (angefachte Störungen, $c_{i}>0$ ) ab. Die niedrigste Reynolds-Zahl, die mit der Indifferenzkurve korrespondiert, wird als Indifferenz-ReynoldsZahl $\operatorname{Re}_{\text {ind }}$ bezeichnet und ist in Abb. 2.3 (siehe auch Abb. 2.2) eingezeichnet. $\mathrm{Re}_{\text {ind }}$ entspricht der Reynolds-Zahl, ab welcher Störungen angefacht werden und es zum Anwachsen primärer Eigenmoden (vgl. Abb. 2.2) kommt. Diese ist von der kritischen

\footnotetext{
${ }^{2}$ Der hier dargestellte Zusammenhang beschreibt die zeitliche Verstärkung. Im Fall der räumlichen Verstärkung ist $k=k_{r}+\mathrm{i} k_{i} \in \mathbb{C}$ und $\omega \in \mathbb{R}$ (siehe hierzu u. a. [68,82]).

${ }^{3}$ Die in der OrR-Sommerfeld-Gleichung auftretenden Längen und Geschwindigkeiten können z. B. durch die Geschwindigkeit $U_{e}$ am Grenzschichtrand und einer Referenz-Grenzschichtdicke $\delta=\sqrt{\frac{\nu x}{U_{e}}}$ entdimensionalisiert werden [68]. In der Literatur sind jedoch auch weitere Möglichkeiten zu finden. Die spezifische Reynolds-Zahl wird dementsprechend mit diesen Größen gebildet, hier z. B. $\operatorname{Re}_{\delta}=\frac{U_{e} \delta}{\nu}$.
} 


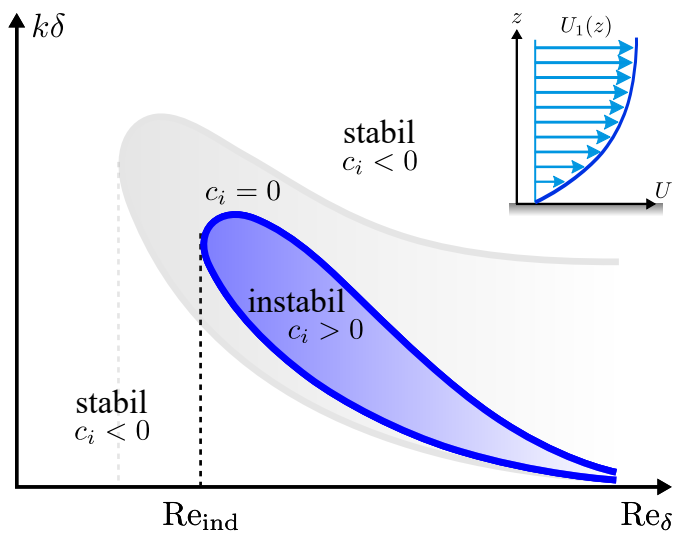

(a) Indifferenzkurve für $U_{1}(z)$

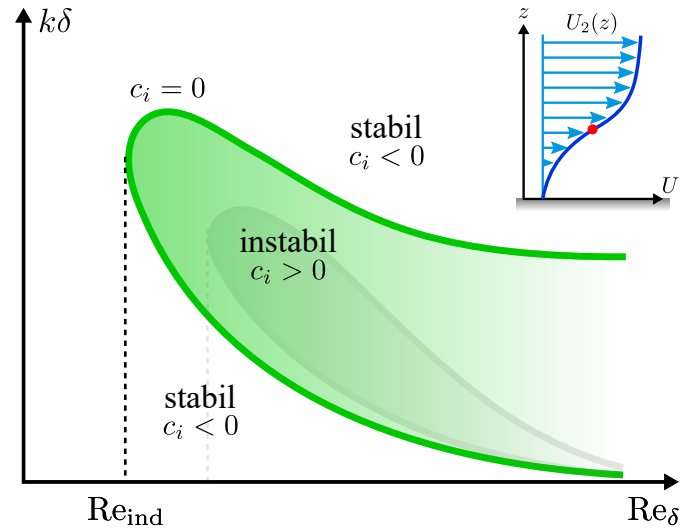

(b) Indifferenzkurve für $U_{2}(z)$

Abbildung 2.3.: Schematische Darstellung der Indifferenzkurven für zwei unterschiedliche Geschwindigkeitsprofile, verändert nach [1]. Die Wellenzahl $k$ wurde mit der Grenzschichtdicke $\delta$ entdimensionalisiert $(k \cdot \delta)$ aufgetragen. (a) zeigt den kleiner ausfallenden Instabilitätsbereich für ein Geschwindigkeitsprofil $U_{1}(z)$ ohne Wendepunkt, wohingegen (b) den großen Instabilitätsbereich für ein Profil $U_{2}(z)$ mit Wendepunkt verdeutlicht.

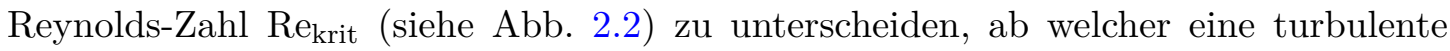
Grenzschicht vorliegt; es gilt $\operatorname{Re}_{\text {ind }}<\operatorname{Re}_{\text {krit }}$. Darüber hinaus zeigt Abb. 2.3, dass nur TS-Wellen mit spezifischen Wellenlängen und Frequenzen auch zu einer Instabilität führen. Externe „erzwungene" Störungen besitzen dabei zumeist ein breites WellenzahlSpektrum, was über eine „erzwungene“ Rezeptivität direkt zu einer Anregung von TS-Wellen mit der passenden $\omega-\lambda$ Kombination führt [78]. Natürliche Störungen weisen hingegen zumeist ein schmalbandiges Spektrum mit Wellenlängen auf, welche sich deutlich von den Instabiltäts-Wellenlängen $\lambda_{\text {TS }}$ unterscheiden. Im Rahmen von „natürlichen“ Rezeptivität-Prozessen kommt es jedoch, wie bereits oben erwähnt, zu einer Anpassung der Wellenlängen [78] und einer Verbreiterung des Spektrums [69]. Dies geschieht in Regionen, wo das mittlere Strömungsfeld eine Anpassung auf einer kleinen Längenskala in Strömungsrichtung vollziehen muss, wie u. a. im Bereich der Vorderkante (siehe hierzu „leading-edge receptivity“ $[69,77,78])$ sowie in Regionen mit einem starken positiven Druckgradienten $[77,78]$.

Der letzte Aspekt korrespondiert dabei auch mit der Abhängigkeit der primären Instabilität der Grenzschicht vom Geschwindigkeitsprofil $U(z)$, wie ein Vergleich von Abb. 2.3(a) mit Abb. 2.3(b) zeigt. Für den in Abb. 2.3(a) dargestellten Fall sind primäre Moden nur in einem begrenzten Reynolds-Zahl-Bereich instabil und für $\operatorname{Re} \rightarrow \infty$ wieder stabil (viskose Instabilität). In Abb. 2.3(b) hingegen existiert für große Reynolds-Zahlen $\operatorname{Re} \rightarrow \infty$ ein ausgedehnter Bereich, in dem ein breites Spektrum an Wellenlängen instabil wird (reibungslose Instabilität). Führt man den Grenzübergang Re $\rightarrow \infty$ auch mathematisch in Gl. (2.1.8) aus und vernachlässigt die Reibungsglieder, so folgt die reibungslose 
Störungsdifferentialgleichung oder auch RAYLEIGH-Gleichung

$$
(U-c)\left(\frac{\mathrm{d}^{2} \varphi}{\mathrm{d} z^{2}}-k^{2} \varphi\right)-\frac{\mathrm{d}^{2} U}{\mathrm{~d} z^{2}} \varphi=0 .
$$

Die OrR-SommerfEld-Gleichung wurde somit auf eine Differentialgleichung 2. Ordnung reduziert, welche die Indifferenzkurve in Abb. 2.3(b) asymptotisch für $\operatorname{Re} \rightarrow \infty$ fortsetzt. Darüber hinaus folgt aus Gl. (2.1.9) das Wendepunktkriterium (für eine Herleitung siehe z. B. [82, S. 3-13 ff.]). Dieses besagt, dass ein Wendepunkt im Geschwindigkeitsprofil $U(z)$ eine notwendige sowie hinreichende Bedingung für die Instabilität von Primärwellen darstellt. Der vernachlässigte Einfluss der Viskosität führt nur zu geringfügigen Änderungen und das Kriterium behält auch bei reibungsbehafteten Strömungen seine Gültigkeit. Dies erklärt auch den größeren Instabilitätsbereich in Abb. 2.3(b) für ein Geschwindigkeitsprofil mit Wendepunkt gegenüber dem kleineren Bereich in Abb. 2.3(a) für ein Profil ohne Wendepunkt. Dabei korreliert die Existenz eines Wendepunktes stark mit dem Druckgradienten der Strömung, was in 2.1.2 ausführlicher diskutiert wird.

Sobald die primären Eigenmoden eine hinreichend große Amplitude (1-2\% der Anströmungsgeschwindigkeit [74]) erreichen, setzt ein sekundärer Mechanismus im Rahmen der laminar-turbulenten Grenzschichttransition ein (vgl. Pfad A in Abb. 2.1 und Abb. 2.2). Einsetzende nichtlineare Prozesse führen zu einer Änderung von Frequenzen und Orientierungen der vorliegenden Störungen, was mit der Ausbildung von Harmonischen einhergeht [71-73]. Dabei treten Resonanzphänomene und Interaktionen der primären Moden auf. Dies ist verbunden mit einem dreidimensionalen (spannweitigem) Energietransfer und der Ausbildung von sekundären (dreidimensionalen) Instabilitäten. $\Lambda$-förmige Wirbel entstehen, welche wiederum gestaffelte Strukturen (C-, H- oder K-Typ) ausbilden [73,83]. Die $\Lambda$-Wirbelstrukturen entwickeln sich stromab in instabile Haarnadel-Strukturen (auch Hairpin-Strukturen). Diese zerfallen und bilden turbulente Spots (siehe „Breakdown“ in Abb. 2.1), was letztendlich zur turbulenten Grenzschicht führt. Eine zusammenfassende Behandlung dieser Aspekte findet sich in [1, S.472 ff.] und umfassend in [83].

\subsubsection{Einfluss des Druckgradienten und Laminarerhaltung}

Im vorherigen Abschnitt wurde bereits das Wendepunktkriterium und der Einfluss auf die primäre Stabilität der laminaren Grenzschicht (siehe Abb. 2.3) diskutiert. Hieraus folgt auch, dass eine Modifikation von $U(z)$ bzw. der Krümmung $\frac{\partial^{2} U}{\partial z^{2}}$ eine Auswirkung auf die Lage der Transition hat. Zielsetzung einer solchen Modifikation ist i.d.R. eine Limitierung des Wachstums von primären Störungen und der Erhalt einer größtmöglichen laminaren Lauflänge der Grenzschicht, also eine Verschiebung der Transition stromabwärts. Technisch wird eine Modifikation von $U(z)$ bzw. eine aktive laminare Strömungskontrolle (engl.: Laminar Flow Control, LFC) durch eine Absaugung der wandnahen Grenzschicht oder 
ein Kühlen (gilt für Luft) der Wand realisiert. LFC erfordert jedoch gemäß Abb. 2.1 eine geringe externe Störungsamplitude [68]. Eine passive bzw. natürliche Möglichkeit stellt die Beeinflussung des Druckgradienten über eine günstige Formgebung des umströmten Körpers oder der Oberfläche dar (engl.: Natural Laminar Flow, NLF). Eine Kombination von aktiven und passiven Technologien wird als hybride laminare Strömungskontrolle (engl.: Hybrid Laminar Flow Control, HLFC) bezeichnet [68, 73, 84].

Wie bereits in 2.1.1 erwähnt, ist in der vorliegenden Arbeit der Druckgradient bedeutsam. Aus der dimensionsbehafteten Grenzschichtgleichung für die ebene, inkompressible und stationäre Strömung (siehe z. B. [1, S. 153])

$$
u \frac{\partial u}{\partial x}+v \frac{\partial u}{\partial z}=-\frac{1}{\rho} \frac{\mathrm{d} p}{\mathrm{~d} x}+\nu \frac{\partial^{2} u}{\partial z^{2}}
$$

leitet sich mit einer Spezifizierung für die Wand (für $z=0: u(x, 0)=v(x, 0)=0$ ) die Wandbindung

$$
\left.\mu \frac{\partial^{2} u}{\partial z^{2}}\right|_{z=0}=\frac{\mathrm{d} p}{\mathrm{~d} x}
$$

ab. Gl. (2.1.11) liefert einen direkten Zusammenhang zwischen der Krümmung des Geschwindigkeitsprofils an der Wand und dem Druckgradienten. Hieraus folgt für einen negative Druckgradienten $\frac{\mathrm{d} p}{\mathrm{~d} x}<0$ (beschleunigte Strömung) eine negative Krümmung an der Wand. Für ein positiven Druckgradienten $\frac{\mathrm{d} p}{\mathrm{~d} x}>0$ (verzögerte Strömung) ist auch die Krümmung positiv. In Abb. 2.4 ist nunmehr die Entwicklung von $\frac{\mathrm{d}^{2} U}{\mathrm{~d} z^{2}}$, sowie

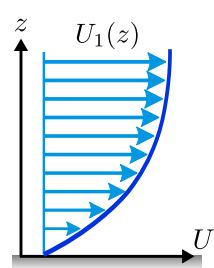

(a) $U(z), \frac{\mathrm{d} U}{\mathrm{~d} z}$ und $\frac{\mathrm{d}^{2} U}{\mathrm{~d} z^{2}}$ für $\frac{\mathrm{dp}}{\mathrm{dx}}<\mathbf{0}$
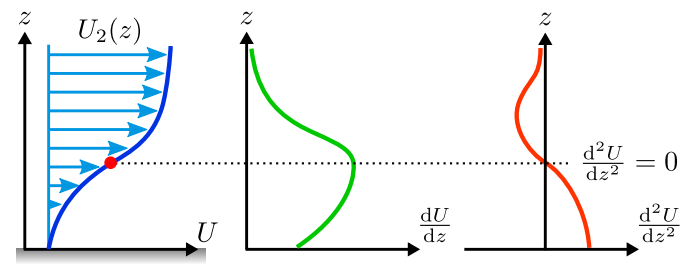

(b) $U(z), \frac{\mathrm{d} U}{\mathrm{~d} z}$ und $\frac{\mathrm{d}^{2} U}{\mathrm{~d} z^{2}}$ für $\frac{\mathrm{dp}}{\mathrm{dx}}>\mathbf{0}$

Abbildung 2.4.: Darstellung der Geschwindigkeitsprofile und deren Ableitungen für einen negativen Druckgradienten in (a) und einen positiven Druckgradienten in (b), nach [1, S.168].

$U(z)$ und $\frac{\mathrm{d} U}{\mathrm{~d} z}$ mit zunehmenden Wandabstand dargestellt. Abb. 2.4(a) zeigt, dass für $\frac{\mathrm{d} p}{\mathrm{~d} x}<0$ auch entfernt von der Wand $\frac{\mathrm{d}^{2} U}{\mathrm{~d} z^{2}}<0$ gültig bleibt und kein Wendepunkt auftritt. Gemäß den in 2.1.1 diskutierten Zusammenhängen wirkt sich ein negativer Druckgradient also stabilisierend auf die laminare Grenzschicht aus. Im Gegensatz dazu folgt aus $\left.\frac{\partial^{2} U}{\partial z^{2}}\right|_{z=0}>0$ ein Wendepunkt, wie Abb. 2.4(b) verdeutlicht. Ein positiver Druckgradient wirkt somit destabilisierend. Dieser Zusammenhang ist im Anhang A.1 auch auf Basis einer Stabilitätsrechnung mit Gl. (2.1.8) und Pohlhausen-Profilen [85] verdeutlicht, wobei der Druckgradient in Form eines Formparameters (PohlHausen-Parameter) variiert wird. 
Bei einem umströmten Körper beeinflusst somit die Druckverteilung maßgeblich die Lage der Grenzschichttransition. Für die (passive) Laminarerhaltung der Grenzschicht ist daher ein ausgedehnter Bereich mit einem negativen Druckgradienten (beschleunigte Strömung) dienlich. Auf diesen physikalischen Aspekten beruht die Auslegung von Laminarflügelprofilen, welche mit einer speziellen Formgebung, im Wesentlichen mit einer großen Dickenrücklage und einem kleinen Nasenradius, das Druckminimum stromabwärts verlagern. Dieser Zusammenhang ist in Abb. 2.5 qualitativ im Vergleich mit einem konventionellen Flügelprofil dargestellt. Der ausgedehnte Bereich mit einem negativen Druckgradienten $\frac{\mathrm{d} c_{p}}{\mathrm{~d} x}<0$ des Laminarprofils führt zu einer Verlagerung der Transitionsposition $x_{\text {tr }}$ stromabwärts (vgl. 2.1.4). So werden lange laminare Laufängen erzielt, was mit einer deutlichen Reduzierung des Widerstands einhergeht. Dieser Effekt ist jedoch sensitiv bzgl. des Anstellwinkels $\alpha$ und lässt sich nur in einen limitierten Bereich realisieren. Hieraus resultiert die für Laminarprofile charakteristische Laminardelle (engl.: drag bucket), wie es im Polardiagramm in Abb. 2.5 ersichtlich ist. Bei einer
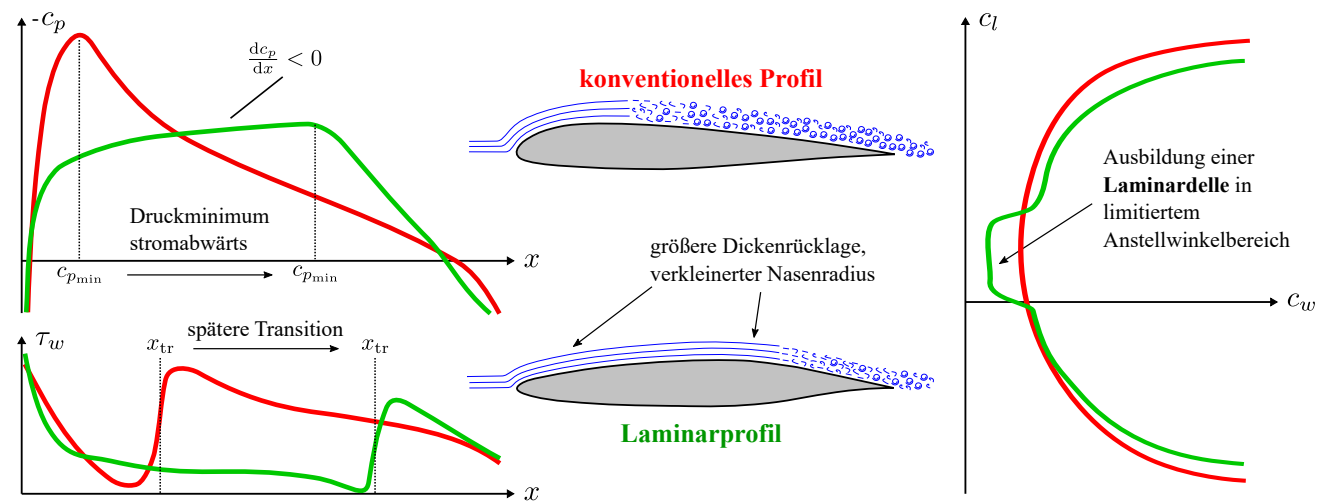

Abbildung 2.5.: Qualitativer Vergleich eines konventionellen Profils mit einem Laminarprofil. Die Druckverteilungen $c_{p}$ und Wandschubspannungsverläufe $\tau_{w}$ sind für die Profiloberseite dargestellt. Die Polaren zeigen die Ausbildung der charakteristischen Laminardelle.

transsonischen Anströmung kommen noch weitere Aspekte hinzu, wie es zum Teil in 2.1.3 disktutiert wird. Transsonische bzw. superkritische Laminarprofile stellen insofern erweiterte Anforderungen und unterliegen einem komplexen Design- und Optimierungsprozess (siehe z. B. [86]). Für das in der vorliegenden Arbeit untersuchte superkritische Laminarflügelprofil CAST 10-2 sind die Entwurfsaspekte in 3.3 aufgeführt.

Ergänzend zur Beeinflussung der Grenzschichttransition sei noch erwähnt, dass ein Druckanstieg auch zu einer Ablösung der Grenzschicht führen kann. Der Ablösepunkt ist definiert durch eine Wandschubspannung die null wird [1, S.150]

$$
\tau_{w}=\left.\eta \frac{\partial u}{\partial z}\right|_{z=0}=0
$$

was gleichbedeutend mit einem verschwindenden Geschwindigkeitsgradienten an der Wand ist. $\eta$ bezeichnet die dynamische Viskosität. Laminare Grenzschichten weisen eine 
deutlich größere Neigung zur Ablösung auf als turbulente Grenzschichten, was bereits aus den unterschiedlichen Geschwindigkeitsprofilen hervorgeht (siehe Abb. 2.2). Der stärkere Impulsaustausch einer turbulenten Grenzschicht führt dem Fluid eine höhere kinetische Energie zu, so dass dieses größere Druckanstiege bewältigen kann bevor es zur Ablösung kommt. Die damit einhergehenden Unterschiede sind u. a. bei der Wechselwirkung der Grenzschicht mit Verdichtungsstößen entscheidend, wie im Folgenden beschrieben.

\subsubsection{Stoß-Grenzschicht-Interaktion}

Transsonische Tragflügelströmungen sind dadurch gekennzeichnet, dass (zumeist auf der Profil-Saugseite) supersonische Strömungsgebiete $(\mathrm{Ma}>1)$ auftreten, welche mit einem Verdichtungsstoß (nahezu normal zur Oberfläche orientiert, engl.: (near-) normal shock) abschließen. Dieser trennt ein stromabwärts liegendes subsonisches Gebiet $(\mathrm{Ma}<1)$ ab [87, S.87]. Verdichtungsstöße interagieren dabei mit der Grenzschicht und beeinflussen maßgeblich die Aerodynamik. Derartige Stoß-Grenzschicht-Interaktionen umfassen ein großes Forschungsgebiet von transsonischen bis hin zu hypersonischen Strömungen. Eine thematische Übersicht findet sich in [2,87-89].
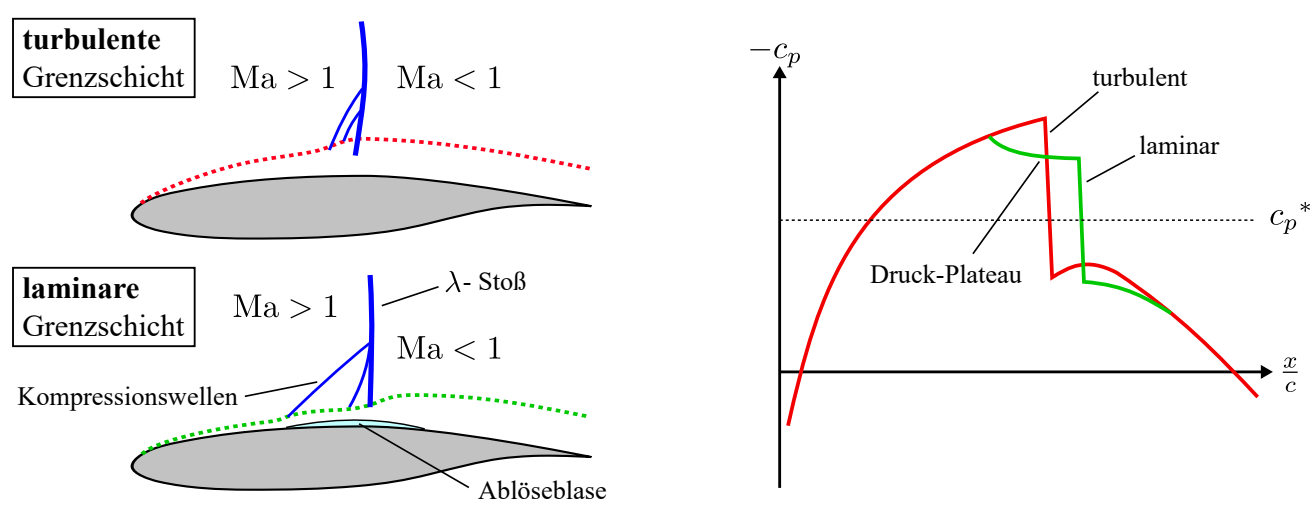

Abbildung 2.6.: Darstellung der Stoß-Interaktion mit einer laminaren und einer turbulenten Grenzschicht sowie deren Auswirkung auf die Druckverteilung, verändert nach [9].

Die Wechselwirkung zwischen Stoß und Grenzschicht wird durch den Stoß induzierten lokalen Druckanstieg und den positiven Druckgradienten hervorgerufen. Hierbei kommt es zu einer Verzögerung und Aufdickung [90-92] sowie zu einer Destabilisierung der Grenzschicht (vgl. 2.1.2). Bei einer laminaren Grenzschicht kommt es nahezu immer zu einer Ablösung [3], bei turbulenten Grenzschichten nur bei einer ausreichenden Stoßstärke [90]. Dementsprechend ist der Zustand der Grenzschicht mit welcher ein Stoß interagiert von entscheidender Bedeutung [2]. Eine Ablösung in Kombination mit einem Normal-Stoß führt zur Stoß-Bifurkation in Wandnähe $[3,90]$. Je nach Zustand der Grenzschicht bilden sich folglich unterschiedliche Stoßmuster und Druckverteilungen aus, was schematisch in Abb. 2.6 dargestellt ist. 
In der vorliegenden Arbeit findet die Interaktion des Verdichtungsstoßes zumeist mit einer laminaren bzw. transitionellen Grenzschicht statt, was sich in den langen laminaren Lauflängen und den ausgedehnten Transitionsbereichen des untersuchten Laminarflügelprofils (siehe 5) begründet. Eine laminare Grenzschicht weist einen subsonischen Bereich auf, durch den der Stoß-induzierte Druckanstieg eine Beeinflussung der stromauf liegenden Grenzschicht bewirkt [93] (vgl. auch [87, S.28 ff.]). Dies führt zu einer Aufdickung (und auch Ablösung) der Grenzschicht stromaufwärts vor dem Stoß, was wiederum zur Ausbildung von Kompressionswellen führt ${ }^{4}$. Diese bilden den vorderen Zweig des Stoßfußes, hinter denen die Strömung noch supersonisch ist [87, S.48]. Ferner schwächt sich der hintere Stoß ab, hinter dem die Strömung dann jedoch subsonisch ist. Mit etwas Abstand zur Wand verschmelzen die Kompressionswellen mit dem Verdichtungsstoß. Die auftretende Stoßbifurkation bildet dabei ein charakteristisches $\lambda$-Muster (typisch für stoßinduzierte Ablösung in transsonischer Strömung [87, S.50]) aus.

Neben einer Ablösung kommt es auch zu einem, mit dem (über kleine Längenskalen lokalisierten) starken Druckanstieg assoziierten Rezeptivitäts-Prozess [78] in der laminaren bzw. (prä-)transitionellen Grenzschicht und einem Anwachsen von Instabilitäten. Folglich setzt im Rahmen der Stoß-Grenzschicht-Interaktion die Transition in der abgelösten Grenzschicht (vgl. 2.1.1) ein. Dies geschieht zumeist in Kombination mit sekundären und dreidimensionalen Instabilitäten [92] (auch KeLvin-Helmholtz Instabilitäten [94]). Die nunmehr turbulente Scherschicht kann durch den größeren Impulsaustausch den starken Druckanstieg ausgleichen, so dass es zum turbulenten Wiederanlegen kommt. Es bildet sich eine laminare Ablöseblase aus, wie in Abb. 2.6 dargestellt. Der laminare Teil der Blase korreliert mit einem charakteristischen Plateau in der Druckverteilung [95, 96], wie es auch die laminare $c_{p}$-Verteilung in Abb. 2.6 zeigt. Einen großen aerodynamischen Einfluss erlangt die Ablösung dann, wenn die Ausdehnung der Ablöseblase die Druckverteilung signifikant beeinflusst, bzw. die Flügelhinterkante erfasst. Insbesondere eine Hinterkantenablösung führt zu erheblichen aerodynamischen Auswirkungen [97] bis hin zum Zusammenbruch der Strömung (engl.: flow breakdown) [93]. Dabei treten durch die Interaktion zwischen Stoß-induzierter Ablöseblase und Hinterkantenablösung verschiedene Ablöseszenarien (Typ-A oder Typ-B Ablösung nach PEARCEY ET AL. [98]) auf, welche sich auch dynamisch verändern können.

Für die Dynamik ist in transonischer Strömung das subsonische Strömungsfeld hinter dem Verdichtungsstoß ein wichtiger Faktor. Dieses ermöglicht es, dass stromabwärts lokalisierte

\footnotetext{
${ }^{4}$ Die Position der Schalllinie $(\mathrm{Ma}=1)$ liegt bei einer turbulenten Grenzschicht durch das vollere Geschwindigkeitsprofil (vgl. Abb. 2.2) in etwa bei $z \approx \mathcal{O}(1 / 10 \delta)$. Bei einer laminaren Grenzschicht liegt die Grenze bei $z \approx \mathcal{O}(1 / 3 \delta)$, womit ein „subsonischer Korridor“ entsteht [93]. Dieser ist im turbulenten Fall entsprechend begrenzt. Die damit verbundene Aufdickung der Grenzschicht beginnt bei einer laminaren Grenzschicht in etwa bei $\Delta x \approx \mathcal{O}(50 \delta)$ stromaufwärts, bei einer turbulenten Grenzschicht bei $\Delta x \approx \mathcal{O}(5 \delta)$ [3]. Da im laminaren Fall die Aufdickung moderater verläuft, bildet sich ein ausgedehntes aber nicht stark konzentriertes Kompressionsfeld aus.
} 
Störungen stromaufwärts wirken [91] und dabei Stoß und verbundene Ablöseblasen sowie Hinterkantenablösungen beeinflussen. Hieraus entstehende Dynamiken können zu großskaligen instationären Strömungsfeldern führen, was in transsonischem Buffet resultieren kann und von großer Bedeutung für die Aeroelastik ist (siehe 2.2.3). Im Allgemeinen sind instationäre Effekte inherent mit Stoß-Grenzschicht Interaktionen bzw. mit deren Wechselwirkung mit dem übrigen Strömungsfeld verbunden [99], insbesondere bei (turbulenten) Ablösungen [2]. Diese Instationaritäten beinhalten eine Vielzahl von weiteren Aspekten, auch außerhalb der Transonik. Untersuchungen hierzu umfassen zumeist Wechselwirkungen von Stößen mit einer turbulenten Grenzschicht (siehe u. a. [100-102]), sie treten aber auch bei laminaren Grenzschichten auf [103].

\subsubsection{Wandschubspannungsverlauf und Definition der Transition}

In den vorherigen Abschnitten wurde mehrfach der Unterschied der Geschwindigkeitsprofile $U(z)$ einer laminaren und turbulenten Grenzschicht angesprochen. Im Verlauf der Transition kommt es folglich zu einer Änderung dieser Profile (vgl. 2.1). Die Wandschubspannung $\tau_{w}$ ist gemäß Gl. (2.1.12) direkt proportional zum Geschwindigkeitsgradienten an der Wand, so dass eine Änderung von $U(z)$ auch eine Änderung von $\tau_{w}$ bewirkt. Da die Wandschubspannung messtechnisch erfasst werden kann (siehe 3.4 und 4.3), werden diese physikalischen Zusammenhänge in der vorliegenden Arbeit zur Identifikation der Grenzschichtvorgänge genutzt.

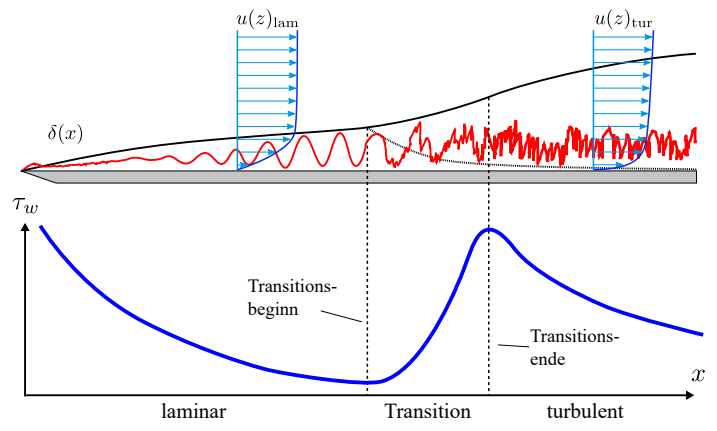

(a) $\tau_{w}(x)$ für natürliche Transition

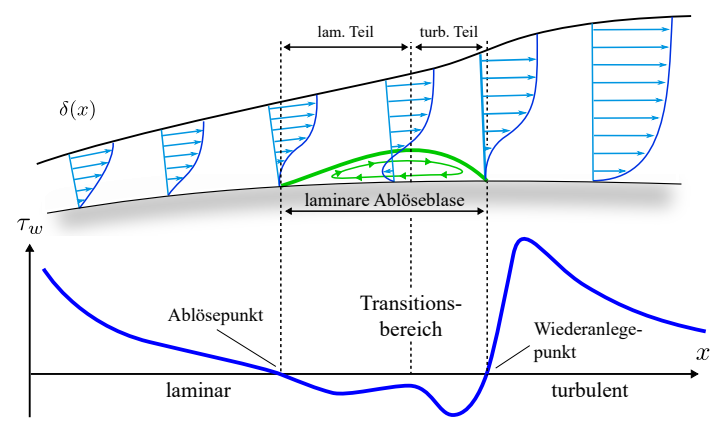

(b) $\tau_{w}(x)$ für Transition mit Ablöseblase

Abbildung 2.7.: Schematische Darstellung des Verlaufes der Wandschubspannung $\tau_{w}(x)$ für eine natürliche Transition (a) und eine Transition über eine laminare Ablöseblase (b), verändert nach $[61,95,96]$.

Für eine natürliche Grenzschichttransition bildet sich ein charakteristischer Verlauf $\tau_{w}(x)$ aus, wie es schematisch in Abb. 2.7(a) dargestellt ist. Mit steigender Lauflänge $x$ wächst die Dicke $\delta(x)$ der laminaren Grenzschicht an, was zu einer Abnahme des Geschwindigkeitsgradienten an der Wand führt. Entsprechend fällt die Wandschubspannung ab. Mit Beginn der Transition, was mit dem Anwachsen erster turbulenter Spots (auch „EMmons-Spots“ [104, S.141 ff.]) in der laminaren Grenzschicht definiert wird [105], 
kommt es zu einem deutlichen Anwachsen der Wandschubspannung infolge der turbulenten Mischbewegung. Die Ausbildung einer viskosen Unterschicht führt zu starken wandnahen Geschwindigkeitsgradienten und entsprechend großen Werten für $\tau_{w}$. Mit steigender Lauflänge wachsen wiederum die turbulente Grenzschicht und die viskose Unterschicht an, womit es zu einem erneuten Abfall von $\tau_{w}$ kommt.

Verläuft die Transition über eine laminare Ablöseblase, wie in Abb. 2.7(b) dargestellt, bleibt der Verlauf von $\tau_{w}(x)$ weitestgehend ähnlich. Im Bereich der Ablösung bildet sich jedoch ein lokales Rückströmungsgebiet aus, so dass der Geschwindigkeitsgradient negativ und damit $\tau_{w}<0$ wird. Der Bereich der Ablösung wird durch den Ablöse- und Wiederanlegepunkt, also gemäß Gl. (2.1.12) durch die Nulldurchgänge von $\tau_{w}$, begrenzt. Der signifikante Anstieg von $\tau_{w}(x)$ korrespondiert in diesem Fall mit dem Wiederanlegen der turbulenten Grenzschicht.

Der mit dem Transitionsvorgang einhergehende Anstieg von $\tau_{w}(x)$ wird in der vorliegenden Arbeit genutzt, um auf Basis von Heißfilmsensoren (vgl. 3.4) und einer neu entwickelten Analyse-Methodik (vgl. 4.3.2 und [14,61]) die Grenzschichtzustände und insbesondere die Transition zu identifizieren/lokalisieren. Zur Vereinfachung wird die Position der Transition $x_{\text {tr }}$ als Punkt ${ }^{5}$ angenommen, welcher mit dem stärksten Anstieg von $\tau_{w}(x)$ identifiziert wird. Dies entspricht der in der vorliegenden Arbeit verwendeten Definition des „Transitionspunktes“. Dieser Punkt korreliert ferner mit dem Maximum des RMS-Wertes der Wandschubspannung [106], welcher zumeist zur Definition und messtechnischen Bestimmung der Transitionsposition herangezogen wird (vgl. auch 4.3). Des Weiteren entspricht dies angenähert der Mitte des Transitionsbereiches und einem Intermittenzfaktor $^{6}$ von $\gamma \approx 0,5$ [105]. Die räumliche Ausdehnung des Transitionsbereiches kann ebenfalls anhand von $\tau_{w}(x)$ abgeschätzt werden. Als Beginn wird das Minimum von $\tau_{w}(x)$ verwendet [105], wohingegen das Maximum eine abgeschlossene Transition markiert (vgl. auch [107] und Abb. 2.7(a)). Jedoch zeigt sich insbesondere bei der Transition über eine Ablöseblase (vgl. Abb. 2.7), dass die Bestimmung einer Transitions-Position und des Transitionsbereiches mit Unsicherheiten verbunden ist. Die Transition verläuft in der abgelösten Scherschicht (vgl. [96]), so dass der maximale Anstieg von $\tau_{q}(x)$ bereits mit einer turbulenten Grenzschicht korreliert. Die eigentliche Transitionsposition liegt leicht stromaufwärts, wie Abb. 2.7(b) verdeutlicht. Eine genauere Aufösung ist jedoch durch die eingesetzte Heißfilm-Messtechnik (vgl. 3.4) nicht möglich, was unter 4.3.2 näher beschrieben ist.

\footnotetext{
${ }^{5}$ Die Transition verläuft immer in einem ausgedehnten Bereich, entsprechend ist der Begriff „Transitionspunkt" strenggenommen nicht ganz korrekt. In der vorliegenden Arbeit bezieht sich dieser konsistent auf einen physikalisch definierten Punkt im Transitionsbereich.

${ }^{6}$ Der Intermittenzfaktor $\gamma$ gibt den Bruchteil der Zeit an, in der die Grenzschichtströmung turbulent ist [1, S.415]. $\gamma=0$ beschreibt eine laminare und $\gamma=1$ eine voll-turbulente Grenzschichtströmung.
} 


\subsection{Physikalische Aspekte der Aeroelastik}

Aeroelastische Probleme umfassen physikalische Wechselwirkungen elastomechanischer Systeme mit der umgebenen Strömung. Auftretende Effekte beruhen dabei auf aerodynamischen Kräften, welche statische und dynamische Verformungen der Struktur hervorrufen, was wiederum zu einer Rückwirkung auf die Aerodynamik führen kann. Verstärkt z. B. diese Rückwirkung die ursprüngliche Verformung, dann tritt eine Instabilität auf. Die möglichen Wechselwirkungen aeroelastischer Systeme lassen sich dabei anschaulich in Form von Funktionsdiagrammen darstellen [108, S.365 ff.], wie es in Abb. 2.8(a) für einen flatternden Tragflügel gezeigt ist. Die Blockelemente entsprechen

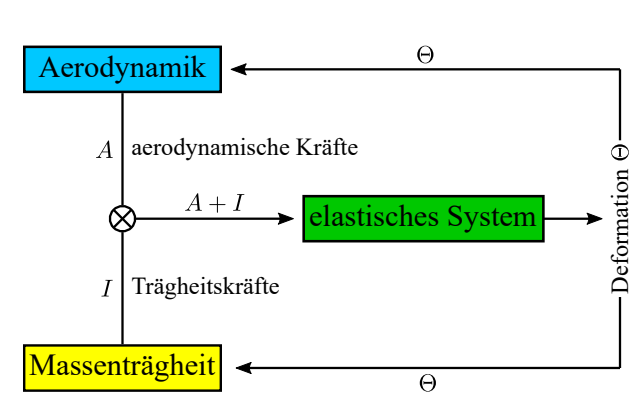

(a) Funktionsdiagramm des flatternden Tragflügels

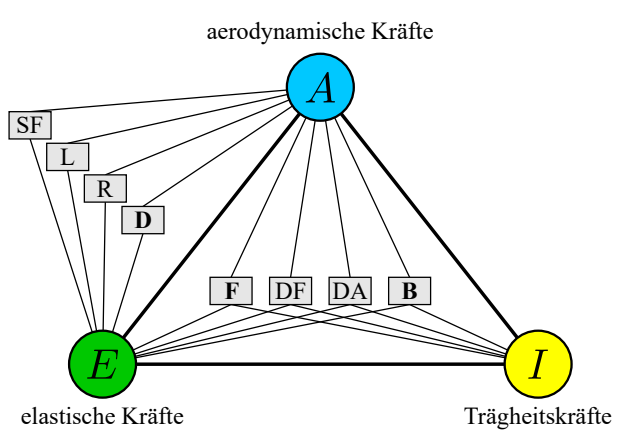

(b) Aeroelastisches Kräftedreieck

Abbildung 2.8.: Aeroelastisches Funktionsdiagramm am Beispiel des flatternden Tragflügels in (a), verändert nach [109, S.10] und [8, S.400] sowie Klassifizierung der aeroelastischen Probleme anhand des Kräftedreiecks in (b), verändert nach [8, S.401].

mathematischen Operatoren $O$, welche (analog zu einer Übertragungsfunktion, vgl. 4.1.3) zu einem Eingangsignal $x_{E}$ ein Ausgangssignal $x_{A}=O \cdot x_{E}$ bereitstellen [109, S.13 ff.]. So führt eine Deformation $\Theta$ (Eingangsgröße) am starren Tragflügel (Operator) zu aerodynamischen Kräften $A$ (Ausgangsgröße). Am bewegten Tragflügel kommen Trägheitskräfte $I$ hinzu. Diese bewirken in Kombination $A+I$ eine Deformation $\Theta$ des elastischen Tragflügels. Letztere wechselwirkt wiederum mit der Aerodynamik und der Massenträgheit, so dass ein geschlossener Rückkopplungsprozess entsteht. Existieren für dieses aeroelastische System nicht triviale Lösungen $\Theta \neq 0$ (für $t \rightarrow \infty$ ), liegt eine Instabilität vor, die als Flattern bezeichnet wird.

Tabelle 2.1.: Klassifizierung der aeroelastischen Probleme gemäß Abb. 2.8(b).

statische aeroelastische Probleme: dynamische aeroelastische Probleme:

- statische Divergenz (D)

- Ruderwirksamkeit (R)

- Auftriebsverteilung (L)

- statische Flugstabilität (SF)
- Flattern (u. Galloping) (F)

- dynamische Flugstabilität (DF)

- dynamische Antwort (DA)

- Buffeting (B) 
Aeroelastische Phänomene unterteilen sich im Allgemeinen in statische und dynamische Probleme, wobei im ersten Fall die Trägheitskräfte verschwinden und die Aerodynamik als stationär angenommen werden kann [8, S.400]. Eine übersichtliche aeroelastische Problemklassifizierung wird anhand des in Abb. 2.8(b) dargestellten aeroelastischen Kräftedreiecks $[110,111]$ vorgenommen. Je nach Zusammenspiel der beteiligten Kräfte folgen daraus die in Tab. 2.1 aufgeführten Probleme, welche hier in ihrer Gesamtheit nicht näher beschrieben werden. Für einen Überblick und eine detailliertere Behandlung sei an die Standardliteratur u. a. $[8,108,109,112,113]$ verwiesen.

In der vorliegenden Arbeit spielt das bereits oben angesprochene und in 2.2.2 näher diskutierte Flatterphänomen die zentrale Rolle. Die Einflüsse der transsonischen Strömung hierauf sind in 2.2.3 diskutiert. Hier tritt zum einen auch die statische Divergenz in den Fokus der Arbeit, welche im „klassischen“ Sinne eine statische Verformung eines Auftriebssystems (schlagartig und bis zum Bruch) bei Erreichen einer kritischen Geschwindigkeit beschreibt [8, S.401], weil die angreifende Luftkraft durch die elastische Rückstellkraft nicht mehr im Gleichgewicht gehalten werden kann. Unter den vorherrschenden physikalischen Randbedingungen tritt eine abgeschwächte Form mit dynamischem Verlauf auf, welche sich in Kombination mit einer einsetzenden Flatterinstabilität verdeutlicht und als schwache Divergenz (engl.: weak divergence) bzw. Flatter-Divergenz-Interaktion (engl.: flutter-divergence interaction) bezeichnet wird [5,114-116]. Zum anderen sind als Konsequenz der transsonischen Anströmung Aspekte des Buffet-Phänomens von Interesse, was bereits in 2.1.3 angesprochen und auch in 2.2.3 näher erläutert wird.

\subsubsection{Grundzüge der instationären Aerodynamik}

Die mit den aeroelastischen Effekten einhergehenden Strukturschwingungen führen zu einer dynamischen Antwort der Aerodynamik. Die Annahme einer stationären Strömung ist somit nicht mehr erfüllt. Dennoch lässt auch der instationäre Fall eine, zumindest für einfache Fälle analytische Behandlung zu, welche die essentiellen Aspekte der instationären Aerodynamik verdeutlicht. Die nachfolgenden Ausführungen basieren dabei weitestgehend auf [8, S.252 ff.].

Es sei ein Tragflügel unendlicher Spannweite in zweidimensionaler, inkompressibler Strömung angenommen. Unter Verwendung der Skeletttheorie (siehe u. a. [117, S.416 ff.]) wird der Flügel durch seine Skelettlinie $z_{s}(x)$ ersetzt. Ferner wird eine schwache Wölbung angenommen ( $z$ sowie $\frac{\mathrm{d} z}{\mathrm{~d} x}$ klein), so dass die Zirkulationsverteilung der gebundenen Wirbel $\gamma(x)$ in ihrer Wirkung einer Platte entsprechen (Anordnung der Wirbelverteilung auf der Profilsehne). Eine als harmonisch angenommene Bewegung der Skelettlinie

$$
z_{s}(x, t)=\hat{z}_{s}(x) \cdot \exp (\mathrm{i} \omega t)
$$


mit der Kreisfrequenz $\omega$ führt nunmehr zu einer Änderung der gebundenen Wirbel $\gamma(x, t)=\hat{\gamma}(x) \cdot \exp (\mathrm{i} \omega t)$, welche eine explizite Zeitabhängigkeit bekommen. Somit ändert sich auch die gesamte Zirkulation um den Tragflügel

$$
\Gamma(t)=\int_{-l}^{l} \gamma(x, t) \mathrm{d} x=\hat{\Gamma} \cdot \exp (\mathrm{i} \omega t)
$$

welche aus der Integration über die Profiltiefe $c=2 l$ folgt. Nach dem Thomson'schen Satz zur zeitlichen Wirbelerhaltung [117, S.87 ff.] muss die Zirkulation jedoch erhalten sein, so dass sich freie Wirbel $\epsilon(x, t)=\hat{\epsilon}(x) \cdot \exp (\mathrm{i} \omega t)$ vom Tragflügel ablösen, welche die zeitliche Änderung der Zirkulation $\frac{\mathrm{d} \Gamma}{\mathrm{d} t}$ ausgleichen. Die Zirkulationsänderung am schwin-

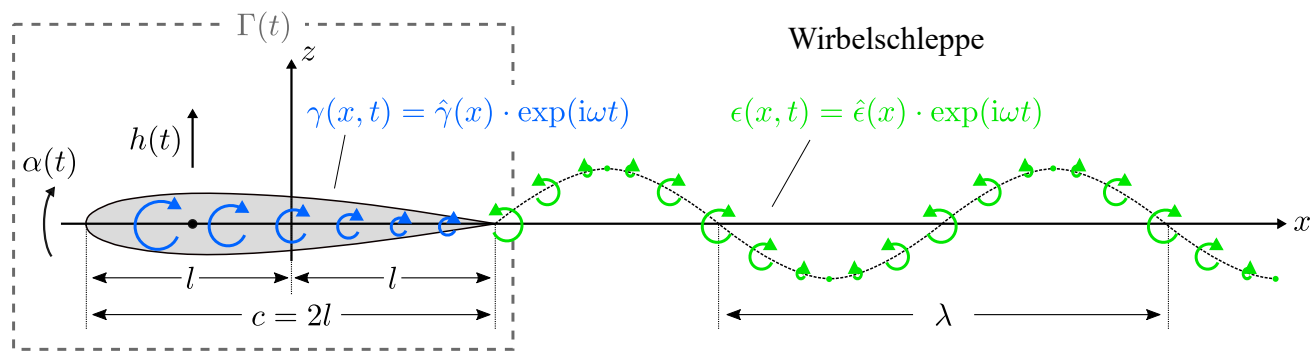

Abbildung 2.9.: Gebundene und freie Wirbelverteilung $\gamma(x, t)$ und $\epsilon(x, t)$ sowie Ausbildung der Wirbelschleppe am schwingenden Flügelprofil (Drehschwingungen $\alpha(t)=\hat{\alpha} \cdot \exp (\mathrm{i} \omega t)$ und Schlagschwingungen $h(t)=\hat{h} \cdot \exp (\mathrm{i} \omega t))$, verändert nach [109, S.252 f.] und [8, S.406].

genden Tragflügel lässt sich also gedanklich mit einem sich permanent wiederholenden Anfahrvorgang eines angestellten Profils vergleichen. Dabei bildet sich eine in Abb. 2.9 dargestellte Wirbelschleppe ${ }^{7}$ mit der Wellenlänge $\lambda$ aus, woraus die reduzierte Frequenz ${ }^{8}$

$$
\omega^{*}=\frac{2 \pi l}{\lambda}=\frac{\omega l}{u_{\infty}}=\frac{\omega \frac{c}{2}}{u_{\infty}}
$$

folgt. Diese kann als Maß für die Instationarität der Strömung interpretiert werden [109, S.262 f.]. Für die Zirkulation eines an der Hinterkante abschwimmenden Wirbelelements $\epsilon(x=l, t)$ folgt somit mit Gl. (2.2.2) unter der Annahme, dass die Strömungsgeschwindigkeit überall $u_{\infty}$ beträgt,

$$
\epsilon(l, t) \mathrm{d} x=\epsilon(l, t) u_{\infty} \mathrm{d} t=-\frac{\mathrm{d}}{\mathrm{d} t}\left[\int_{-l}^{l} \gamma(x, t) \mathrm{d} x\right] \mathrm{d} t=-\frac{\mathrm{d} \Gamma}{\mathrm{d} t} \mathrm{~d} t
$$

$\epsilon(l, t) \mathrm{d} x$ entspricht also genau der Zirkulation, nur mit umgedrehtem Vorzeichen, welche sich im Zeitintervall $\mathrm{d} t$ am Tragflügel geändert hat. Für ein Wirbelelement, welches in der Zeit $\tau=\frac{\xi-l}{u_{\infty}}$ von der Hinterkante $x=l$ zum Punkt $x=\xi$ gelangt ist, lässt sich

\footnotetext{
${ }^{7}$ Abweichend zum restlichen Teil der vorliegenden Arbeit wird für die theoretische Betrachtung der Ursprung des Koordinatensystems in die halbe Flügeltiefe gelegt, wie es in der Literatur [109,118,119] hierzu üblich ist. Jedoch wird der Schlag $h$ bei einer Bewegung des Flügels nach oben in Richtung $z$ positiv gewählt, entsprechend den Messergebnissen in dieser Arbeit. Ein positiver Anstellwinkel $(\alpha$ positiv) beschreibt einheitlich ein Aufdrehen des Flügels im Uhrzeigersinn.

${ }^{8}$ In der vorliegenden Arbeit wird die reduzierte Frequenz auf die halbe Flügeltiefe $l=\frac{c}{2}$ bezogen.
} 
Gl. (2.2.4) zusammen mit Gl. (2.2.2) und (2.2.3) auch ausdrücken zu

$$
\epsilon(\xi, t+\tau) u_{\infty}=-\frac{\mathrm{d} \Gamma}{\mathrm{d} t} \quad \Leftrightarrow \quad \epsilon(\xi, t)=-\frac{\mathrm{i} \omega}{u_{\infty}} \Gamma(t) \exp \left(-\frac{\mathrm{i} \omega \xi}{u_{\infty}}\right) \exp \left(\mathrm{i} \omega^{*}\right) .
$$

Der induzierte Abwind $w(x, t)$ folgt dann nach dem BIOT-SAVART'schen Gesetz (siehe u. a. [117, S.93 ff.]) aus $\gamma(x, t)$ und $\epsilon(x, t)$ zu

$$
w(x, t)=-\frac{1}{2 \pi}\left[\oint_{-l}^{l} \frac{\gamma(\xi, t)}{x-\xi} \mathrm{d} \xi+\int_{l}^{\infty} \frac{\epsilon(\xi, t)}{x-\xi} \mathrm{d} \xi\right] .
$$

Das erste Integral in Gl. (2.2.6) besitzt eine Singularität an der Stelle $\xi=x$, so dass von dem Integral der ChauchY'sche Hauptwert ${ }^{9}$ zu nehmen ist. Ferner muss der Abwind $w$ die kinematische Strömungsbedingung

$$
w=\frac{\partial z_{s}}{\partial t}+u_{\infty} \frac{\partial z_{s}}{\partial x}
$$

erfüllen, welche im Anhang A.2 hergeleitet ist. Einsetzen von Gl. (2.2.1) in (2.2.7) und Gleichsetzen mit (2.2.6) führt dann zu einer Integralgleichung 2. Art ${ }^{10}$

$$
\mathrm{i} \omega z_{s}+u_{\infty} \frac{\partial z}{\partial x}=-\frac{1}{2 \pi}\left[\oint_{-l}^{l} \frac{\gamma(\xi, t)}{x-\xi} \mathrm{d} \xi+\int_{l}^{\infty} \frac{\epsilon(\xi, t)}{x-\xi} \mathrm{d} \xi\right]
$$

Die Lösung von Gl. (2.2.8), mit dem Ziel die Wirbelverteilung $\gamma(x, t)$ zu erhalten, entspricht der zweiten Hauptaufgabe der Skeletttheorie (vgl. [117, S.431 ff.]), wobei eine analytische Lösung der Integralgleichung (2.2.8) mathematisch länglich und mühsam ist. Die Lösungswege von Küssner [118] und Schwarz [119] sind im Anhang A.3 skizziert.

Ist jedoch $\gamma(x, t)$ bekannt, so folgt aus dem Satz von KUTTA-JoukowskY die instationäre Druckdifferenz $\Delta p(x, t)$. Der Auftrieb und das Moment um $x_{A}$ (Abstand des Drehpunktes vom Flügeltiefenmittelpunkt) bzgl. eines Flügelschnitts der Breite $\mathrm{d} y$ folgen dann gemäß

$$
\begin{aligned}
\frac{F_{L}(t)}{\mathrm{d} y} & =\int_{-l}^{l} \Delta p(x, t) \mathrm{d} x=\int_{-l}^{l} \rho_{\infty} u_{\infty} \gamma(x, t) \mathrm{d} x, \\
\frac{M_{\alpha}(t)}{\mathrm{d} y} & =-\int_{-l}^{l} \Delta p(x, t)\left(x-x_{A}\right) \mathrm{d} x=-\int_{-l}^{l} \rho_{\infty} u_{\infty} \gamma(x, t)\left(x-x_{A}\right) \mathrm{d} x .
\end{aligned}
$$

Für den schwingenden Tragflügel mit den zwei Freiheitsgraden Schlag $h$ und Drehung $\alpha$ sind die instationären aerodynamischen Kräfte, aufbauend auf der Lösung von Gl. (2.2.8) im Anhang A.4 abgeleitet und hier nicht näher aufgeführt.

\footnotetext{
${ }^{9}$ Der Chauchy'sche Hauptwert ist in der vorliegenden Arbeit durch $£$ angedeutet.

${ }^{10}$ Die Integrationsgrenze des letzten Integrals über die freie Wirbelschicht in Gl. (2.2.8) erstreckt sich bis ins Unendliche. Dies verdeutlicht eine Art „Gedächtnis“ der Strömung bzgl. sämtlicher voraus gegangener Bewegungen des Tragflügels, von denen die aerodynamischen Kräfte und Momente abhängen. Zur mathematischen Behandlung ist folglich die Annahme einer harmonischen Bewegung zweckmäßig.
} 
Die essentiellen physikalischen Aspekte verdeutlichen sich darin, dass die resultierenden instationären aerodynamischen Kräfte sich in einen nichtzirkulatorischen und einen zirkulatorischen Anteil aufspalten (vgl. Gl. (A.4.5) und Gl. (A.4.6) in A.4). Letzterer Teil ist dabei mit der Theodorsen-Funktion $C\left(\omega^{*}\right)$ in Abhängigkeit von der reduzierten Frequenz $\omega^{*}$ verknüpft [120]. $C\left(\omega^{*}\right) \in \mathbb{C}$ setzt sich zusammen aus HANKEL'schen Zylinderfunktionen 2. Art der Ordnung 0 und 1 gemäß

$$
C\left(\omega^{*}\right)=F\left(\omega^{*}\right)+\mathrm{i} G\left(\omega^{*}\right)=\frac{H_{1}^{(2)}\left(\omega^{*}\right)}{H_{1}^{(2)}\left(\omega^{*}\right)+\mathrm{i} H_{0}^{(2)}\left(\omega^{*}\right)}
$$

und ergibt sich u. a. als Teil ${ }^{11}$ der Lösung der Integralgleichung (2.2.8). In Abb. 2.10 ist der Real- und Imaginärteil von $C\left(\omega^{*}\right)$ dargestellt. Insbesondere für aeroelastische

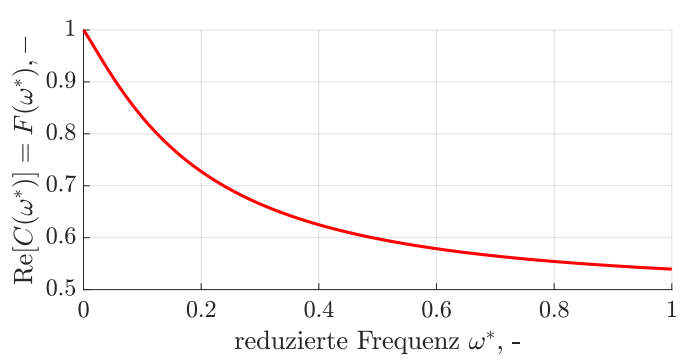

(a) Realteil $F\left(\omega^{*}\right)$

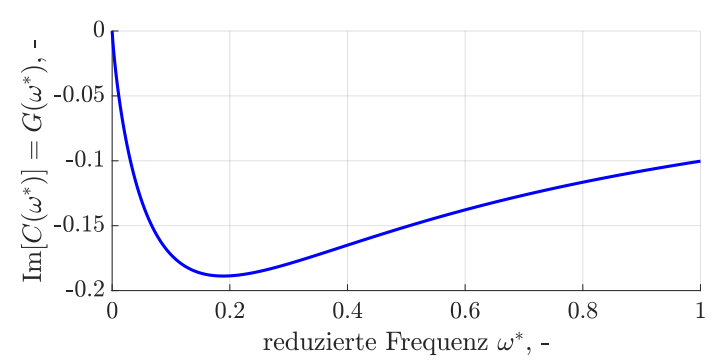

(b) Imaginärteil $G\left(\omega^{*}\right)$

Abbildung 2.10.: Grafische Darstellung des Realteils $F\left(\omega^{*}\right)$ in (a) und des Imaginärteils $G\left(\omega^{*}\right)$ in (b) der Theodorsen-Funktion $C\left(\omega^{*}\right)$ in Abhängigkeit der reduzierten Frequenz.

Probleme wie das Flattern ist die Abhängigkeit der zirkulatorischen Terme von der reduzierten Frequenz entscheidend. Die ThEodorsen-Funktion fungiert dabei wie eine Übertragungsfunktion (vgl. 4.1.3) zwischen der eingehenden Profilbewegung und der instationären aerodynamischen Antwort. Nach Abb. 2.10(a) nimmt der Realteil $F\left(\omega^{*}\right)$, also die Amplitude der zirkulatorischen Terme, mit steigender reduzierter Frequenz ab. Der negative Imaginärteil $G\left(\omega^{*}\right)$, dargestellt in Abb. 2.10(b), verdeutlicht darüber hinaus eine frequenzabhängige Phasendifferenz, was zu einem Nacheilen der aerodynamischen Kräfte zur Profilbewegung führt.

Dies wird umso deutlicher bei einer zur THEODORSEN-Funktion äquivalenten Betrachtung im Zeitbereich. Nach [121] folgt aus der Fourier-Transformation (vgl. 4.1.1) von $C\left(\omega^{*}\right)$ die WAGNER-Funktion [122]

$$
\phi(\tau)=\frac{1}{\mathrm{i}} \int_{-\infty}^{\infty} \frac{C\left(\omega^{*}\right)}{\omega^{*}} \exp \left(\mathrm{i} \omega^{*} \tau\right) \mathrm{d} \omega^{*}, \quad \text { mit } \quad \tau=\frac{t u_{\infty}}{l}
$$

\footnotetext{
${ }^{11}$ Die TheOdorsen-Funktion leitet sich, ebenso wie die Küssner'sche Wirbelschleppenfunktion $T\left(\omega^{*}\right)$ (vgl. A.3), aus der Behandlung des instationären Problems des harmonisch schwingenden Tragflügels ab. Jedoch folgt $C\left(\omega^{*}\right)$ aus der Behandlung des Problems mit der Methode der konformen Abbildungen [120] und $T\left(\omega^{*}\right)=2 C\left(\omega^{*}\right)-1$ aus der Singularitätenmethode $[118,119]$.
} 
$\phi(\tau)$ beschreibt dabei das Anwachsen der Zirkulation bzw. des Auftriebs bei einem instantanen Anstellwinkelsprung. Dies ist gleichbedeutend mit einem Anfahrvorgang eines angestellten Tragflügels, welcher aus der Ruhe impulsartig auf eine konstante Geschwindigkeit $u$ beschleunigt wird. In Abb. 2.11 ist eine Approximation der WAGNER-Funktion dargestellt. Die anfängliche Zirkulation des gebundenen Wirbels ist null, besitzt jedoch

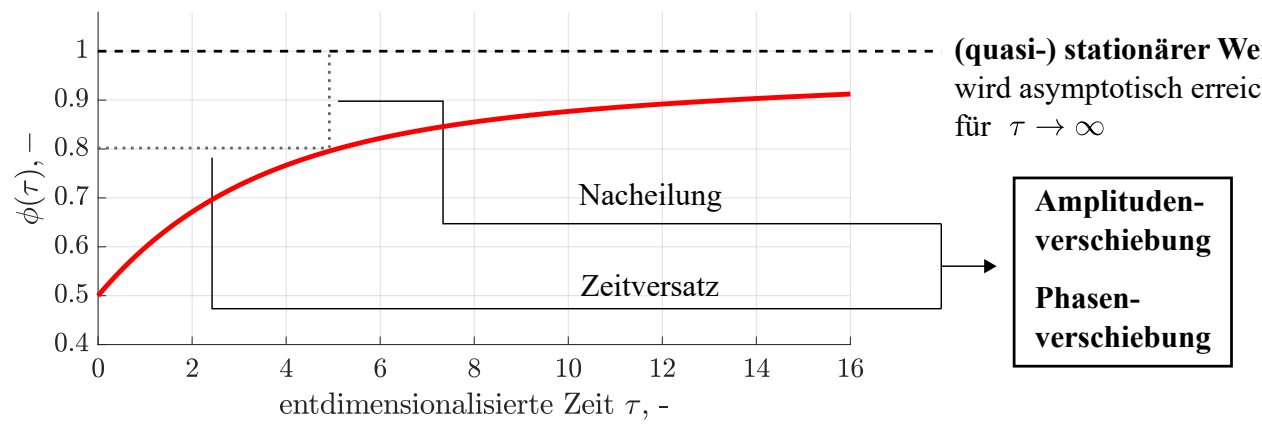

Abbildung 2.11.: JonES-Approximation der WAGNER-Funktion und resultierende Effekte bzgl. der instationären aerodynamischen Kräfte. Da ein expliziter Ausdruck für $\phi(\tau)$ nicht existiert, wurde hier auf die Approximation $\phi(\tau) \approx 1-\sum_{n} a_{n} \exp \left(-b_{n} \tau\right)$ [123] mit den Koeffizienten $a_{1}=0,165 ; a_{2}=0,335 ; b_{1}=0,041$ und $b_{2}=0,320$ [124] zurückgegriffen.

eine anfängliche unendliche Wachstumsrate [125] (vgl. auch [122]). Hieraus folgt, dass der anfängliche Auftrieb der Hälfte $\phi(\tau=0)=0,5$ des stationären Auftriebs entspricht, wie in Abb. 2.11 dargestellt. Das Anwachsen der Zirkulation führt zu einem langsamen Anwachsen des Auftriebs mit der Zeit $\tau$ (bzw. äquivalent mit der zurückgelegten Strecke in halben Flügeltiefen). Anschaulich lässt sich dieser Sachverhalt auch damit erklären, dass ein gedachter Anstellwinkelsprung oder auch ein Anfahrvorgang einen Anfahrwirbel produziert, welcher zunächst die gebundene Zirkulation (infolge der zeitlichen Wirbelerhaltung) und damit den Auftrieb reduziert. Schwimmt der Anfahrwirbel weg, nimmt die Wirkung auf die gebundene Zirkulation ab. Der stationäre Auftrieb wird dann asymptotisch erreicht $\phi(\tau \rightarrow \infty)=1$. Es resultiert hieraus somit eine Nacheilung und ein Zeitversatz des Auftriebs im Vergleich zum stationären Fall. Dies wiederum führt zu den zwei essentiellen Aspekten der instationären Aerodynamik, einer Amplituden- und einer Phasenverschiebung der Kräfte in Bezug zur Bewegung ${ }^{12}$. Dabei ist insbesondere die Phasenverschiebung von großer Bedeutung für Flatterphänomene, wie es im folgendem Abschnitt diskutiert wird.

\footnotetext{
${ }^{12}$ Es sei angemerkt, dass die Theodorsen-Funktion eine harmonische Bewegung des aeroelastischen Systems voraussetzt. Diese Annahmen sind im Allgemeinen nur erfüllt, wenn $u_{\infty}=0$ (Dämpfung vernachlässigt), eine externe harmonische Anregung erfolgt, oder das System selbsterregte harmonische Schwingungen ausführt, es also flattert. Eine beliebige Systembewegung kann jedoch als eine Superposition von Bewegungsschritten aufgefasst werden. Diese wiederum können als kleine Änderung der Bewegung (des Anstellwinkels) bei einer gegebenen Anfangsströmung aufgefasst werden. Das zeitliche Verhalten der aus den Bewegungsschritten resultierenden Kräfte kann also ebenfalls aus einer Superposition derselben gewonnen werden. Dem folgend lässt sich die Wagner-Funktion in Gl. (A.4.5) und Gl. A.4.6 mithilfe eines Duhamel-Integrals als Faltung einbauen und die instationären Kräfte auch auf beliebige Systembewegungen erweitern (siehe z. B. [126, S.285 ff.] und [127, S.114 ff.]).
} 


\subsubsection{Flattern}

In 2.2 wurde Flattern als dynamisches aeroelastisches Problem bereits angesprochen und anhand des Funktionsdiagramms Abb. 2.8(a) in den Grundzügen diskutiert. Dabei resultiert aus dem dynamischen Zusammenspiel von Aerodynamik und Trägheitskräften eine Deformation des elastischen Systems. Flattern charakterisiert also eine selbsterregte Schwingung des aeroelastischen Gesamtsystems bei einer konstanten Anströmung [109, S.482]. Das schwingende System entzieht dem Fluidstrom folglich Energie und kompensiert bzw. überwiegt die dissipativen Energieverluste infolge der Systemdämpfung. Die Strömungsgeschwindigkeit hinter dem Profil muss daher geringer als vor dem Profil sein.

Anhand der Aerodynamik lässt sich Flattern in zwei Kategorien unterteilen [109, S.480 f.] Dem „klassischen“ Flattern liegt eine potentialtheoretische Annahme der Aerodynamik zugrunde. Viskositätseffekte werden ignoriert und die Flatterproblematik weitestgehend linearisiert betrachtet. Diese Flatterform ist, neben der analytischen Zugänglichkeit auch deshalb von Bedeutung, da die auftretenden Schwingungen durch ein teils rapides exponentielles Amplitudenwachstum gekennzeichnet sind. Dabei muss die Schwingungsbewegung translatorische und rotatorische Anteile haben. In der Praxis führt ein solches Flattern in aller Regel zum Versagen (Bruch) der Struktur. Werden hingegen Grenzschichteffekte und Ablösevorgänge mit berücksichtigt, treten Flatterprobleme der zweiten Kategorie auf, welche oftmals als „Abreißflattern“ bezeichnet werden. Diesen überaus komplexen und vielschichtigen Flatterphänomenen gemein ist eine nichtlineare Aerodynamik. Die vorhandenen Nichtlinearitäten führen i. A. zu einer Amplitudenbegrenzung. Das aeroelastische System führt Grenzzyklusschwingungen (engl.: Limit Cycle Oscillation, LCO) aus. Diese Flatterphänomene treten u. a. bei transsonischer Anströmung auf und werden in 2.2.3 näher behandelt.

Am Flattervorgang sind im Allgemeinen mehrere Freiheitsgrade des aeroelastischen Systems beteiligt, wobei für eine physikalisch anschauliche Erklärung bereits ein generisches Tragflügelmodell mit den zwei Freiheitsgraden Schlag- und Drehung ausreichend ist, wie es auch experimentell in der vorliegenden Arbeit betrachtet wird (vgl. 3.2.2 und 7). In Abb. 2.12 ist die Energiebilanz eines Tragflügels dargestellt, der für zwei kinematische Phasenbedingungen gekoppelte Schlag- und Drehschwingungen ausführt. Dabei können für kleine reduzierte Frequenzen die aerodynamischen Kräfte als quasistationär angenommen werden (vgl. $C\left(\omega^{*}=0\right)$ in Abb. 2.10), d.h. der Auftrieb folgt unmittelbar der Drehbewegung. In Abb. 2.12(a) sind beide Bewegungen $h$ und $\alpha$ in Phase. Jeweils zwischen Nulldurchgang und Umkehrpunkt der Bewegung sind Auftriebskraft und Bewegungsrichtung des Flügels gleichgerichtet. Es wird also positive Arbeit verrichtet, die Strukturschwingung entzieht der Strömung Energie. Zwischen 


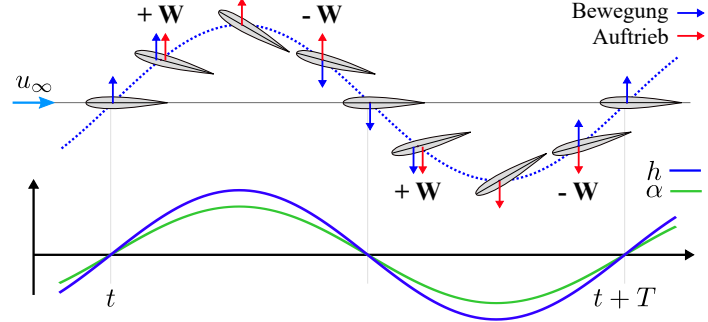

(a) Ausgeglichene Energiebilanz über eine Periode

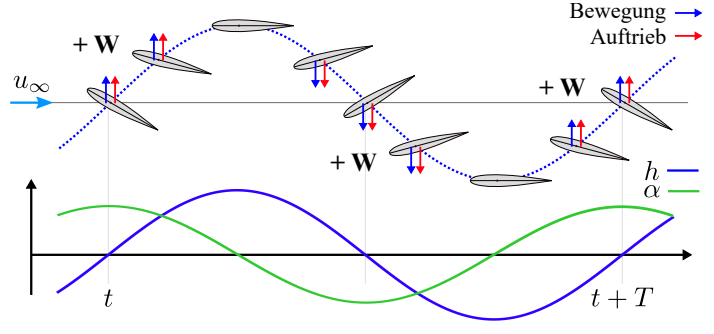

(b) Positive Energiebilanz über eine Periode

Abbildung 2.12.: Schematische Darstellung der Energiebilanz eines schwingenden Tragflügels mit den zwei Freiheitsgraden Schlag und Drehung für zwei verschiedene Phasenbeziehungen zwischen $h$ und $\alpha$. In (a) beträgt $\Delta \varphi_{\alpha, h}=0^{\circ}$, in (b) gilt $\Delta \varphi_{\alpha, h}=90^{\circ}$.

Umkehrpunkt und Nulldurchgang sind jedoch Kraft- und Bewegungsrichtung entgegengesetzt und der Schwingung wird Energie entzogen. Die Energiebilanz über eine Periode $W_{\mathrm{Per}}=\frac{1}{T} \cdot \int_{t}^{t+T} W(t) \mathrm{d} t$ ist gleich null und das aeroelastische System stabil. In Abb. 2.12(b) eilt hingegen die Drehbewegung dem Schlag um $\Delta \varphi_{\alpha, h}=90^{\circ}$ voraus. Hierdurch sind Bewegungsrichtung und Auftrieb über die gesamte Schwingungsperiode gleichgerichtet und es wird kontinuierlich Arbeit am System verrichtet, $W_{\text {Per }}>0$. Das Resultat ist eine selbsterregte Schwingung, die als „klassisches“ Biege-Torsionsflattern bezeichnet wird.

Abb. 2.12 stellt, u. a. durch die quasistationäre Annahme der aerodynamischen Kräfte eine Vereinfachung dar. Die physikalische Bedeutung der Energiebilanz bleibt jedoch erhalten, auch im Falle nichtlinearer Flatterphänomene. Für die Vollständigkeit sind lediglich neben den kinematischen Phasenbeziehungen zwischen $h$ und $\alpha$ auch die jeweiligen Phasenbeziehungen zu den aerodynamischen Kräften und deren Amplituden zu beachten, was die Bedeutung der in 2.2.1 angesprochenen essentiellen Aspekte der instationären Aerodynamik unterstreicht. Stellen sich untereinander Systembeziehungen derart ein, dass $W_{\text {Per }}>0$ gilt, liegt eine Instabilität vor. Flattern wird also durch das Zusammenspiel von Phasen- und Amplitudenbeziehungen zwischen Bewegung und den daraus induzierten aerodynamischen Käften gesteuert. Die hieraus resultierenden Energiebilanzen „steuern und regulieren" das aeroelastische System als selbsterregten Oszillator.

Mathematisch stellt das Flatterphänomen ein Eigenwertproblem dar. Die aeroelastische Bewegungsgleichung geht mit dem harmonischen Ansatz $q=\hat{q} \exp (\lambda t)$ über in

$$
\mathbf{M} \ddot{q}+\mathbf{D} \dot{q}+\mathbf{K} q=\mathbf{L}\left(\ddot{q}, \dot{q}, q, \omega^{*}\right) \stackrel{q=\hat{q} \exp (\lambda t)}{\rightarrow}\left[\lambda^{2} \mathbf{M}+\lambda \mathbf{D}+\mathbf{K}+\mathbf{A}\left(\omega^{*}\right)\right] \hat{q}=0 .
$$

Für dieses komplexe Eigenwertproblem werden nichttriviale Lösungen gesucht (vgl. 2.2). Dabei beschreibt $\mathbf{M}$ die Massenmatrix, $\mathbf{D}$ die (viskose) Dämpfungsmatrix, $\mathbf{K}$ die Steifigkeitsmatrix und $q$ den Vektor der generalisierten Koordinaten. Die aerodynamischen 
Kräfte $\mathbf{L}$ gehen in die Luftkraftmatrix $\mathbf{A}$ ein. Der Realteil der resultierenden komplexen Eigenwerte $\lambda$ kann als Dämpfung und der Imaginärteil als Frequenz der jeweiligen Systemfreiheitsgrade interpretiert werden (vgl. [8, S.417] und [128, S. 108]). Als schwierig gestaltet sich jedoch die implizite Abhängigkeit der Luftkräfte von der reduzierten Frequenz $\omega^{*}$ (vgl. 2.2.1), so dass eine Lösung von Gl. (2.2.13) nur iterativ bei einer Vorgabe von $\omega^{*}$ möglich ist. Verschiedene Lösungsverfahren existieren hierfür, welche unter anderem in [128, S.109 ff.] und [129, S.130 ff.] genauer erläutert sind.

Die Ergebnisse einer Flatteranalyse werden im Allgemeinen in einem Frequenz- und Dämpfungsdiagramm über der Anströmungsgeschwindigkeit $u_{\infty}$ dargestellt, welche aus den berechneten Eigenwerten resultieren [128, S.125 ff.]. Für ein generisches aeroelastisches System eines Tragflügels mit den Freiheitsgraden Schlag und Drehung sind in Abb. 2.13 beispielhafte Diagramme dargestellt. Wie Abb. 2.13(a) zeigt, nähren sich die

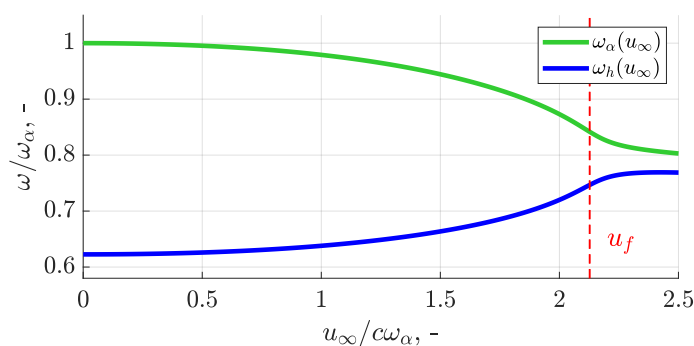

(a) Frequenzverlauf

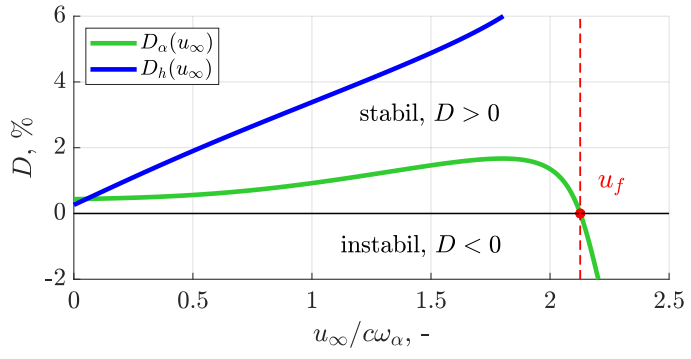

(b) Dämpfungsverlauf

Abbildung 2.13.: Beispielhafte Frequenz- (a) und Dämpfungsverläufe (b) für ein generisches aeroelastisches System mit zwei Freiheitsgraden. Die dargestellten Ergebnisse basieren auf einem inkompressiblen Zeitbereichsverfahren [130].

beiden Eigenfrequenzen $\omega_{h}$ und $\omega_{\alpha}$ mit steigender Strömungsgeschwindigkeit an. Dies begründet sich insbesondere durch den Einfluss der aerodynamischen Steifigkeit, welche eine geschwindigkeitsabhängige Frequenzänderung zur Folge hat. Ferner verdeutlicht sich eine zunehmende aerodynamische Kopplung der Freiheitsgrade. Die resultierende Schwingungsform des aeroelastischen Systems, insbesondere die Flatterschwingung ist folglich eine Kombination (komplexe Linearkombination [8, S.417]) der beteiligten Moden (Ausnahme: Ein-Freiheitsgrad-Flattern ${ }^{13}$ ). Die in Abb. 2.13(b) dargestellten Dämpfungsverläufe der Freiheitsgrade $D_{h}$ und $D_{\alpha}$ zeigen hingegen ein divergentes Verhalten zueinander. Die Dämpfung des Schlags nimmt kontinuierlich zu, wohingegen die Dämpfung der Drehung erst moderat anwächst und dann abrupt abfällt. $D_{\alpha}$ weist für $u_{\infty}=u_{f}$ einen Nulldurchgang auf, wobei $D=0$ den stabilen $(D>0)$ vom instabilen

\footnotetext{
${ }^{13}$ Bei einer Potentialströmung lässt sich zeigen, dass reine Schlagschwingungen stets aerodynamisch gedämpft sind [109, S.485]. Reine Torsionsschwingungen können hingegen nur instabil werden, wenn die Drehachse vor dem c/4-Punkt des Tragflügels liegt [109, S.487]. Dies ist bei praktischen Anwendungen im Allgemeinen nicht gegeben. In transsonischer Anströmung (und auch unterkritischer, subsonischer Anströmung mit Ablösung [30-32]) verhält sich dies anders. Hier kann es auch unter physikalisch/technisch relevanten Randbedingungen zum Ein-Freiheitsgrad-Flattern kommen [131].
} 
$(D<0)$ Bereich abgrenzt. Der Nulldurchgang definiert somit den Flatterpunkt und $u_{f}$ die Flattergeschwindigkeit. Oberhalb $u_{f}$ kommt es zum selbsterregten Aufschwingen des Tragflügels, was infolge des steil abfallenden Dämpfungsdurchgangs mit einer schnell anwachsenden Amplitude einhergeht und als „moderates" oder gar „explosives" Flattern bezeichnet wird [132]. Ein flach verlaufender Nulldurchgang würde hingegen mit einem „milden“ Flatterfall korrespondieren.

\subsubsection{Aeroelastische Instabilitäten unter transsonischer Strömung}

Unter transsonischer Anströmung behalten die in den vorherigen Abschnitten 2.2.1 und 2.2.2 beschriebenen grundlegenden Aspekte, wie das Auftreten von Phasen- und Amplitudenverschiebungen der instationären aerodynamischen Kräfte sowie die energetische Betrachtung des Flatterphänomens, ihre physikalische Bedeutung bei. Ein entscheidender Unterschied der Transonik besteht jedoch darin, dass das instationäre Strömungsfeld nicht mehr unabhängig von der stationären Strömung betrachtet werden kann [9, S.19]. Transsonische Strömungen und die transsonischen aerodynamischen Kräfte sind inherent nichtlinear [5-7]. Dies hat zur Konsequenz, dass eine Linearisier- und Superponierbarkeit der Luftkräfte und Moden, wie dies in 2.2.1 und auch zur Lösung von Gl. (2.2.13) angenommen wird, nicht mehr gültig ist [7]. Lediglich für kleine Amplituden ist dies möglich [8, S.419]. Die Nichtlinearitäten begründen sich nicht zuletzt in dem Auftreten von Verdichtungsstößen und einer Stoß-Grenzschicht-Interaktion (vgl. 2.1.3) [6], woraus für die Aeroelastik zwei wichtige Konsequenzen folgen; der Transonic Dip und die nichtlineare aeroelastische Systemreaktion. Dies ist schematisch in Abb. 2.14 dargestellt.

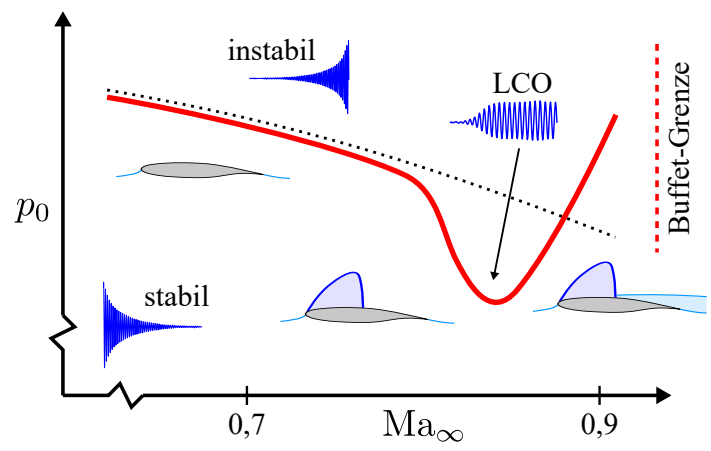

(a) Transonic Dip

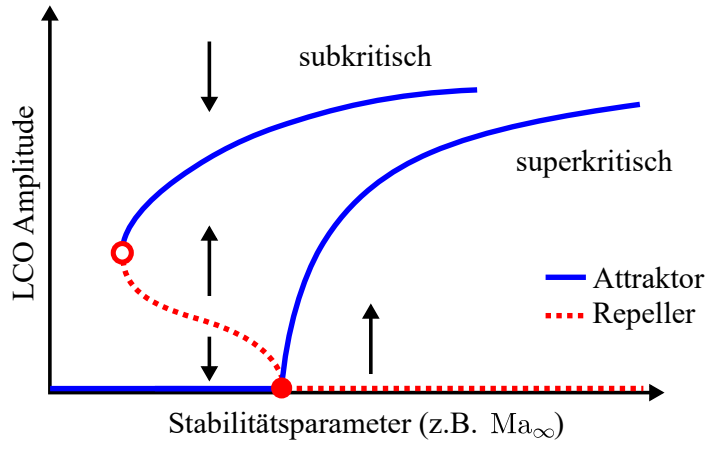

(b) Nichtlineare aeroelastische Systemreaktion

Abbildung 2.14.: Schematische Darstellung des Transonic Dip und charakteristische Strömungsfelder in (a), verändert nach [28], [133, S.256 f.], [8, S.419] und [134]. In (b) ist die typische nichtlineare Reaktion eines aeroelastischen Systems in Kombination mit LCO's dargestellt, verändert nach [63].

Die erste Konsequenz verdeutlicht sich bei einem Auftragen der Flattergrenze über der Mach-Zahl Ma $a_{\infty}$ und dem Ruhedruck $p_{0}$, was in Abb. 2.14(a) gezeigt ist. Hierbei fällt 
ein deutlicher Einbruch der Stabilitätsgrenze auf, was als Transonic Dip bezeichnet wird. Linearisierte aerodynamische Methoden (gestrichelte schwarze Linie in Abb. 2.14(a)) sind nicht in der Lage eine korrekte Vorhersage dieser transsonischen Flattergrenze zu treffen $[28,135]$. Eine vollständige Erklärung des Transonic Dip ist ein offenes Diskussionsthema [6], nicht zuletzt aufgrund des „ziemlich merkwürdigen“ Verhaltens der instationären Aerodynamik [9]. Eine essentielle physikalische Rolle spielt jedoch die Stoßbewegung [8, S.419], was anhand eines Flügels mit einem Drehfreiheitsgrad in Abb. 2.15 erläutert ist.
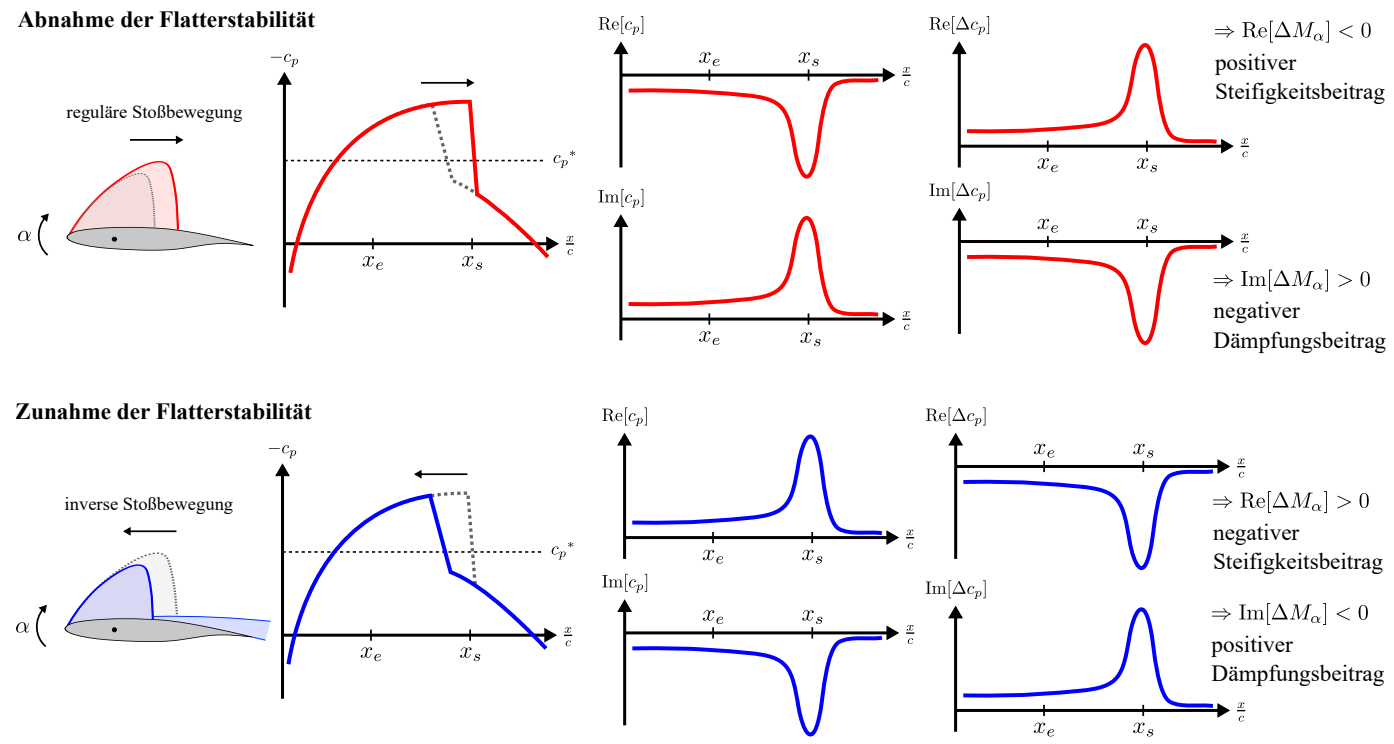

Abbildung 2.15.: Schematische Darstellung des Verhaltens der instationären Druckbeiwerte und den resultierenden Beiträgen zum instationären Nickmoment in Abhängigkeit der Stoßbewegung, verändert nach [8, S.419].

Bei einer regulären Stoßbewegung, wie diese bei anliegender Strömung und geringen transsonischen Mach-Zahlen (linke Seite des Dips) angenommen wird, wandert der Verdichtungsstoß beim aufdrehenden Tragflügel auf der Oberseite stromabwärts (Abb. 2.15 oben). Beim Zudrehen folgt eine Stoßbewegung stromaufwärts. Der instationäre Druckbeiwert (siehe 4.2.3) weist somit einen negativen Realteil $\operatorname{Re}\left[c_{p, \alpha}\right]<0$ (lokaler instationärer Druckabfall) um die Stoßposition $x_{s}$ herum auf. Dies geht mit einem positiven Imaginärteil $\operatorname{Im}\left[c_{p, \alpha}\right]>0$ einher. Der lokale instationäre Auftrieb, also die Druckdifferenz $\Delta c_{p}$ zwischen Ober- und Unterseite, hat entgegengesetzte Vorzeichen. Somit gilt $\operatorname{Re}\left[\Delta c_{p, \alpha}\right]>0$ (lokaler Auftrieb) und $\operatorname{Im}\left[\Delta c_{p, \alpha}\right]<0$ (Nacheilung des Auftriebs, vgl. 2.2.1). Liegt dieser Auftriebsanstieg hinter der Drehachse $x_{e}$, so resultiert ein zudrehender Anteil $\Delta M_{\alpha}$ zum instationären aerodynamischen Moment, also ein negativer Realteil $\operatorname{Re}\left[\Delta M_{\alpha}\right]<0$. Dieser trägt positiv zur aerodynamischen Steifigkeit bei. Der positive Imaginärteil $\operatorname{Im}\left[\Delta M_{\alpha}\right]>0$ wirkt hingegen negativ auf die aerodynamische Dämpfung ein und führt somit zur Abnahme der Flatterstabilität (linker, abfallender Ast des Transonic Dip). Für höhere Mach-Zahlen tritt hingegen eine Stoß-induzierte Ablösung auf (vgl. 2.1.3), die zu einer 
inversen Stoßbewegung führt (Abb. 2.15 unten). Dementsprechend drehen sich die Vorzeichen der Real- und Imaginärteile um. Als Ergebnis folgt $\operatorname{Re}\left[\Delta M_{\alpha}\right]>0$, entsprechend einer Abnahme der aerodynamischen Steifigkeit und $\operatorname{Im}\left[\Delta M_{\alpha}\right]<0$, was positiv zur aerodynamischen Dämpfung beiträgt. Dies führt zum Anstieg der Flattergrenze und begründet den rechten ansteigenden Ast des Dips.

Im Allgemeinen ist die Beschaffenheit des Transonic Dip nicht universell. Die Ausprägung und Position zeigt u. a. Abhängigkeiten vom Anstellwinkel [136], der Profildicke und der Temperatur (Beeinflussung des Massenverhältnis) [6,7,135]. Die zweidimensionale Darstellung der Flattergrenze wird daher auch auf eine drei- (oder mehrdimensionale) Flatter-Grenz-Fläche erweitert, um den Parametereinflüssen Rechnung zu tragen (siehe z. B. $[6,7,137])$. Neuere Untersuchungen haben des Weiteren die Bedeutung des Zustandes der Grenzschicht auf das Flatterverhalten und den Transonic Dip herausgestellt [20,23,24], wie auch die vorliegende Arbeit zeigt.

Als zweite Konsequenz führt die nichtlineare transsonische Aerodynamik zur Amplitudenbegrenzung der selbsterregten Flatterschwingungen und damit zum Auftreten von LCOs. Auch hier spielt die instationäre Stoßbewegung eine wesentliche Rolle, wobei ein Übergang der Stoßbewegung von Typ-A zu Typ-B nach TiJdeman [138] sowie eine Strömungsablösung an der Hinterkante mit der Amplitudenbegrenzung in Verbindung gebracht wird $[5,26,139]$. Die nichtlineare Systemreaktion wird dabei durch eine superoder subkritische Hopf-Bifurkation ${ }^{14}$ beschrieben [141], wie es in Abb. 2.14(b) dargestellt ist. Der superkritische Fall wird auch als „guter" LCO und der subkritische als „schlechter" LCO bezeichnet $[139,141,142]$. Letzteres resultiert aus dem potentiellen abrupten Anstieg der LCO-Amplitude [143] zwischen dem Hopf-Bifurkationspunkt (geschlossener roter Kreis in Abb. 2.14(b)) und dem Sattel-Knoten-Bifurkationspunkt (offener roter Kreis in Abb. 2.14(b), vgl. auch Sattel-Knoten Bifurkation von Zyklen [140, S.254 f.]). Des Weiteren folgt aus diesem Systemverhalten eine Hysterese bei einer Variation des Stabilitätsparameters $[63,141,141]$. Eine ausreichend große Störung, wie z. B. durch Böen, Wirbelschleppen oder starke Turbulenzen $[27,28]$ wäre ausreichend, um das System über den Repeller auf den oberen Attraktor zu überführen. Das aeroelastische System würde sich somit auch unterhalb der „klassischen“ Stabilitätsgrenze (entsprechend dem Hopf-Bifurkations-Punkt) zu einem LCO mit großen Amplituden aufschwingen. In einigen Fällen treten auch „geschachtelte“ LCOs $[26,144]$ bzw. Kombinationen von subkritischen und Sattel-Knoten Bifurkationen [145] auf. Dies lässt sich auch auf komplexere, nichtlineare Systemdynamiken erweitern [141,142], welche außerhalb der Thematik dieser Arbeit liegen.

\footnotetext{
${ }^{14}$ Für eine genaue Definition eines Limit Cycles sowie einer Hopf-Bifurkation sei an [140, S.198 ff. und
} S.252 ff.] verwiesen. 
LCOs können auch infolge struktureller Nichtlinearitäten des aeroelastischen Systems auftreten [142,146] und sind nicht nur auf den transsonischen Strömungsbereich beschränkt. Aerodynamische Nichtlinearitäten setzen in der transsonischen Aerodynamik i. A. schon bei deutlich kleineren Amplituden ein, als im Vergleich zur Sub- und Supersonik [147, S.194]. Dementsprechend sind die LCO-Amplituden vergleichsweise niedrig, was eine experimentelle Untersuchung überhaupt erst ermöglicht. Unter realen Randbedingungen würden auch bei zunächst exponentiell anwachsenden und als „klassisch“ bezeichneten Flatterfällen amplitudenbegrenzende aerodynamische Nichtlinearitäten auftreten, spätestens bei einer Ablösung der Strömung. Diese liegen jedoch zumeist außerhalb eines für die Struktur erträglichen Bereiches und entziehen sich somit auch einer experimentellen Beobachtung. Es sind somit Denkweisen naheliegend, die sowohl LCOs wie auch „klassisches“ Flattern als Bifurkation auffassen, wobei Flattern als instabiler LCO verstanden wird [148]. „Klassisches“ Flattern wird somit als eine Art „Grenzfall“ in die nichtlineare Welt eingebettet.

Für höhere Mach-Zahlen treten bei transsonischer Anströmung Buffet-Phänomene auf, wie die in Abb. 2.14(a) dargestellte Buffet-Grenze verdeutlicht. Buffeting umfasst eine Vielzahl dynamischer aeroelastischer Probleme, welche aus Strömungsabriss und Wirbelbildung resultieren [8, S.423]. Dabei wird transsonisches Buffeting im klassischen Sinne als dynamisches Antwortproblem gesehen (vgl. 2.2), also als erzwungene Schwingung der Struktur unter der instabilen Buffet-Strömung [149], bzw. der Einwirkung der Stoß-Grenzschicht-Interaktion (vgl. 2.1.3) und den daraus resultierenden oszillierenden aerodynamischen Kräften. Neuere Untersuchungen führen hingegen die physikalischen Mechanismen auf eine instabile „Fluid-Mode“ und deren mögliche Kopplung mit der Struktur zurück [149-151]. Hieraus ergeben sich neue Diskussionspunkte und Ansätze für die aeroelastische Problembetrachtung. 


\section{Experimenteller Aufbau}

Das folgende Kapitel beschreibt die Komponenten des experimentellen Aufbaus. Eine detailliertere Darlegung von einzelnen Aspekten und Methodiken, insbesondere der Berechnung von Strömungsparametern, der Messung von Strukturparametern und eine Diskussion von Ungenauigkeiten ist dem Anhang B zu entnehmen.

\subsection{Transsonischer Windkanal}

Die experimentellen Untersuchungen wurden im Transsonischen Windkanal Göttingen (TWG) durchgeführt, welcher von der Stiftung Deutsch-Niederländische Windkanäle (DNW) betrieben wird. Der Windkanal Göttinger Bauart, dargestellt in Abb. 3.1, ermöglicht kontinuierliche Anströmungen im subsonischen, transsonischen und supersonischen Geschwindigkeitsbereich. Für die Versuche wurde die adaptive Messstrecke mit

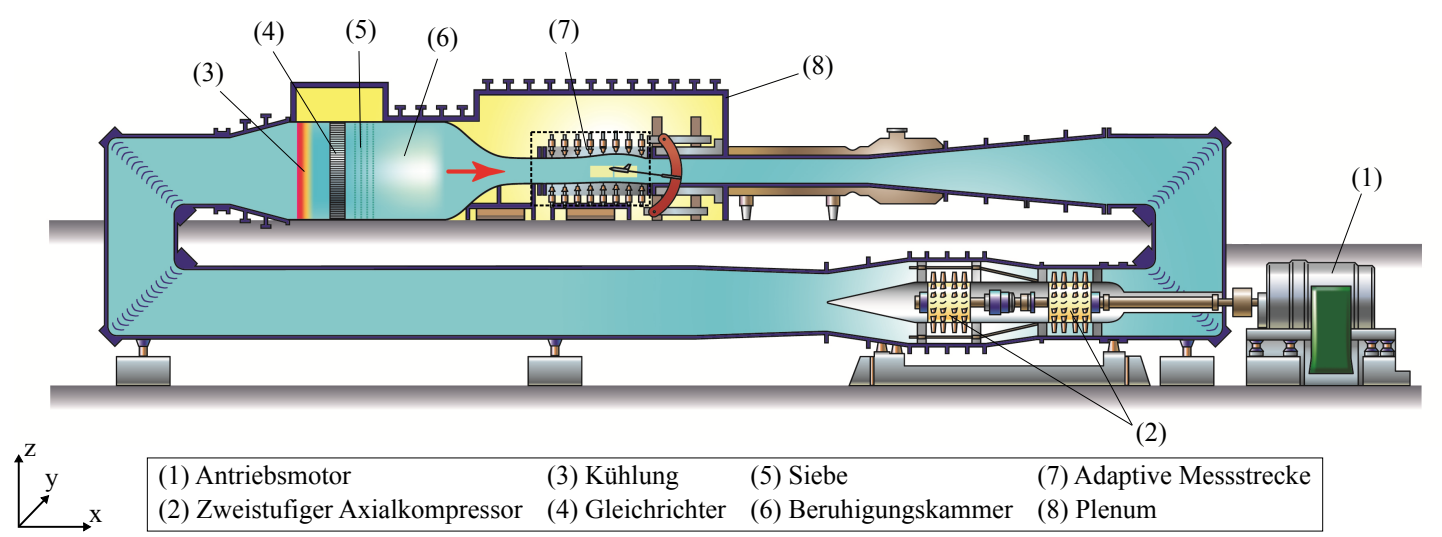

Abbildung 3.1.: Schematische Darstellung des DNW-TWG mit der adaptiven Messstrecke, verändert nach interner Kommunikation mit DNW. Bezogen auf das Windkanalkoordinatensystem erfolgt die Anströmung entlang der x-Achse.

einem Querschnitt von $1 \mathrm{~m} \times 1 \mathrm{~m}$ und einer Länge von 4,5 $\mathrm{m}$ eingesetzt. Es kann ein Mach-Zahl-Bereich von $0,3 \lesssim \mathrm{Ma}_{\infty} \lesssim 0,9$ abgedeckt werden. Die Messstrecke verfügt über eine adaptive Boden- und Deckenwand. Basierend auf Messungen der stationären Wanddrücke entlang der Mittellinie der Messtrecke wird mithilfe der CHAUCHY'schen 
Integralforme $^{1}$ in einem einstufigen Algorithmus [152] iterativ eine, den Stromlinien angepasste Wandkontur berechnet. Es wird eine zweidimensionale Adaption an das stationäre Strömungsfeld durchgeführt, um wandinduzierte Störungen zu minimieren ${ }^{2}$. Die Messstrecke des Windkanals befindet sich in einem abgeschlossenen Plenum, in welchem der Plenumsdruck $p_{K}$ und somit der Ruhedruck $p_{0}$ von $30 \mathrm{kPa}$ bis $150 \mathrm{kPa}$ variiert werden kann. Die Ruhetemperatur $T_{0}$ wird mithilfe eines Kühlers geregelt. Ein Trockner gewährleistet einen niedrigen Taupunkt, um Kondensation zu vermeiden. Basierend auf einer Bezugs-Flügeltiefe von $c=0,3$ m werden Reynolds-Zahlen von $0,5 \cdot 10^{6} \lesssim \operatorname{Re}_{\infty} \lesssim 5,4 \cdot 10^{6}$ erreicht. Die Mach-Zahl wird durch eine stufenweise Anpassung der Verdichter-Drehzahl und eine kontinuierlich regelbare Änderung des Diffusorwinkels stromab der Messstrecke in einer Größenordnung von $\mathcal{O}\left(10^{-3}\right)$ variiert. Eine Erläuterung zur Messung und Berechnung der Strömungsparamter sowie eine Diskussion der Messfehler findet sich in B.1 im Anhang.

\subsection{Versuchsstände}

Zur aerodynamischen und aeroelastischen Untersuchung des Laminarflügelprofils (siehe 3.3) wurden zwei Versuchsstände eingesetzt. Der Nickversuchsstand (NVS) ermöglichte die Charakterisierung der stationären und instationären Aerodynamik des Laminarflügels unter zwangserregten Nickschwingungen. Der Flatterversuchsstand (FVS) wurde zur Untersuchung von selbsterregten Schwingungen eingesetzt. Beide Versuchsstände waren jeweils in der adaptiven Messstrecke des DNW-TWG installiert.

\subsubsection{Nickversuchsstand für zwangserregte Profilschwingungen}

Der NVS des DLR - Instituts für Aeroelastik, dargestellt in Abb. 3.2, wurde für die aerodynamische Charakterisierung des Laminarflügelprofils eingesetzt. Der Versuchsstand besteht aus zwei hydraulischen Aktuatoreinheiten, welche auf einem Support außerhalb der Windkanalwände befestigt sind. Es können sowohl 2D-Modelle (beidseitiger Betrieb) wie auch 3D-Modelle (einseitiger Betrieb) untersucht werden. Die Aktuatoreinheiten setzen sich jeweils aus einem hydraulischen Drehzylinder, einer Falten- bzw. Ausgleichskupplung, einer Sicherheitssperre und einem Lager mit einer Vollwelle zusammen. Die Anbindung des Windkanalmodells erfolgt über piezoelektrische Waagen (siehe 3.4.6) sowie Balken. Letztere dienen als Zielfäche für Lasertriangulatoren (siehe 3.4.4) sowie zur Anbringung von Zusatzmassen zur Verschiebung des Schwerpunktes (vgl. auch 3.2.2).

\footnotetext{
${ }^{1}$ Dem Adaptionsvefahren liegt die Annahme einer zweidimensionalen Strömung zugrunde. Ferner wird angenommen, dass auftretende supersonische Strömungsgebiete lokal auf das Windkanalmodell beschränkt sind und die Strömung an den adaptiven Wänden subsonisch ist.

${ }^{2}$ Einflüsse der Seitenwände stellen dreidimensionale Störungen dar, welche durch die Adaption ebenfalls günstig beeinflusst, jedoch nicht vollständig minimiert werden.
} 
Der Versuchsstand ermöglicht es, dass Windkanalmodell für stationäre Untersuchungen bei einem konstanten Anstellwinkel $\alpha$ zu fixieren. Zur Untersuchung der instationären Aerodynamik kann das Windkanalmodell in zwangserregte Nickschwingungen $\alpha(t)=$ $\hat{\alpha} \cdot \sin (\omega t)+\bar{\alpha}$ versetzt werden. Die Hydraulikzylinder können zu diesem Zweck ein maximales Drehmoment von jeweils $1 \mathrm{kNm}$ bei einem Betriebsdruck von 280 bar erzeugen. Der mittlere Anstellwinkel kann in einem Bereich von $-50^{\circ} \lesssim \bar{\alpha} \lesssim+50^{\circ}$ variiert werden. Ebenso ist eine Schwingungsamplitude $\hat{\alpha}$ von bis zu $50^{\circ}$ möglich, welche jedoch mit zunehmender Schwingungsfrequenz $\omega$ begrenzt ist.

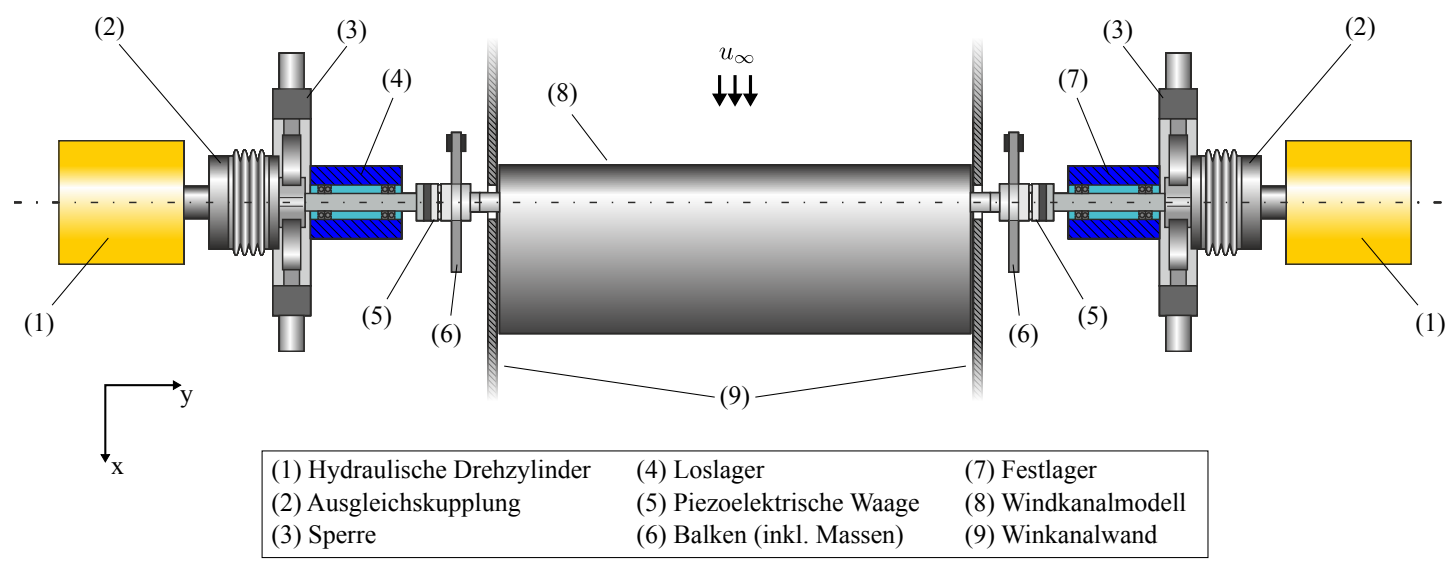

Abbildung 3.2.: Schematische Darstellung des NVS, verändert nach Zeichnung von T. Büte.

Die Sollbewegung des Modells wird mithilfe eines Funktionsgenerators an die Hydraulikregelung (Labtronic $8800 \mathrm{ml}$ von Instron) übergeben, welche die Servo-Ventile der Hydraulikzylinder steuert. Ein Abgleich der Bewegung erfolgt über jeweils ein Potentiometer pro Aktuatoreinheit. Die PID-Parameter der Regelung wurden auf die Trägheitskäfte des Windkanalmodells abgestimmt. Eigenschwingungen des Hydrauliksystems werden durch Druckölspeicher ausgeglichen. Im 2D-Betrieb sind beide Hydraulikzylinder synchronisiert. Eine Komparatorschaltung gleicht beide Potentiometer ab und schaltet die Dynamik bei einer Grenzwertüberschreitung (mögliche Torsion des Modells) aus.

\subsubsection{Flatterversuchstand für selbsterregte Profilschwingungen}

Zur Untersuchung der aeroelastischen Eigenschaften des Laminarflügelmodells wurde der FVS des DLR - Instituts für Aeroelastik eingesetzt. Eine schematische Darstellung ist in Abb. 3.3 gezeigt. Konzipiert für 2D-Windkanalversuche, setzt sich der Versuchsstand aus zwei gegenüberliegenden, identischen Federsystemen zusammen. Jedes dieser Federsysteme, dargestellt in Abb. 3.4(a), besteht aus einer Rechteck-Parallelfeder (vgl. [154, S. 495 f.]) und einem Torsions- bzw. Kreuzfedergelenk. Die Systeme sind außerhalb der Windkanalwände angebracht und über das Windkanalmodell miteinander verbunden. Die Federn sind somit parallel verschaltet. Die elastische Modellaufhängung generiert ein klassisches 


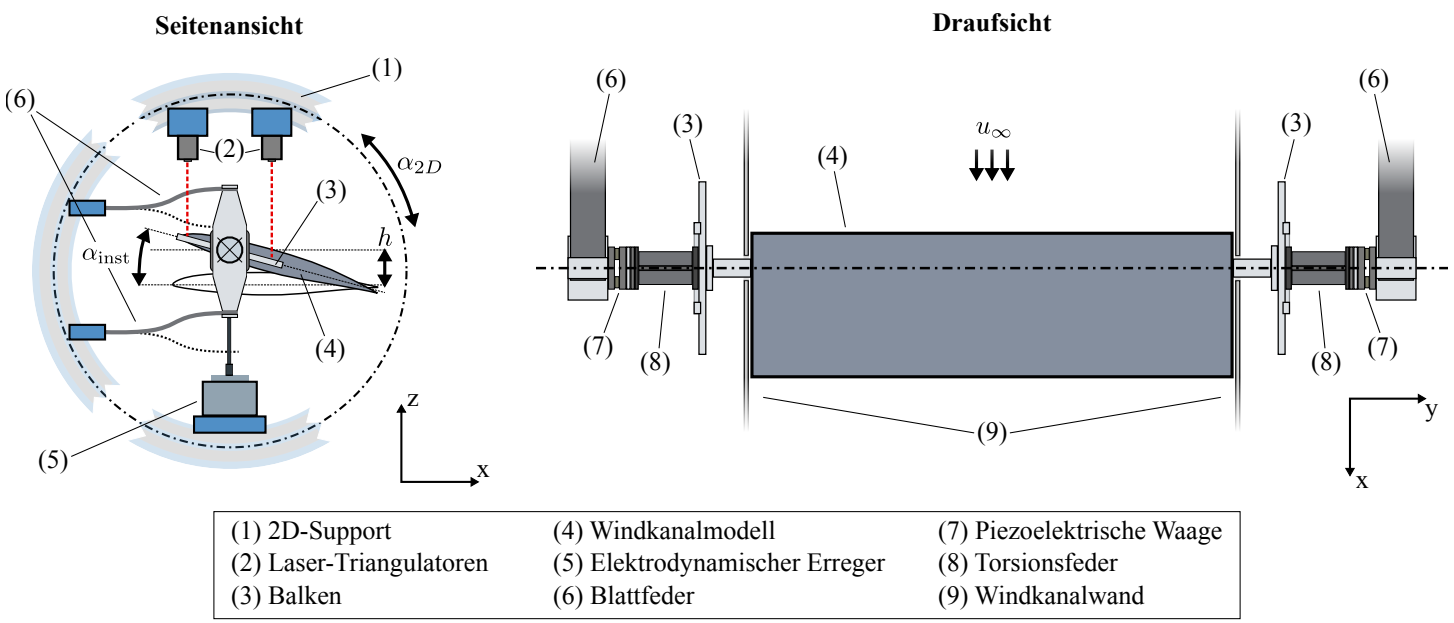

Abbildung 3.3.: Schematische Darstellung des FVS in unterschiedlichen Perspektiven, verändert nach [59] und basierend auf [26] und [153].

Biege-Torsions-Flattersystem [109, S. 488 f.] mit den zwei Starrkörper-Freiheitsgraden (engl.: Degree of Freedom, DoF) Schlag $h$ und Drehung $\alpha$. Die Federkonstante $k_{h}$ und Eigenfrequenz $f_{h}$ des Schlag-DoF kann durch eine Änderung der Blattfederlängen variiert werden, die Federsteifigkeit $k_{\alpha}$ und Eigenfrequenz $f_{\alpha}$ des Dreh-DoF durch einen Austausch der Kreuzfedergelenke. Ferner kann der Schlag mechanisch gesperrt werden, wie es im Rahmen der vorliegenden Arbeit u. a. erfolgte. Die aeroelastische 2-DoF Konfiguration wurde somit auf ein 1-DoF System mit einer Starrkörper-Drehung reduziert (vgl. 7.1). Zur optischen Messung der Modellbewegung sind außerhalb der Windkanalwände wiederum zwei Balken angebracht, die als Zielfäche für die Lasertriangulatoren (vgl. 3.4.4) dienen. Ebenso können zusätzliche Massen zur Änderung des Schwerpunkts und Trägheitsmomentes an die Balken angebracht werden.

Abhängig von den Strömungsparametern kommt es zu einer Änderung der aerodynamischen Lasten. Insbesondere eine Änderung des aerodynamischen Moments führt in Kombination mit der elastischen Aufhängung zu einer Verdrehung des Modells. Der Einstellwinkel des Windkanalmodells $\alpha_{0}$ (Anstellwinkel ohne Anströmung) verändert sich folglich. Zur Justierung des mittleren Anstellwinkels $\bar{\alpha}$ wird daher der FVS in den 2D-Support des DNW-TWG eingebaut, welcher in Abb. 3.4(b)) dargestellt ist. Der gesamte Versuchsstand kann hiermit um den Winkel $\alpha_{2 D}$ rotiert werden (siehe Abb. 3.3), um ein aeroelastisches Gleichgewicht für einen vorgesehenen Wert von $\bar{\alpha}$ einzustellen.

Die Bewegung des Windkanalmodells wird am FVS über zwei unabhängige Sicherheitssysteme kontrolliert. Zum einen sind auf beiden Seiten des Versuchsstands pneumatische Bremsen installiert. Diese können manuell oder automatisch geschlossen werden, sobald ein $\alpha$-Grenzwert überschritten wird. Zum anderen sind elektrodynamische Erreger (vgl. Abb. 3.3) mit den Schlagfedersystemen verbunden. Die Erreger werden durch 


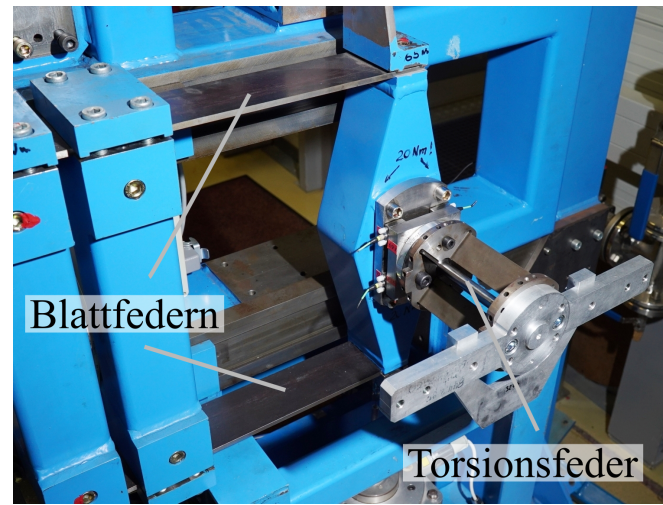

(a) Federsystem des FVS

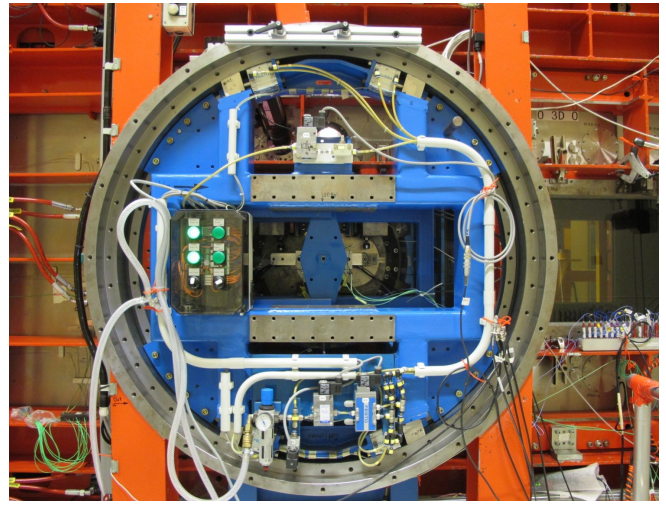

(b) 2D-Support des DNW-TWG

Abbildung 3.4.: Federsystem des FVS (a), bestehend aus zwei Blattfedern und einer Torsionsfeder, die über einen Schlagkasten und eine piezoelektrische Waage verbunden sind. Ostseite des FVS (b), eingebaut in den 2D-Support des DNW-TWG.

einen echtzeitfähigen Digital-Signal-Prozessor (DSP) angesteuert, der ein zur Schlaggeschwindigkeit $\dot{h}$ proportionales Spannungssignal ausgibt. Diese wird in Echtzeit aus den Signalen der Lasertriangulatoren (vgl. 3.4.4) berechnet. Eine Veränderung der Phase dieses Signals ermöglicht es, die Schlagbewegung anzuregen (in Phase) oder zu dämpfen $\left(180^{\circ}\right.$-Phasenversatz). Ist der Reglerkreis offen, wird das aeroelastische System nicht beeinflusst. Die Flatterregelung ist jedoch nur wirksam, sofern die auftretende Modellbewegung bei aeroelastischen Instabilitäten eine ausreichende Schlag-Komponente aufweist. Wird der Schlag gesperrt (1-DoF System), ist das Erregersystem wirkungslos. Weitere Informationen zur Flatterregelung finden sich in [27, 28].

Tabelle 3.1.: Strukturmechanische Parameter des FVS.

\begin{tabular}{llll}
\hline Parameter/ Beschreibung & Notation & Einheit & Wert \\
\hline Steifigkeit Schlagfedersystem & $k_{h}$ & $\mathrm{kN} / \mathrm{m}$ & $835,19 \pm 0,33$ \\
Steifigkeit Torsionsfedersystem & $k_{\alpha}$ & $\mathrm{Nm} / \mathrm{rad}$ & $6555,32 \pm 6,09$ \\
Masse $^{1}$ Schlagfedersystem & $m_{h, s}^{*}$ & $\mathrm{~kg}$ & $8,05 \pm 0,12$ \\
Trägheitsmoment $^{1}$ Torsionsfedersystem & $I_{\alpha, s}^{*}$ & $10^{-3} \mathrm{~kg} \cdot \mathrm{m}^{2}$ & $4,37 \pm 1,27$ \\
schlagschwingende Masse $^{2}$ & $m_{h, F V S}$ & $\mathrm{~kg}$ & $20,98 \pm 0,12$ \\
drehschwingendes Trägheitsmoment $^{2}$ & $I_{\alpha, F V S}$ & $10^{-3} \mathrm{~kg} \cdot \mathrm{m}^{2}$ & $26,92 \pm 1,28$ \\
\hline
\end{tabular}

${ }^{1}$ als generalisierte Masse/ Trägheitsmoment bestimmt (siehe Anhang B.2)

${ }^{2}$ bezogen auf gesamten Versuchsaufbau ohne das Windkanalmodell

Die strukturmechanischen Parameter des FVS wurden in separaten Messungen untersucht. So wurden zum einen die Massen und Trägheitsmomente sämtlicher schlag- und drehschwingender Komponenten gemessen, was in [155] dokumentiert ist. Die Federsteifigkeiten des FVS wurden durch statische Auslenkungen experimentell bestimmt. Die statische Linearität der Struktur wurde dabei nachgewiesen. Ferner wurden die Massen von fest verbauten schwingenden Komponenten durch eine Frequenzverstimmung als 
generalisierte Massen und Trägheitsmomente ermittelt. Die detaillierte Beschreibung der Methodik sowie ausführliche Ergebnisse der Untersuchungen sind in B.2 zu finden. Eine Zusammenfassung der relevanten Parameter ist in Tab. 3.1 enthalten. Weitere strukturmechanische Parameter wie Eigenfrequenzen, Eigenmoden und Dämpfungen wurden für die aeroelastischen Gesamtsysteme bestimmt (vgl. 7.1).

\subsection{Laminarflügelprofil CAST 10-2}

Gegenstand der Untersuchungen war das superkritische Flügelprofil CAST 10-2, welches aus einer Entwicklung von überkritischen Tragflügelprofilen für Verkehrsflugzeuge der Dornier GmbH in Zusammenarbeit mit der DFVLR-(AVA) Göttingen hervorging [156, 157]. Die Profil-Koordinaten sind u. a. in [158-161] zu finden. Neben den allgemeinen Entwursforderungen an ein superkritisches Profil ${ }^{3}$ wurde bei der Auslegung des CAST 10-2 ein möglichst kleines Nickmoment (geringes Rear Loading und Minimierung des Trimmwiderstands) sowie eine möglichst große Hinterkantendicke (Unterbringung eines Klappensystems) angestrebt. Die Profilgeometrie ist in Abb. 3.5 dargestellt. Das Profil weist eine maximale Dicke von $\frac{d}{c}=12,1 \%$ bei einer Dickenrücklage von $\frac{x_{d}}{c}=45 \%$, eine Hinterkanten-Dicke von $\left.\frac{d}{c}\right|_{H K}=0,5 \%$ und einen Hinterkantenwinkel von $\delta_{H K}=14^{\circ}$ auf $[156,161]$. Die Hinterkante selbst liegt unterhalb der Referenzlinie $\frac{z}{c}=0$ (vgl. Abb 3.5). Hieraus folgt ein Voreinstellwinkel von $0,88^{\circ}$ zwischen der Referenzlinie und der Profilsehne (definierte Verbindungslinie zwischen Vorderkante und der Mitte der Hinterkante) [165]. Als Bezugslinie (für Anstellwinkel, Profiltiefe, etc.) wird in der vorliegenden Arbeit jedoch die Referenzlinie (gestrichelte Linie in Abb. 3.5 oben) verwendet, entsprechend einem Einstellwinkel von $0^{\circ}$ in Abb. 3.5. Der theoretische Auslegungspunkt beträgt $\mathrm{Ma}_{\infty}=0,76$ bei $\alpha=0,3^{\circ}$ mit $c_{l}=0,595[158,166]$.

Frühere Windkanaluntersuchungen bestätigten eine ausgeprägte Sensitivität und starke Abhängigkeit der aerodynamischen Charakteristiken des CAST 10-2 Profils von der Reynolds-Zahl sowie der Lage des laminar-turbulenten Grenzschichtumschlags $[156,158,166]$. Diese Sensitivität ist bei kleineren Reynolds-Zahlen besonders ausgeprägt.

\footnotetext{
${ }^{3}$ Die Profilgeometrie des CAST 10-2 entspricht weitestgehend den Entwurfsforderungen eines klassischen superkritischen Profils. Zielsetzung hierbei ist ein wirtschaftlicher Reiseflug bei transsonischer Strömung und eine Verschiebung der Divergenz-Mach-Zahl (Drag-Rise-Grenze) zu höheren Flug-MachZahlen. Ferner gilt es Stoß-induzierte Instabilitätsprobleme (Buffet) zu vermeiden. Dies erfordert, neben der generellen Vermeidung von Grenzschichtablösung, eine Minimierung der Ausdehnung des supersonischen Geschwindigkeitsfeldes um das Tragflügelprofil in Verbindung mit einer möglichst geringen Kontraktionsrate des Überschallgebietes. Hieraus resultiert eine Abschwächung des Verdichtungstoßes und eine Reduzierung der durch den Stoß induzierten Energieverluste [162]. Aus diesen Anforderungen wurden Profilgeometrien entwickelt, welche sich im Vergleich zu konventionellen Profilen im Wesentlichen durch eine signifikante Reduzierung der Oberseitenkrümmung, eine Aufdickung der Vorderkante und eine zur Hinterkante verlagerte Wölbung auszeichnen [163,164].
} 
Ursächlich hierfür ist eine, abhängig von der Transitionslage auftretende Grenzschichtaufdickung und eine resultierende Änderung der effektiven Profilform. Dies geht einher mit einer starken Beeinflussung des Hinterkantendrucks und der Stoßlage [167]. Des Weiteren
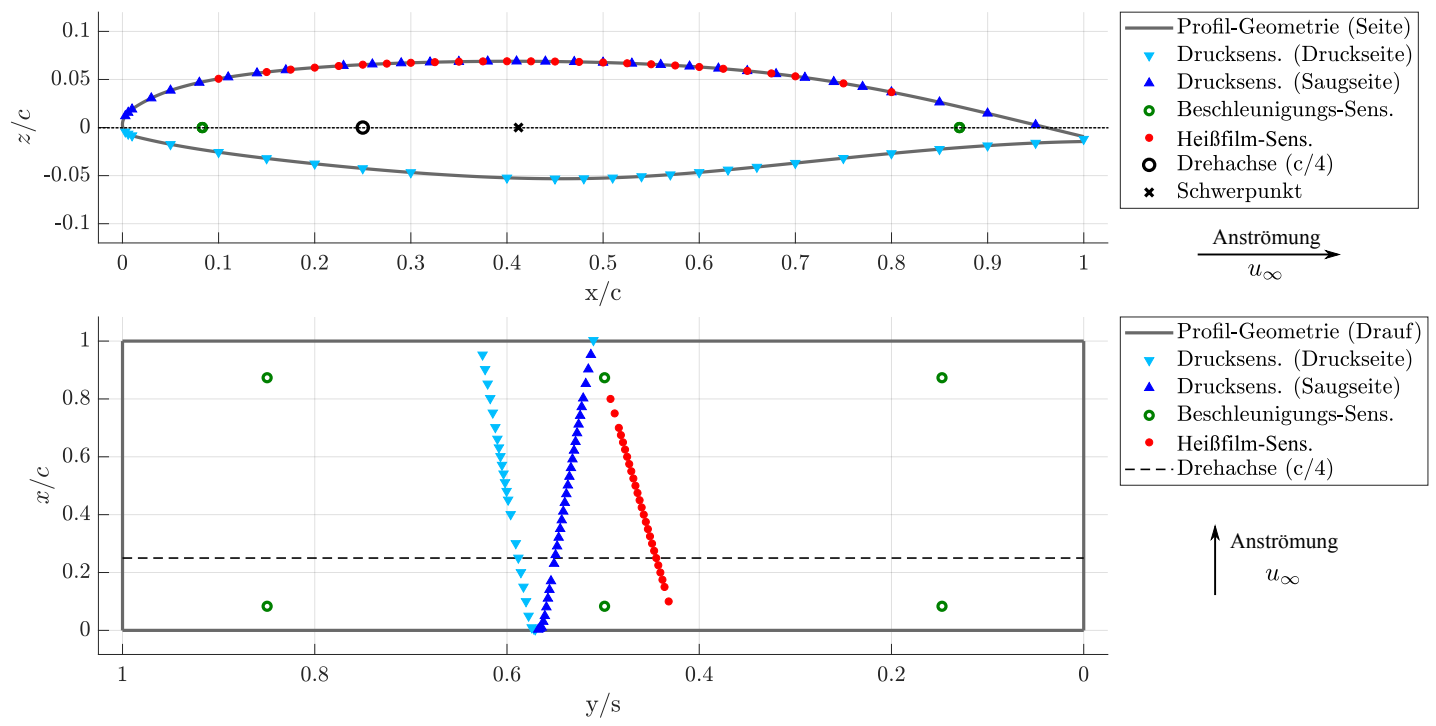

Abbildung 3.5.: Profilgeometrie des superkritischen CAST 10-2 Laminarflügels und Sensorverteilung des Windkanalmodells, verändert nach [59].

deuteten bereits frühere Winkanaltest bei Reynoldzahlen von $\operatorname{Re}_{\infty} \approx 2,4 \cdot 10^{6}$ im Bereich der Auslegungs-Mach-Zahl und natürlicher Transition die aerodynamischen Eigenschaften eines Laminarflügels (vgl. 2.1.2) an [158]. Untersuchungen im T2 Kryo-Windkanal der ONERA konnten die Laminarflügeleigenschaften des CAST 10-2 Profils auch für höhere Reynolds-Zahlen $\operatorname{Re}_{\infty} \lesssim 8 \cdot 10^{6}$ nachweisen $[168,169]$. Gemäß [170] wurde eine Laminardelle bei Messungen in der NAE High Reynolds Number Test Facility nahe der Design-Mach-Zahl des CAST 10-2 Profils sogar bei Reynolds-Zahlen bis $15 \cdot 10^{6}$ beobachtet. Messungen bei vergleichbaren Reynolds-Zahlen im transsonischen Kryo-Windkanal (TCT) der NASA, zusammengefasst in [171], zeigten lediglich eine Übereinstimmung der Ergebnisse bei fixierter Transition. Dies wurde auf einen höheren Turbulenzgrad des TCT im Vergleich zum T2 Kryo-Kanal zurückgeführt. Neuere numerische [20,23] und experimentelle Untersuchungen $[13,19,59]$ bei $\operatorname{Re}_{\infty} \approx 2 \cdot 10^{6}$ und transsonischer Strömung bestätigten die Laminarflügeleigenschaften des CAST 10-2 Profil und wiesen lange laminare Lauflängen und ausgedehnte Transitionsbereiche nach.

Das für die Messungen verwendete 2D-Windkanalmodell weist eine Flügeltiefe von $c=0,3 \mathrm{~m}$ und eine Spannweite von $s=0,997 \mathrm{~m}$ auf, entsprechend einer Flügelstreckung von $\Lambda=\frac{s}{c} \approx 3,3$. Dies entspricht zugleich dem ungefähren Verhältnis zwischen dem Abstand der Windkanalwände und der Flügeltiefe. Das Modell wurde in Schalenbauweise aus carbonfaserverstärktem Kunstoff (CFK) hergestellt. Zur Modellversteifung wurde bei $\frac{x}{c}=33 \%$ ein Holm einlaminiert. Die Anbindung an die Versuchsstände erfolgt über zwei 
Aluminium-Fußstücke. Der so definierte Drehpunkt bzw. die elastische Achse liegt bei $e=$ c/4. Die Weiteren strukturmechanischen Parameter des CAST 10-2 Windkanalmodells sind in Tab. 3.2 aufgelistet. Die Messung des Modell-Schwerpunkts $s_{M}$ sowie des ModellTrägheitsmomentes $I_{M}$ ist in [155] dokumentiert. Ferner wurde die Profilkontur des

Tabelle 3.2.: Strukturmechanische Parameter des CAST 10-2 Windkanalmodells.

\begin{tabular}{llll}
\hline Parameter/ Beschreibung & Notation & Einheit & Wert \\
\hline Masse & $m_{M}$ & $\mathrm{~kg}$ & $4,62 \pm 0,04$ \\
Schwerpunkt ${ }^{1}$ (hinter VK) & $s_{M}$ & $\mathrm{~mm}$ & $123,57 \pm 0,52$ \\
Trägheitsmoment (bzgl. $e$ ) & $I_{M_{e}}$ & $10^{-3} \mathrm{~kg} \cdot \mathrm{m}^{2}$ & $37,89 \pm 3,37$ \\
Trägheitsmoment (bzgl. $\left.s_{M}\right)^{2}$ & $I_{M_{s}}$ & $10^{-3} \mathrm{~kg} \cdot \mathrm{m}^{2}$ & $26,98 \pm 3,85$ \\
\hline
\end{tabular}

${ }^{1}$ entspricht $(41,19 \pm 0,17) \%$ der Flügeltiefe $c$

2 folgt aus dem Steiner'schen Satz [172, S. 146]

Windkanalmodells vermessen, da dieses bereits in vorherigen Messungen (ALF-3 [13,19] und ALF-4 [24]) zum Einsatz kam. Die Ergebnisse der Vermessung sind in B.3 dargestellt. Eine relevante Abweichung von der Modell-Sollkontur liegt nicht vor. Insbesondere sind keine scharfen Kanten und Unstetigkeiten auf der Oberseite des Modells vorhanden. Eine mögliche Störung der Grenzschichtströmung kann ausgeschlossen werden.

\subsection{Messtechnik und Sensorik}

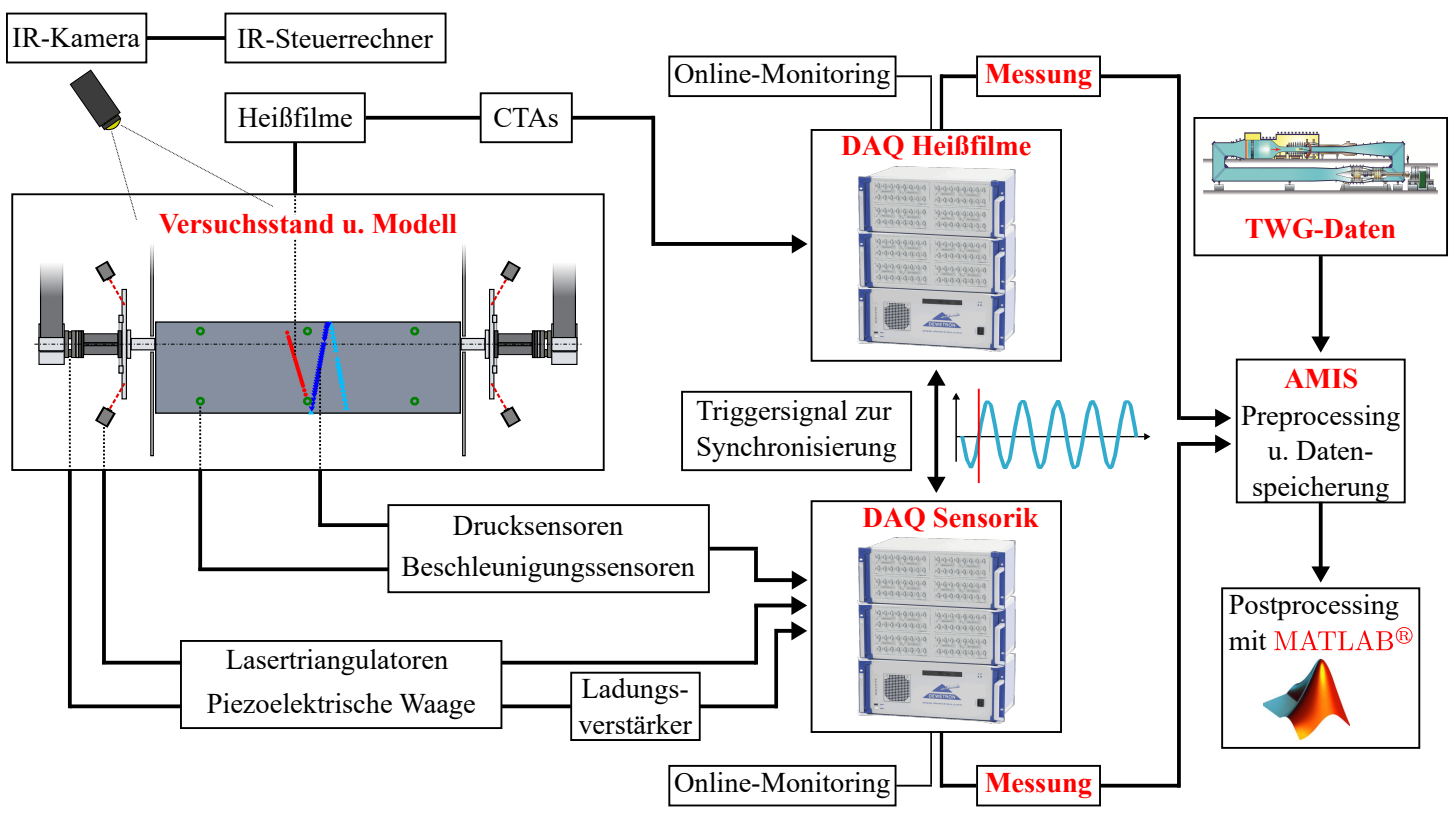

Abbildung 3.6.: Schematische Darstellung der Messtechnik und Datenerfassung. Einzelne Steuerungs- und Taktgebungsprozesse innerhalb der Messkette sind nicht dargestellt. 
Die Messtechnik und Datenerfassung ist für die durchgeführten Versuche und die verschiedenen Versuchsstände weitestgehend identisch. Eine schematische Übersicht ist in Abb. 3.6 dargestellt. Die einzelnen Komponenten sind nachfolgend detaillierter beschrieben, ebenso die umfangreiche Sensorik mit der das Windkanalmodell ausgestattet wurde. Die Verteilung der Sensoren ist in Abb. 3.5 dargestellt. Eine allgemeine Diskussion von Messungenauigkeiten ist in B.4 zu finden.

\subsubsection{Drucksensoren}

Das Windkanalmodell verfügt über 60 instationäre, temperaturkompensierte Druckdifferenzsensoren (Kulite XCQ-093D) mit einem Messbereich von $\pm 34,5 \mathrm{kPa}$. Hiermit erfolgte eine Messung der Druckverteilungen und eine sich anschließende Berechnung der aerodynamischen Beiwerte (siehe 4.2). Die Sensoren sind um den Mittelschnitt des Modells in einem Winkel von $10^{\circ}$ gegenüber der Anströmung angeordnet (vgl. Abb. 3.5). Eine gegenseitige Beeinflussung der Druckbohrungen durch die jeweilig induzierten Nachlaufströmungen wird so vermieden. Die Sensoren sind mithilfe von Halterungen in der Innenseite der Modellschalen direkt mit den Druckbohrungen $(\varnothing \approx 0,3 \mathrm{~mm})$ in den Modellschalen verbunden. Das Volumen zwischen Sensormembran und Druckbohrung ist auf ein Minimum reduziert, um eine Veränderung des Übertragungsverhaltens ${ }^{4} \mathrm{zu}$ vermeiden. Dennoch auftretenden Änderungen des dynamischen Verhaltens werden als vernachlässigbar klein angesehen (vgl. [174, S. 41 ff.]).

Die Drucksensoren wurden im Versuchsbetrieb täglich kalibriert. Hierfür wurden Kalibrierdrücke zwischen $\pm 21 \mathrm{kPa}$ auf die Referenzdruckseite aufgebracht. Die Sensorsensitivitäten wurden dann anhand einer kubischen Funktion bestimmt, womit auch eventuelle Abweichungen der Sensorlinearität berücksichtigt werden. Im Windkanalbetrieb wurde der statische Druck als Referenzdruck verwendet. So entspricht der gemessene Differenzdruck der Sensoren $\Delta p(x, t)$ direkt der Druckdifferenz zwischen $p_{\infty}$ und dem lokal vorliegenden Druck $p(x, t)$ an der Stelle $x$ des Windkanalmodells. Dies ermöglicht eine direkte Umrechnung in den dimensionslosen Druckbeiwert

$$
c_{p}(x, t)=\frac{\Delta p(x, t)}{q_{\infty}}=\frac{p(x, t)-p_{\infty}}{\frac{1}{2} \rho_{\infty} u_{\infty}^{2}}
$$

mit dem dynamischen Druck $q_{\infty}=\frac{1}{2} \rho_{\infty} u_{\infty}^{2}$, der Dichte $\rho_{\infty}$ und der Geschwindigkeit $u_{\infty}$ der ungestörten Anströmung (vgl. B.1). Die Genauigkeit der gemessenen Druckbeiwerte beträgt im für die Versuche relevanten Strömungsbereich $\sigma_{c_{p}} \leq 0,01$ (siehe B.4.1).

\footnotetext{
${ }^{4}$ Die Zuleitungen zwischen Sensoren und Druckbohrungen wirken wie ein Verzögerungsglied bzw. ein Filter. Ein Verfahren zur Signalkorrektur, basierend auf einer dynamischen Kalibrierung und Signal-Dekonvolution mit einer ermittelten Übertragungsfunktion, findet sich in [173, S. 57 f.]
} 


\subsubsection{Beschleunigungssensoren}

Im Bereich der Vorderkante $(x / c=0,0833)$ sowie der Hinterkante $(x / c=0,8733)$ sind sechs piezoelektrische Beschleunigungssensoren (PCB 352C22/ACS-97) im Windkanalmodell eingebaut (siehe Abb. 3.5). Die Beschleunigungssensoren haben eine Sensitivität von $1 \mathrm{mV} /\left(\mathrm{m} / \mathrm{s}^{2}\right)$ mit einem Messbereich von $\pm 4900 \mathrm{~m} / \mathrm{s}^{2}$. Der Frequenzbereich beträgt $1 \mathrm{~Hz}$ bis $10 \mathrm{kHz}$ bei einer Resonanzfrequenz $\geq 50 \mathrm{kHz}$ und einer Nichtlinearität von $\leq 1 \%$. Die Sensoren sind über die Modellspannweite gleichmäßig verteilt $(y / s=\{0,15 ; 0,5 ; 0,85\})$. Weitere (baugleiche) Beschleunigungssensoren waren an den Versuchsständen angebracht.

\subsubsection{Heißfilm-Anemometrie}

Zur Auflösung des Grenzschichtverhaltens ist auf der Saugseite des Windkanalmodells ein Heißfilm-Sensor-Array (engl.: multiple hot-film sensor, MHFS) installiert. Dieses besteht aus insgesamt 26 Heißfilm-Sensoren (siehe Abb. 3.5). Die Sensoren sind in Nähe des Mittelschnitts über eine Flügeltiefe von $\frac{x}{c}=10 \%$ bis $\frac{x}{c}=80 \%$ in einem Winkel von $10^{\circ}$ zur Anströmung verteilt. Letzteres dient der Vermeidung von gegenseitigen Störungen durch die Temperaturgrenzschichten, welche durch die konvektive Wärmeabfuhr im Nachlauf der einzelnen Heißfilm-Sensoren auftreten. Die Sensoren bestehen aus einer dünnen Nickelschicht $(1,45 \mathrm{~mm} \times 0,1 \mathrm{~mm} \times 0,2 \mu \mathrm{m})$ und sind auf einer $50 \mu \mathrm{m}$ dicken Polyimid-Folie aufgebracht. Die MHFS-Folie (Sonderanfertigung Senflex, Tao of Systems Integration Inc.) wurde in eine $100 \mu \mathrm{m}$ tiefe, entsprechend der Folie dimensionierte Aussparung in der Modell-Oberschale mit doppelseitigem Klebeband (Dicke $50 \mu \mathrm{m}$ ) eingeklebt. Verbliebene Spalte wurden verspachtelt und geschliffen, so dass ein glatter Übergang zwischen Modell und Folie entstand. Über verkupferte Leitungen auf der Folie sowie eine Verkabelung innerhalb des Modells wurden die Sensoren mit KonstantTemperatur-Anemometern (engl.: Constant Temperatur Anemometer, CTA) verbunden. Das MHFS Array kann als nicht-intrusiv angesehen werden. Jeder Sensor wurde in eine Wheatstones'sche Brückenschaltung integriert und mit einem Betriebswiderstand $R_{S} \approx 11,8 \Omega$ (Sensor-Nennwiderstand $R_{0} \approx 9,1 \Omega$ bei Umgebungstemperatur) auf eine Betriebstemperatur $T_{S}$ geregelt (Brücke im Gleichgewicht). Die Heißfilme wurden mit einem Überhitzungsverhältnis von $a=\frac{R_{S}}{R_{0}}=1,3$ betrieben, resultierend in einer Temperaturdifferenz von $\Delta T=T_{S}-T_{a} \approx 100 \mathrm{~K}$ zwischen Sensor- und Umgebungstemperatur $T_{a}$ [14,61]. Zur Gewährleistung konstanter Testbedingungen wurden die Widerstände bzw. das Überhitzungsverhältnis jedes einzelnen Sensors während des Versuchsbetriebes täglich überprüft und an die aktuellen Umgebungstemperaturen angepasst.

Infolge sich ändernder Grenzschichtströmungen kommt es zu einer Änderung des lokalen Wärmeübergangs (vgl. 3.4.5). Dies hat eine Änderung von $T_{S}$ bzw. $R_{S}$ zur Folge und die 
Brücke gerät aus dem Gleichgewicht. Die Anemometer-Elektronik stellt dieses Gleichgewicht durch eine Änderung der Brückenspannung $U$ sofort wieder her ${ }^{5}$. Dementsprechend korrelieren die Spannungssignale der CTAs mit der lokalen Wandschubspannung und lassen einen Rückschluss auf den Zustand der Grenzschicht zu. Die Brückenspannungen der 26 CTAs wurden aufgezeichnet und nach einer weiterführenden Datenverarbeitung (siehe 4.3 und $[14,61]$ ) zur Identifikation des stationären und instationären Grenzschichtverhaltens genutzt. Die Aufzeichnung wurde mit einem separaten Datenerfassungssystem mit einer Abtastfrequenz von $120 \mathrm{kHz}$ durchgeführt. Um diese hohe Abtastfrequenz auch vollumfänglich nutzen zu können, wurden die Sensoren vor jedem Messtag mit einem Rechteckwellen-Test abgeglichen. Hierbei wurde die Stabilität der CTA-Schaltungen überprüft und eine Grenzfrequenz von $\sim 60 \mathrm{kHz}$ eingestellt.

\subsubsection{Lasertriangulatoren}

Die (Starrkörper-) Bewegung des Windkanalmodells wurde mit vier Lasertriangulatoren (Flatterversuch: Micro-Epsilon optoNCDT 1605 mit LD 1605-20 und \pm 10 mm Messbereich; Nickversuch: Micro-Epsilon optoNCDT 1607 mit LD 1607-50 und $\pm 25 \mathrm{~mm}$ Messbereich) nicht-intrusiv gemessen. Jeweils zwei Triangulatoren pro Seite wurden stromauf und stromab der Drehachse an die Versuchsstände installiert. Als Ziel dienten dabei die mit den Modellfußstücken verbundenen Balken (vgl. 3.2).

Die Lasertriangulatoren arbeiten nach dem Prinzip der optischen Triangulation. Ein Laserpunkt wird an einer Zielfläche gestreut. Das reflektierte Licht wird mithilfe einer Linse auf eine Positions-Sensitive-Diode fokussiert. Diese liefert ein zum Ziel-Abstand direkt proportionales, analoges Spannungs- bzw. Abstandssignal $d_{L_{i}}$. Hieraus berechnet sich dann die Schlagbewegung $h(t)$ des Windkanalmodells zu

$$
h(t)=\frac{h_{\mathrm{ost}}(t)+h_{\mathrm{west}}(t)}{2} \quad \text { mit } \quad h_{\mathrm{ost} / \mathrm{west}}(t)=\frac{d_{L_{1 / 3}}+d_{L_{2 / 4}}}{2} .
$$

Der zeitabhängige Anstellwinkel bzw. die Nickbewegung des Modells $\alpha(t)=\bar{\alpha}+\alpha_{\text {inst }}(t)$ setzt sich aus einem mittleren Anstellwinkel $\bar{\alpha}$ und einem instationären Winkelanteil $\alpha_{\text {inst }}(t)$ zusammen. Letzterer wird wiederum aus den Lasersignalen gemäß

$$
\alpha_{\text {inst }}(t)=\frac{\alpha_{\text {ost }}(t)+\alpha_{\text {west }}(t)}{2} \quad \text { mit } \quad \alpha_{\text {ost } / \text { west }}(t)=\tan ^{-1}\left(\frac{d_{L_{2 / 4}}-d_{L_{1 / 3}}}{\Delta x_{L}}\right)
$$

berechnet, wobei $\Delta x_{L}$ den Abstand der Laserzielpunkte der jeweiligen Seite beschreibt. Der mittlere Anstellwinkel $\bar{\alpha}$ besteht aus dem Einbauwinkel $\alpha_{0}{ }^{6}$ und dem Einstellwinkel

\footnotetext{
${ }^{5}$ Sofern die Brückenverstärkung ausreichend groß ist, wird das Gleichgewicht instantan wieder hergestellt, so dass $T_{S} \approx$ const. ( $\rightarrow$ Constant Temperature Anemometry, CTA).

${ }^{6}$ Der Winkel beim Einbau des Windkanalmodells wurde mit einer Profilschablone und einem Clinotronic Neigungsmessgerät bestimmt (siehe B.4.2).
} 
des Modells in dem jeweiligen Versuchsstand. Im NVS wird $\bar{\alpha}$ über die Hydraulikregelung eingestellt und von den Lasertriangulatoren erfasst, so dass diese direkt $\alpha(t)$ messen (vgl. 3.2.1). Im FVS messen die Laser nur den instationären Anteil. Der mittlere Anstellwinkel wird über den Einbauwinkel $\alpha_{0}$ und den Winkel des 2D-Supports $\alpha_{2 D}$ bestimmt (vgl. 3.2.2). Der zeitabhängige Anstellwinkel folgt dann aus der Addition der Winkelanteile. Dementsprechend ist auch die Messgenauigkeit unterschiedlich. Bei Flatterversuchen wird der Messfehler des Anstellwinkels mit $\sigma_{\alpha}=0,09^{\circ}$ abgeschätzt. Bei Nickversuchen ist der Fehler mit $0,07^{\circ}$ etwas geringer. Für das Schlagsignal wird ein Fehler von $\sigma_{h}=4 \cdot 10^{-5} \mathrm{~m}$ abgeschätzt. Die Verzögerungszeit der Lasertriangulatoren liegt in der Größenordnung $<\mathcal{O}\left(10^{-4}\right)$ s [175]. Fehler infolge einer Phasenverschiebung sind vernachlässigbar.

\subsubsection{Infrarotthermographie}

Die Grenzschicht der Saugseite des Windkanalmodells wurde zusätzlich mit einer hochauflösenden Langwellen-Infrarot (LWIR) Wärmebildkamera (FLIR A655sc) beobachtet. Basierend auf der Reynolds-Analogie besteht zwischen dem lokalen Wärmeübergang und der lokalen Wandschubspannung ein Zusammenhang. In Abhängigkeit vom Grenzschichtzustand kommt es zu einer Änderung der Wandschubspannung und somit auch zu einer Änderung des Wärmeübergangs. Die Transition von einer laminaren Grenzschicht (geringere Wandschubspannung $\rightarrow$ geringerer Wärmeübergang) zu einer turbulenten Grenzschicht (höhere Wandschubspannung $\rightarrow$ höherer Wärmeübergang) wird im Bild der LWIR-Kamera durch auftretende Temperaturkontraste ersichtlich [176,177]. Die hierfür erforderliche Temperaturdifferenz zwischen Windkanalmodell und Strömung wurde durch eine Steuerung der Ruhetemperatur im Windkanal realisiert.

Die LWIR-Kamera wurde zur räumlichen Lokalisierung des laminar-turbulenten Grenzschichtumschlags unter stationären Strömungsbedingungen genutzt ${ }^{7}$. Zum anderen diente sie der Überprüfung der Heißfilm-Anemometer und zur Verifizierung der Wirksamkeit der aufgebrachten Transitionsstreifen zur Transitionsfixierung. Ferner konnten durch Modellverunreinigungen oder Mikroschäden der Modelloberfläche induzierte Turbulenzkeile identifiziert und lokalisiert werden, um die Ursachen zu beheben.

\subsubsection{Piezoelektrische Waage}

Mithilfe von zwei zwischen den Versuchsständen und dem Windkanalmodell integrierten piezoelektrischen Waagen (vgl. 3.2 und $[180,181]$ ) wurden die aerodynamischen Kräfte und Trägheitskräfte gemessen. Die Waagen wurden ausschließlich zur Überwachung der Gesamtlasten während des Versuchsbetriebes eingesetzt, um Belastungsgrenzen der

\footnotetext{
${ }^{7}$ Ein methodische Erweiterung zur Lokalisierung von Transitionsvorgängen unter instationären Strömungen mithilfe von LWIR-Thermographie ist in $[178,179]$ beschrieben.
} 
Mechanik und des Windkanalmodells nicht zu überschreiten. Zur eigentlichen Messung der aerodynamischen Kräfte wurden die Drucksensoren (vgl. 3.4.1) verwendet. Diese erfassen keine zusätzlichen Trägheitskomponenten und sind daher, insbesondere zur instationären Messung aerodynamischer Kräfte, besser geeignet.

\subsubsection{Datenerfassung und Aufbereitung}

Die Datenerfassung wurde mithilfe der AMIS III (Anlage zur Messung instationärer Signale) des DLR - Instituts für Aeroelastik durchgeführt. Wie in Abb. 3.6 dargestellt, kamen zwei getrennte Datenerfassungssysteme der Firma Dewetron zum Einsatz (Dewetron DEWE-818 Steuerrechner in Kombination mit DEWE-51-PCI-128 Frontends mit 24-bit Delta-Sigma Analog-Digital-Wandlern). Ein System wurde für die Erfassung der Heißfilm-Anemometer eingesetzt, ein weiteres System erfasste die Daten der Druck- und Beschleunigungssensoren sowie der Lasertriagulatoren und der piezoelektrischen Waagen. Die Systeme wurde jeweils redundant betrieben, so dass ein System zur Datenerfassung bzw. Messung eingesetzt wurde und ein gespiegeltes System zur Überwachung der Messsignale im Versuchsbetrieb diente. Zur zeitlichen Synchronisierung der Messsysteme wurde ein Generatorsignal als gemeinsames Triggersignal verwendet. Die Erfassung der Heißfilme wurde mit einer Abtastrate von $120 \mathrm{kHz}$ durchgeführt (vgl. 3.4.3). Die übrigen Signale wurden während des Flatterversuches mit 2,4 kHz abgetastet. Die Abtastung während des Nickversuches erfolgte phasenstarr mit 128 Punkten pro Periode. Die interne Steuerungsarchitektur und Taktgebung der Messysteme für eine phasenstarre Abtastung ist im Detail in [173, S. 55 f.] beschrieben. Kanal- und Strömungsdaten (TWG-Daten) wurden separat für jeden Messpunkt von Seiten des DNW zur Verfügung gestellt und direkt in der AMIS-Anlage verarbeitet (vgl. B.1). Die Strömungsgrößen $T_{0}, p_{0}, p_{K}$ und $p_{\infty}$ wurden zusätzlich mithilfe der Dewetron aufgezeichnet und gemäß den in B.1 angegebenen Zusammenhängen in weitere Strömungsgrößen umgerechnet. So wurden auch die zeitlichen Entwicklungen einzelner Strömungsparameter aufgezeichnet. Die hierfür eingesetzte Sensorik besitzt jedoch nicht die notwendige Frequenzauflösung, um eine vollständige instationäre Messung zu gewährleisten. Des Weiteren enthalten diese zeitabhängigen Strömungsgrößen keine windkanalspezifischen Korrekturen.

Sämtliche Signale werden in der AMIS-Anlage einem Preprocessing unterzogen, was u. a. eine Verrechnung mit Kalibrierungskoeffizienten und Sensitivitäten sowie eine Driftkorrektur und Nullpunktkompensation der Messdaten enthält. Darüber hinaus erfolgt bereits eine erste Verrechnung einzelner Messdaten und eine Datenspeicherung. Das weiterführende Postprocessing der Daten wird dann mit MATLAB ${ }^{\circledR}$ realisiert. Die eingesetzten Analyseverfahren und Methodiken sind in Kapitel 4 beschrieben. 



\section{Datenanalyse und Methodik}

Das folgende Kapitel enthält eine Beschreibung der Methodiken zur Analyse der experimentellen Daten. Die numerische Implementierung zur Anwendung auf diskrete Zeitsignale sowie ergänzende Informationen sind dem Anhang C zu entnehmen.

\subsection{Datenanalyse im Frequenzbereich}

Ein großer Teil der Datenanalyse erfolgt im Frequenzbereich. Die eingesetzten mathematischen Methoden sind im Folgenden dargestellt. Die numerische Umsetzung ist in C.1 und C.2 beschrieben, ergänzende Informationen sind des Weiteren in C.3 dargelegt.

\subsubsection{Fourier-Reihe und Fourier-Transformation}

Für die Signal-Transformation vom Zeit- in den Frequenzbereich wird eine FourierAnalyse durchgeführt. Eine periodische Funktion $f(t)=f(t+T)$ mit der Periodendauer $T$ und der Kreisfrequenz $\omega$ kann mithilfe der Fourier-Reihe als Summe von harmonischen Teilschwingungen dargestellt werden. In reeller Form

$$
f(t)=f_{0}+\sum_{n=1}^{\infty} \hat{f}_{n} \sin \left(n \omega t+\varphi_{n, i}\right) \quad \text { bzw. } \quad f(t)=f_{0}+\sum_{n=1}^{\infty} \hat{f}_{n} \cos \left(n \omega t+\varphi_{n, j}\right)
$$

beschreibt $f_{0}$ anschaulich den Mittelwert (Gleichanteil, DC-Wert) von $f(t)$ [182, S. 21] und die Summe der verbleibenden Terme den Wechselanteil (AC-Wert). Die FourierKoeffizienten $\hat{f}_{n}$ stellen die Amplitudenanteile der Grund- $(n=1)$ und Oberschwingungen $(n>1)$ bzw. höheren Harmonischen dar und bilden das diskrete Frequenzspektrum von $f(t)\left[183\right.$, S.13 f.]. Die Nullphasenwinkel $\varphi_{n, i}$ bzw. $\varphi_{n, j}$ unterschieden sich je nach Wahl der Fourier-Reihen Darstellung. Mithilfe der Euler'schen Formel $\exp (\mathrm{i} \omega t)=\cos (\omega t)+\mathrm{i} \sin (\omega t)$ lässt sich Gl. (4.1.1) in komplexer Form ausdrücken durch

$$
f(t)=\sum_{n=-\infty}^{\infty} \hat{f}_{n} \exp (\mathrm{i} n \omega t) \quad \text { mit } \quad \hat{f}_{n}=\frac{1}{T} \int_{0}^{T} f(t) \exp (-\mathrm{i} n \omega t) \mathrm{d} t .
$$


Führt man in Gl. (4.1.2) den Grenzübergang ${ }^{1} T \rightarrow \infty$ aus (siehe z.B. [184, S.350] oder [182, S.29 ff.]), lässt sich eine Fourier-Analyse auch für aperiodische Funktionen formulieren. Dies führt zur Fourier-Transformation (Vorfaktoren hier gemäß [184, S.350])

in den Zeitbereich:

$$
f(t)=\frac{1}{\sqrt{2 \pi}} \int_{-\infty}^{\infty} F(\mathrm{i} \omega) \exp (\mathrm{i} \omega t) \mathrm{d} \omega,
$$

in den Frequenzbereich: $\quad F(\mathrm{i} \omega)=\frac{1}{\sqrt{2 \pi}} \int_{-\infty}^{\infty} f(t) \exp (-\mathrm{i} \omega t) \mathrm{d} t$.

$F(\mathrm{i} \omega)=\mathcal{F}[f(t)]$ bezeichnet die Fourier-Transformierte ${ }^{2}$ von $f(t)$. Gl. (4.1.4) transformiert also die Funktion $f(t)$ aus dem Zeit- in den Frequenzbereich und liefert ein kontinuierliches Fourier- bzw. Frequenzspektrum (spektrale Amplitudendichte). Gl. (4.1.3) führt die Fourier-Transformation invers aus und bildet $F(\mathrm{i} \omega)$ wieder in den Zeitbereich $f(t)=$ $\mathcal{F}^{-1}[F(\mathrm{i} \omega)]$ ab.

Da Messsignale nun nicht als kontinuierliche Funktionen, sondern als diskrete, endliche Zeitreihen zur Verfügung stehen, wird für die numerische Berechnung der FourierTransformation auf die diskrete (DFT), bzw. auf die schnelle Fourier-Transformation (engl.: Fast-Fourier-Transformation, FFT) zurückgegriffen (siehe C.1).

\subsubsection{Korrelationsfunktionen und Leistungsdichtespektren}

Neben dem Fourierspektrum einer Funktion bzw. eines Zeitsignals ist auch die spektrale Leistungsdichte von Interesse. Hierfür sei zunächst für die Zeitsignale $x(t)$ und $y(t)$ die Autokorrelationsfunktion (AKF) [182, Anhang B S.24]

$$
r_{x x}(\tau)=\lim _{T \rightarrow \infty} \frac{1}{2 T} \int_{-T}^{T} x(t) \cdot x(t+\tau) \mathrm{d} t
$$

und die Kreuzkorrelationsfunktion (KKF) [182, Anhang B S.31]

$$
r_{x y}(\tau)=\lim _{T \rightarrow \infty} \frac{1}{2 T} \int_{-T}^{T} x(t) \cdot y(t+\tau) \mathrm{d} t
$$

definiert. Die Analogie zur Faltung $(*)$ ist gegeben durch $r_{x x}(\tau)=x(t) * x(-t)$ und $r_{x y}(\tau)=x(t) * y(-t)[185$, S.140 und S.170]. Nach dem WiEnER'schen Satz (auch WiEnERChintschin-Theorem) [185, S.145 f.] folgt durch Fourier-Transformation von Gl. (4.1.5) und Gl. (4.1.6) sowie Beachtung des Faltungssatzes $\mathcal{F}[f * g]=\mathcal{F}[f] \mathcal{F}[g][184$, S.353] das Autoleistungsdichtespektrum (engl.: Power-Spectral-Density, PSD)

$$
S_{x x}(\omega)=\int_{-\infty}^{\infty} r_{x x}(\tau) \cdot \exp (-\mathrm{i} \omega t) \mathrm{d} \tau=X(-\mathrm{i} \omega) \cdot X(\mathrm{i} \omega)=X^{*}(\mathrm{i} \omega) \cdot X(\mathrm{i} \omega)
$$

\footnotetext{
${ }^{1}$ Der Grenzübergang setzt voraus, dass $f(t)$ absolut integrierbar ist, also $\int_{-\infty}^{\infty}|f(t)| \mathrm{d} t<\infty$.

${ }^{2}$ Die Schreibweise $F(\mathrm{i} \omega)$ verdeutlicht die Verwandtschaft zur Laplace-Transformation (vgl. [182, S.31]).
} 
sowie das Kreuzleistungsdichtespektrum ${ }^{3}$ (engl.: Cross-Power-Spectral-Density, CPSD)

$$
S_{x y}(\omega)=\int_{-\infty}^{\infty} r_{x y}(\tau) \cdot \exp (-\mathrm{i} \omega t) \mathrm{d} \tau=X(-\mathrm{i} \omega) \cdot Y(\mathrm{i} \omega)=X^{*}(\mathrm{i} \omega) \cdot Y(\mathrm{i} \omega)
$$

$X^{*}(\mathrm{i} \omega)$ bezeichnet die komplex konjungierte Fourier-Transformierte von $x(t)$. Es gilt im Übrigen $S_{x x}(\omega) \in \mathbb{R}$, wohingegen $S_{x y}(\omega) \in \mathbb{C}$ und $S_{x y}(\omega)=S_{y x}(-\omega)=S_{y x}^{*}(\omega)$.

Sowohl das Auto- wie auch das Kreuzleistungsdichtespektrum sind für die Berechnung von Transferfunktionen (siehe 4.1.3) bzw. instationären aerodynamischen Beiwerten (siehe 4.2.3) essentiell. Auch die Darstellung von spektralen Signalanteilen erfolgt in der vorliegenden Arbeit stets als PSD. Die numerischen Berechnungen werden wiederum auf Basis der DFT/FFT mithilfe der WeLch-Methode [186, S.211 ff.] durchgeführt. Standardmäßig wird eine Hanning-Fensterfunktionen mit einer Überlappung von 62, 5 \% bei variabler Signalsegmentierung eingesetzt (siehe C.2).

\subsection{3. Übertragungsfunktion}

Bei einem linearen, zeitinvarianten System (LTI-System), wie es im Folgendem angenommen wird, ist das Ausgangssignal $y(t)$ bei beliebiger Anregung durch die Faltung des Eingangssignals $x(t)$ mit der Impulsantwort (Systemfunktion) des Systems $h(t)$ gemäß

$$
y(t)=x(t) * h(t)=\int_{-\infty}^{\infty} x(\tau) \cdot h(t-\tau) \mathrm{d} \tau
$$

verknüpft [182, S.76 f.]. Hierbei enthält $h(t)$ sämtliche Informationen des LTI-Systems bzgl. dessen Übertragungsverhaltens und beschreibt dieses vollständig. Wendet man nunmehr das Faltungstheorem an, so lässt sich Gl. (4.1.9) im Frequenzbereich durch eine Multiplikation der Fourier-Transformierten darstellen zu

$$
Y(\mathrm{i} \omega)=X(\mathrm{i} \omega) \cdot H(\mathrm{i} \omega) \quad \Leftrightarrow \quad H(\mathrm{i} \omega)=\frac{Y(\mathrm{i} \omega)}{X(\mathrm{i} \omega)} .
$$

Damit ist eine einfache Berechnung der Übertragungsfunktion ${ }^{4} H(\mathrm{i} \omega)$ durch den Quotienten der Fourier-Transformierten des Ausgangs- und Eingangssignals gegeben.

Messsignale sind jedoch i. d. R. mit Rauschen überlagert, so dass es sich empfiehlt, für die Berechnung der Übertragungsfunktion Gl. (4.1.10) mit $\frac{X^{*}(\mathrm{i} \omega)}{X^{*}(\mathrm{i} \omega)}$ zu erweitern. Ein

\footnotetext{
${ }^{3}$ Häufig wird das Kreuzleistungsdichtespektrum auch mit $S_{x y}=X \cdot Y^{*}$ berechnet, u. a. in der Software MATLAB ${ }^{\circledR}$. Es gilt dann die Relation $S_{x y}=S_{y x}^{*}$, was bei der Berechnung von Transferfunktionen (vgl. 4.1.3) berücksichtigt werden muss. Andernfalls ist das Vorzeichen des Imaginärteils und damit der Phasendifferenz (vgl. C.8) vertauscht.

${ }^{4}$ Streng genommen beschreibt $H(\mathrm{i} \omega)$ den Frequenzgang. Die Übertragungsfunktion $H(s)$ würde anstelle der Fourier- die Laplace-Transformation erfordern. Unter der Annahme, dass alle LaplaceTransformierten auf der $\mathrm{i} \omega$-Achse konvergieren $(\operatorname{Re}[s]=0)$, ist mit einer einfachen Substitution $s \leftrightarrow \mathrm{i} \omega$ der Frequenzgang $H(\mathrm{i} \omega)$ in die Übertragungsfunktion $H(s)$ überführbar und umgekehrt [182, S.80].
} 
Vergleich mit Gl. (4.1.7) und (4.1.8) zeigt dabei, dass die Übertragungsfunktion nunmehr aus dem Quotienten des Kreuz- und Autoleistungsdichtespektrums der Signale $x(t)$ und $y(t)$ besteht, also durch eine Korrelationsanalyse gemäß

$$
H_{1}(\mathrm{i} \omega)=\frac{X^{*}(\mathrm{i} \omega) \cdot Y(\mathrm{i} \omega)}{X^{*}(\mathrm{i} \omega) \cdot X(\mathrm{i} \omega)}=\frac{S_{x y}(\omega)}{S_{x x}(\omega)}
$$

berechnet wird. Dabei beschreibt Gl. (4.1.11) einem $\mathrm{H}_{1}$-Schätzer, welcher sich bei einem ungestörten Eingangssignal anbietet. Diese Zweckmäßigkeit von Gl. (4.1.11) gegenüber (4.1.10) ist ausführlicher in C.3 dargelegt. In der vorliegenden Arbeit werden Übertragungsfunktionen auf dieser Basis berechnet. Für die numerische Implementierung erfolgt wiederum ein Rückgriff auf die WelCH-Methode mit den in C.2 und C.3 genannten Spezifikationen sowie einer Mittelwertbildung über mehrere Signalsegmente.

Stellt man $H(\mathrm{i} \omega) \in \mathbb{C}$ durch den Realteil $\operatorname{Re}[H(\mathrm{i} \omega)]$ und Imaginärteil $\operatorname{Im}[H(\mathrm{i} \omega)]$ dar, führt dies zu dem Amplitudengang

$$
|H(\mathrm{i} \omega)|=\sqrt{\operatorname{Re}[H(\mathrm{i} \omega)]^{2}+\operatorname{Im}[H(\mathrm{i} \omega)]^{2}}
$$

und dem Phasengang

$$
\arg [H(\mathrm{i} \omega)]=\arctan \left(\frac{\operatorname{Im}[H(\mathrm{i} \omega)]}{\operatorname{Re}[H(\mathrm{i} \omega)]}\right)
$$

was die bevorzugten Darstellungsvarianten von $H(\mathrm{i} \omega)$ in der vorliegenden Arbeit sind.

\subsubsection{Integration und Differentiation im Frequenzbereich}

Aus den Eigenschaften der Fourier-Transformation [184, S.351 ff.] folgt für die FourierTransformation einer Ableitung $f^{\prime}(t)$ einer Funktion $f(t)$

$$
\mathcal{F}\left[f^{\prime}(t)\right]=i \omega \mathcal{F}[f(t)] \quad \Rightarrow \quad f^{\prime}(t)=\mathcal{F}^{-1}[i \omega \mathcal{F}[f(t)]]
$$

Gemäß Gl. (4.1.14) kann somit die Differentiation einer Funktion im Zeitbereich durch eine Multiplikation der Fourier-Transformierten mit $i \omega$ im Frequenzbereich und anschließende Rücktransformation in den Zeitbereich erfolgen. Somit ist auch die numerische Differentiation einer Zeitreihe mit Gl. (4.1.14) auf eine Berechnung der DFT/FFT (vgl. C.1) zurückzuführen. Des Weiteren folgt aus Gl. (4.1.14) auch, dass

$$
\mathcal{F}\left[f^{\prime}(t)\right]=i \omega \mathcal{F}\left[\int f^{\prime}(t) \mathrm{d} t\right] \Leftrightarrow \int g(t) \mathrm{d} t=\mathcal{F}^{-1}\left[\frac{1}{\mathrm{i} \omega} \mathcal{F}[g(t)]\right]
$$

mit $f^{\prime}(t)=g(t)$. Entsprechend kann auch die numerische Integration einer Zeitreihe auf eine Berechnung der Fourier-Transformierten bzw. DFT/FFT zurückgeführt werden. 


\subsection{Berechnung der aerodynamischen Beiwerte}

Nachfolgend ist die Berechnung der aerodynamischen Beiwerte und des aerodynamischen Energieeintrags dargelegt. Die numerische Implementierung ist in C.5 und C.7 dargestellt. Ergänzende Berechnungen sind in C.4 und C.6 beschrieben.

\subsubsection{Aerodynamische Beiwerte und stationäre Derivativa}

Die aerodynamischen Beiwerte werden durch eine Integration der gemessenen Druckbeiwerte $c_{p}$ (vgl. 3.4) entlang der Oberfläche des Windkanalmodells berechnet. Die resultierende Druckkraft ergibt sich somit zu

$$
\vec{F}(t)=\left(\begin{array}{c}
F_{A}(t) \\
F_{N}(t)
\end{array}\right)=-q_{\infty} c \oint c_{p}(t) \hat{n} \mathrm{~d} A
$$

mit dem Einheits-Normalenvektor $\hat{n}$ zum jeweiligen Flächenelement $\mathrm{d} A$ des Tragflügels. Für die Berechnung wird zunächst eine Interpolation der Profilkontur und Druckverteilung mit anschließender Diskretisierung vorgenommen (siehe C.4). Hierin begründet sich auch eine zusätzliche Multiplikation mit der Flügeltiefe $c$ in Gl. (4.2.1). Die Auswertung von Gl. (4.2.1) erfolgt dann ebenfalls in diskreter Form (siehe C.5). Die Berechnung wird im modellfesten Koordinatensystem durchgeführt. Zur Berücksichtigung des Anstellwinkels $\alpha(t)$ müssen die zur Profil-Bezugslinie (vgl. 3.3) horizontale Axialkraft $F_{A}$ und die vertikale Normalkraft $F_{N}$ ins Laborkoordinatensystem transformiert werden. Die Druckwiderstandskraft $F_{D}$ und die Auftriebskraft $F_{L}$ folgen damit zu

$$
\left(\begin{array}{c}
F_{D}(t) \\
F_{L}(t)
\end{array}\right)=\mathbf{D}(\alpha) \vec{F}(t) \quad \text { mit } \quad \mathbf{D}(\alpha)=\left(\begin{array}{cc}
\cos (\alpha) & \sin (\alpha) \\
-\sin (\alpha) & \cos (\alpha)
\end{array}\right) \text {. }
$$

Das aerodynamische Nickmoment $M_{\alpha}$ folgt analog zu Gl. (4.2.1) aus einer Multiplikation der lokalen Druckkraft mit dem zugehörigen Hebelarm $\vec{\epsilon}$ (Abstand zwischen Bezugspunkt, hier c/4-Punkt des Windkanalmodells, und dem jeweiligem Flächenelement) gemäß

$$
M_{\alpha}(t)=-q_{\infty} c^{2} \oint c_{p}(t) \operatorname{det}(\vec{\epsilon}, \hat{n}) \mathrm{d} A .
$$

Ein negatives Nickmoment beschreibt ein „Zudrehen“ $(\alpha \downarrow)$ des Flügels, ein positives Moment ein „Aufdrehen“ ( $\alpha \uparrow)$.

Zur Messung des Gesamtwiderstands des Tragflügels kam im Rahmen der Nickschwingungsversuche ein Nachlaufrechen im Windkanal zum Einsatz. Ausgehend von den Gesamtdruck- bzw. Impulsverlusten wurde hiermit die Widerstandskraft $F_{W}$ berechnet. 
Für den Auftriebsbeiwert $c_{l}$, den Druckwiderstandsbeiwert $c_{d}$, den Widerstandsbeiwert $c_{w}$ und den Nickmomentenbeiwert $c_{m}$ gilt damit

$$
c_{l}(t)=\frac{F_{L}(t)}{q_{\infty} c s}, \quad c_{d}(t)=\frac{F_{D}(t)}{q_{\infty} c s}, \quad c_{w}=\frac{F_{W}}{q_{\infty} c s} \text { und } \quad c_{m}(t)=\frac{M_{\alpha}(t)}{q_{\infty} c^{2} s} .
$$

Neben den aerodynamischen Beiwerten sind auch deren Abhängigkeiten von den Strömungsgrößen relevant. So lässt sich der Auftriebs- und Nickmomentenbeiwert in der Umgebung des Anstellwinkels $\alpha_{i}$ in einer Taylor-Entwicklung ausdrücken durch

$$
c_{l} \approx c_{l}\left(\alpha_{i}\right)+\left.\frac{\partial c_{l}}{\partial \alpha}\right|_{\alpha_{i}} \Delta \alpha \quad \text { und } \quad c_{m} \approx c_{m}\left(\alpha_{i}\right)+\left.\frac{\partial c_{m}}{\partial \alpha}\right|_{\alpha_{i}} \Delta \alpha
$$

Das dabei auftretende stationäre Auftriebsderivativ $\frac{\partial c_{l}}{\partial \alpha}$ und das Derivativ des Nickmoments $\frac{\partial c_{m}}{\partial \alpha}$ beschreiben die Änderung der Beiwerte in Abhängigkeit des Anstellwinkels. Die stationären Derivativa stellen den Grenzfall der instationären Beiwerte (vgl. 4.2.3) für $\omega^{*} \rightarrow 0$ dar und liefern eine erste Abschätzung von aeroelastischen Stabilitätseigenschaften. Die Berechnung erfolgt hierbei, abweichend zu 4.1.4, im Zeitbereich.

\subsubsection{Aerodynamischer Energie- und Leistungseintrag}

Für aeroelastische Fragestellungen ist es relevant, inwieweit die Strömung mit der Struktur Energie austauscht [12, 27, 28, 139, 173]. Zur Berechnung der Energiebilanz wird die Bewegung der einzelnen Flächenelemente d $A$ des Windkanalmodells durch eine affine Transformation aus der gemessenen Schlag- und Drehbewegung $h(t)$ und $\alpha(t)$ rekonstruiert (siehe C.6). Die Verschiebung eines Flächenelementes, das sich mit der Geschwindigkeit $\vec{v}(t)$ bewegt, ist dann gegeben durch

$$
\mathrm{d} \vec{r}(t)=\vec{v}(h(t), \alpha(t)) \mathrm{d} t .
$$

Die kleinstmögliche Verschiebung wird durch den kleinstmöglichen aufzulösenden Zeitversatz $\mathrm{d} t=1 / f_{s}$ vorgegeben. Dieser ist wiederum durch die Abtastrate $f_{s}$ der Messdatenerfassung festgelegt. Die Arbeit $\mathrm{d} W$, welche in dem Zeitintervall $\mathrm{d} t$ pro Flächenelement verrichtet wird, folgt dann aus dem Skalarprodukt der jeweiligen Druckkraft $\mathrm{d} \vec{F}$ und der Verschiebung $\mathrm{d} \vec{r}$. Für die gesamte Energie gilt dann mit Gl. (4.2.1)

$$
W(t)=-q_{\infty} c^{2} \oint c_{p}(t) \hat{n}(t) \cdot \mathrm{d} \vec{r}(t) \mathrm{d} A
$$

wobei nunmehr auch der Normalenvektor $\hat{n}(t)$ infolge der affinen Transformation eine explizite Zeitabhängigkeit erhalten hat und den Anstellwinkel berücksichtigt (vgl. Gl. (4.2.2)). Die diskretisierte Form von Gl. (4.2.7) ist in C.7 dargestellt. Im Hinblick auf aeroelastische 
Instabilitäten ist die Energiebilanz über eine Schwingungsperiode mit Periodendauer $T$

$$
W_{\mathrm{Per}}=\frac{1}{T} \cdot \int_{t}^{t+T} W(t) \mathrm{d} t
$$

ausschlaggebend, wobei oftmals nur der instationäre Druckanteil $c_{p}(t)-\overline{c_{p}}[12,28]$ in Gl. (4.2.7) berücksichtigt wird. Unter der vereinfachten Annahme eines strukturell

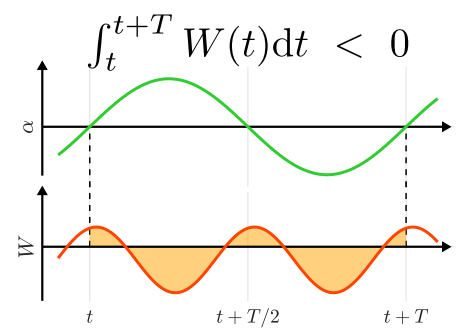

(a) negative Energiebilanz

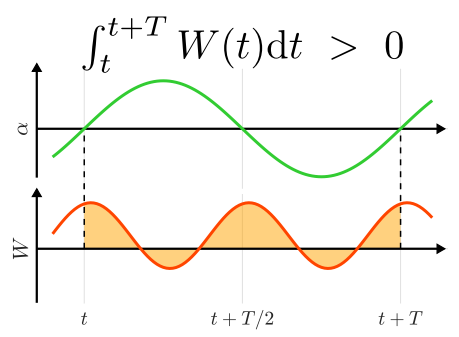

(b) positive Energiebilanz

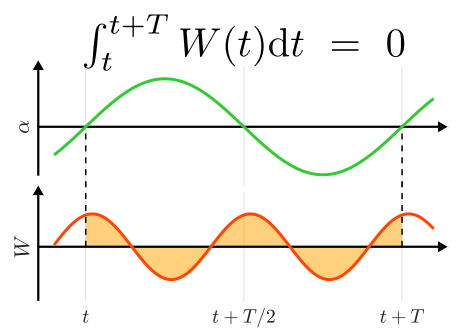

(c) ausgeglichene Energiebilanz

Abbildung 4.1.: Schematische Darstellung der aerodynamischen Energiebilanz über eine Periode der Bewegung. Unter Annahme einer dämpfungsfreien Struktur entspricht (a) einem gedämpften, (b) einem angefachten und (c) einem aeroelastischen System im Grenzzyklus.

dämpfungsfreien Systems, lassen sich dann anhand der Energiebilanz die Systemzustände klassifizieren. Ist diese gemäß Abb. 4.1(a) negativ, wird dem System Energie entnommen. Die Bewegung der Struktur führt somit der Strömung Energie zu und wird gedämpft, dass aeroelastische System ist stabil. Ist hingegen die Energiebilanz positiv, wie in Abb. 4.1(b) dargestellt, führt die Strömung der Struktur Energie zu und das aeroelastische System ist instabil, die Bewegungsgamplituden wachsen an. Ist die Energiebilanz über eine Periode null, wie in Abb. 4.1(c) gezeigt, befindet sich das System in einem Gleichgewicht. Dies würde einem Grenzzyklusfall (LCO) entsprechen.

Zur genaueren Differenzierung des Energieeintrages bietet es sich an, die einzelnen aerodynamischen Leistungen zu betrachten $[27,28,187]$. Die Leistungen des Auftriebs $P_{l}$ und des Nickmomentes $P_{m}$ folgen direkt aus Gl. (4.2.2) und (4.2.3) mit den jeweiligen zeitlichen Änderungen der Modellbewegung zu

$$
P_{l}(t)=\frac{\mathrm{d} h}{\mathrm{~d} t} F_{L}(t) \quad \text { und } \quad P_{m}(t)=\frac{\mathrm{d} \alpha}{\mathrm{d} t} M_{\alpha}(t)
$$

Zur qualitativen Beschreibung der aerodynamischen Leistungsanteile ist auch eine Darstellung in der entdimensionalisierten Form

$$
P_{l}(t) \sim \frac{\mathrm{d}(h / c)}{\mathrm{d} t}\left[c_{l}(t)-\bar{c}_{l}\right] \quad \text { und } \quad P_{m}(t) \sim \frac{\mathrm{d} \alpha}{\mathrm{d} t}\left[c_{m}(t)-\overline{c_{m}}\right]
$$

ausreichend, wobei die Schlagbewegung mit der Flügeltiefe $c$ entdimensionalisiert wird und nur die instationären Anteile der aerodynamischen Beiwerte betrachtet werden [27,62]. Die gesamte aerodynamische Leistung ist dann $P_{\text {aero }}(t)=P_{l}(t)+P_{m}(t)$. 
Im realen aeroelastischen System ist die strukturelle Dämpfung zu berücksichtigen. Durch Dissipation wird der Struktur kontinuierlich Energie entzogen. Die Energie oder auch Leistung der Dämpfung $P_{d}$ ist folglich negativ. Daher gilt für ein reales aeroelastisches System, dass dieses stabil (gedämpft) ist, wenn $\int_{t}^{t+T} P_{\text {aero }} \mathrm{d} t<\int_{t}^{t+T} P_{d} \mathrm{~d} t$ (vgl. Abb. 4.1(a)) ist. Ein instabiles System ist gegeben, wenn $\int_{t}^{t+T} P_{\text {aero }} \mathrm{d} t>\int_{t}^{t+T} P_{d} \mathrm{~d} t$ (vgl. Abb. 4.1(b)) gilt. Ein LCO tritt dann auf, wenn sich die aerodynamische und die dissipierte Energie ausgleichen, also $\int_{t}^{t+T} P_{\text {aero }} \mathrm{d} t=\int_{t}^{t+T} P_{d} \mathrm{~d} t$ (vgl. Abb. 4.1(c)).

\subsubsection{Instationäre aerodynamische Beiwerte}

Zur Identifizierung des Einflusses der Modellbewegung auf die aerodynamischen Kräfte werden die instationären aerodynamischen Beiwerte $c_{i, \alpha}$ betrachtet. Diese werden als Übertragungsfunktion gemäß Gl. (4.1.11) berechnet. Hierbei dient der jeweilige aerodynamische Beiwert $c_{i}$ als Ausgangsfunktion und das Anstellwinkelsignal $\alpha(t)$ als Eingangsfunktion ${ }^{5}$. Da im Allgemeinen $\alpha(t)$ als rauschärmer angenommen wird, erfolgt die Berechnung der instationären Beiwerte mit einem $H_{1}$-Schätzer (vgl. C.3). Für die instationären Beiwerte gilt somit:

$$
\begin{array}{rr}
\text { instationärer Druckbeiwert } & c_{p, \alpha}=\frac{A^{*}(\mathrm{i} \omega) \cdot C_{P}(\mathrm{i} \omega)}{A^{*}(\mathrm{i} \omega) \cdot A(\mathrm{i} \omega)}=\frac{S_{\alpha c_{p}}(\omega)}{S_{\alpha \alpha}(\omega)} \\
\text { instationärer Auftriebsbeiwert } & c_{l, \alpha}=\frac{A^{*}(\mathrm{i} \omega) \cdot C_{L}(\mathrm{i} \omega)}{A^{*}(\mathrm{i} \omega) \cdot A(\mathrm{i} \omega)}=\frac{S_{\alpha c_{l}}(\omega)}{S_{\alpha \alpha}(\omega)} \\
\text { instationärer Momentenbeiwert } & c_{m, \alpha}=\frac{A^{*}(\mathrm{i} \omega) \cdot C_{M}(\mathrm{i} \omega)}{A^{*}(\mathrm{i} \omega) \cdot A(\mathrm{i} \omega)}=\frac{S_{\alpha c_{m}}(\omega)}{S_{\alpha \alpha}(\omega)}
\end{array}
$$

$A(\mathrm{i} \omega)$ bezeichnet die Fourier-Transformierte des Anstellwinkelsignals $\alpha(t)$. Die numerische Berechnung wird mit der WelCH-Methode und den, in C.2 genannten Spezifikationen durchgeführt. Die Darstellung der instationären Beiwerte $c_{i, \alpha}$ erfolgt dann als Amplitudenund Phasengang gemäß Gl. (4.1.12) und (4.1.13). Zur Interpretation sei auf die in C.8 beschriebene Vorzeichenkonvention bei Phasendifferenzen verwiesen.

\subsection{Identifikation der Grenzschichttransition}

Einen inhärenten Bestandteil der strömungsmechanischen Vorgänge am Laminarflügel stellt die laminar-turbulente Grenzschichttransition (vgl. 2.1) dar. Eine nähere Untersuchung des Grenzschichtverhaltens erfolgt mithilfe der Heißfilme bzw. CTAs (vgl. 3.4).

Die Identifikation von Grenzschichtzuständen mithilfe von Heißfilmen geschieht zumeist anhand von charakteristischen Verläufen der Spannungssignale [188]. Statistische Methoden, wie Standardabweichungen, RMS-Werte und Schiefen der Spannungszeitreihen

\footnotetext{
${ }^{5}$ Die mit $\alpha$ gebildeten instationären Beiwerte werden auch als Nickderivativa bezeichnet.
} 
können die Identifikation unterstützen [12,13, 189, 190]. Jedoch sind diese Verfahren weitestgehend auf stationäre Messdaten beschränkt. Auch können sie bei transsonischer Strömung mit einhergehender Stoß-Grenzschicht-Wechselwirkung die physikalische Interpretierbarkeit verlieren. Die Lokalisierung einer instationären Grenzschichttransition muss auf dieser Basis aufwendig manuell erfolgen und ist daher auf kleine Datenausschnitte beschränkt [12,13]. Automatisierte Algorithmen auf Basis der Schiefe ermöglichen auch die Auswertung größerer Datenmengen [191-193]. Diese sind jedoch weitestgehend auf eine subsonische Anströmung, große Amplituden sowie geringe Frequenzen der Bewegung von Flügelprofil und Grenzschichttransition ausgelegt. Ferner kommen diese Methoden bei zumeist zwangserregten Profilschwingungen mit konstanten Amplituden zum Einsatz, womit eine Ensemble- bzw. Phasenmittelung über mehrere Schwingungsperioden möglich ist. Eine zeitaufgelöste Lokalisierung der Transition entfällt.

Aeroelastische Systeme weisen zumeist andere Randbedingungen auf, wie u. a. kleine, zeitabhängige Amplituden und hohe Frequenzen. Im Rahmen der vorliegenden Arbeit wurde daher ein physikalisch motiviertes Verfahren entwickelt [14,61], welches unter den vorliegenden Randbedingungen, neben einer stationären Identifikation der Grenzschichtzustände, auch eine automatisierte, zeitaufgelöste Lokalisierung der instationären Grenzschichttransition auf Basis von CTA-Signalen ermöglicht.

\subsubsection{Quasiwandschubspannung}

Die Grundlage des Verfahrens bildet die Reynolds-Analogie und die daraus abgeleitete Korrelation zwischen dem Impuls- und Wärmetransfer in viskoser Strömung. Hieraus folgt eine Verbindung zwischen der lokalen Wandschubspannung $\tau_{w}$ (vgl. 2.1) und dem Wärmefluss $\dot{Q}_{F}$ an einem Heißfilmsensor. Letzterer wird bestimmt durch die dem Heißfilmsensor zugeführte elektrische Leistung $P(t)=\frac{U(t)^{2}}{R_{S}}$ und der Temperaturdifferenz $\Delta T$ (vgl. 3.4). Die Wandschubspannung lässt sich damit zusammenfassen und als Funktion von $U(t)$ und $\Delta T$ nach [194] ausdrücken zu

$$
\tau_{w}=A\left(\frac{U(t)^{2}-B^{2}}{\Delta T}\right)^{3}
$$

Hierbei sind $A$ und $B$ Funktionen der Temperaturdifferenz, welche bei kleinen Änderungen von $\Delta T$ als Konstanten ${ }^{6}$ angenommen werden können. Eine Herleitung des funktionalen Zusammenhangs ist in [61] dargestellt. Für eine quantitative Messung von Wandschubspannungen mit Heißfilmen werden diese Konstanten mithilfe einer Kalibrierung bestimmt. Bei den vorliegenden experimentellen Randbedingungen ist eine Kalibrierung eines MHFS Arrays jedoch nicht möglich. Dementsprechend stehen nur

\footnotetext{
${ }^{6}$ Angemerkt sei die Ähnlichkeit von Gl. (4.3.1) zum KING'schen Gesetz.
} 
qualitative Werte für die Wandschubspannung zur Verfügung. Gl. (4.3.1) kann jedoch in einen Ausdruck umgewandelt werden, der semi-quantitative Werte für $\tau_{w}$ liefert und sich ähnlich verhält wie die reale Wandschubspannung. Diese Quasi-Wandschubspannung $\tau_{q}$ nach $[195,196]$ ermöglicht somit eine physikalisch motivierte Interpretation der Heißfilmsignale und setzt diese in direkten Bezug zum Grenzschichtverhalten.

$\mathrm{Zu}$ diesem Zweck wird in Gl. (4.3.1) die Konstante $B$ durch die Nullspannung $U_{0}$ der Heißfilmsensoren ersetzt. Da $\tau_{w}$ ohne Anströmung verschwindet, wird dies ebenfalls von der Quasi-Wandschubspannung gefordert. Entsprechend muss $U(t)^{2}-B^{2}=0$ gelten, da $A \neq 0$ gilt und $\Delta T$ nicht divergent ist. Ohne Anströmung $\left(u_{\infty}=0\right)$ gilt $U(t)=U_{0}$ und somit $B=U_{0}$. Ferner ist der Wärmefluss $\dot{Q}_{F}$ zwischen dem Heißfilmelement und der Umgebung proportional zur Temperaturdifferenz $\Delta T$. Gleiches gilt für den Wärmefluss $\dot{Q}_{S}$ zwischen dem Heißfilmelement und dem Trägersubstrat bzw. Windkanalmodell. Unter der Annahme, dass die Modelltemperatur der Umgebungstemperatur entspricht, folgt zusammen mit der Energiebilanz ${ }^{7}$ eines Heißfilmsensors

$$
\sum \dot{Q}=\underbrace{m_{S} c_{S} \frac{\mathrm{d} T_{S}}{\mathrm{~d} t}}_{=0}+\dot{Q}_{F}+\dot{Q}_{S}-\frac{U(t)^{2}}{R_{S}}=0 \Rightarrow U(t)^{2}=R_{S}\left(\dot{Q}_{F}+\dot{Q}_{S}\right) \sim \Delta T .
$$

Da die Temperaturdifferenz $\Delta T$ auch ohne Anströmung besteht, folgt aus Gl. (4.3.2) $\Delta T \sim U_{0}^{2}$. Setzt man dies in Gl. (4.3.1) ein, so folgt die semi-quantitative QuasiWandschubspannung

$$
\tau_{w} \sim \tau_{q}=\left(\frac{U(t)^{2}-U_{0}^{2}}{U_{0}^{2}}\right)^{3} .
$$

Die Konstante $A$ aus Gl. (4.3.1) geht in die Proportionalitätskonstante aus Gl. (4.3.2) mit ein. Somit wurden alle Konstanten durch direkte Messgrößen ersetzt, was keine weitere Kalibrierung erfordert. Die Beziehung zwischen $\tau_{q}$ und $\tau_{w}$ ist nicht exakt. Jedoch verhält sich die Quasi-Wandschubspannung ähnlich wie die reale Wandschubspannung, so dass $\tau_{q}$ auf identische Weise interpretiert werden kann.

\subsubsection{Identifikation der Grenzschichtzustände}

Die Identifikation der Grenzschichtzustände auf Basis des charakteristischen Verlaufes der Wandschubspannung $\tau_{w}(x)$ wurde bereits in 2.1.4 beschrieben. Dieser Verlauf zeigt sich nunmehr gemäß Gl. (4.3.3) auch im Verlauf von $\tau_{q}(x)$, ebenso wie der mit der Transition assoziierte starke Anstieg. Ein Unterschied besteht jedoch im Fall einer Ablösung der Grenzschicht. Eine Ablösung geht mit einer Wandschubspannung $\tau_{w}(x) \leq 0$ einher. Die

\footnotetext{
${ }^{7}$ Bei der Energiebilanz des Heißfilmsensors verschwindet der Wärmekapazitätsterm, da bei der CTA $T_{S}=$ const. gilt und damit $\frac{\mathrm{d} T_{S}}{\mathrm{~d} t}=0$. Des Weiteren gilt $R_{S}=$ const., womit der Sensorwiderstand als Proportionalitätskonstante aufgefasst werden kann.
} 
eingesetzten Heißfilm-Sensoren sind jedoch nicht richtungssensitiv und erkennen somit keine Rückströmung (vgl. [197, S.184 ff.]). Folglich gilt $U(t)^{2}-U_{0}{ }^{2}>0$ und damit auch $\tau_{q}>0$. Jedoch liefern Quasi-Wandschubspannungen nahe Null einen Hinweis auf eine mögliche Ablösung der Grenzschicht. Es empfiehlt sich jedoch dies durch weitere Methoden (z.B. Plateaus in Druckverteilungen $c_{p}(x)$ ) zu stützen. Ferner ist eine korrekte Identifizierung der Transitionsposition im Falle einer Ablösung eingeschränkt. Die Transition verläuft bei einer laminaren Ablöseblase innerhalb der abgelösten Scherschicht. Der Anstieg von $\tau_{q}(x)$, der zur Bestimmung der Transitionsposition genutzt wird, korrespondiert bereits mit einer turbulenten, wieder angelegten Grenzschicht (vgl. 2.1.4). Für diesen Fall wird die Position der Transition tendenziell etwas zu weit stromab detektiert.

\subsubsection{Datenverarbeitung und Transitionsdetektion}

Die Datenverarbeitung der Heißfilmsignale und die Methodik zur Lokalisierung der Transition sind schematisch in Abb. 4.2 dargestellt. Für jeden Heißfilm-Sensor werden die Nullspannungen $U_{0}$ und die Spannungen unter Anströmung $U(t)$ aufgezeichnet. Infolge variierender Umgebungstemperaturen $T_{a}$ im Windkanal wird eine Temperaturkorrektur

$$
U_{\text {corr }}=U \cdot \sqrt{\frac{T_{S}-T_{0}}{T_{S}-T_{a}}}
$$

angewendet [198]. Die Korrektur wird auf die Referenztemperatur $T_{0}$ bezogen, die der Umgebungstemperatur der jeweils letzten Einstellung des Überhitzungsverhältnisses der Heißfilme (vgl. 3.4) entspricht. Mit Gl. (4.3.3) folgt dann aus den korrigierten Werten $\tau_{q}$.

Bei stationären Messreihen (ruhendes Tragflügelmodell) wird für jeden Heißfilm-Sensor an der Stelle $x$ aus der zugehörigen Zeitreihe $\tau_{q}(t)$ der zeitliche Mittelwert $\overline{\tau_{q}}$ bestimmt (siehe Abb. 4.2 oben). Auf dieser Basis wird der räumliche Gradient des Verlaufs der mittleren Quasi-Wandschubspannungen sowie das Maximum des Gradienten gemäß

$$
\bar{\tau}_{q}^{\prime}(x)=\frac{\mathrm{d} \bar{\tau}_{q}(x)}{\mathrm{d} x}, \quad{\overline{\tau_{q}}}^{\prime}\left(x_{t r}\right)=\max \left[\bar{\tau}_{q}^{\prime}(x)\right]
$$

berechnet. Das Maximum, lokalisiert an der Stelle $x_{t r}$, korrespondiert mit dem stärksten Anstieg von $\overline{\tau_{q}}(x)$ und lokalisiert die Grenzschichttransition (vgl. 4.3.2). Abschließend werden die Ergebnisse manuell mit den Spannungszeitschrieben der Heißfilme sowie den Druckverteilungen $c_{p}(x)$ auf Plausibilität überprüft. Ein Verdichtungsstoß, dessen Position mit der eines Heißfilm-Sensors übereinstimmt, kann u. U. zu einem weiteren Anstieg von $\tau_{q}$ führen und sich in einer inkorrekten Lokalisierung der Transitionsposition wiederspiegeln. Des Weiteren kann die Ausdehnung des Transitionsbereichs anhand der Breite des Anstiegs manuell bestimmt werden. 


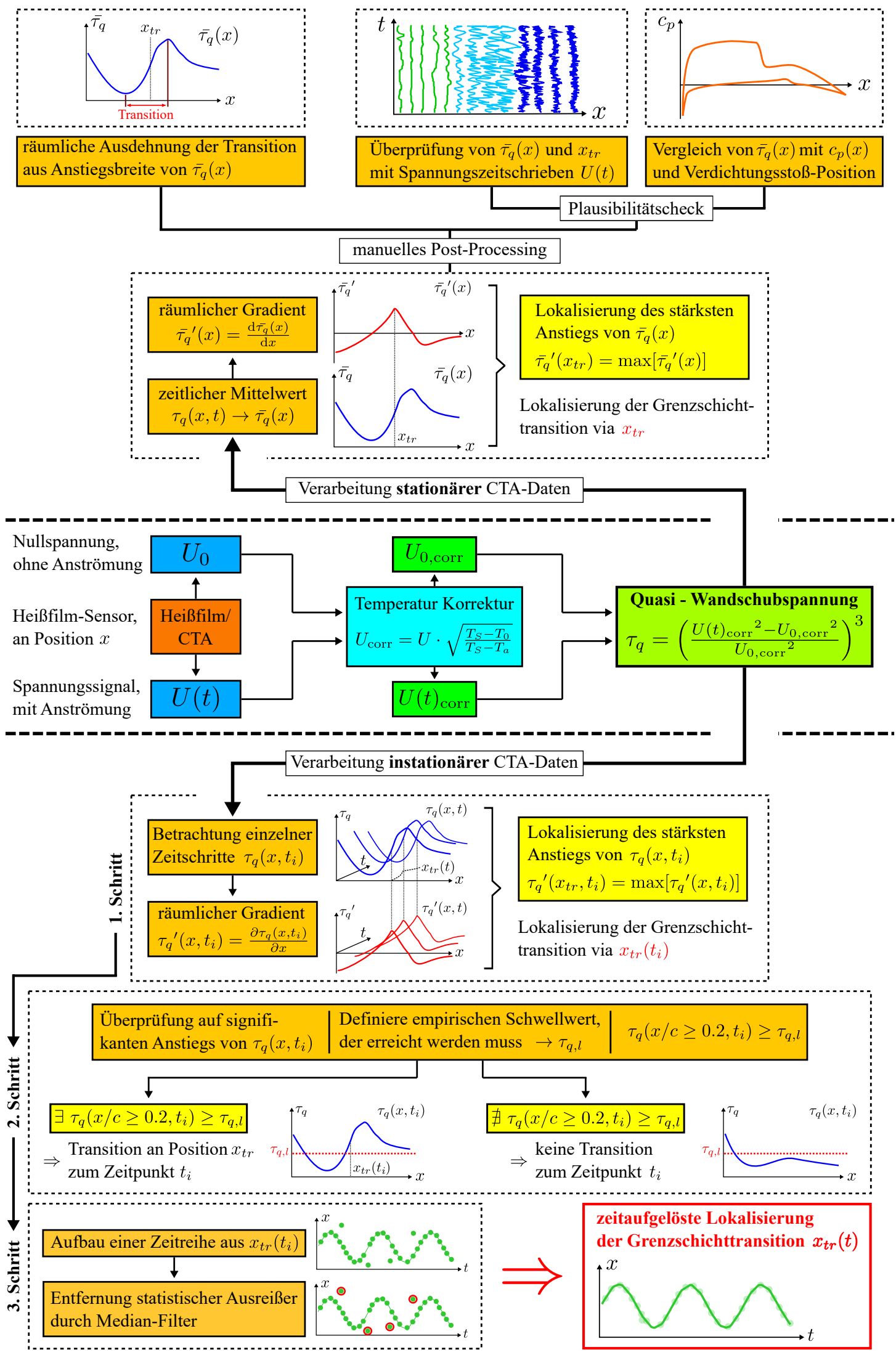

Abbildung 4.2.: Flussdiagramm zur Verarbeitung der Heißfilmsignale und Detektion der stationären und instationären Position der laminar-turbulenten Grenzschichttransition auf Basis der Quasi-Wandschubspannung, verändert nach [14]. 
Im Fall von instationären Messreihen (bewegtes Tragflügelmodell) ist ein manuelles PostProcessing nicht mehr möglich. Die Signalverarbeitung ist anfangs zwar vergleichbar, es werden jedoch keine zeitlichen Mittelwerte mehr betrachtet. Die Verläufe $\tau_{q}\left(x, t_{i}\right)$ werden zu jedem Zeitpunkt $t_{i}$ ausgewertet ${ }^{8}$ (siehe Abb. 4.2 unten). Die automatisierte Datenanalyse erfolgt nunmehr mit einem dreistufigen Algorithmus. Anhand von Gl. (4.3.5) wird im ersten Schritt die zeitaufgelöste Position der Grenzschichttransition $x_{t r}\left(t_{i}\right)$ ermittelt, weitestgehend analog zum Vorgehen bei stationären Messreihen. Zum Teil tritt jedoch keine Transition innerhalb des Sensorbereiches auf, sofern eine laminare Grenzschicht vorliegt. Dennoch auftretende geringe Anstiege von $\tau_{q}(x)$ werden im ersten Schritt jedoch als inkorrekte Transitionsposition identifiziert und müssen im zweiten Schritt entfernt werden. Hierfür wird gefordert, dass $\tau_{q}\left(x, t_{i}\right)$ einen empirisch definierten Schwellwert $\tau_{q, l}$ erreicht bzw. überschreitet, damit ein Anstieg von $\tau_{q}\left(x, t_{i}\right)$ als signifikant gilt und mit einer Transition in Verbindung gesetzt wird. Aus Gründen der Zweckmäßigkeit werden hierbei nur Werte $\tau_{q}(x / c \geq 0.2)$ betrachtet. Sofern die notwendige Bedingung $\tau_{q}\left(x / c \geq 0.2, t_{i}\right) \geq \tau_{q, l}$ nicht erfüllt ist, wird die zuvor bestimmte Transitionsposition $x_{t r}\left(t_{i}\right)$ verworfen und eine laminare Grenzschicht angenommen. Ist die Bedingung hingegen erfüllt, wird $x_{t r}\left(t_{i}\right)$ als Position der Grenzschichttransition übernommen und im dritten Schritt des Algorithmus mit weiteren Positionen zur Zeitreihe $x_{t r}(t)$ kombiniert. Abschließend werden vereinzelte Ausreißer in der Zeitreihe heraus gefiltert. Diese begründen sich u. a. in einem bei gegebenen Randbedingungen auftretenden Doppelstoß-System am CAST 10-2 Laminarflügelprofil, was zu fehlerhaft lokalisierten Transitionspositionen führen kann. Auch treten vereinzelte Überschwinger im Verlauf von $\tau_{q}\left(x, t_{i}\right)$ auf. Derartige Ausreißer werden als statistische Signalausreißer betrachtet und mit einem Median-Filter ${ }^{9}$ entfernt. Die finale zeitaufgelöste Position der Grenzschichttransition $x_{t r}(t)$ kann nunmehr mit den in 4.1 beschrieben Verfahren weiter analysiert werden und erstmalig auch in einem größeren Umfang in Relation zur Bewegung des Modells sowie der aerodynamischen Kräfte gesetzt werden.

${ }^{8}$ Aufgrund der hohen Abtastsrate der CTA's (vgl. 3.4) erfolgt zumeist ein Downsampling bzw. eine Datenbetrachtung bei zeitidentischen Abtastpunkten mit der übrigen Sensorik. Bei einer verbleibenden, vollkommen ausreichenden Zeitauflösung beschleunigt dies die Datenverarbeitung erheblich.

${ }^{9}$ Die Fensterlänge des Medianfilters sollte so kurz wie möglich gewählt werden, um eine ausreichende Unterdrückung der Ausreißer zu erzielen, jedoch die Signalverzerrung und einhergehende Phasenverschiebung von $x_{t r}(t)$ so gering wie möglich zu halten. 



\section{Aerodynamisches Verhalten bei statischen Anstellwinkeln}

Im Vorfeld der dynamischen Untersuchungen wurde mithilfe des NVS (vgl. 3.2.1) die stationäre Aerodynamik des CAST 10-2 Laminarflügelprofils eingehender untersucht. Es wurden stationäre Polaren für einen Mach-Zahl-Bereich von 0,73 bis 0,76 bei einer Variation des Anstellwinkels $\alpha$ von $-1^{\circ}$ bis $2^{\circ}$ vermessen. Der Ruhedruck betrug während diesen Messungen $p_{0}=54 \mathrm{kPa}$, woraus eine weitestgehend konstante Reynolds-Zahl von $\operatorname{Re}_{\infty} \approx 2 \cdot 10^{6}$ resultierte. Die Messungen wurden bei einer freien Grenzschichttransition (keine Transitionsfixierung) durchgeführt.

Die Auswahl der Versuchsparameter beruhte auf vorherigen Windkanaluntersuchungen $[12,13,19]$ sowie den Ergebnissen des ersten Flatterversuchs mit dem CAST 10-2 Laminarflügelprofil [24]. So ist insbesondere der Bereich zwischen $\mathrm{Ma}_{\infty}=0,73$ bis $\mathrm{Ma}_{\infty}=0,75$ von Interesse. Zum einen wurden unter diesen Randbedingungen neuartige Flattermechanismen beobachtet (vgl. 1.1 und [59]). Zum anderen konnten in vorherigen Versuchen keine Messungen in diesem Bereich (ausgenommen $\mathrm{Ma}_{\infty}=0,75$ ) durchgeführt werden. Auftretende Modellschwingungen wurden durch den alten Nickversuchsstand (siehe z.B. [17, 199]) nicht kompensiert, so dass eine statische und dynamische WinkelPositionierung des Windkanalmodells nicht möglich war. Durch den erstmaligen Einsatz des neuen NVS (vgl. 3.2.1) konnte nunmehr auch dieser Bereich eingehender untersucht werden.

Das folgende Kapitel stellt die Ergebnisse der stationären Messungen, insbesondere das Verhalten der aerodynamischen Kräfte, der Druckverteilungen und der Grenzschicht dar. Es werden erste instationäre Effekte diskutiert, welche bereits bei statischen Anstellwinkeln im Rahmen der Messungen beobachtet wurden.

\subsection{Verhalten der aerodynamischen Kräfte}

Der Verlauf der aerodynamischen Kräfte ist in Abb. 5.1 für die vier untersuchten Mach-Zahlen dargestellt. Diese wurden gemäß der in 4.2.1 beschriebenen Methodik, ausgehend von den gemessenen Drücken, in aerodynamische Beiwerte umgerechnet. Die 
Widerstandsbeiwerte basieren auf der stationären Messung des Nachlaufrechens. Die dargestellten Messwerte entsprechen den Mittelwerten der aufgezeichneten Zeitreihen, die vertikalen Fehlerbalken bilden die jeweilige einfache Standardabweichung ab. Der Fehler des Anstellwinkels folgt aus den Anmerkungen in 3.4.4. Die schwarz gestrichelte Linie in den nachfolgenden Abbildungen markiert einen Anstellwinkel von $\alpha=0^{\circ 1}$.

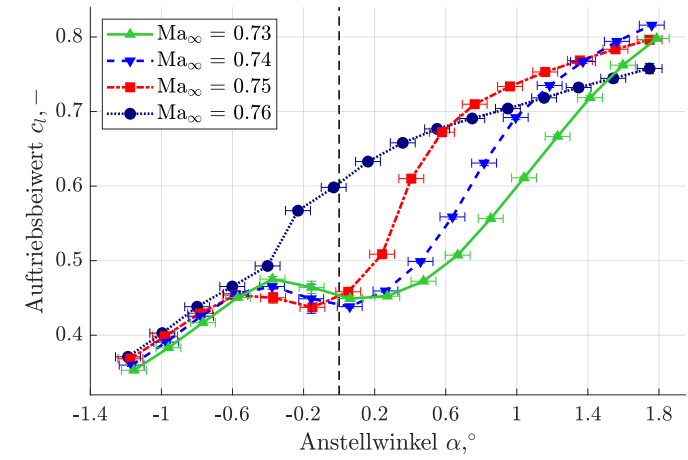

(a) Auftriebsbeiwert $c_{l}(\alpha)$

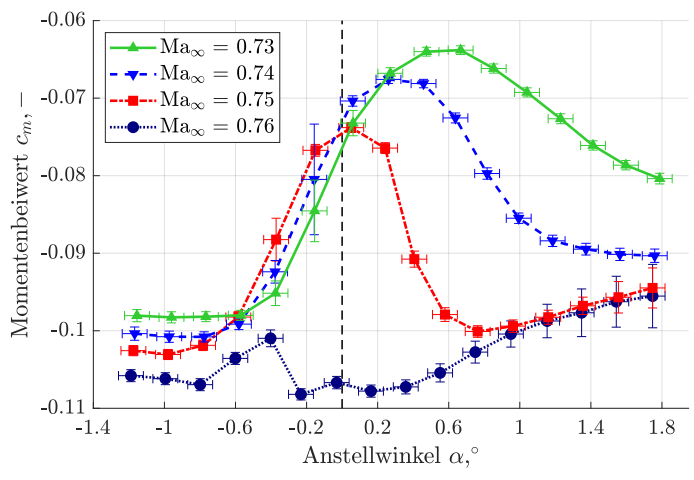

(c) Momentenbeiwert $c_{m}(\alpha)$

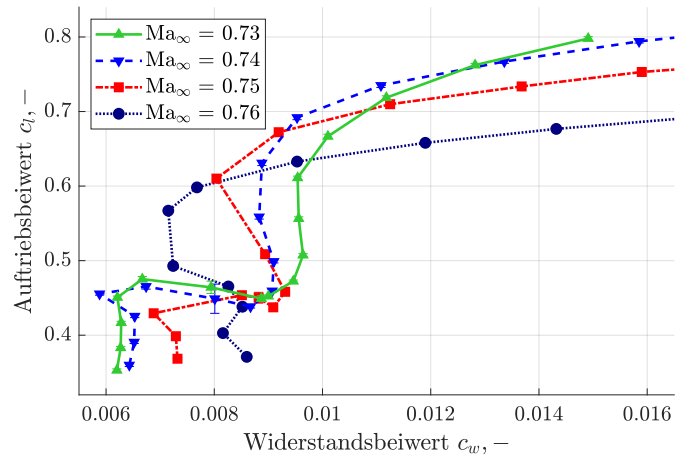

(b) Profilpolare $c_{l}\left(c_{w}\right)$

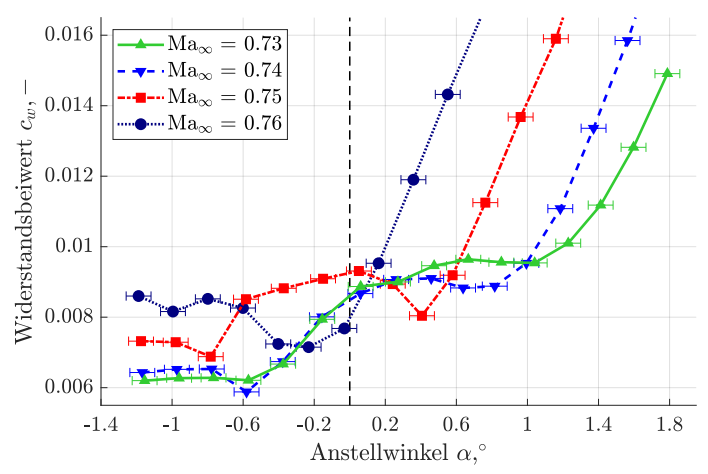

(d) Widerstandsbeiwert $c_{w}(\alpha)$

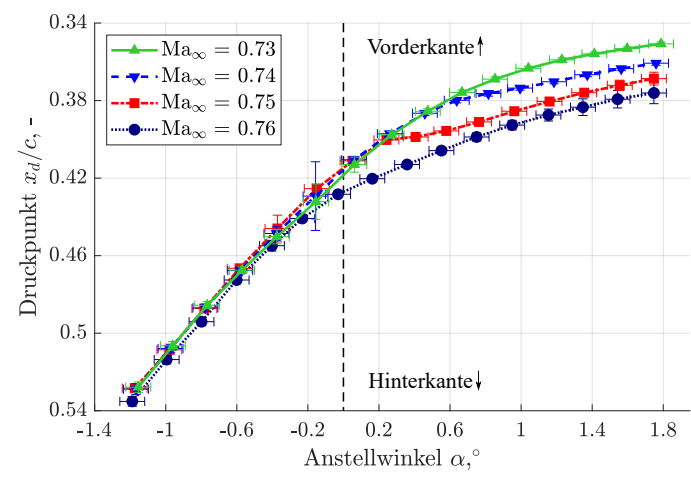

(e) Druckpunktposition $x_{d}(\alpha)$

Abbildung 5.1.: Verlauf der stationären Auftriebs- $c_{l}(\alpha)$ (a), Momenten- $c_{m}(\alpha)$ (c) und Widerstandsbeiwerte $c_{w}(\alpha)$ (d) sowie Profilpolaren $c_{l}\left(c_{w}\right)$ (b) und Position des Druckpunktes $x_{d}(\alpha)$ (e) für $\mathrm{Ma}_{\infty}=0,73$ bis $\mathrm{Ma}_{\infty}=0,76$.

\footnotetext{
${ }^{1}$ Um einen Anstellwinkel von $\alpha=0^{\circ}$ herum wurden neuartige Flatterphänomene am CAST 10-2 Profil beobachtet. Ferner konzentrierten sich auch die im Rahmen der vorliegenden Arbeit durchgeführten aeroelastischen Untersuchungen auf diesen Anstellwinkelbereich (vgl. 6 und 7).
} 
Wie Abb. 5.1(a) zeigt, bildet sich für $\mathrm{Ma}_{\infty}=0,73$ ein Auftriebsplateau ( $c_{l} \approx$ const.) im Bereich von $-0,4^{\circ} \lesssim \alpha \lesssim 0,4^{\circ}$ aus. Dieses Plateau korrespondiert mit dem Übergangsbereich (Zunahme $c_{w}(\alpha)$ bei $c_{l}(\alpha) \approx$ const.) der zugehörigen Laminardelle, welche in Abb. 5.1(b) zu erkennen ist. Dies verdeutlicht ferner die Laminarflügel-Charakteristiken des CAST 10-2 Profils für $\operatorname{Re}_{\infty} \approx 2 \cdot 10^{6}$. Mit steigender Mach-Zahl nimmt die Ausdehnung des Plateaus ab, es bleibt jedoch auch für $\mathrm{Ma}_{\infty}=0,74$ und $\mathrm{Ma}_{\infty}=0,75$ erhalten. Der mittlere Anstellwinkel $\alpha \approx 0^{\circ}$ liegt für diese Mach-Zahlen somit innerhalb des Übergangsbereiches der Laminardelle. Die Polaren in Abb. 5.1(b) sowie der Verlauf der Widerstandsbeiwerte in Abb. 5.1(d) zeigen des Weiteren, dass der minimale Widerstandsbeiwert mit steigender Mach-Zahl ansteigt. Entsprechend nimmt die Ausdehnung der Laminardelle und des Übergangsbereichs ab. Für $\mathrm{Ma}_{\infty}=0,76$ ist die ursprüngliche Laminardelle und somit auch der Übergangsbereich vollständig verschwunden. Hierfür tritt ein Bereich mit reduziertem Widerstand bei größeren Auftriebsbeiwerten $0,5 \lesssim c_{l} \lesssim 0,6$ bzw. Anstellwinkeln $-0,4^{\circ} \lesssim \alpha \lesssim 0^{\circ}$ auf, der oberhalb von $c_{l} \gtrsim 0,6$ bzw. $\alpha \gtrsim 0^{\circ}$ in einen starken Anstieg des Widerstands übergeht, wie Abb. 5.1(b) und (d) zeigen. Für kleinere Mach-Zahlen setzt die Zunahme von $c_{w}(\alpha)$ erst bei größeren $c_{l}$ bzw. $\alpha$ ein.

Der deutlichste Einfluss von $\mathrm{Ma}_{\infty}$ zeigt sich im Verlauf des Momentenbeiwertes, dargestellt in Abb. 5.1(c). Für $\mathrm{Ma}_{\infty}<0,76$ steigt der $c_{m}(\alpha)$-Verlauf, ausgehend von einem Momentenbeiwert um $c_{m} \approx-0,1$ herum, ab $\alpha \approx-0,6^{\circ}$ stark an. Das zudrehende Moment $\left(c_{m}<0\right)$ reduziert sich entsprechend, was annähernd mit dem Beginn des Auftriebsplateaus übereinstimmt. Das Ende des Plateaus (Ende des Übergangsbereichs der Laminardelle) korrespondiert hingegen in etwa mit dem geringsten zudrehenden Momentenbeiwert. Für $\mathrm{Ma}_{\infty}=0,73$ wird dieser Wert bei $\alpha \approx 0,6^{\circ}$ erreicht, für $\mathrm{Ma}_{\infty}=0,75$ bei $\alpha \approx 0^{\circ}$. Oberhalb dieser Anstellwinkel kommt es dann, abhängig von $\mathrm{Ma}_{\infty}, \mathrm{zu}$ einer starken Reduzierung von $c_{m}(\alpha)$, also einer Zunahme des zudrehenden Moments. $c_{m}\left(\mathrm{Ma}_{\infty}=0,75\right)$ fällt innerhalb von $0^{\circ} \lesssim \alpha \lesssim 0,6^{\circ}$ rapide fast wieder auf das ursprüngliche Niveau ab. Moderater sinken hingegen $c_{m}\left(\mathrm{Ma}_{\infty}=0,74\right)$ und $c_{m}\left(\mathrm{Ma}_{\infty}=0,73\right)$, welche für $\alpha \approx 1,8^{\circ}$ auch höhere $c_{m}$-Werte (kleinere zudrehende Nickmomente) erreichen. Dennoch zeigt sich für alle drei Mach-Zahlen eine ausgeprägte Sensitivität von $c_{m}$ bzgl. $\alpha$. Kleine Änderungen des Anstellwinkels führen zu teils deutlichen Unterschieden im Nickmoment. Für $\mathrm{Ma}_{\infty}=0,76$ ist diese Änderung im Verlauf von $c_{m}(\alpha)$ hingegen bedeutend geringer. In Abb. 5.1(c) ist lediglich eine gemäßigte Abnahme des zudrehenden Moments mit steigendem Anstellwinkel zu erkennen. Der mit dem Übergangsbereich der Laminardelle verknüpfte Anstieg des $c_{m}(\alpha)$-Verlaufs bleibt für $\mathrm{Ma}_{\infty}=0,76$ aus.

Das Verhalten der Beiwerte spiegelt sich auch in der Position des Druckpunktes ${ }^{2}$ wieder, dargestellt in Abb. 5.1(e). Die negativen Momente für $\alpha \approx-1,2^{\circ}$ äußern sich in einem,

\footnotetext{
${ }^{2}$ Der Druckpunkt berechnet sich nach $[117, \mathrm{~S} .385$ f. $]$ zu $x_{d}(\alpha)=\left[-\frac{c_{m}(\alpha)}{c_{l}(\alpha)}+\frac{1}{4}\right] \cdot c$.
} 
hinter dem Flügeltiefenmittelpunkt liegenden Druckpunkt. Dieser ist zunächst nahezu identisch für alle Mach-Zahlen. Der annähernd lineare Anstieg von $c_{l}$ (siehe Abb. 5.1(a)) bei einem konstanten Verlauf von $c_{m}$ (siehe Abb. 5.1(c)) führt bis $\alpha \approx-0,6$ zu einer ebenfalls linearen und einheitlichen Bewegung des Druckpunktes stromaufwärts. Mit dem Beginn des Übergangsbereiches der Laminardelle verlangsamt sich die Druckpunktwanderung stromauf zunächst für $\mathrm{Ma}_{\infty}=0,76$ infolge des stärkeren Anstiegs von $c_{l}\left(\mathrm{Ma}_{\infty}=0,76\right)$. Für $0,73 \leq \mathrm{Ma}_{\infty} \leq 0,75$ wandert die Druckpunktposition bis $\alpha \approx 0,2^{\circ}$ identisch weiter stromauf. Hier verlangsamt sich nun auch die Verschiebung des Druckpunktes für $\mathrm{Ma}_{\infty}=0,75$, entsprechend dem beginnenden Abfall (Anstieg des zudrehenden Moments) von $c_{m}\left(\mathrm{Ma}=0,75_{\infty}\right)$ für $\alpha \gtrsim 0,2^{\circ}$ (siehe Abb. 5.1(c)). Für $\mathrm{Ma}_{\infty}=0,73$ und $\mathrm{Ma}_{\infty}=0,74$ ist der Abfall von $c_{m}$ hinzu größeren Anstellwinkeln verschoben, was sich in der nochmals verzögerten Verlangsamung der Druckpunktbewegung widerspiegelt. Die Wanderung von $x_{d}$ ist somit umso ausgeprägter, je kleiner $\mathrm{Ma}_{\infty}$ ist.

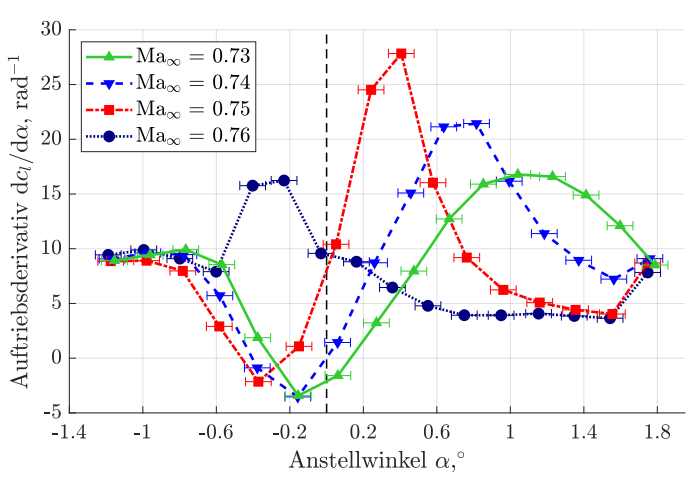

(a) Stationäres Auftriebsderivativ $\frac{\mathrm{d} c_{l}}{\mathrm{~d} \alpha}$

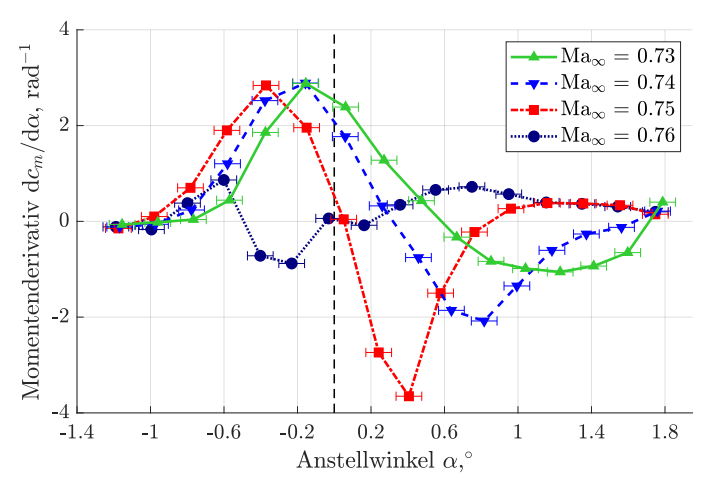

(b) Stationäres Momentenderivativ $\frac{\mathrm{d} c_{m}}{\mathrm{~d} \alpha}$

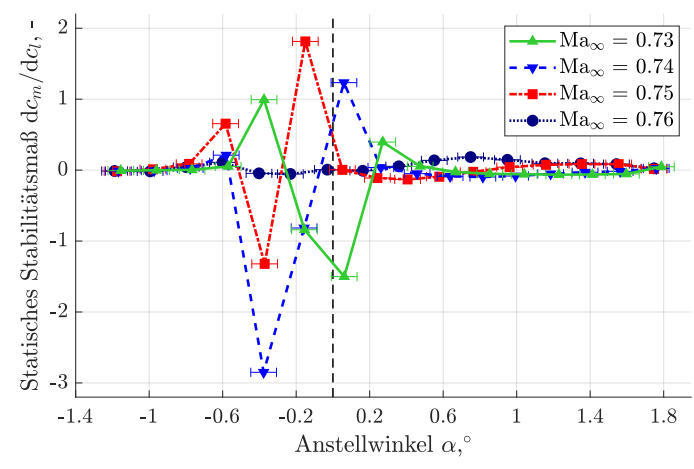

(c) Statisches Stabilitätsmaß $\frac{\mathrm{d} c_{m}}{\mathrm{~d} c_{l}}$

Abbildung 5.2.: Verlauf der stationären Auftriebs- $\frac{\mathrm{d} c_{l}}{\mathrm{~d} \alpha}$ (a) und Momentenderivativa $\frac{\mathrm{d} c_{m}}{\mathrm{~d} \alpha}$ (b) sowie des statischen Stabilitätsmaß $\frac{\mathrm{d} c_{m}}{\mathrm{~d} c_{l}}$ (c) für $\mathrm{Ma}_{\infty}=0,73$ bis $\mathrm{Ma}_{\infty}=0,76$.

Die Sensitivität der stationären aerodynamischen Kräfte bei einer Änderung des Anstellwinkels zeigt sich noch deutlicher in den stationären Derivativa $\frac{\mathrm{d} c_{l}}{\mathrm{~d} \alpha}$ und $\frac{\mathrm{d} c_{m}}{\mathrm{~d} \alpha}$ (vgl. 4.2.1), dargestellt in Abb. 5.2. Für $\mathrm{Ma}_{\infty}=0,73$ bis $\mathrm{Ma}_{\infty}=0,75$ sind ausgeprägte Änderungen der Derivativa zu erkennen. Dies bringt zum einen die nichtlineare Aerodynamik des 
Laminarflügelprofils zum Ausdruck. Zum anderen liefern die Derivativa einen Hinweis zur statischen Längsstabilität des Profils. Dabei ist die Übertragbarkeit auf instationäre Vorgänge nicht trivial, jedoch können die nichtlinearen, stark schwankenden Verläufe als Hinweis für eine aeroelastische Instabilität interpretiert werden. So wechselt insbesondere das Momentenderivativ die Vorzeichen, ändert also zwischen einer statischen Stabilität $\left(\frac{\mathrm{d} c_{m}}{\mathrm{~d} \alpha}<0\right)^{3}$ und einer statischen Instabilität $\left(\frac{\mathrm{d} c_{m}}{\mathrm{~d} \alpha}>0\right)$. Ferner sind die Nichtlinearitäten auch ein erster Hinweis bzgl. einer Amplitudenbegrenzung von Flatterinstabilitäten in dem entsprechenden Anstellwinkelbereich (vgl. [26]). Dabei ist auch hier die Verbindung zum Übergang der Laminardelle erkennbar. So fällt auch die Änderung von $c_{l}$ und $c_{m}$ für $\mathrm{Ma}_{\infty}=0,76$ vergleichsweise gering aus. Zusammengefasst ist dieser Sachverhalt in Abb. 5.2(c), wo das aus den Derivativa berechnete statische Stabilitätsmaß $\frac{\mathrm{d} c_{m}}{\mathrm{~d} c_{l}}[117, \mathrm{~S} .385]$ aufgetragen ist. Dabei zeigt sich sehr deutlich, dass dieses für $\mathrm{Ma}_{\infty}=0,76$ nahe null verläuft. Für die übrigen Mach-Zahlen kommt es jedoch im Bereich $-0,6^{\circ} \lesssim \alpha \lesssim 0,2^{\circ}$ zu erheblichen Abweichungen, was einer Wanderung des Neutralpunktes entspricht. So liegt auch hier, wie bereits für $\frac{\mathrm{d} c_{m}}{\mathrm{~d} \alpha}$ ein Wechsel zwischen einer statischen Längsstabilität $\left(\frac{\mathrm{d} c_{m}}{\mathrm{~d} c_{l}}<0\right)$ und einer statischen Instabilität vor $\left(\frac{\mathrm{d} c_{m}}{\mathrm{~d} c_{l}}>0\right)$.

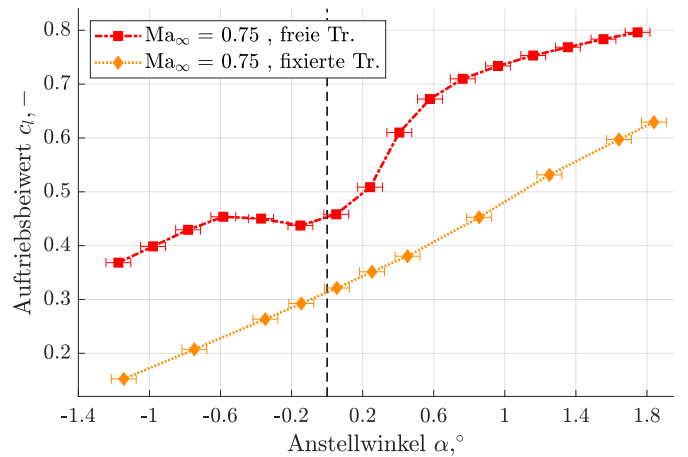

(a) Vergleich Auftriebsbeiwert $c_{l}(\alpha)$

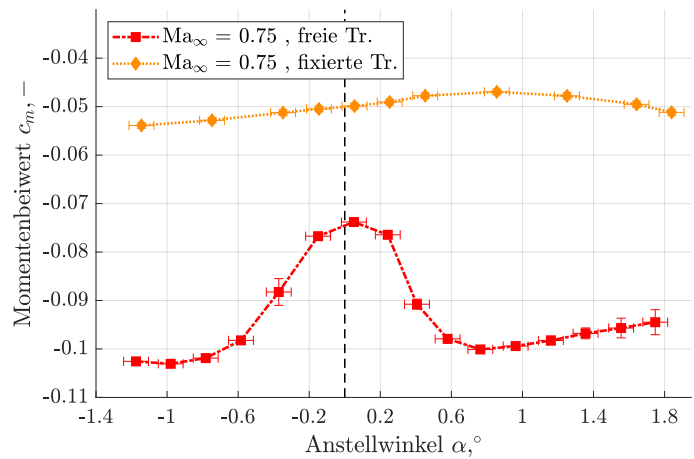

(b) Vergleich Momentenbeiwert $c_{m}(\alpha)$

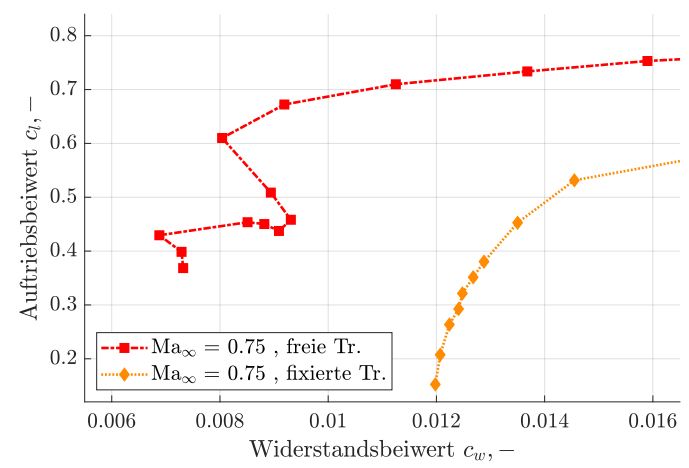

(c) Vergleich Polare $c_{l}\left(c_{w}\right)$

Abbildung 5.3.: Verläufe des Auftriebs- $c_{l}(\alpha)$ (a) und Momentenbeiwertes $c_{m}(\alpha)$ (b) sowie der Profilpolaren $c_{l}\left(c_{w}\right)$ (c) für $\mathrm{Ma}_{\infty}=0,75$ bei freier und fixierter Transition. Die Messungen mit fixierter Transition erfolgten bereits im Rahmen früherer Messungen (siehe [13,17]).

\footnotetext{
$3 \frac{\mathrm{d} c_{m}}{\mathrm{~d} \alpha}<0$ weist auf selbsterregte Schwingungen mit begrenzter Amplitude hin [9, S.90] (vgl. auch [26]).
} 
Der Zusammenhang der aerodynamischen Nichtlinearitäten und der damit einhergehenden Sensitivität bzgl. des Anstellwinkels mit dem Übergangsbereich der Laminardelle legt nahe, dass diese ein Resultat der vorherrschenden Grenzschichtzustände am CAST 10-2 Profil sind. In Abb. 5.3 sind die Verläufe von $c_{l}(\alpha)$ (Abb. 5.3(a)) und $c_{m}(\alpha)$ (Abb. 5.3(b)) sowie die Profilpolaren $c_{l}\left(c_{w}\right)$ (Abb. 5.3(c)) für $\mathrm{Ma}_{\infty}=0,75$ für eine freie und eine, an der Vorderkante fixierte Transition gegenübergestellt. Neben einer Verschiebung von $\Delta c_{l} \approx 0,2$ hin zu kleineren Auftriebsbeiwerten und einer Reduzierung des zudrehenden Nickmomentes um $\Delta c_{m} \approx 0,05$ für eine turbulente Grenzschicht zeigt sich deutlich, dass der ausgeprägte nichtlineare Bereich entfällt. Für eine turbulente Grenzschicht nimmt $c_{l}(\alpha)$ annähernd linear zu. Der Verlauf des Nickmomentes $c_{m}(\alpha)$ bleibt hingegen nahezu konstant. Dabei ist in Abb. 5.3(c) eindeutig zu erkennen, dass die Laminardelle für eine turbulente Grenzschicht vollständig verschwindet und der Widerstandsbeiwert insgesamt deutlich erhöht ist. Dies bestätigt den direkten Zusammenhang der nichtlinearen stationären Aerodynamik mit dem Auftreten der Laminardelle und somit mit dem laminaren bzw. transitionellen Zustand der Grenzschicht. Zu ähnlichen Ergebnissen kamen auch frühere experimentelle [12,13] und auch numerische Untersuchungen [20,23].

\subsection{Verhalten der Druckverteilung}

Die Druckverteilungen der stationären Polaren sind in Abb. 5.4 abgebildet und die Druckbeiwerte der Flügeloberseite als Konturplot dargestellt. Die Ergebnisse entsprechen den Mittelwerten der aufgezeichneten Zeitreihen der Drucksensoren (vgl. 3.4.1), welche zuvor gemäß Gl. (3.4.1) in $c_{p}$-Werte umgerechnet wurden. Auf eine zusätzliche Darstellung von Fehlern (vgl. B.4.1) wurde aus Gründen der Übersichtlichkeit verzichtet. Die schwarz gestrichelten Linien in Abb. 5.4 markieren den kritischen Druckbeiwert $c_{p}^{* 4}$.

Die Druckverteilungen der Flügelunterseite entwickeln sich für alle vier Mach-Zahlen bei einer Variation des Anstellwinkels weitestgehend identisch. Für alle Konfigurationen bleibt die Druckseite des Laminarflügelprofils stoßfrei. Der dort einheitlich auftretende Druckanstieg im Bereich $x / c \approx 0,64$ hängt mit der laminar-turbulenten Grenzschichttransition $[19,20,23]$ zusammen. Die Profil-Druckseite spielt im Hinblick auf die aerodynamischen Effekte somit nur eine sekundäre Rolle. Signifikante Unterschiede und Effekte treten hingegen auf der Saugseite des Profils auf.

Abb. 5.4(a) zeigt die Druckverteilung für $\mathrm{Ma}_{\infty}=0$, 73. Für diese Mach-Zahl ist das Profil bis $\alpha \approx 0,4^{\circ}$ stoßfrei. Es liegen zwar für $\alpha \lesssim 0,4^{\circ}$ bereits supersonische Gebiete vor, wie die Konturlinie $c_{p}^{*}$ in Abb. 5.4(a) (oben) verdeutlicht, diese sind jedoch nur schwach

\footnotetext{
${ }^{4}$ Der kritische Druckbeiwert berechnet sich nach [200, S.73] unter der Annahme einer isentropen Strömung zu $c_{p}^{*}=-\frac{2}{\kappa \cdot \mathrm{Ma}_{\infty}^{2}} \cdot\left[1-\left(\frac{2}{\kappa+1}+\frac{\kappa-1}{\kappa+1} \cdot \mathrm{Ma}_{\infty}^{2}\right)^{\frac{\kappa}{\kappa-1}}\right]$.
} 


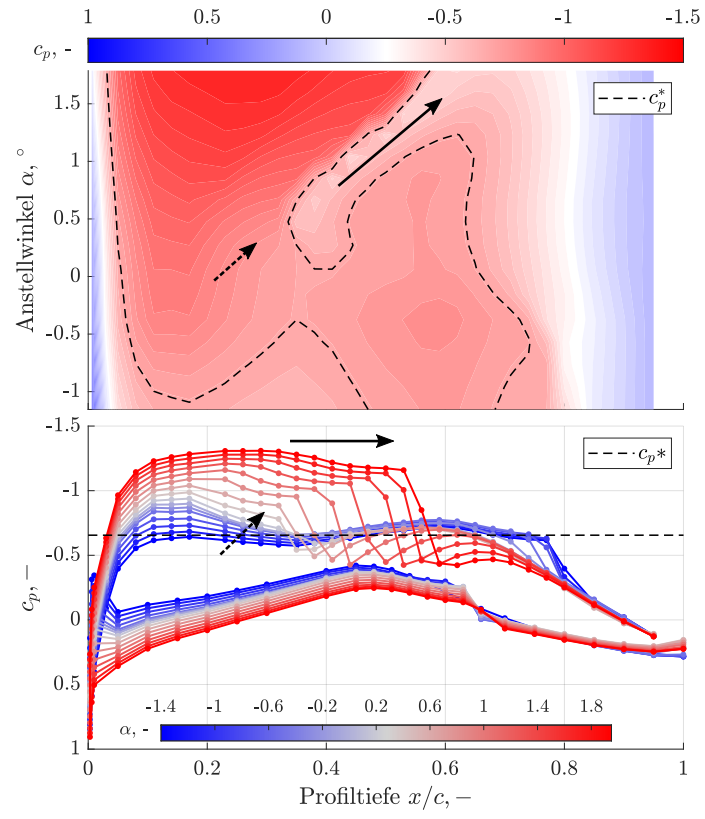

(a) Druckverteilung für $\mathrm{Ma}_{\infty}=0,73$

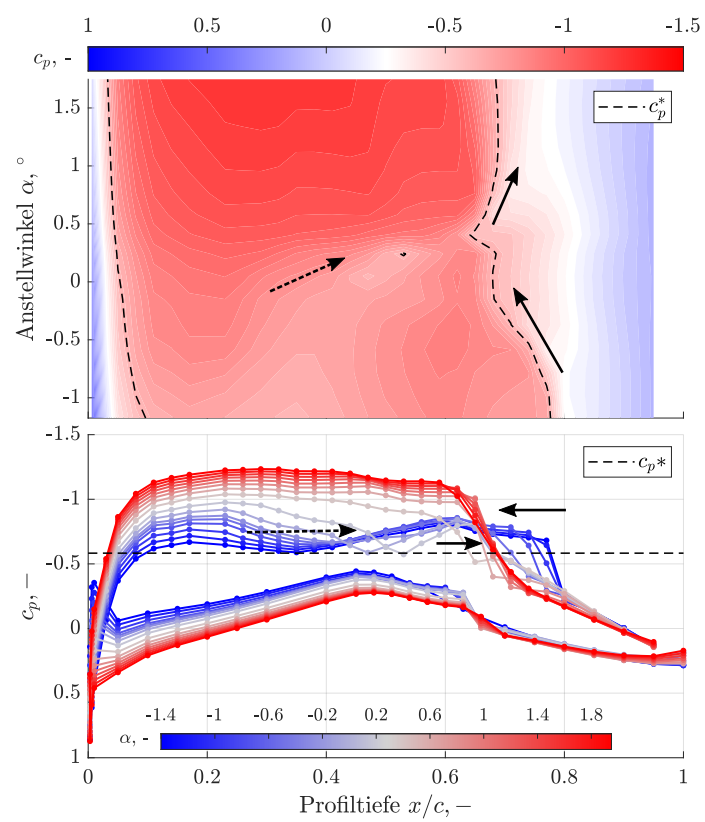

(c) Druckverteilung für $\mathrm{Ma}_{\infty}=0,75$

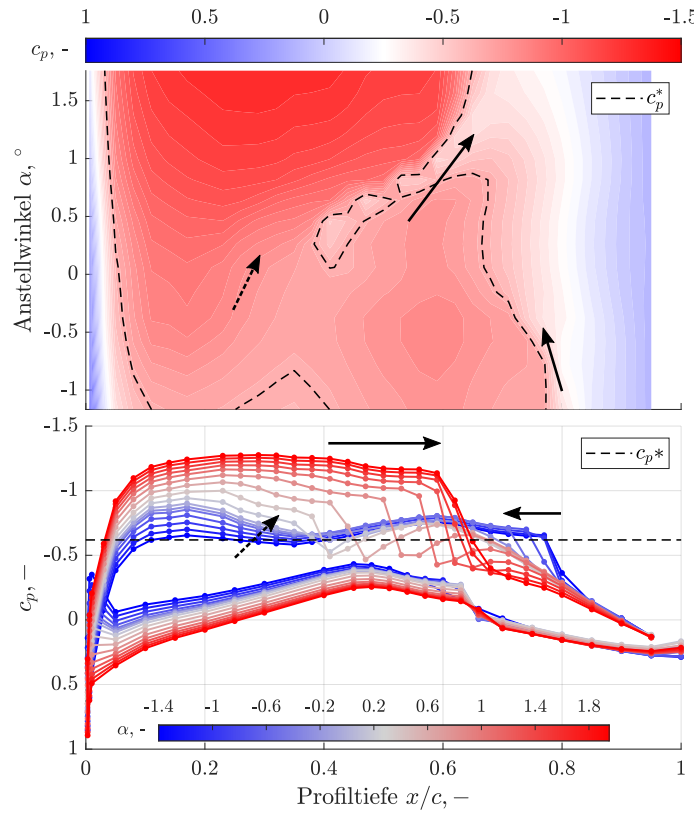

(b) Druckverteilung für $\mathrm{Ma}_{\infty}=0,74$

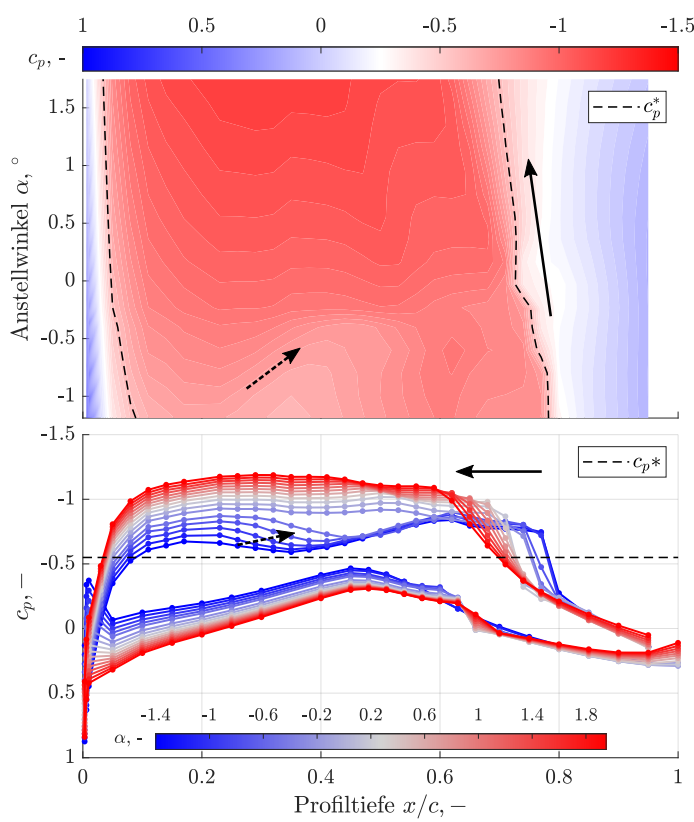

(d) Druckverteilung für $\mathrm{Ma}_{\infty}=0,76$

Abbildung 5.4.: Druckverteilungen (jeweils unten) und Konturplots der Druckbeiwerte der Profil-Saugseite (jeweils oben) für die stationären Polaren. Die gestrichelten Pfeile markieren die Ausdehnung von Überschallgebieten und die Bewegung der Druckanstiege. Die durchgezogenen Pfeile zeigen dann die Bewegung von Verdichtungsstößen an.

ausgeprägt. Die Druckanstiege um $x / c \approx 0,3$ verlaufen flach, was auf eine isentrope Rekompression hindeutet. Steilere Druckanstiege treten lediglich im hinteren Flügeltiefenbereich bei $x / c \approx 0,75$ für $\alpha \lesssim-0,6^{\circ}$ auf. Das dort ebenfalls nur schwach ausgeprägte supersonische Gebiet liefert jedoch auch hier keinen Hinweis auf Verdichtungsstöße. Die sich im Vorfeld ausbildenden $c_{p}$-Plateaus weisen vielmehr auf eine Ablöseblase hin 
(vgl. 2.1.3), welche für $\alpha>-0,6^{\circ}$ verschwindet. Das im vorderen Flügeltiefenbereich $x / c \lesssim 0,3$ auftretende supersonische Gebiet dehnt sich jedoch mit steigendem Anstellwinkel aus (gestrichelte Pfeile in Abb. 5.4(a)), was den Anstieg von $c_{m}(\alpha)$ (vgl. 5.1(c)) für diesen $\alpha$-Bereich erklärt. Zwischen $0,2^{\circ} \lesssim \alpha \lesssim 0,4^{\circ}$ bildet sich dann ein schwacher Stoß bei $x / c \approx 0,35$ aus, was mit dem Ende des Übergangsbereiches der Laminardelle übereinstimmt. Für größere $\alpha$ nimmt die Ausdehnung des supersonischen Gebietes (über den Drehpunkt bei $c / 4$ ) zu, was zu dem Anstieg des Auftriebs (vgl. 5.1(a)) und zum erneuten Abfall des Momentes (vgl. 5.1(c)) führt. Die zunehmende Stoßstärke ab $\alpha \approx 1^{\circ}$ korreliert mit der deutlichen Zunahme des Widerstands (vgl. Abb. 5.1(b) und (d)). Zusätzlich deutet die Ausbildung von Druck-Plateaus im Vorfeld des Stoßes wiederum auf laminare Ablöseblasen hin. Mit steigendem Anstellwinkel wandert der Stoß für $\mathrm{Ma}_{\infty}=0,73$ regulär stromabwärts (durchgezogene Pfeile in Abb. 5.4(a)).

Ein vergleichbares Verhalten der Druckverteilungen zeigt sich auch für $\mathrm{Ma}_{\infty}=0,74$, dargestellt in Abb. 5.4(b). Die erhöhte Anströmungsgeschwindigkeit führt jedoch zu ausgeprägteren Überschallgebieten, so dass Verdichtungsstöße bereits für kleinere Anstellwinkel auftreten. Dies verdeutlicht sich zum einen im hinteren Flügeltiefenbereich. Die lokalen Druckanstiege bei $x / c \approx 0,75$ deuten für $\alpha \lesssim-0,4^{\circ}$ nunmehr auf schwache Stöße hin, die wiederum mit einem stromauf liegenden Druckplateau (Hinweis auf Ablöseblase) einhergehen. Der Stoß wandert mit steigendem Anstellwinkel invers, also stromauf (durchgezogene Pfeile in Abb. 5.4(b)), schwächt sich ab und geht in eine stoßfreie Rekompression über. Zum anderen ist auch das vordere supersonische Gebiet ausgeprägter und schließt bereits für $\alpha \approx 0,2^{\circ}$ mit einem Verdichtungsstoß bei $x / c \approx 0,4$ ab. Analog zu $\mathrm{Ma}_{\infty}=0,73$ wächst dieses Gebiet für steigende $\alpha$ an (gestrichelte Pfeile in Abb. 5.4(b)) und der Stoß bewegt sich stromabwärts (schwarze Pfeile in Abb. 5.4(b)). Für $\alpha \gtrsim 1,4^{\circ}$ deutet sich jedoch bereits eine Verlangsamung der Stoßbewegung um $x / c \approx 0,63$ an. Die Verläufe der aerodynamischen Beiwerte (vgl. 5.1) korrelieren dabei in analoger Weise mit der Druckverteilung, wie bereits für $\mathrm{Ma}_{\infty}=0,73$ geschildert.

Für $\mathrm{Ma}_{\infty}=0,75$ sind beide supersonischen Gebiete ineinander übergegangen, wie Abb. 5.4(c) (oben) zeigt. Dieses schließt für $\alpha \approx-1,2^{\circ}$ mit einem Stoß bei $x / c \approx 0,79$ ab, welcher invers stromauf wandert (durchgezogener Pfeil in Abb. 5.4(c)). Mit Eintritt in den Übergangsbereich der Laminardelle (vgl. Abb. 5.1) schwächt sich dieser wiederum ab, bleibt aber erhalten. Im ehemaligen, vorderen supersonischen Gebiet liegt immer noch ein Druckanstieg um $x / c \approx 0,3$ vor, welcher sich jedoch nicht mehr zu einem Stoß ausbildet. Der Druckanstieg wandert stromabwärts (gestrichelter Pfeil in Abb. 5.4(c)), baut sich für $0^{\circ} \lesssim \alpha \lesssim 0,2^{\circ}$ um $x / c \approx 0,5$ deutlich auf und verschmilzt für $\alpha \approx 0,4^{\circ}$ mit dem stromauf laufenden Stoß bei $x / c \approx 0,64$. Der Verdichtungsstoß wandert bis $\alpha \approx 0,6^{\circ}$ regulär stromabwärts und bleibt dann für $\alpha \gtrsim 0,8^{\circ}$ stationär bei $x / c \approx 0,68$ 
stehen. Der Zusammenhang mit den Beiwerten (vgl. 5.1) erklärt sich wiederum mit der Ausdehnung des Überschallgebietes, analog $\mathrm{zu} \mathrm{Ma}_{\infty}=0,73$ und $\mathrm{Ma}_{\infty}=0,74$.

Die Druckverteilungen für $\mathrm{Ma}_{\infty}=0,76$ sind in Abb. 5.4(d) dargestellt. Es treten weiterhin Druckanstiege im Bereich von 0,3 $\lesssim x / c \lesssim 0,4$ auf (gestrichelter Pfeil in Abb. 5.4(d)). Diese interagieren jedoch nicht mehr mit den auftretenden Verdichtungsstößen im hinteren Bereich des Flügels. Für $\alpha \approx-1,2$ bildet sich ein Stoß bei $x / c \approx 0,78$ aus, welcher für steigende Anstellwinkel kontinuierlich invers stromauf wandert (schwarzer Pfeil in Abb. 5.4(d)). Dabei deuten Plateaus in der Druckverteilung im Vorfeld des Stoßes wiederum auf eine Ablösung hin. Die ausgeprägte inverse Stoßbewegung lässt darüber hinaus vermuten, dass sich die Ablösung bis zur Hinterkante erstreckt. Die Stoßdynamik ist für $\mathrm{Ma}_{\infty}=0,76$ insgesamt deutlich reduziert. Die Stoßposition bewegt sich innerhalb eines kleineren Bereiches von $0,7 \lesssim x / c \lesssim 0,78$. Das Überschallgebiet ist homogener und erstreckt sich über einen längeren Flügeltiefenbereich, was sich in der deutlichen Abnahme der nichtlinearen Verläufe der Beiwerte (vgl. 5.1) für $\mathrm{Ma}_{\infty}=0,76$ wiederspiegelt.
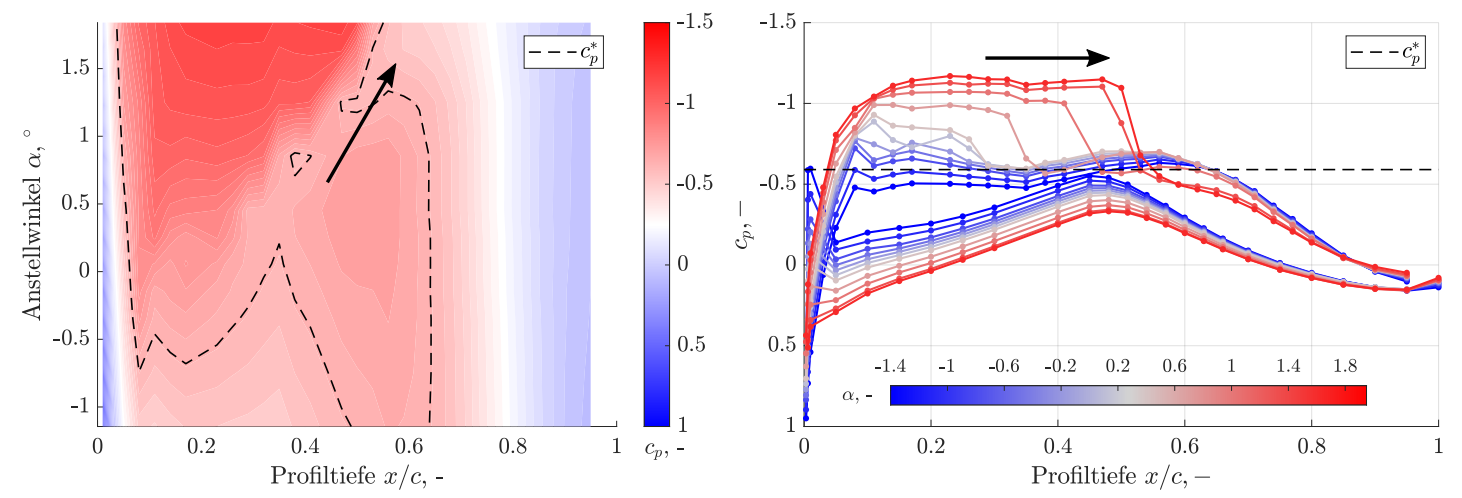

Abbildung 5.5.: Konturplot der Druckbeiwerte der Profil-Saugseite und Druckverteilung für $\mathrm{Ma}_{\infty}=0,75$ bei einer fixierten Transition.

Um den Einfluss des Grenzschichtzustands zu verdeutlichen, sind in Abb. 5.5 zusätzlich die Druckverteilungen für eine fixierte Transition für $\mathrm{Ma}_{\infty}=0,75$ dargestellt, welche mit den Beiwertverläufen in Abb. 5.3 korrespondieren. Deutlich zu erkennen ist die Ausbildung eines Überschallgebietes in der vorderen Flügeltiefenhälfte, welches kleiner ausfällt als im Vergleich zur freien Transition (vgl. Abb. 5.4(c)). Ein weiteres Überschallgebiet im hinteren Flügeltiefenbereich ist kaum ausgebildet. Dies erklärt die vergleichsweise reduzierten Auftriebsbeiwerte und höheren Nickmomente (vgl. Abb. 5.3). Ein Verdichtungsstoß tritt erst bei höheren Anstellwinkeln $\alpha \gtrsim 0,8^{\circ}$ im Bereich $0,35 \lesssim x / c \lesssim 0,55$ auf, welcher regulär stromabwärts wandert (durchgezogener Pfeil in Abb. 5.5). Die Druckbeiwerte hinter dem Stoß fallen hier jedoch kaum unter $c_{p}^{*}$ ab, was für eine schallnahe Strömungsgeschwindigkeit auch hinter dem Stoß spricht. Insgesamt zeigt sich auch für eine fixierte Transition eine deutlich reduzierte Druck- und Stoßdynamik, was mit dem nahezu linearem Verhalten der aerodynamischen Beiwerte (vgl. 5.3) zusammenhängt. 


\subsection{Verhalten der Grenzschicht}

Das Grenzschichtverhalten wurde auf Basis der CTA-Daten (vgl. 3.4.3) und entwickelten Analysemethodik (vgl. 4.3 sowie [14,61]) untersucht. In Abb. 5.6 sind die ermittelten QuasiWandschubspannungen $\tau_{q}(x, \alpha)$ und die räumlichen Gradienten $\frac{\mathrm{d} \tau_{q}}{\mathrm{~d} x}$ als Konturplots für $0,73 \leq \mathrm{Ma}_{\infty} \leq 0,76$ dargestellt. Die ermittelte Transitionsposition $x_{t r}$ ist als gestrichelte schwarze Linie eingezeichnet. Ferner sind Bereiche $\tau_{q} \leq 0,02$, also Bereiche nahe Null markiert. Wie in 4.3.2 und [14] diskutiert, liefern diese einen Hinweis auf eine Ablösung der Grenzschicht. Dabei sei auf die Unsicherheiten der detektierten Transitionspositionen im Falle einer laminaren Ablöseblase hingewiesen (siehe 2.1.4 und 4.3.2).

Abb. 5.6(a) und (b) zeigen das Grenzschichtverhalten für $\mathrm{Ma}_{\infty}=0,73$. Bis $\alpha \lesssim-0,6$ liegt die Position der Transition bei $x / c \approx 0,8$, wo sie mit dem auftretenden Druckanstieg korrespondiert (vgl. 5.2 und Abb. 5.4(a)). Stromauf deuten Plateaus in der $c_{p}$-Verteilung auf eine laminare Ablöseblase hin, was von dem in Abb. 5.6(a) markierten Bereich $\tau_{q} \leq$ 0,02 bestätigt wird. Für $-0,4^{\circ} \lesssim \alpha \lesssim 0,2^{\circ}$ wandert $x_{t r}$ dann bis $x / c \approx 0,35$ stromauf, wo die Transition bis $\alpha \approx 0,8^{\circ}$ verbleibt. Die Bewegung von $x_{t r}$ korrespondiert dabei mit dem Übergangsbereich der Laminardelle und dem Anstieg von $c_{w}$ (vgl. Abb. 5.1(b) und (d)). Der für $0,2^{\circ} \lesssim \alpha \lesssim 0,4^{\circ}$ einsetzende Stoß spiegelt sich in den größeren Gradienten $\frac{\mathrm{d} \tau_{q}}{\mathrm{~d} x}$ in Abb. 5.6(b) wieder. Es treten auch hier stromauf der Transitionslinie Bereiche $\tau_{q} \leq 0,02$ auf, die auf eine Stoß-Grenzschicht-Interaktion mit einer laminaren Ablöseblase hindeuten. Für größere Anstellwinkel bewegt sich, neben dem Stoß, auch die Transitionsposition stromabwärts. Dabei ist zu erkennen, dass Stoß- und Transitionsposition zusammenhängen (vgl. Verläufe von $x_{t r}$ und $c_{p}^{*}$ in Abb. 5.6(a) und Abb. 5.4(a)).

Analog zur $c_{p}$-Verteilung zeigt sich in Abb. 5.6(c) und (d) auch für die Grenzschicht bei $\mathrm{Ma}_{\infty}=0,74$ ein ähnliches Verhalten wie für $\mathrm{Ma}_{\infty}=0,73$. Die Transition wandert jedoch bereits für $\alpha \approx-0,6^{\circ}$ bis $x / c \approx 0,45$ stromauf. Die Zunahme des Druckanstiegs im vorderen supersonischen Gebiet (vgl. Abb. 5.4(b)) führt zu einer weiteren Transitionsverlagerung stromauf. $\mathrm{Ab} 0^{\circ} \lesssim \alpha \lesssim 0,2^{\circ}$ setzt wieder eine Stoß-Grenzschicht-Interaktion mitsamt der Ausbildung einer laminaren Ablöseblase ein. Mit dem Stoß bewegt sich die Transition und die Ablöseblase für $\alpha \geq 0,4^{\circ}$ stromab. Für $\alpha \geq 1,4^{\circ}$ stellt sich dann eine weitestgehend feste Transitionslage um $x / c \approx 0,6$ ein, wie es auch für die Stoßposition in Abb. 5.4(b) zu erkennen ist. Es zeigt sich folglich, dass die Bewegungen von Stoß- und Transitionsposition weitestgehend zusammen erfolgen und miteinander gekoppelt sind.

Für $\mathrm{Ma}_{\infty}=0,75$ kommt es in Kombination mit dem bestehenden Druckanstieg um $x / c \approx 0,3$ herum (vgl. 5.4(c)) zu einem Sprung der Transitionsposition von $x / c \approx 0,8$ bei $\alpha \approx-1^{\circ}$ hinzu $x / c \approx 0,42$ für $\alpha \approx-0,8^{\circ}$, wie Abb. 5.6(e) und Abb. 5.6(f) zeigen. Die Ausdehnung des supersonischen Gebietes sorgt jedoch bereits im Bereich $0^{\circ} \lesssim \alpha \lesssim 0,2^{\circ}$ 


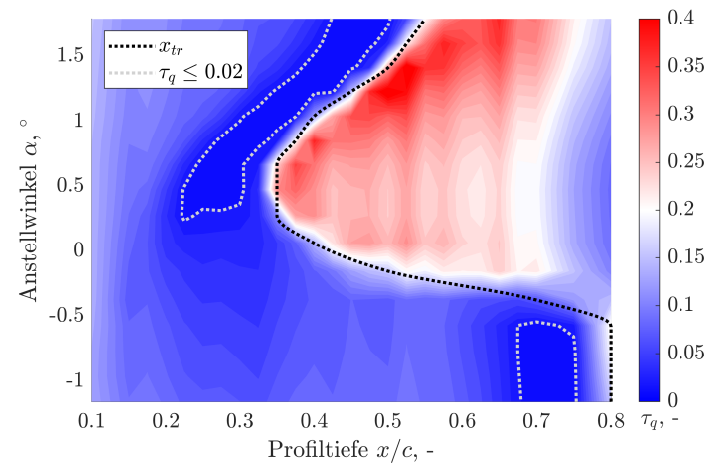

(a) $\tau_{q}$ für $\mathrm{Ma}_{\infty}=0,73$

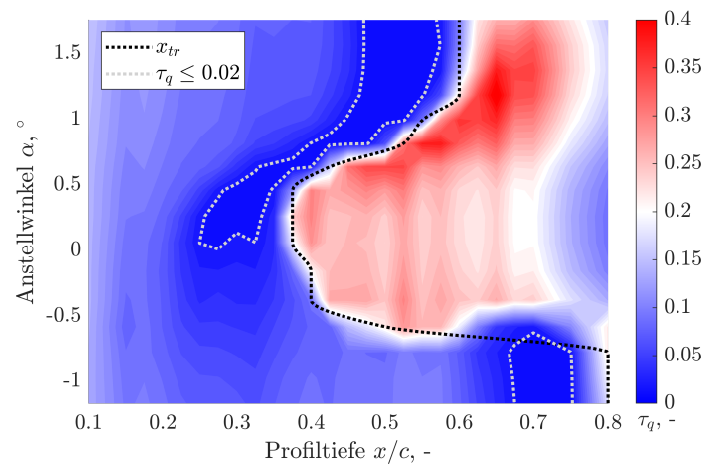

(c) $\tau_{q}$ für $\mathrm{Ma}_{\infty}=0,74$

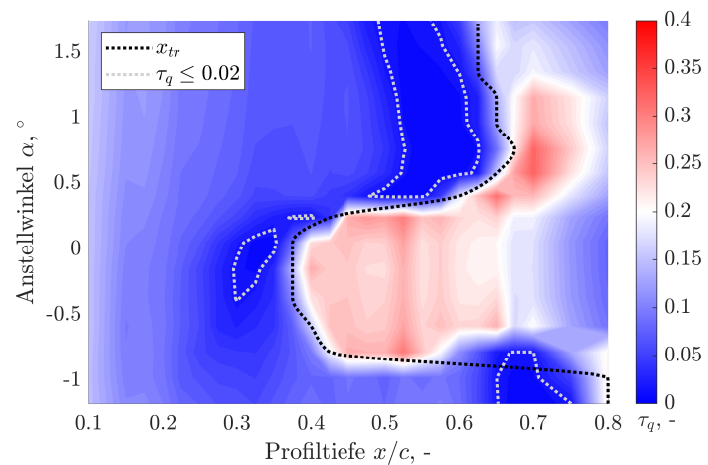

(e) $\tau_{q}$ für $\mathrm{Ma}_{\infty}=0,75$

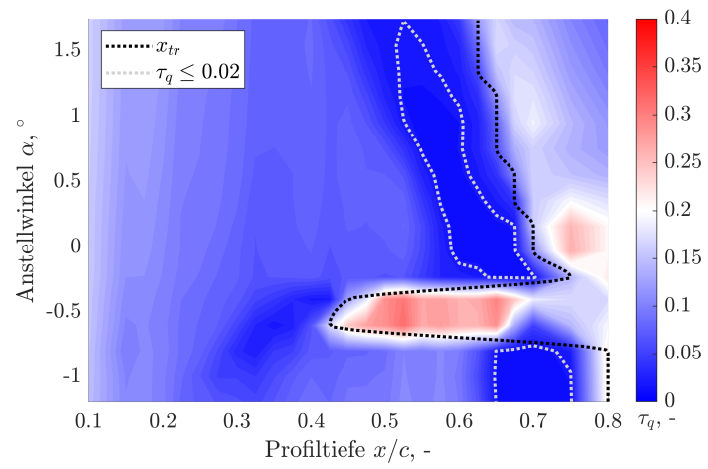

(g) $\tau_{q}$ für $\mathrm{Ma}_{\infty}=0,76$

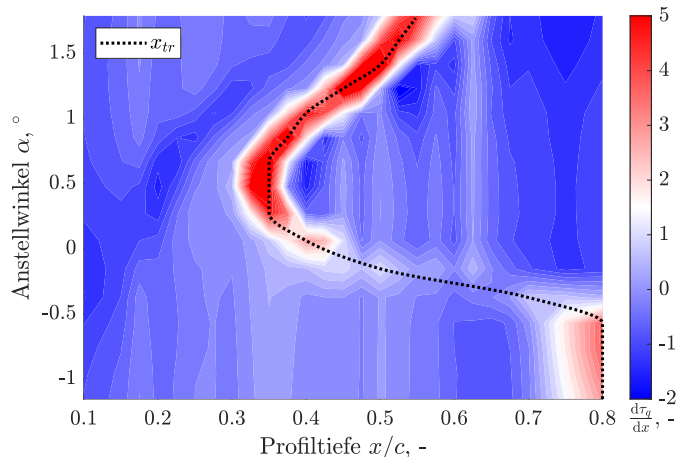

(b) $\frac{\mathrm{d} \tau_{q}}{\mathrm{~d} x}$ für $\mathrm{Ma}_{\infty}=0,73$

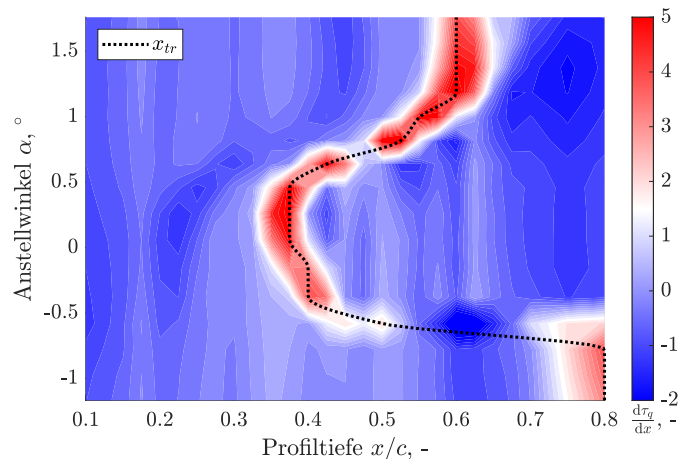

(d) $\frac{\mathrm{d} \tau_{q}}{\mathrm{~d} x}$ für $\mathrm{Ma}_{\infty}=0,74$

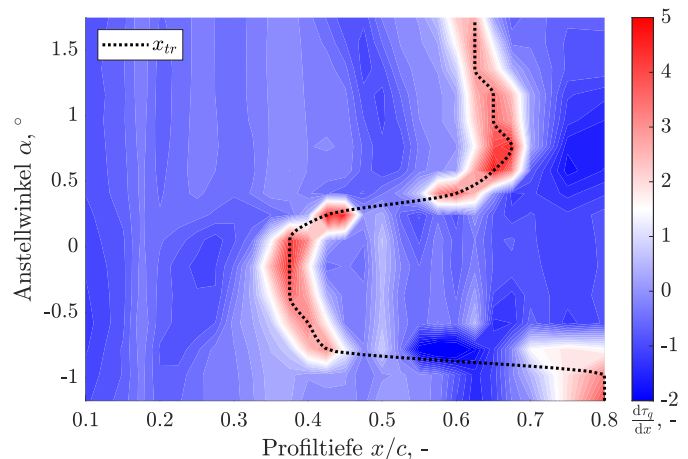

(f) $\frac{\mathrm{d} \tau_{q}}{\mathrm{~d} x}$ für $\mathrm{Ma}_{\infty}=0,75$

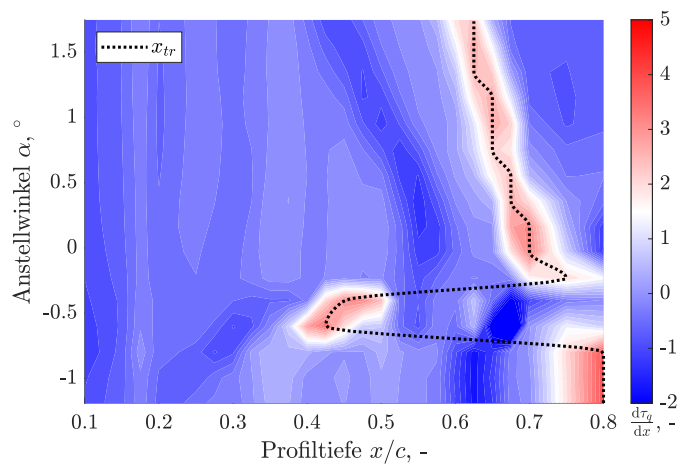

(h) $\frac{\mathrm{d} \tau_{q}}{\mathrm{~d} x}$ für $\mathrm{Ma}_{\infty}=0,76$

Abbildung 5.6.: Verteilung der Quasi-Wandschubspannung $\tau_{q}(\alpha)$ und des räumlichen Gradienten $\frac{\mathrm{d} \tau_{q}}{\mathrm{~d} x}$. Ferner sind die Transitionsposition $x_{t r}$ und Bereiche mit $\tau_{q} \leq 0,02$ markiert. 
für eine zunächst moderate Bewegung von $x_{t r}$ stromabwärts. Mit der Ausbildung des Stoßes bei $x / c \approx 0,64$ für $\alpha \approx 0,4^{\circ}$ bewegt sich auch die Transition abrupt stromabwärts. Es kommt zur Stoß-Grenzschicht-Interaktion und der Ausbildung einer laminaren Ablöseblase. Die erneute Bewegung der Transition stromaufwärts für $\alpha>0,6^{\circ}$ deutet, gemäß der beobachteten Kopplung beider Bewegungen, bereits auf eine einsetzende inverse Stoßbewegung hin, wie sie für $\mathrm{Ma}_{\infty} \approx 0,76$ deutlich erkennbar ist.

Das Grenzschichtverhalten für $\mathrm{Ma}_{\infty} \approx 0,76$ ist in Abb. 5.6(g) und Abb. 5.6(h) dargestellt. Der für $\alpha \lesssim-0,4$ vorliegende Druckanstieg um $0,3 \lesssim x / c \lesssim 0,4$ herum (vgl. Abb. 5.4(d)) sorgt im Bereich $-0,6^{\circ} \lesssim \alpha \lesssim-0,4^{\circ}$ zu einem abrupten Sprung der Transition stromaufwärts. Mit $\alpha \geq-0,2^{\circ}$ verschwindet der Druckanstieg und auch die Transition springt wieder zurück. Über den gesamten weiteren Anstellwinkelbereich liegt dann eine Stoß-Grenzschicht-Interaktion in Kombination mit einer Ablöseblase vor. Dementsprechend bewegt sich auch $x_{t r}$ stromauf, der inversen Stoßbewegung (vgl. Abb. 5.4(d)) folgend. Es zeigt sich somit erneut die Kopplung beider Bewegungen.

In Abb. 5.7(a) sind die ermittelten Transitionspositionen $x_{t r}$ zusammengefasst. Abb. 5.7(b) stellt die Änderung von $x_{t r} / c$ bei einer Änderung des Anstellwinkels als Gradient $\frac{\mathrm{d} x_{t r} / c}{\mathrm{~d} \alpha}$ dar, analog zu den stationären Derivativen (vgl. Abb. 5.2). So wird aus Abb. 5.7(a) noch-

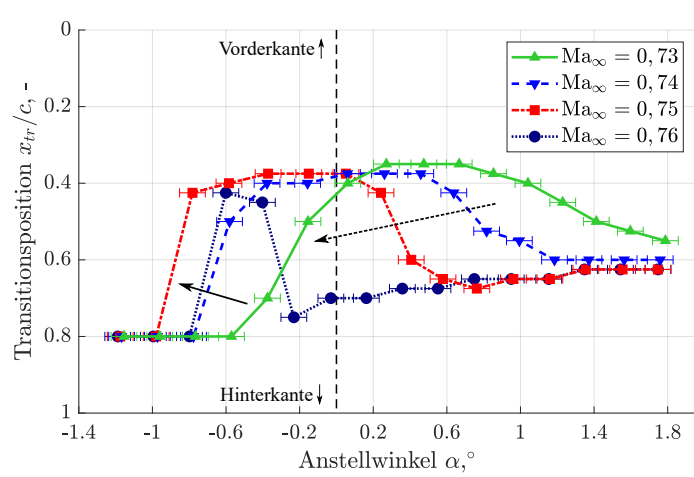

(a) Transitionsposition $x_{t r} / c$

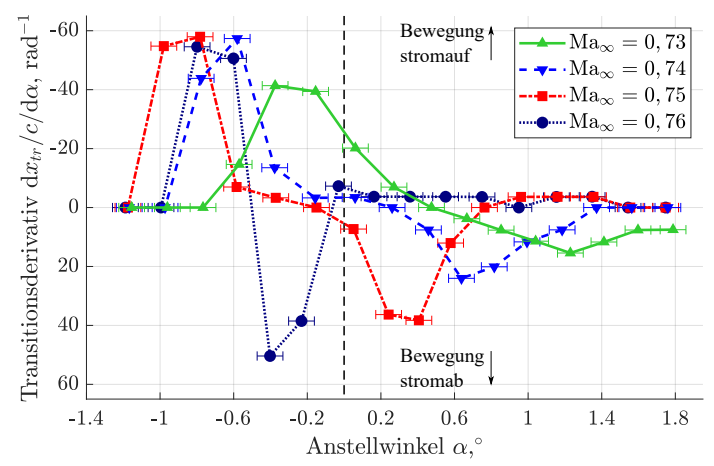

(b) Transitionsgradient $\frac{\mathrm{d} x_{t r} / c}{\mathrm{~d} \alpha}$

Abbildung 5.7.: Transitionspositionen (a) und Transitionsgradienten (b) für die stationären Polaren. Die gestrichelte schwarze Linie markiert einen Anstellwinkel von $\alpha=0^{\circ}$.

mals deutlich, dass mit steigender Mach-Zahl die Transition für kleinere Anstellwinkel stromauf wandert (durchgezogener Pfeil in Abb. 5.7(a)), sich jedoch die Ausdehnung der Transitionslinie und somit der turbulenten Grenzschicht über einen kleineren Anstellwinkelbereich erstreckt (gestrichelter Pfeil in Abb. 5.7(a)). Dies hängt einerseits mit der Ausprägung der Laminardelle zusammen, wie Abb. 5.1(b) zeigt. Andererseits führt dies auch zu den großen Gradienten in Abb. 5.7(b), welche die rapide Bewegung der Transition stromauf $\left(\frac{\mathrm{d} x_{t r} / c}{\mathrm{~d} \alpha}<0\right)$ und stromab $\left(\frac{\mathrm{d} x_{t r} / c}{\mathrm{~d} \alpha}>0\right)$ zum Ausdruck bringen. Die 
Gradienten verdeutlichen dabei die Sensitivität der Grenzschicht, bzw. der Transitionsposition bzgl. einer Änderung des Anstellwinkels. So kann Abb. 5.7(b), analog zu den Derivativa der aerodynamischen Beiwerte in Abb. 5.2, als Hinweis für eine Instationarität der Grenzschicht im Übergangsbereich der Laminardelle interpretiert werden.

\subsection{Instationäre Effekte bei statischen Anstellwinkeln}

Die stationären Derivativa der aerodynamischen Beiwerte (vgl. Abb. 5.2) sowie die Gradienten der Transitionsposition (vgl. Abb. 5.7(b)) deuten bereits auf aerodynamische Instabilitäten hin, verbunden mit einer Instationarität des Strömungsfeldes. Dies spiegelt sich auch in den erhöhten Standardabweichungen der aerodynamischen Beiwerte, insbesondere für $c_{m}$ im Bereich $-0.4^{\circ} \lesssim \alpha \lesssim 0^{\circ}$ bei $\mathrm{Ma}_{\infty}=0,73$ und $\mathrm{Ma}_{\infty}=0,74$ wieder (vgl. Fehlerbalken in Abb. 5.1). Instationäre Effekte treten folglich auch in den Druckverteilungen und Quasi-Wandschubspannungen auf, was die Standardabweichungen von $c_{p}$ und $\tau_{q}$ in Abb. 5.8 und Abb. 5.9 verdeutlichen.

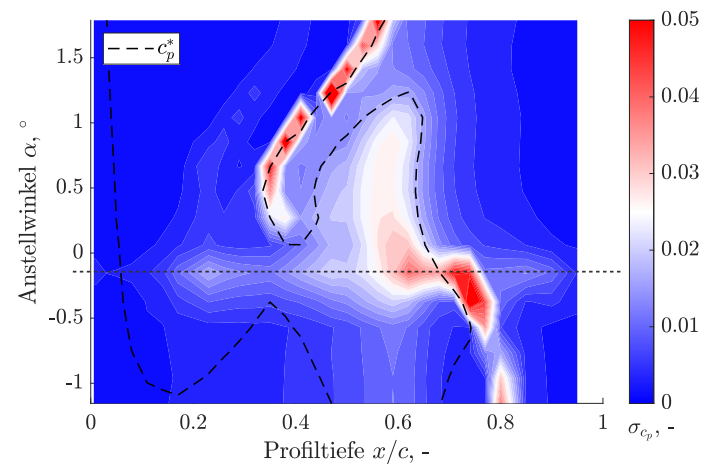

(a) $\sigma_{c_{p}}$ für $\mathrm{Ma}_{\infty}=0,73$

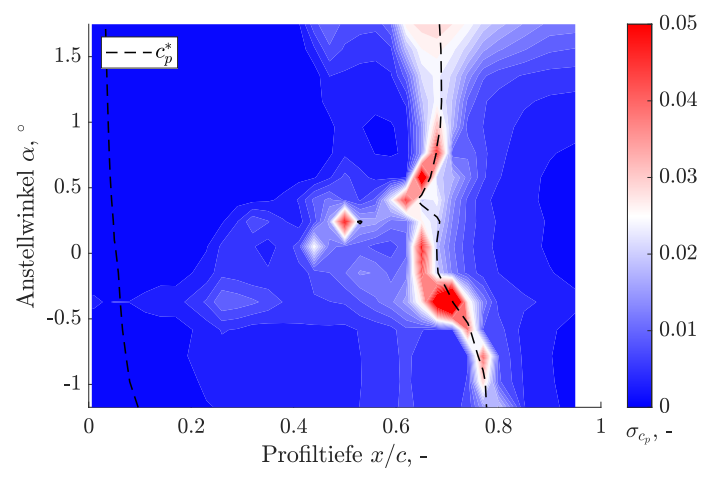

(c) $\sigma_{c_{p}}$ für $\mathrm{Ma}_{\infty}=0,75$

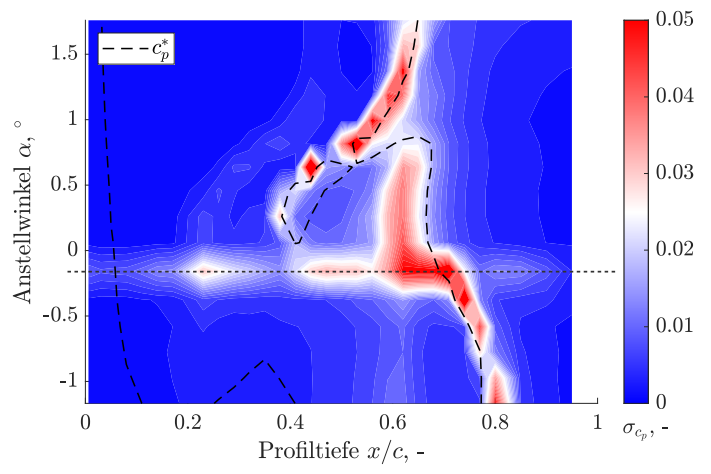

(b) $\sigma_{c_{p}}$ für $\mathrm{Ma}_{\infty}=0,74$

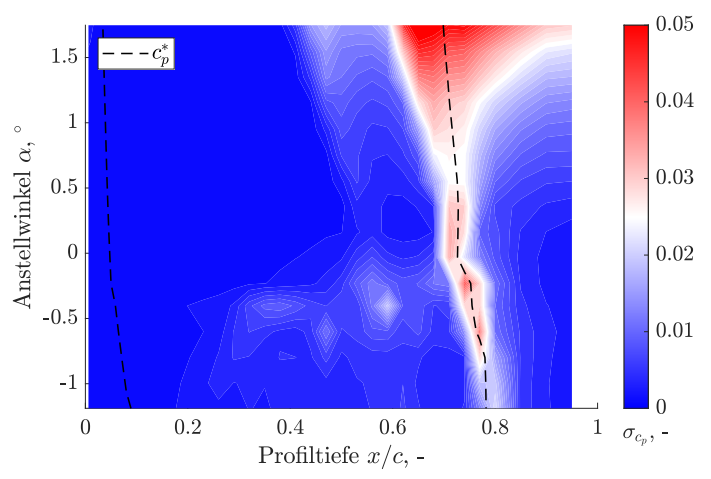

(d) $\sigma_{c_{p}}$ für $\mathrm{Ma}_{\infty}=0,76$

Abbildung 5.8.: Standardabweichung $\sigma_{c_{p}}$ der Druckbeiwerte der Profil-Saugseite für die stationären Polaren.

Wie die rötlich eingefärbten Gebiete in Abb. 5.8 zeigen, ist $\sigma_{c_{p}}$ entlang der Konturlinie $c_{p}^{*}$ erhöht, was in Teilen auf lokale Schwankungen der Stoßposition hindeutet. Für 
$\mathrm{Ma}_{\infty}=0,76$ tritt zusätzlich ein Bereich mit erhöhten $\sigma_{c_{p}}$-Werten für Anstellwinkel $\alpha \gtrsim 0,75^{\circ}$ auf, der sich in Richtung Profil-Hinterkante ausdehnt. Dies weist auf eine beginnende Ablösung an der Hinterkante hin, wie es bereits die inverse Stoßbewegung vermuten lässt (vgl. Abb. 5.4(d)). $\tau_{q}$ fällt zwar nicht auf Werte nahe null ab (vgl. Abb. 5.6(g)), jedoch verläuft $\tau_{q}(x)$ insgesamt bei kleineren Werten als im Vergleich zu $\mathrm{Ma}_{\infty}<0,76$ (vgl. Abb. 5.6(a) bis 5.6(e)). Eine Ablösung an der Hinterkante ist daher naheliegend. Die zeitlichen Verläufe $c_{p}(t)$ und die Schwankungen von $\tau_{q}(t)$ bei hohen Anstellwinkeln für $\mathrm{Ma}_{\infty}=0,76$ unterstützen dies, wie Abb. D.1 im Anhang zeigt.

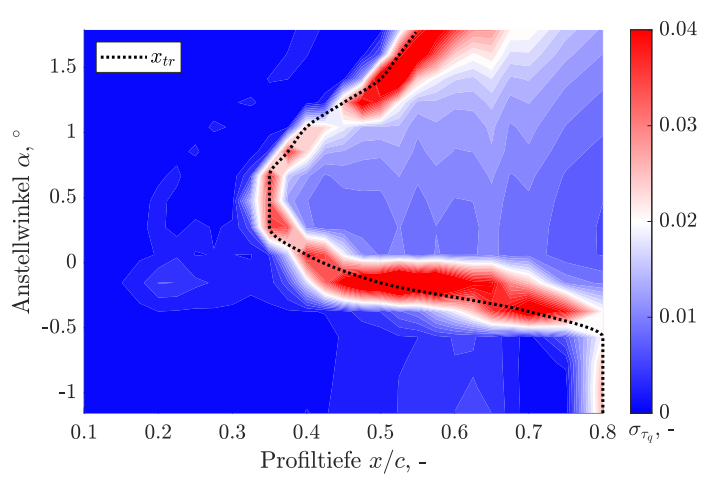

(a) $\sigma_{\tau_{q}}$ für $\mathrm{Ma}_{\infty}=0,73$

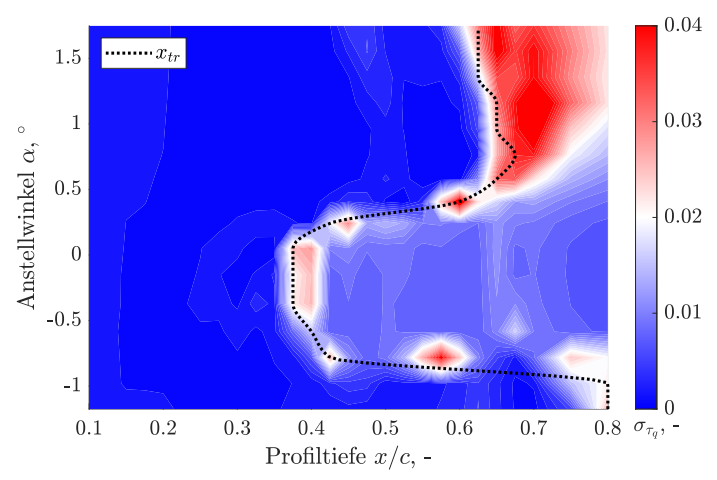

(c) $\sigma_{\tau_{q}}$ für $\mathrm{Ma}_{\infty}=0,75$

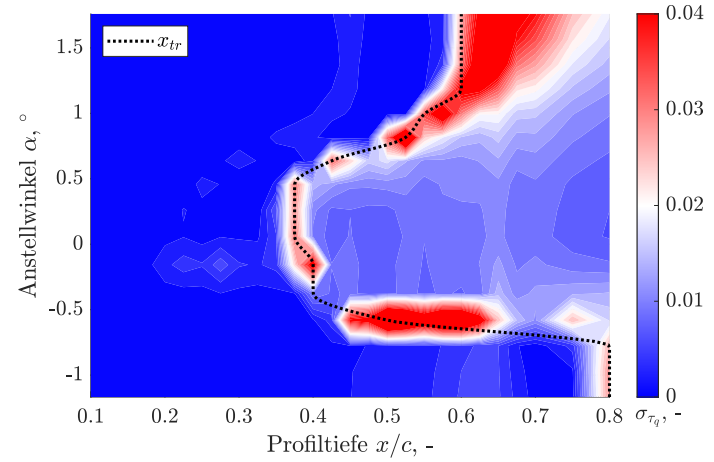

(b) $\sigma_{\tau_{q}}$ für $\mathrm{Ma}_{\infty}=0,74$

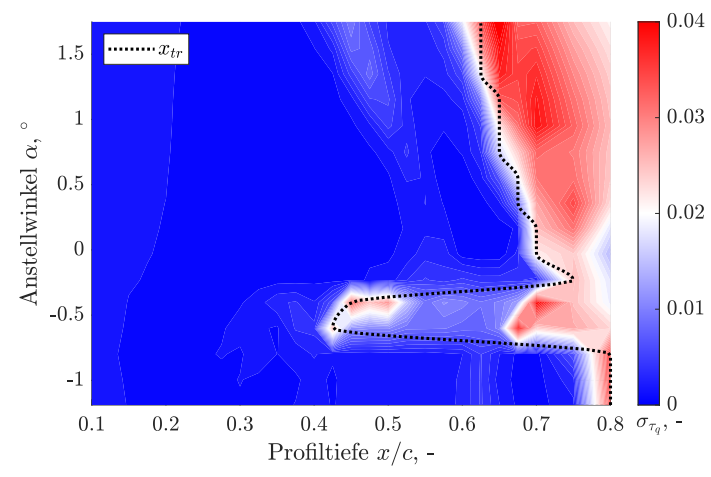

(d) $\sigma_{\tau_{q}}$ für $\mathrm{Ma}_{\infty}=0,76$

Abbildung 5.9.: Standardabweichung $\sigma_{\tau_{q}}$ der Quasi-Wandschubspannung der Profil-Saugseite für die stationären Polaren.

Auch die Standardabweichungen von $\tau_{q}$, dargestellt in Abb. 5.9, sind stromab der Transitionslinie $x_{t r}$ in Teilen deutlich erhöht. Eine trichterförmige Ausdehnung höherer Werte von $\sigma_{\tau_{q}}$ in Richtung Hinterkante ist bereits für $\mathrm{Ma}_{\infty}=0,73 \mathrm{ab} \alpha \gtrsim 1^{\circ}$ zu beobachten. Für größere Mach-Zahlen tritt dies dann bereits bei kleineren Anstellwinkeln auf. Bei $\mathrm{Ma}_{\infty}=0,76$ ist $\sigma_{\tau_{q}}$ im turbulenten Grenzschichtbereich fast über den gesamten untersuchten Anstellwinkelbereich erhöht. Für alle Mach-Zahlen sind die Werte vergleichsweise zwei- bis dreimal höher als im turbulenten Grenzschichtbereich bei Anstellwinkeln im Übergangsbereich der Laminardelle. Demnach führt eine Interaktion der Grenzschicht mit einem (ausreichend starken) Verdichtungsstoß zu einem Anstieg von $\sigma_{\tau_{q}}$ 
in der turbulenten Grenzschicht. Darüber hinaus nehmen die Standardabweichungen der Quasi-Wandschubspannung entlang der Transitionslinien lokale Maxima an. Gemäß der Auswertungsmethodik und dem Zusammenhang mit einem Intermittenzfaktor von $\gamma=0,5$ ist dies zu erwarten (vgl. 2.1.4 und 4.3). Für $\mathrm{Ma}_{\infty} \approx 0,73$ um $\alpha \approx-0,2^{\circ}$ und für $\mathrm{Ma}_{\infty} \approx 0,74 \mathrm{um} \alpha \approx-0,6^{\circ}$ treten darüber hinaus erhöhte $\sigma_{\tau_{q}}$-Werte über einen ausgedehnten Profiltiefenbereich auf. Hier zeichnet sich eine verstärkte Bewegung der Position der Grenzschichttransition ab. Die Transitionsgradienten weisen für diese $(\alpha$, $\mathrm{Ma}_{\infty}$ )-Werte ihre Minima auf (maximale Bewegung stromauf, vgl. Abb. 5.7(b)).
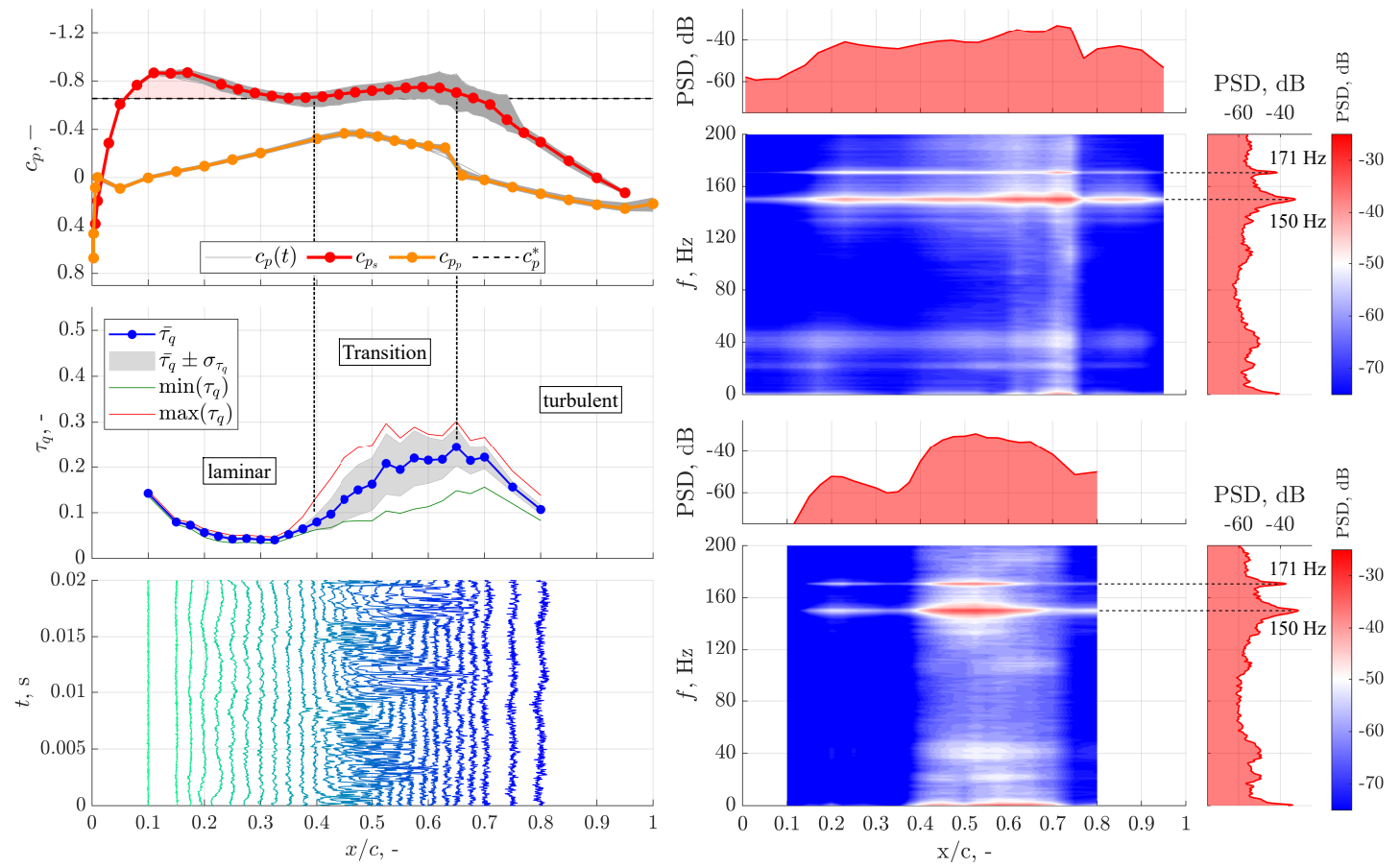

Abbildung 5.10.: Druckverteilung, Quasi-Wandschubspannung, CTA-Zeitreihen und Leistungsdichtespektren für $\mathrm{Ma}_{\infty}=0,73$ und $\alpha=-0,15^{\circ}$.

Die Instationaritäten gehen dabei mit Frequenzanteilen einher, die in den Spektren der Druckverteilungen und der Quasi-Wandschubspannungen deutlich hervortreten. Dies ist für $\mathrm{Ma}_{\infty}=0,73$ und $\alpha=-0,15^{\circ}$ in Abb. 5.10 dargestellt. Diese zeigt oben links die gemittelte Druckverteilung ( $c_{p_{s}}$ für Saug- und $c_{p_{p}}$ für Druckseite) und den zeitlichen Verlauf $c_{p}(t)$. Darunter ist der Verlauf der mittleren Quasi-Wandschubspannung $\bar{\tau}_{q}$ mit der Schwankungsbreite $\pm \sigma_{\tau_{q}}$ und der Verlauf der minimalen und maximalen Werte dargestellt. In der unteren Grafik ist ein Ausschnitt der Spannungszeitschriebe der Heißfilm-Anemometer abgebildet. Rechts oben ist die PSD der Druckbeiwerte der FlügelSaugseite gezeigt, rechts unten die PSD der Quasi-Wandschubspannungen. Die PSDs am Rand der Konturplots stellen jeweils die projizierten Maximalwerte dar.

Der zeitliche Verlauf der Druckverteilung $c_{p}(t)$ und die Schwankungen der QuasiWandschubspannung verdeutlichen die Instationarität der Strömung bei $\mathrm{Ma}_{\infty}=0,73$ 
und $\alpha=-0,15^{\circ}$. Es treten Frequenzanteile bei $150 \mathrm{~Hz}$ und $171 \mathrm{~Hz}$ (abgeschwächt bei $40 \mathrm{~Hz}$ ) in Erscheinung, welche in Abb. 5.10 markiert sind. Die Leistungsdichte der QuasiWandschubspannung steigt lokal insbesondere im Transitionsbereich bei $0,4 \lesssim x / c \lesssim 0,65$ an, was eine Verbindung der Frequenzanteile mit der Grenzschichtransition nahelegt. Verdichtungsstöße treten bei diesen Randbedingungen kaum auf, wie die Druckverteilung in Abb. 5.10 zeigt. Klassisches Buffet, welches mit einer ausgeprägten Stoßdynamik einhergeht (vgl. 2.2.3), liegt demnach nicht vor.
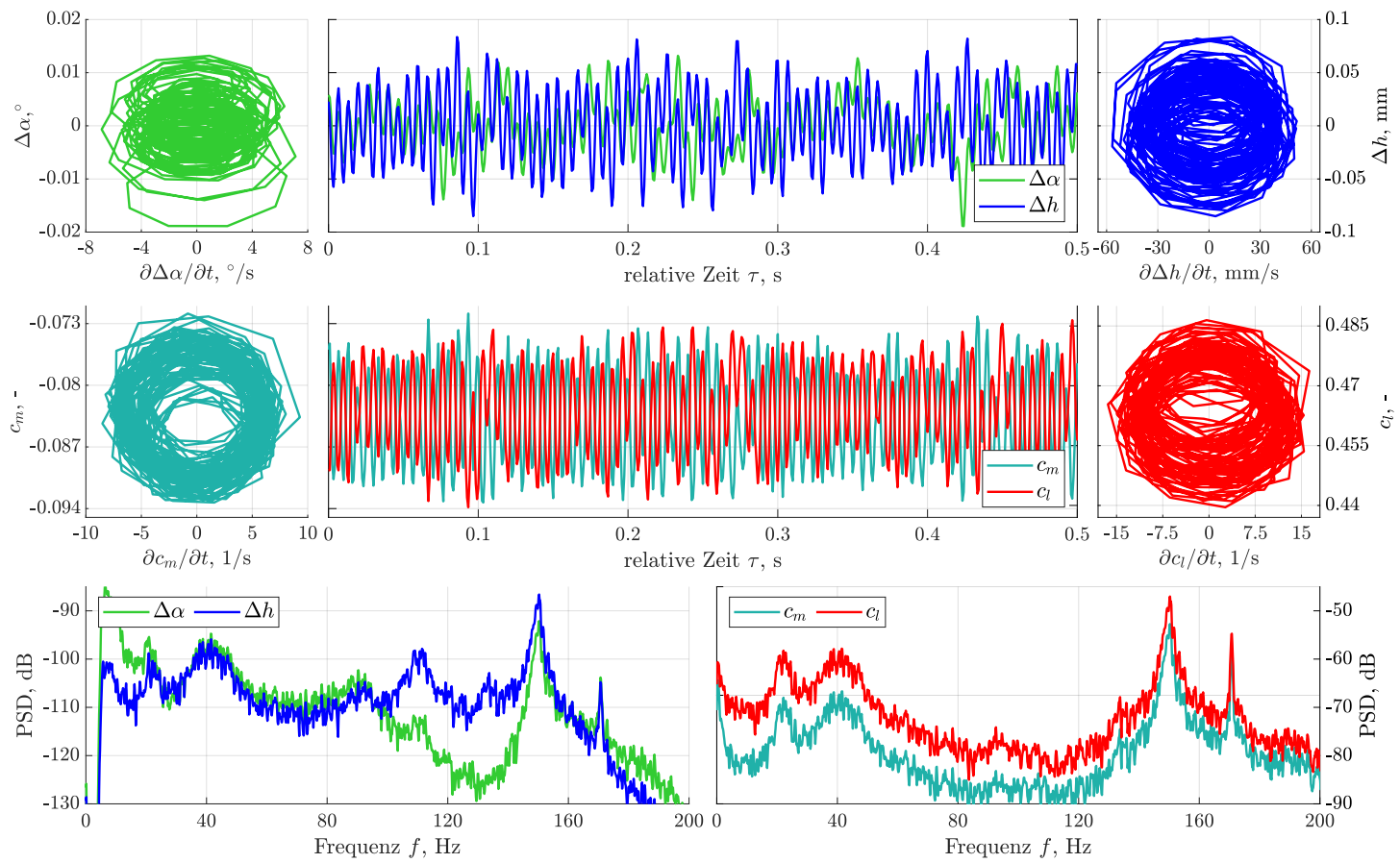

Abbildung 5.11.: Bewegung des Modellmittelschnitts $\Delta \alpha$ und $\Delta h$ und Zeitreihenauschnitt der aerodynamischen Beiwerte $c_{m}$ und $c_{l}$ sowie Leistungsdichtespektren für $\mathrm{Ma}_{\infty}=0,73$ und $\alpha=-0,15^{\circ}$.

Aerodynamisch wirken sich die Instationaritäten dennoch deutlich aus, wie die starken Oszillationen der aerodynamischen Beiwerte in Abb. 5.11 zeigen. Die Phasendiagramme verdeutlichen, dass sich annähernd aerodynamische Grenzzyklen ausbilden. Insbesondere das Nickmoment weist dabei vergleichsweise große Amplituden auf. Die Spektren von $c_{m}$ und $c_{l}$ zeigen die gleichen Frequenzanteile wie die PSDs in Abb. 5.10. Dabei sei angemerkt, dass die auftretenden Frequenzen weder in Beziehung zu Eigenfrequenzen des Windkanalmodells (vgl. 7.1) stehen, noch mit möglichen Windkanalresonanzen (vgl. F.3) korrespondieren. Das Windkanalmodell wird unter den aerodynamischen Lasten zwar zur Schwingung angeregt, jedoch sind die auftretenden Modellbewegungen vernachlässigbar klein, wie Abb. 5.11 oben zeigt. Die Standardabweichung der Drehbewegung des Modellmittelschnitts beträgt für den dargestellten Zeitausschnitt $\sigma_{\alpha}<0,01^{\circ}$, für die Schlagbewegung ist $\sigma_{h}<0,04 \mathrm{~mm}$. Die Oszillationen der Aerodynamik sind somit nicht das Resultat von Modellschwingungen, sondern sie sind selbsterregt. 
Die Instationaritäten und Frequenzanteile sind dabei stark vom Anstellwinkel abhängig. Für $\mathrm{Ma}_{\infty}=0,73$ sind in Abb. D.2 bis D.5 jeweils Ergebnisse bei einem etwas geringeren bzw. höheren Anstellwinkel dargestellt. Bei $\alpha=-0,37^{\circ}$ treten Frequenzanteile um $145 \mathrm{~Hz}$ herum auf. Für $\alpha=0,06^{\circ}$ finden sich deutlich abgeschwächte Frequenzanteile um $44 \mathrm{~Hz}$ und $143 \mathrm{~Hz}$. Bei einer weiteren Änderung des Anstellwinkels $(\alpha \uparrow \downarrow)$ verschwinden die Frequenzanteile.

Vergleichbar verhält es sich auch bei höheren Mach-Zahlen. Für $\mathrm{Ma}_{\infty}=0,74$ sind Ergebnisse in Abb. D.6 bis Abb. D.11 und für $\mathrm{Ma}_{\infty}=0,75$ in Abb. D.12 bis Abb. D.17 im Anhang dargestellt. Dominierende Frequenzanteile treten hier vorwiegend bei kleineren Frequenzen $f<40 \mathrm{~Hz}$ und etwas geringeren Anstellwinkeln auf. Zum Teil sind höhere Harmonische (vgl. Abb. D.8) und eine verstärkte Anregung der Modellstruktur (vgl. Abb. D.9) zu beobachten. Mit steigender Mach-Zahl konzentrieren sich dabei die Instationaritäten zunehmend auf die Druckverteilungen (vgl. Abb. D.14).

Zusammenfassend zeigt sich, dass auftretende Instationaritäten wiederum im Zusammenhang mit der Laminardelle bzw. einer freien Grenzschichttransition stehen. Verschwindet die Laminardelle, so treten auch keine Frequenzanteile mehr in Erscheinung, wie es für $\mathrm{Ma}_{\infty}=0,76$ oder auch für $\mathrm{Ma}_{\infty}=0,75$ bei einer fixierten Transition der Fall ist.

\subsection{Zusammenfassung der stationären Aerodynamik}

Die Ergebnisse der stationären Messungen bestätigen erneut die Laminarflügel-Charakteristik des CAST 10-2 Tragflügelprofils. Für $\operatorname{Re}_{\infty} \approx 2 \cdot 10^{6}$ und $0,73 \lesssim \mathrm{Ma}_{\infty} \lesssim 0,75$ bildet sich eine Laminardelle aus. Mit steigender Mach-Zahl nimmt die Ausdehnung ab, für $\mathrm{Ma}_{\infty}=0,76$ oder bei einer fixierten Transition ist diese nicht mehr zu beobachten.

Die aerodynamischen Beiwerte sowie die stationären Derivativa weisen im Übergangsbereich der Laminardelle deutliche Nichtlinearitäten auf. Es zeigt sich eine hohe Sensitivität der Beiwerte, insbesondere von $c_{m}$ bezüglich $\alpha$. Die Schwankungen der Derivativa und des statischen Stabilitätsmaß liefern erste Hinweise auf Instabilitäten sowie eine mögliche Amplitudenbegrenzung im Übergangsbereich. Dabei zeigt sich die Verbindung der Nichtlinearitäten und Fluktuationen mit der freien Grenzschichttransition.

Aerodynamische Vorgänge konzentrieren sich auf die Profil-Saugseite. Im vorderen Flügeltiefenbereich bildet sich ein supersonisches Gebiet und ein Druckanstieg aus, welcher für alle $\mathrm{Ma}_{\infty}$ eine teils rapide Bewegung der Transition stromauf induziert. So zeigt sich auch für die Grenzschichttransition eine ausgeprägte Sensitivität bzgl. des Anstellwinkels. Ferner deutet dies eine Instationarität der Grenzschicht im Übergangsbereich der Laminardelle an. Für $\mathrm{Ma}_{\infty} \geq 0,74$ bildet sich aus dem Druckanstieg ein Verdichtungsstoß aus, 
welcher für steigende Anstellwinkel regulär stromab wandert. $\mathrm{Ab} \mathrm{Ma}_{\infty} \gtrsim 0,75$ tritt dann eine inverse Stoßbewegung auf, was auf eine beginnende Ablösung an der Hinterkante hindeutet. Im Zusammenspiel mit der Transition liegt eine Stoß-Grenzschicht-Interaktion in Kombination mit einer laminaren Ablöseblase vor. Transition- und Stoßposition sind dabei miteinander verbunden.

Instationäre Effekte treten bereits bei statischen Anstellwinkeln auf und sind mit diskreten Frequenzanteilen verbunden. Deutlich ersichtlich sind diese nur in einem begrenzten Anstellwinkelbereich, der wiederum mit dem Übergangsbereich der Laminardelle verknüpft ist. Die Frequenzanteile unterscheiden sich dabei stark in Abhängigkeit der Mach-Zahl. Lokal konzentrieren sich die maximalen Leistungsdichten für $\mathrm{Ma}_{\infty}=0,73$ deutlich um die Position der Grenzschichttransition, wohingegen sich mit steigender Mach-Zahl eine Lokalisierung in Richtung Verdichtungsstoß abzeichnet. Gleichwohl zeigt sich eine allgemeine Verbindung der Instationaritäten mit einer freien Grenzschichttransition. Das Verschwinden der Instationaritäten für $\mathrm{Ma}_{\infty}=0,76$ oder eine fixierte Transition bestätigen dies. Die Ergebnisse legen ferner nahe, dass es sich hierbei um selbsterregte Schwingungen der Aerodynamik handelt, was die Hinweise bzgl. aerodynamischer Instabilitäten des CAST 10-2 Laminarflügels im Übergangsbereich der Laminardelle unterstützt. Eine Abschätzung von Resonanzfrequenzen (vgl. F.3) in der geschlossenen Messstrecke des DNW-TWG schließt ferner eine Beteiligung von möglichen Windkanalresonanzen aus. 


\section{Aerodynamisches Verhalten bei zwangserregten Nickschwingungen}

Die Untersuchungen zur instationären Aerodynamik des CAST 10-2 Laminarflügelprofils schließen sich direkt an die stationären Messungen an. Mithilfe des NVS (vgl. 3.2.1) wurde das Laminarflügel-Modell in zwangserregte Nickschwingungen mit diskreten Amplituden $\hat{\alpha}$ von $(0,10 \pm 0,02)^{\circ},(0,25 \pm 0,02)^{\circ}$ und $(0,50 \pm 0,02)^{\circ}$ versetzt. Die Frequenzen wurden in einem Bereich von $13 \mathrm{~Hz} \leq f_{\alpha} \leq 73 \mathrm{~Hz}$ variiert, entsprechend einer reduzierten Frequenz von $0,05 \lesssim \omega^{*} \lesssim 0,27$. Der mittlere Anstellwinkel ${ }^{1}$ betrug für diskrete Frequenzvariationen $\bar{\alpha}=(0,05 \pm 0,02)^{\circ}$. Die instationären Messungen wurden somit direkt im oberen Übergangsbereich der Laminardelle $\left(c_{l} \approx 0,45\right)$ durchgeführt. Zusätzlich wurden Frequenzsweeps für verschiedene mittlere Anstellwinkel von $-0,8^{\circ} \lesssim \bar{\alpha} \lesssim 0,9^{\circ}$ aufgezeichnet. Sämtliche Messungen erfolgten bei einer Mach-Zahl von $0,73 \leq \mathrm{Ma}_{\infty} \leq 0,75$ und einem Ruhedruck von $p_{0}=54 \mathrm{kPa}$. Die Reynolds-Zahl betrug somit wieder $\operatorname{Re}_{\infty} \approx 2 \cdot 10^{6}$, so dass eine direkte Vergleichbarkeit zu den stationären Polaren (vgl. 5) gegeben ist.

Das folgende Kapitel behandelt die Ergebnisse dieser instationären Messungen. Einerseits werden die instationären Beiwerte und die Energiebilanzen zwischen Strukturbewegung und Strömung dargestellt. Andererseits wird das instationäre Verhalten der Druckbeiwerte und der Grenzschicht, bzw. das Zusammenspiel von Verdichtungsstößen und der laminarturbulenten Grenzschichttransition diskutiert.

\subsection{Instationäre Derivativa der aerodynamischen Beiwerte}

Ausgehend von den Zeitreihen der aerodynamischen Beiwerte, welche wie zuvor gemäß der in 4.2.1 dargestellten Methodik ermittelt wurden, folgen zusammen mit dem Bewegungssignal des Windkanalmodells die instationären Derivativa. Hierfür wurde das instationäre Anstellwinkelsignal $\alpha_{\text {inst }}(t)$ gemäß Gl. (3.4.3) als Eingangssignal verwendet. Die Berechnung der instationären Derivativa erfolgte dann mittels eines $H_{1}$-Schätzers gemäß den Anmerkungen in 4.2.3.

\footnotetext{
${ }^{1}$ Bei instationären Messungen (bewegte Windkanalmodelle) erfolgt eine Adpation der Winkanalwände (vgl. 3.1) an das stationäre Strömungsfeld des mittleren Modell-Anstellwinkels. Für moderat schwingende Windkanalmodelle führt dies zur Minimierung der verbleibenden Wandinterferenz [27].
} 
In Abb. 6.1 sind die instationären Auftriebs- und Momentenderivativa $c_{l, \alpha}$ und $c_{m, \alpha}$ als Betrag bzw. Amplituden- (Abb. 6.1(a) und Abb. 6.1(c)) und Phasengang (Abb. 6.1(b) und Abb. 6.1(d)) dargestellt. Diese wurden mit einer diskreten Frequenzvariation bei einer Schwingungsamplitude von $\hat{\alpha}=0,10^{\circ}$ bestimmt.

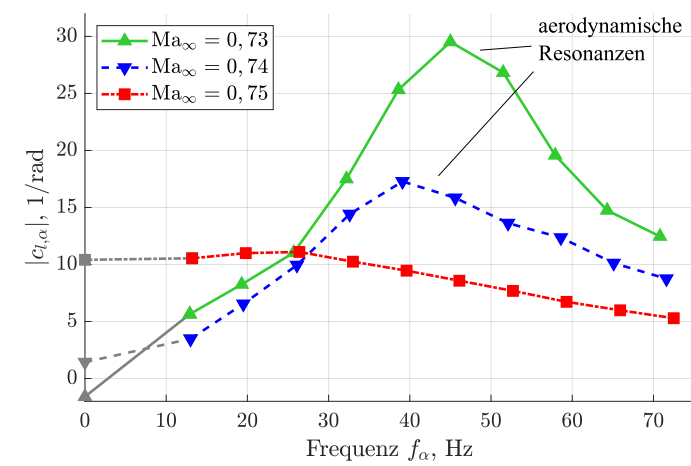

(a) Amplitude $\left|c_{l, \alpha}\right|$ des Auftriebs

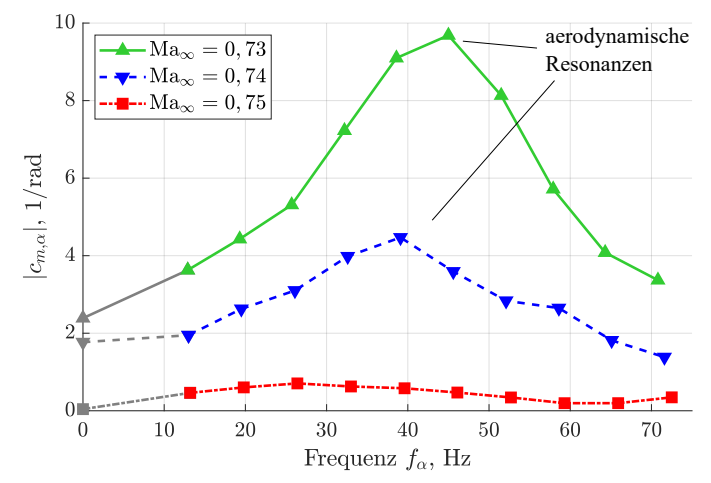

(c) Amplitude $\left|c_{m, \alpha}\right|$ des Moments

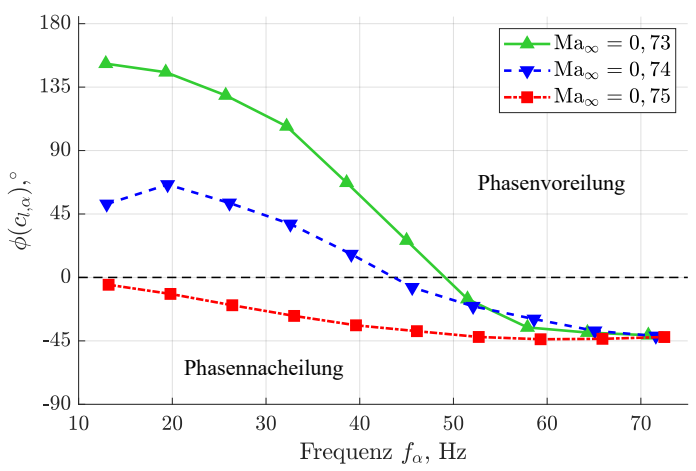

(b) Phase $\phi\left(c_{l, \alpha}\right)$ des Auftriebs

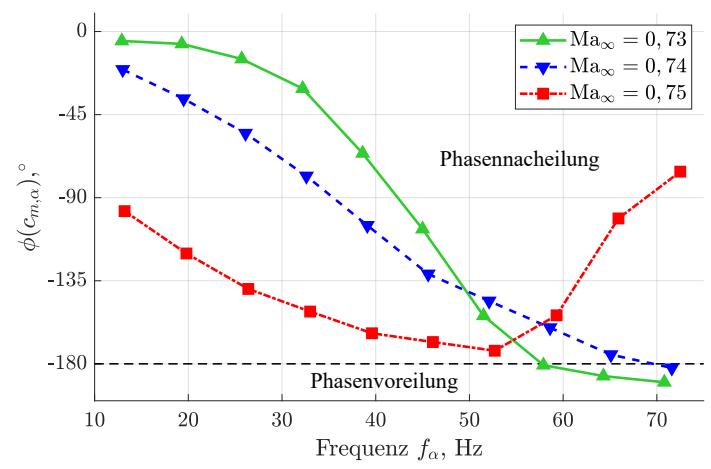

(d) Phase $\phi\left(c_{m, \alpha}\right)$ des Moments

Abbildung 6.1.: Instationäre Auftriebs- und Momentenderivativa in Betrag und Phase, gemessen mit einer Frequenzvariation mit $\hat{\alpha}=0,10^{\circ}$ um $\bar{\alpha}=0,05^{\circ}$ (vgl. auch [62]) bei freier Grenzschichttransition. Zum Vergleich sind zusätzlich die stationären Derivativa $\left(\omega^{*} \rightarrow 0\right)$ für $\bar{\alpha} \approx 0,05^{\circ}$ aus Abb. 5.2(a) und 5.2(b) in (a) und (c) dargestellt.

Die Amplituden der Derivativa des Auftriebs und des Nickmoments weisen deutlich erkennbare Maxima bei einer Frequenz von $f_{\alpha} \approx 45 \mathrm{~Hz}\left(\omega^{*} \approx 0,18\right)$ für $\mathrm{Ma}_{\infty}=0,73$ und bei $f_{\alpha} \approx 39 \mathrm{~Hz}\left(\omega^{*} \approx 0,15\right)$ für $\mathrm{Ma}_{\infty}=0,74$ auf. Diese aerodynamischen Resonanzen zeigen dabei eine große Ähnlichkeit zu aerodynamischen Resonanzen in transsonischer, abgelöster Strömung mit einer turbulenten Grenzschicht [201,202]. Ferner stimmen die Frequenzen der Resonanzen in Teilen auch mit den Frequenzanteilen der instationären Effekte überein, welche bereits bei statischen Anstellwinkeln beobachtet wurden (vgl. 5.4). So ist auch plausibel, dass für $\mathrm{Ma}_{\infty}=0,75$ in Abb. 6.1 keine Resonanz ersichtlich ist. Der mittlere Anstellwinkel der Frequenzvariation liegt außerhalb des Bereiches, in dem gemäß der stationären Resultate auch für $\mathrm{Ma}_{\infty}=0,75$ mit einer Resonanz zu rechnen ist. Die damit verbundenen Anstellwinkeleffekte sind anhand der durchgeführten Frequenzsweeps weiter unten näher erläutert. 
Neben den Amplituden weisen auch die Phasenverläufe in Abb. 6.1 Besonderheiten auf. Für $\mathrm{Ma}_{\infty}=0,73$ und $\mathrm{Ma}_{\infty}=0,74$ tritt bis zu einer Frequenz von $45-50 \mathrm{~Hz}$ $\left(\omega^{*} \approx 0,18-0,2\right)$ eine Phasenvoreilung des Auftriebs auf, wie die Verläufe $\phi\left(c_{l, \alpha}\right)>0^{\circ}$ in Abb. 6.1(b) zeigen. Die instationäre Aerodynamik weicht hier deutlich von der klassischen Theorie ab (vgl. 2.2.1). Für $\mathrm{Ma}_{\infty}=0,75$ zeigt sich hingegen eine Nacheilung des Auftriebs. Zusätzlich deutet sich für $\mathrm{Ma}_{\infty}=0,73$ und $\mathrm{Ma}_{\infty}=0,74$ eine Phasenvoreilung des Moments $\phi\left(c_{m, \alpha}\right)<-180^{\circ}$ für Frequenzen $f_{\alpha} \gtrsim 58 \mathrm{~Hz}\left(\omega^{*} \gtrsim 0,23\right)$ in Abb. 6.1(d) an. Dies weist auf ein potentielles 1-DoF-Flattern des Dreh-Freiheitsgrades hin [126, S.622]. Vorherige Untersuchungen beobachteten ein vergleichbares Phasenverhalten des Nickmoments, jedoch für andere Mach-Zahlen und Anstellwinkel [13, 20, 23,55].

Ferner deuten die Derivativa eine Amplitudenabhängigkeit an, wie ein Vergleich von Abb. 6.1 mit den Ergebnissen der Frequenzvariation mit $\hat{\alpha}=0,25^{\circ}$, dargestellt in E.1 in Abb. E.1, zeigt. Für $\mathrm{Ma}_{\infty}=0,74$ und $\mathrm{Ma}_{\infty}=0,75$ sind die Unterschiede gering. Bei $\mathrm{Ma}_{\infty}=0,73$ fällt hingegen die maximale Amplitude des Auftriebs $\left|c_{l, \alpha}\right|$ und des Moments $\left|c_{m, \alpha}\right|$ um ca. $50 \%$ ab. Die aerodynamische Resonanz ist stark reduziert, was auf eine nichtlineare Amplitudenabhängigkeit hinweist und die Sensitivität der beobachteten Effekte bzgl. einer Änderung des Anstellwinkels verdeutlicht.

Die Abhängigkeiten der instationären Effekte vom Anstellwinkel schließen sich den vorangegangenen Beobachtungen der stationären Messungen (vgl. 5) an. Der deutliche Einfluss von $\alpha$ auf die aerodynamischen Resonanzen und Phaseneffekte bestätigt sich beispielhaft für $\mathrm{Ma}_{\infty}=0,73$ in Abb. 6.2 und Abb. 6.3. Dort sind die Derivativa dargestellt, welche durch Frequenzsweeps von $0 \mathrm{~Hz}$ bis $70 \mathrm{~Hz}$ mit einer Schwingungsamplitude von $\hat{\alpha}=$ $0,08^{\circ}$ bei einer Variation des mittleren Anstellwinkels $\bar{\alpha}$ ermittelt wurden. Aus Gründen der Übersichtlichkeit sind die Amplituden- und Phasengänge in Abb. 6.2 als Konturplots dargestellt. In Abb. 6.3(a), (c), (e) und (g) sind die Ergebnisse nochmals separat für jeden Wert von $\bar{\alpha}$ aufgetragen. Sowohl für den Auftrieb (Abb. 6.2(a) und Abb. 6.3(a)) wie auch für das Moment (Abb. 6.2(c) und Abb. 6.3(e)) tritt eine aerodynamische Resonanz auf. Maximale Werte werden bei $f_{\alpha} \approx 50 \mathrm{~Hz}\left(\omega^{*} \approx 0,2\right)$ erreicht. Diese sind im Vergleich zur diskreten Messung (vgl. Abb. 6.1) zu etwas höheren Frequenzen verschoben. Die aerodynamische Resonanz tritt in einem mittleren Anstellwinkelbereich von $-0,2^{\circ} \lesssim \bar{\alpha} \lesssim$ $0,2^{\circ}$ auf. Dies fällt wiederum mit dem Übergangsbereich der Laminardelle zusammen (vgl. Abb. 5.1). Auch die Phasenvoreilung des Auftriebs beschränkt sich auf diesen Bereich, wie Abb. 6.2(b) und Abb. 6.3(c) zeigen. Außerhalb des Übergangsbereichs der Laminardelle liegt eine Phasennacheilung vor. Die Voreilung des Nickmoments tritt ebenfalls nur für den angesprochenen Anstellwinkelbereich auf. In Abb. 6.2(d $)^{2}$ verdeutlicht sich

\footnotetext{
${ }^{2}$ In Abb. 6.2(d) ist der Bereich oberhalb von $\bar{\alpha} \geq 0,5^{\circ}$ ausgespart. Die hier gemessenen Nickmomente weisen z. T. ein kleines Signal-Rausch-Verhältnis auf, so dass eine valide Phasenbestimmung nur eingeschränkt möglich ist. In Abb. 6.3(g) sind diese Werte jedoch ersichtlich.
} 
dies anhand der deutlich angestiegenen Werte für $\phi\left(c_{m, \alpha}\right)$, welche einen Phasensprung $\left(\phi\left(c_{m, \alpha}\right)<-180^{\circ}\right)$ anzeigen. Letzteres ist in Abb. 6.3(g) noch deutlicher zu erkennen.

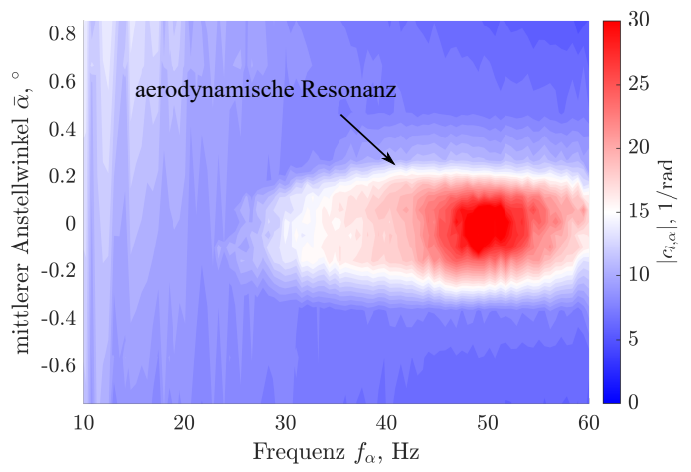

(a) Amplitude $\left|c_{l, \alpha}\right|$

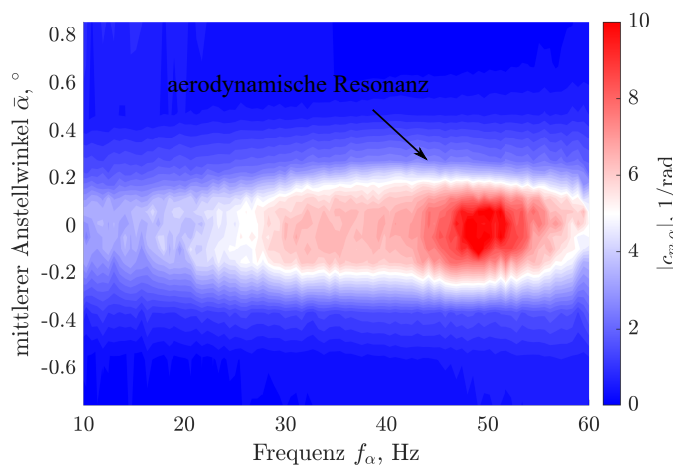

(c) Amplitude $\left|c_{m, \alpha}\right|$

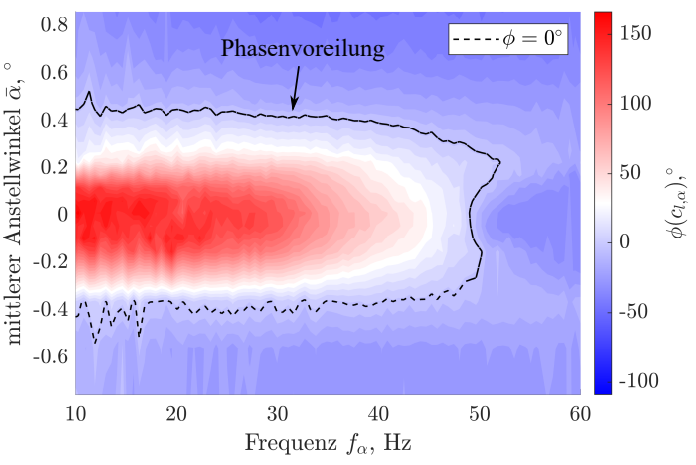

(b) Phase $\phi\left(c_{l, \alpha}\right)$

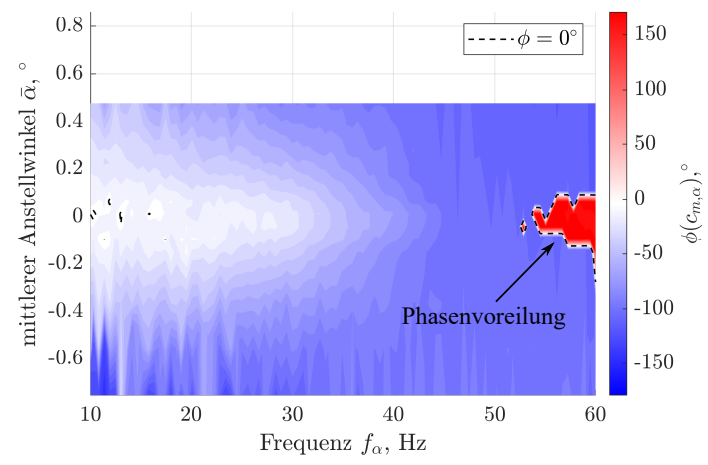

(d) Phase $\phi\left(c_{m, \alpha}\right)$

Abbildung 6.2.: Abhängigkeiten der aerodynamischen Derivativa bei $\mathrm{Ma}_{\infty}=0,73$ vom mittleren Anstellwinkel $\bar{\alpha}$, gemessen mithilfe von Frequenzsweeps mit $\hat{\alpha}=0,08^{\circ}$ bei einer freien Grenzschichttransition.

Ein vergleichbares Verhalten liegt auch für $\mathrm{Ma}_{\infty}=0,74$ und $\mathrm{Ma}_{\infty}=0,75$ vor. Die zugehörigen Derivativa sind im Anhang in Abb. E.2 und Abb. E.3 dargestellt. Zusammenfassend ist hier zu beobachten, dass sich die aerodynamischen Resonanzen mit steigender Mach-Zahl hinzu tieferen Frequenzen verschieben. Ebenso nimmt auch der mittlere Anstellwinkel etwas ab, bei dem Resonanzen zu beobachten sind. Auch hier stimmt dies mit dem Übergangsbereich der Laminardelle und der, bereits in 5 beobachteten Verschiebung des Selbigen hinzu kleineren Anstellwinkeln bei einem Anstieg von $\mathrm{Ma}_{\infty}$ überein. Ebenso tritt eine Phasenvoreilung des Auftriebs auch für $\mathrm{Ma}_{\infty}=0,74$ und $\mathrm{Ma}_{\infty}=0,75 \mathrm{im}$ Übergangsbereich auf. Eine Phasenvoreilung des Nickmoments ist hier ebenfalls für alle Mach-Zahlen bei hohen Frequenzen zu beobachten. Für höhere Anstellwinkel tritt zudem eine erneute Voreilung von $c_{m, \alpha}$ auf. Die zugehörigen Anstellwinkel liegen dabei umso tiefer, je höher die Mach-Zahl ist. Diese Anstellwinkel stimmen ferner mit einem negativen stationären Momentenderivativ $\frac{\mathrm{d} c_{m}}{\mathrm{~d} \alpha}<0$ überein (vgl. Abb 5.2(b)). So zeigt sich, zusätzlich zum Übergangsbereich, eine Phasenvoreilung von $c_{m, \alpha}$ ab $\mathrm{Ma}_{\infty}=0,73$ 

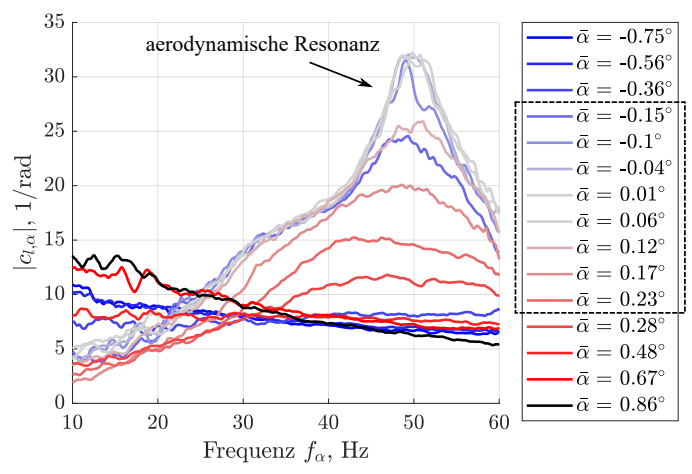

(a) Amplitude $\left|c_{l, \alpha}\right|$, freie Transition
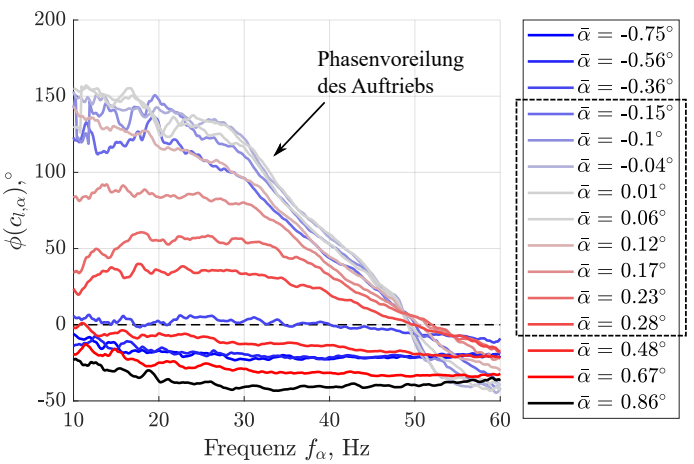

(c) Phase $\phi\left(c_{l, \alpha}\right)$, freie Transition

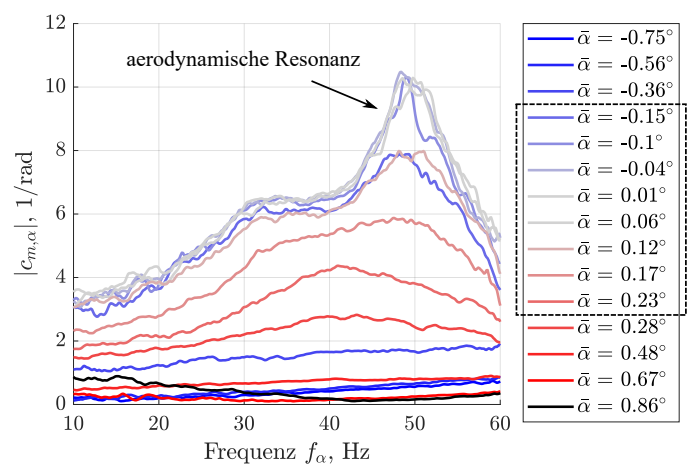

(e) Amplitude $\left|c_{m, \alpha}\right|$, freie Transition

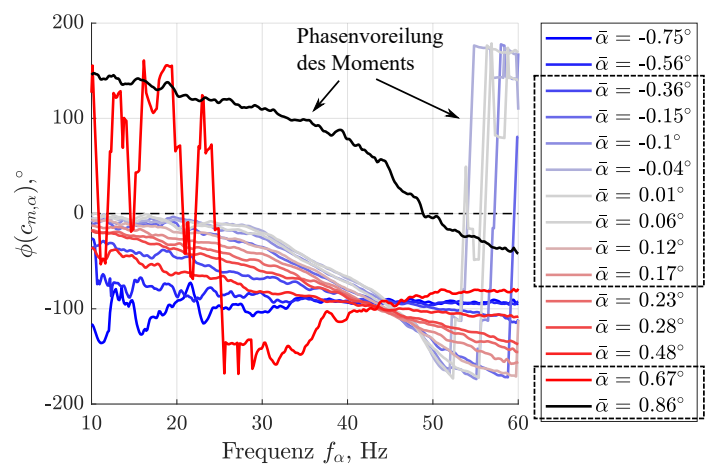

(g) Phase $\phi\left(c_{m, \alpha}\right)$, freie Transition

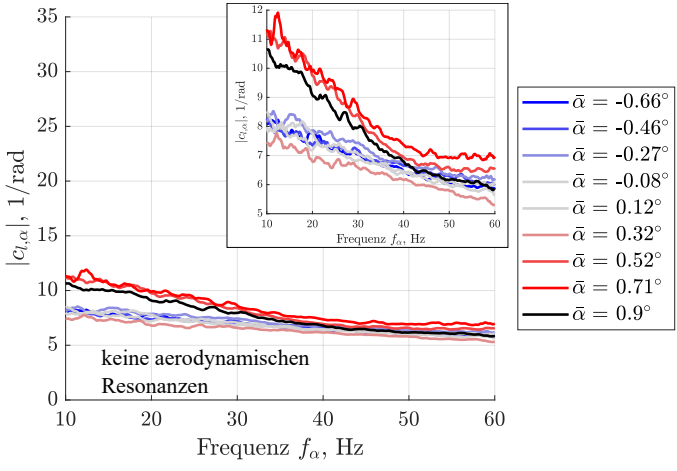

(b) Amplitude $\left|c_{l, \alpha}\right|$, fixierte Transition

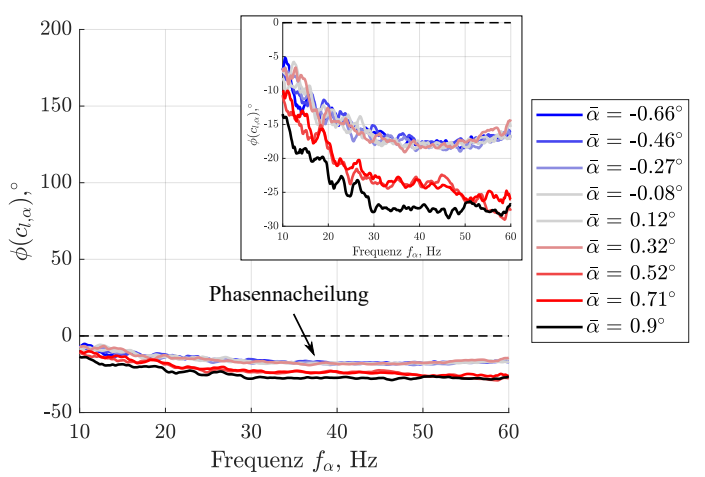

(d) Phase $\phi\left(c_{l, \alpha}\right)$, fixierte Transition

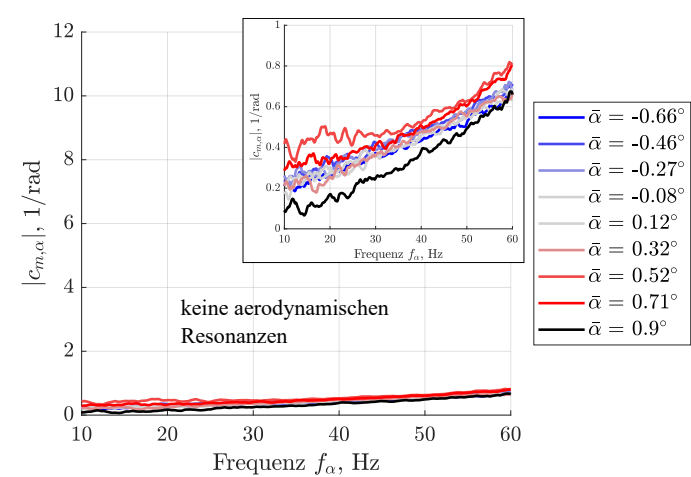

(f) Amplitude $\left|c_{m, \alpha}\right|$, fixierte Transition

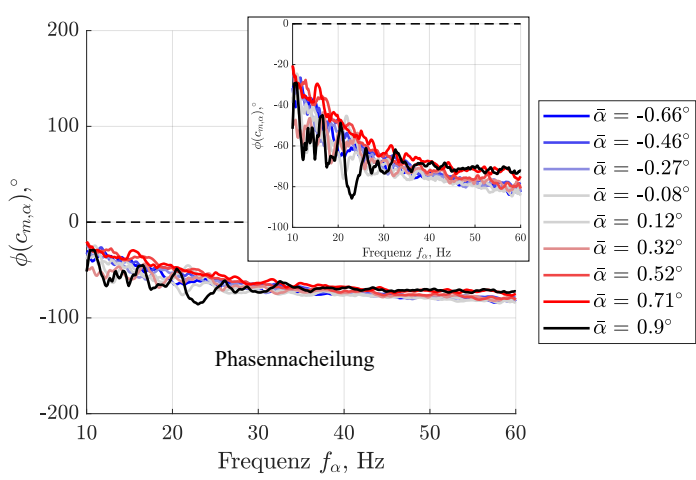

(h) Phase $\phi\left(c_{m, \alpha}\right)$, fixierte Transition

Abbildung 6.3.: Instationäre Derivativa in Betrag und Phase für eine freie (a, c, e und g) und fixierte Grenzschichttransition (b, d, f und h), gemessen mithilfe von Frequenzsweeps mit $\hat{\alpha}=0,08^{\circ}$ bei $\mathrm{Ma}_{\infty}=0,73$. 
für $\bar{\alpha} \gtrsim 0,6^{\circ}$, bei $\mathrm{Ma}_{\infty}=0,74 \mathrm{ab} \bar{\alpha} \gtrsim 0,4^{\circ}$ und für $\mathrm{Ma}_{\infty}=0,75 \mathrm{ab} \bar{\alpha} \gtrsim 0,2^{\circ}$. Sowohl die Phasenvoreilung wie auch das negative Momentenderivativ weisen auch hier auf ein potentielles Flattern des Dreh-Freiheitsgrades hin (vgl. [126, S.622], [9, S.90] und [26]).

Auch für die instationären Effekte liegt eine direkte Verbindung zur freien Grenzschichttransition vor. Die Amplitudengänge der Derivativa weisen für eine turbulente Grenzschicht keine aerodynamischen Resonanzen auf. Für $\mathrm{Ma}_{\infty}=0,73$ ist dies in Abb. 6.3(b) und (f) ersichtlich, für die weiteren Mach-Zahlen sind die Ergebnisse in Abb. E.2(b) und (f) sowie in Abb. E.3(b) und (f) im Anhang E.1 dargestellt. Auch die Phasen, dargestellt in Abb. 6.3(d) und (h) für $\mathrm{Ma}_{\infty}=0,73$ sowie in Abb. E.2(d), (h) und Abb. E.3(d), (h) für $\mathrm{Ma}_{\infty}=0,74$ und $\mathrm{Ma}_{\infty}=0,75$, verlaufen für alle Anstellwinkel und Mach-Zahlen vergleichbar und gemäß den theoretischen Erwartungen. Es liegt sowohl für den Auftrieb, wie auch für das Moment eine Phasennacheilung vor. Eine Phasenvoreilung als Hinweis auf mögliche aeroelastische Instabilitäten tritt nicht auf.

Abschließend sei erwähnt, dass auch in numerischen Untersuchungen des CAST 10-2 Laminarflügelprofils aerodynamische Resonanzen für reduzierte Frequenzen von $0,15 \lesssim$ $\omega^{*} \lesssim 0,2$ bei einer Mach-Zahl von $\mathrm{Ma}_{\infty}=0,72$ und einem mittleren Anstellwinkel von $\bar{\alpha}=0^{\circ}$ nachgewiesen wurden [20]. Eine höhere Frequenzauflösung schränkte den Frequenzbereich genauer auf eine Resonanz bei etwa $\omega^{*} \approx 0,18$ ein und bestätigte darüber hinaus eine Verschiebung der Resonanzspitze hin zu kleineren Frequenzen mit zunehmender Mach-Zahl [23]. Die starke Einflussnahme des mittleren Anstellwinkels wurde ebenfalls bestätigt. Für eine Mach-Zahl von $\mathrm{Ma}_{\infty}=0,73$ stellten sich keine stabilen Lösungen des stationären Strömungsfeldes infolge einer stark wechselnden Transitionsposition und eines periodischen Auftretens von Verdichtungsstößen ein [20]. Erst mit der Entwicklung eines neueren Transitionsmodells ( $\gamma$-Transitionsmodell $[55,56]$ ), konnte auch eine aerodynamische Resonanz für $\mathrm{Ma}_{\infty}=0,73$ bei einer reduzierten Frequenz von $\omega^{*} \approx 0,23$ nachgewiesen werden [57]. So wurde auch bestätigt, dass Resonanzen an eine freie Grenzschichttransition gebunden sind. Insgesamt sind jedoch die instationären Strömungsphänomene am CAST 10-2 Laminarflügel in der Nähe des Übergangsbereiches der Laminardelle numerisch nur schwer zu erfassen.

\subsection{Energiebetrachtung}

Zur Untersuchung des Energietransfers zwischen Strömung und Struktur bzw. deren Bewegung, wurde der Energietransfer im Mittelschnitt des Laminarflügelmodells mit der in 4.2.2 beschriebenen Methodik berechnet. Dabei hat sich herausgestellt, dass hierfür die Grundannahme eines unendlich steifen Windkanalmodells nicht mehr haltbar ist. So führt eine Berechnung der Energie mit einer reinen Nickbewegung, basierend auf 

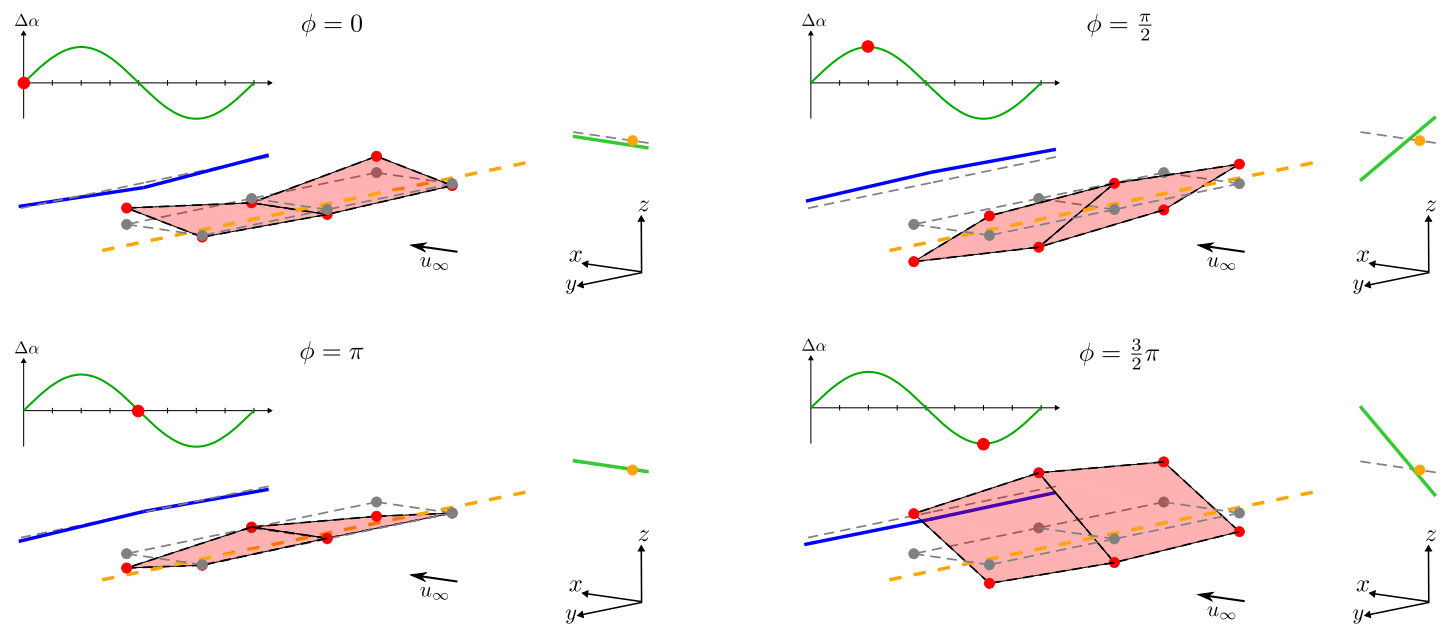

Abbildung 6.4.: Schematische Darstellung der Schwingungsmode des Windkanalmodells bei einer zwangserregten Nickschwingung für vier ausgewählte Phasenwinkel $\phi$ einer Periode, hier beispielhaft für $\mathrm{Ma}_{\infty}=0,73$ um $\bar{\alpha}=0,05^{\circ}$ mit $\hat{\alpha}=0,5^{\circ}$ und $f_{\alpha}=51,5 \mathrm{~Hz}$.

den Lasersignalen (vgl. 3.4.4), zu unzureichenden Ergebnissen [62]. In Abb. 6.4 ist die Schwingungsmode des Windkanalmodells bei einer zwangserregten Nickschwingung für die vier Phasenwinkel $\phi=0$ bzw. $\phi=2 \pi, \phi=\frac{\pi}{2}, \phi=\pi$ und $\phi=\frac{3}{2} \pi$ einer Periode dargestellt. Die Bewegung des Modells wurde anhand der verbauten Beschleunigungssensoren (vgl. 3.4.2) mit einer zweifachen Integration im Frequenzbereich (4.1.4) rekonstruiert. Die in Abb. 6.4 eingezeichnete blaue Linie stellt dabei die Hubbewegung auf Höhe der Drehachse bei $c / 4$ dar, welche als orange gestrichelte Linie dargestellt ist. Die grüne Linie repräsentiert die Drehbewegung im Mittelschnitt. Die grau gestrichelten Linien verdeutlichen die Mittellage. Zur Veranschaulichung sind die Auslenkungen 30-fach überhöht dargestellt. Abb. 6.4 zeigt, dass die vorliegende Schwingungsmode aus einer Starrkörper-Nickschwingung und einer zusätzlichen strukturellen Biegung des Modells samt der Aufhängung im NVS besteht. Aus Letzterer resultiert eine zusätzliche Hubbewegung des Mittelschnitts, welche für die Energiebetrachtung von Relevanz ist. Folglich werden hierfür, abweichend zu den sonstigen Auswertungen, nicht die Bewegungssignale der Lasertriangulatoren verwendet, sondern es wird die Bewegung des Mittelschnitts auf Basis der dort verbauten Beschleunigungssensoren berechnet ${ }^{3}$.

In Abb. 6.5 sind die auf diese Weise berechneten und gemittelten Energietransfers $\overline{\frac{1}{T} \int_{t}^{t+T} W(t) \mathrm{d} t}$ dargestellt, welche über eine Schwingungsperiode zwischen Strukturbewegung und Aerodynamik ausgetauscht werden. Hierfür wurde zunächst der jeweilige Energietransfer pro Schwingungsperiode $\frac{1}{T} \int_{t}^{t+T} W(t) \mathrm{d} t$ für die gesamte Zeitreihe $W(t)$

\footnotetext{
${ }^{3}$ Die elastische Verformung des Windkanalmodells sowie die sichtbare Phasennacheilung des Anstellwinkels an den Endseiten des Modells (siehe äußere Schnitte für $\phi=0$ und $\phi=\pi$ in Abb. 6.4) wirft die Frage auf, inwieweit das Winkelsignal der Lasertriangulatoren valide Aussagen über die Verformung des Mittelschnitts macht. Ein Vergleich der Bewegungssignale beider Messtechniken zeigt hier, dass die Phasenverschiebung weniger als $0,1^{\circ}$ beträgt und auch die mittlere Amplitudenabweichung mit $0,02^{\circ}$ deutlich innerhalb des Fehlerintervalls beider Messtechniken liegt.
} 
berechnet, welcher dann über alle Schwingungsperioden gemittelt wurde. Die in Abb. 6.5 eingezeichneten Fehlerbalken stellen die zugehörige Standardabweichung dar. Die grau hinterlegten Linien entsprechen zum Vergleich den Energien, welche auf Basis der Lasersignale berechnet wurden, also ohne Berücksichtigung der zusätzlichen Hubbewegung im Mittelschnitt. Hierbei zeigt sich, dass insbesondere bei höheren Frequenzen eine deutliche Abweichung auftritt und die Energieeinträge zu klein ausfallen. Der aus einer Hubbewegung resultierende Energieeintrag trägt also signifikant zur gesamten Energiebilanz bei. Negative Werte für $\overline{\frac{1}{T} \int_{t}^{t+T} W(t) \mathrm{d} t}$ bedeuten einen Energietransfer von der Struktur in die Strömung, positive Werte entsprechen folglich einem Energieeintrag aus der Strömung in die Struktur. Letzteres deutet dabei auf eine selbsterregte Schwingung und somit auf eine aeroelastische Instabilität hin.

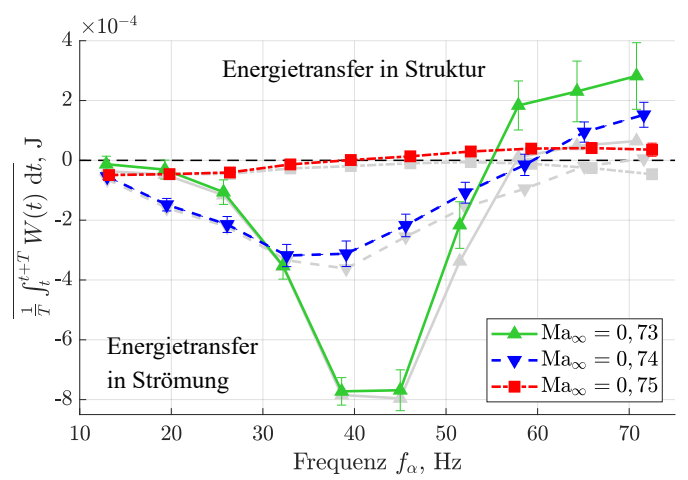

(a) Energietransfer für $\hat{\alpha}=0,1^{\circ}$

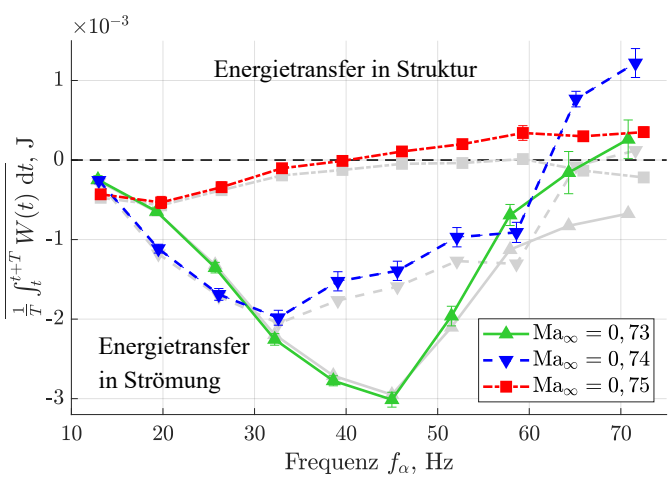

(b) Energietransfer für $\hat{\alpha}=0,25^{\circ}$

Abbildung 6.5.: Periodengemittelter Energietransfer zwischen Struktur und Strömung für eine freie Grenzschichttransition, gemessen und berechnet auf Basis einer diskreten Frequenzvariation um $\bar{\alpha}=0,05^{\circ}$ mit $\hat{\alpha}=0,1^{\circ}$ in (a) und $\hat{\alpha}=0,25^{\circ}$ in (b).

So zeigt Abb. 6.5, dass für $\hat{\alpha}=0,1^{\circ}$ (Abb. 6.5(a)) und $\hat{\alpha}=0,25^{\circ}$ (Abb. 6.5(b)) im Bereich der aerodynamischen Resonanz (vgl. 6.1) verstärkt Energie aus der Strukturbewegung in die Strömung übergeht. Gemäß der deutlichen Ausprägung der Resonanzkurve für $\mathrm{Ma}_{\infty}=$ 0,73 (vgl. Abb. 6.1 und Abb. E.1) ist hier auch der Energietransfer am größten. Der reduzierte Energietransfer für $\mathrm{Ma}_{\infty}=0,74$ stimmt mit den abgeflachten Resonanzkurven in Abb. 6.1 und Abb. E.1 überein. Für $\mathrm{Ma}_{\infty}=0,75$ fällt der Energieeintrag in die Strömung in beiden Fällen dementsprechend am geringsten aus.

Für $\mathrm{Ma}_{\infty}=0,73$ und $\mathrm{Ma}_{\infty}=0,74$ treten bei Frequenzen oberhalb von $55 \mathrm{~Hz}$ bis $60 \mathrm{~Hz}$ positive Werte für den Energietransfer auf, hier verrichtet die Strömung Arbeit an der Struktur. Bei größeren Schwingungsamplituden zeigt sich eine Verschiebung zu etwas höheren Frequenzen, insbesondere für $\mathrm{Ma}_{\infty}=0,73$, wie der Vergleich von Abb. 6.5(a) und (b) erkennen lässt. Dies kann wiederum als Hinweis auf eine Amplitudenbegrenzung gedeutet werden. Für $\mathrm{Ma}_{\infty}=0,75$ liegt hingegen bereits ab ca. $40 \mathrm{~Hz}$ ein Energieeintrag in die Struktur vor, welcher auch unabhängig von der Schwingungsamplitude ist. Insgesamt 
nehmen also die Frequenzen mit steigender Mach-Zahl ab, für welche $\overline{\frac{1}{T} \int_{t}^{t+T} W(t) \mathrm{d} t}>0$ gilt. Damit weisen die Ergebnisse auf aeroelastische Instabilitäten mit $f_{\alpha} \gtrsim 55 \mathrm{~Hz}$ hin, wobei zu erwarten ist, dass sich die Frequenzen mit steigender Mach-Zahl reduzieren.

In Ergänzung zu den diskreten Ergebnissen sind in Abb. 6.6 sowie in Abb. E.4 im Anhang E.2 die Energietransfers für $\mathrm{Ma}_{\infty}=0,73$ bzw. $\mathrm{Ma}_{\infty}=0,74$ und $\mathrm{Ma}_{\infty}=$ 0,75 für verschiedene mittlere Anstellwinkel $\bar{\alpha}$ dargestellt. Diese wurden auf Basis der Frequenzsweeps ermittelt. Zur Berechnung wurden die Zeiten $t_{i}$ und $t_{i}+T$ der einzelnen Schwingungsperioden eines jeweiligen Frequenzsweeps bestimmt und über jede Periode die gemittelte Energie $\frac{1}{T_{i}} \int_{t_{i}}^{t_{i}+T_{i}} W(t) \mathrm{d} t$ berechnet. Zur Rauschunterdrückung wurden die Verläufe der gemittelten Energien mit einem SAVITZKY-GolAY-Filter [203] geglättet.

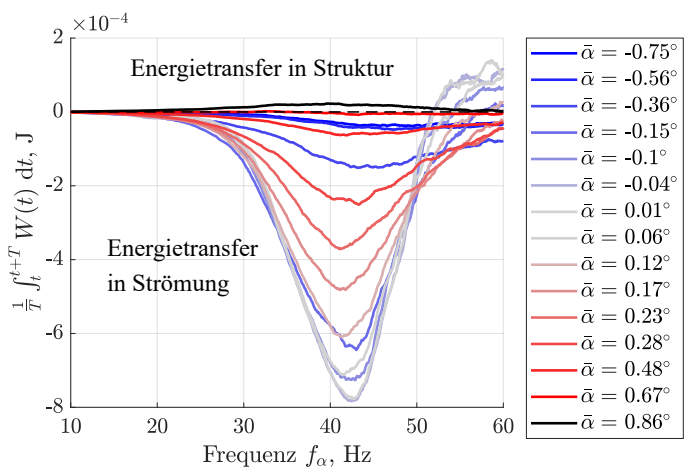

(a) Energietransfer 2D, freie Transition

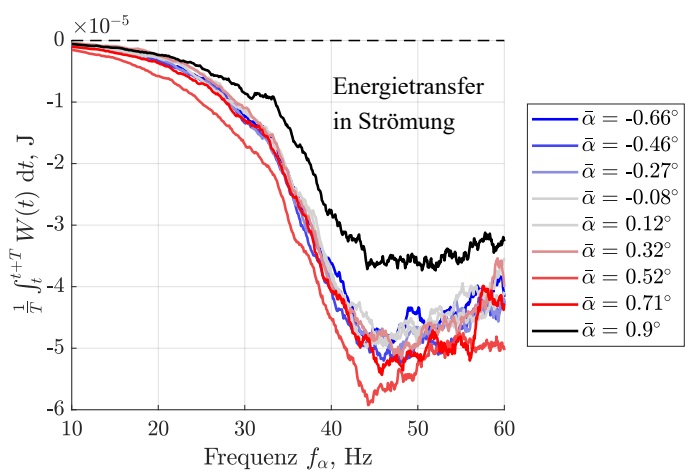

(c) Energietransfer 2D, fixierte Transition

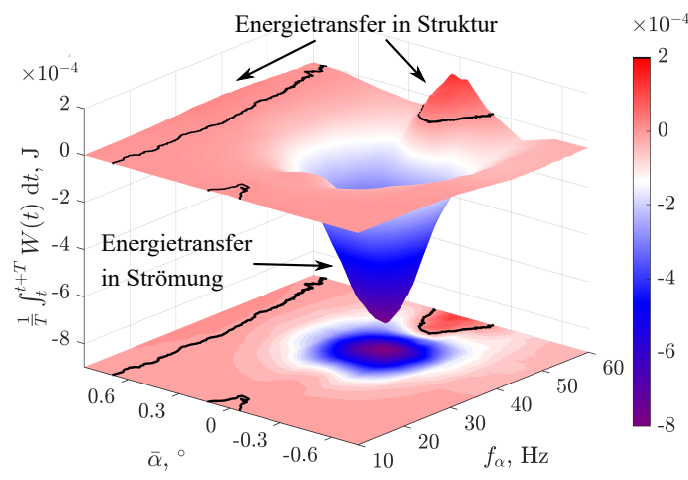

(b) Energietransfer 3D, freie Transition

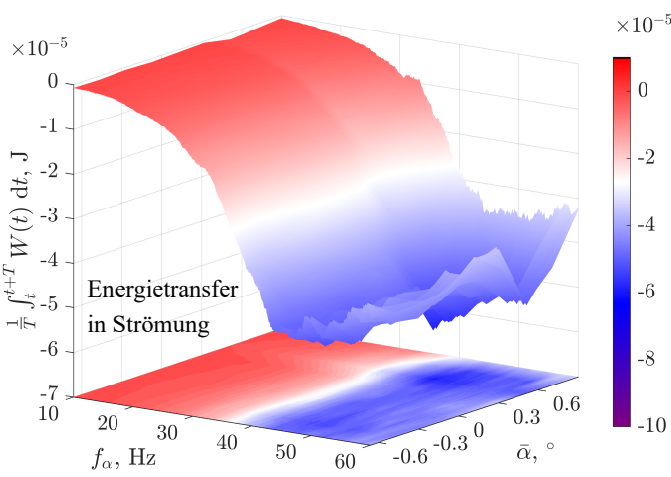

(d) Energietransfer 3D, fixierte Transition

Abbildung 6.6.: Energietransfer pro Schwingungsperiode zwischen Struktur und Strömung für eine freie ( $a$ und b) sowie eine fixierte Grenzschichttransition (c und d), gemessen mithilfe von Frequenzsweeps mit $\hat{\alpha}=0,08^{\circ}$ bei $\mathrm{Ma}_{\infty}=0,73$. Zum Zwecke der besseren Darstellung sind die 3D Grafiken der verschiedenen Grenzschichtzustände gedreht.

Abb. 6.6(a) zeigt die Ergebnisse für $\mathrm{Ma}_{\infty}=0,73$ bei einer freien Grenzschichttransition in zweidimensionaler Darstellung. In Abb. 6.6(b) sind die Ergebnisse nochmals dreidimensional visualisiert. Deutlich zu erkennen ist dabei die Ausbildung eines „Energietrichters“ in der Nähe der aerodynamischen Resonanz. Ein direkter Vergleich der Frequenzen zeigt 
hier, dass ein Energieminimum um $43 \mathrm{~Hz}$ herum auftritt. Das Maximum der aerodynamischen Resonanz liegt für die kontinuierlichen Messungen jedoch bei ca. $50 \mathrm{~Hz}$ und somit bei leicht erhöhten Frequenzen (vgl. Abb. 6.3 und Abb. 6.2). Jedenfalls wird in diesem Bereich der Strömung Energie zugeführt. Für Frequenzen oberhalb von ca. $52 \mathrm{~Hz}$ dreht sich das Vorzeichen um und es bildet sich ein Gebiet aus, in dem ein Energietransfer in die Struktur erfolgt. Entsprechend sind hier, wie bereits zuvor erwähnt, aeroelastische Instabilitäten zu erwarten. Besonderes deutlich wird dabei aus Abb. 6.6(a) und Abb. 6.6(b), dass sich sowohl der „Energietrichter" sowie die Energiezufuhr in Richtung Struktur wieder auf mittlere Anstellwinkel $\bar{\alpha}$ beschränkt, die mit dem Übergangsbereich der Laminardelle korrelieren. Für $\bar{\alpha} \gtrsim 0,7^{\circ}$ liegt noch ein weiteres Gebiet vor, in dem Energie in die Struktur eingetragen wird. Dabei gehen die positiven Energieeinträge in beiden Gebieten mit einer Phasenvoreilung des Nickmoments einher (vgl. Abb. 6.3(g)).

Ferner sind in Abb. 6.6(c) und Abb. 6.6(d) die Ergebnisse für eine fixierte Grenzschichttransition dargestellt. Im direkten Vergleich zur freien Transition zeigt sich, dass kein „Energietrichter" auftritt. Dies ist angesichts der Ergebnisse der instationären Beiwerte für eine fixierte Transition (vgl. 6.3) und dem Ausbleiben einer aerodynamischen Resonanz zu erwarten. Zum anderen ist über den gesamten Frequenz- und Anstellwinkelbereich die Energie pro Periode negativ. Für eine turbulente Grenzschicht sind somit keine aeroelastischen Instabilitäten unter den vorliegenden Randbedingungen zu erwarten.

Analog verhalten sich die Zusammenhänge auch für $\mathrm{Ma}_{\infty}=0,74$ und $\mathrm{Ma}_{\infty}=0,75$. Hierfür sind die Ergebnisse in Abb. E.4 im Anhang E.2 dargestellt. In beiden Fällen bildet sich für eine freie Grenzschichttransition ebenfalls ein „Energietrichter“ aus. Wie auch die aerodynamischen Resonanzen (vgl. 6.1 und Abb. E.2 sowie Abb. E.3) verschiebt sich dieser mit steigender Mach-Zahl hin zu etwas kleineren Frequenzen und geringeren mittleren Anstellwinkeln. An den „Energietrichter“ schließt sich für höhere Frequenzen oberhalb von ca. $45 \mathrm{~Hz}$ ein Gebiet an, in dem wiederum Energie von der Strömung in die Struktur eingebracht wird. Dabei deutet sich auch hier an, dass mit einer Abnahme der Frequenzen von potentiellen aeroelastischen Instabilitäten zu rechnen ist, wenn die Mach-Zahl erhöht wird. Darüber hinaus tritt auch für $\mathrm{Ma}_{\infty} \geq 0,74$ ein zweites Gebiet mit positiven Energiewerten bei höheren Anstellwinkeln auf. Dieses vergrößert sich mit steigender Mach-Zahl, so dass Energie über einen größeren Frequenzbereich für immer kleinere Anstellwinkel in die Struktur eingebracht wird. So gehen für $\mathrm{Ma}_{\infty}=0,75$ die bei $\mathrm{Ma}_{\infty}=0,73$ und $\mathrm{Ma}_{\infty}=0,74$ noch getrennten Bereiche ineinander über. Dies hängt erneut mit der Phasenvoreilung des Momentes zusammen, welche mit steigender Mach-Zahl bei kleineren mittleren Anstellwinkeln auftritt (vgl. 6.1).

Für eine fixierte Grenzschichttransition treten auch bei $\mathrm{Ma}_{\infty}=0,74$ und $\mathrm{Ma}_{\infty}=$ 0,75 keine ausgewiesenen Energieminima auf, welche in einer Verbindung zu einer 
aerodynamischen Resonanz stehen (siehe Abb. E.4(c), (d), (g) und (h)). Es treten auch keine Bereiche in Erscheinung, in denen ein positiver Energietransfer vorliegt. Insgesamt weisen somit die Ergebnisse auch für höhere Mach-Zahlen auf eine Stabilität des aeroelastischen Systems hin, sofern eine turbulente Grenzschicht vorliegt.

\subsection{Instationäres Druckverhalten}

Zur Veranschaulichung des instationären Verhaltens der Druckverteilung wird, analog zu den instationären Derivativa (vgl. 6.1), auf eine Berechnung der instationären Druckbeiwerte gemäß Gl. 4.2.11 zurückgegriffen. Abweichend hierzu wurden jedoch zunächst die Druckdifferenzen $\Delta c_{p}=c_{p, p}-c_{p, s}$ zwischen Druck- und Saugseite berechnet, wozu die in C.4 beschriebene Interpolation der Druckverteilung verwendet wurde. Positive Werte von $\Delta c_{p}$ resultieren somit in einer positiven, nach oben gerichteten Normalkraft. Die Verteilung von $\Delta c_{p}$ kann folglich annähernd als Auftriebsverteilung über der Flügelebene interpretiert werden.

In Abb. 6.7 sind die instationären Druckdifferenzen für einen exemplarischen Frequenzsweep bei $\bar{\alpha}=0,01^{\circ}$ und $\mathrm{Ma}_{\infty}=0,73$ dargestellt. Die Randbedingungen sind vergleichbar mit der diskreten Frequenzvariation für $\mathrm{Ma}_{\infty}=0,73$ (vgl. 6.1). Deutlich zu erkennen ist eine Reduzierung der Phase $\phi\left(\Delta c_{p, \alpha}\right)$ mit steigender Frequenz. Dies geht aus dem Konturplot in Abb. 6.7(b) und aus der dreidimensionalen Darstellung in Abb. 6.7(c) sowie den jeweiligen Schnitten bei vier ausgewählten Schwingungsfrequenzen hervor. Der gesamte Phasenverlauf der instationären Auftriebsverteilung senkt sich kontinuierlich ab (Pfeile in Abb. 6.7(b) und (c)). Die Amplitude der instationären Druckdifferenz $\left|\Delta c_{p, \alpha}\right|$ weist zwei Bereiche mit erhöhten Werten auf. So sind zum einen die Amplituden in einem Flügeltiefenbereich von $0,65 \lesssim \frac{x}{c} \lesssim 0,72$ entlang dem gesamten Frequenzbereich erhöht. Zum anderen zeigt sich in einem Frequenzbereich zwischen $40 \mathrm{~Hz}$ und $53 \mathrm{~Hz}$ eine weitere, deutliche Zunahme der Amplituden stromauf einer Flügeltiefe von $x / c \approx 0,7$, entsprechend der aerodynamischen Resonanz (vgl. 6.1).

Zur genaueren Identifizierung dieser Sachverhalte sind in Abb. 6.8 die Druckverteilungen der Profil-Saugseite für drei Schwingungsperioden des Frequenzsweeps dargestellt, die jeweils mit den Frequenzschnitten in Abb. 6.7(c) korrespondieren. Die schwarzen Punkte markieren die Position $x_{s}$ von automatisiert detektierten Verdichtungsstößen ${ }^{4}$. Die Druckverteilungen verdeutlichen, dass die erhöhten Amplitudenwerte entlang einer

\footnotetext{
${ }^{4}$ Für die automatisierte Detektion von Verdichtungsstößen wurde eine lineare Interpolation der Druckverteilung vorgenommen und der Gradient $\frac{\partial c_{p, s}}{\partial(x / c)}$ an den Schnittpunkten mit $c_{p}^{*}$ ausgewertet. Basierend auf dem Vorzeichen und der jeweiligen Steigung (es wird ein empirischer Schwellwert von $\frac{\partial c_{p, s}}{\partial(x / c)} \geq 3,22$ zur Abgrenzung von vermeintlich stoßfreien Druckanstiegen verwendet) wird die Position $x_{s}$ (schwarze Punkte in Abb. 6.8) sowie die Stoßstärke (skaliert Punktgröße in Abb. 6.8) bestimmt. Weitere Informationen zur automatisierten Stoßdetektion finden sich in [62].
} 


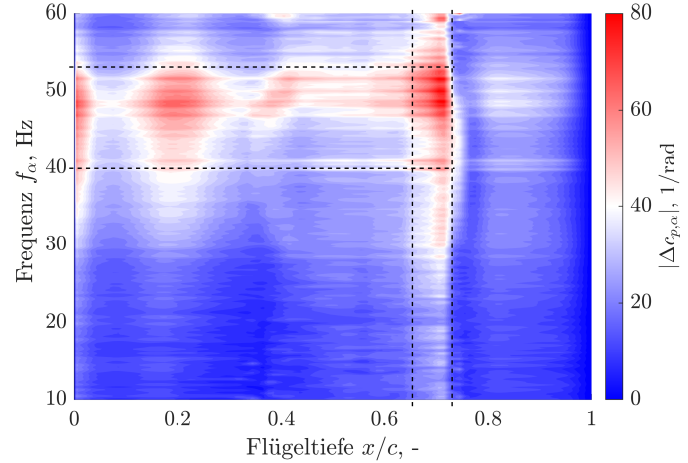

(a) Amplitude $\left|\Delta c_{p, \alpha}\right|$

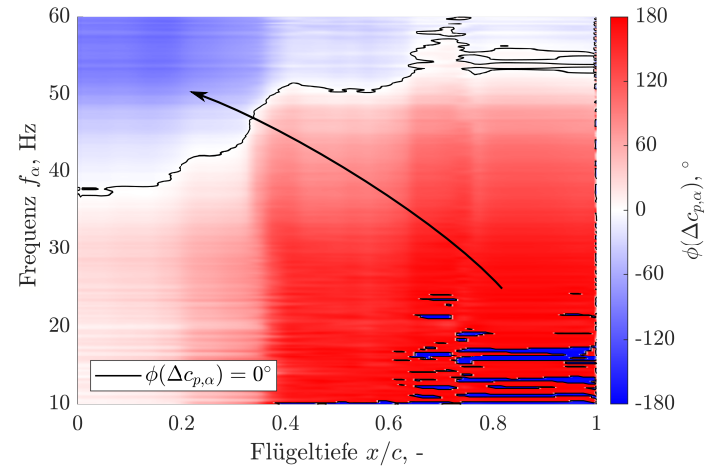

(b) Phase $\phi\left(\Delta c_{p, \alpha}\right)$
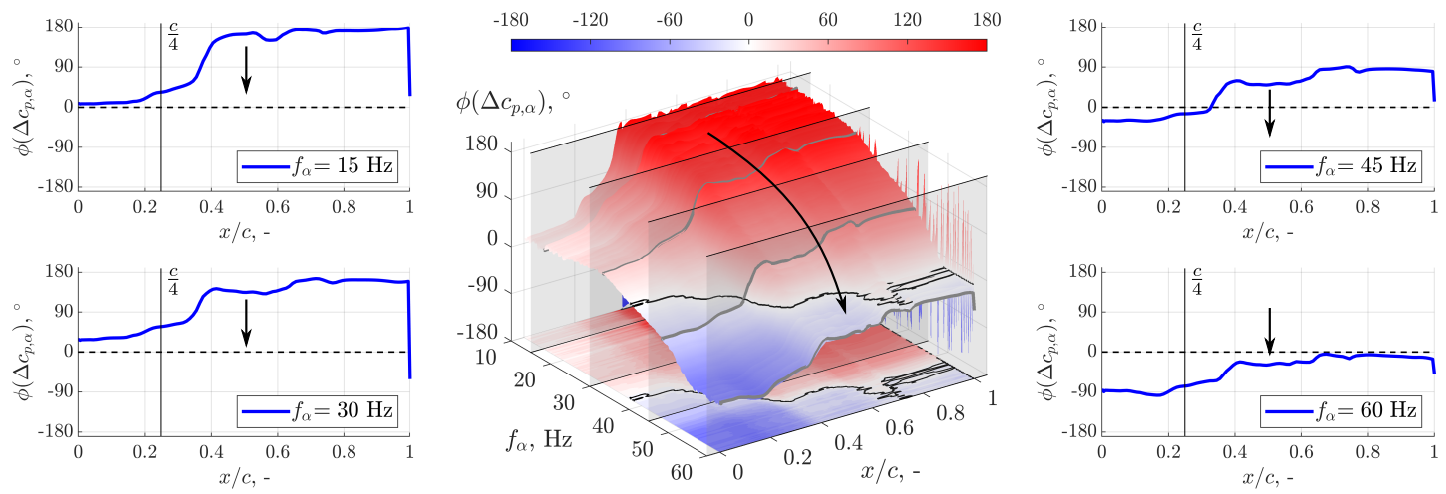

(c) Phase $\phi\left(\Delta c_{p, \alpha}\right)$ in 3D und Frequenzschnitte für $15 \mathrm{~Hz}, 30 \mathrm{~Hz}, 45 \mathrm{~Hz}$ und $60 \mathrm{~Hz}$

Abbildung 6.7.: Instationäre Druckdifferenz $\Delta c_{p, \alpha}$ in Betrag (a) und Phase (b) sowie 3D-Darstellung der Phase samt Frequenzschnitte (c) für eine freie Grenzschichttransition, gemessen mithilfe eines Frequenzsweeps mit $\hat{\alpha}=0,08^{\circ}$ bei $\mathrm{Ma}_{\infty}=0,73$ und $\bar{\alpha}=0,01^{\circ}$.

Flügeltiefe von $x / c \approx 0,69$ in Abb. 6.7(a) mit dem Auftreten von Verdichtungsstößen im Bereich $0,6 \lesssim x / c \lesssim 0,65$ und der damit einhergehenden Instationarität der Strömung korrelieren. Dies ist für alle vier Frequenzen in Abb. $6.8 \mathrm{zu}$ beobachten ${ }^{5}$.

So treten für $f_{\alpha} \approx 15 \mathrm{~Hz}$, dargestellt in Abb. 6.8(a), schwache Verdichtungsstöße bei einer mittleren Stoßposition von $\bar{x}_{s} \approx 0,64 c$ auf. Die Stoßposition ist weitestgehend stationär, eine ausgeprägte Stoßdynamik hat noch nicht eingesetzt ${ }^{6}$. Ebenso ist noch kein periodisches Verhalten der Druckbeiwerte ersichtlich. Wie Abb. 6.7(c) links oben zeigt, eilt jedoch die instationäre Druckdifferenz für $f_{\alpha} \approx 15 \mathrm{~Hz}$ der Flügelbewegung vollständig voraus. Hieraus resultiert auch die Phasenvoreilung des Auftriebs $c_{l, \alpha}$ sowie eine Phasennacheilung des Moments $c_{m, \alpha}$ (vgl. Abb. 6.3(c) und (g)).

\footnotetext{
${ }^{5}$ Es sei hier angemerkt, dass die Verdichtungsstöße großenteils nur sehr schwach sind. Die Steigung des Druckanstieges variiert, so dass eine Fluktuation von Stößen und isentropen Rekompressionen vorliegt. Bei einer Phasenmittelung der Druckbeiwerte, wie es für diskrete Frequenzvariationen durchgeführt wird, mitteln sich daher nicht kontinuierlich auftretende, schwache Verdichtungsstöße heraus (vgl. 6.4)

${ }^{6}$ Die hohe Stoßdichte in Abb. 6.8(a) ist nicht physikalisch, sondern begründet sich in der hohen Abtastfrequenz und der großen Anzahl an Datenpunkten im dargestellten Zeitfenster.
} 


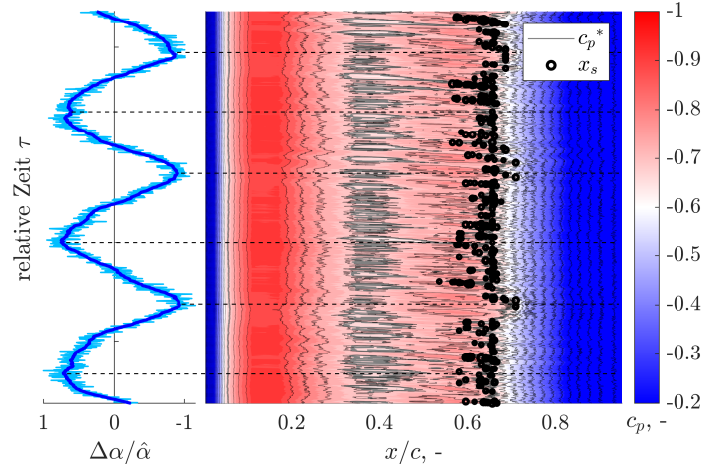

(a) $c_{p, s}$ für $f_{\alpha} \approx 15 \mathrm{~Hz}$

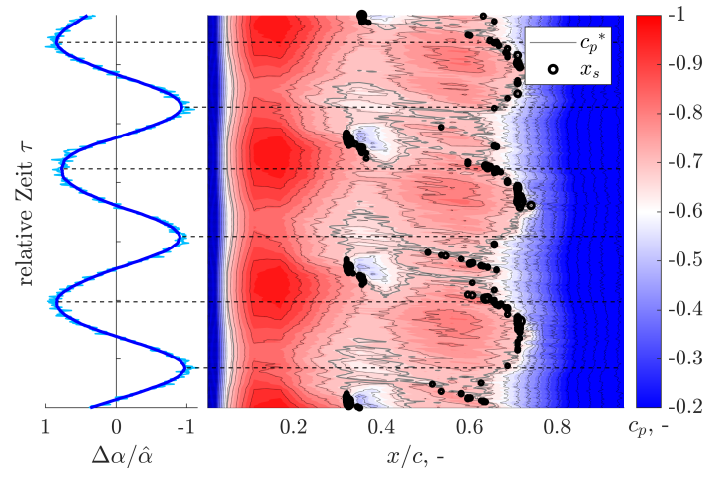

(c) $c_{p, s}$ für $f_{\alpha} \approx 45 \mathrm{~Hz}$

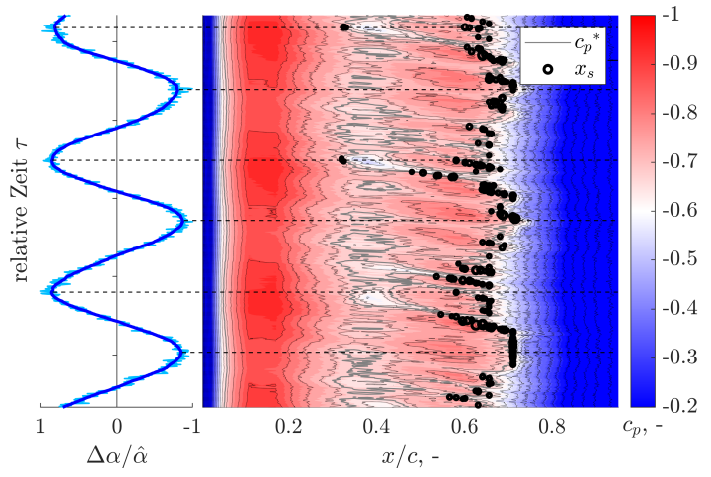

(b) $c_{p, s}$ für $f_{\alpha} \approx 30 \mathrm{~Hz}$

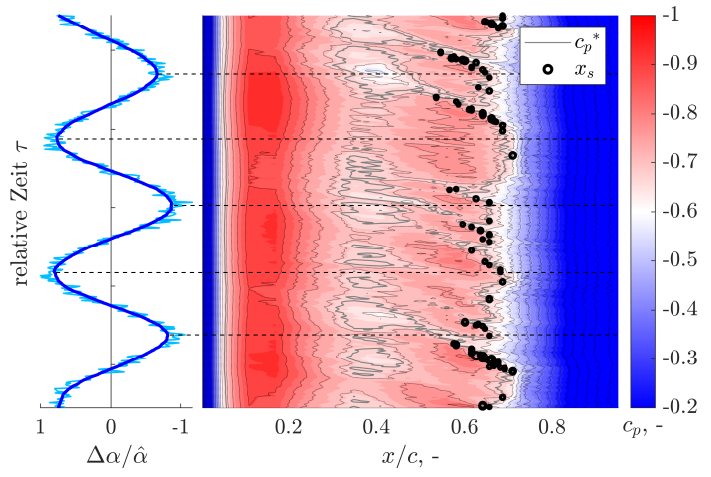

(d) $c_{p, s}$ für $f_{\alpha} \approx 60 \mathrm{~Hz}$

Abbildung 6.8.: Druckverteilungen der Profil-Saugseite $c_{p, s}$ für einzelne Zeitausschnitte, die mit den jeweiligen Frequenzschnitten des Frequenzsweeps mit $\hat{\alpha}=0,08^{\circ}$ bei $\mathrm{Ma}_{\infty}=0,73$ und $\bar{\alpha}=0,01^{\circ}$ korrespondieren.

Mit steigender Frequenz treten Stöße weiter stromauf auf, wie es für $f_{\alpha} \approx 30 \mathrm{~Hz}$ in Abb. 6.8(b) zu erkennen ist. Die Streuung der Stoßpositionen nimmt zu, konzentriert sich jedoch noch auf einen Bereich um $x / c \approx 0,65$. Es zeigt sich eine periodische Ausprägung des vorderen Überschallgebietes stromauf von $x / c \approx 0,3$, welches bereits in den stationären Druckverteilungen (vgl. 5.4(a)) beobachtet wurde. Saugspitzen treten kurz vor dem Erreichen der maximalen Auslenkungen von $\Delta \alpha / \hat{\alpha}$ auf. Dies zeigt sich auch in der Phasenvoreilung der Druckdifferenz, dargestellt in Abb. 6.7(c) links unten. Der Phasenverlauf ist stromab der Drehachse gesunken, nimmt jedoch im Vergleich zu $f_{\alpha}=15 \mathrm{~Hz}$ im Bereich der Saugspitzen höhere Werte an. Die Voreilung von $c_{l, \alpha}$ und die Nacheilung von $c_{m, \alpha}$ (vgl. Abb. 6.3(c) und (g)) bleiben erhalten.

Bei $f_{\alpha} \approx 45 \mathrm{~Hz}$ verdeutlicht sich in der Druckverteilung in Abb. 6.8(c) die aerodynamische Resonanz. Es treten weiterhin Verdichtungsstöße im hinteren Flügeltiefenbereich bei $\overline{x_{s}}=0,68$ auf, jedoch schließt nunmehr auch das vordere Überschallgebiet zeitweise mit Stößen bei $x / c \approx 0,34$ ab. Die gesamte Druckverteilungweist eine sichtbare Periodizität auf, was den Anstieg der Amplitude $\left|\Delta c_{p, \alpha}\right|$ in Abb. 6.7(a) für diesen Frequenzbereich erklärt. Die Saugspitzen für $f_{\alpha} \approx 45 \mathrm{~Hz}$ treten nun hinter der maximalen Auslenkung der 
Flügelbewegung auf, was die Änderung der Phase verdeutlicht. Es liegt eine Nacheilung des Auftriebs im vorderen Flügeltiefenbereich vor, was Abb. 6.7(c) rechts oben mit $\phi\left(\Delta c_{p, \alpha}\right)(x / c<0,25)<0$ bestätigt. Das resultierende lokale Moment vor dem Drehpunkt eilt nach und liefert einen positiven aerodynamischen Dämpfungsbeitrag. Dies wird durch die erhöhten Amplituden im vorderen Flügeltiefenbereich verstärkt. Der weitere Phasenverlauf der Druckdifferenz hat sich deutlich zu kleineren Phasenwinkeln verschoben. Es liegt jedoch stromab des Drehpunktes noch eine Voreilung des Auftriebs vor, was sich in den integralen Werten $c_{l, \alpha}$ und $c_{m, \alpha}$ (vgl. Abb. 6.3(c) und (g)) widerspiegelt. Für $c_{m, \alpha}$ resultiert hieraus eine Nacheilung, die einen weiteren Beitrag zur Dämpfung bewirkt. Dieser wirkt sich durch den Phasenversatz von $\phi\left(\Delta c_{p, \alpha}\right)(x / c>0,25) \approx 90^{\circ}$ über die volle Schwingungsperiode aus. Die starke aerodynamische Dämpfung erklärt somit auch die auftretenden „Energietrichter“ (vgl. 6.2).

Für $f_{\alpha} \approx 60 \mathrm{~Hz}$ treten bei den geringen Schwingungsamplituden keine Stöße im vorderen Bereich des Flügels auf, wie Abb. 6.8(d) zeigt. Diese konzentrieren sich wieder auf den hinteren Flügeltiefenbereich mit einer mittleren Stoßposition von $\bar{x}_{s} \approx 0.64$. Die Druckverteilung weicht zeitweise von einer periodischen Struktur ab. Die Saugspitzen lassen jedoch eine weitere Phasenverschiebung erkennen und treten jetzt um den Nulldurchgang der zudrehenden Nickbewegung auf. Dies spiegelt sich in einer Nacheilung von $\phi\left(\Delta c_{p, \alpha}\right)(x / c<0,25) \approx-90^{\circ}$ wider, zu erkennen in Abb. 6.7(c) rechts unten. Die Phasenbeziehung maximiert die aerodynamische Dämpfung stromauf $\frac{c}{4}$. Wie Abb. 6.7(a) zeigt, hat sich hier jedoch die Amplitude $\left|\Delta c_{p, \alpha}\right|$ reduziert, so dass der zur Dämpfung beitragende lokale instationäre Auftriebsanteil gering ist. Die Phase der Druckdifferenz stromab des Drehpunkts hat sich ebenfalls reduziert und eilt nun der Bewegung nach. Dies führt zur Nacheilung von $c_{l, \alpha}$ und einer Voreilung von $c_{m, \alpha}(\operatorname{vgl}$. Abb. 6.3(c) und (g)). Insbesondere die Nacheilung des lokalen Auftriebs (Voreilung des lokalen Moments) hinter $\frac{c}{4}$ generiert dabei einen negativen Dämpfungsbeitrag. Entlang der gesamten Flügeltiefe führt somit die Phasen- und Amplitudenverteilung von $\Delta c_{p, \alpha}$ zu einem Energieeintrag in die Struktur und deutet eine aeroelastische Instabilität an (vgl. 6.2).

Die Betrachtung der instationären Druckdifferenzen eines vergleichbaren Frequenzsweeps für eine fixierte Grenzschichttransition zeigt, warum für eine turbulente Grenzschicht keine Instabilitäten zu erwarten sind. In Abb. 6.9 sind die Ergebnisse eines Frequenzsweeps bei $\mathrm{Ma}_{\infty}=0,73$ mit einem leicht reduzierten mittleren Anstellwinkel von $\bar{\alpha}=-0,08^{\circ}$ für eine fixierte Transition dargestellt. So zeigt sich zum einen, dass die in Abb. 6.9(a) dargestellte Amplitude $\left|\Delta c_{p, \alpha}\right|$ im Vergleich zur freien Grenzschichttransition (vgl. 6.7) insgesamt deutlich reduziert ist. Dies weist auf eine geringere Instationarität der Strömung hin, die sich hauptsächlich auf den vorderen Bereich des Laminarflügels stromauf des Drehpunktes konzentriert. Die Druckverteilung weist lediglich im Bereich der Transitionsfixierung bei $\frac{x}{c} \approx 0,075$ geringe periodische Strukturen auf, was die dort erhöhten Amplitudenwerte 


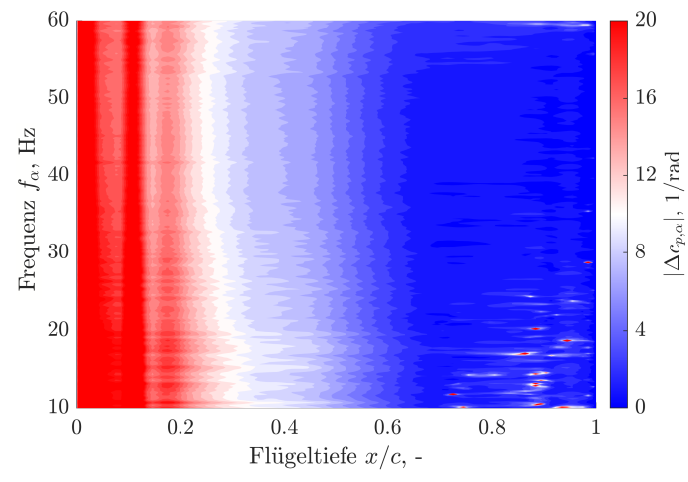

(a) Amplitude $\left|\Delta c_{p, \alpha}\right|$

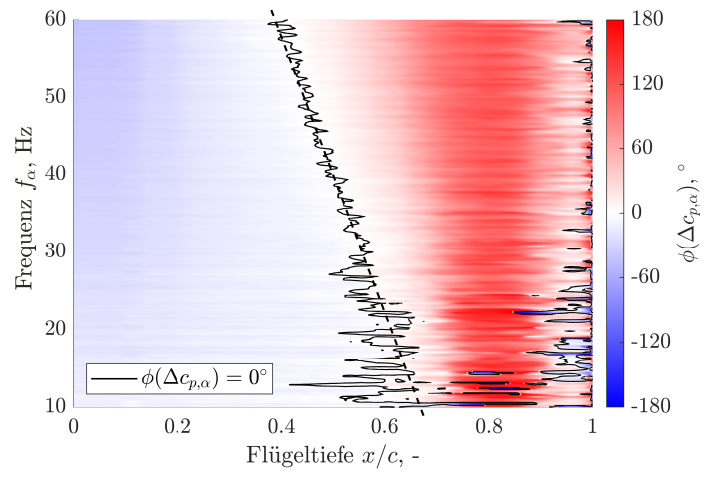

(b) Phase $\phi\left(\Delta c_{p, \alpha}\right)$
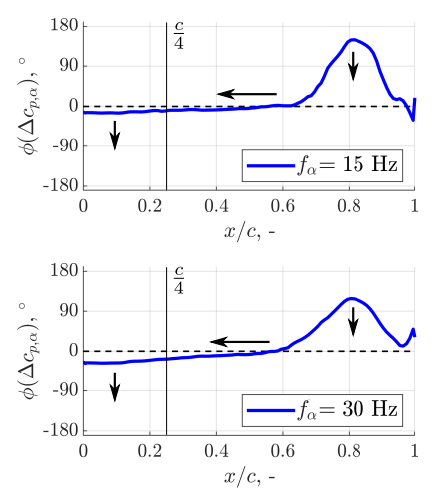
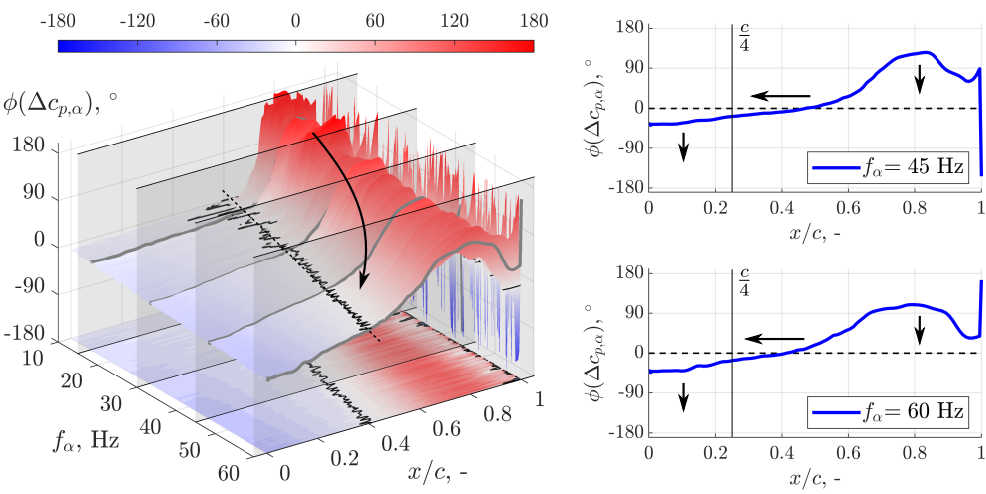

(c) Phase $\phi\left(\Delta c_{p, \alpha}\right)$ in 3D und Frequenzschnitte für $15 \mathrm{~Hz}, 30 \mathrm{~Hz}, 45 \mathrm{~Hz}$ und $60 \mathrm{~Hz}$

Abbildung 6.9.: Instationäre Druckdifferenz $\Delta c_{p, \alpha}$ in Betrag (a) und Phase (b) sowie 3DDarstellung der Phase samt Frequenzschnitte (c) für eine fixierte Grenzschichttransition, gemessen mithilfe eines Frequenzsweeps mit $\hat{\alpha}=0,08^{\circ}$ bei $\mathrm{Ma}_{\infty}=0,73$ und $\bar{\alpha}=-0,08^{\circ}$.

erklärt. Das Profil bleibt jedoch über den gesamten Frequenzbereich stoßfrei, so dass auch der Amplitudenverlauf weitestgehend unverändert bleibt. Die Phasenverteilung in Abb. 6.9(b) und (c) zeigt hingegen deutlichere Änderungen. Die Phasenvoreilung von $\Delta c_{p, \alpha}$ im hinteren Flügeltiefenbereich um $\frac{x}{c} \approx 0,8$ nimmt kontinuierlich ab, die Phasennacheilung im vorderen Flügeltiefenbereich zu. Ebenso verschiebt sich der PhasenNulldurchgang mit steigender Frequenz annährend linear stromauf, wie Abb. 6.9(b) und Abb. 6.9(c) zeigen. Die Kombination aus Amplituden- und Phasenverteilung führt zu einer zunehmenden aerodynamischen Dämpfung mit steigender Frequenz. Entsprechend nimmt auch der Energietransfer von der Struktur in die Strömung mit steigender Frequenz zu (vgl. Abb 6.6(c) und (d)) und das aeroelastische System bleibt stabil.

Weitestgehend analog verhält sich dies auch für $\mathrm{Ma}_{\infty}=0,74$ und $\mathrm{Ma}_{\infty}=0,75$. Bei einer freien Grenzschichttransition verschiebt sich mit steigender Frequenz die Phase $\phi\left(\Delta c_{p, \alpha}\right)$ zu kleineren Werten, so dass stromauf der Drehachse sowie in Teilen des hinteren Flügeltiefenbereichs eine Nacheilung der Druckdifferenz vorliegt. Dies ist für $\mathrm{Ma}_{\infty}=0,74$ in Abb. E.5 und für Ma $\mathrm{a}_{\infty}=0,75$ in Abb. E.6 in E.3 dargestellt. Wesentliche Unterschiede zeigen sich im Verhalten der Stoßdynamik. So setzt für $\mathrm{Ma}_{\infty}=0,74$ ein 
Verdichtungsstoß im vorderen Flügeltiefenbereich bereits bei kleineren Frequenzen ein und tritt verstärkt auf, wie Abb. E.5(d)-(g) verdeutlicht. Für $\mathrm{Ma}_{\infty}=0,75$ schließt das vordere Überschallgebiet lediglich für $f_{\alpha} \approx 30 \mathrm{~Hz}$ vereinzelt mit einem Stoß ab, was in Abb. E.6(e) zu erkennen ist. Dabei hängt auch hier die Stoßdynamik im vorderen Flügelbereich mit dem dortigen Anstieg der Amplituden $\left|\Delta c_{p, \alpha}\right|$, dargestellt in Abb. E.5(a) und Abb. E.6(a), und mit den aerodynamischen Resonanzen zusammen. Darüber hinaus deutet sich bei hohen Frequenzen für $f_{\alpha} \approx 60 \mathrm{~Hz}$ sowohl für $\mathrm{Ma}_{\infty}=0,74$ und besonders für $\mathrm{Ma}_{\infty}=0,75$ an, dass auftretende Verdichtungsstöße im hinteren Flügeltiefenbereich $\left(\overline{x_{s}} \approx 0,66\right.$ für $\mathrm{Ma}_{\infty}=0,74$ und $\overline{x_{s}} \approx 0,68$ für $\left.\mathrm{Ma}_{\infty}=0,75\right)$ einen stabilisierenden Effekt haben. Dies wird besonderes in Abb. E.6(c) rechts unten deutlich, wo eine ausgeprägte Phasenvoreilung des lokalen instationären Auftriebs auf Höhe der Stoßposition vorliegt. In Kombination mit hohen Amplitudenwerten (vgl. Abb. E.6(a)) liegt ein Dämpfungsbeitrag vor. Letzterer hebt jedoch die destabilisierende Wirkung der Phasenvoreilung im übrigen Flügeltiefenbereich stromab des Drehpunktes nicht auf, was den positiven Energieeintrag in die Struktur erklärt (vgl. Abb. E.4). Ebenso bestätigt sich auch für $\mathrm{Ma}_{\infty}=0,74$ und $\mathrm{Ma}_{\infty}=0,75$, dass bei einer turbulenten Grenzschicht keine Instabilitäten zu erwarten sind. Die Ergebnisse sind hierfür in Abb. E.7 dargestellt. Diese verhalten sich vollkommen analog zu $\mathrm{Ma}_{\infty}=0,73$. Die Amplituden- und Phasenbeziehungen von $\Delta c_{p, \alpha}$ tragen über den gesamten Frequenzbereich zu einer Dämpfung des aeroelastischen Systems bei.

Abschließend sind in Abb. E.8 bis Abb. E.10 in E.3 Amplituden $\left|\Delta c_{p, \alpha}\right|$ und Phasen $\phi\left(\Delta c_{p, \alpha}\right)$ in Abhängigkeit von $\bar{\alpha}$ für $\mathrm{Ma}_{\infty}=0,73$ bis $\mathrm{Ma}_{\infty}=0,75$ dargestellt, um den Einfluss des Anstellwinkels auf die instationäre Druckverteilung zu verdeutlichen. So spiegelt sich zum einen die aerodynamische Resonanz in erhöhten Amplitudenwerten $\left|\Delta c_{p, \alpha}\right|$ wieder. Diese konzentriert sich, abhängig von $\mathrm{Ma}_{\infty}$ und $f_{\alpha}$, auf mittlere Anstellwinkelbereiche, die mit dem Übergangsbereich der Laminardelle korrespondieren. Zum anderen ist eine Reduzierung der Phase von $\Delta c_{p, \alpha}$ hauptsächlich auf diesen Anstellwinkelbereich konzentriert. Am deutlichsten wird dies für Ma = 0,73 in Abb. E.8(h). Dort erstreckt sich im Bereich von $-0,2^{\circ} \lesssim \bar{\alpha} \lesssim 0,1^{\circ}$ ein Korridor über die gesamte Flügeltiefe, in dem $\phi\left(\Delta c_{p, \alpha}\right)<0$ gilt. Die für eine aeroelastische Instabilität bedeutsame Phasenvoreilung zeigt folglich eine Abhängigkeit vom Anstellwinkel. Vergleichbares lässt sich auch für $\mathrm{Ma}=0,74$ und $\mathrm{Ma}=0,75$ beobachten, jedoch verschoben hin zu kleineren Anstellwinkeln und damit in Übereinstimmung mit den bisherigen Beobachtungen.

\subsection{Instationäres Transitionsverhalten}

Zur Untersuchung des zeitabhängigen Transitionsverhaltens wurde die Analysemethodik (vgl. 4.3 sowie $[14,61]$ ) zur Auswertung von instationären CTA-Daten eingesetzt. Hierzu konnten nur die Messdaten der diskreten Frequenzvariationen verwendet werden. Im 
Rahmen der Messungen der Frequenzsweeps bildeten sich lokale Turbulenzkeile entlang der Heißfilmsensoren, so dass diese einer permanenten turbulenten Grenzschichtströmung ausgesetzt waren und bzgl. des Transitionsverhaltens keine validen Daten liefern.

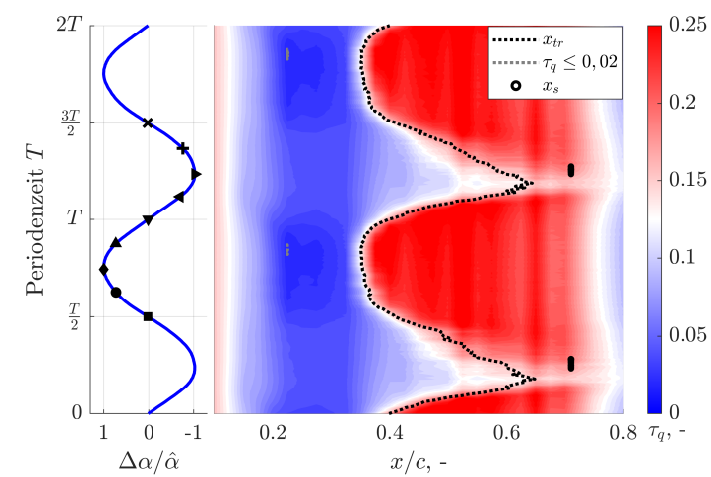

(a) $x_{t r}$ für $f=12,9 \mathrm{~Hz}$

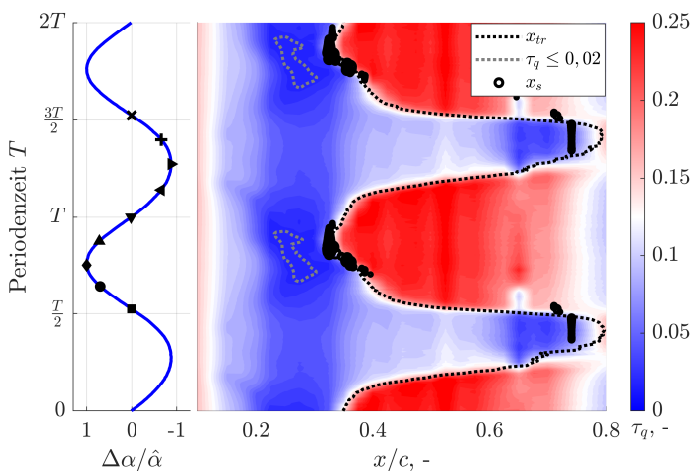

(c) $x_{t r}$ für $f=32,2 \mathrm{~Hz}$

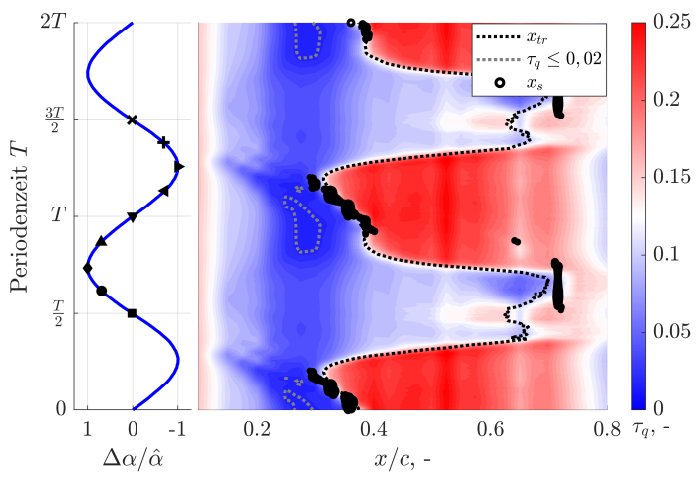

(e) $x_{t r}$ für $f=51,5 \mathrm{~Hz}$

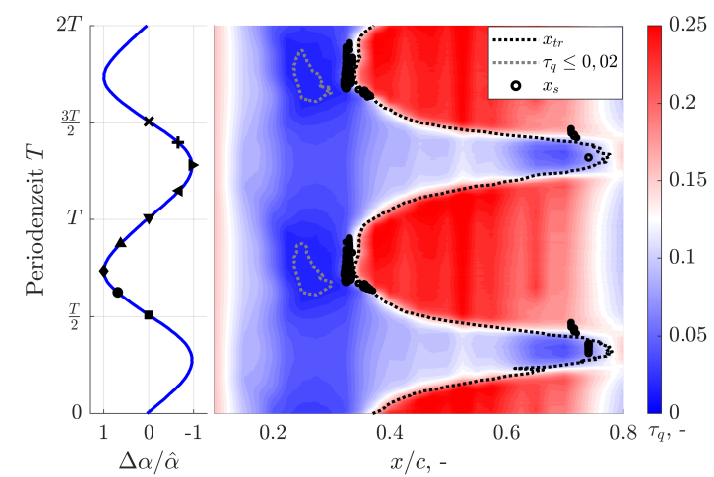

(b) $x_{t r}$ für $f=25,7 \mathrm{~Hz}$

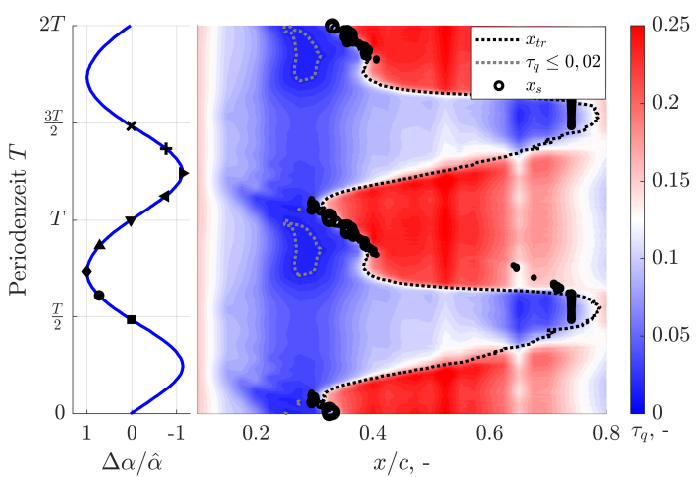

(d) $x_{t r}$ für $f=45,0 \mathrm{~Hz}$

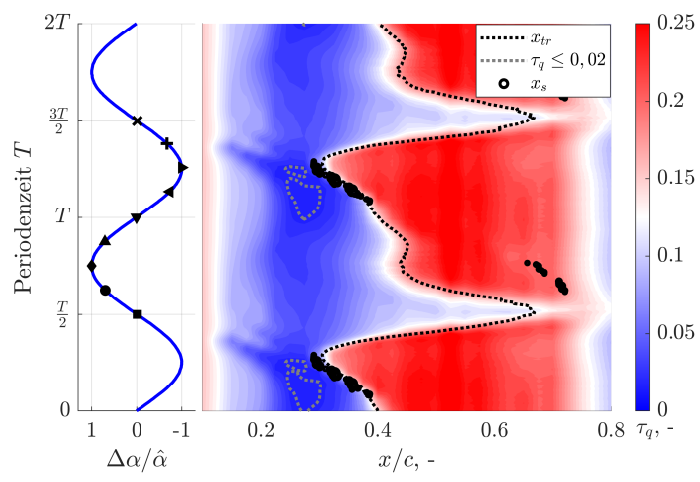

(f) $x_{t r}$ für $f=64,3 \mathrm{~Hz}$

Abbildung 6.10.: Phasengemittelte Quasi-Wandschubspannung $\tau_{q}$ für zwei Schwingungsperioden, gemessen und berechnet auf Basis einer diskreten Frequenzvariation um $\bar{\alpha}=0,05^{\circ}$ mit $\hat{\alpha}=0,25^{\circ}$ bei $\mathrm{Ma}_{\infty}=0,73$.

In Abb. 6.10 sind die Verläufe der Quasi-Wandschubspannungen $\tau_{q}$ für verschiedene Frequenzen bei einer Schwingungsamplitude von $\hat{\alpha}=0,25^{\circ}$ für $\mathrm{Ma}_{\infty}=0,73$ dargestellt. Diese wurden über drei Schwingungsperioden phasengemittelt. Ferner sind die Verläufe der ebenfalls phasengemittelten Transitionsposition $x_{t r}$ eingezeichnet und Bereiche $\tau_{q} \leq$ 
0,02 markiert (Hinweis auf Grenzschihtablösungen, vgl. 4.3.2 und 5.3). Zur Identifikation einer Stoß-Grenzschicht-Interaktion sind in Abb. 6.10 zusätzlich die Positionen $x_{s}$ der automatisiert detektierten Verdichtungsstöße eingetragen (vgl. 6.3), welche auf den phasengemittelten Druckverteilungen beruhen.

Abb. 6.10 zeigt deutlich, dass für $\mathrm{Ma}_{\infty}=0,73$ bereits bei Amplituden von $\hat{\alpha}=0,25^{\circ}$ eine ausgeprägte Bewegung der Transitionslage von ca. $50 \%$ der Flügeltiefe im Bereich $0,3 \lesssim \frac{x}{c} \lesssim 0,8$ auftritt. Dies bestätigt die bereits in 5.3 diskutierte Sensitivität der Transitionsposition bzgl. einer Änderung des Anstellwinkels. Dabei zeigt das dynamische Verhalten der Grenzschicht eine Abhängigkeit von der Schwingungsfrequenz. So ist zum einen der Verlauf der Transitionslinie unterschiedlich ausgeprägt. Zum anderen tritt ein mit steigender Frequenz zunehmender Phasenversatz der Position der Grenzschichttransition zur Profilbewegung auf, wie es bereits für die aerodynamischen Beiwerte und die Druckverteilungen beobachtet wurde. Gleiches gilt auch für die auftretenden Verdichtungsstöße, wobei Abb. 6.10 eine Phasenvoreilung der Transitionsbewegung gegenüber dem dynamischen Verhalten der Stöße vermuten lässt. Gerade bei höheren Frequenzen, wie z.B. für $f_{\alpha}=64,3 \mathrm{~Hz}$ in Abb. 6.10(f) ist deutlich ersichtlich, dass zunächst die Transition stromauf wandert und die Verdichtungsstöße auf die Transitionsposition „aufspringen“. Vergleichbare Effekte wurden bereits im Rahmen von selbsterregten Schwingungen des Laminarflügels untersucht [62]. Der Zusammenhang zwischen Stoß- und Transitionsposition wurde auch bei den stationären Messungen beobachtet (vgl. 5.3). Dabei deuten die niedrigen Werte für $\tau_{q}$ stromauf der Stoß-Grenzschicht-Interaktionen (vgl. Bereiche $\tau_{q} \leq 0,02$ in Abb. 6.10) auf laminare Ablöseblasen hin, wie sie für nahezu den gesamten Frequenzbereich um $\frac{x}{c} \approx 0,3$ herum periodisch auftreten. Laminare Ablöseblasen sind gemäß der stationären Ergebnisse sowie der Beobachtungen in $[14,61,62]$ auch bei einer Stoß-Interaktion mit einer laminaren Grenzschicht im hinteren Flügeltiefenbereich bei $\frac{x}{c} \approx 0,7 \mathrm{zu}$ erwarten. Die Quasi-Wandschubspannungen in Abb. 6.10 weisen jedoch in den betroffenen Gebieten Werte $\tau_{q}>0,02$ auf. Es zeigt sich dennoch eine Reduzierung von $\tau_{q}$ stromauf der Verdichtungsstöße, so dass auch hier mit Ablöseblasen zu rechnen ist. Infolge der Phasenmittelung ist der empirische Schwellwert von 0,02 demnach kritisch zu bewerten. Die Diskrepanz zwischen Stoßposition und Transitionslinie im hinteren Flügeltiefenbereich, wie diese insbesondere in Abb. 6.10(c) und (d) vorliegt, resultiert aus dem großen Abstand der Heißfilmsensoren im Bereich 0,7 $\leq \frac{x}{c} \leq 0,8$ (vgl. Abb 3.5). Die räumliche Aufösungsgenauigkeit der Transitionsposition ist hierdurch begrenzt. Der minimale Fehler für $x_{t r}$ ist hier mit dem vorliegenden Sensorabstands von 0,05c abzuschätzen, was die Diskrepanz von Stoß- und Transitionsposition erklärt.

In Abb. 6.11 sind die ermittelten Verläufe der Transitionspositionen in Abhängigkeit des Anstellwinkels zusammenfassend dargestellt, wobei die Analogie zu Lissajousfiguren [185, S.81 f.] angemerkt sei. Neben der Transitionsbewegung für $\hat{\alpha}=0,25^{\circ}$ sind 


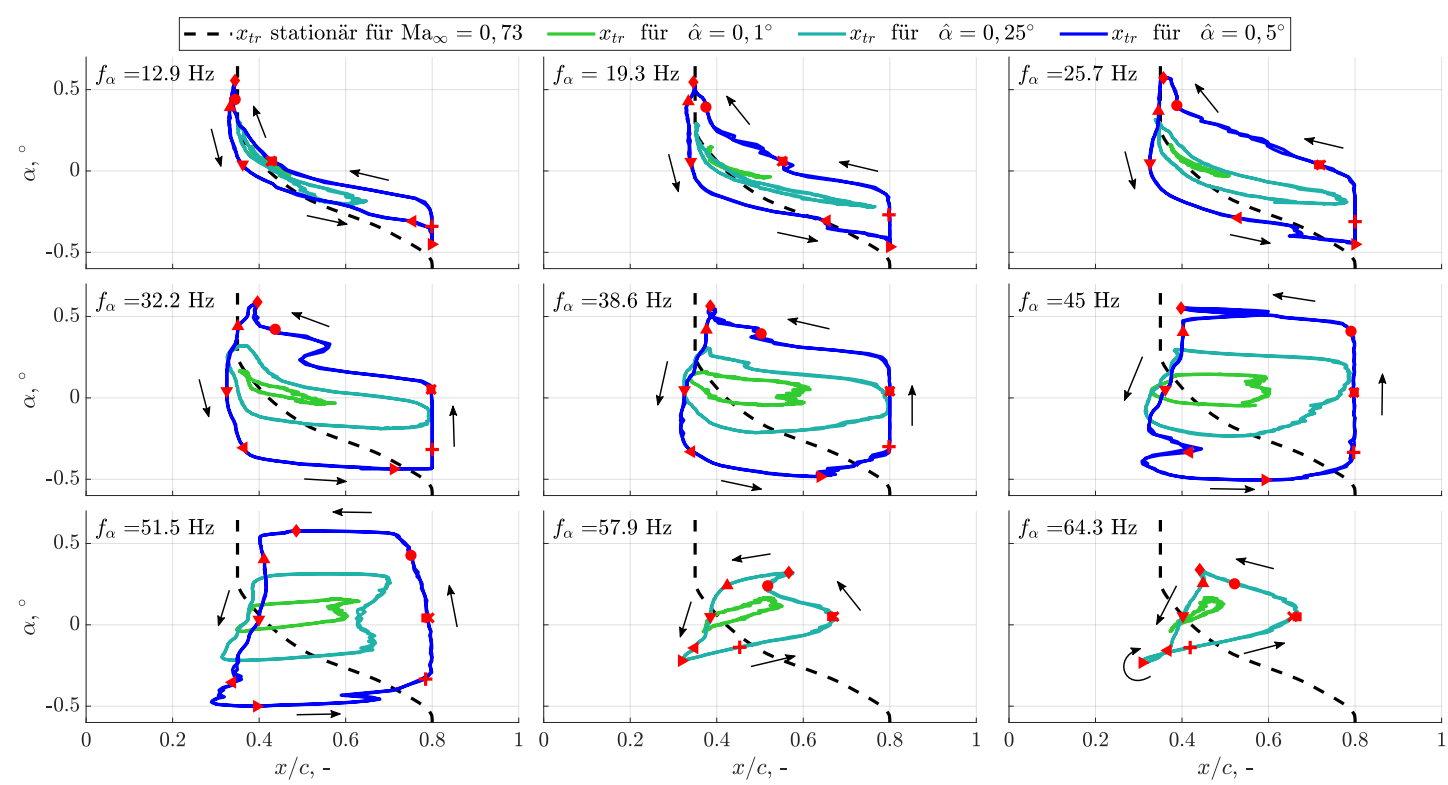

Abbildung 6.11.: Ermittelte Transitionsbewegung für die, bei $\mathrm{Ma}_{\infty}=0,73 \mathrm{um} \bar{\alpha}=0,05^{\circ}$ durchgeführten Frequenzvariationen mit $\hat{\alpha}=0,1^{\circ}, \hat{\alpha}=0,25^{\circ}$ und $\hat{\alpha}=0,5^{\circ}$. Für die letzte Amplitude wurden keine Messungen bei Frequenzen oberhalb von $52 \mathrm{~Hz}$ durchgeführt.

auch die Ergebnisse der Frequenzvariationen mit $\hat{\alpha}=0,1^{\circ}$ und $\hat{\alpha}=0,5^{\circ}$ aufgetragen. Die gestrichelte Linie in Abb. 6.11 markiert die stationäre Position der Transition für $\mathrm{Ma}_{\infty}=0,73$ (vgl. 5.3 und Abb. 5.6(a)). So zeigt sich zum einen eine Zunahme der Transitionsbewegung mit steigender Schwingungsamplitude. Die Transitionlage wandert jedoch auch bei Amplituden von $\hat{\alpha}=0,5^{\circ}$ nicht über $\frac{x}{c} \approx 0,3$ weiter stromauf. Auch stromab scheint eine Limitierung der Bewegung der Transitionsposition bei $\frac{x}{c} \approx 0,8$ vorzuliegen. Jedoch endet hier der Aufösungsbereich der Heißfilmsensoren. Ergebnisse bei selbsterregten LCOs unter vergleichbaren Randbedingungen deuten hier auf eine teils unterbrochene Transitionsbewegung hin [14,61,62], entsprechend einer zeitweisen vollständigen laminaren Grenzschicht entlang der gesamten Flügeltiefe. Folglich sind auch Transitionsbewegungen über $\frac{x}{c} \approx 0,8$ bzw. vollständige laminare Phasen zu vermuten. Ferner zeigt sich in diesem Bereich erneut eine Diskrepanz zwischen Stoß- und Transitionsposition, was die Verteilungen der Quasi-Wandschubspannung für $\hat{\alpha}=0,1^{\circ}$ und insbesondere für $\hat{\alpha}=0,5^{\circ}$, dargestellt im Anhang E.4 in Abb. E.11 und Abb. E.12 zeigen.

Abb. 6.11 verdeutlicht die Instationarität der Bewegung der Transitionslage. Für kleine Schwingungsfrequenzen von $f_{\alpha}=12,9 \mathrm{~Hz}$ (Abb.6.11 links oben) verläuft die dynamische Position der Transition $x_{t r}(t)$ annährend entlang der stationären Position. Dabei liegt eine reguläre Bewegung der Transitionlage vor, also eine Bewegung von $x_{t r}$ stromauf bei einem Aufdrehen $(\alpha \uparrow)$ des Flügels und einer Bewegung stromab beim Zudrehen $(\alpha \downarrow)$. Dieser Zusammenhang spiegelt sich in der nach links geneigten Orientierung der Kurven wieder. Erste Abweichungen von der stationären Transitionsposition treten jedoch bereits 
im hinteren Flügeltiefenbereich für $\hat{\alpha}=0,5^{\circ}$ (blaue Kurven in Abb. 6.11) auf. Diese Abweichungen nehmen mit steigender Schwingungsfrequenz für alle drei Schwingungsamplituden immer deutlicher zu. Das „Aufblähen“ der Transitionsverläufe spiegelt den zunehmenden Phasenversatz zwischen $x_{t r}$ und $\alpha$ wieder. Bei einem Phasenversatz von $\Delta \varphi>\frac{\pi}{2}$ nimmt die Ausdehnung wieder ab, jedoch neigt sich nunmehr die Orientierung der Kurven nach rechts. Dies geschieht in etwa ab einer Frequenz von $f_{\alpha}=38,6 \mathrm{~Hz}$. Hier verläuft auch die Transitionsbewegung im vorderen Flügeltiefenbereich invers, wie bereits Abb. 6.10 erkennen lässt. So bewegt sich z.B. für $f_{\alpha}=51,5 \mathrm{~Hz}$ (Abb. 6.11 links unten) die Transitionposition während des Zudrehens $\left(\alpha \approx 0,5^{\circ} \searrow \alpha \approx-0,5^{\circ}\right)$ von $0,4 c \mathrm{zu}$ $0,3 c$ stromauf, um dann abrupt stromab bis $0,8 c$ zu springen. Noch ausgeprägter ist diese inverse Transitionsbewegung für $f_{\alpha}=57,9 \mathrm{~Hz}$ und $f_{\alpha}=64,3 \mathrm{~Hz}$ (Abb. 6.11 mittig und links unten). Vermehrt bewegt sich $x_{t r}$ auch noch im ersten Teil des Aufdrehens des Flügels invers, was bereits ab $f_{\alpha}=38,6 \mathrm{~Hz}$ zu beobachten ist. Zusätzlich verdeutlicht die Form der Kurven den nichtlinearen Charakter der Transitionsbewegung, wobei in einzelnen Abschnitten einer Schwingungsperiode sogar ein Hystereseverhalten vorliegt.

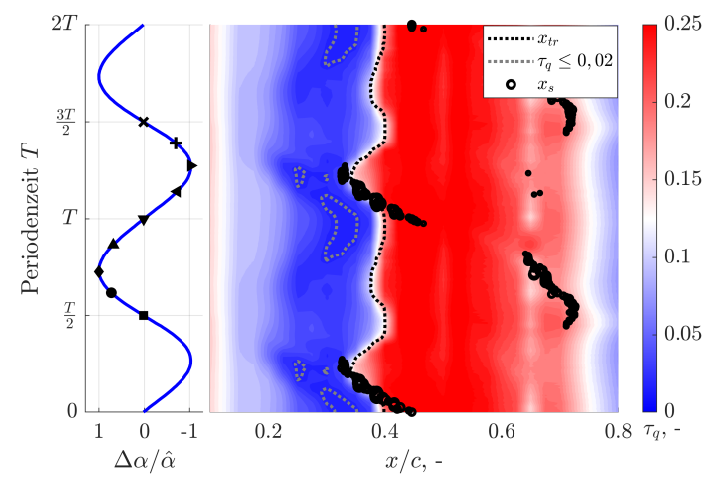

(a) $x_{t r}$ für $\mathrm{Ma}_{\infty}=0,74$ bei $f_{\alpha}=52,1 \mathrm{~Hz}$

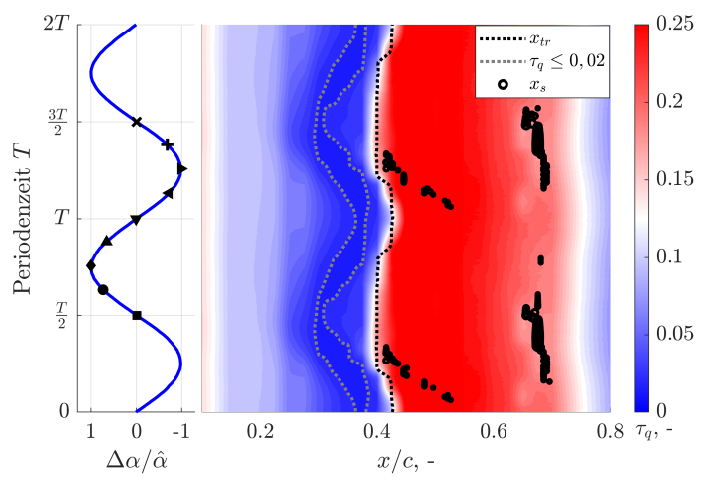

(b) $x_{t r}$ für $\mathrm{Ma}_{\infty}=0,75$ bei $f_{\alpha}=52,7 \mathrm{~Hz}$

Abbildung 6.12.: Phasengemittelte Quasi-Wandschubspannungen sowie Stoß- und Transitionspositionen $x_{t r}$ und $x_{s}$, gemessen und berechnet auf Basis diskreter Frequenzvariationen um $\bar{\alpha}=0,05^{\circ}$ mit $\hat{\alpha}=0,25^{\circ}$ bei $\mathrm{Ma}_{\infty}=0,74$ (a) und $\mathrm{Ma}_{\infty}=0,75$ (b).

Für höhere Mach-Zahlen $\mathrm{Ma}_{\infty}=0,74$ bzw. $\mathrm{Ma}_{\infty}=0,75$ fällt die Bewegung der Transitionsposition deutlich geringer aus. Diese beschränkt sich für die untersuchten Frequenzen und Amplituden $\left(f_{\alpha, \max } \approx 70 \mathrm{~Hz}, \hat{\alpha}_{\max }=0,25^{\circ}\right)$ auf eine Flügeltiefe von $0,3 \lesssim \frac{x}{c} \lesssim 0,45$ und beträgt mehrheitlich weniger als $5 \%$ der Flügeltiefe. In Abb. 6.12 sind die phasengemittelten Positionen der Transition sowie der Quasi-Wandschubspannung für zwei exemplarische Fälle bei $f_{\alpha} \approx 52 \mathrm{~Hz}$ und $\hat{\alpha}=0,25^{\circ}$ dargestellt. Weitere Ergebnisse sind in Abb. E.13 sowie Abb. E.14 in E.4 gezeigt. Dort sind auch die Bewegungen der Transitionspositionen in Abb. E.15 und Abb. E.16 zusammengefasst.

Abb. 6.12 verdeutlicht die nur geringfügig ausgeprägte Bewegung der Transitionsposition, die sich auf einen Bereich nahe der stationären Transitionslage konzentriert (vgl. 
Abb. 5.6(c), (e) sowie Abb. E.15 und Abb. E.16). Der mittlere Anstellwinkel der durchgeführten Frequenzvariationen liegt jedoch auch außerhalb des Bereiches, indem die stationären Ergebnisse eine starke Transitionsbewegung vermuten lassen (vgl. insbesondere Abb. 5.7). Es zeigt sich dennoch eine schwache periodische Bewegung von $x_{t r}$ und $x_{s}$ sowie derjenigen Gebiete, die auf eine laminare Ablöseblase hindeuten. Ferner ist eine zunehmende Phasenverschiebung von $\tau_{q}$ bzw. $x_{t r}$ mit steigender Schwingungsfrequenz für beide Mach-Zahlen erkennbar (vgl. Abb. 5.6(c) und (e)), analog zu Ma $a_{\infty}=0,73$.

Für Ma $=0,74$ wird auch die periodische Stoßdynamik besonders deutlich, wie Abb. 6.12(a) zeigt (vgl. auch Abb. E.13). Im periodischen Wechsel treten Verdichtungsstöße im Bereich um $0,4 c$ sowie um $0,65 c$ auf, welche zeitweise unterbrochen sind. Es handelt sich somit um eine Typ-B Stoßbewegung nach TiJdeman und SEebass [138]. Ferner verläuft die Stoßbewegung im hinteren Flügeltiefenbereich weitestgehend invers. Diese bewegt sich während des Aufdrehens des Flügels $(\alpha \uparrow)$ stromauf und deutet auf eine mögliche Hinterkantenablösung hin, welche jedoch durch die Druckverteilungen nicht bestätigt wird. Gleichwohl werden die Typ-B Stoßbewegung als auch eine Hinterkantenablösung in Verbindung mit einer Amplitudenbegrenzung von aeroelastischen Instabilitäten gebracht $[5,26]$, was gleichbedeutend mit einem positiven Beitrag dieser Aspekte zur aerodynamischen Dämpfung ist. Dabei sei erwähnt, dass auch für $\mathrm{Ma}_{\infty}=0,73$ und $\hat{\alpha}=0,5^{\circ}$ sowohl eine Typ-B Stoßbewegung sowie invers wandernde Verdichtungsstöße auftreten. Für $\mathrm{Ma}_{\infty}=0,74$ findet die Interaktion der Verdichtungsstöße im hinteren Flügeltiefenbereich dabei ausschließlich mit einer turbulenten Grenzschicht statt. Im vorderen Bereich liegt eine Interaktion mit einer laminaren Grenzschicht vor. Hier deuten die geringen Quasi-Wandschubspannungen wieder auf periodisch auftretende laminare Ablöseblasen hin, wie Abb. E.13 zeigt.

Für $\mathrm{Ma}_{\infty}=0,75$ sind laminare Ablöseblasen nicht mehr direkt mit dem Auftreten von Verdichtungsstößen verbunden. Jedoch verläuft gemäß Abb. 6.12(b) hier die Transition, zumindest für Schwingungsfrequenzen von $f_{\alpha} \gtrsim 46 \mathrm{~Hz}$ (vgl. Abb. E.14) immer über einer laminare Ablöseblase. Dabei ist deutlich zu erkennen, dass die Blase gemäß der Bewegung des Laminarflügels ebenfalls eine periodische bzw. harmonische Bewegung ausführt. Die Stoßdynamik hat sich im Vergleich zu $\mathrm{Ma}_{\infty}=0,74$ hingegen abgeschwächt. Verdichtungsstöße konzentrieren sich vermehrt auf den hinteren Flügeltiefenbereich, wie es bereits in 6.3 beobachtet wurde. Die Stoßposition ist dabei weitestgehend stationär. Lediglich bei größeren Schwingungsfrequenzen ist eine marginale inverse Bewegung stromauf zu erkennen. Im vorderen Flügeltiefenbereich treten nur schwache Stöße auf, die bei kleineren Schwingungsfrequenzen auch mit dem Auftreten einer laminaren Ablöseblase korrelieren (vgl. Abb. E.14). Zeitweise treten auch Stöße simultan auf, so dass ein DoppelStoß-System vorliegt. Die Position der Transition konzentriert sich für $\mathrm{Ma}_{\infty}=0,75$ ausschließlich auf einen Bereich von $0,4 \lesssim \frac{x}{c} \lesssim 0,45$, wobei für Frequenzen ab $f_{\alpha} \approx 40 \mathrm{~Hz}$ 
die Dynamik der Transitionsposition verlangsamt scheint, wie Abb. E.14 und Abb. E.16 zeigen. Es ist keine Änderung in der periodischen Bewegungsform von $x_{t r}$ mehr ersichtlich und auch die Änderung der Phasenverschiebung von $\tau_{q}$ und $x_{t r}$ bzgl. der Bewegung des Laminarflügels bei einer Änderung der Frequenz scheint für $\mathrm{Ma}_{\infty}=0,75$ reduziert.

\subsection{Zusammenfassung der instationären Aerodynamik}

Die Beobachtungen zur instationären Aerodynamik des CAST 10-2 Laminarflügels entsprechen den Erwartungen, die sich aus den vorherigen stationären Ergebnissen ergeben (vgl. 5). Wie bereits die instationären Effekte bei statischen Anstellwinkeln vermuten ließen, liegen für ausgewählte Frequenzen und Anstellwinkelbereiche ausgeprägte Instationaritäten vor. Diese verdeutlichen sich in aerodynamischen Resonanzen, welche je nach Mach-Zahl bei Frequenzen zwischen $30 \mathrm{~Hz}$ bis $50 \mathrm{~Hz}$ auftreten und insbesondere für $\mathrm{Ma}_{\infty}=0,73$ stark in Erscheinung treten. Dabei zeigt sich ein direkter Zusammenhang der Resonanzen mit einem Anstellwinkelbereich, welcher mit dem Übergangsbereich der Laminardelle korrespondiert. Entsprechend treten die Resonanzen mit steigender Mach-Zahl auch bei kleineren Anstellwinkeln auf, wobei auch die Frequenzen abnehmen (vgl. 5.4). So ist auch das Auftreten der aerodynamischen Resonanzen direkt mit einer freien Grenzschichttransition verknüpft, für eine turbulente Grenzschicht sind keine Resonanzen identifizierbar.

Bei einer freien Transition und abhängig von der Mach-Zahl tritt für Anstellwinkel, die mit dem Übergangsbereich der Laminardelle korrespondieren, eine Voreilung des Auftriebs auf. Diese Voreilung besteht bis zu Schwingungsfrequenzen von 40-50 Hz, welche in etwa mit den Eigenfrequenzen der aerodynamischen Resonanzen korrelieren. Bei höheren Frequenzen liegt dann eine Voreilung des Nickmomentes vor, was auf eine potentielle 1-DoF-Flatterinstabilität des Dreh-Freiheitsgrades hinweist. Letzteres tritt nochmals bei höheren Anstellwinkeln für einen erweiterten Frequenzbereich auf. Die Anstellwinkel, für die eine erneute Phasenvoreilung des Moments eintritt, nehmen mit steigender Mach-Zahl kontinuierlich ab. Auch diese Dynamiken sind nur bei einer freien Grenzschichttransition beobachtbar.

An die aerodynamischen Resonanzen schließen sich Bereiche an, in denen Energie in die Strömung transferiert wird. Es bilden sich „Energietrichter“ aus, welche eine aerodynamische Dämpfung des Systems zum Ausdruck bringen. Für größere Frequenzen oder auch für höhere Anstellwinkel kehrt sich dies um. Es wird Energie aus der Strömung entzogen, was sich in „Energiehügeln“ darstellen lässt. Letzteres deutet auf aeroelastische Instabilitäten hin. Der Energietransfer in die Struktur ist für alle Mach-Zahlen mit der Phasenvoreilung des Nickmomentes und ebenso mit einer freien Grenzschichttransition 
gekoppelt. Für eine erzwungene turbulente Grenzschicht hingegen liegt für den gesamten Anstellwinkel- und Frequenzbereich ein Energietransfer in die Strömung vor, was zu einer aeroelastischen System-Stabilität unter diesen Randbedingungen führt.

Das Phasenverhalten der integralen aerodynamischen Beiwerte $c_{l, \alpha}$ und $c_{m, \alpha}$ folgt aus dem Verhalten der instationären Druckbeiwerte $c_{p, \alpha}$ bzw. der Druckdifferenz $\Delta c_{p, \alpha}$. Für alle Mach-Zahlen reduziert sich die Phase $\phi\left(\Delta c_{p, \alpha}\right)$ mit steigender Frequenz, so dass aus einer anfänglichen Voreilung von $\Delta c_{p, \alpha}$ eine zunehmende Nacheilung wird. Stromauf des Drehpunktes resultiert hieraus ein nacheilender Beitrag zum Moment, welcher einen positiven aerodynamischen Dämpfungsbeitrag liefert. Stromab des Drehpunktes führt die Nacheilung von $\Delta c_{p, \alpha}$ zu einem voreilenden Momentenbeitrag, der sich aerodynamisch anfachend auswirkt. Die Verteilung der Phasenwinkel sowie die Kombination mit den Amplitudenwerten entlang der Flügeltiefe verhält sich abhängig von der Schwingungsfrequenz dabei so, dass im Bereich der „Energietrichter“ bzw. in der Nähe der aerodynamischen Resonanzen eine starke aerodynamische Dämpfung (Energietransfer in Strömung, Phasenwinkel $\left.\phi\left(\Delta c_{p, \alpha}\right)(x / c>0,25) \approx 90^{\circ}\right)$ vorliegt. Bei höheren Frequenzen bzw. Anstellwinkeln ist hingegen eine aerodynamische Anfachung (Energietransfer in Struktur, Phasenwinkel $\left.\phi\left(\Delta c_{p, \alpha}\right)(x / c>0,25)<0^{\circ}\right)$ zu beobachten. Für alle Mach-Zahlen ist ein derartiges Phasenverhalten wiederum ausschließlich bei einer freien Grenzschichttransition festzustellen. Im turbulenten Fall bewegt sich die Phasenverteilung mit steigender Frequenz in Richtung $\left.\phi\left(\Delta c_{p, \alpha}\right)(x / c \lessgtr 0,25) \approx \mp 90^{\circ}\right)$, entsprechend nimmt die aerodynamische Dämpfung zu. Ferner konzentriert sich dieses Phasenverhalten primär wieder auf Anstellwinkel, die im Übergangsbereich der Laminardelle liegen.

Die entwickelte Analysemethodik zur Auswertung der Heißfilm-Sensoren ermöglichte erstmalig und in einem größeren Umfang die Aufösung der Dynamik der laminar-turbulenten Grenzschichttransition unter den vorliegenden Randbedingungen. Für $\mathrm{Ma}_{\infty}=0,73$ offenbart sich so die bereits bei kleinen Schwingungsamplituden vorliegende Transitionsbewegung von bis zu 50 \% der Flügeltiefe. Für größere Amplitude nimmt die Bewegung weiter zu. Dabei verdeutlicht sich die Instationarität und Dynamik der Transitionsposition, welche mit steigender Schwingungsfrequenz zunehmend von dem stationären Verlauf abweicht, einen wachsenden Phasenversatz zur Bewegung des Laminarflügels aufweist und zeitweise sogar invers verläuft (Bewegung von $x_{t r}$ stromauf wenn Flügel zudreht und v. v.). Dabei zeigt sich eine nichtlineare Charakteristik der Transitionsbewegung, was sich u. a. in einem Hystereseverhalten wiederspiegelt. Für größere Mach-Zahlen liegt eine deutlich reduzierte Transitionsbewegung vor, wobei schon die stationären Transitionspositionen in dem untersuchten mittleren Anstellwinkelbereich keine ausgeprägte Transitionsbewegung vermuten lassen. Eine periodische Transitionsbewegung sowie ein wachsender Phasenversatz zur Profilbewegung mit steigender Frequenz wird dennoch bestätigt. Ferner bilden sich verstärkt laminare Ablöseblasen aus, welche ebenfalls eine 
periodische Dynamik zeigen. Ebenso tritt, insbesondere für $\mathrm{Ma}_{\infty}=0,74$, eine verstärkte Stoßdynamik auf (Typ-B nach TiJdeman und SeEbass [138]), die mit der Grenzschicht wechselwirkt. Für $\mathrm{Ma}_{\infty}=0,73$ zeigt sich sogar eine Voreilung der Transitionsbewegung zur Stoßbewegung. Die Transitionslage gibt die Stoßposition vor.

Die Ergebnisse liefern damit Einsichten über die kausale Verkettung der einzelnen aerodynamischen und strukturellen Aspekte im Hinblick auf aeroelastische Instabilitäten. So begründen sich diese in erster Linie in einem (positiven) Energieeintrag aus der Strömung in die Struktur. Voraussetzung dafür ist wiederum eine passende Phasen- sowie Amplitudenbeziehung zwischen Strukturbewegung und den resultierenden aerodynamischen Beiwerten. Da diese als integrale Werte aus der Druckverteilung resultieren, müssen bereits die einzelnen Druckanteile eine entsprechende Phasen- und Amplitudenbeziehung aufweisen. Diese Zusammenhänge sind, wie oben gezeigt, für das CAST 10-2 Laminarflügelprofil unter spezifischen Randbedingungen erfüllt. Dabei zeigt sich in allen Aspekten, dass hierfür eine freie Grenzschichttransition eine notwendige Bedingung darstellt und damit auch für das Einsetzen einer aeroelastischen Instabilität des Laminarflügels. Jedoch ist aus den bisherigen Ergebnissen nicht zu klären, ob eine freie Grenzschichttransition auch verantwortlich für eine aeroelastische Instabilität unter den betrachteten Randbedingungen ist, also die Grenzschichttransition und deren dynamisches Verhalten als Ursache und die weiteren Effekte als Wirkung auftreten. 


\section{Aeroelastisches Verhalten}

Zum Abschluss der vorliegenden Arbeit wurde das aeroelastische Verhalten des CAST 10-2 Laminarflügels für zwei aeroelastische Konfigurationen mithilfe des FVS (vgl. 3.2.2) untersucht. Die Messungen erfolgten in einem Bereich von $0.3 \leq \mathrm{Ma}_{\infty} \leq 0.8$ und $40 \leq$ $p_{0} \leq 75 \mathrm{kPa}$, resultierend in Reynolds-Zahlen von $1,15 \cdot 10^{6} \lesssim \operatorname{Re}_{\infty} \lesssim 2,83 \cdot 10^{6}$. Basierend auf den Erfahrungen des ersten Flatterversuchs [24] sowie den Ergebnissen der stationären und instationären Aerodynamik (vgl. 5 und 6), fanden die Messungen überwiegend bei einem mittleren Anstellwinkel von $\bar{\alpha} \approx 0^{\circ}$ und einer freien Grenzschichttransition statt.

Das folgende Kapitel liefert eine Übersicht über das aeroelastische Verhalten des Laminarflügels bei den genannten Randbedingungen. Die aeroelastischen Stabilitätgrenzen (Transonic Dips) und auftretende Flatterfälle beider Konfigurationen werden diskutiert. Die nichtlineare Systemantwort bei einer Änderung der Mach-Zahl und des Anstellwinkels wird beschrieben. Anhand eines repräsentativen LCOs wird die instationäre Aerodynamik genauer charakterisiert. Weitere Informationen finden sich im Anhang F sowie in diversen Veröffentlichungen [14,59-63], auf denen ein Großteil des Kapitels basiert.

\subsection{Aeroelastische Konfigurationen}

Die zwei Konfigurationen umfassten ein klassisches Biege-Torsions-Flattersystem mit den zwei Starrkörper-Freiheitsgraden (2-DoF) Schlag und Drehung sowie ein Ein-FreiheitsgradSystem (1-DoF) mit einer Starrkörper-Drehung. Letzteres ging durch mechanische Sperrung der Schlagfedern aus der ersten Konfiguration hervor (vgl. 3.2.2).
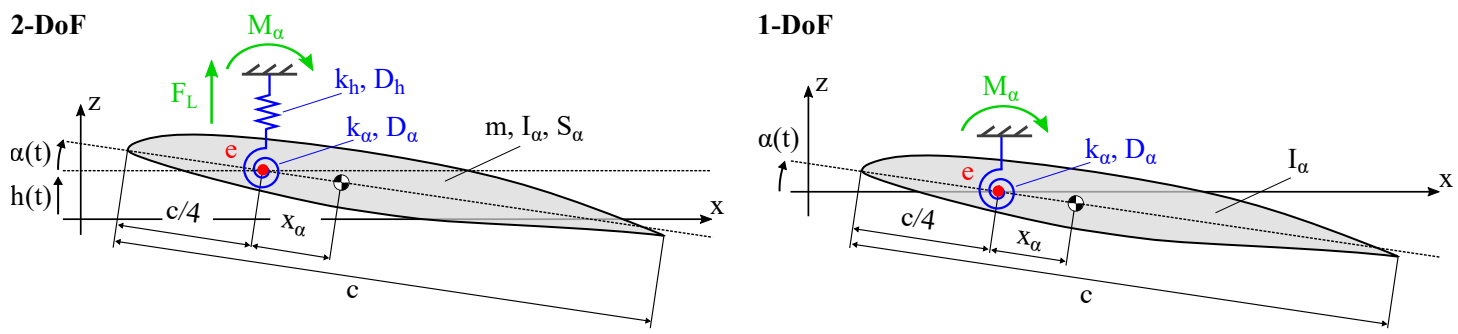

Abbildung 7.1.: Schematische Darstellung der Strukturmodelle der zwei aeroelastischen Konfigurationen mit zwei (links) und einem (rechts) Freiheitsgrad, verändert nach [59]. 
Die Strukturmodelle beider Konfigurationen sind in Abb. 7.1 dargestellt. Die StrukturParameter folgen dabei aus der Kombination der Parameter des FVS (siehe Tab. 3.1) und des CAST 10-2 Windkanalmodells (siehe Tab. 3.2) und sind für die aeroelastischen Gesamtsysteme in Tab. 7.1 aufgelistet. Der Schwerpunkt $s$ des 2-DoF Systems ist hinter der elastischen Achse $e$ lokalisiert. Hieraus folgt eine strukturelle Kopplung der beiden Freiheitsgrade Schlag und Drehung durch das statische Massenmoment $S_{\alpha}=m x_{\alpha}$. Die

Tabelle 7.1.: Strukturmechanische Parameter der aeroelastischen Konfigurationen.

\begin{tabular}{llll}
\hline Parameter & Notation & 2-DoF System & 1-DoF System \\
\hline Steifigkeit Schlag & $k_{h}, \mathrm{kN} / \mathrm{m}$ & $835,19 \pm 0,33$ & - \\
Steifigkeit Drehung & $k_{\alpha}, \mathrm{Nm} / \mathrm{rad}$ & $6555,32 \pm 6,09$ & $6555,32 \pm 6,09$ \\
Masse $^{1}$ & $m, \mathrm{~kg}$ & $25,60 \pm 0,13$ & - \\
elastische Achse, Drehpunkt $^{1}$ & $e / c,-$ & 0,25 & 0,25 \\
Schwerpunkt $^{1}$ & $s / c,-$ & $0,279 \pm 0,01$ & - \\
Abstand $s \mathrm{zu} e$ & $x_{\alpha} / c,-$ & $0,029 \pm 0,01$ & - \\
statisches Massenmoment & $S_{\alpha}, \mathrm{kg} \cdot \mathrm{m}$ & $0,224 \pm 0,13$ & - \\
Trägheitsmoment $^{2}$ & $I_{\alpha}, 10^{-3} \mathrm{~kg} \cdot \mathrm{m}^{2}$ & $64,81 \pm 3,60$ & $64,81 \pm 3,60$ \\
\hline
\end{tabular}

${ }^{1}$ bezogen auf das aeroelastische Gesamtsystem

${ }^{2}$ bezogen auf die elastische Achse, den Drehpunkt $e=c / 4$

Eigenfrequenzen, Dämpfungen und Schwingungsmoden beider Konfigurationen wurden durch Vibrationstests bestimmt. Abb. 7.2 zeigt die PSDs der hierzu augezeichneten Beschleunigungssignale. Die identifizierten Eigenfrequenzen sind in Tab. 7.2 eingetragen. Die Dämpfungen wurden anhand von Ausklingkurven der jeweiligen Schwingungsmoden bestimmt. Das Verfahren ist in F.1 beschrieben. Die Ergebnisse sind als LEHR'sche Dämpfungsmaße $D_{i}$ in Tab. 7.2 aufgelistet. Die Schwingungsmoden sind in Abb. F.3 (2-DoF System) und Abb. F.4 (1-DoF System) in F.2 dargestellt.

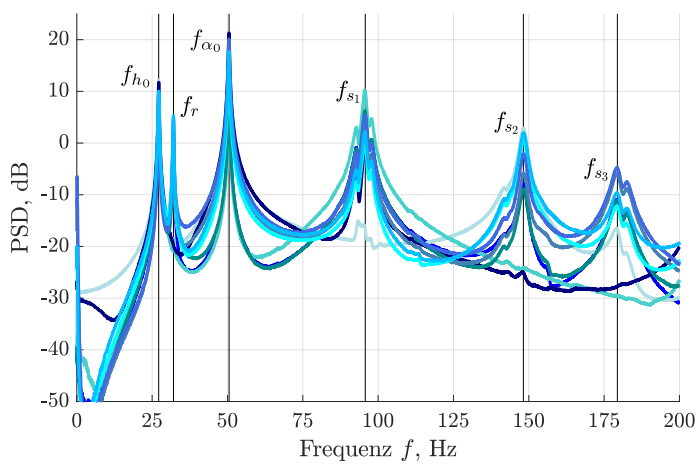

(a) Eigenfrequenzen der 2-DoF Konfiguration

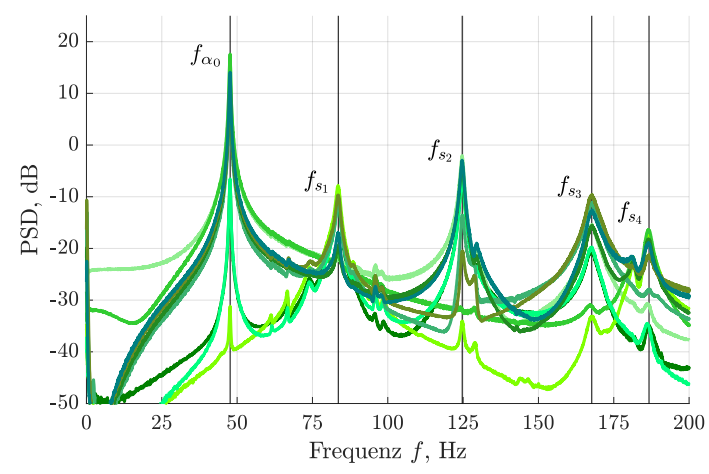

(b) Eigenfrequenzen der 1-DoF Konfiguration

Abbildung 7.2.: Eigenfrequenzen der zwei aeroelastischen Konfigurationen mit zwei (a) und einem (b) Freiheitsgrad. Die PSDs basieren auf den Signalen der Beschleunigungssensoren. 
Die experimentell vorgegebenen Starrkörper-Eigenfrequenzen liegen für das 2-DoF System bei $f_{h_{0}}=27,2 \mathrm{~Hz}$ (Schlag) und $f_{\alpha_{0}}=50,5 \mathrm{~Hz}$ (Drehung). Die weitere StarrkörperEigenfrequenz bei $f_{r}=32,1 \mathrm{~Hz}$ entspricht einer Rollbewegung des Flügelmodells und ist ein Artefakt der zweidimensionalen Modell-Aufhängung. Bei der 1-DoF Konfiguration liegt die Eigenfrequenz der Drehung etwas tiefer bei $f_{\alpha_{0}}=47,7 \mathrm{~Hz}$. Dabei sei angemerkt, dass die eigentliche Starrköper-Mode zusätzlich einen sehr schwachen strukturellen Biegeanteil aus Windkanalmodell und Einspannung enthält. Die weiteren strukturellen Schwingungsmoden sind deutlich von den Starrkörpermoden getrennt und bzgl. des aeroelastischen Verhaltens von nachrangiger Bedeutung. Ferner wurde eine mögliche Interaktion der Starrkörper-Eigenfrequenzen mit auftretenden Windkanalresonanzen ausgeschlossen, was in F.3 und [62] dargelegt ist.

Tabelle 7.2.: Schwingungsmoden, Eigenfrequenzen (vgl. Abb. 7.2) und Dämpfungen der zwei aeroelastischen Konfigurationen als Ergebnisse der Vibrationstests.

\begin{tabular}{ccccc}
\hline System & Bez. & Eigenmode & $\mathbf{f} \pm \sigma_{\mathbf{f}}, \mathrm{Hz}$ & $\mathbf{D} \pm \sigma_{\mathbf{D}}, \%$ \\
\hline 2-DoF & $f_{h_{0}}$ & Schlag & $27,2 \pm 0,1$ & $0,47 \pm 0,03$ \\
$" "$ & $f_{r}$ & Rollen & $32,1 \pm 0,2$ & $0,36 \pm 0,03$ \\
$"$ " & $f_{\alpha_{0}}$ & Drehung & $50,5 \pm 0,1$ & $0,34 \pm 0,01$ \\
$"$ & $f_{s_{1}}$ & 1. Biegung + Drehung & $95,6 \pm 0,1$ & $0,56 \pm 0,03$ \\
$"$ & $f_{s_{2}}$ & 2. Biegung + 1. Torsion & $148,2 \pm 0,2$ & $0,76 \pm 0,07$ \\
$"$ & $f_{s_{3}}$ & 1. Torsion & $179,3 \pm 0,3$ & $0,64 \pm 0,10$ \\
\hline 1-DoF & $f_{\alpha_{0}}$ & Drehung & $47,7 \pm 0,1$ & $0,39 \pm 0,01$ \\
$"$ & $f_{s_{1}}$ & 1. Biegung + Drehung & $83,5 \pm 0,1$ & $0,92 \pm 0,11$ \\
$"$ & $f_{s_{2}}$ & 2. Biegung & $124,6 \pm 0,2$ & $0,48 \pm 0,01$ \\
$"$ & $f_{s_{3}}$ & 1. Torsion & $167,6 \pm 0,2$ & $0,89 \pm 0,18$ \\
$"$ & $f_{s_{4}}$ & 3. Biegung & $186,5 \pm 0,2$ & $0,82 \pm 0,11$ \\
\hline
\end{tabular}

\subsection{Aeroelastische Stabilität der 2-DoF Konfiguration}

Beginnend bei einer Mach-Zahl von $\mathrm{Ma}_{\infty}=0,5$ wurde durch Erhöhung von $\mathrm{Ma}_{\infty}$ bei $p_{0}=$ const. der Transonic Dip des Laminarprofils für die 2-DoF Konfiguration bis $\mathrm{Ma}_{\infty} \approx 0,8$ nochmals eingehender untersucht. Eine erste Messung des Dips unter selbigen Randbedingungen erfolgte bereits im Rahmen des ersten Flatterversuchs [24]. Die Ergebnisse der erneuten Messung sind in Abb. 7.3 dargestellt. Die Stabilitätsgrenze ${ }^{1}$ ist als graue, interpolierte Linie eingezeichnet. Verschiedene Flatterfälle wurden innerhalb des Transonic Dips beobachtet. In Abb. 7.4 sind die Zeitreihen des instationären Anstellwinkel$\Delta \alpha$ und Schlaganteils $\Delta h$ des Modellmittelschnitts (gemessen mithilfe der dort verbauten

\footnotetext{
${ }^{1}$ Die Bestimmung der Flatter- bzw. Stabilitätsgrenze ist immer mit Unsicherheiten behaftet und als Übergangsbereich und nicht als scharfe Trennung zwischen stabil | instabil zu interpretieren (vgl. [60]).
} 
Beschleunigungssensoren, vgl. 3.4.2) für vier repräsentative Flattertypen dargestellt. Die Fälle korrespondieren mit den schwarz umrandeten Punkten in Abb. 7.3.

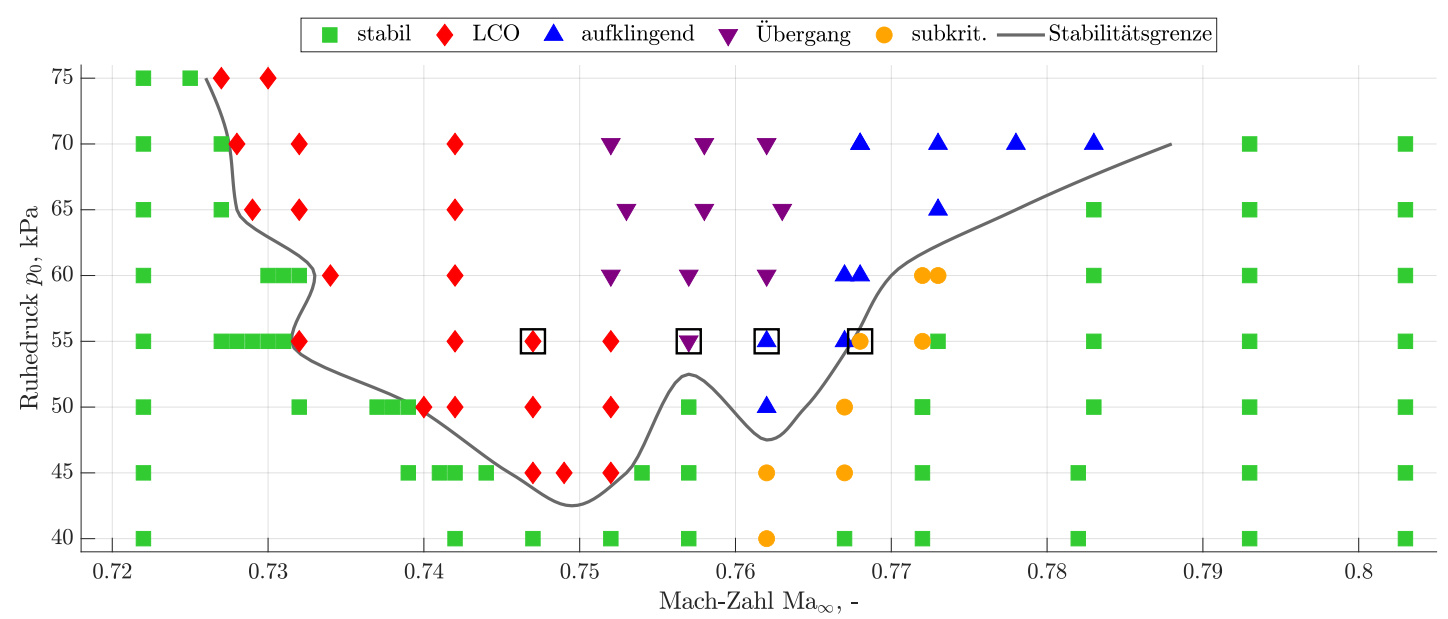

Abbildung 7.3.: Stabilitätsdiagramm der aeroelastischen 2-DoF Konfiguration bei einen mittleren Anstellwinkel $\bar{\alpha} \approx 0^{\circ}$ und freier Grenzschichttransition, verändert nach [59].

Im vorderen Bereich des Transonic Dips treten für $0,73 \lesssim \mathrm{Ma}_{\infty} \lesssim 0,75$ Grenzzyklusschwingungen auf, die durch den Dreh-Freiheitsgrad dominiert werden (rote Marker in Abb. 7.3). Diese Flatterfälle waren die Motivation zur vorliegenden Arbeit (vgl. 1.1). Repräsentative Zeitreihen sind in Abb. 7.4(a) dargestellt und verdeutlichen den nahezu sinusförmigen LCO von $\Delta \alpha$ und den nur geringen Schlaganteil. Eine genauere Diskussion dieses Flattermechanismus erfolgt für die aeroelastische 1-DoF Konfiguration in 7.3.

Für $0,75 \lesssim \mathrm{Ma}_{\infty} \lesssim 0,76$ tritt ein Übergangsgebiet mit LCOs auf, die eine abnehmende Dreh-Amplitude aufweisen (violette Marker in Abb. 7.3). Diese wird zunehmend instationär, was zu einer Verschmierung der LCOs führt, wie Abb. 7.4(b) zeigt. Es kommt zu einem zeitweisen und mit steigender Mach-Zahl zunehmenden Anstieg der Schlag-Amplitude, bis der LCO verschwindet und der Schlag aufklingt.

Im hinteren Bereich des Dips treten für $0,76 \lesssim \mathrm{Ma}_{\infty} \lesssim 0,78$ Schlag-dominierte Flatterfälle auf (blaue Marker in Abb. 7.3). Wie Abb. 7.4(c) zeigt, wächst die Amplitude kontinuierlich an und muss mithilfe der Bremsen bzw. Erreger (vgl. 3.2.2) gestoppt werden (in Abb. 7.4(c) und (d) rot markiert). Geschachtelte LCOs oder solche mit einer größeren Grenzamplitude (siehe z. B. $[26,27,135,139,144]$ ) wurden nicht beobachtet.

Für Ruhedrücke $p_{0} \lesssim 60 \mathrm{kPa}$ treten im Bereich $0,76 \lesssim \mathrm{Ma}_{\infty} \lesssim 0,775$ zusätzlich subkritische Flatterfälle auf (orange Marker in Abb. 7.3). Das dynamische Antwortverhalten des aeroelastischen Systems ist hier durch eine subkritische Hopf-Bifurkation beschrieben. Das System ist zunächst stabil. Erst eine Anregung mit den elektrodynamischen 
Erregern über einen Schwellwert (siehe Repeller in Abb. 2.14(b)) führt zu einer Instabilität, wie in Abb. 7.4(d) dargestellt. Diese Flatterfälle wurden für die Interpolation der Stabilitätsgrenze in Abb. 7.3 nicht mit berücksichtigt.
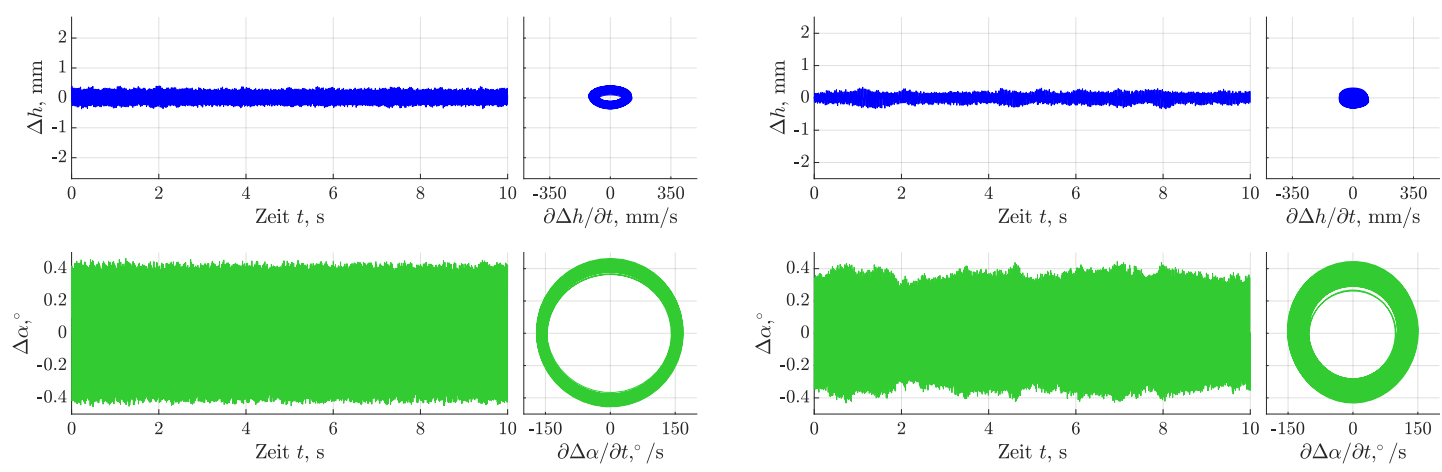

(a) LCO bei $\mathrm{Ma}_{\infty}=0,747$

(b) LCO im Übergang bei $\mathrm{Ma}_{\infty}=0,757$
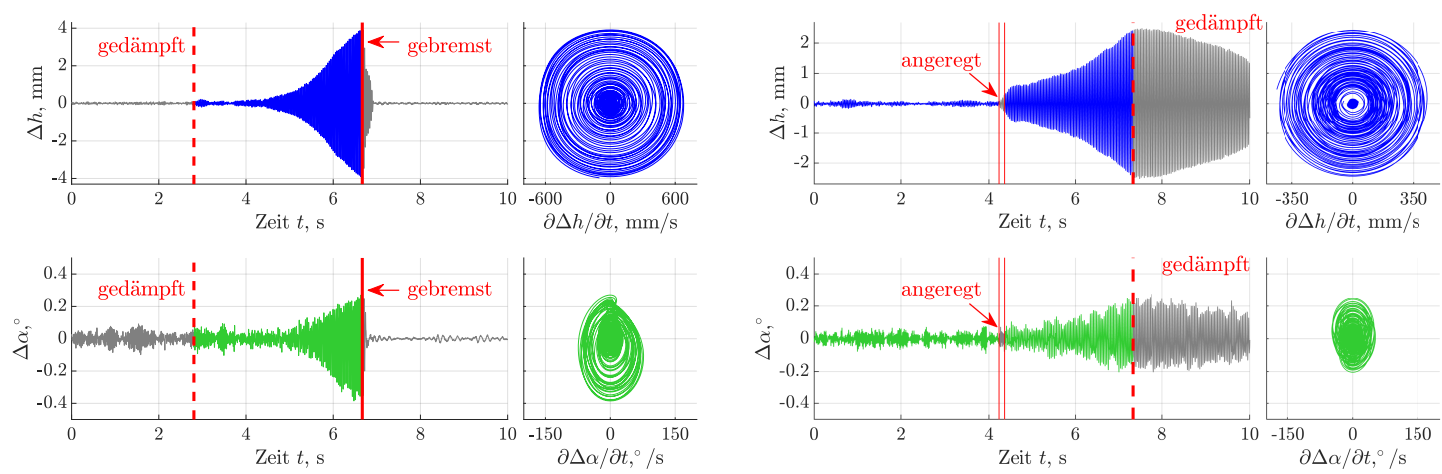

(c) aufklingendes Flattern bei $\mathrm{Ma}_{\infty}=0,762$

(d) subkrit. Flattern bei $\mathrm{Ma}_{\infty}=0,768$

Abbildung 7.4.: Zeitreihen und Phasenraumdarstellungen der vier unterschiedlichen Flatterfälle der 2-DoF Konfiguration für $p_{0}=55 \mathrm{kPa}$, verändert nach [59].

Die Besonderheit des aeroelastischen Verhaltens, insbesondere der LCOs, zeigt sich in den Frequenzverläufen der Starrkörper-Moden, dargestellt in Abb. 7.5. Die Schlagfrequenz $f_{h}$ verläuft weitestgehend konstant nahe der Eigenfrequenz $f_{h_{0}}$, die Drehfrequenz $f_{\alpha}$ ändert sich hingegen deutlich. Für $0,72 \lesssim \mathrm{Ma}_{\infty} \lesssim 0,73$ steigt $f_{\alpha}$ um mehr als $30 \%$ gegenüber $f_{\alpha_{0}}$ auf fast $66 \mathrm{~Hz}$ bei $\mathrm{Ma}_{\infty}=0,731$ an. Für $\mathrm{Ma}_{\infty}=0,732$ treten dann erstmalig LCOs auf, die durch die hohen Drehfrequenzen auffallen. Diese Frequenzanteile treten auch in den Wasserfallspektren in Abb. 7.5(a) hervor. Dort sowie in den, durch die Balken in Abb. 7.5(b) gekennzeichneten 3dB-Abfällen, zeigt sich ferner die hohe Dämpfung des Dreh-Freiheitsgrades im Bereich $0,7 \lesssim \mathrm{Ma}_{\infty} \lesssim 0,73$ und der abrupte Dämpfungsabfall vor dem Einsetzen der LCOs. Solange diese dann vorliegen, fällt $f_{\alpha}$ wieder kontinuierlich ab. Dieser Bereich ist in Abb. 7.5(b) rot hinterlegt (Übergangsbereich violett, aufklingende Flatterfälle blau). Mit dem Auftreten von aufklingenden Flatterfällen ab $\mathrm{Ma}_{\infty} \approx 0,76$ ist dann $f_{\alpha}$ wieder in der Nähe von $f_{\alpha_{0}}$. Als Flatterfrequenz tritt nun $f_{h}$ hervor, wie Abb. 7.5(a) zeigt. 


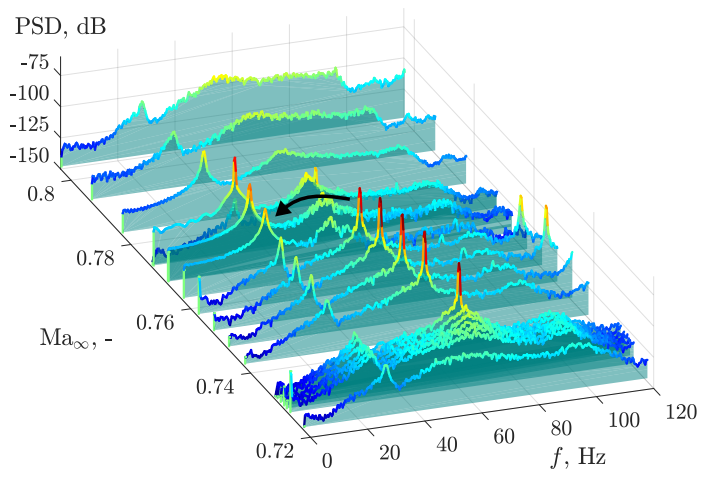

(a) Wasserfallspektren der 2-DoF Konfiguration

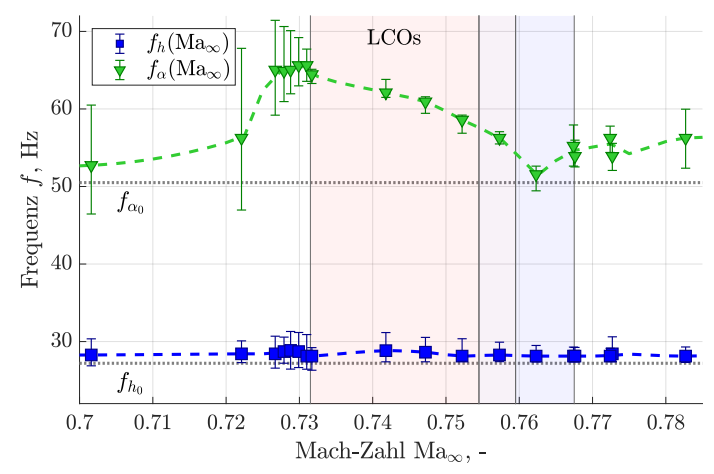

(b) Frequenzverlauf der 2-DoF Konfiguration

Abbildung 7.5.: Wasserfallspektren eines Beschleunigungssensors (a) und Verlauf der Schlag$f_{h}\left(\mathrm{Ma}_{\infty}\right)$ und Drehfrequenz $f_{\alpha}\left(\mathrm{Ma}_{\infty}\right)$ (b) für $p_{0}=55 \mathrm{kPa}$, verändert nach [59]. Die Balken markieren den 3dB-Abfall der ausgewerteten Frequenzpeaks.

Die in 2.2.2 und Abb. 2.13 beschriebene Annäherung der Schlag- und Drehfrequenz eines klassischen Biege-Torsions-Flatterns bleibt somit aus. Es liegt vielmehr ein stückweise divergenter Frequenzverlauf vor. Klassische Methoden zur Vorhersage von FlatterInstabilitäten, wie z. B. das ZIMMERMAN-WEISSENBURGER-Interpolationsverfahren [132], würden hier versagen (vgl. auch [25]). Jedoch bestätigen die Frequenzverläufe zumindest qualitativ die Beobachtungen der instationären Aerodynamik bzw. die der Energiebetrachtungen (vgl. 6.2). So setzen Instabilitäten und hier insbesondere LCOs bei, im Vergleich zu $f_{\alpha_{0}}$ deutlich erhöhten Frequenzen ein, die mit steigender Mach-Zahl absinken.

Des Weiteren wird auch der in Kap. 6 diskutierte Einfluss der Grenzschichttransition auf die Flatterinstabilität bestätigt. Abb. 7.6(a) zeigt die Stabilitätsgrenze ${ }^{2}$ (blaue Linie) des Laminarflügels für $\bar{\alpha} \approx 0^{\circ}$ bei einer fixierten Transition. Die Messungen wurden im Rahmen des ersten Flatterversuchs mit dem CAST 10-2 Profil durchgeführt [24]. Zum Vergleich ist auch die Stabilitätsgrenze bei einer freien Transition, basierend auf Abb. 7.3, als graue Linie in Abb. 7.6(a) eingezeichnet. Die Verschiebung des Transonic Dips infolge der Grenzschichttransition zu kleineren Mach-Zahlen und Ruhedrücken ist klar ersichtlich, wie es bereits experimentell in [24] und numerisch in [20,22,23] beschrieben wurde. Es zeigt sich auch, dass nur aufklingende, durch den Schlag dominierte Flatterfälle für eine turbulente Grenzschicht vorliegen. Diese gleichen weitestgehend den aufklingenden Flatterfällen bei freier Transition, wie es die Zeitreihen eines turbulenten Flatterfalls in Abb. 7.6(b) zeigen. Auch die mittleren Schlag- bzw. Flatterfrequenzen sowie Phasendifferenzen zwischen Schlag und Drehung sind mit $\bar{f}_{h} \approx 28,5 \mathrm{~Hz}$ sowie $\bar{\Delta}_{h, \alpha} \approx-174^{\circ}$ bei freier und $\bar{f}_{h} \approx 28,8 \mathrm{~Hz}$ sowie $\bar{\Delta}_{\varphi_{h, \alpha}} \approx-169^{\circ}$ bei fixierter Transition vergleichbar und ähneln den beobachteten Flatterfällen am superkritischen NLR 7301 Profil (vgl. [26-28]).

\footnotetext{
${ }^{2}$ Abweichend zu dem Stabilitätsdiagramm in [24, Abb. 14], wurden hier indifferente Messpunkte (stark fluktuierende Amplituden, jedoch kein Aufklingen oder LCO) als stabil gewertet.
} 


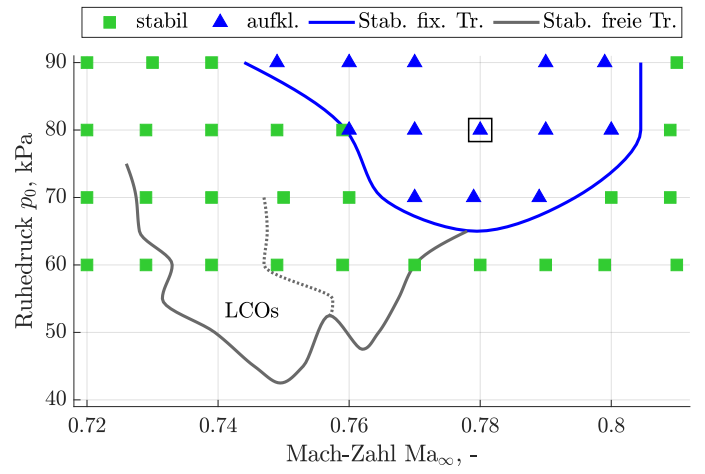

(a) Stabilitätsdiagramm, fixierte Transition
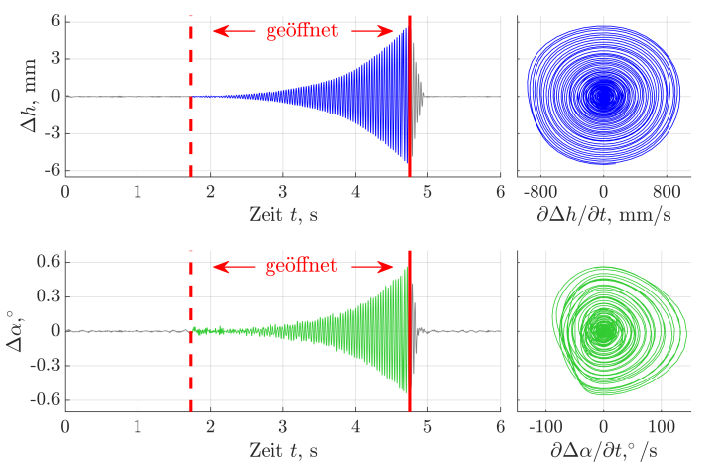

(b) Flatterfall bei $\mathrm{Ma}_{\infty}=0,78$ und $p_{0}=80 \mathrm{kPa}$

Abbildung 7.6.: Stabilitätsdiagramm der 2-DoF Konfiguration bei $\bar{\alpha} \approx 0^{\circ}$ und einer fixierten Transition in (a), verändert nach [24], sowie Zeitreihen eines exemplarischen Flatterfalls (b).

Es tritt somit auch bei einer turbulenten Grenzschicht eine Flatterinstabilität auf, jedoch bei höheren Mach-Zahlen und Ruhedrücken. So bestätigen sich die Ergebnisse der instationären Aerodynamik 6, die eine Stabilität für $p_{0}=54 \mathrm{kPa}$ bis mindestens $\mathrm{Ma}_{\infty}=0,75$ bei turbulenter Grenzschicht erwarten ließen. Es zeigt sich auch, dass die hier beobachteten LCOs nur bei einer freien Grenzschichttransition auftreten. Dies bestätigt die Hypothese, dass eine freie Grenzschichttransition eine notwendige Bedingung für Flatterinstabilitäten des Laminarflügels darstellt. Jedoch werden die Instabilitäten auf die beobachteten LCOs eingegrenzt. Dieser Flattermechanismus lässt sich demnach als „Transitionsflattern“ bezeichnen. So tritt nicht nur eine Verschiebung des Transonic Dips bei freier Grenzschichttransition auf, sondern ein weiterer Flattertyp kommt hinzu und bewirkt eine zusätzliche Ausdehnung des Dips.

\subsection{Aeroelastische Stabilität der 1-DoF Konfiguration}

Für die aeroelastische 1-DoF Konfiguration wurde in analoger Weise zu 7.2 die Stabilität untersucht, jedoch für einen kleineren Ruhedruckbereich. Die Ergebnisse sind in Abb. 7.7 dargestellt. So treten auch für diese Konfiguration Instabilitäten auf, die bei kleineren Mach-Zahlen zunächst begrenzte Amplituden aufweisen (rote Marker in Abb. 7.7). Mit steigender Mach-Zahl wachsen die Amplituden jedoch stark an (blaue Marker in Abb. 7.7) und es tritt ein teils „explosives“ Flattern auf.

Die Entwicklung des Flatterverhaltens ist für $p_{0}=54 \mathrm{kPa}$ in Abb. 7.8 anhand der Dreh(grün) und Schlagbewegungen (blau) des Modell-Mittelschnitts dargestellt. Obwohl die Schlag-Starrkörpermode mechanisch blockiert war, liegt im Modellmittelschnitt eine Schlagbewegung vor, ähnlich wie bei den zwangerregten Nickschwingungen (vgl. 6.2 und auch [62]). Dabei wies bereits die Eigenmode der Drehschwingung einen schwachen Biegeanteil auf (vgl. 7.1), der hier nunmehr deutlich sichtbar wird. 


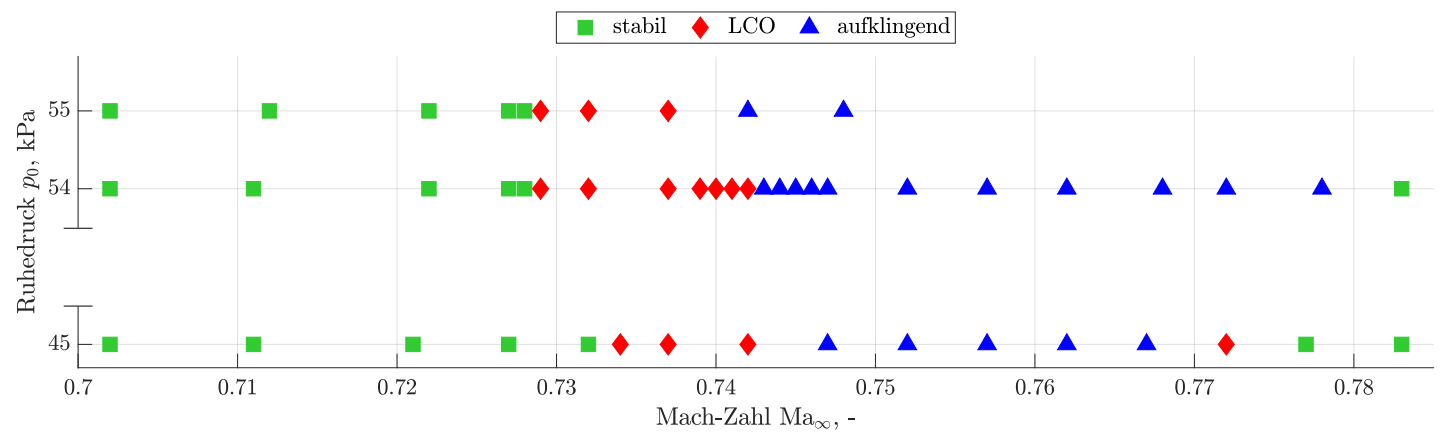

Abbildung 7.7.: Stabilitätsdiagramm der aeroelastischen 1-DoF Konfiguration bei einem mittleren Anstellwinkel $\bar{\alpha} \approx 0^{\circ}$ und freier Grenzschichttransition, verändert nach [59].

Wie Abb. 7.8 zeigt, tritt bei $\mathrm{Ma}_{\infty}=0,729$ ein LCO des Laminarflügels mit Amplituden von $\hat{\alpha} \approx 0,74^{\circ}$ und $\hat{h} \approx 0,99 \mathrm{~mm}$ auf (jeweils oberhalb der Zeitreihen in Abb. 7.8 notiert). Mit steigender Mach-Zahl wachsen die Amplituden des LCO an. Für $\mathrm{Ma}_{\infty}=0,743$ wird der Grenzzyklus instabil und die Amplituden klingen auf, so dass die Bewegung des Windkanalmodells mithilfe der Bremse gestoppt werden muss (Schließen: durchgezogene rote Linie, Öffnen: gestrichelte rote Linie). Die Anfachungsrate der Amplitude erreicht für $\mathrm{Ma}_{\infty} \approx 0,755$ ein Maximum, wo ein „explosives“ Flattern vorliegt. Eine weitere Erhöhung von $\mathrm{Ma}_{\infty}$ führt dann zu einem „moderaten“ Flattern bis hin zur erneuten Stabilität des aeroelastischen Systems für $\mathrm{Ma}_{\infty}=0,783$.

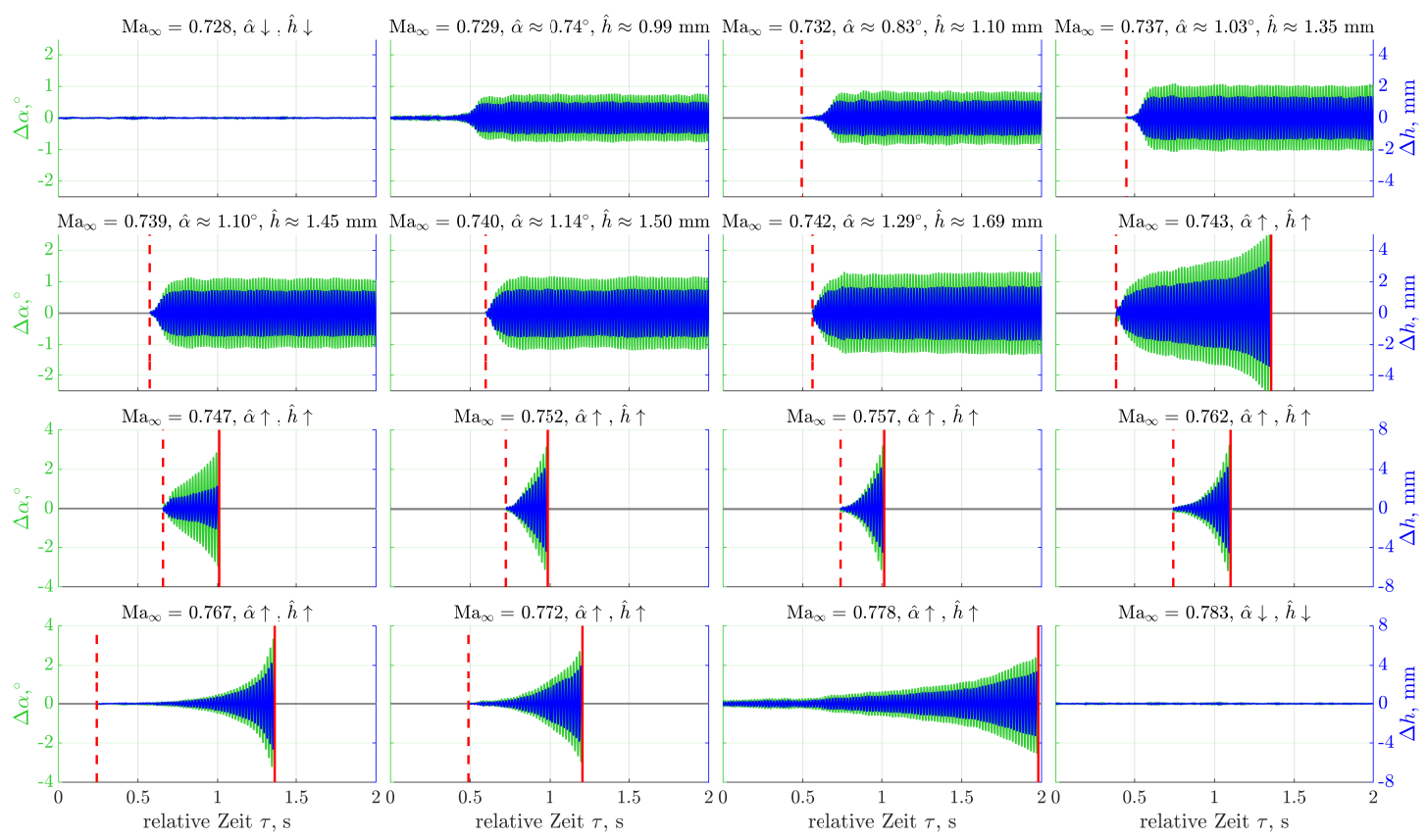

Abbildung 7.8.: Zeitreihen-Entwicklung von $\Delta \alpha$ und $\Delta h$ der aeroelastischen 1-DoF Konfiguration für $p_{0}=54 \mathrm{kPa}, \bar{\alpha} \approx 0^{\circ}$ und eine freie Grenzschichttransition, verändert nach [59].

Im Vergleich zur 2-DoF-Konfiguration (vgl. 7.2) zeigt sich, dass eine Reduktion des Schlag-Freiheitsgrades zu einem ausgedehnteren instabilen Bereich (größerer $\mathrm{Ma}_{\infty^{-}}$und 
$p_{0}$-Bereich) führt. Die auftretenden LCOs weisen größere Amplituden auf und der Drehanteil bei aufklingenden Flatterfällen nimmt deutlich zu. Es deutet sich somit eine stabilisierende Wirkung des Schlag-Starrkörper-Freiheitsgrades an. Mit Blick auf die LCOs bzw. das Transitionsflattern zeigen sich aber auch Analogien zwischen beiden aeroelastischen Konfigurationen, die eine Übertragbarkeit der Untersuchungsergebnisse untereinander rechtfertigen.

\subsubsection{Vergleichbarkeit zur 2-DoF Konfiguration}

In Abb. 7.9 sind die Bewegungen des Modellmittelschnitts sowie die PSDs von $\Delta \alpha$ und $\Delta h$ für je einen LCO beider aeroelastischer Konfigurationen bei gleichen Randbedingungen dargestellt. Die Amplituden der LCOs unterscheiden sich und auch die Flatterfrequenz ist für die 1-DoF Konfiguration um $5 \mathrm{~Hz}$ niedriger. Entscheidend ist aber, dass die Phasenbeziehungen zwischen Schlag und Drehung mit $\Delta \varphi_{h, \alpha} \approx-67^{\circ}$ (2-DoF) bzw. $\varphi_{h, \alpha} \approx-64^{\circ}$ (1-DoF) bei beiden Konfigurationen fast identisch sind. Die mit den LCOs korrespondierenden Schwingungsmoden sind demnach einander ähnlich. Dies wird auch durch einen Vergleich der Eigenvektoren beider Schwingungsmoden mithilfe des MAC-Kriteriums (engl.: Modal Assurance Criterion) [204] bestätigt, welches eine Übereinstimmung von über $86 \%$ liefert.
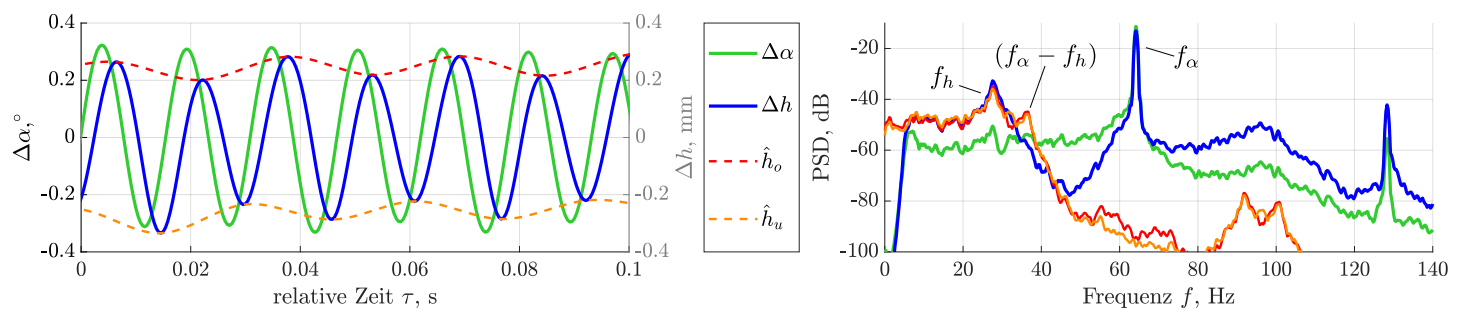

(a) LCO der 2-DoF-Konfiguration bei $\mathrm{Ma}_{\infty}=0,732$ und $p_{0}=55 \mathrm{kPa}, f_{\alpha} \approx 64,2 \mathrm{~Hz}$ und $\Delta \varphi_{h, \alpha} \approx-67^{\circ}$
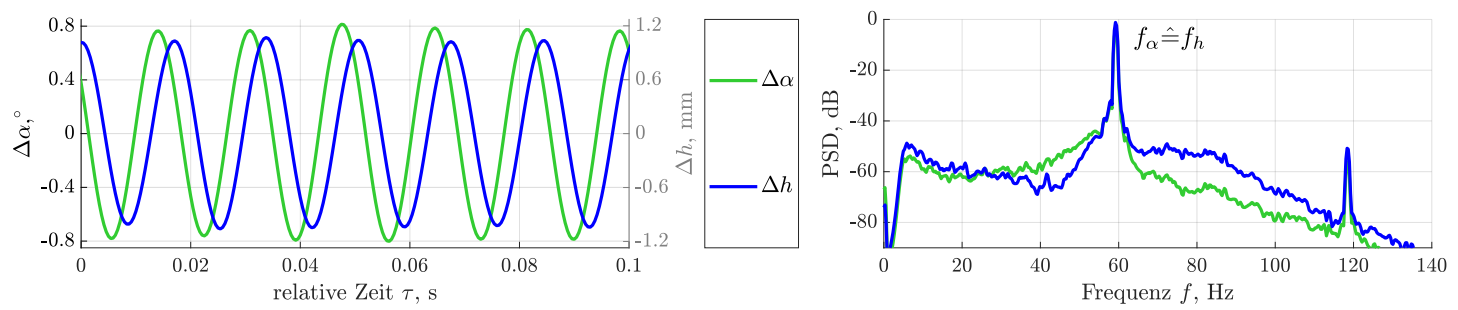

(b) LCO der 1-DoF-Konfiguration bei $\mathrm{Ma}_{\infty}=0,732$ und $p_{0}=55 \mathrm{kPa}, f_{\alpha} \approx 59,2 \mathrm{~Hz}$ und $\Delta \varphi_{h, \alpha} \approx-64^{\circ}$

Abbildung 7.9.: Vergleich zweier LCOs der aeroelastischen Konfigurationen. In (a) ist die Bewegung des Modellmittelschnitts, aufgeteilt in Schlag $\Delta h$ und Drehung $\Delta \alpha$, für das 2-DoF-System dargestellt, (b) zeigt die Bewegung für einen LCO des 1-DoF-Systems.

Ferner wird in Abb. 7.9(a) die Auswirkung des Schlag-Starrkörper-Freiheitsgrades bei der 2-DoF-Konfiguration deutlich. Dieser Freiheitsgrad zeichnet sich neben der dominierenden Dreh- bzw. Flatterfrequenz $f_{\alpha} \approx 64,2 \mathrm{~Hz}$ auch in der Schlagbewegung des Mittelschnitts 
ab, wie der Frequenzanteil bei $f_{h} \approx 27,6 \mathrm{~Hz}$ im PSD von $\Delta h$ (blaue Kurve in Abb. 7.9(a) rechts) zeigt. Der Starrkörper-Schlaganteil führt zu einer Schwebung der Schlagbewegung des Mittelschnitts. So tritt in den PSDs der Einhüllenden $\hat{h}_{o}$ und $\hat{h}_{u}$ von $\Delta h$ (rote und orange Kurven in Abb. 7.9), neben dem Schlaganteil $f_{h}$, auch ein Frequenzanteil $f_{\alpha}-f_{h}=36,6 \mathrm{~Hz}$ auf. Dies entspricht der Modulationsfrequenz zweier überlagerter Schwingungen (siehe z.B. [205, S.6 ff.]). So ist anzunehmen, dass diese Schwebung zur Stabilisierung des aeroelastischen Systems beiträgt und sowohl eine Versteifung (höhere Frequenzen) als auch geringere LCO-Amplituden für die 2-DoF Konfiguration bewirkt. Eine genauere Untersuchung ist jedoch nicht Gegenstand der vorliegenden Arbeit. Es zeigt aber, dass auch für das 2-DoF System kein substantieller Beitrag der Schlag-Starrkörpermode zum Transitionsflattern vorliegt. Zusammengenommen mit der Reduktion des Schlag-Freiheitsgrades für die 1-DoF Konfiguration und den auch hier beobachteten, vergleichbaren LCOs, kann eine anfänglich vermutete Beteiligung von höheren Harmonischen des Schlag-Freiheitsgrades am Transitionsflattern (vgl. 1.1) somit eindeutig ausgeschlossen werden.

Des Weiteren bestätigt sich auch bei der 1-DoF-Konfiguration die Notwendigkeit einer freien Grenzschichttransition für eine aeroelastische Instabilität bzw. das Transitionsflattern. Auch für die 1-DoF Konfiguration wurde eine Fixierung der Grenzschichttransition auf der Ober- und Unterseite des CAST 10-2 Profils vorgenommen. Für $p_{0}=55 \mathrm{kPa}$ wurde die Stabilität bis $\mathrm{Ma}_{\infty}=0,8$ untersucht, wobei keinerlei aeroelastische Instabilitäten auftraten. Erst für $\mathrm{Ma}_{\infty}>0,8$ deuteten einsetzende Vibrationen auf eine beginnende Strömungsablösung und Buffet (vgl. 2.2.3) hin. Die hier auftretenden Frequenzen lagen mit $80 \mathrm{~Hz}$ bis $85 \mathrm{~Hz}$ in der Nähe der strukturellen Biege-Eigenfrequenz $f_{s_{1}}$ (vgl. 7.1), so dass von einer weiteren Erhöhung der Mach-Zahl oder des Ruhedrucks abgesehen wurde.

Abschließend zeigt sich auch im Frequenzverlauf $f_{\alpha}\left(\mathrm{Ma}_{\infty}\right)$ der 1-DoF-Konfiguration, dargestellt in Abb. 7.10, eine weitere qualitative Übereinstimmung zum Transitionsflattern der 2-DoF-Konfiguration. Im Vorfeld der LCOs tritt ebenfalls ein deutlicher aber etwas moderaterer Anstieg von $f_{\alpha}$ auf, da bereits $f_{\alpha}\left(\mathrm{Ma}_{\infty}=0,7\right)$ deutlich über $f_{\alpha_{0}}$ liegt. Für $\mathrm{Ma}_{\infty}=0,728$ erreicht $f_{\alpha}$ maximal $61 \mathrm{~Hz}$, wiederum kurz bevor LCOs auftreten. Dies entspricht einem Anstieg von fast $28 \%$ gegenüber $f_{\alpha_{0}} . f_{\alpha, \max }$ liegt ca. $5 \mathrm{~Hz}$ unterhalb der maximalen Frequenz der 2-DoF Konfiguration. Dies zeigte sich bereits in Abb. 7.9 und ist vermutlich auf den Einfluss der Schlag-Starrkörper-Mode zurückzuführen. Im Bereich der LCOs sinkt die Frequenz dann wieder ab, bis die Grenzzyklen instabil werden.

Bei den aufklingenden Flatterfällen treten dann Unterschiede auf. Bei der 2-DoF Konfiguration werden die Flattermoden und -frequenzen der aufklingenden Flatterfälle durch die Starrkörper-Schlagbewegung dominiert. Ferner ändert sich die Phasenbeziehung zwischen der Schlag- und Drehbewegung des Mittelschnitts. Sofern LCOs vorliegen, bewegt sich 


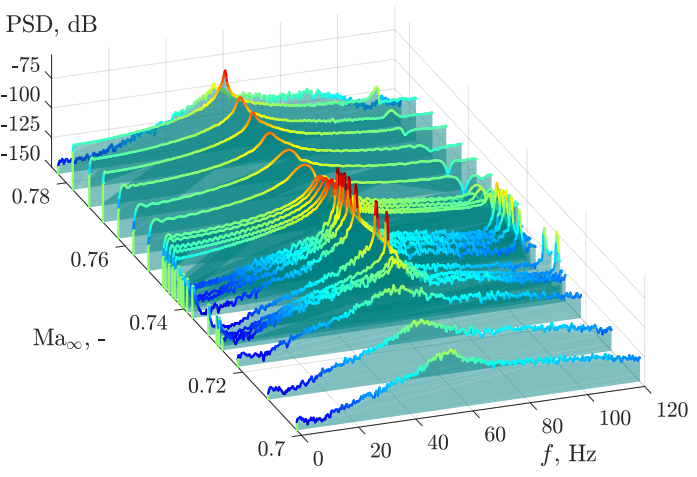

(a) Wasserfallspektren der 1-DoF Konfiguration

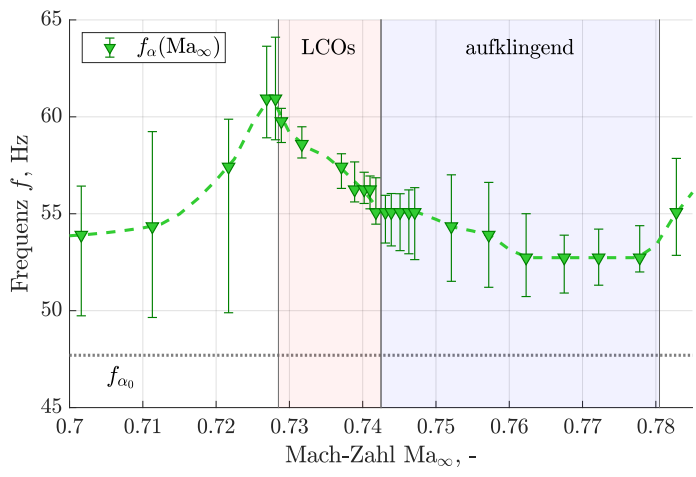

(b) Frequenzverlauf der 1-DoF Konfiguration

Abbildung 7.10.: Wasserfallspektren eines ausgewählten Beschleunigungssensors (a) und Verlauf der Drehfrequenz $f_{\alpha}\left(\mathrm{Ma}_{\infty}\right)$ (b) für $p_{0}=54 \mathrm{kPa}$, verändert nach [59]. Die Balken markieren den 3dB-Abfall der ausgewerteten Frequenzpeaks.

$\Delta \varphi_{h, \alpha}$ für beide Konfigurationen im Bereich von $-60^{\circ} \lesssim \Delta \varphi_{h, \alpha} \lesssim-80^{\circ}$, welche tendenziell größer (also negativer) wird, wenn die Mach-Zahl ansteigt und/oder der Ruhedruck abnimmt. Dieser Trend setzt sich für die 1-DoF Konfiguration auch bei aufklingenden Flatterfällen fort. Für die 2-DoF Konfiguration hingegen kommt es mit dem Einsetzen von aufklingenden Flatterfällen und der Änderung der Flatterfrequenz zu einer erheblichen Änderung der Phasendifferenz hin zu $-165^{\circ} \lesssim \Delta \varphi_{h, \alpha} \lesssim-175^{\circ}$, ungeachtet des Zustands der Grenzschicht (vgl. 7.2 und Abb. 7.6). Eine Vergleichbarkeit beider Konfigurationen scheint hier nicht mehr gegeben zu sein. Eine genauere Untersuchung ist jedoch nicht mehr Gegenstand der vorliegenden Arbeit.

Im Hinblick auf das Transitionsflattern (LCOs) weisen somit beide aeroelastische Konfigurationen ähnliche Charakteristiken und Schwingungsformen auf. Ein Vergleich dieses spezifischen Flatterverhaltens untereinander ist somit gerechtfertigt. Weitere Untersuchungen zum Transitionsflattern konzentrieren sich im Folgenden auf die 1-DoF Konfiguration, zumal eine größere Analogie zum Aufbau der zwangserregten Nickschwingungen (vgl. 3.2.1 und 6) besteht.

\subsubsection{Einfluss der Mach-Zahl}

Zur genaueren Identifikation des aeroelastischen Systemverhaltens während des Transitionsflatterns wurde eine experimentelle Bifurkationsanalyse durchgeführt. Für die 1-DoF Konfiguration wurde bei einem Ruhedruck von $p_{0}=55 \mathrm{kPa}$ die Mach-Zahl systematisch variiert. Das genaue experimentelle Vorgehen dazu ist in [63] beschrieben.

In Abb. 7.11 sind die LCO-Amplituden der Drehung $\hat{\alpha}$ und Schlagbewegung $\hat{h}$ des Modell-Mittelschnitts dargestellt. Beginnend mit $\mathrm{Ma}_{\infty}=0,729$ tritt bei einer weiteren Erhöhung der Mach-Zahl eine selbsterregte Schwingung auf (vgl. auch Abb. 7.8), was 


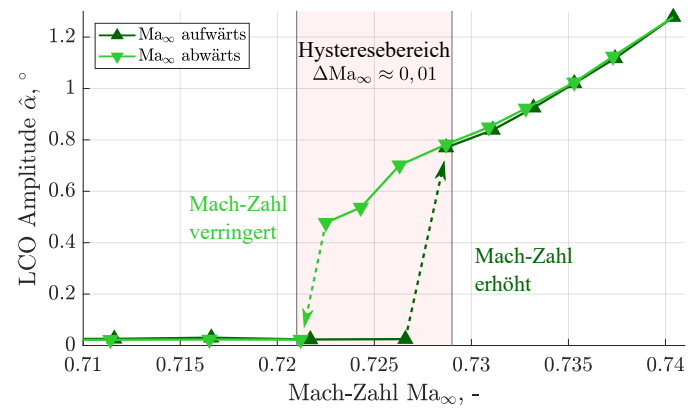

(a) LCO Amplituden der Dreh-Bewegung

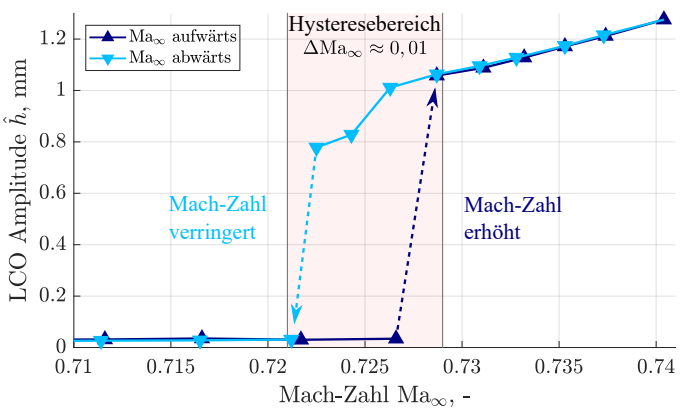

(b) LCO Amplituden der Schlag-Bewegung

Abbildung 7.11.: Bifurkationsdiagramm der Drehung (a) und des Schlags (b) bei einer Variation der Mach-Zahl für $\bar{\alpha} \approx 0^{\circ}$ und $p_{0}=55 \mathrm{kPa}$, verändert nach [63].

auf einen Bifurkationspunkt im Bereich $0,727 \lesssim \mathrm{Ma}_{\infty} \lesssim 0,729$ hinweist. Mit Zunahme der Mach-Zahl wachsen die LCO-Amplituden annähernd linear an und nehmen bei einer Reduzierung von $\mathrm{Ma}_{\infty}$ in selbem Maße wieder ab. Das aeroelastische System verbleibt jedoch auch für $\mathrm{Ma}_{\infty}<0,729$ in einem stabilen Grenzzyklus. Im Bereich 0,721 $\mathrm{Ma}_{\infty} \lesssim 0,723$ tritt dann eine Sattel-Knoten-Bifurkation auf und das Flattern klingt ab. Im Bereich 0,721 $\lesssim \mathrm{Ma}_{\infty} \lesssim 0,729$ tritt somit eine Hysterese auf (rot hinterlegt in Abb. 7.11) und das Systemverhalten beim Transitionsflattern wird durch eine subkritische Hopf-Bifurkation beschrieben (vgl. Abb. 2.14(b)). Für eine weiterführende Diskussion der Hysterese sowie möglicher Konsequenzen für die aeroelastische Stabilitätsgrenze sei an $[60,63]$ verwiesen.

\subsubsection{Einfluss des Anstellwinkels}

Eine vergleichbare Bifurkationsanalyse wurde bei einer Variation des mittleren Anstellwinkels $\bar{\alpha}$ für $\mathrm{Ma}_{\infty}=0,73$ und $p_{0}=55 \mathrm{kPa}$ durchgeführt. Experimentell wurde dies mithilfe des 2D-Supports realisiert (vgl. 3.2.2). Die Adaption der Windkanalwände wurde einmalig bei einem mittleren Anstellwinkel von $\bar{\alpha}=0^{\circ}$ ausgeführt. Die LCO Amplituden der Drehbewegung sind in Abb. 7.12(a) dargestellt, die Schlag-Amplituden finden sich in Abb. F.6(a) in F.4. LCOs treten bei den Randbedingungen in einem Bereich von $-0,2^{\circ} \lesssim \bar{\alpha} \lesssim 0,4^{\circ}$ auf. Auch hier zeigt sich, dass die Systemdynamik durch subkritische Bifurkationen beschrieben wird, mit $\bar{\alpha}$ als Stabilitätsparameter. Es liegen zwei Hysteresebereiche bei $-0,4^{\circ} \lesssim \bar{\alpha} \lesssim 0,0^{\circ}$ sowie $0,25^{\circ} \lesssim \bar{\alpha} \lesssim 0,6^{\circ}$ vor (rot hinterlegt in Abb. 7.12(a)). Der Übergang zwischen den beiden Zuständen (gestrichelte Pfeile in Abb. 7.12(a)) tritt dabei in Kombination mit einer Flatter-Divergenz-Interaktion (vgl. 2.2 sowie [5,114-116]) auf. Bei einer inkrementellen Veränderung des mittleren Anstellwinkels von $\Delta \bar{\alpha} \approx 0,1^{\circ}$ ändert sich $\bar{\alpha}$ bei den Übergängen um ca. $0,2^{\circ}$. Der zusätzliche Anteil von $\Delta \bar{\alpha} \approx 0,1^{\circ}$ resultiert aus der Flatter-Divergenz-Interaktion, wie es in Abb. 7.12(b) für das Aufklingen in einen LCO gezeigt ist. 


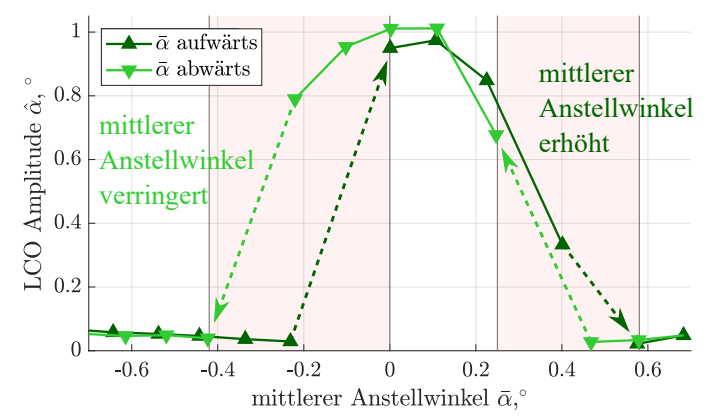

(a) LCO Amplituden der Dreh-Bewegung

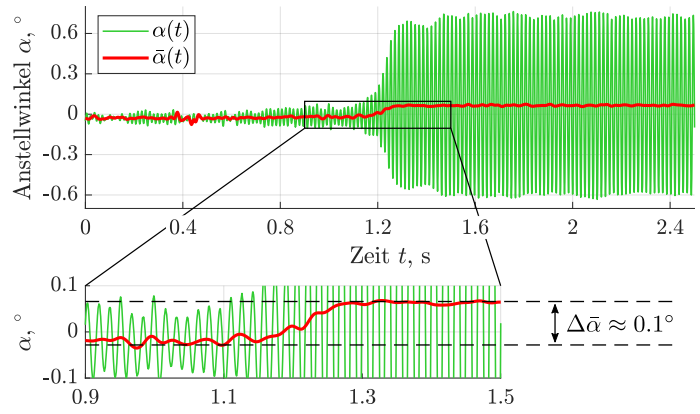

(b) Flatter-Divergenz-Interaktion

Abbildung 7.12.: Bifurkationsdiagramm der Drehung (a) bei einer Variation des mittleren Anstellwinkels $\bar{\alpha}$ sowie beispielhafte Flatter-Divergenz-Interaktion beim Aufklingen in einen LCO bei $\mathrm{Ma}_{\infty}=0,729$ und $p_{0}=54 \mathrm{kPa}$, verändert nach $[60,63]$.

Das zugehörige Frequenzverhalten des aeroelastischen Systems ist anhand der Wasserfallspektren in Abb. 7.13(a) verdeutlicht. Die identifizierten Frequenzen sind in Abb. F.6(b) im Anhang dargestellt. In Verbindung mit dem Transitionsflattern kommt es für $-0,2^{\circ} \lesssim \bar{\alpha} \lesssim 0,4^{\circ}$ zu einem sprunghaften Anstieg der Frequenz (Pfeile in Abb. 7.13(a)), was die lokale Abgrenzung des „Transitionsflatterns“ zeigt. Ein Vergleich des Anstellwinkelbereichs mit den Ergebnissen der stationären Aerodynamik (vgl. 5) bestätigt abschließend auch die Verbindung zum Übergangsbereich der Laminardelle. Für $\mathrm{Ma}_{\infty}=0,73$ ist in Abb. 7.13(b) die Polare nochmals dargestellt. Der Bereich, in dem LCOs auftreten, ist rot eingefärbt. Der Zusammenhang zwischen Übergangsbereich der Laminardelle und dem Transitionsflattern ist für $\mathrm{Ma}_{\infty}=0,73$ dabei offensichtlich.

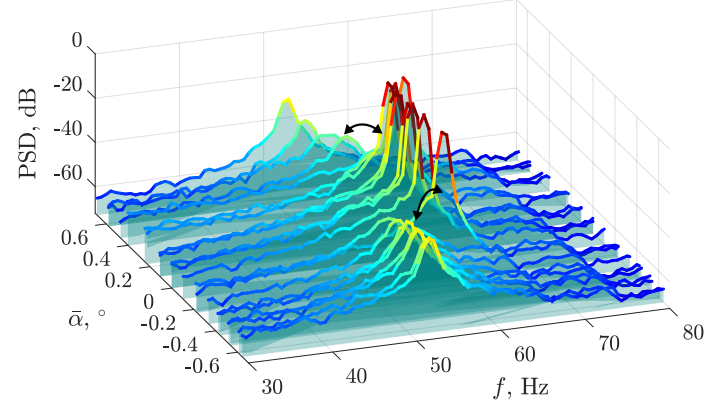

(a) Spektren der $\bar{\alpha}$-Variation

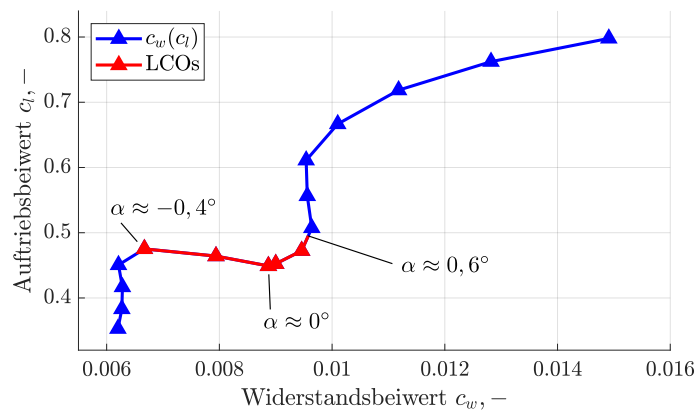

(b) Polare für $\mathrm{Ma}_{\infty}=0,73$

Abbildung 7.13.: Spektren bei einer Variation des mittleren Anstellwinkels $\bar{\alpha}$ in (a) sowie Polare mit rot markierter LCO-Bereich in (b), verändert nach $[60,63]$.

Die direkte Messung des aeroelastischen Verhaltens bestätigt insgesamt die ausgeprägte Sensitivität des CAST 10-2 Laminarprofils bzgl. des Anstellwinkels, wie es bereits für die stationäre und instationäre Aerodynamik (vgl. 5 und 6) beobachtet wurde. Hieraus leitet sich auch eine starke Abhängigkeit des Transonic Dips vom Anstellwinkel ab. Ferner sei die gute Übereinstimmung der beobachteten LCOs mit den Ergebnissen des 
Energieeintrags für $\mathrm{Ma}_{\infty}=0,73$ (vgl. 6.2 und Abb. 6.6) angemerkt. Die zwangserregten Nickschwingungen deuten auf Instabilitäten im Bereich von $-0,2^{\circ} \lesssim \bar{\alpha} \lesssim 0,15^{\circ}$ bei Frequenzen von $f_{\alpha} \gtrsim 52 \mathrm{~Hz}$ hin, was sich hier bestätigt. Der weitere LCO bei $\alpha \approx 0,4^{\circ}$ (vgl. Abb. 7.12(a)) weist eine Flatterfrequenz von $64 \mathrm{~Hz}$ auf (vgl. Abb. F.6(b)) und wird somit nicht mehr von den in 6.2 untersuchten Schwingungsfrequenzen abgedeckt.

\subsection{Grenzzyklusschwingung der 1-DoF Konfiguration}

Die abschließende Diskussion der instationären Aerodynamik während einer aeroelastischen Instabiliät des Laminarprofils basiert auf einem Grenzzyklus, der im Eingangsbereich des Transonic Dips bei $\mathrm{Ma}_{\infty}=0,732, p_{0}=55 \mathrm{kPa}$ und $\bar{\alpha}=0,06^{\circ}$ auftrat. Der LCO ist vergleichbar mit der bereits in [14,59-62] diskutierten Grenzzyklusschwingung. Er zeichnet sich jedoch dadurch aus, dass die Modellbewegung zunächst durch die Flatterbremse unterdrückt wurde und dann, nach dem Öffnen der Bremse, auch das Aufklingen und der Übergang in den LCO aufgezeichnet wurde. In Abb. 7.14 sind die Zeitreihen der Bewegung des Mittelschnitts sowie der aerodynamischen Beiwerte dargestellt.

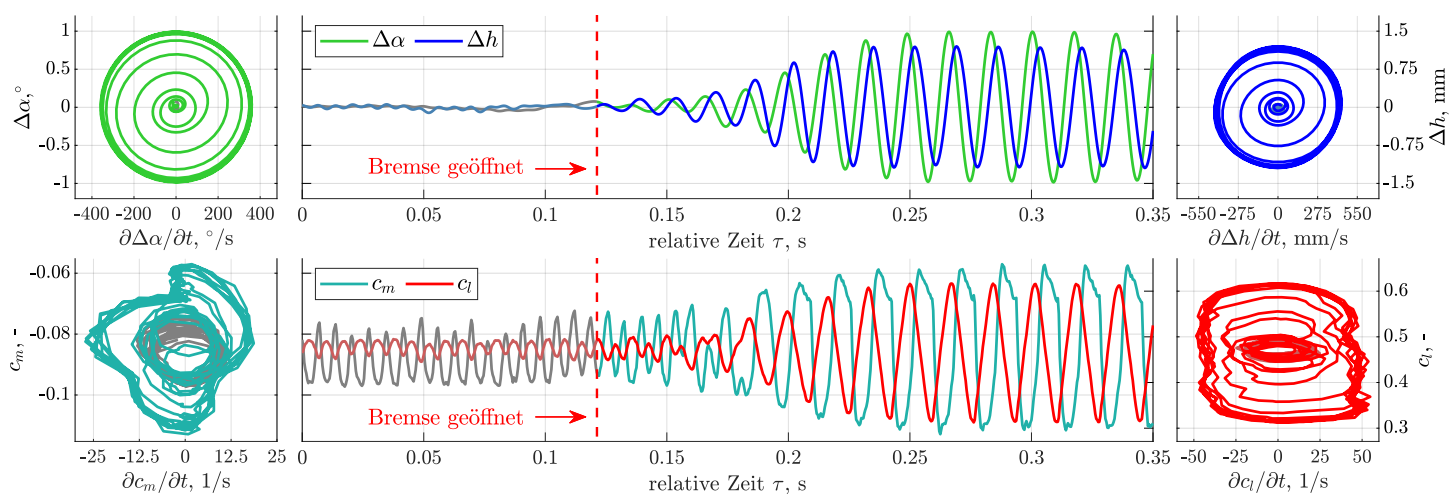

Abbildung 7.14.: LCO bei $\mathrm{Ma}_{\infty}=0,732, p_{0}=55 \mathrm{kPa}$ und $\bar{\alpha}=0,06^{\circ}$. Oben ist die Drehund Schlagbewegung $\Delta \alpha$ und $\Delta h$ des Modellmittelschnitts als Zeitreihe sowie Phasenraumdarstellung gezeigt, unten sind die aerodynamischen Beiwerte $c_{m}$ und $c_{l}$ aufgetragen.

Deutlich ersichtlich ist die nahezu sinusförmige Drehbewegung des Modellmittelschnitts im Grenzzyklus. Die Drehamplitude des LCO beträgt $\hat{\alpha} \approx 0,99^{\circ}$, die Schlagamplitude $\hat{h} \approx 1,19 \mathrm{~mm}$. Im gebremsten Zustand befindet sich das Modell nahezu vollständig in Ruhe $\left(\hat{\alpha}<0,01^{\circ}\right.$ und $\left.\hat{h}<0,05 \mathrm{~mm}\right)$. Die aerodynamischen Kräfte zeigen hingegen ein nichtlineares Verhalten, was insbesondere in der Phasenraumdarstellung in Abb. 7.14 deutlich wird und auch bei einem vergleichbaren LCO beobachtet wurde [59]. Das Nickmoment schwingt im LCO mit einer Amplitude von $\hat{c_{m}} \approx 0,027$, der Auftrieb mit $\hat{c}_{l} \approx 0,15$. Ferner liegen Schwingungen der aerodynamischen Kräfte auch dann vor, wenn sich das Windkanalmodell noch in Ruhe befindet. Diese Instationaritäten äußern sich insbesondere im Nickmoment und sind vergleichbar mit den instationären Effekten, 
welche bereits in 5.4 beschrieben wurden. Diese aerodynamischen LCOs sind nicht auf eine Modellbewegung zurückzuführen. Die Amplitude des Nickmoments beträgt im gebremsten Zustand $\hat{c_{m}} \approx 0,01$. Gemäß den stationären Verläufen $c_{m}(\alpha)$ (vgl. Abb. 5.1(c)) würde dies eine Anstellwinkelamplitude von mindestens $\hat{\alpha} \approx 0,25^{\circ}$ erfordern. Dies bestätigt, dass es sich um selbsterregte Schwingungen der Aerodynamik handelt (vgl. 5.4).

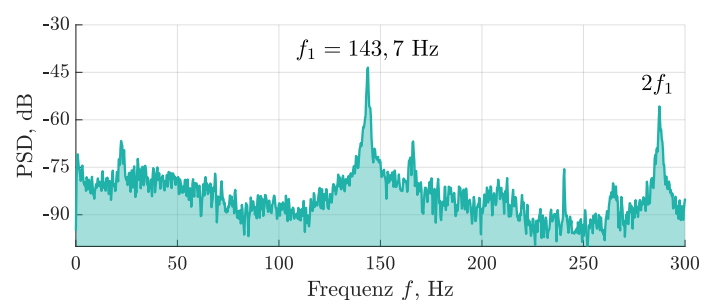

(a) PSD von $c_{m}$, Bremse zu

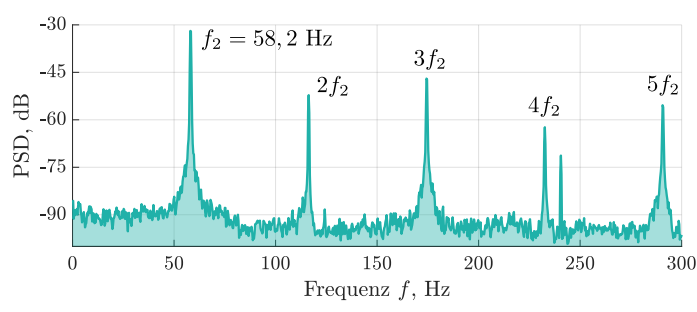

(b) PSD von $c_{m}$, Bremse auf

Abbildung 7.15.: Leistungsdichtespektren des Nickmoments bei $\mathrm{Ma}_{\infty}=0,73, p_{0}=55 \mathrm{kPa}$ und $\bar{\alpha}=0,06^{\circ}$. (a) zeigt das PSD von $c_{m}$ bei geschlossener Bremse, (b) in offenem Zustand.

Dabei weisen die aerodynamischen Schwingungen vergleichbare Frequenzen zu 5.4 auf, wie es die PSDs des Nickmoments in Abb. 7.15 zeigen. Im gebremsten Zustand liegt die dominierende Schwingungsfrequenz der aerodynamischen Kräfte bei $f_{1}=143,7 \mathrm{~Hz}$ $\left(\omega^{*} \approx 0,56\right)$, dargestellt in Abb. 7.15(a). Nach Öffnung der Bremse (rote gestrichelte Linie in Abb. 7.14) kommt es zu einem Sprung der Frequenzen. Im aeroelastischen Grenzzyklus liegt die dominierende Frequenz dann für die Kräfte sowie für die Modellbewegung bei $f_{2}=58,2 \mathrm{~Hz}\left(\omega^{*} \approx 0,23\right)$. Der nichtlineare Charakter der Aerodynamik verdeutlicht sich in den höheren Harmonischen $n f_{2}$ in Abb. 7.15(b).

\subsubsection{Verhalten von Stoß und Transition}

Eine Betrachtung der Grenzschichtdynamik während des in Abb. 7.14 dargestellten Zeitausschnitts verdeutlicht die Ursachen für die aerodynamischen Schwingungen. In Abb. 7.16 ist der Verlauf der Quasi-Wandschubspannung $\tau_{q}(t)$, die Position $x_{t r}(t)$ des laminar-turbulenten Grenzschichtumschlags (vgl. 4.3) sowie Bereiche $\tau_{q} \leq 0,02$ (Hinweis auf Ablösung) dargestellt. Ein direkter Vergleich von $\tau_{q}(t)$ und $x_{t r}(t)$ mit den Spannungssignalen der CTAs ist Abb. F.7 in F.5 zu entnehmen.

Wie Abb. 7.16 zeigt, tritt bereits im gebremsten Zustand (Modell in Ruhe) eine ausgeprägte periodische Bewegung der Transitionsposition über ca. $40 \%$ der Flügeltiefe im Bereich $0.4 \lesssim x / c \lesssim 0,8$ auf. Diese weist die gleiche Schwingungsfrequenz auf wie die aerodynamischen Kräfte. Sobald die Bremse geöffnet wird, deutet sich auch in der Transitionsbewegung ein Übergang an. Diese ist kurzzeitig aperiodisch und geht im aeroelastischen Grenzyklus wieder in eine periodische Bewegung über. Im ausgebildeten LCO schwingt die Transitionsposition dann mit der Flatterfrequenz $f_{2}=58,2 \mathrm{~Hz}$ in 


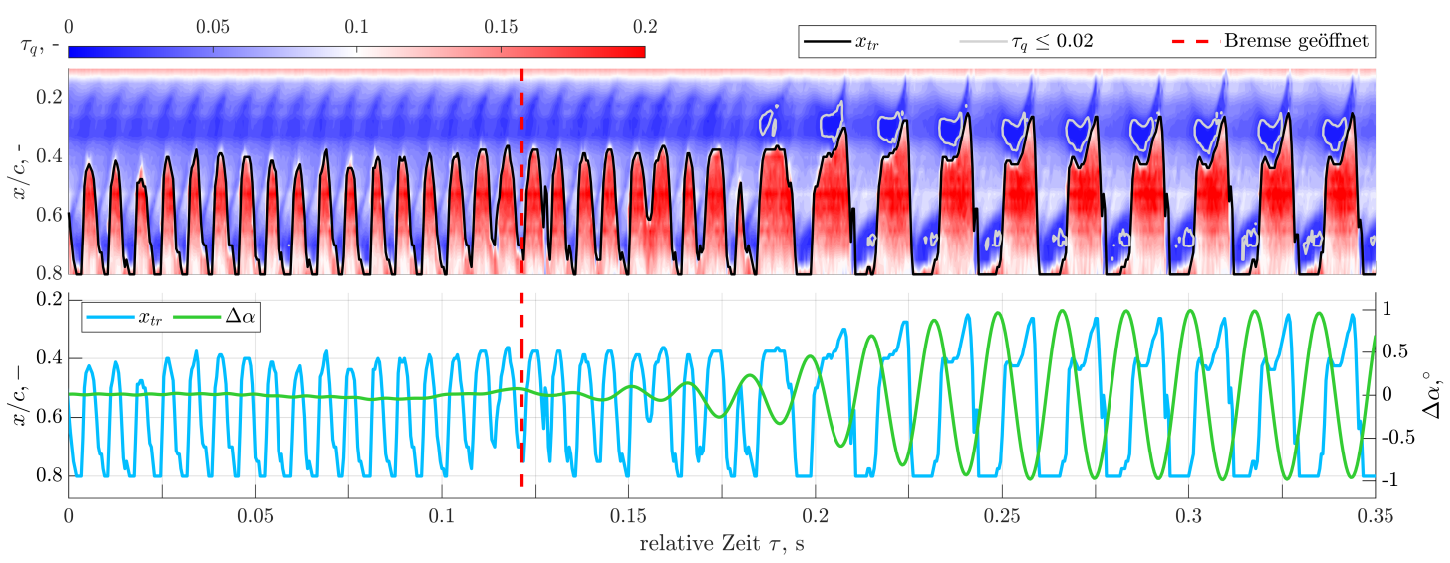

Abbildung 7.16.: Verlauf der Quasi-Wandschubspannung $\tau_{q}(t)$ (oben) und der Transitionsposition $x_{t r}(t)$ (unten) während des LCO bei $\mathrm{Ma}_{\infty}=0,732, p_{0}=55 \mathrm{kPa}$ und $\bar{\alpha}=0,06^{\circ}$. Die Profiltiefe $x / c$ ist in Ordinatenrichtung invers aufgetragen, nach oben entspricht in Richtung Profil-Vorderkante, nach unten in Richtung Profil-Hinterkante.

einem Bereich von $0.25 \lesssim x / c \lesssim 0,8$. Die Muster, welche sich zwischen laminarer und turbulenter Grenzschicht ausbilden, sind dabei vergleichbar mit den Ergebnissen der durchgeführten Frequenzvariationen (vgl. Abb. 6.10 und Abb. 6.11) und zeugen vom nichtlinearen Charakter der Transitionsbewegung.

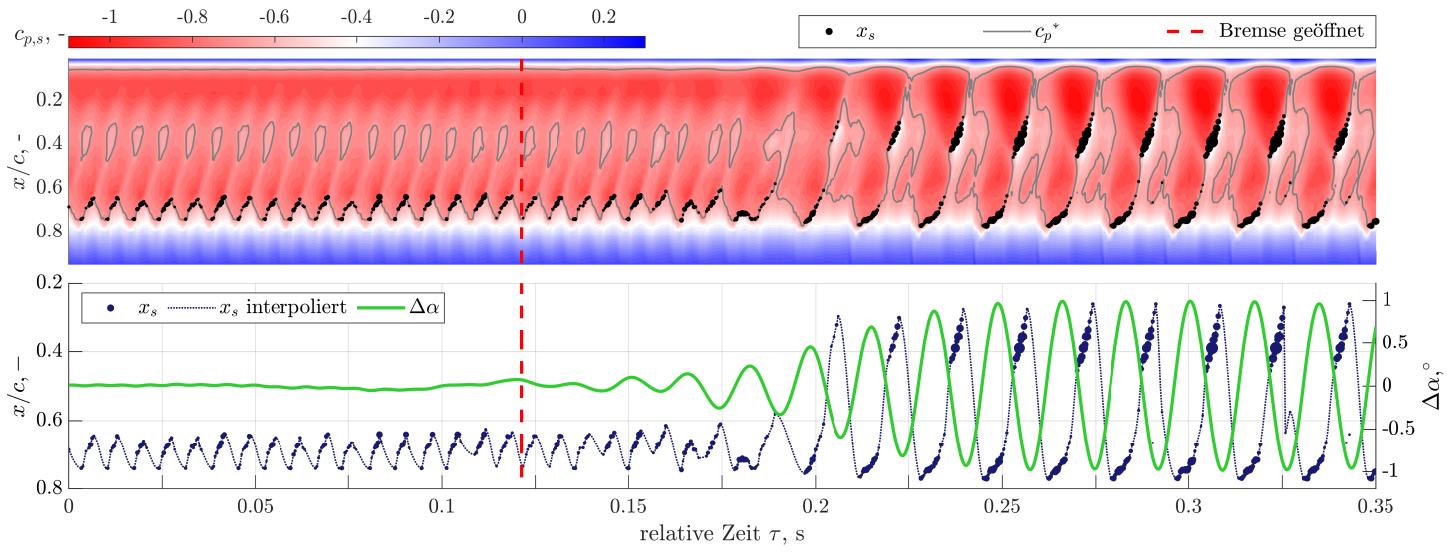

Abbildung 7.17.: Verlauf der Druckverteilung $c_{p, s}(t)$ (oben) sowie Positionen der Verdichtungsstöße $x_{s}(t)$ und interpolierte Stoßbewegung (unten) während des LCOs bei $\mathrm{Ma}_{\infty}=0,732, p_{0}=55 \mathrm{kPa}$ und $\bar{\alpha}=0,06^{\circ}$. Die Größe der Stoßmarker skaliert mit der Stoßstärke. Die Profiltiefe $x / c$ ist in Ordinatenrichtung invers aufgetragen, nach oben entspricht in Richtung Profil-Vorderkante, nach unten in Richtung Profil-Hinterkante.

Der zugehörige zeitliche Verlauf der Druckverteilung der Profil-Saugseite ist in Abb. 7.17 dargestellt, zusammen mit den detektierten Verdichtungsstößen bzw. deren Positionen $x_{s}(t)$ (vgl. 6.3). Periodische Änderungen von $c_{p, s}(t)$ treten ebenfalls bereits am ruhenden Modell auf, die Dynamik ist jedoch im Vergleich zur Transitionsbewegung schwächer ausgeprägt. Dennoch kommt es zur Ausbildung von schwachen Verdichtungsstößen, welche im stationären Fall (vgl. 5.2 und 5.4) durch eine Mittelung nicht ersichtlich sind. Die Stöße führen eine periodische Bewegung im Bereich $0.64 \lesssim x / c \lesssim 0,74$ aus, welche 
zeitweise unterbrochen ist. Es liegt somit bereits im gebremsten Zustand eine Typ-B Stoßbewegung nach TiJdEman und SEeBass [9] vor (vgl. auch 6.3). Deutlich sichtbar ist einmal mehr ein Übergangsbereich, sobald die Flatterbremse geöffnet wird. Während die LCO-Amplitude ansteigt, verschmilzt das vordere Unterschallgebiet um $x / c \approx 0,4$ (Bereiche in Abb. 7.17 durch $c_{p}{ }^{*}$ abgegrenzt) mit dem hinteren Druckanstieg. Es bilden sich periodische Überschallgebiete auf der Flügeloberseite aus, welche im Wechsel mit Stößen um $x / c \approx 0,7$ und $x / c \approx 0,35$ herum abschließen. Insgesamt liegt im LCO wieder eine Typ-B Stoßbewegung vor, die im hinteren Flügeltiefenbereich in Teilen invers verläuft. Ähnliche Ergebnisse zeigen auch die Frequenzvariationen (vgl. 6.3 und 6.4).

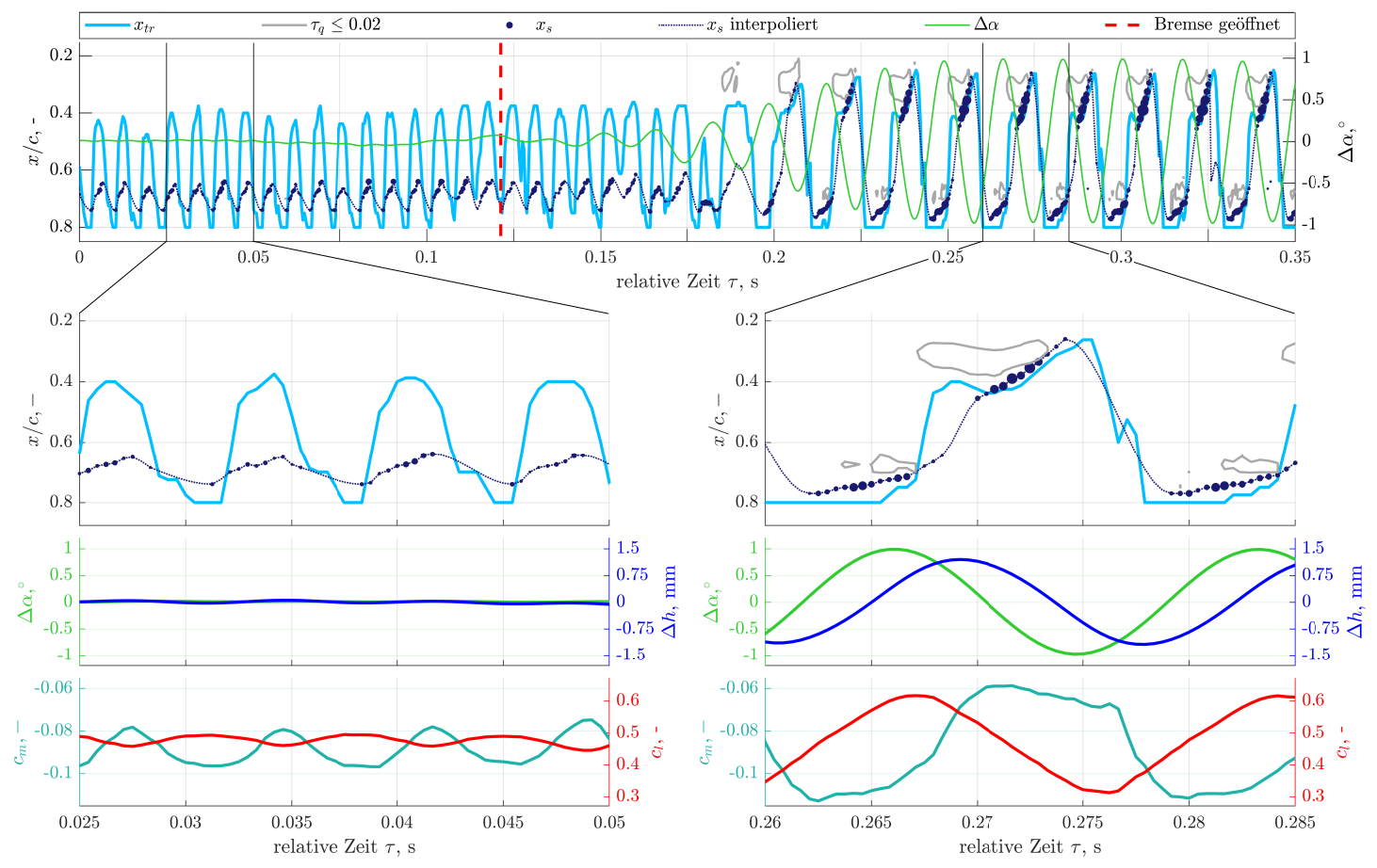

Abbildung 7.18.: Transitions- und Stoßbewegung zur Darstellung der Stoß-GrenzschichtInteraktion während des LCOs bei $\mathrm{Ma}_{\infty}=0,732, p_{0}=55 \mathrm{kPa}$ und $\bar{\alpha}=0,06^{\circ}$ sowie Zeitausschnitte samt Modellbewegung und aerodynamischen Beiwerten. Die Profiltiefe $x / c$ ist in den zwei oberen Grafiken in Ordinatenrichtung invers aufgetragen, nach oben entspricht in Richtung Profil-Vorderkante, nach unten in Richtung Profil-Hinterkante.

Stoß- und Transitionsbewegung sind zusammenfassend nochmals in Abb. 7.18 aufgetragen. Zusätzlich sind Gebiete markiert, welche auf eine Ablösung hindeuten $\left(\tau_{q} \leq 0,02\right)$. Darüber hinaus sind kurze Zeitausschnitte vor und nach der Öffnung der Bremse (rot gestrichelte Linie in Abb. 7.18) vergrößert dargestellt, wobei zusätzlich auch die Modellbewegung und die aerodynamischen Kräfte mit aufgeführt sind. Bzgl. der Interaktion von Stoß und Grenzschicht zeigt sich sofort, dass es im Rahmen der aerodynamischen LCOs (Bremse zu) keine Hinweise auf eine Ablösung der Grenzschicht gibt. Stoß- und Transitionsposition sind hier klar voneinander getrennt, der laminar-turbulente Grenzschichtumschlag findet weiter stromauf statt. Die Interaktion der auftretenden und 
ohnehin nur schwachen Verdichtungsstöße findet mit einer turbulenten Grenzschicht statt, was ebenfalls gegen eine Ablösung spricht (vgl. 2.1.3). Dies ändert sich im aeroelastischen LCO (Bremse auf). Hier zeigt sich weitestgehend eine Übereinstimmung von Stoß- und Transitionsposition sowohl im hinteren Flügeltiefenbereich um $x / c \approx 0,7$ (bzgl. der hier auftretenden Diskrepanzen zwischen $x_{t r}$ und $x_{s}$ sei an 6.4 verwiesen) als auch weiter stromauf bei $x / c \approx 0,35$. Die Interaktion der nunmehr in Teilen auch stärkeren Verdichtungsstöße findet hier mit einer laminaren Grenzschicht statt. Die damit einhergehende größere Ablöseneigung wird durch die niedrigen Quasi-Wandschubspannungen bestätigt, welche auf laminare Ablöseblasen hinweisen. Ferner ist wiederum zu beobachten, dass insbesondere im aeroelastischen LCO die Stoßbewegung von der Transition vorgegeben und gesteuert wird, wie es bereits die instationären Messungen in 6.4 zeigen und für einen vergleichbaren LCO in [62] diskutiert ist. Zur Quantifizierung dieser Sachverhalte sind in Tab. 7.3 die Phasenbeziehungen der in Abb. 7.18 dargestellten Größen in Bezug zur Dreh- $\Delta \alpha$ bzw. Transitionsbewegung $x_{t r}{ }^{3}$ eingetragen. So bestätigt die Phasenbeziehung zwischen Transitions- und Stoßbewegung $\Delta \varphi_{x_{s}, x_{t r}}$, dass sowohl während der aerodynamischen LCOs (Bremse zu, Phase bei $f_{1}=143,7 \mathrm{~Hz}$ ) wie auch im aeroelastischen LCO (Bremse auf, Phase bei $f_{2}=58,2 \mathrm{~Hz}$ ) die Bewegung der Transition die der Stoßposition vorauseilt. Im aeroelastischen LCO ist die Phasendifferenz jedoch gering. Sichtbare Unterschiede in den Bewegungen äußern sich auch vielmehr in den nichtlinearen Bewegungsanteilen. Diese werden bei der Phasenbestimmung nicht mit berücksichtigt, ebenso wenig wie das zeitweise Aussetzen des Verdichtungsstoßes gemäß der vorliegenden Typ-B-Stoßbewegung.

Tabelle 7.3.: Phasenbeziehungen zwischen Transitions- und Stoßbewegung $x_{t r}(t)$ und $x_{s}(t)$, aerodynamischen Kräften $c_{m}(t)$ und $c_{l}(t)$ sowie Modellbewegung im Mittelschnitt $\Delta \alpha(t)$ und $\Delta h(t)$ für den LCO bei $\mathrm{Ma}_{\infty}=0,73, p_{0}=55 \mathrm{kPa}$ und $\bar{\alpha}=0,06^{\circ}$.

\begin{tabular}{ccc}
\hline Phasendifferenz & Bremse zu, $f_{1}=143,7 \mathbf{H z}$ & Bremse auf, $f_{2}=58,2 \mathbf{H z}$ \\
\hline$\Delta \varphi_{\Delta h, \Delta \alpha}$ & $47^{\circ}$ & $-66^{\circ}$ \\
$\Delta \varphi_{x_{t r}, \Delta \alpha}$ & $91^{\circ}$ & $-131^{\circ}$ \\
$\Delta \varphi_{x_{s}, \Delta \alpha}$ & $64^{\circ}$ & $-138^{\circ}$ \\
$\Delta \varphi_{c_{m}, \Delta \alpha}$ & $45^{\circ}$ & $-135^{\circ}$ \\
$\Delta \varphi_{c_{l}, \Delta \alpha}$ & $-130^{\circ}$ & $-20^{\circ}$ \\
\hline$\Delta \varphi_{x_{s}, x_{t r}}$ & $-27^{\circ}$ & $-7^{\circ}$ \\
$\Delta \varphi_{c_{m}, x_{t r}}$ & $-46^{\circ}$ & $-4^{\circ}$ \\
$\Delta \varphi_{c_{l}, x_{t r}}$ & $139^{\circ}$ & $111^{\circ}$ \\
\hline
\end{tabular}

Im aeroelastischen LCO (Bremse auf, Modell elastisch) sind Transition $x_{t r}(t)$ und Nickmoment $c_{m}(t)$ nahezu in Phase. Eine Bewegung der Transition stromauf führt somit

\footnotetext{
${ }^{3}$ Für eine Berechnung und Deutung der Phasenbeziehungen wird bzgl. der Transitions- und Stoßposition eine Bewegung stromauf als Zunahme und eine Bewegung stromab als Abnahme interpretiert.
} 
zu einer Zunahme des Moments. Dies geht einher mit einem sich ausdehnenden und periodisch auftretenden Überschallgebiet im vorderen Flügeltiefenbereich, wie es für einen vergleichbaren LCO in $[14,62,155]$ diskutiert ist. Damit verbunden sind negative $c_{p}$-Werte, die zu einem lokalen Auftriebsanstieg vor der Drehachse führen und das zudrehende Moment $\left(c_{m}<0\right)$ periodisch reduzieren. Das Auftreten und die Ausdehnung des Überschallgebietes bedingt dabei auch die Ausbildung von Verdichtungsstößen.

Im aerodynamischen LCO (Bremse zu, Modell fixiert) liegt hingegen eine deutliche Voreilung der Transitionsposition zum Nickmoment vor. Dies trifft auch in Bezug zur Drehbewegung des Laminarprofils zu, wo die Phasendifferenz $\Delta \varphi_{x_{t r}, \Delta \alpha}$ über $90^{\circ}$ beträgt. Ferner liegt auch eine Voreilung des Nickmoments bzgl. $\Delta \alpha$ vor, was als Hinweis für eine aeroelastische Instabilität bereits in 6 diskutiert wurde. Im ausgebildeten aeroelastischen LCO ändert sich die Phasenbeziehung dann deutlich, $c_{m}$ eilt $\Delta \alpha$ hinterher. Folglich liegt auch eine Nacheilung der Transitions- und Stoßbewegung zur Modell-Drehbewegung vor.

Bzgl. des Auftriebs $c_{l}$ tritt vor und nach dem Öffnen der Bremse eine Voreilung gegenüber der Transitionsbewegung auf. In Bezug zur Drehbewegung eilt der Auftrieb durchgehend nach, jedoch ändert sich hier die Phasenbeziehung deutlich von $\Delta \varphi_{c_{l}, \Delta \alpha} \approx-130^{\circ} \mathrm{im}$ gebremsten Zustand hin zu $\Delta \varphi_{c_{l}, \Delta \alpha} \approx-20^{\circ}$ im offenen Zustand. Ähnlich verhält es sich auch bei der Phasenbeziehung zwischen der Dreh- und Schlagbewegung, welche sich ebenfalls deutlich von einer Voreilung von $\Delta h$ mit $\Delta \varphi_{\Delta h, \Delta \alpha} \approx 47^{\circ}$ zu einer Nacheilung mit $\Delta \varphi_{\Delta h, \Delta \alpha} \approx-66^{\circ}$ verschiebt.

\subsubsection{Energie- und Leistungsbetrachtung}

Die Auswirkungen der Phasenbeziehungen und deren Änderungen vom aerodynamischen hin zum aeroelastischen LCO werden bei einer Energie- bzw. Leistungsbetrachtung ersichtlich. Basierend auf den in 4.2.2 beschriebenen Grundlagen ist in Abb. 7.19 der Energietransfer zwischen Struktur und Strömung für den zuvor betrachteten LCO dargestellt. Der Energietransfer fluktuiert dabei annähernd um die Nulllage. Für den mittleren

Energieeintrag pro Schwingungsperiode gilt jedoch $\frac{1}{T} \int_{t}^{t+T} W(t) \mathrm{d} t>0$ (rote Kurve in Abb. 7.19). Dabei zeigt sich einerseits, dass bereits im aerodynamischen LCO die Phasenlagen zwischen aerodynamischen Kräften und Struktur einen positiven Energieeintrag liefern (Abb. 7.19 links unten). Dies ist angesichts der aerodynamischen Anregung der Strukturbewegung zu erwarten, bestätigt aber auch, dass aus der Instabilität der Aerodynamik eine aeroelastische Instabilität resultieren kann. Sobald die Bremse geöffnet wird, steigt der Energieeintrag an, was zum Anwachsen der Bewegungsamplituden führt. Der Energieeintrag erreicht ein Maximum in der Nähe des Wendepunktes beim Einschwingen in den LCO. Der sich anschließende Rückgang des Energieeintrages führt 
dann zur Amplitudenbegrenzung und somit zum aeroelastischen Grenzzyklus. Der konstante aerodynamische Energieeintrag (Abb. 7.19 rechts unten) gleicht die dissipativen Energieverluste infolge der Systemdämpfung aus.

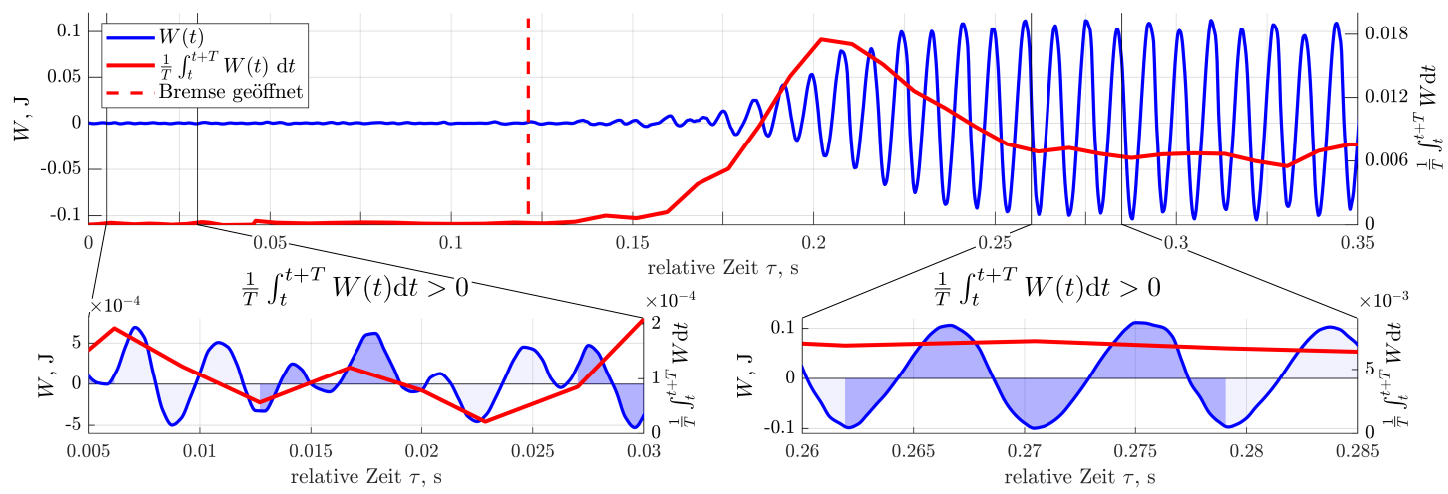

Abbildung 7.19.: Aerodynamischer Energietransfer zwischen Struktur und Strömung $W(t)$ sowie mittlerer Energieeintrag pro Periode $\frac{1}{T} \int_{t}^{t+T} W(t) \mathrm{d} t$ während des LCOs bei $\mathrm{Ma}_{\infty}=$ $0,732, p_{0}=55 \mathrm{kPa}$ und $\bar{\alpha}=0,06^{\circ}$. Die Zeitausschnitte stellen Bereiche vor und nach dem Öffnen der Flatterbremse (rot gestrichelte Linie) vergrößert dar.

In Abb. 7.20 sind die Beiträge des Nickmoments und des Auftriebs zum aerodynamischen Energieeintrag anhand einer qualitativen Leistungsbetrachtung von $P_{m}$ und $P_{l}$ genauer aufgeschlüsselt. Wie in 4.2.2 beschrieben, sind die Leistungsanteile gemäß Gl. (4.2.10) in entdimensionalisierter Form dargestellt. Die Auftragung erfolgt in Phasenraumdiagrammen, wobei die Leistungen wie auch die zeitliche Änderung der Drehbewegung $\partial \Delta \alpha / \partial t$ mit der Amplitude der Drehbewegung $\partial \widehat{\Delta \alpha / \partial} t$ normalisiert sind, analog zu [27]. Dies ermöglicht eine einfache Unterscheidung zwischen Aufdrehen $(\partial \Delta \alpha / \partial t / \partial \widehat{\Delta \alpha / \partial t}>0)$ und Zudrehen $(\partial \Delta \alpha / \partial t / \partial \widehat{\Delta \alpha / \partial} t<0)$ des Flügels. Das in Abb. 7.20 betrachtete Zeitfenster ist in drei Zeitausschnitte $\Delta t_{i}$ unterteilt. Der erste Zeitausschnitt $\Delta t_{1}$ (Abb. 7.20(a) und (b)) betrifft den aufgezeichneten Zeitraum des aerodynamischen LCOs bei geschlossener Flatterbremse. Der Zeitausschnitt $\Delta t_{2}$ (Abb. 7.20(c) und (d)) umfasst den aufklingenden LCO, welcher bereits in Abb. 7.14 bis 7.19 betrachtet wurde. Abschließend deckt $\Delta t_{3}$ (Abb. 7.20(e) und (f)) den Zeitraum des ausgebildeten aeroelastischen Grenzzyklus ab.

Im aerodynamischen LCO liegt eine breite Streuung der aerodynamischen Leistungen $P_{m, \Delta t_{1}}$ und $P_{l, \Delta t_{1}}$ vor (graue Kurven in Abb. 7.20(a) und (b)), was sich in der nur zeitweisen periodischen Bewegung des Windkanalmodells begründet. Eine auf Basis des gefilterten Bewegungssignals durchgeführte Periodenmittelung der Leistungen $\bar{P}_{m, \Delta t_{1}}$ und $\bar{P}_{l, \Delta t_{1}}$ bestätigt jedoch, dass ein positiver Energieeintrag durch das Nickmoment erfolgt, wie es die zuvor diskutierte Phasenvoreilung vermuten ließ. Der Auftrieb weist hingegen einen geringen negativen Leistungsbeitrag pro Periode auf. Für die aerodynamische Gesamtleistung $P_{\text {aero }}=P_{m}+P_{l}$ gilt insgesamt $\bar{T}_{\Delta t_{1}}^{-1} \int_{0}^{\bar{T}_{\Delta t_{1}}} \bar{P}_{\text {aero, } \Delta t_{1}} \mathrm{~d} t>0$, entsprechend des positiven Energieeintrags in Abb. 7.19. $\bar{P}_{\text {aero, } \Delta t_{1}}$ ist in Abb. F.8 in F.6 dargestellt. 


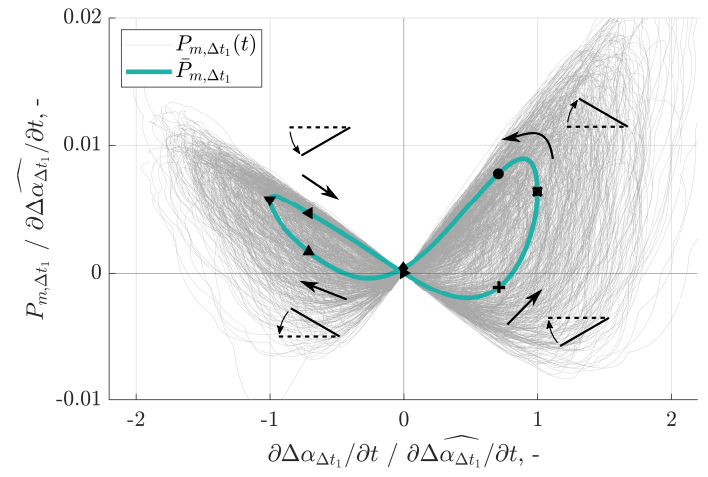

(a) Leistung des Moments im Zeitausschnitt $\Delta t_{1}$, es gilt $\bar{T}_{\Delta t_{1}}^{-1} \int_{0}^{\bar{T}_{\Delta t_{1}}} \bar{P}_{m, \Delta t_{1}} \mathrm{~d} t>0$

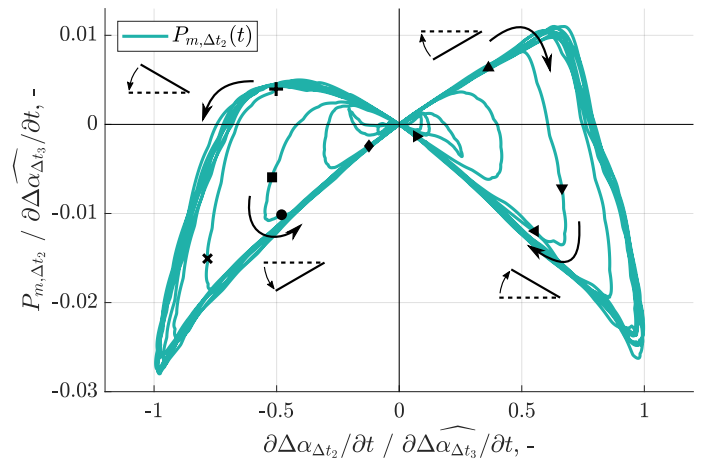

(c) Leistung des Moments im Zeitausschnitt $\Delta t_{2}$

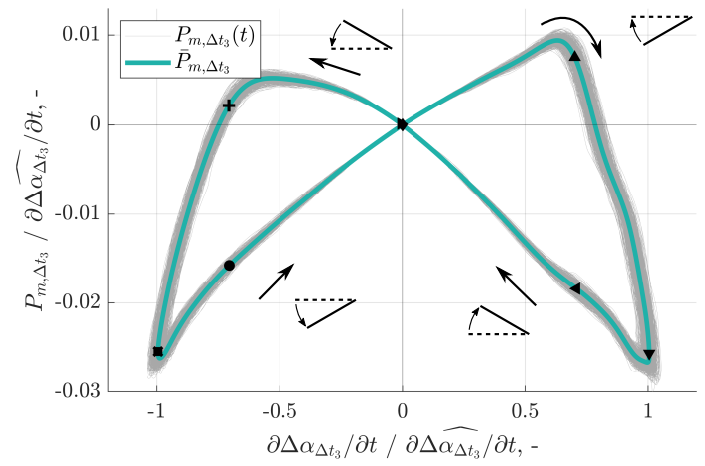

(e) Leistung des Moments im Zeitausschnitt $\Delta t_{3}$, es gilt $\bar{T}_{\Delta t_{3}}^{-1} \int_{0}^{\bar{T}_{\Delta t_{3}}} \bar{P}_{m, \Delta t_{3}} \mathrm{~d} t<0$

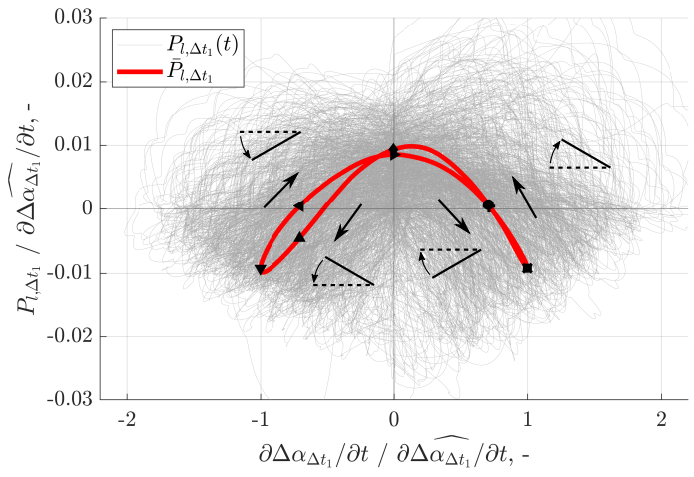

(b) Leistung des Auftriebs im Zeitausschnitt $\Delta t_{1}$, es gilt $\bar{T}_{\Delta t_{1}}^{-1} \int_{0}^{\bar{T}_{\Delta t_{1}}} \bar{P}_{l, \Delta t_{1}} \mathrm{~d} t<0$

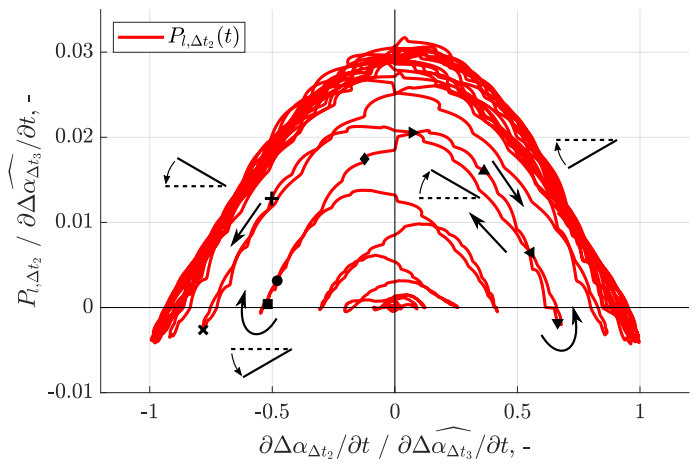

(d) Leistung des Auftriebs im Zeitausschnitt $\Delta t_{2}$

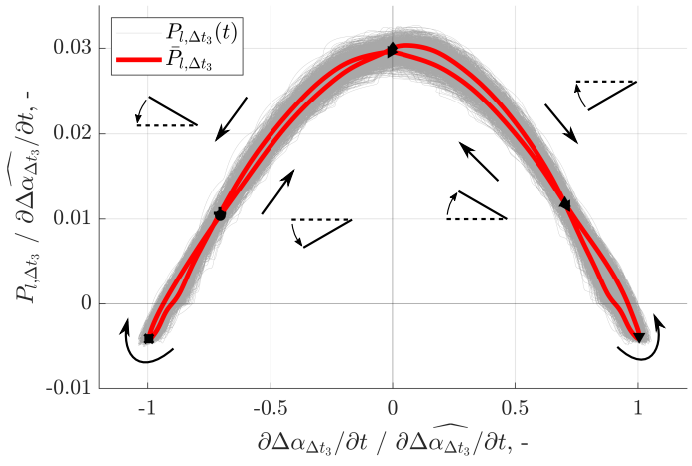

(f) Leistung des Auftriebs im Zeitausschnitt $\Delta t_{3}$, es gilt $\bar{T}_{\Delta t_{3}}^{-1} \int_{0}^{\bar{T}_{\Delta t_{3}}} \bar{P}_{l, \Delta t_{3}} \mathrm{~d} t>0$

Abbildung 7.20.: Leistung des Nickmoments $P_{m}$ und des Auftriebs $P_{l}$ für drei Zeitausschnitte $\Delta t_{i}$ während des LCOs bei $\mathrm{Ma}_{\infty}=0,732, p_{0}=55 \mathrm{kPa}$ und $\bar{\alpha}=0,06^{\circ}$. Die Pfeile und Markierungen geben die Richtung der Drehbewegung an, die schwarzen Punkte markieren jeweils $45^{\circ}$-Schritte einer Periode.

Die Streuung der aerodynamischen Leistungen im aeroelastischen LCO, dargestellt in Abb. 7.20(e) und (f), sind deutlich geringer. Ferner zeigt sich, dass hier nunmehr der Auftrieb maßgeblich zum positiven Energieeintrag beiträgt, was aus der Voreilung des Auftriebs gegenüber der Schlagbewegung resultiert (vgl. Tab. 7.3). Das Moment eilt der Drehbewegung im aeroelastischen LCO deutlich nach und wirkt sich über weite Teile 
der Schwingungsperiode dämpfend aus bzw. leistet einen negativen Energiebeitrag. Die aerodynamische Gesamtleistung (siehe Abb. F.8) ist wiederum positiv.

Während der Aufklingphase in den aeroelastischen LCO ändern sich folglich die Leistungseinträge, wie es in Abb. 7.20(c) und (d) ersichtlich ist. Die Normierung erfolgt hier mit der Drehamplitude $\partial \widehat{\Delta \alpha_{\Delta t_{3}}} / \partial t$ des aeroelastischen LCOs. Mit steigender Amplitude (Anstieg von $|\partial \Delta \alpha / \partial t / \partial \widehat{\Delta \alpha / \partial} t|)$ nimmt der Leistungseintrag durch den Aufrieb zu, wie die parabelförmige positive Ausdehnung von $P_{l, \Delta t_{2}}(t)$ in Abb. 7.20(d) zeigt. Im Gegensatz dazu wirkt das Nickmoment zunehmend dämpfend und entzieht der Struktur Energie. Insbesondere während des Auf- und Zudrehens des Flügels aus der Mittellage heraus ist $P_{m, \Delta t_{2}}(t)<0$. Diese Gebiete dehnen sich mit steigender LCO-Amplitude schneller aus als Bereiche, in denen auch das Moment zu einem positiven Energieeintrag beiträgt. Dies ist kurzzeitig beim Zu- und Aufdrehen aus den maximalen Auslenkungen heraus der Fall, wie es in Abb. 7.20(c) zu erkennen ist. In der Bilanz führt dies zu einem Anstieg der Dämpfung infolge des Nickmoments mit steigender Amplitude und damit zu einer Begrenzung des gesamten aerodynamischen Energieeintrages. Das bedeutet letztgenannter Effekt ist hauptsächlich für die Begrenzung der Schwingungsamplitude und damit für den aeroelastischen LCO verantwortlich, wie es für den hier betrachteten LCO in [62] genauer dargelegt ist (vgl. auch [27,28,187]).

Die anteilige Änderung der Leistungs- bzw. Energieeinträge vom aerodynamischen LCO hin zum aeroelastischen LCO (Änderung mit steigender Schwingungsamplitude) resultiert aus einer Änderung der Phasenbeziehungen (vgl. Tab. 7.3). Diese erfolgt während des Aufklingens in den aeroelastischen LCO und ist in [62] für die LCO-Frequenz $f_{2}$ mithilfe der Hilbert-Transformation (liefert instationäre Phasenbeziehungen) gezeigt. Dabei trägt insbesondere die signifikante Änderung von $\Delta \varphi_{c_{m}, \Delta \alpha}$ zwischen Drehbewegung und Nickmoment zur Amplitudenbegrenzung bei.

Insgesamt geht aus der Energie- bzw. Leistungsbetrachtung des LCO hervor, dass die Phasenvoreilung des Nickmoments einen anfänglichen Energieeintrag bewirkt und initial für eine aeroelastische Instabilität verantwortlich zu sein scheint. Dies deckt sich mit den Ergebnissen in 6, wo ein positiver Energieeintrag ebenfalls mit einer Phasenvoreilung des Moments in Verbindung steht. Jedoch bezieht sich die Phasenvoreilung hier zunächst auf den aerodynamischen LCO bei einer Frequenz von $f_{1}=143,7 \mathrm{~Hz}$. Die durchgeführten zwangserregten Nickschwingungen konzentrieren sich hingegen u. a. auf Frequenzen, die der Schwingungsfrequenz des aeroelastischen LCOs $f_{1}=58,2 \mathrm{~Hz}$ entsprechen. Gleichwohl zeigt sich auch hier die essentielle Rolle der freien Grenzschichttransition, welche als ursächlich für die aerodynamische Instabilität anzunehmen ist und dann zu einer aeroelastischen Instabilität, dem Transitionsflattern führt. 


\subsection{Zusammenfassung des aeroelastischen Verhaltens}

Die durchgeführten Flatterversuche bestätigen erneut die Existenz von aeroelastischen Instabilitäten am CAST 10-2 Laminarflügel unter den gegebenen Randbedingungen. Bei der Untersuchung des klassischen Biege-Torsions-Flatterverhaltens (2-DoF-Konfiguration) wurden für eine freie Grenzschichttransition verschiedene Flatterfälle beobachtet, was insgesamt die Komplexität des aeroelastischen Verhaltens des Laminarflügels zeigt. Ein differenzierter Vergleich dieser Flatterfälle mit Messungen bei einer fixierten Grenzschichttransition bestätigt die Verschiebung des Transonic Dips hin zu kleineren Mach-Zahlen und Ruhedrücken. Vielmehr zeigt sich, dass die im Eingang des Transonic Dips beobachteten LCOs ausschließlich bei freier Grenzschichttransition auftreten und sich sowohl bzgl. der Phasenbeziehung zwischen Schlag und Drehung als auch bzgl. der Flatterfrequenzen von den anderen Flatterfällen klar unterscheiden. Eine freie Grenzschichttransition führt demnach nicht nur zu einer Verschiebung des Transonic Dips, sondern auch zu einem zusätzlichen Flattertyp, dem „Transitionsflattern“. Für diesen Fall stellt die freie Grenzschichttransition eine notwendige Bedingung dar, womit die Ergebnisse der Untersuchungen zur instationären Aerodynamik (vgl. 6.5) bestätigt werden.

Das Transitionsflattern wurde ebenfalls bei einer aeroelastischen 1-DoF-Konfiguration nachgewiesen und die Vergleichbarkeit zur aeroelastischen 2-DoF-Konfiguration aufgezeigt. Ein substantieller Beitrag des Schlag-Starrkörper-Freiheitsgrades und insbesondere auch von höheren Harmonischen an diesem Flattermechanismus kann ausgeschlossen werden. Es deutet sich jedoch eine stabilisierende Wirkung der Schlag-Starrkörpermode bei der 2-DoF-Konfiguration an.

Für beide Konfigurationen setzt das Transitionsflattern für $p_{0} \approx 55 \mathrm{kPa}$ bei $\mathrm{Ma}_{\infty} \approx$ 0,73 ein, entsprechend einer Reynolds-Zahl von $\operatorname{Re}_{\infty} \approx 2 \cdot 10^{6}$. Dieses geht mit einem abrupten Dämpfungsabfall sowie einem deutlichen Anstieg der Dreh- bzw. Flatterfrequenz einher. Eine experimentelle Bifurkationsanalyse zeigt ferner, dass die aeroelastische Systemantwort durch eine subkritische Hopf-Bifurkation beschrieben wird. Es tritt eine Hysterese sowohl bei einer Variation der Mach-Zahl als auch des mittleren Anstellwinkels auf. Bei $\mathrm{Ma}_{\infty}=0,73$ zeigt sich dabei auch, dass der Anstellwinkelbereich, in welchem Transitionsflattern auftritt, mit dem Übergangsbereich der Laminardelle weitestgehend identisch ist. Es liegt eine gute Übereinstimmung mit den Ergebnissen der instationären Aerodynamik, insbesondere der Energiebetrachtung (vgl. 6.2) vor.

Eine abschließende Betrachtung der instationären Aerodynamik eines repräsentativen LCOs offenbart die ausgeprägte Dynamik des laminar-turbulenten Grenzschichtumschlags im Vorfeld und während des Transitionsflatterns. Sofern sich das Modell in Ruhe befindet, treten LCOs der aerodynamischen Kräfte, insbesondere des Nickmomentes auf. Diese 
sind mit den in 5.4 geschilderten instationären Effekten vergleichbar. Die LCOs gehen einher mit einer ausgeprägten Bewegung der Transitionsposition über ca. $40 \%$ der Flügeltiefe. Verdichtungsstöße treten in Form einer Typ-B-Stoßbewegung im hinteren Flügeltiefenbereich auf. Sowohl die Stoßstärke als auch die Bewegung sind jedoch im Vergleich zur Dynamik der Transitionsposition vergleichsweise gering.

Bei Freigabe der Starrkörper-Drehung kommt es zu einem Übergang der Transitionswie auch der Stoßbewegung. Die Interaktion mit der nunmehr vorliegenden Elastizität der Struktur (spontane Reduzierung der Steifigkeit) äußert sich in einer kurzzeitigen aperiodischen Transitions- und Stoßbewegung sowie einem Sprung der Frequenzen. Im aeroelastischen LCO nimmt die Typ-B Stoßbewegung dann deutlich zu. Dabei kommt es im hinteren Flügeltiefenbereich teilweise zu invers verlaufenden Stößen und im vorderen Bereich zur Stoß-Grenzschicht-Interaktion und laminaren Ablöseblasen. Ferner bestätigt sich im aeroelastischen LCO, den Ergebnissen der instationären Messungen (vgl. 6.4) folgend, der Eindruck, dass die Transitionsbewegung die Stoßbewegung vorgibt und steuert. Dies belegt auch die durchgehende Phasenvoreilung gegenüber der Stoßbewegung und dem Nickmoment.

Die Phasenvoreilung des Nickmomentes gegenüber der angeregten Drehbewegung führt im aerodynamischen LCO zu einem positiven aerodynamischen Energieeintrag. Die aerodynamische Instabilität kann somit auch direkt zu einer aeroelastischen Instabilität führen. Im aeroelastischen LCO wirkt das Moment hingegen weitestgehend dämpfend (negative Energiebilanz). Hauptsächlich führt die Auftriebsvoreilung gegenüber der Schlagbewegung des Mittelschnitts dem Grenzzyklus Energie zu. Die Änderung der Energieeinträge basiert dabei auf einer Änderung der Phasenbeziehungen während des Aufklingens in den aeroelastischen LCOs. Hierin begründet sich die Amplitudenbegrenzung.

Grundsätzlich bestätigen die aeroelastischen Untersuchungen die essentielle Rolle der laminar-turbulenten Grenzschichttransition bzgl. der aeroelastischen Instabilitäten des Laminarflügels. Insbesondere für das Transitionsflatterns zeigen die Ergebnisse eine gute Übereinstimmung mit den Messungen zur stationären und instationären Aerodynamik unter zwangserregten Nickschwingungen. Die Ergebnisse des Flatterversuches bestätigen eine freie Grenzschichttransition als notwendige Bedingung für das Transitionsflattern. Ferner legen die Ergebnisse nahe, dass die Transition auch als ursächlich für die aerodynamische Instabilität und das Transitionsflattern anzunehmen ist. 


\section{Diskussion und Ausblick}

Wechselwirkungen zwischen einem transsonischen Strömungsfeld, der laminar-turbulenten Grenzschichttransition und einer elastischen Struktur können zu neuartigen aeroelastischen Instabilitäten führen. Ein Zusammenspiel dieser Komponenten tritt an dem superkritischen CAST 10-2 Laminarprofil auf und wurde in der vorliegenden Arbeit untersucht, ein neuartiger Flattermechanismus wurde identifiziert. Windkanalexperimente mit zwangserregten Nickschwingungen und selbsterregten Flatterschwingungen wurden durchgeführt. Die Untersuchungen fanden schwerpunktmäßig bei einer freien Grenzschichttransition in einem Mach-Zahl-Bereich von $0,73 \lesssim \mathrm{Ma}_{\infty} \lesssim 0,75$ bei einer Reynolds-Zahl von $\operatorname{Re}_{\infty} \approx 2 \cdot 10^{6}$ statt.

Im Rahmen dieser Arbeit wurde der neuartige Flattermechanismus auf Mach-Zahlen und Anstellwinkel eingegrenzt, die mit dem oberen Übergangsbereich der Laminardelle des CAST 10-2 Flügelprofils zusammenfallen. Die essentielle Rolle der laminar-turbulenten Grenzschichttransition an diesem Flattermechanismus wurde aufgezeigt und eine freie Grenzschichttransition als hierfür notwendige Bedingung herausgearbeitet. Der Flattermechanismus wurde als Transitionsflattern identifiziert. Eine experimentelle Bifurkationsanalyse zeigte des Weiteren, dass bei einer Variation der Mach-Zahl und des mittleren Anstellwinkels Hysteresen auftreten. Das aeroelastische Systemverhalten tritt demnach als subkritische Hopf-Bifurkation in Erscheinung. Ferner führt eine laminar-turbulente Grenzschichttransition nicht nur zu einer Verschiebung des Transonic Dips hin zu kleineren Mach-Zahlen und Ruhedrücken, sondern der Dip wird durch den neuen Flattertyp zusätzlich erweitert. Es kommt somit zu einer Vergrößerung des instabilen Bereiches.

Mithilfe von zwangserregten Nickschwingungen wurde nachgewiesen, dass für Strömungsparameter, bei denen das Transitionsflattern auftritt, ein positiver Energietransfer von der Strömung in die Struktur stattfindet. Der positive Energieeintrag geht mit einer Phasenvoreilung des Nickmomentes bzw. einem hierzu führenden Phasen- und Amplitudenverhalten der instationären Druckbeiwerte einher. Ein entsprechendes Verhalten liegt bei einer turbulenten Grenzschicht nicht vor. Für diese wird ein negativer Energieeintrag und somit eine positive aerodynamische Systemdämpfung nachgewiesen. Eine aeroelastische Instabilität wurde somit für eine turbulente Grenzschicht und den untersuchten Strömungsbereich ausgeschlossen. Erstmalig wurde auch das instationäre Verhalten bzw. 
die nichtlineare Dynamik der Position der laminar-turbulenten Grenzschichttransition mitsamt der Stoß-Grenzschicht-Interaktion aufgelöst. Dies wurde durch eine, im Rahmen dieser Arbeit entwickelte Auswertungsmethodik von Heißfilm-Anemometrie-Daten ermöglicht. Einerseits zeigte sich so eine Phasenvoreilung der Position der laminarturbulenten Grenzschichttransition gegenüber der Bewegung des Verdichtungsstoßes und eine damit einhergehende Steuerung der Stoßlage und Stoßdynamik. Andererseits konnten aerodynamische Grenzzyklusschwingungen, die bei festen Anstellwinkeln im oberen Übergangsbereich der Laminardelle beobachtet wurden, auf eine Oszillation der Transitionsposition zurückgeführt werden. Somit wurde die Existenz eines Transitionsbuffets nachgewiesen und dessen Interaktion mit dem aeroelastischen System gezeigt. Der Übergang von einem aerodynamischen in einen aeroelastischen Grenzzyklus wurde experimentell aufgelöst. Dabei unterstützen diese Beobachtungen die Hypothese, dass eine Instationarität des laminar-turbulenten Grenzschichtumschlags die Ursache für den neuartigen Flattermechanismus darstellt und im Allgemeinen zu aerodynamischen und aeroelastischen Instabilitäten führen kann.

Für detailliertere Zusammenfassungen der einzelnen Ergebnisse dieser Arbeit sei auf die, jeweils am Ende der Kapitel zu findenden Abschnitte 5.5, 6.5 und 7.5 verwiesen. Dieses abschließende Kapitel dient dazu, die Ergebnisse der Arbeit im Hinblick auf eine aeroelastische Instabilität mit oder auch durch eine Instationarität der laminar-turbulenten Grenzschichttransition nochmals zusammenfassend zu diskutieren und zukünftige Arbeiten zu motivieren.

\subsection{Diskussion}

Bereits das aerodynamische Verhalten bei statischen Anstellwinkeln (vgl. 5) weist im Übergangsbereich der Laminardelle auf Instabilitäten des Strömungsfeldes hin. Unter anderem treten aerodynamische Nichtlinearitäten auf und es liegt eine hohe Sensitivität der aerodynamischen Beiwerte bzgl. einer Änderung des Anstellwinkels vor. Ein Zusammenhang zwischen den Nichtlinearitäten und einer freien Grenzschichttransition wurde bereits in vorherigen Untersuchungen $[13,23]$ bestätigt. Eine veränderte Position des Grenzschichtumschlags führt infolge der unterschiedlichen Verdrängungsdicke zwischen einer laminaren und turbulenten Grenzschicht zu einer Änderung der effektiven Profilgeometrie [16]. Die Folge ist eine Änderung der aerodynamischen Kräfte.

Naheliegend ist, dass die Sensitivität der aerodynamischen Beiwerte auf eine Instationarität der Position des laminar-turbulenten Grenzschichtumschlags zurückzuführen ist. Frühere Untersuchungen $[156,158,166]$ wiesen bereits eine starke Abhängigkeit der 
aerodynamischen Charakteristiken des CAST 10-2 Profils von der Lage der Grenzschichttransition und der Reynolds-Zahl nach. Eine Erklärung für eine Instationarität der Transitionsposition verdeutlicht dabei Abb. 8.1. Dort sind die Druckverteilungen und

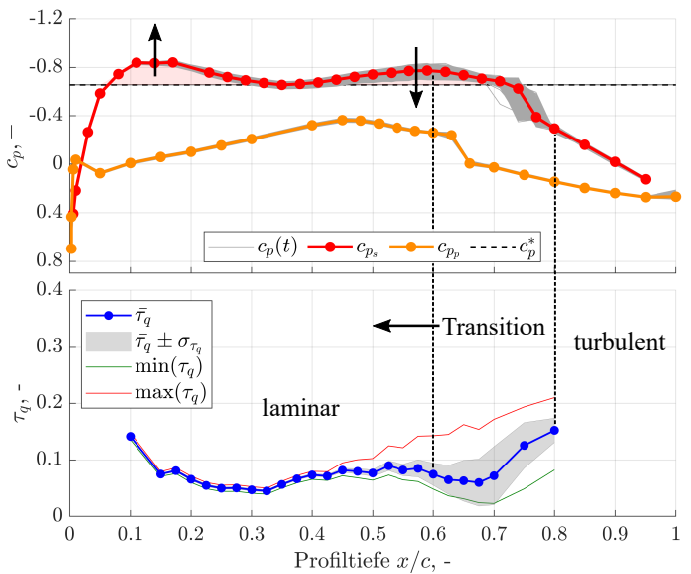

(a) $\alpha=-0,37^{\circ}$

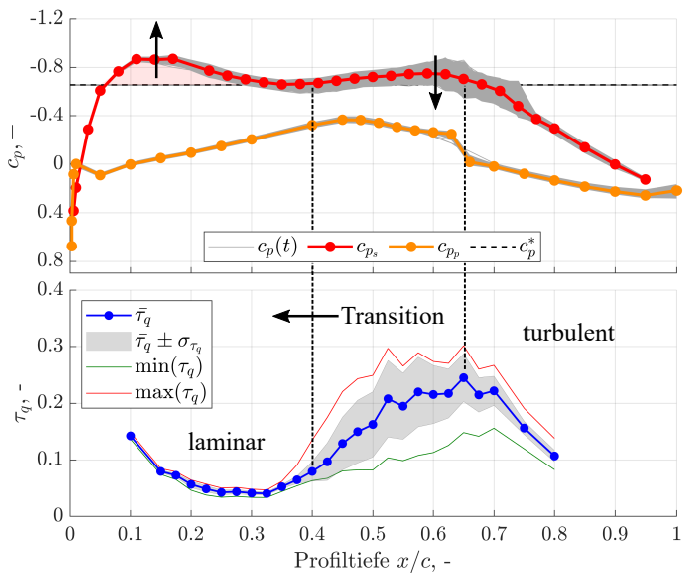

(b) $\alpha=-0,15^{\circ}$

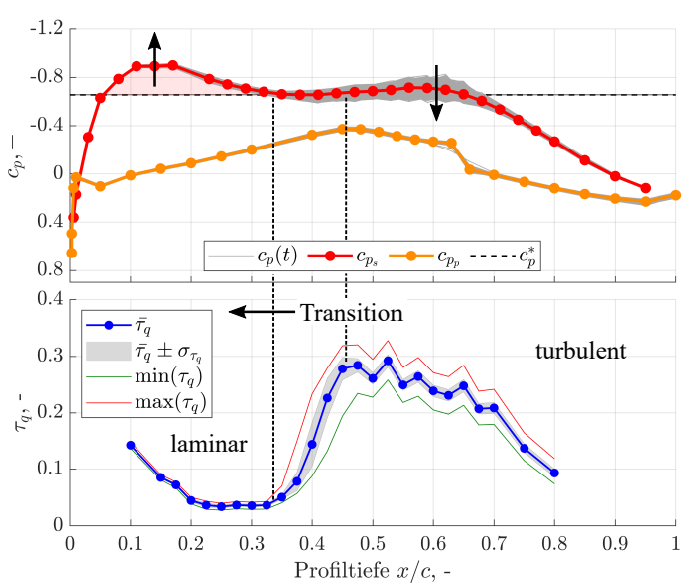

(c) $\alpha=0,06^{\circ}$

Abbildung 8.1.: Druckverteilungen und Verläufe der Quasi-Wandschubspannung für drei Anstellwinkel im oberen Übergangsbereich der Laminardelle bei $\mathrm{Ma}_{\infty}=0,73$ und $p_{0}=$ $54 \mathrm{kPa}$ (siehe auch Abb. 5.10, D.2 und D.4).

Verläufe der Quasi-Wandschubspannung für drei Anstellwinkel im oberen Übergangsbereich der Laminardelle des CAST 10-2 Profils für $\mathrm{Ma}_{\infty}=0,73$ dargestellt. Für alle in Abb. 8.1 dargestellten Anstellwinkel bildet sich im vorderen Flügeltiefenbereich bei $0,05 \lesssim \frac{x}{c} \lesssim 0,35$ auf der Profil-Saugseite ein lokales supersonisches Gebiet $\left(\mathrm{Ma}_{x}>1\right.$ bzw. $\left.c_{p}<c_{p}^{*}\right)$ aus. Im Bereich $0,175 \lesssim \frac{x}{c} \lesssim 0,35$ wird die Strömung durch einen positiven Druckgradienten $\frac{\partial c_{p}}{\partial x}>0$ verzögert und erreicht bei $x / c \approx 0,35$ wieder $\mathrm{Ma}_{x} \approx 1\left(c_{p} \approx c_{p}^{*}\right)$. Dies begünstigt die Rezeptivität und TS-Moden in der laminaren Grenzschicht werden angefacht (vgl. 2.1.1 und 2.1.2). Stromab von $x / c \approx 0,35$ liegt jedoch wieder ein negativer Druckgradient $\frac{\partial c_{p}}{\partial x}<0$ vor, der wiederum stabilisierend auf die Grenzschicht einwirkt und die laminar-turbulente Grenzschichttransition verzögert. Ein ansteigender Anstellwinkel 
führt zu einem Anwachsen des vorderen Überschallgebietes und einer Zunahme des positiven Druckgradienten. Gleichzeitig nimmt der hintere negative Druckgradient ab. Dies ist in Abb. 8.1 durch Pfeile gekennzeichnet. Das Resultat ist eine Bewegung der Grenzschichttransition stromaufwärts, was am Verlauf der Quasi-Wandschubspannung in Abb. 8.1 zu erkennen ist. Die Druckgradienten und deren Änderungen im dargestellten Anstellwinkelbereich sind jedoch gering. Deutlich ausgeprägter ist hingegen die Positionsänderung der Transition (Verschiebung von $\bar{\tau}_{q}$ in Abb. 8.1) und auch die starke Schwankung ( $\bar{\tau}_{q} \pm \sigma_{\tau_{q}}$ in Abb. 8.1), insbesondere für $\alpha=-0,15^{\circ}$ (Abb. 8.1(b)). Die Schwankungen gehen mit deutlichen Frequenzanteilen einher (vgl. 5.4), die in Verbindung mit einer Schwingung der Transitionslage stehen (vgl. 7.4.1).

In dem in Abb. 8.1 dargestellten Anstellwinkelbereich reicht somit bereits eine kleine Änderung der Druckgradienten aus, um die Lage der Grenzschichttransition deutlich zu ändern. Es ist somit anzunehmen, dass im oberen Übergangsbereich der Laminardelle diese moderat verlaufenden Druckgradienten bzw. die allgemeine Beschaffenheit des Druckfeldes die Grenzschichttransition in einen kritischen Zustand bringt, wo bereits eine kleine Störung zu einer deutlichen Verschiebung der Transitionsposition führt. Diese ändert wiederum das Druckfeld, worauf die Transitionsposition erneut reagiert und es gegebenenfalls zu einer Selbsterregung der Bewegung des laminar-turbulenten Grenzschichtumschlags kommt. Eine solche Schwingung der Transitionsposition geht dann mit einer dynamischen Änderung der Verdrängungsdicke und der effektiven Profilgeometrie einher. Dies wirkt sich, zusammen mit der Änderung des Druckfeldes, direkt auf die aerodynamischen Kräfte und somit auch auf die Aeroelastik aus.

Die Ergebnisse der vorliegenden Arbeit bestätigen dies. So führt die in Abb. 8.1 sichtbare Bewegung der Transition stromauf zu einer zunehmenden Aufdickung der Grenzschicht stromab. Es kommt zu einer Entwölbung des Profils bzw. zu einer Auftriebsabnahme im hinteren Flügeltiefenbereich. Stationär begründet sich hiermit die Reduzierung des zudrehenden Nickmomentes im Übergangsbereich der Laminardelle, wie es für diesen Anstellwinkelbereich auftritt (vgl. 5.1). Instationär erklärt dies die ausgeprägte Schwingung des Nickmomentes, wie sie bei den aerodynamischen LCOs zu beobachten ist (vgl. 5.4, D.1 und 7.4).

Dabei liefern die Ergebnisse der zwangserregten Nickschwingungen einen wesentlichen Einblick in die kausale Verkettung der einzelnen Aspekte, die zu aeroelastischen Instabilitäten und insbesondere zum Transitionsflattern führen. So wurden Bereiche lokalisiert, in denen ein Energietransfer in die Struktur erfolgt (vgl. 6.2). Die mittleren Anstellwinkel und Frequenzen bei bzw. mit denen das Transitionsflattern auftritt, wiesen dazu eine gute Übereinstimmung auf (vgl. 7.3.3). Als Ursache für den Energieeintrag in die Struktur konnte eine Phasenvoreilung des Nickmomentes identifiziert werden. Dieses begründet 
sich wiederum auf einem entsprechenden Phasenverhalten der instationären Druckbeiwerte (vgl. 6.3). Es wurde ferner direkt gezeigt, dass sowohl ein positiver Energieeintrag wie auch das notwendige Phasenverhalten der Druckbeiwerte nur dann auftritt, wenn eine laminare bzw. transitionelle Grenzschicht vorliegt. Hieraus leitet sich auch ab, dass eine freie Grenzschichttransition eine notwendige Bedingung für das Transitionsflattern darstellt, was durch die Flattermessungen bestätigt wurde. Inwieweit eine freie Grenzschichttransition vielmehr auch die eigentliche Ursache für die aeroelastische Instabilität darstellt, ist nicht eindeutig zu klären.

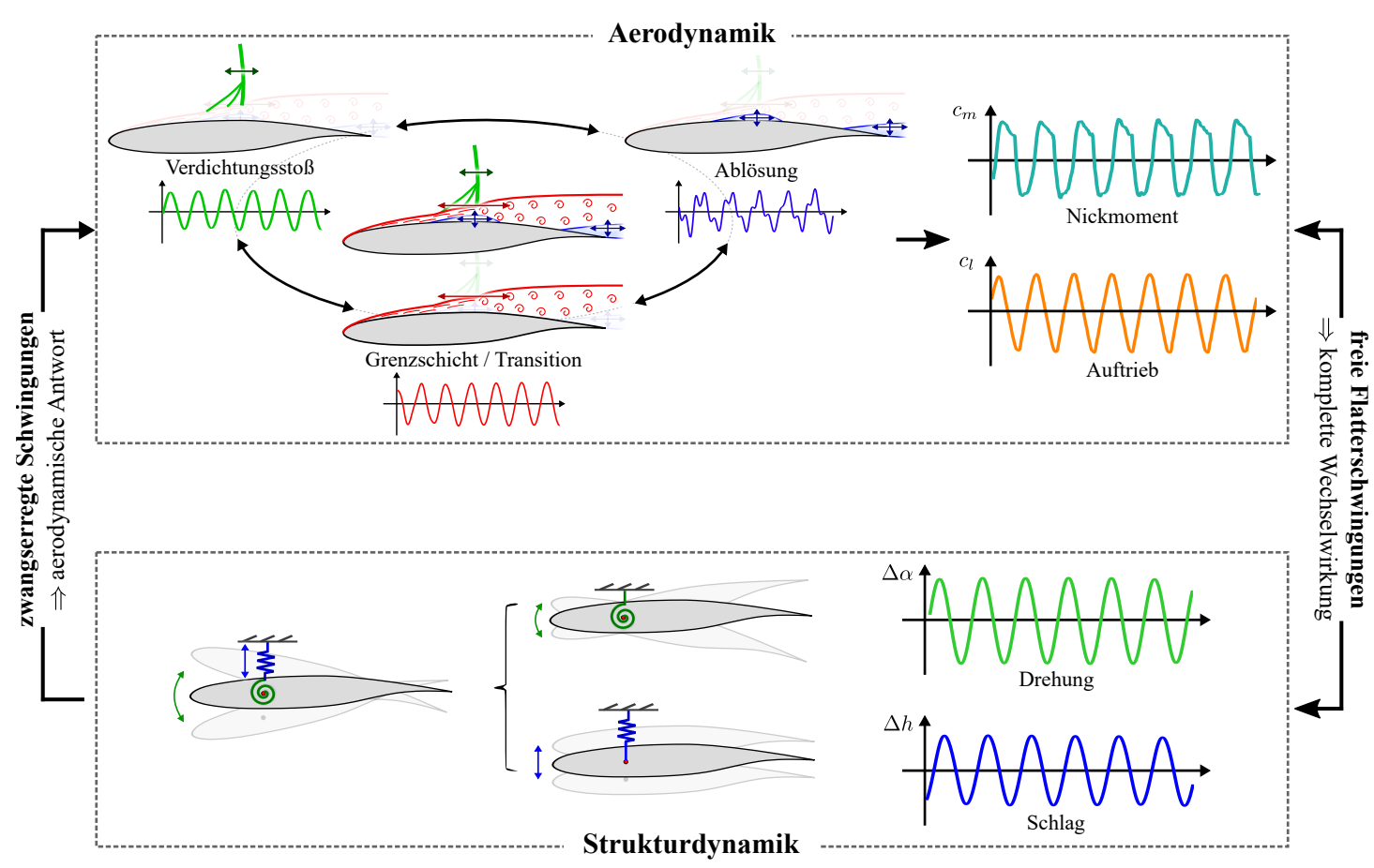

Abbildung 8.2.: Schematische Darstellung des aeroelastischen Systems und Aufspaltung in aerodynamische und strukturelle Schwinger. Die Wechselwirkungen untereinander sind durch Pfeile veranschaulicht. Ferner ist verdeutlicht, welche Wechselwirkungen der einzelnen Komponenten durch die jeweiligen experimentellen Methodiken untersucht werden können.

Ein aeroelastisches System besteht aus miteinander gekoppelten aerodynamischen und strukturellen Schwingern. Eine Aufspaltung eines solchen Systems ist schematisch in Abb. 8.2 dargestellt. Das strukturelle System (Abb. 8.2 unten) besteht dabei idealisiert aus den zwei Freiheitsgraden Schlag und Drehung. Das aerodynamische System (Abb. 8.2 oben) ist idealisiert durch die drei, bereits in der Einleitung 1 angesprochenen aerodynamischen Komponenten: Grenzschicht samt Grenzschichttransition, Verdichtungsstoß und Ablösung. Diese drei Komponenten können als nichtlineare Schwinger angesehen werden, wie die aerodynamischen LCOs (vgl. 5.4 und 7.4) zeigen. Die Schwinger wechselwirken miteinander und lassen sich demnach als gekoppelte Fluid-Schwinger beschreiben. Aus dem Zusammenspiel resultieren die integralen aerodynamischen Beiwerte wie Auftrieb $c_{l}$ und Nickmoment $c_{m}$, die dann auf die hier lineare Struktur (vgl. 3.2.2 und B.2) einwirken. 
Es entsteht eine sehr komplexe aeroelastische Wechselwirkung, wie sie in der vorliegenden Arbeit untersucht wurde.

Die hier eingesetzten experimentellen Methodiken sind jedoch dahingehend limitiert, gezielt einzelne Wechselwirkungen bzw. Komponenten des in Abb. 8.2 dargestellten Systems isoliert zu untersuchen. So führen zwangserregte Nick- bzw. Strukturschwingungen zu einer aerodynamischen Antwort, die aus einer Wechselwirkung aller Fluid-Schwinger resultiert. Freie bzw. selbsterregte Flatterschwingungen lassen dann zusätzlich auch noch eine Wechselwirkung mit den Strukturschwingern zu. Einzelne Schwinger können zwar ausgeschaltet werden, wie es beispielsweise mit der Grenzschichttransition durch eine Transitionsfixierung gemacht wurde. Hieraus lässt sich jedoch nur die Notwendigkeit einer freien Transition für das Transitionflattern in dem gekoppelten Gesamtsystem schlussfolgern. Es ist jedoch nicht möglich, Ursache und Wirkung klar auszuweisen.

Die Ergebnisse der vorliegenden Arbeit liefern jedoch Hinweise dafür, dass eine Instationarität der Transitionslage auch die Ursache für das Transitionsflattern darstellt. So wird diese Hypothese durch das instationäre Verhalten der Grenzschichttransition (vgl. 6.4) und der Voreilung der Transitionsposition gegenüber der Stoßbewegung gestützt. Sowohl bei zwangserregten Nickschwingungen wie auch bei untersuchten LCOs (vgl. 7.4.1 und [62]) zeigt sich, dass die Stoßposition durch die Transitionslage vorgegeben wird.

Auch die aerodynamischen Resonanzen (vgl. 6.1) und die aerodynamischen LCOs (vgl. 5.4 und 7.4) unterstützen die Hypothese. So weisen Erstere große Ähnlichkeit zu aerodynamischen Resonanzen auf, wie sie im Rahmen von Stoß-Buffet-Phänomenen beobachtet wurden [201,202]. Die aerodynamischen LCOs sind dann der direkte Nachweis für selbsterregte Schwingungen des Strömungsfeldes. Die hier beobachteten Strömungsinstabilitäten setzen jedoch allesamt bereits bei mittleren Anstellwinkeln um $\bar{\alpha} \approx 0^{\circ}$ und Mach-Zahlen von $\mathrm{Ma}_{\infty} \approx 0,73$ ein. Die Instabilitäten treten somit bei deutlich kleineren Anstellwinkeln und Mach-Zahlen auf, als es bei typischen Stoß-Buffet-Schwingungen der Fall ist. Die Auflösung der Bewegung der Grenzschichttransition während eines aerodynamischen LCOs zeigt für $\mathrm{Ma}_{\infty} \approx 0,73$ vielmehr auch, dass es primär die Lage der Grenzschichttransition ist, die ausgeprägte Schwingungen ausführt und die Dynamik des Verdichtungsstoßes ein sekundärer Effekt ist (vgl. 7.4.1). Diese Beobachtungen zeigen, dass es sich nicht um ein klassisches Stoß-Buffet handelt, sondern um ein Transitionsbuffet. Genau dieser Zusammenhang zwischen einem Transitionsbuffet und einem Transitionsflattern weist nunmehr Parallelen zu neueren Forschungsergebnissen auf, die transsonische Flattermechanismen auf eine Kopplung bzw. ein Frequenz-Lock-In zwischen einer Strukturmode und einer instabilen Fluid-Mode zurückführen [149-151]. Auch der unter 7.4 aufgelöste Übergang von einem aerodynamischen in einen aeroelastischen LCO zeigt dabei Analogien zu entsprechenden numerischen Ergebnissen in [149,151]. Diese Analogien weisen darauf 
hin, dass die Instationarität der Transitionsposition als instabile Fluid-Mode ursächlich zum Transitionsflattern am CAST 10-2 Laminarflügel führt. Eine abschließende Prüfung dieser Hypothese und den damit verknüpften Fragen erfordert eine isolierte Untersuchung einzelner Komponenten und Wechselwirkungen. Die hieraus folgenden neuen Ansatzund Ausgangspunkte für weiterführende Arbeiten sind im Folgenden kurz motiviert.

Es bleibt jedoch abschließend festzuhalten, dass zusätzlich zu der Identifizierung des Transitionsflatterns am CAST 10-2 Laminarprofil, die Ergebnisse der Arbeit deutlich machen, dass die laminar-turbulente Grenzschichttransition eine essentiellen Stellenwert bzgl. des aerodynamischen und aeroelastischen Verhaltens einnimmt. Aus den Ergebnissen der Arbeit leitet sich die Notwendigkeit ab, für eine vollständige aeroelastische Systembewertung, experimentell sowie numerisch, die laminar-turbulente Grenzschichttransition zu berücksichtigen.

\subsection{Ausblick}

Bisherige experimentelle Methodiken sind dahingehend limitiert, dass immer eine Wechselwirkung mehrerer Komponenten eines aeroelastischen Systems betrachtet bzw. angeregt wird. Für eine genauere Untersuchung der zuvor genannten Aspekte sowie zur Klärung der angeführten Hypothese, dass die Instationarität der Transitionsposition ursächlich zu aeroelastischen Instabilitäten führt, wäre im ersten Schritt eine Untersuchung der aerodynamischen Antwort bei einer weitestgehend isolierten Anregung der Transitionsbewegung von Interesse. Eine zwangserregte Bewegung der Grenzschichttransition wäre hierfür notwendig.

Eine experimentelle Umsetzung einer solchen Zwangserregung wäre durch den Einsatz von nichtthermischen Plasma Aktuatoren denkbar. Diese generieren einen sogenannten Ionen-Wind parallel zur Oberfläche, womit die Geschwindigkeitsprofile der Grenzschicht modifiziert werden können [206]. Plasma-Aktuatoren auf Basis von dielektrisch behinderten Entladungen (engl.: dielectric barrier discharge, DBD) werden bereits erfolgreich zur aktiven Strömungskontrolle eingesetzt [207-209]. DBD-Plasma Aktuatoren ermöglichen u. a. eine Modifizierung der Position des laminar-turbulenten-Grenzschichtumschlags [210]. Eine Verzögerung [211-213] wie auch eine Begünstigung [214,215] der Transition kann erzielt werden. So können DBD-Plasma Aktuatoren als elektrisch steuerbares Transitionstripping eingesetzt werden [213]. Eine instationäre Ansteuerung der Aktuatoren bei harmonischen Nickschwingungen von Flügelprofilen ist ebenfalls bereits erfolgt [216, 217]. Eine gepulste bzw. frequenzgesteuerte Fixierung der Transition und damit eine zwangserregte Bewegung dieser sollte somit experimentell umsetzbar sein. Numerisch wurde ein 
Transitionstripping mithilfe von DBD-Plasma Aktuatoren für das CAST 10-2 Laminarprofil unter transsonischer Strömung bereits simuliert [65].

Eine Messung der aerodynamischen Antwort auf eine zwangserregte Transitionsbewegung würde Aufschluss darüber geben, inwieweit die Transitionsbewegung weitere aerodynamische Komponenten steuert bzw. beeinflusst und ob eine Isolierung einzelner Fluid-Schwinger aus dem aerodynamischen System (vgl. Abb. 8.2 oben) überhaupt möglich ist. Dies umfasst gegebenenfalls auch eine genauere Auflösung der kausalen Verkettung der jeweiligen aerodynamischen Komponenten, also zwischen einer Bewegung der laminar-turbulenten Grenzschichttransition mitsamt einer Aufdickung der Grenzschicht, dem instationärem Druckfeld inklusive Ausbildung eines Verdichtungsstoßes und den daraus resultierenden aerodynamischen Kräften. Die Ergebnisse der vorliegenden Arbeit legen für die Durchführung solcher Messungen eine Variation der Frequenz der Transitionsbewegung bei Strömungsparametern nahe, die dem Eingangsbereich des Transonic Dips entsprechen. Von besonderem Interesse wären hierbei mittlere Profil-Anstellwinkel im oberen Übergangsbereich der Laminardelle sowie Frequenzen der Transitionsbewegung im Bereich der Flatterfrequenzen, der aerodynamischen Resonanzen und der aerodynamischen Grenzzyklen. Deutliche aerodynamische Antworten wären eine erste Bestätigung der Hypothese, dass aus einer Instationarität der Transitionslage auch eine aeroelastische Instabilität resultieren kann.

Im zweiten Schritt bietet eine Kombination der zwangserregten Transitionsbewegung mit einer zwangserregten Bewegung der Struktur die Möglichkeit, den Energietransfer zwischen Struktur und Strömung eingehender zu untersuchen. So könnten direkte Hinweise auf aeroelastische Instabilitäten aufgelöst und die angeführte Hypothese bestätigt werden. Ferner bietet eine Kombination der zwangserregten Schwingungen die Möglichkeit, Frequenz Lock-In Prozesse zu untersuchen. Die Interaktion zwischen dem Transitionsbuffet und der elastischen Struktur könnten genauer geklärt und der große Frequenzunterschied zwischen den aerodynamischen und aeroelastischen LCOs mitsamt den hohen Flatterfrequenzen des Transitionsflatterns erklärt werden.

Als abschließender Punkt sei noch erwähnt, dass eine essentielle Frage bei allen Untersuchungen und Ergebnissen der vorliegenden Arbeit der Einfluss der Reynolds-Zahl ist. Insbesondere für die praktische Anwendung ist die Übertragbarkeit der Ergebnisse auf flugrealistische Reynolds-Zahlen von großer Relevanz. Typische Reynolds-Zahlen von Transportflugzeugen liegen eine Größenordnung über den Reynolds-Zahlen der hier durchgeführten Experimente. Dabei ist anzunehmen, dass mit steigender Reynolds-Zahl infolge der Abnahme der Grenzschichtdicke die aerodynamischen Auswirkungen einer Bewegung der Grenzschichttransition ebenfalls abnehmen, wie es auch umfassend in [16] 
diskutiert ist. Dennoch zeigten bereits frühere Experimente am CAST 10-2 Profil, das Laminarflügeleigenschaften und die damit verbundenen aerodynamischen Nichtlinearitäten auch bei größeren Reynolds-Zahlen (bis $\operatorname{Re}_{\infty} \sim 15 \cdot 10^{6}$ ) auftreten [168-170]. Weiterhin besteht bei hohen Reynolds-Zahlen eine große Lücke an Daten von instationären Experimenten und somit auch bei der Validierung von numerischen Verfahren. Eine eindeutige Abschätzung des Einflusses des laminar-turbulenten Grenzschichtumschlags auf das aeroelastische Verhalten bei hohen Reynolds-Zahlen ist folglich bisher nicht möglich. So werden zukünftige Untersuchungen darauf hinzielen, genau diese Lücke zu schließen. Den Anfang hierzu hat jüngst ein Versuch mit erstmalig durchgeführten zwangserregten Nickschwingungen an einem Laminarprofil bei subsonischer Strömung unter kryogenen Bedingungen gemacht [218]. Es wurden Reynolds-Zahlen bis $\operatorname{Re}_{\infty} \approx 14 \cdot 10^{6}$ erreicht. Versuche bei vergleichbaren Reynolds-Zahlen und transsonischer Strömung sollen folgen. 



\section{Literaturverzeichnis}

[1] Schlichting, H., Gersten, K.: Grenzschicht-Theorie, 10 Aufl. Springer-Verlag (2006). doi:10.1007/3-540-32985-4

[2] Dolling, D.S.: Fifty Years of Shock-Wave/Boundary-Layer Interaction Research: What Next? AIAA Journal 39(8), 1517-1531 (2001). doi:10.2514/2.1476

[3] Liepmann, H., Roshko, A., Dhawan, S.: On Reflection of Shock Waves from Boundary Layers. Technical Note TN 2334, National Advisory Committee for Aeronautics (1951)

[4] Barnes, C.J., Visbal, M.R.: Numerical exploration of the origin of aerodynamic enhancements in [low-Reynolds number] corrugated airfoils. Physics of Fluids 25(11) (2013). doi:10.1063/1.4832655

[5] Bendiksen, O.O.: Role of shock dynamics in transonic flutter. In: AIAA Dynamics Specialists Conference, 16-17 April, Dallas, TX, USA (1992). doi:10.2514/6.19922121

[6] Bendiksen, O.O.: Transonic flutter and the nature of the transonic dip. In: CEAS/AIAA/AIAE International Forum on Aeroelasticity and Structural Dynamics, Vol. 2, S. 273-286 (2001)

[7] Bendiksen, O.O.: Transonic flutter prediction. In: International Forum on Aeroelasticity and Structural Dynamics (IFASD), 4-6 June, Amsterdam, The Netherlands (2003)

[8] Försching, H., Tichy, L.: Grundlagen der Aeroelastik. In: C.C. Rossow, K. Wolf, P. Horst (Hgg.) Handbuch der Luftfahrzeugtechnik, Kap. 4.5, S. 399-429. Carl Hanser Verlag München (2014)

[9] Tijdeman, H.: Investigations of the Transonic Flow around Oscillating Airfoils. Diss., Technische Universität Delft, Delft, Niederlande (1977)

[10] Arnal, D., Archambaud, J.P.: Laminar-Turbulent Transition Control: NLF, LFC, HLFC. In: Advances in Laminar Turbulent Transition Modelling, AVT-151 RTO 
AVT/VKI Lecture Series, 9 -12 June, The von Karman Institute, Rhode St. Genèse, Belgium (2008)

[11] Holmes, B.J., Obara, C.J.: Observations and implications of natural laminar flow on practical airplane surfaces. Journal of Aircraft 20(12), 993-1006 (1983). doi: $10.2514 / 3.48203$

[12] Mai, H., Hebler, A.: Aeroelasticity of a laminar wing. In: International Forum on Aeroelasticity and Structural Dynamics (IFASD), 26-30 June, Paris, France (2011)

[13] Hebler, A., Schojda, L., Mai, H.: Experimental investigation of the aeroelastic behaviour of a laminar airfoil in transonic flow. In: International Forum on Aeroelasticity and Structural Dynamics (IFASD), 24-26 June, Bristol, United Kingdom (2013)

[14] Braune, M., Koch, S.: Application of hot-film anemometry to resolve the unsteady boundary layer transition of a laminar airfoil experiencing limit cycle oscillations. Experiments in Fluids 61(68), 1-15 (2020). doi:10.1007/s00348-020-2907-y

[15] Seitz, A., Hübner, A., Risse, K.: The DLR TuLam project: design of a short and medium range transport aircraft with forward swept NLF wing. CEAS Aeronautical Journal 11, 449-459 (2020). doi:10.1007/s13272-019-00421-1

[16] Tichy, L., Mai, H., Fehrs, M., Nitzsche, J., Hebler, A.: Risk analysis for flutter of laminar wings. In: International Forum on Aeroelasticity and Structural Dynamics (IFASD), 25-28 June, Como, Italy (2017)

[17] Hebler, A.: Aeroelastik am Laminarflügel - Versuchsbericht ALF-1. Interner Bericht IB 232-2012 J 03, DLR, Institut für Aeroelastik (2012)

[18] Hebler, A.: Aeroelastik am Laminarflügel - Versuchsbericht ALF-2. Interner Bericht IB 232-2013 J 04, DLR, Institut für Aeroelastik (2013)

[19] Hebler, A.: Aeroelastik am Laminarflügel - Versuchsbericht ALF-3. Interner Bericht IB 232-2013 J 12, DLR, Institut für Aeroelastik (2013)

[20] Fehrs, M.: Influence of transitional flows at transonic mach numbers on the flutter speed of a laminar airfoil. In: International Forum on Aeroelasticity and Structural Dynamics (IFASD), 24-26 June, Bristol, United Kingdom (2013)

[21] Fehrs, M., van Rooij, A.C.L.M., Nitzsche, J.: Flutter Prediction in the Transonic Flight Regime with the $\gamma-\operatorname{Re}_{\theta}$ Transition Model. In: 6th European Conference on Computational Fluid Dynamics (ECFD VI), 20-25 July, Barcelona, Spain (2014) 
[22] van Rooij, A.C.L.M., Wegner, W.: Numerical investigation of the flutter behaviour of a laminar supercritical airfoil. In: A. Dillmann, G. Heller, E. Krämer, H.P. Kreplin, W. Nitsche, U. Rist (Hgg.) New Results in Numerical and Experimental Fluid Mechanics IX, Notes on Numerical Fluid Mechanics and Multidisciplinary Design, Vol. 124, S. 33-41. Springer (2014). doi:10.1007/978-3-319-03158-3_4

[23] Fehrs, M., van Rooij, A.C.L.M., Nitzsche, J.: Influence of boundary layer transition on the flutter behavior of a supercritical airfoil. CEAS Aeronautical Journal 6(2), 291-303 (2015). doi:10.1007/s13272-014-0147-7

[24] Hebler, A.: Experimental assessment of the flutter stability of a laminar airfoil in transonic flow. In: International Forum on Aeroelasticity and Structural Dynamics (IFASD), 25-28 June, Como, Italy (2017)

[25] Jelicic, G., Schwochow, J., Govers, Y., Hebler, A., Böswald, M.: Real-time assessment of flutter stability based on automated output-only modal analysis. In: International Conference on Noise and Vibration Engineering (ISMA), 15.-17. Sept., Leuven, Belgien (2014)

[26] Schewe, G., Mai, H., Dietz, G.: Nonlinear effects in transonic flutter with emphasis on manifestations of limit cycle oscillations. Journal of Fluids and Structures 18(1), 3-22 (2003). doi:10.1016/S0889-9746(03)00085-9

[27] Dietz, G., Schewe, G., Mai, H.: Experiments on heave/pitch limit-cycle oscillations of a supercritical airfoil close to the transonic dip. Journal of Fluids and Structures 19, 1-16 (2004). doi:10.1016/j.jfluidstructs.2003.07.019

[28] Dietz, G., Schewe, G., Mai, H.: Amplification and amplitude limitation of heave/pitch limit-cycle oscillations close to the transonic dip. Journal of Fluids and Structures 22(4), 505-527 (2006). doi:10.1016/j.jfluidstructs.2006.01.004

[29] van de Vooren, I.A.I., Bergh, H.: Spontaneous Oscillations of an Aerofoil due to Instability of the Laminar Boundary Layer. Report F. 96, National Aeronautical Research Institute, Amsterdam (1951)

[30] Poirel, D., Harris, Y., Benaissa, A.: Self-sustained aeroelastic oscillations of a NACA0012 airfoil at low-to-moderate Reynolds numbers. Journal of Fluids and Structures 24(5), 700-719 (2008). doi:10.1016/j.jfluidstructs.2007.11.005

[31] Poirel, D., Yuan, W.: Aerodynamics of laminar separation flutter at a transitional Reynolds number. Journal of Fluids and Structures 26(7-8), 1174-1194 (2010). doi:10.1016/j.jfluidstructs.2010.06.005 
[32] Poirel, D., Métivier, V., Dumas, G.: Computational aeroelastic simulations of self-sustained pitch oscillations of a NACA0012 at transitional Reynolds numbers. Journal of Fluids and Structures 27(8), 1262-1277 (2011). doi:10.1016/j.jfluidstructs.2011.05.009

[33] Poirel, D., Mendes, F.: Experimental Small-Amplitude Self-Sustained Pitch-Heave Oscillations at Transitional Reynolds Numbers. AIAA Journal 52(8), 1581-1590 (2014). doi:10.2514/1.J052541

[34] Métivier, V., Dumas, G., Poirel, D.: Aeroelastic Dynamics of a NACA 0012 Airfoil at Transitional Reynolds Numbers. In: 39th AIAA Fluid Dynamics Conference, 22-25 June, San Antonio, Texas (2009). doi:10.2514/6.2009-4034

[35] Khalil, M., Poirel, D., Sarkar, A.: Probabilistic parameter estimation of a fluttering aeroelastic system in the transitional reynolds number regime. Journal of Sound and Vibration 332(15), 3670-3691 (2013). doi:10.1016/j.jsv.2013.02.012

[36] Yuan, W., Poirel, D., Wang, B.: Simulations of Pitch-Heave Limit-Cycle Oscillations at a Transitional Reynolds Number. AIAA Journal 51(7), 1716-1732 (2013). doi:10.2514/1.J052225

[37] Poels, A., Rudmin, D., Benaissa, A., Poirel, D.: Localization of Flow Separation and Transition Over a Pitching NACA0012 Airfoil at Transitional Reynolds Numbers Using Hot-Films. Journal of Fluids Engineering 137(12) (2015). doi:10.1115/1.4031008

[38] Yuan, W., Poirel, D., Wang, B., Benaissa, A.: Effect of Freestream Turbulence on Airfoil Limit-Cycle Oscillations at Transitional Reynolds Numbers. Journal of Aircraft 52(4), 1214-1225 (2015). doi:10.2514/1.C032807

[39] Barnes, C.J., Visbal, M.R.: High-Fidelity LES Simulations of Self-Sustained Pitching Oscillations on a NACA0012 Airfoil at Transitional Reynolds Numbers. In: 54th AIAA Aerospace Sciences Meeting, 4-8 January, San Diego, California, USA (2016). doi:10.2514/6.2016-1353

[40] Barnes, C.J., Visbal, M.R.: Aeroelastic Response of an Airfoil at Transitional Reynolds Numbers. In: 46th AIAA Fluid Dynamics Conference,13-17 June, Washington, D.C., USA (2016). doi:10.2514/6.2016-3634

[41] Barnes, C.J., Visbal, M.R.: Self-Sustained Pitch-Heave Oscillations and Gust Response of an Airfoil at Transitional Reynolds Numbers. In: 55th AIAA Aerospace Sciences Meeting, AIAA SciTech Forum, 9-13 January, Grapevine, Texas, USA (2017). doi:10.2514/6.2017-1001 
[42] Barnes, C.J., Visbal, M.R.: On the Role of Flow Transition in Laminar Separation Flutter. Journal of Fluids and Structures 77, 213-230 (2018). doi:10.1016/j.jfluidstructs.2017.12.009

[43] Barnes, C.J., Visbal, M.R.: Stiffness Effects on Laminar Separation Flutter. Journal of Fluids and Structures 91 (2019). doi:10.1016/j.jfluidstructs.2019.102767

[44] Barnes, C.J., Visbal, M.R.: Mitigation of Laminar Separation Flutter Using PlasmaBased Actuators. In: Flow Control Conference, AIAA AVIATION Forum, 25-29 June, Atlanta, Georgia, USA (2018). doi:10.2514/6.2018-3523

[45] Houwink, R., Kraan, A.N., Zwaan, R.J.: Wind-Tunnel Study of the Flutter Characteristics of a Supercritical Wing. Journal of Aircraft 19(5), 400-405 (1982). doi:10.2514/3.57409

[46] Erickson, L.L., Gambucci, B.J., Wilcox, P.R.: Part II - Thick High-Aspect-Ratio Wing. In: NASA Space Shuttle Technology Conference, Volume III - Dynamics and Aeroelasticity, NASA TM X-2274, S. 201-229. National Aeronautics and Space Administration, Washington, D.C., USA (1971)

[47] Erickson, L.L.: Transonic Single-Mode Flutter and Buffet of a low Aspect Ratio Wing having a Subsonic Airfoil Shape. Nasa tn d-7346, National Aeronautics and Space Administration, Washington, D. C., USA (1974)

[48] Mabey, D.G., Ashill, P.R., Welsh, B.L.: Aeroelastic oscillations caused by transitional boundary layers and their attenuation. Journal of Aircraft 24(7), 463-469 (1987). doi:10.2514/3.45502

[49] Ericsson, L.E.: Comment on "Aeroelastic oscillations caused by transitional boundary layers and their attenuation". Journal of Aircraft 25(10), 975-976 (1988). doi:10.2514/3.45690

[50] Ericsson, L.E.: Transition Effects on Airfoil Dynamics and the Implications for Subscale Tests. Journal of Aircraft 26(12), 1051-1058 (1989). doi:10.2514/3.45880

[51] Ericsson, L.E.: Moving wall effects in unsteady flow. Journal of Aircraft 25(11), 977-990 (1988). doi:10.2514/3.45691

[52] Mabey, D.G., Ashill, P.R.: Comment on "Transition Effects on Airfoil Dynamics and Implications for Subscale Tests". Journal of Aircraft 29(3), 519-520 (1988). doi:10.2514/3.46195

[53] Schewe, G., Deyhle, H.: Experiments on Transonic Flutter of a Two-Dimensional Supercritical Wing with emphasis on the non-linear effects. In: Proceedings of the 
Royal Aeronautical Society Conference on Unsteady Aerodynamics, 17-18 July, London, UK (1996)

[54] Schewe, G., Knipfer, A., Mai, H., Dietz, G.: Experimental and Numerical Investigation of Nonlinear Effects in Transonic Flutter. Interner Bericht IB 232 - $2002 \mathrm{~J}$ 01, DLR, Institut für Aeroelastik (2002)

[55] Fehrs, M., Nitzsche, J., Hebler, A.: Cfd-based flutter prediction for high reynolds number flows with free boundary layer transition. In: International Forum on Aeroelasticity and Structural Dynamics (IFASD), 25-28 June, Como, Italy (2017)

[56] Fehrs, M.: Boundary Layer Transition in External Aerodynamics and Dynamic Aeroelastic Stability. Diss., Technische Universität Carolo-Wilhelmina zu Braunschweig (2018)

[57] Fehrs, M., Helm, S., Kaiser, C.: Numerical investigation of unsteady transitional boundary layer flows. In: International Forum on Aeroelasticity and Structural Dynamics (IFASD), 9-13 June, Savannah, Georgia, USA (2019)

[58] Hebler, A., Thormann, R.: Flutter prediction of a laminar airfoil using a doublet lattice method corrected by experimental data. In: A. Dillmann, G. Heller, E. Krämer, C. Wagner, C. Breitsamter (Hgg.) New Results in Numerical and Experimental Fluid Mechanics X, Notes on Numerical Fluid Mechanics and Multidisciplinary Design, Vol. 132, S. 445-455. Springer (2016). doi:10.1007/978-3-319-27279-5_39

[59] Braune, M., Hebler, A.: Experimental investigation of transonic flow effects on a laminar airfoil leading to limit cycle oscillations. In: Applied Aerodynamics Conference, AIAA AVIATION Forum, June 25-29, Atlanta, Georgia (2018). doi:10.2514/6.2018-3641

[60] Braune, M., Hebler, A.: Sensitivity of single degree of freedom limit cycle flutter of a laminar airfoil and resulting uncertainties of the transonic dip. In: A. Dillmann, G. Heller, E. Krämer, C. Wagner, C. Tropea, S. Jakirlic (Hgg.) New Results in Numerical and Experimental Fluid Mechanics XII, Notes on Numerical Fluid Mechanics and Multidisciplinary Design, Vol. 142, S. 481-490. Springer (2020). doi:10.1007/978-3-030-25253-3_46

[61] Braune, M., Koch, S.: Application of Hot-film Anemometry to resolve the Unsteady Boundary Layer Transition of a Laminar Airfoil experiencing Limit Cycle Oscillations. In: 15th International Conference on Fluid Control, Measurements and Visualization (FLUCOME), May 27-30, Naples, Italy (2019) 
[62] Braune, M., Hebler, A.: Mechanisms of Transonic Single Degree of Freedom Flutter of a Laminar Airfoil. In: International Forum on Aeroelasticity and Structural Dynamics (IFASD), 9-13 June, Savannah, Georgia, USA (2019)

[63] Braune, M., Hebler, A.: Hysteretic response of a laminar airfoil undergoing single degree of freedom limit cycle oscillations in transonic flow. In: International Forum on Aeroelasticity and Structural Dynamics (IFASD), 9-13 June, Savannah, Georgia, USA (2019)

[64] Barnes, C.J., Visbal, M.R.: Transonic Effects on a Laminar Airfoil in SmallAmplitude Pitch Oscillation. In: AIAA AVIATION Forum, 15-19 June, Virtual Event (2020). doi:10.2514/6.2020-3043

[65] Barnes, C.J., Visbal, M.R.: Investigation of Flow Transition Effects on a Transonic Laminar Airfoil in Small-Amplitude Pitch Oscillation. In: AIAA Scitech Forum, 19-21 January, Virtual Event (2021). doi:10.2514/6.2021-1650

[66] Prandtl, L.: Über Flüssigkeitsbewegung bei sehr kleiner Reibung. Sonderabdruck des III. Internationalen Mathematiker-Kongresses Heidelberg 1904. In: A. Dillmann (Hrsg.) Prandtl, L., Betz, A.: Vier Abhandlungen zur Hydrodynamik und Aerodynamik, Göttinger Klassiker der Strömungsmechanik Bd. 3, S. 1-8. Universitätsverlag Göttingen (2010). doi:10.17875/gup2010-106

[67] Saric, W.S.: Laminar-Turbulent Transition: Fundamentals. In: Special Course on Skin Friction Drag Reduction, AGARD Report 786, S. 4/1 - 4/32. Advisory Group for Aerospace Research \& Development, Neuilly Sur Seine, France (1992)

[68] Reed, H.L., Saric, W.S., Arnal, D.: Linear Stability Theory Applied to Boundary Layers. Annual Review of Fluid Mechanics 28, 389-428 (1996). doi:10.1146/annurev.fl.28.010196.002133

[69] Saric, W., White, E., Reed, H.: Boundary-layer receptivity to freestream disturbances and its role in transition. In: 30th AIAA Fluid Dynamics Conference, June 28 - July 1, Norfolk, Virginia, USA (1999). doi:10.2514/6.1999-3788

[70] Reshotko, E.: Paths to Transition in Wall Layers. In: Advances in Laminar Turbulent Transition Modelling, AVT-151 RTO AVT/VKI Lecture Series, 9 -12 June, The von Karman Institute, Rhode St. Genèse, Belgium (2008)

[71] Reshotko, E.: Stability theory as a guide to the evaluation of transition data. AIAA Journal 7(6) (1969). doi:10.2514/3.5279

[72] Reshotko, E.: Boundary-Layer Stability and Transition. Annual Review of Fluid Mechanics 8, 311-349 (1976). doi:10.1146/annurev.fl.08.010176.001523 
[73] Reshotko, E.: Boundary Layer Instability, Transition and Control. In: 32nd AIAA Aerospace Sciences Meeting and Exhibit, 10-13 January, Reno, Nevada, USA (1994). doi:10.2514/6.1994-1

[74] Kachanov, Y.S.: Physical Mechanisms of Laminar-Boundary-Layer Transition. Annual Review of Fluid Mechanics 26, 411-482 (1994). doi:10.1146/annurev.fl.26.010194.002211

[75] Morkovin, M.V.: Critical Evaluation of Transition from Laminar to Turbulent Shearlayers with Emphasis on Hypersonically Travelling Bodies. Technical Report AFFDL-TR-08-149, Air Force Flight Dynamics Laboratory, Air Force Systems Command Wright-Patterson Airforce Base, Ohio, USA (1969)

[76] Morkovin, M.V.: Recent Insight into Instability and Transition to Turbulence in Open-Flow Systems. Contractor Report NASA CR-181693, ICASE Report No. 88-44, NASA Langley Research Center, Hampton, Virginia, USA (1988)

[77] Kerschen, E.J.: Boundary Layer Receptivity Theory. Final Report AFSOR Grant No. 90-0065, Air Force Office of Scientific Research, AFOSR/PKA, Bolling Air Force Base, Washington, D.C., USA (1993)

[78] Saric, W.S., Reed, H.L., Kerschen, E.J.: Boundary-Layer Receptivity to Freestream Disturbances. Annual Review of Fluid Mechanics 34, 291-319 (2002). doi:10.1146/annurev.fluid.34.082701.161921

[79] Tumin, A.: Flow Instabilities and Transition. In: R. Blockley, W. Shyy (Hgg.) Encyclopedia of Aerospace Engineering, Kap. Incompressible Flows and Aerodynamics. John Wiley \& Sons (2010). doi:10.1002/9780470686652.eae013

[80] Reshotko, E.: Transient growth: A factor in bypass transition. Physics of Fluids 13(5), 1067-1075 (2001). doi:10.1063/1.1358308

[81] Saric, W.S.: Introduction to Linear Stability. In: Advances in Laminar Turbulent Transition Modelling, AVT-151 RTO AVT/VKI Lecture Series, 9 -12 June, The von Karman Institute, Rhode St. Genèse, Belgium (2008)

[82] Mack, L.M.: Boundary-Layer Linear Stability Theory. In: Special Course on Stability and Transition of Laminar Flow, AGARD Report 709, S. 3/1 - 3/81. Advisory Group for Aerospace Research \& Development, Neuilly Sur Seine, France (1984)

[83] Arnal, D.: Description and Prediction of Transition in Two-Dimensional, Incompressible Flow. In: Special Course on Stability and Transition of Laminar Flow, 
AGARD Report 709, S. 2/1 - 2/71. Advisory Group for Aerospace Research \& Development, Neuilly Sur Seine, France (1984)

[84] Joslin, R.D.: Aircraft Laminar Flow Control. Annual Review of Fluid Mechanics 30, 1-29 (1998). doi:10.1146/annurev.fluid.30.1.1

[85] Pohlhausen, K.: Zur näherungsweisen Integration der Differentialgleichung der laminaren Grenzschicht. Zeitschrift für Angewandte Mathematik und Mechanik 1(4), 252-290 (1921). doi:10.1002/zamm.19210010402

[86] Han, Z.H., Chen, J., Zhang, K.S., Xu, Z.M., Zhu, Z., Song, W.P.: Aerodynamic Shape Optimization of Natural-Laminar-Flow Wing Using Surrogate-Based Approach. AIAA Journal 56(7), 2579-2593 (2018). doi:10.2514/1.J056661

[87] Babinsky, H., et al.: Shock Wave-Boundary-Layer Interactions. Cambridge University Press (2011). doi:10.1017/CBO9780511842757

[88] Delery, J., Marvin, J.G.: Shock-Wave Boundary Layer Interactions. AGARD-AG 280. Advisory Group for Aerospace Research \& Development, Neuilly Sur Seine, France (1986)

[89] Gaitonde, D.V.: Progress in shock wave/boundary layer interactions. Progress in Aerospace Sciences 72, 89-99 (2015). doi:10.1016/j.paerosci.2014.09.002

[90] Inger, G.R.: Shock-Boundary Layer Interaction Effects in Transonic Flow Fields. Final Report to Office of Naval Research N00014-75-C-0456, Virginia Polytechnic Institute and State University, Blacksburg, VA, USA (1980)

[91] Delery, J., Dussauge, J.P.: Some physical aspects of shock wave/boundary layer interactions. Shock Waves 19(453), 453-468 (2009). doi:10.1007/s00193-009-0220-z

[92] Sandham, N.D.: Shock-Wave/Boundary-Layer Interactions. In: Engine Intake Aerothermal Design: Subsonic to High Speed Applications, RTO-EN-AVT-195 (2011)

[93] Elsenaar, A.: Separation in transonic flow: a shocking experience. Report NLRTP-1997-151, National Aerospace Laboratory NLR (1997)

[94] Malkiel, E., Mayle, R.E.: Transition in a separation bubble. ASME Journal of Turbomachinery 118(4), 752-759 (1996). doi:10.1115/1.2840931

[95] Roberts, W.B.: Calculation of Laminar Separation Bubbles and Their Effect on Airfoil Performance. AIAA Journal 18(1), 25-31 (1980). doi:10.2514/3.50726 
[96] Lee, D., et al.: Mechanisms of surface pressure distribution within a laminar separation bubble at different Reynolds numbers. Physics of Fluids 27(2) (2015). doi:10.1063/1.4913500

[97] Stanewsky, E., Little, B.H.: Separation and Reattachment in Transonic Airfoil Flow. AIAA Journal of Aircraft 8(12), 952-958 (1971). doi:10.2514/3.59192

[98] Pearcey, H.H., Osborne, J., Haines, A.B.: The interaction between local effects at the shock and rear separation - a source of significant scale effects in wind-tunnel tests on airfoils and wings. In: Transonic Aerodynamics, AGARD Conference Proceedings No. 35, S. 11/1 - 11/23. Advisory Group for Aerospace Research \& Development, Neuilly Sur Seine, France (1968)

[99] Ligrani, P.M., McNabb, E.S., Collopy, H., Anderson, M., Marko, S.M.: Recent investigations of shock wave effects and interactions. Advances in Aerodynamics 2(4) (2020). doi:10.1186/s42774-020-0028-1

[100] Pirozzoli, S., Larsson, J., Nichols, J.W., Bernardini, M., Morgan, B.E., Lele, S.K.: Analysis of unsteady effects in shock/boundary layer interactions. In: Center for Turbulence Research Proceedings of the Summer Program, Center for Turbulence Research, Stanford University and NASA Ames Research Center, Stanford, CA, USA, S. 153-164 (2010)

[101] Grilli, M., Schmid, P.J., Hickel, S., Adams, N.A.: Analysis of unsteady behaviour in shockwave turbulent boundary layer interaction. Journal of Fluid Mechanics 700, 16-28 (2012). doi:10.1017/jfm.2012.37

[102] Estruch-Samper, D., Chandola, G.: Separated shear layer effect onshockwave/turbulent-boundary-layer interaction unsteadiness. Journal of Fluid Mechanics 848, 154-192 (2018). doi:10.1017/jfm.2018.350

[103] Sansicaa, A., Sandham, N.D., Hu, Z.: Forced response of a laminar shock-induced separation bubble. Physics of Fluids 26(9) (2014). doi:10.1063/1.4894427

[104] Mayle, R.E.: Elements of Transitional Boundary-Layer Flow, 2. Aufl. Logos Verlag Berlin (2018)

[105] Arnal, D., Casalis, G., Houdeville, R.: Practical Transition Prediction Methods: Subsonic and Transonic Flows. In: Advances in Laminar Turbulent Transition Modelling, AVT-151 RTO AVT/VKI Lecture Series, 9 -12 June, The von Karman Institute, Rhode St. Genèse, Belgium (2008)

[106] Kreplin, H.P., Höhler, G.: Application of the Surface Hot Film Technique for Laminar Flow Investigations. In: First European Forum on Laminar Flow Technology, 
March 16-18, Hamburg, Germany, DGLR-Bericht 1992-06, S. 123-131. Deutsche Gesellschaft für Luft und Raumfahrt e.V (1992)

[107] Gaudet, L., Betts, C.J., Ashill, P.R.: Experimental Investigation of BoundaryLayer Transition on a Natural Laminar Flow Aerofoil. In: First European Forum on Laminar Flow Technology, March 16-18, Hamburg, Germany, DGLR-Bericht 1992-06, S. 132-140. Deutsche Gesellschaft für Luft und Raumfahrt e.V (1992)

[108] Fung, Y.C.: An Introduction to the Theory of Aeroelasticity. Dover Publications, Inc. New York (1993)

[109] Försching, H.W.: Grundlagen der Aeroelastik. Springer-Verlag Berlin Heidelberg (1974). doi:10.1007/978-3-642-48285-4

[110] Collar, A.R.: The Expanding Domain of Aeroelasticity. The Aeronautical Journal 50(428), 613-636 (1946). doi:10.1017/S0368393100120358

[111] Collar, A.R.: The first fifty years of aeroelasticity. Aerospace S. 12-20 (1978)

[112] Wright, J.R., Cooper, J.E.: Introduction to Aircraft Aeroelasticity and Loads, 2. Aufl. John Wiley \& Sons, Ltd. (2015). doi:10.1002/9781118700440

[113] Dowell, E.H., et al.: A Modern Course in Aeroelasticity, Solid Mechanics and Its Applications, Vol. 217, 5. Aufl. Springer International Publishing (2015). doi:10.1007/978-3-319-09453-3

[114] Kousen, K., Bendiksen, O.: Nonlinear aspects of the transonic aeroelastic stability problem. In: 29th AIAA Structures, Structural Dynamics and Materials Conference, 18-20 April, Williamsburg, VA, USA (1988). doi:10.2514/6.1988-2306

[115] Bendiksen, O.O.: Nonlinear Flutter and Divergence in Transonic Flow. In: European Forum on Aeroelasticity and Structural Dynamics, 17-19 April, Aachen, Deutschland, S. 669-686 (1989)

[116] Bendiksen, O.O.: Nonunique Solutions in Transonic Aeroelasticity. In: International Forum on Aeroelasticity and Structural Dynamics (IFASD), 17-20 June, Rome, Italy (1997)

[117] Schlichting, H., Truckenbrodt, E.: Aerodynamik des Flugzeuges, Erster Band, 3. Aufl. Springer-Verlag Berlin Heidelberg (2001). doi:10.1007/978-3-642-56911-1

[118] Küssner, H.G.: Zusammenfassender Bericht über den instationären Auftrieb von Flügeln. Luftfahrtforschung 13, 410-424 (1936) 
[119] Schwarz, L.: Berechnung der Druckverteilung einer harmonisch sich verformenden Tragfläche in ebener Strömung. Luftfahrtforschung 17, 379-386 (1940)

[120] Theodorsen, T.: General Theory of Aerodynamic Instability and the Mechanism of Flutter. NACA Technical Report 496, National Advisory Committee for Aeronautics. Langley Aeronautical Lab., Langley Field, VA, USA (1935)

[121] Garrick, I.E.: On some reciprocal relations in the theory of nonstationary flows. NACA Technical Report 629, National Advisory Committee for Aeronautics. Langley Aeronautical Lab., Langley Field, VA, USA (1938)

[122] Wagner, H.: Über die Entstehung des dynamischen Auftriebes von Tragflügeln. Zeitschrift für Angewandte Mathematik und Mechanik 5(1), 17-35 (1925). doi:10.1002/zamm.19250050103

[123] Rodden, W.P., Stahl, B.: A strip method for prediction of damping in subsonic wind tunnel and flight flutter tests. Journal of Aircraft 6(1), 9-17 (1969). doi:10.2514/3.43994

[124] Jones, R.T.: The unsteady lift of a wing of finite aspect ratio. NACA Technical Report 681, National Advisory Committee for Aeronautics. Langley Aeronautical Lab., Langley Field, VA, USA (1940)

[125] Bai, C., Li, J., Li, S., Li, S., Wu, Z.: Review of theoretical achievements for starting flow problem for all Mach numbers. Chinese Journal of Aeronautics 32(1), 78-91 (2018). doi:10.1016/j.cja.2018.10.009

[126] Bisplinghoff, R.L., Ashley, H., Halfman, R.L.: Aeroelasticity, 1 Aufl. Dover Publications (1996)

[127] Bisplinghoff, R.L., Ashley, H.: Principles of Aeroelasticity. John Wiley and Sons, Inc., New York, London (1962)

[128] Schwochow, J.: Die aeroelastische Stabilitätsanalyse - ein praxisnaher Ansatz zur intervalltheoretischen Betrachtung von Modellierungsunsicherheiten am Flugzeug. Diss., Universität Kassel (2012)

[129] Hodges, D.H., Pierce, G.A.: Introduction to Structural Dynamics and Aeroelasticity. Cambridge University Press (2002). doi:10.1017/CBO9780511809170

[130] Braune, M.: Untersuchung des Pfeilungseinflusses auf aeroelastische Instabilitäten bei kleinen Reynoldszahlen. Masterarbeit, Georg-August-Universität Göttingen (2016) 
[131] Bendiksen, O.O.: Transonic single-degree-of-freedom flutter and natural mode instabilities. In: 54th AIAA/ASME/ASCE/AHS/ASC Structures, Structural Dynamics, and Materials Conference, April 8-11, Boston, Massachusetts (2013). doi:10.2514/6.2013-1593

[132] Zimmerman, N.H., Weissenburger, J.T.: Prediction of flutter onset speed based on flight testing at subcritical speeds. Journal of Aircraft 1(4), 190-202 (1964). doi:10.2514/3.43581

[133] Försching, H.: Aeroelastische Probleme in kompressibler Unterschall- und transsonischer Strömung. In: H.U. Meier (Hrsg.) Die deutsche Luftfahrt - Die Pfeilflügelentwicklung in Deutschland bis 1945, S. 233-260. Bernard \& Graefe Verlag, Bonn (2006)

[134] Voss, R., Tichy, L., Thormann, R.: A ROM based Flutter Prediction Process and its Validation with a new Reference Model. In: International Forum on Aeroelasticity and Structural Dynamics (IFASD), 26-30 June, Paris, France (2011)

[135] Bendiksen, O.O.: Review of unsteady transonic aerodynamics: Theory and applications. Progress in Aerospace Sciences 47(2), 135-167 (2011). doi:10.1016/j.paerosci.2010.07.001

[136] Edwards, J.W., Bennett, R.M., Jr., W.W., Seidel, D.A.: Time-marching transonic flutter solutions including angle-of-attack effects. Journal of Aircraft 20(11), 899-906 (1983). doi:10.2514/3.48190

[137] Kholodar, D.B., Dowell, E.H., Thomas, J.P., Hall, K.C.: Improved Understanding of Transonic Flutter: A Three-Parameter Flutter Surface. Journal of Aircraft 41(4) (2004). doi:10.2514/1.467

[138] Tijdeman, H., Seebass, R.: Transonic flow past oscillating airfoils. Annual Review of Fluid Mechanics 12, 181-222 (1980). doi:10.1146/annurev.fl.12.010180.001145

[139] Bendiksen, O.O.: Transonic Limit Cycle Flutter/LCO. In: 45th AIAA/ASME/ASCE/AHS/ASC Structures, Structural Dynamics \& Materials Conference, 19 - 22 April, Palm Springs, California (2004). doi:10.2514/6.2004-1694

[140] Strogatz, S.H.: Nonlinear Dynamics and Chaos, 2. Aufl. Westview Press (2015)

[141] Dowell, E., Edwards, J., Strganac, T.: Nonlinear Aeroelasticity. Journal of Aircraft 40(5), 857-874 (2003). doi:10.2514/2.6876 
[142] Dowell, E.: Some recent advances in nonlinear aeroelasticity: Fluid-structure interaction in the 21st century. In: 51st AIAA/ASME/ASCE/AHS/ASC Structures, Structural Dynamics, and Materials Conference, Orlando, Florida (2010). doi:10.2514/6.2010-3137

[143] Pettit, C.L.: Uncertainty Quantification in Aeroelasticity: Recent Results and Research Challenges. Journal of Aircraft 41(5), 1217-1229 (2004). doi:10.2514/1.3961

[144] Bendiksen, O.O.: Nested limit cycles in transonic flutter. In: International Forum on Aeroelasticity and Structural Dynamics (IFASD), 25-28 June, Como, Italy, S. 425-435 (2017)

[145] Eaton, A., Howcroft, C., Coetzee, E., Neild, S., Lowenberg, M., Cooper, J.: Numerical continuation of limit cycle oscillations and bifurcations in high-aspect-ratio wings. Aerospace 5(3) (2018). doi:10.3390/aerospace5030078

[146] Thomas, J.P., Dowell, E.H., Hall, K.C.: Nonlinear Inviscid Aerodynamic Effects on Transonic Divergence, Flutter, and Limit-Cycle Oscillations. AIAA Journal 40(4), 638-646 (2002). doi:10.2514/2.1720

[147] Dowell, E.H., Ilgamov, M.: Studies in Nonlinear Aeroelasticity. Springer-Verlag New York (1988). doi:10.1007/978-1-4612-3908-6

[148] Henshaw, M., et al.: Non-linear aeroelastic prediction for aircraft applications. Progress in Aerospace Sciences 43(4-6), 65-137 (2007). doi:10.1016/j.paerosci.2007.05.002

[149] Gao, C., Zhang, W., Li, X., Liu, Y.: Mechanism of frequency lock-in in transonic buffeting flow. Journal of Fluid Mechanics 818, 528-561 (2017). doi:10.1017/jfm.2017.120

[150] Gao, C., Zhang, W., Ye, Z.: A new viewpoint on the mechanism of transonic single-degree-of-freedom flutter. Aerospace Science and Technology 52, 144-156 (2016). doi:10.1016/j.ast.2016.02.029

[151] Gao, C., Zhang, W.: Transonic aeroelasticity: A new perspective from the fluid mode. Progress in Aerospace Sciences 113 (2020). doi:10.1016/j.paerosci.2019.100596

[152] Wedemeyer, E., Taylor, N.J., Holst, H.: Adaptive wall techniques. In: B. Ewald (Hrsg.) Wind Tunnel Wall Corrections, AGARD-AG-336, Kap. 10, S. 1-48. Advisory Group for Aerospace Research \& Development (1998)

[153] Braune, M., Wiggen, S.: Experimental investigation of flutter mechanisms depending on sweep under subsonic flow and low reynolds numbers. In: A. Dillmann, 
G. Heller, E. Krämer, C. Wagner, S. Bansmer, R. Radespiel, R. Semaan (Hgg.) New Results in Numerical and Experimental Fluid Mechanics XI, Notes on Numerical Fluid Mechanics and Multidisciplinary Design, Vol. 136, S. 411-420. Springer (2018). doi:10.1007/978-3-319-64519-3_37

[154] Niemann, G., Winter, H., Höhn, B.R.: Maschinenelemente, 4 Aufl. Springer-Verlag Berlin Heidelberg (2005). doi:10.1007/b137557

[155] Braune, M., Büte, T.: Schwerpunkt- und Trägheitsmomentenmessung an Windkanalmodellen. Interner Bericht DLR-IB-AE-GO-2018-196, DLR, Institut für Aeroelastik (2018)

[156] Stanewsky, E., Zimmer, H.: Entwicklung und Windkanalerprobung von drei überkritischen Tragflügelprofilen für Verkehrsflugzeuge. In: 7. Jahrestagung der DGLR, 17.-19. September, Kiel, Deutschland (1974)

[157] Stanewsky, E., Zimmer, H.: Development and Wind Tunnel Investigation of Three Supercritical Airfoil Profiles for Transport Aircraft. Technical Memorandum NASA TM-75840, NASA, Washington, D.C., United States (1980)

[158] Stanewsky, E.: Windkanalerprobung von drei überkritischen Profilen: Ergebnisse für die Profile CAST 10-2 und CAST 12-1. DFVLR-AVA Bericht 25174 C 14, DFVLR-AVA (1974)

[159] Dress, D.A., McGuire, P.D., Stanewsky, E., Ray, E.J.: High Reynolds Number Tests of the CAST 10-2/DOA 2 Airfoil in the Langley 0.3-Meter Transonic Cryogenic Tunnel - Phase I. Technical Memorandum NASA TM 84620, NASA Langley Research Center, Hampton, VA, United States (1983)

[160] Dress, D.A., Stanewsky, E., McGuire, P.D., Ray, E.J.: High Reynolds Number Tests of the CAST 10-2/DOA 2 Airfoil in the Langley 0.3-Meter Transonic Cryogenic Tunnel - Phase II. Technical Memorandum NASA TM 86273, NASA Langley Research Center, Hampton, VA, United States (1984)

[161] Freimuth, P., Stanewsky, E., Demurie, F., Ray, E., Johnson, C.: Messungen am Profil CAST 10-2/DOA 2 im 0,3-m Transonic Cryogenic Tunnel von NASA-Langley bei hohen Reynoldszahlen. DFVLR-AVA Bericht IB 222-86 A 40, DFVLR-AVA, Institut für Experimentelle Strömungsmechanik (1986)

[162] Whitcomb, R.T., Clark, L.R.: An Airfoil Shape for Efficient Flight at Supercritical Mach Numbers. Technical Report NASA TM X-1109, NASA Langley Research Center, Hampton, VA, United States (1965) 
[163] Whitcomb, R.T.: Review of NASA supercritical airfoils. In: International Council of the Aeronautical Sciences (ICAS), 9th-Congress, 25-30 August, Haifa, Israel (1974)

[164] Whitcomb, R.T.: Advanced Transonic Aerodynamic Technology. In: Advances in Engineering Science, Proceedings of the 13th Ann. Meeting of Soc. of Eng. Sci., 1-3 November, Hampton, VA, United States, Vol. 4, S. 1521-537. NASA Langley Research Center, Hampton, VA, United States (1976)

[165] Mineck, R.E.: Comparison of NAE Porous Wall and NASA Adaptive Wall Test Results Using the NAE CAST-10 Airfoil Model. In: E.J. Ray, A.S. Hill (Hgg.) CAST10-2/DOA 2 Airfoil Studies Workshop Results, NASA Conference Publication 3052. NASA Langley Research Center, Hampton, VA, United States (1988)

[166] Stanewsky, E., Demurie, F., Ray, E., Johnson, C.: High Reynolds number tests of the CAST-10-2/DOA 2 transonic airfoil at ambient and cryogenic temperature conditions. In: AGARD Fluid Dynamics Panel Symposium Wind Tunnels and Testing Techniques (AGARD CP-348), 26-29 September, Cesme, Turkey (1983)

[167] Sobieczky, H., Stanewsky, E.: The design of transonic airfoils under consideration of shock wave boundary layer interaction. DFVLR-AVA Bericht IB 251 - 76 A 26, DFVLR-AVA (1976)

[168] Blanchard, A., Seraudie, A., Breil, J.F.: Main Results of CAST-10 Airfoil Tested in T2 Cryogenic Wind Tunnel. In: E.J. Ray, A.S. Hill (Hgg.) CAST-10-2/DOA 2 Airfoil Studies Workshop Results, NASA Conference Publication 3052. NASA Langley Research Center, Hampton, VA, United States (1988)

[169] Thibert, J.J.: Test Data Analysis and Theory - Experiment Comparisons. In: E.J. Ray, A.S. Hill (Hgg.) CAST-10-2/DOA 2 Airfoil Studies Workshop Results, NASA Conference Publication 3052. NASA Langley Research Center, Hampton, VA, United States (1988)

[170] Chan, Y.Y.: Investigation of CAST-10/DOA 2 Airfoil in NAE High Reynolds Number Two-Dimensional Test Facility. In: E.J. Ray, A.S. Hill (Hgg.) CAST-102/DOA 2 Airfoil Studies Workshop Results, NASA Conference Publication 3052. NASA Langley Research Center, Hampton, VA, United States (1988)

[171] Ray, E.J., Hill, A.S. (Hgg.): CAST-10-2/DOA 2 Airfoil Studies Workshop Results, NASA Conference Publication 3052. NASA Langley Research Center, Hampton, VA, United States (1988) 
[172] Demtröder, W.: Experimentalphysik 1, 5 Aufl. Springer-Verlag Berlin Heidelberg (2008). doi:10.1007/978-3-540-79295-6

[173] Wiggen, S.: Bewegungsinduzierte instationäre Luftkräfte bei wirbeldominierter Strömung. Diss., Technische Universität Carolo-Wilhelmina zu Braunschweig (2016)

[174] Mai, H.: Untersuchung der Druckverteilungen eines schwingenden Ringflügels in transsonischer Strömung. Diss., Georg-August-Universität Göttingen (2011)

[175] Micro-Epsilon Messtechnik GmbH \& Co. KG, Koenigbacher Str. 15, 94496 Ortenburg, Deutschland: Instruction Manual optoNCDT 1605

[176] Richter, K., Schülein, E.: Boundary-layer transition measurements on hovering helicopter rotors by infrared thermography. Experiments in Fluids 55(7) (2014). doi:10.1007/s00348-014-1755-Z

[177] Crawford, B.K., Duncan Jr., G.T., West, D.E., Saric, W.S.: Laminar-Turbulent Boundary Layer Transition Imaging Using IR Thermography. Optics and Photonics Journal 3(3), 233-239 (2013). doi:10.4236/opj.2013.33038

[178] Raffel, M., Merz, C.B.: Differential Infrared Thermography for Unsteady BoundaryLayer Transition Measurements. AIAA Journal 52(9), 2090-2093 (2014). doi:10.2514/1.J053235

[179] Raffel, M., Merz, C.B., Schwermer, T., Richter, K.: Differential infrared thermography for boundary layer transition detection on pitching rotor blade models. Experiments in Fluids 56(2), 30 (2015). doi:10.1007/s00348-015-1905-y

[180] Dietz, G., Schewe, G., Kießling, F., Sinapius, M.: Limit-Cycle Oscillation Experiments at a Transport Aircraft Wing Model. In: International Forum on Aeroelasticity and Structural Dynamics (IFASD), 4-6 June, Amsterdam, The Netherlands (2003)

[181] Hufnagel, K., Schewe, G.: Force and moment measurement. In: C. Tropea, A.L. Yarin, J.F. Foss (Hgg.) Springer Handbook of Experimental Fluid Mechanics, S. 563-616. Springer Berlin Heidelberg (2007). doi:10.1007/978-3-540-30299-5_8

[182] Meyer, M.: Signalverarbeitung: Analoge und digitale Signale, Systeme und Filter, 7. Aufl. Springer Vieweg (2014). doi:10.1007/978-3-658-02612-7

[183] Jäger, H., Mastel, R., Knaebel, M.: Technische Schwingungslehre, 8. Aufl. Springer Vieweg (2013). doi:10.1007/978-3-8348-2099-0 
[184] Korsch, H.J.: Mathematische Ergänzungen zur Einführung in die Physik, 4. Aufl. Binomi Verlag (2007)

[185] Guicking, D.: Schwingungen, 1. Aufl. Springer Vieweg (2016). doi:10.1007/978-3658-14136-3

[186] Brandt, A.: Noise and Vibration Analysis, 1. Aufl. Wiley (2011). doi:10.1002/9780470978160

[187] van Rooij, A., Nitzsche, J., Dwight, R.: Energy budget analysis of aeroelastic limit-cycle oscillations. Journal of Fluids and Structures 69, 174-186 (2017). doi:10.1016/j.jfluidstructs.2016.11.016

[188] Lee, T., Basu, S.: Measurement of unsteady boundary layer developed on an oscillating airfoil using multiple hot-film sensors. Experiments in Fluids 25(2), 108-117 (1998). doi:10.1007/s003480050214

[189] Richter, K., Koch, S., Gardner, A.: Influence of oscillation amplitude and Mach number on the unsteady transition on a pitching rotor blade airfoil. In: AHS 69th Annual Forum, 21-23 May, Phoenix, Arizona (2013)

[190] Richter, K., Koch, S., Gardner, A., Mai, H., Klein, A., Rohardt, C.H.: Experimental Investigation of Unsteady Transition on a Pitching Rotor Blade Airfoil. Journal of the American Helicopter Society 59(1) (2014). doi:10.4050/JAHS.59.012001

[191] Richter, K., Koch, S., Goerttler, A., Lütke, B., Wolf, C.C., Benkel, A.: Unsteady Boundary Layer Transition on the DSA-9A Rotor Blade Airfoil. In: 41st European Rotorcraft Forum, 1-4 September, Munich, Germany (2015)

[192] Richter, K., Wolf, C.C., Gardner, A., Merz, C.B.: Detection of Unsteady Boundary Layer Transition Using Three Experimental Methods. In: 54th AIAA Aerospace Sciences Meeting, 4-8 January, San Diego, California, USA (2016). doi:10.2514/6.2016-1072

[193] Goerttler, A., Gardner, A., Richter, K.: Unsteady Boundary Layer Transition Detection by Automated Analysis of Hot Film Data. In: A. Dillmann, G. Heller, E. Krämer, C. Wagner, S. Bansmer, R. Radespiel, R. Semaan (Hgg.) New Results in Numerical and Experimental Fluid Mechanics XI, Notes on Numerical Fluid Mechanics and Multidisciplinary Design, Vol. 136, S. 387-395. Springer (2017). doi:10.1007/978-3-319-64519-3_35

[194] Bellhouse, B.J., Schultz, D.L.: Determination of mean and dynamic skin friction, separation and transition in low-speed flow with a thin-film heated element. Journal of Fluid Mechanics 24(2), 379-400 (1966). doi:10.1017/S0022112066000715 
[195] Hodson, H.P.: Boundary-Layer Transition and Separation Near the Leading Edge of a High-Speed Turbine Blade. Journal of Engineering for Gas Turbines and Power 107(1), 127-134 (1985). doi:10.1115/1.3239672

[196] Hodson, H.P., Huntsman, I., Steele, A.B.: An Investigation of Boundary Layer Development in a Multistage LP Turbine. Journal of Turbomachinery 116(3), 375-383 (1994). doi:10.1115/1.2929424

[197] Nitsche, W., Brunn, A.: Strömungsmesstechnik, 2. Aufl. Springer-Verlag (2006). doi:10.1007/3-540-32487-9

[198] Hultmark, M., Smits, A.J.: Temperature corrections for constant temperature and constant current hot-wire anemometers. Measurement Science and Technology 21(10) (2010). doi:10.1088/0957-0233/21/10/105404

[199] Wiggen, S.: Verbesserungspotential der Lagerung und Antriebswelle des Nickversuchsstands. Interner Bericht IB 232-2013 J 05, DLR, Institut für Aeroelastik (2013)

[200] Radespiel, R.: Grundlagen der Aerodynamik. In: C.C. Rossow, K. Wolf, P. Horst (Hgg.) Handbuch der Luftfahrzeugtechnik, Kap. 2.2, S. 57-83. Carl Hanser Verlag München (2014)

[201] Nitzsche, J., Brouwers, K.: A Numerical Study on Aerodynamic Resonance Phenomena in Transonic Airfoil Flow. In: Deutscher Luft- und Raumfahrtkongress (DLRK), 23 - 25 September, Darmstadt, Deutschland (2008)

[202] Nitzsche, J.: A numerical study on aerodynamic resonance in transonic separated flow. In: International Forum on Aeroelasticity and Structural Dynamics (IFASD), 21-25 June, Seattle, WA, USA (2009)

[203] Savitzky, A., Golay, M.J.E.: Smoothing and Differentiation of Data by Simplified Least Squares Procedures. Analytical Chemistry 36(8), 1627-1639 (1964). doi:10.1021/ac60214a047

[204] Allemang, R.J.: The modal assurance criterion - twenty years of use and abuse. Sound \& Vibration 37(8), 14-21 (2003)

[205] Magnus, K., Popp, K., Sextro, W.: Schwingungen, 9. auflage Aufl. Springer Vieweg (2013). doi:10.1007/978-3-8348-2575-9

[206] Seraudie, A., Aubert, E., Naudé, N., Cambronne, J.: Effect of plasma actuators on a flat plate laminar boundary layer in subsonic conditions. In: 3rd AIAA Flow Control 
Conference, 5-8 June, San Francisco, California, USA (2006). doi:10.2514/6.20063350

[207] Moreau, E.: Airflow control by non-thermal plasma actuators. Journal of Physics D: Applied Physics 605(40), 605-636 (2007). doi:10.1088/0022-3727/40/3/S01

[208] Corke, T.C., Post, M.L., Orlov, D.M.: Single dielectric barrier discharge plasma enhancedaerodynamics: physics, modeling and applications. Experiments in Fluids 46, 1-26 (2009). doi:10.1007/s00348-008-0582-5

[209] Corke, T.C., Enloe, C.L., Wilkinson, S.P.: Dielectric Barrier Discharge Plasma Actuators for Flow Control. Annual Review of Fluid Mechanics 42, 505-529 (2010). doi:10.1146/annurev-fluid-121108-145550

[210] Joussot, R., Weber, R., Leroy, A., Hong, D.: Transition control using a single plasma actuator. International Journal of Aerodynamics 3(1/2/3), 26-46 (2013). doi:10.1504/IJAD.2013.050912

[211] Grundmann, S., Tropea, C.: Experimental transition delay using glow-discharge plasma actuators. Experiments in Fluids 42, 653-657 (2007). doi:10.1007/s00348007-0256-8

[212] Grundmann, S., Tropea, C.: Active cancellation of artificially introduced Tollmien-Schlichting waves using plasma actuators. Experiments in Fluids 44, 795-806 (2008). doi:10.1007/s00348-007-0436-6

[213] Grundmann, S., Tropea, C.: Experimental damping of boundary-layer oscillations using DBD plasma actuators. International Journal of Heat and Fluid Flow 30, 394-402 (2009). doi:10.1016/j.ijheatfluidflow.2009.03.004

[214] Porter, C., McLaughlin, T., Enloe, C., Font, G., Roney, J., Baughn, J.: Boundary Layer Control Using a DBD Plasma Actuator. In: 45th AIAA Aerospace Sciences Meeting and Exhibit, 8-11 January, Reno, Nevada, USA (2007). doi:10.2514/6.2007786

[215] Magnier, P., Boucinha, V., Dong, B., Weber, R., Leroy-Chesneau, A., Hong, D.: Experimental Study of the Flow Induced by a Sinusoidal Dielectric Barrier Discharge Actuator and Its Effects on a Flat Plate Natural Boundary Layer. Journal of Fluids Engineering 131(1) (2009). doi:10.1115/1.3026722

[216] Post, M., Corke, T.: Separation Control using Plasma Actuators - Stationary and Oscillating Airfoils. In: 42nd AIAA Aerospace Sciences Meeting and Exhibit, 5-8 January, Reno, Nevada, USA (2004). doi:10.2514/6.2004-841 
[217] Post, M.L., Corke, T.C.: Separation Control Using Plasma Actuators: Dynamic Stall Vortex Control on Oscillating Airfoil. AIAA Journal 44(12), 3125-3135 (2006). doi:10.2514/1.22716

[218] Mai, H., Hebler, A., Koch, S.: High Reynolds Number Testing of a Laminar Airfoil under Forced Harmonic Pitching Oscillations at Cryogenic Conditions. In: International Forum on Aeroelasticity and Structural Dynamics (IFASD), 9-13 June, Savannah, Georgia, USA (2019)

[219] Schlichting, H., Ulrich, A.: Zur Berechnung des Umschlages laminar/turbulent. In: Jahrbuch 1942 der Deutschen Luftfahrtforschung, S. 8-35. R. Oldenbourg (1942)

[220] Schlichting, H., Truckenbrodt, E.: Aerodynamik des Flugzeuges, Zweiter Band, 3. Aufl. Springer-Verlag Berlin Heidelberg (2001). doi:10.1007/978-3-642-56911-2

[221] Brunton, S.L., Rowley, C.W.: Empirical state-space representations for Theodorsen's lift model. Journal of Fluids and Structures 38, 174-186 (2013). doi:10.1016/j.jfluidstructs.2012.10.005

[222] Fahrmeier, L., Künstler, R., Pigeot, I., Tutz, G.: Statistik, 6 Aufl. Springer-Verlag Berlin Heidelberg (2007). doi:10.1007/978-3-540-69739-8

[223] Försching, H.: Kritischer Vergleich der Methoden zur experimentellen Bestimmung der generalisierten Massen von Eigenschwingungsformen elastomechanischer Systeme. Tech. Rep. 88, VDI (1965)

[224] Wyler AG: Operator's Manual Clinotronic PLUS

[225] Micro-Epsilon Messtechnik GmbH \& Co. KG, Koenigbacher Str. 15, 94496 Ortenburg, Deutschland: Instruction Manual optoNCDT 1607

[226] Wiggen, S.: Verbesserungspotential der Lagerung und Antriebswelle des Nickversuchsstands. Interner Bericht IB 232-2013 J 05, DLR, Institut für Aeroelastik (2013)

[227] Werner, M.: Digitale Signalverarbeitung mit MATLAB ${ }^{\circledR}$, 4. Aufl. Vieweg + Teubner (2009). doi:10.1007/978-3-8348-9243-0

[228] Herlufsen, H.: Dual Channel FFT Analysis (Part I). Brüel \& Kjær (1984)

[229] Fritsch, F.N., Carlson, R.E.: Monotone piecewise cubic interpolation. SIAM Journal on Numerical Analysis 17(2), 238-246 (1980) 
[230] Basu, B.C., Hancock, G.J.: The unsteady motion of a two-dimensional aerofoil in incompressible inviscid flow. Journal of Fluid Mechanics 87(1), 159-178 (1978). doi:10.1017/S0022112078002980

[231] Katz, J., Weihs, D.: Wake rollup and the kutta condition for airfoils oscillating at high frequency. AIAA Journal 19(12), 1604-1606 (1981). doi:10.2514/3.60097

[232] Poling, D.R., Telionis, D.P.: The response of airfoils to periodic disturbances - the unsteady kutta condition. AIAA Journal 24(2), 193-199 (1986). doi:10.2514/3.9244

[233] Satyanarayana, B., Davis, S.: Experimental studies of unsteady trailing-edge conditions. AIAA Journal 16(2), 125-129 (1978). doi:10.2514/3.60869

[234] Ikeda, T., Atobe, T., Konishi, Y., Nagai, H., Asai, K.: Numerical study of windtunnel acoustic resonance induced by two-dimensional airfoil flow at low reynolds number. In: 29th Congress of the International Council of the Aeronautical Sciences (ICAS), 7-12 September 2014, St. Petersburg, Russia (2014)

[235] Voss, R.: Wind tunnel wall corrections. Report 336, AGARD (1998)

[236] Runyan, H.L., Woolston, D.S., Rainey, A.G.: Theoretical and experimental investigation of the effect of tunnel walls on the forces on an oscillating airfoil in two-dimensional subsonic compressible flow. Technical Note 3416, National Advisory Commitee for Aeronautics (1955) 


\section{Nomenklatur}

\section{Lateinische Buchstaben}

$a$

A

A

$b$

$B$

$\vec{B}$

$c$

$c_{d}$

$c_{l}$

$c_{l, \alpha}$

$c_{m}$

$c_{m, \alpha}$

$c_{p}$

$c_{p}{ }^{*}$

$c_{p, \alpha}$

$c_{w}$

$C\left(\omega^{*}\right)$

$d$

$d_{L}$

D

D

e

$\vec{E}$

$f$

$\hat{f}$

$f_{s}$

$F$ bzw. $\vec{F}$

$F_{A}$

Koeffizienten, Überhitzungsverhältnis, Beschleunigung

Flügelfläche, Konstante, Amplitude

Luftkraftmatrix, Drehmatrix der Modellbewegung

Koeffizient, halbe Höhe der Windkanal-Messstrecke

Konstante

Bezugspunkt für Drehmoment

Wellengeschwindigkeit, Flügeltiefe, spezifische Wärmekapazität

Druckwiderstandsbeiwert

Auftriebsbeiwert

instationärer Auftriebsbeiwert, bezogen auf $\alpha$

Nickmomentenbeiwert

instationärer Momentenbeiwert, bezogen auf $\alpha$

Druckbeiwert

kritischer Druckbeiwert

instationärer Druckbeiwert, bezogen auf $\alpha$

Widerstandsbeiwert

THEODORSEN-Funktion

Profildicke, Dämpfungskoeffizient

Abstandssignal Lasertriangulator

LEHR'sches Dämpfungsmaß

Dämpfungsmatrix, Drehmatrix

Drehpunkt bzw. elastische Achse

Drehpunkt des Modells

Frequenz, allgemeine Funktion, Hüllkurve

Fourier-Koeffizient

Abtastrate

Kraft bzw. Kraftvektor

Axialkraft (horizontal) 


\begin{tabular}{|c|c|}
\hline$F_{D}$ & Druckwiderstandskraft \\
\hline$F_{L}$ & Auftriebskraft \\
\hline$F_{N}$ & Normalkraft (vertikal) \\
\hline$F_{W}$ & Widerstandskraft \\
\hline $\mathcal{F}[f(t)]$ & Fourier-Transformierte von $f(t)$, auch $\mathcal{F}[f(t)]=F(\mathrm{i} \omega)$ \\
\hline$F\left(\omega^{*}\right)$ & Realteil der THEODORSEN-Funktion \\
\hline$g$ & allgemeine Funktion, Erdbeschleunigung \\
\hline$G\left(\omega^{*}\right)$ & Imaginärteil der THEODORSEN-Funktion \\
\hline$h$ & Schlagbewegung, Systemfunktion \\
\hline $\mathbf{H}$ & Translationsmatrix der Modellbewegung \\
\hline$H\left(\omega^{*}\right)$ & HANKEL'sche Zylinderfunktion \\
\hline$H(\mathrm{i} \omega)$ & Übertragungsfunktion \\
\hline i & imaginäre Einheit \\
\hline$I$ & Trägheitsmoment \\
\hline $\operatorname{Im}[$ ] & Imaginärteil \\
\hline$k$ & Wellenzahl, Steifigkeit, Anzahl Signalelemente \\
\hline $\mathbf{K}$ & Steifigkeitsmatrix \\
\hline$l$ & halbe Flügeltiefe \\
\hline $\mathbf{L}$ & aerodynamische Kräfte \\
\hline$m$ & Masse, diskrete Frequenz \\
\hline$M$ & Drehmoment \\
\hline M & Massenmatrix \\
\hline$M_{\alpha}$ & Nickmoment \\
\hline $\mathrm{Ma}$ & Mach-Zahl \\
\hline$\hat{n}$ & Einheits-Normalenvektor \\
\hline$N$ & Anzahl der Abtastwerte \\
\hline$O$ & mathematischer Operator \\
\hline$p$ & Druck \\
\hline$P$ & Leistung, Koeffizienten \\
\hline$q$ & generalisierte Koordinate, dynamischer Druck \\
\hline$Q$ & Wärme \\
\hline$\vec{r}$ & allgemeiner Verschiebungsvektor \\
\hline$r_{x x}$ & Autokorrelationsfunktion von $x$ \\
\hline$r_{x y}$ & Kreuzkorrelationsfunktion von $x$ und $y$ \\
\hline$R$ & elektrischer Widerstand \\
\hline$R_{s}$ & spezifische Gaskonstante \\
\hline $\operatorname{Re}$ & Reynolds-Zahl \\
\hline $\operatorname{Re}[]$ & Realteil \\
\hline
\end{tabular}


$s$

$\vec{s}$

$S_{A}$

$S_{\alpha}$

$S_{x x}$

$S_{x y}$

$\mathrm{Su}$

$t$

T

$\mathbf{T}$

$T\left(\omega^{*}\right)$

$u$

$U$

$v, V$

$\vec{v}$

$w$

$w[n]$

W

$x$

$x[n]$

$x_{\alpha}$

$x_{A}$

$\tilde{x}_{A}$

$x_{d}$

$x_{E}$

$x_{L}$

y

$z$

$z_{s}$

Spannweite, Schwerpunkt, Sensitivität

Profilkontur

Skalierungskonstante

statisches Massenmoment

Autoleistungsdichtespektrum von $x$

Kreuzleistungsdichtespektrum von $x$ und $y$

Sutherlandkonstante

Zeit

Periodendauer, Temperatur

Translationsmatrix

KÜSSNER'sche Wirbelschleppenfunktion

Strömungsgeschwindigkeit in x-Richtung

Strömungsgeschwindigkeit in x-Richtung, elektrische Spannung

Strömungsgeschwindigkeit in y-Richtung

allgemeiner Geschwindigkeitsvektor

Strömungsgeschwindigkeit in z-Richtung, Abwind

(diskrete) Fensterfunktion

Strömungsgeschwindigkeit in z-Richtung, Energie

Koordinate in x-Richtung, Zeitsignal, Auslenkung

zeitdiskretes Signal

Abstand des Schwerpunktes zur elastischen Achse

Ausgangsgröße, Abstand des Drehpunktes vom

Flügeltiefenmittelpunkt

entdimensionalisierter Drehpunktabstand $\tilde{x}_{A}=x_{A} / l$

Dickenrücklage, Druckpunkt

Eingangsgröße

Position Lasertriangulator

Koordinate in y-Richtung, Zeitsignal

Koordinate in z-Richtung

Skelettlinie

\section{Griechische Buchstaben}

$\alpha \quad$ Anstellwinkel, Drehbewegung

$\gamma$

Intermittenzfaktor, Zirkulationsverteilung (gebundenen Wirbel),

Abklingkonstante

$\Gamma \quad$ Zirkulation

Grenzschichtdicke, Hinterkantenwinkel, DiRAC'sche Deltafunktion 
Differenz, instationärer Anteil, anteilige Größe, Laplace-Operator Zirkulationsverteilung (freie Wirbel)

Verbindungsvektor

Stützstelle eines Profil-Flächenelementes in z-Richtung

Deformation, Substitutionsvariable

dynamische Viskosität

Isentropenexponent

Wellenlänge, Eigenwert

Streckung, PohlHausen-Parameter

kinematische Viskosität

Koordinate in $\mathrm{x}$-Richtung

Dichte

Standardabweichung, Fehlergröße

relative Zeit, entdimensionalisierte Zeit

Quasi-Wandschubspannung

Wandschubspannung

Amplitudenfunktion, Phasenwinkel

Phasenwinkel, Phasengang

WAGNER-Funktion

Stützstelle eines Profil-Flächenelementes in x-Richtung

Ortsvektor eines Profil-Flächenelementes

Stromfunktion, Profilkoordinate in x-Richtung

allgemeine Funktion

Kreisfrequenz

reduzierte Frequenz

\section{Subskripte}

$\begin{array}{ll}{[]_{0}} & \text { Ruhegröße, Ausgangsgröße } \\ {[]_{2 D}} & \text { bezogen auf den 2D-Support } \\ {[]_{a}} & \text { bezogen auf die Umgebung } \\ {[]_{\text {aero }}} & \text { bezogen auf die Aerodynamik } \\ {[]_{c_{p}}} & \text { bezogen auf den Druckbeiwert } \\ {[]_{e}} & \text { bezogen auf den Drehpunkt bzw. die elastische Achse } \\ {[]_{f}} & \text { bezogen auf den Flatterpunkt } \\ {[]_{h}} & \text { bezogen auf den Schlag } \\ {[]_{K}} & \text { bezogen auf das Windkanalplenum } \\ {[]_{l}} & \text { bezogen auf den Auftrieb }\end{array}$




$\begin{array}{ll}{[]_{m}} & \text { bezogen auf das Nickmoment } \\ {[]_{M}} & \text { bezogen auf das Windkanalmodell } \\ {[]_{r}} & \text { Referenzgröße } \\ {[]_{s}} & \text { bezogen auf den Verdichtungsstoß, den Schwerpunkt, } \\ & \text { einen Sensor } \\ {[]_{S}} & \text { bezogen auf Heißfilm-Sensor im Betriebszustand } \\ {[]_{\operatorname{tr}}} & \text { bezogen auf die Grenzschichttransition } \\ {[]_{Z}} & \text { bezogen auf einen Zusatz } \\ {[]_{\alpha}} & \text { bezogen auf die Drehung } \\ {[]_{\infty}} & \text { bezogen auf die freie Anströmung }\end{array}$

\section{Superskripte}

$\begin{array}{ll}{[]^{-1}} & \text { inverse Operation } \\ {[]^{*}} & \text { komplexe Konjugation, generalisierte Größe } \\ {[\overline{[}]} & \text { Mittelwert } \\ {[\hat{[]}} & \text { Einheitsvektor, Amplitude } \\ {[]^{\prime}} & \text { allgemeine Ableitung } \\ {[]^{\prime}} & \text { Ableitung nach der Zeit } \\ {[\overrightarrow{[]}} & \text { vektorielle Größe }\end{array}$

\section{Abkürzungen}

$\mathrm{AKF}$

AMIS

AVA

CFK

CPSD

CTA

DAQ

DBD

DFT

DFVLR
Autokorrelationsfunktion

Anlage zur Messung instationärer Signale

Aerodynamische Versuchsanstalt

Carbonfaserverstärkter Kunststoff

Kreuzleistungsdichtespektrum

(engl.: Cross-Power-Spectral-Density)

Konstant-Temperatur-Anemometrie

(engl.: Constant Temperature Anemometry)

Datenerfassung (engl.: Data Acquisition)

Dielektrisch behinderte Entladungen

(engl.: dielectric barrier discharge)

diskrete Fourier-Transformation

(engl.: Discrete Fourier Transformation)

Deutsche Forschungs- und Versuchsanstalt für Luft-

und Raumfahrt e. V. 


\begin{tabular}{|c|c|}
\hline DLR & Deutsches Zentrum für Luft- und Raumfahrt e. V. \\
\hline DNW & Deutsch Niederländische Windkanäle \\
\hline DoF & Freiheitsgrad (engl.: Degree of Freedom) \\
\hline DSP & Digital-Signal-Prozessor \\
\hline \multirow[t]{2}{*}{ FFT } & schnelle Fourier-Transformation \\
\hline & (engl.: Fast Fourier Transformation) \\
\hline FTA & Fourier-Transformation für Abtastsignale \\
\hline FVS & Flatterversuchsstand \\
\hline \multirow[t]{2}{*}{ HLFC } & hybride laminare Strömungskontrolle \\
\hline & (engl.: Hybrid Laminar Flow Control) \\
\hline ILES & Implizite Large-Eddy-Simulationen \\
\hline IR & Infrarot \\
\hline KKF & Kreuzkorrelationsfunktion \\
\hline $\mathrm{LCO}$ & Grenzzyklusschwingung (engl.: Limit Cycle Oscillation) \\
\hline LFC & laminare Strömungskontrolle (engl.: Laminar Flow Control) \\
\hline LTI & Linear Zeitinvariant (engl.: Linear Time-Invariant) \\
\hline LWIR & Langwellen-Infrarot \\
\hline MAC & Modal Assurance Criterion \\
\hline MHFS & Multiple Hot-Film Sensor \\
\hline NACA & National Advisory Committee for Aeronautics \\
\hline NAE & National Aeronautical Establishment \\
\hline NASA & National Aeronautics and Space Administration \\
\hline NLF & natürliche laminare Strömung (engl.: Natural Laminar Flow) \\
\hline NVS & Nickversuchsstand \\
\hline ONERA & Office National d'Études et de Recherches Aérospatiales \\
\hline PID & Proportional-Integral-Derivative \\
\hline PSD & Autoleistungsdichtespektrum (engl.: Power-Spectral-Density) \\
\hline RANS & Reynolds-gemittelte Navier-Stokes \\
\hline & (engl.: Reynolds Averaged Navier-Stokes) \\
\hline RMS & Effektivwert (engl.: Root Mean Square) \\
\hline TCT & Transonic Cryogenic Tunnel \\
\hline TS & TOLLMiEn-SChLiChting \\
\hline TWG & Transsonischer Windkanal Göttingen \\
\hline
\end{tabular}




\section{Abbildungsverzeichnis}

1.1. Darstellung der Wechselwirkung eines transsonischen Strömungsfeldes mit einer elastischen Struktur . . . . . . . . . . . . . . . . . 1

1.2. Beispielhafte Flatterfälle des CAST 10-2 Laminarfügels . . . . . . . . . 3

2.1. Darstellung des laminar-turbulenten Transitionsprozesses . . . . . . . . 12

2.2. Darstellung der Grenzschichttransition an einer ebenen Platte . . . . . . 13

2.3. Indifferenzkurven für verschiedene Geschwindigkeitsprofile . . . . . . . . 16

2.4. Geschwindigkeitsprofile und deren Ableitungen für verschiedene Druckgradienten . . . . . . . . . . . . . . . . . . 18

2.5. Vergleich eines konventionellen Flügelprofils mit einem Laminarprofil . . 19

2.6. Stoß-Interaktion mit einer laminaren und turbulenten Grenzschicht . . . 20

2.7. Verlauf der Wandschubspannung während einer Transition . . . . . . . . 22

2.8. Funktionsdiagramm und aeroelastisches Kräfte-Dreieck . . . . . . . . . . 24

2.9. Wirbelschleppe und Wirbelverteilung am schwingenden Flügelprofil . . . 26

2.10. Grafische Darstellung der Theodorsen-Funktion $C\left(\omega^{*}\right) \ldots \ldots$

2.11. Jones-Approximation der WAGner-Funktion . . . . . . . . . . . . . 29

2.12. Energiebilanz eines schwingenden Tragflügels . . . . . . . . . . . . . . 31

2.13. Flatterdiagramme eines generischen aeroelastischen Systems . . . . . . . 32

2.14. Darstellung des Transonic Dip und der nichtlinearen Systemreaktion . . 33

2.15. Verhalten der instationären Druckbeiwerte mit Stoßbewegung . . . . . . 34

3.1. Darstellung des TWG . . . . . . . . . . . . . . . . . . 37

3.2. Darstellung des NVS . . . . . . . . . . . . . . . . . . . . 39

3.3. Darstellung des FVS . . . . . . . . . . . . . . . . . . 40

3.4. Federsystem des FVS und 2D-Support . . . . . . . . . . . . . . . . . 41

3.5. Profilgeometrie und Sensorverteilung des CAST 10-2 Windkanalmodells 43

3.6. Darstellung der Messkette und Datenerfassung . . . . . . . . . . . . . 44

4.1. Darstellung der aerodynamischen Energiebilanz . . . . . . . . . . . . 57

4.2. Flussdiagramm zur Verarbeitung der Heißfilmsignale . . . . . . . . . . . 62

5.1. Verlauf der stationären, aerodynamische Beiwerte, Polaren und Position des Druckpunktes . . . . . . . . . . . . . . 66 
5.2. Stationäre aerodynamische Derivativa und statisches Stabilitätsmaß . . 68

5.3. Aerodynamische Beiwerte für freie und fixierte Transition . . . . . . . . 69

5.4. Druckverteilungen der stationären Polaren . . . . . . . . . . . . . . . 71

5.5. Druckverteilungen für $\mathrm{Ma}_{\infty}=0,75$ bei fixierter Transition . . . . . . . 73

5.6. Verteilung der Quasi-Wandschubspannung für die stationären Polaren . 75

5.7. Transitionsposition und Transitionsgradient für die stationären Polaren $\quad 76$

5.8. Standardabweichung der Druckbeiwerte für die stationären Polaren . . . 77

5.9. Standardabweichung der Quasi-Wandschubspannung für die stationären Polaren . . . . . . . . . . . . . . . . . . . . . 78

5.10. Druckverteilung, Quasi-Wandschubspannung, CTA-Zeitreihen und Leistungsdichtespektren für $\mathrm{Ma}_{\infty}=0,73$ und $\alpha=-0,15^{\circ} \ldots \ldots \ldots$

5.11. Modellbewegung $\Delta \alpha$ und $\Delta h$, aerodynamische Beiwerte $c_{m}$ und $c_{l}$ und Spektren für $\mathrm{Ma}_{\infty}=0,73$ und $\alpha=-0,15^{\circ} \ldots \ldots \ldots$

6.1. Instationäre Derivativa, gemessen mit einer Frequenzvariation mit $\hat{\alpha}=$ $0,10^{\circ}$ um $\bar{\alpha}=0,05^{\circ} \ldots \ldots \ldots \ldots \ldots \ldots$

6.2. Abhängigkeiten der instationären Derivativa bei $\mathrm{Ma}_{\infty}=0,73$ vom mittle-

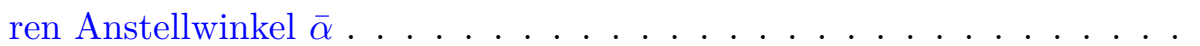

6.3. Instationäre Derivativa für eine freie und fixierte Grenzschichttransition bei $\mathrm{Ma}_{\infty}=0,73 \ldots \ldots \ldots \ldots \ldots \ldots$

6.4. Darstellung der Schwingungsmode des Windkanalmodells bei einer zwangserregten Nickschwingung . . . . . . . . . . . . . . . . . . . .

6.5. Energietransfer bei diskreter Frequenzvariation um $\bar{\alpha}=0,05^{\circ}$ mit $\hat{\alpha}=0,1^{\circ}$

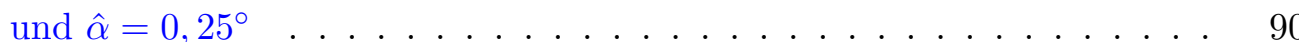

6.6. Energietransfer zwischen Struktur und Strömung für $\mathrm{Ma}_{\infty}=0,73$. . .

6.7. Instationäre Druckdifferenz bei $\hat{\alpha}=0,08^{\circ}, \bar{\alpha}=0,01^{\circ}$ und $\mathrm{Ma}_{\infty}=0,73$ für eine freie Grenzschichttransition . . . . . . . . . . . .

6.8. Druckverteilungen der Frequenzsweeps mit $\hat{\alpha}=0,08^{\circ}$ bei $\mathrm{Ma}_{\infty}=0,73$ und $\bar{\alpha}=0,01^{\circ}$

6.9. Instationäre Druckdifferenz bei $\hat{\alpha}=0,08^{\circ}, \bar{\alpha}=-0,08^{\circ}$ und $\mathrm{Ma}_{\infty}=0,73$ für eine fixierte Grenzschichttransition . . . . . . . . . . . . .

6.10. Phasengemittelte Quasi-Wandschubspannung für diskrete Frequenzvariation um $\bar{\alpha}=0,05^{\circ}$ mit $\hat{\alpha}=0,25^{\circ}$ bei $\mathrm{Ma}_{\infty}=0,73 \ldots \ldots \ldots$

6.11. Transitionsbewegung bei $\mathrm{Ma}_{\infty}=0,73$ für Frequenzvariationen um $\bar{\alpha}=$ $0,05^{\circ}$ mit $\hat{\alpha}=0,1^{\circ}, \hat{\alpha}=0,25^{\circ}$ und $\hat{\alpha}=0,5^{\circ} \ldots \ldots \ldots 10 \ldots \ldots$

6.12. Phasengemittelte Quasi-Wandschubspannung für diskrete Frequenzvariation um $\bar{\alpha}=0,05^{\circ}$ mit $\hat{\alpha}=0,25^{\circ}$ bei $\mathrm{Ma}_{\infty}=0,74$ und $\mathrm{Ma}_{\infty}=0,75 \ldots$

7.1. Strukturmodelle der zwei aeroelastischen Konfigurationen . . . . . . . . 
7.2. Eigenfrequenzen der aeroelastischen Konfigurationen

7.3. Stabilitätsgrenze der aeroelastischen 2-DoF Konfiguration bei freier Transition ........................... . . 110

7.4. Flatterfälle der 2-DoF Konfiguration . . . . . . . . . . . . . . . . . . . 111

7.5. Frequenzverlauf der aeroelastischen 2-DoF Konfiguration . . . . . . . . . 112

7.6. Stabilitätsgrenze und Flatterfall der 2-DoF Konfiguration bei fixierter

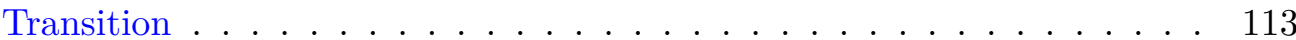

7.7. Stabilitätsgrenze der 1-DoF Konfiguration bei freier Transition . . . . . 114

7.8. Entwicklung des Flatterverhaltens der 1-DoF Konfiguration . . . . . . . 114

7.9. Vergleich zweier LCOs der aeroelastischen Konfigurationen . . . . . . 115

7.10. Frequenzverlauf der aeroelastischen 1-DoF Konfiguration . . . . . . . . 117

7.11. Bifurkationsverhalten bei einer Mach-Zahl-Variation der aeroelastischen 1-DoF-Konfiguration . . . . . . . . . . . . . . . . .

7.12. Bifurkationsverhalten bei einer Anstellwinkel-Variation der aeroelastischen 1-DoF-Konfiguration ...................

7.13. Freqeunzspektren bei Anstellwinkel-Variation der aeroelastischen 1-DoFKonfiguration . . . . . . . . . . . . . . . . . . . .

7.14. LCO bei $\mathrm{Ma}_{\infty}=0,73, p_{0}=55 \mathrm{kPa}$ und $\bar{\alpha}=0,06^{\circ}$..........

7.15. Leistungsdichtespektren des Nickmoments bei $\mathrm{Ma}_{\infty}=0,73, p_{0}=55 \mathrm{kPa}$ und $\bar{\alpha}=0,06^{\circ}$

7.16. Quasi-Wandschubspannung und Transitionsposition während des LCOs bei $\mathrm{Ma}_{\infty}=0,73, p_{0}=55 \mathrm{kPa}$ und $\bar{\alpha}=0,06^{\circ} \ldots . . . . . . . .$.

7.17. Druckverteilung während des LCOs bei $\mathrm{Ma}_{\infty}=0,73, p_{0}=55 \mathrm{kPa}$ und $\bar{\alpha}=0,06^{\circ}$

7.18. Stoß-Grenzschicht-Interaktion während des LCOs bei $\mathrm{Ma}_{\infty}=0,732$, $p_{0}=55 \mathrm{kPa}$ und $\bar{\alpha}=0,06^{\circ}$

7.19. Aerodynamischer Energietransfer während des LCOs bei $\mathrm{Ma}_{\infty}=0,732$,

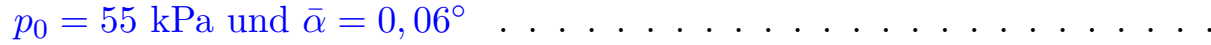

7.20. Aerodynamischer Leistungseintrag während des LCOs bei $\mathrm{Ma}_{\infty}=0,732$, $p_{0}=55 \mathrm{kPa}$ und $\bar{\alpha}=0,06^{\circ}$

8.1. Druckverteilungen und Quasi-Wandschubspannungen für drei Anstellwinkel im oberen Übergangsbereich der Laminardelle bei $\mathrm{Ma}_{\infty}=0,73 .$.

8.2. Darstellung des aeroelastischen Systems und Aufspaltung in aerodynamische und strukturelle Schwinger . . . . . . . . . . . . . . . .

A.1. Pohlhausen-Profile und zugehörige Indifferenzkurven . . . . . . . . . . 180

A.2. Bewegung der Skelettlinie am schwingenden Tragflügelprofil . . . . . . . 183

A.3. Induzierter Abwind des schwingenden Tragflügelprofils . . . . . . . . . . 184 
B.1. Messfehler der Mach-Zahl, der statischen Temperatur, der dynamischen Viskosität und der Reynolds-Zahl . . . . . . . . . . . . . . . . . . . . 189

B.2. Federkennlinien der Schlagfedern des FVS . . . . . . . . . . . . . . . 192

B.3. Generalisierte Massen der Schlagfedersysteme . . . . . . . . . . . . . . 193

B.4. Federkennlinien der Torsionsfedern des FVS . . . . . . . . . . . . . . . . 194

B.5. Generalisierte Trägheitsmomente der Torsionsfedern . . . . . . . . . . . 195

B.6. Vermessung der Profilgeometrie entlang der Flügeltiefe . . . . . . . . . . 197

B.7. Vermessung der Profilgeometrie entlang der Spannweite . . . . . . . . 198

B.8. Beispielhafte Kalibrierung der Kulite-Drucksensoren (XCQ-093D) . . . . 199

B.9. Messfehler des dynamischen Drucks und des Druckbeiwertes . . . . . . . 200

B.10.Messfehler des instationären Winkelanteils und Fehler des Anstellwinkels bei Flatterversuchen . . . . . . . . . . . . . . . . . . . . . 203

B.11.Lineare Regression der Winkelkalibrierung und Messfehler des Anstellwinkels bei Nickversuchen . . . . . . . . . . . . . . . . . . . . . . . 204

C.1. Darstellung zur Auswirkung der Profilkoordinaten-Substitution . . . . . 211

C.2. Darstellung der Diskretisierung der Profiloberfläche . . . . . . . . . . . . 211

C.3. Grafische Veranschaulichung zur Definition der Phasenverschiebung . . . 215

C.4. Argand-Diagramm zur Darstellung der Übertragungsfunktion $H(\mathrm{i} \omega)$. . 216

D.1. Druckverteilungen und Quasi-Wandschubspannungen für $\alpha=1,54^{\circ}$ und $\alpha=1,75^{\circ}$ bei $\mathrm{Ma}_{\infty}=0,76 \ldots \ldots \ldots \ldots$

D.2. Druckverteilung, Quasi-Wandschubspannung, CTA-Zeitreihen und Leistungsdichtespektren für $\mathrm{Ma}_{\infty}=0,73$ und $\alpha=-0,37^{\circ} \ldots \ldots 218$

D.3. Modellbewegung, aerodynamische Beiwerte und Spektren für $\mathrm{Ma}_{\infty}=0,73$

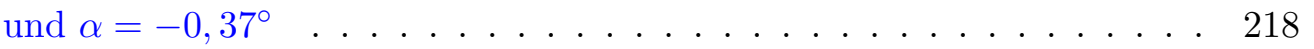

D.4. Druckverteilung, Quasi-Wandschubspannung, CTA-Zeitreihen und Leistungsdichtespektren für $\mathrm{Ma}_{\infty}=0,73$ und $\alpha=0,06^{\circ} \ldots \ldots$. . .

D.5. Modellbewegung, aerodynamische Beiwerte und Spektren für $\mathrm{Ma}_{\infty}=0,73$ und $\alpha=0,06^{\circ} \ldots \ldots \ldots \ldots \ldots \ldots \ldots \ldots$

D.6. Druckverteilung, Quasi-Wandschubspannung, CTA-Zeitreihen und Leistungsdichtespektren für $\mathrm{Ma}_{\infty}=0,74$ und $\alpha=-0,38^{\circ} \ldots \ldots$. .

D.7. Modellbewegung, aerodynamische Beiwerte und Spektren für $\mathrm{Ma}_{\infty}=0,74$ und $\alpha=-0,38^{\circ} \ldots \ldots \ldots \ldots \ldots \ldots \ldots$

D.8. Druckverteilung, Quasi-Wandschubspannung, CTA-Zeitreihen und Leistungsdichtespektren für $\mathrm{Ma}_{\infty}=0,74$ und $\alpha=-0,16^{\circ} \ldots \ldots$

D.9. Modellbewegung, aerodynamische Beiwerte und Spektren für $\mathrm{Ma}_{\infty}=0,74$ und $\alpha=-0,16^{\circ} \ldots \ldots \ldots \ldots \ldots \ldots$ 
D.10.Druckverteilung, Quasi-Wandschubspannung, CTA-Zeitreihen und Leistungsdichtespektren für $\mathrm{Ma}_{\infty}=0,74$ und $\alpha=0,06^{\circ} \ldots \ldots 22$

D.11.Modellbewegung, aerodynamische Beiwerte und Spektren für $\mathrm{Ma}_{\infty}=0,74$ und $\alpha=0,06^{\circ} \ldots \ldots \ldots \ldots \ldots \ldots \ldots \ldots$

D.12.Druckverteilung, Quasi-Wandschubspannung, CTA-Zeitreihen und Leistungsdichtespektren für $\mathrm{Ma}_{\infty}=0,75$ und $\alpha=-0,58^{\circ} \ldots \ldots$.

D.13.Modellbewegung, aerodynamische Beiwerte und Spektren für $\mathrm{Ma}_{\infty}=0,75$ und $\alpha=-0,58^{\circ} \ldots \ldots \ldots \ldots \ldots \ldots \ldots \ldots$

D.14.Druckverteilung, Quasi-Wandschubspannung, CTA-Zeitreihen und Leistungsdichtespektren für $\mathrm{Ma}_{\infty}=0,75$ und $\alpha=-0,37^{\circ} \ldots \ldots$. .

D.15.Modellbewegung, aerodynamische Beiwerte und Spektren für $\mathrm{Ma}_{\infty}=0,75$ und $\alpha=-0,37^{\circ}$

D.16.Druckverteilung, Quasi-Wandschubspannung, CTA-Zeitreihen und Leistungsdichtespektren für $\mathrm{Ma}_{\infty}=0,75$ und $\alpha=-0,15^{\circ} \ldots \ldots \ldots$

D.17.Modellbewegung, aerodynamische Beiwerte und Spektren für $\mathrm{Ma}_{\infty}=0,75$ und $\alpha=-0,15^{\circ} \ldots \ldots \ldots \ldots \ldots \ldots \ldots$

E.1. Instationäre Derivativa aus Frequenzvariation mit $\hat{\alpha}=0,25^{\circ} \ldots \ldots$.

E.2. Instationäre Derivativa für eine freie und fixierte Grenzschichttransition bei $\mathrm{Ma}_{\infty}=0,74 \ldots \ldots \ldots \ldots \ldots \ldots$

E.3. Instationäre Derivativa für eine freie und fixierte Grenzschichttransition bei $\mathrm{Ma}_{\infty}=0,75 \ldots \ldots \ldots \ldots \ldots \ldots$

E.4. Energietransfer zwischen Struktur und Strömung für $\mathrm{Ma}_{\infty}=0,74$ und

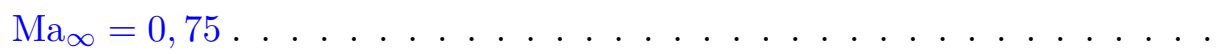

E.5. Instationäre Druckdifferenz und Druckverteilungen eines Frequenzsweeps mit $\hat{\alpha}=0,08^{\circ}$ bei $\mathrm{Ma}_{\infty}=0,74$ und $\bar{\alpha}=0,07^{\circ}$

E.6. Instationäre Druckdifferenz und Druckverteilungen eines Frequenzsweeps mit $\hat{\alpha}=0,08^{\circ}$ bei $\mathrm{Ma}_{\infty}=0,75$ und $\bar{\alpha}=-0,15^{\circ}$

E.7. Instationäre Druckdifferenz für eine fixierte Grenzschichttransition bei $\mathrm{Ma}_{\infty}=0,74$ und $\mathrm{Ma}_{\infty}=0,75$

E.8. Abhängigkeit der instationären Druckdifferenz von $\bar{\alpha}$ bei $\mathrm{Ma}_{\infty}=0,73$.

E.9. Abhängigkeit der instationären Druckdifferenz von $\bar{\alpha}$ bei $\mathrm{Ma}_{\infty}=0,74$.

E.10.Abhängigkeit der instationären Druckdifferenz von $\bar{\alpha}$ bei $\mathrm{Ma}_{\infty}=0,75$.

E.11.Phasengemittelte Quasi-Wandschubspannung für diskrete Frequenzvariation um $\bar{\alpha}=0,05^{\circ}$ mit $\hat{\alpha}=0,1^{\circ}$ bei $\mathrm{Ma}_{\infty}=0,73 \ldots \ldots \ldots$

E.12.Phasengemittelte Quasi-Wandschubspannung für diskrete Frequenzvariation um $\bar{\alpha}=0,05^{\circ}$ mit $\hat{\alpha}=0,5^{\circ}$ bei $\mathrm{Ma}_{\infty}=0,73 \ldots \ldots \ldots$

E.13.Phasengemittelte Quasi-Wandschubspannung für diskrete Frequenzvariation um $\bar{\alpha}=0,05^{\circ}$ mit $\hat{\alpha}=0,25^{\circ}$ bei $\mathrm{Ma}_{\infty}=0,74 \ldots \ldots$. . . . 
E.14.Phasengemittelte Quasi-Wandschubspannung für diskrete Frequenzvariation um $\bar{\alpha}=0,05^{\circ}$ mit $\hat{\alpha}=0,25^{\circ}$ bei $\mathrm{Ma}_{\infty}=0,75 \ldots \ldots 240$

E.15. Transitionsbewegung für die Frequenzvariationen bei $\mathrm{Ma}_{\infty}=0,74 \mathrm{um}$

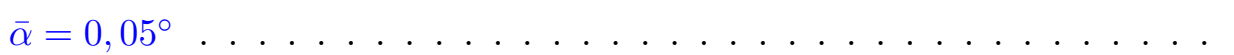

E.16. Transitionsbewegung für die Frequenzvariationen bei $\mathrm{Ma}_{\infty}=0,75 \mathrm{um}$

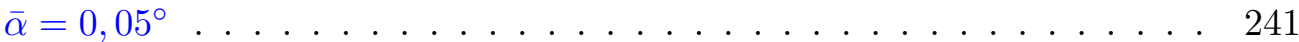

F.1. Darstellung der Signalfilterung zur Dämpfungsbestimmung . . . . . . . 243

F.2. Veranschaulichung der Dämpfungsbestimmung . . . . . . . . . . . . . 244

F.3. Schwingungsmoden der aeroelastischen 2-DoF Konfiguration . . . . . . . 246

F.4. Schwingungsmoden der aeroelastischen 1-DoF Konfiguration . . . . . . . 247

F.5. Windkanalresonanzfrequenzen für die geschlossene Messstrecke des DNWTWG ............. 249

F.6. Bifurkationsdiagramm der Schlagbewegung und Frequenzverlauf bei einer Variation des mittleren Anstellwinkels . . . . . . . . . . . . . . . . . . 249

F.7. Zeitreihen des LCO bei $\mathrm{Ma}_{\infty}=0,73, p_{0}=55 \mathrm{kPa}$ und $\bar{\alpha}=0,06^{\circ} \ldots .250$

F.8. Aerodynamischer Gesamt-Leistungseintrag $P_{m}+P_{l}$ während des LCOs bei $\mathrm{Ma}_{\infty}=0,732, p_{0}=55 \mathrm{kPa}$ und $\bar{\alpha}=0,06^{\circ} \ldots \ldots \ldots 251$ 


\section{Tabellenverzeichnis}

2.1. Klassifizierung der aeroelastischen Probleme . . . . . . . . . . . . . 24

3.1. Strukturmechanische Parameter des FVS . . . . . . . . . . . . . 41

3.2. Strukturmechanische Parameter des CAST 10-2 Windkanalmodells . . . 44

7.1. Strukturmechanische Parameter der aeroelastischen Konfigurationen . . 108

7.2. Schwingungsmoden, Eigenfrequenzen und Dämpfungen der aeroelastischen Konfigurationen . . . . . . . . . . . . . . . . . . . . . . . 109

7.3. Phasenbeziehungen während des aerodynamischen und aeroelastischen LCOs bei $\mathrm{Ma}_{\infty}=0,73, p_{0}=55 \mathrm{kPa}$ und $\bar{\alpha}=0,06^{\circ} \ldots \ldots \ldots 124$

B.1. Konstanten zur Berechnung der Strömungsparameter . . . . . . . . . . . 187

B.2. Federkonstanten der Schlagfedern des FVS . . . . . . . . . . . . . . . . . 192

B.3. Generalisierte Massen der Schlagfedersysteme . . . . . . . . . . . . . . . 193

B.4. Federkonstanten der Torsionsfedern des FVS . . . . . . . . . . . . . . 195

B.5. Generalisierte Trägheitsmomente der Torsionsfedern . . . . . . . . . . . 196

B.6. Abweichung der Profilgeometrie entlang der Flügeltiefe . . . . . . . . . . 197

B.7. Abweichung der Profilgeometrie entlang der Spannweite . . . . . . . . . 198

F.1. Abklingkoeffizienten und LEHR'sche Dämpfungsmaße der aeroelastischen Konfigurationen . . . . . . . . . . . . . . . . . . . . . 245 

Anhang 



\section{A. Ergänzungen zu Kapitel 2}

\section{A.1. Stabilitätsrechnung mit variierendem Pohlhausen-Parameter}

Der Einfluss des Druckgradienten auf die Stabilität der laminaren Grenzschicht kann mit einer Stabilitätsrechnung mithilfe von Gl. (2.1.8) verdeutlicht werden. Die Geschwindigkeitsprofile der laminaren Plattengrenzschicht $U(x, z)$ werden hierfür in Form einer einparametrigen Kurvenschar durch Pohlhausen-Profile [85]

$$
\frac{U(x, z)}{U_{e}}=2 \eta-2 \eta^{3}+\eta^{4}+\frac{\Lambda}{6}\left(\eta-3 \eta^{2}+3 \eta^{3}-\eta^{4}\right), \quad \text { mit } \quad \eta=\frac{z}{\delta}
$$

approximiert. Der Formparameter $\Lambda$ (PohlHausen-Parameter)

$$
\Lambda=\frac{\delta^{2}}{\nu} \frac{\mathrm{d} U_{e}}{\mathrm{~d} x}=-\frac{\delta^{2}}{\mu U_{e}} \frac{\mathrm{d} p}{\mathrm{~d} x}
$$

kann mithilfe der Wandbindung nach Gl. (2.1.11) als entdimensionalisierter Druckgradient interpretiert werden. Entsprechend folgt für $\Lambda<0$ ein Druckanstieg und für $\Lambda>0$ ein Druckabfall. Die aus Gl. (A.1.1) folgende Schar an Geschwindigkeitsprofilen ist in Abb. A.1(a) dargestellt. Der Formparameter ist dabei in einem Bereich von $\Lambda \in$ [-12:12] physikalisch limitiert. Für $\Lambda>12$ würde die Geschwindigkeit innerhalb der Grenzschicht größer als die Geschwindigkeit der Außenströmung $U_{e}$. In die andere Richtung entspricht hingegen $\Lambda=-12$ dem Ablösepunkt der Grenzschicht, jenseits dessen die Grenzschichtgleichungen ihre Gültigkeit verlieren. Aus Abb. A.1(a) wird die Ausbildung von Wendepunkten für eine verzögerte Strömung $(\Lambda<0)$ deutlich.

Die Ergebnisse der Stabilitätsrechnung mit den PohlHausen-Profilen als Ansatz für $U(z)$ sind in Abb. A.1(b) dargestellt. Diese basieren auf frühen analytischen Stabilitätsrechnungen ${ }^{1}$ durch [219]. $\operatorname{Re}_{\text {ind }}(\Lambda)$ nimmt für einen kleiner werdenden Formparameter deutlich ab. Ein Druckanstieg führt folglich zu einem früheren Anwachsen von primären Eigenmoden.

\footnotetext{
${ }^{1}$ In [219] wurde anstelle eines Polynomansatzes 4. Ordnung (P4-Verfahren) gemäß Gl. (A.1.1) ein erweitertes Pohlhausen-Verfahren mit einem Polynom 6. Grades (P6-Verfahren) verwendet. Hiermit wurde eine genauere Approximation der Geschwindigkeitsprofile $U(z)$ und insbesondere von $\frac{\mathrm{d} U}{\mathrm{~d} z}$ und $\frac{\mathrm{d}^{2} U}{\mathrm{~d} z^{2}}$ erzielt. Qualitativ sind die Ergebnisse zwischen dem P4- und dem P6-Verfahren jedoch identisch. Abb. A.1(b) zeigt daher nur qualitative Ergebnisse ohne Zahlenwerte.
} 


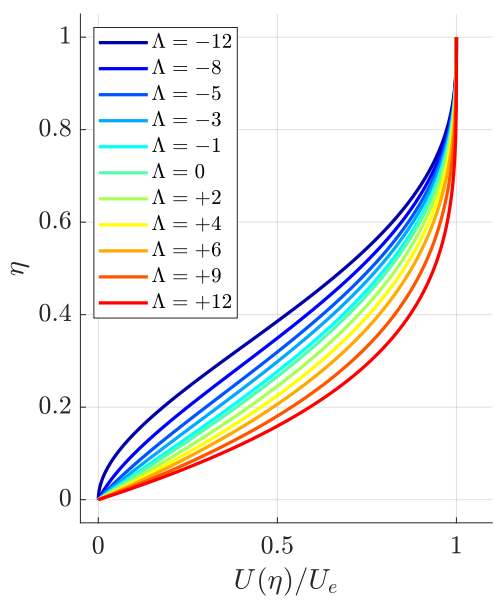

(a) Pohlhausen-Profile

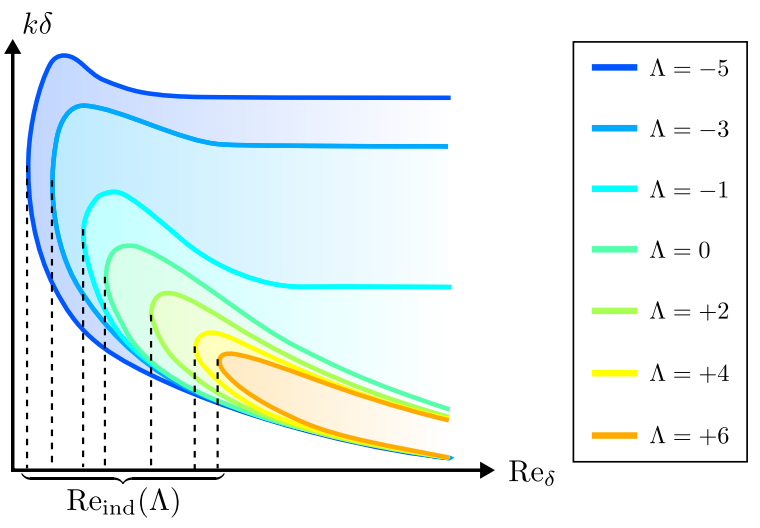

(b) Indifferenzkurve für verschiedene $\Lambda$

Abbildung A.1.: Pohlhausen-Profile gemäß Gl. (A.1.1) für die laminare Grenzschicht (a) und qualitative Indifferenzkurven als Ergebnisse der Stabilitätsrechnung auf Basis des Pohlhausen-Verfahrens (b), verändert nach [1, S.447] und [219].

Zum anderen nimmt auch der instabile Bereich und damit das angefachte Störungsspektrum mit abnehmenden $\Lambda$ signifikant zu, zumal auch der Rezeptivitätsprozess durch positive Druckgradienten begünstigt wird [77,78]. So tritt für $\Lambda<0$ eine reibungslose Instabilität für Re $\rightarrow \infty$ auf. Für $\Lambda \geq 0$ laufen die Zweige der Indifferenzkurven gegen $k \delta=0$ zusammen [219] (vgl. 2.1.1).

\section{A.2. Kinematische Strömungsbedingung}

Ein durch eine Skelettlinie $z_{s}(x, t)$ ersetztes Tragflügelprofil fordert als Randbedingung die Undurchdringlichkeit des Profils. Im stationären Fall heißt dies, dass die Normalkomponente der Strömungsgeschwindigkeit auf der Profilobefläche verschwinden muss [220, S.19 f.]. Im instationären Fall (also bei einem bewegten Modell) muss somit gewährleistet sein, dass die Normalkomponente der Geschwindigkeitsvektoren der Profilverformung $\vec{v}_{P}$ und der Strömungsgeschwindigkeit auf der Profiloberfläche $\vec{u}_{P}$ identisch sind [119].

Der Geschwindigkeitsvektor der Profilverformung folgt aus der Bewegung der Skelettlinie in z-Richtung in einfacher Weise gemäß

$$
\vec{v}_{P}=\left(0, \frac{\partial z_{s}}{\partial t}\right)^{T}
$$

Der Geschwindigkeitsvektor der Strömung an der Profiloberfläche ist gegeben durch

$$
\vec{u}_{P}=\left(u_{\infty}+u_{P}, w_{P}\right)^{T} .
$$


Dabei ist $u_{\infty}$ die Anströmungsgeschwindigkeit, $u_{P}$ die in x-Richtung durch die Profilverformung induzierte Geschwindigkeitskomponente und $w_{P}$ die induzierte Komponente in z-Richtung. Ferner werden $u_{P}$ und $w_{P}$ als klein gegenüber der Anströmung $u_{\infty}$ angenommen. Ein Normalenvektor zur Skelettlinie ist gegeben durch $\vec{n}_{P}=\left(-\frac{\partial z_{s}}{\partial x}, 1\right)^{T}$. Die oben geforderte Randbedingung führt damit zu

$$
\begin{aligned}
\vec{v}_{P} \cdot \vec{n}_{P} & =\vec{u}_{P} \cdot \vec{n}_{P} \\
\Leftrightarrow \quad \frac{\partial z_{s}}{\partial t} & =-\left(u_{\infty}+u_{P}\right) \frac{\partial z_{s}}{\partial x}+w_{P} .
\end{aligned}
$$

Unter Vernachlässigung von $u_{P}$ gegenüber $u_{\infty}$ folgt aus Gl. (A.2.4) die kinematische Strömungsbedingung für den bewegten Tragflügel bzw. für den induzierten Abwind w:

$$
w=w_{p}=\frac{\partial z_{s}}{\partial t}+u_{\infty} \frac{\partial z_{s}}{\partial x}
$$

\section{A.3. Zur Lösung der Integralgleichung GI. (2.2.8)}

Die Berechnung der gebundenen Zirkulationsverteilung $\gamma(x, t)$ erfordert die Lösung der Integralgleichung Gl. (2.2.8), wie diese in 2.2.1 für den harmonisch schwingenden Flügel hergeleitet ist. Ein Lösungsansatz nach Küssner [118] basiert dabei auf einem Fourier-Reihenansatz für den Abwind bei einer beliebigen Skelettverformung gemäß

$$
w(\Theta, t)=-u_{\infty} \exp (\mathrm{i} \omega t)\left[P_{0}+2 \sum_{1}^{\infty} P_{n} \cos (n \Theta)\right] .
$$

Dabei wird $\frac{x}{l}$ durch $\cos (\Theta)$ substituiert, mit $\Theta=\pi$ an der Flügelvorderkante und $\Theta=0$ an der Hinterkante [109, S.255] (auch [108, S.408], abweichend zu [118, 119]). Für die Koeffizienten gilt

$$
\begin{aligned}
& P_{0}=-\frac{1}{\pi u_{\infty}} \exp (-\mathrm{i} \omega t) \int_{0}^{\pi} w(\Theta, t) \mathrm{d} \Theta \\
& P_{n}=-\frac{1}{\pi u_{\infty}} \exp (-\mathrm{i} \omega t) \int_{0}^{\pi} w(\Theta, t) \cos (n \Theta) \mathrm{d} \Theta .
\end{aligned}
$$

Die Verteilung der gebundenen Wirbel wird durch die Birnbaum-ACKERMann'sche Ansatzfunktion $^{2}$ (vgl. auch [117, S.420 ff.])

$$
\gamma(\Theta, t)=u_{\infty} \exp (\mathrm{i} \omega t)\left[2 a_{0} \frac{1-\cos (\Theta)}{\sin (\Theta)}+4 \sum_{1}^{\infty} a_{n} \frac{\sin (n \Theta)}{n}\right]
$$

\footnotetext{
${ }^{2}$ Es gilt: $[1-\cos (\Theta)] / \sin (\Theta)=\tan (\Theta / 2)$
} 
ausgedrückt [118]. Gl. (A.3.3) genügt dabei der KUTTA-Bedingung eines glatten Abflusses an der Hinterkante ${ }^{3}$. Durch Einsetzen von Gl. (A.3.3) in die Integralgleichung Gl. (2.2.8) gelingt zusammen mit Gl. (2.2.5) (in [118] in leicht abgewandelter Form) eine Lösung des Integrals (siehe Gl. (26) in [118]). Über einen Vergleich der Lösung mit Gl. (A.3.1) folgen die Koeffizienten $a_{0}$ und $a_{n}$ nach [109, S.255] bzw. [108, S.408] zu

$$
\begin{aligned}
& a_{0}=\frac{\mathrm{i} H_{0}^{(2)} P_{1}+H_{1}^{(2)} P_{0}}{\mathrm{i} H_{0}^{(2)}+H_{1}^{(2)}}=\frac{1+T\left(\omega^{*}\right)}{2}\left(P_{0}+P_{1}\right)-P_{1}, \\
& a_{n}=\frac{\mathrm{i} \omega^{*}}{2} P_{n-1}+n P_{n}-\frac{\mathrm{i} \omega^{*}}{2} P_{n+1} .
\end{aligned}
$$

Die auftretenden Funktionen $H_{0}^{(2)}$ und $H_{1}^{(2)}$ bezeichnen HANKEL'sche Zylinderfunktionen 2. Art der Ordnung 0 und 1. Die Funktion

$$
T\left(\omega^{*}\right)=\frac{H_{1}^{(2)}\left(\omega^{*}\right)-\mathrm{i} H_{0}^{(2)}\left(\omega^{*}\right)}{H_{1}^{(2)}\left(\omega^{*}\right)+\mathrm{i} H_{0}^{(2)}\left(\omega^{*}\right)}=2 C\left(\omega^{*}\right)-1
$$

ist die KÜssneR'sche Wirbelschleppenfunktion und steht in direktem Zusammenhang mit der in 2.2.1 dargestellten Theodorsen-Funktion $C\left(\omega^{*}\right)$ nach [120]. Mit Gl. (2.2.9) und (2.2.10) folgt dann aus der Wirbelverteilung $\gamma(\Theta, t)$ der instationäre Auftrieb zu

$$
\frac{F_{L}(t)}{\mathrm{d} y}=\rho_{\infty} u_{\infty} l \int_{0}^{\pi} \gamma(\Theta, t) \sin (\Theta) \mathrm{d} \Theta=\pi \rho_{\infty} u_{\infty}^{2} l \exp (\mathrm{i} \omega t)\left(2 a_{0}+2 a_{1}\right)
$$

und das instationäre Moment bzgl. des $c / 4$-Punktes $\left(x_{A}=-\frac{1}{2}\right) \mathrm{zu}$

$$
\begin{aligned}
\frac{M_{\alpha}(t)}{\mathrm{d} y} & =\rho_{\infty} u_{\infty} l^{2} \int_{0}^{\pi} \gamma(\Theta, t)\left[\frac{1}{2}-\cos (\Theta)\right] \sin (\Theta) \mathrm{d} \Theta \\
& =\pi \rho_{\infty} u_{\infty}{ }^{2} l^{2} \exp (\mathrm{i} \omega t)\left(a_{1}-\frac{a_{2}}{2}\right) .
\end{aligned}
$$

$\mathrm{Zu}$ analogen Ergebnissen führte auch eine geschlossenen Lösung der Integralgleichung Gl. (2.2.8) nach Schwarz [119]. Durch umschreiben von Gl. (2.2.8) in die Form (vgl. Gl. (24) und (28) in [119])

$$
g(x)=\frac{1}{2 \pi} \oint_{-1}^{1} \frac{f(\xi)}{x-\xi} \mathrm{d} \xi
$$

lässt sich ausnutzen, dass Gl. (A.3.8) eine eindeutige Lösung in der Form

$$
f(x)=-\frac{2}{\pi} \sqrt{\frac{1-x}{1+x}} \oint_{-1}^{1} \frac{g(\xi)}{x-\xi} \sqrt{\frac{1+\xi}{1-\xi}} \mathrm{d} \xi
$$

\footnotetext{
${ }^{3}$ Für $\Theta=0$ (Hinterkante) folgt aus Gl. (A.3.3) $\gamma(\Theta=0)=0$, mit $\lim _{\Theta \rightarrow 0}\left[\frac{1-\cos (\Theta)}{\sin (\Theta)}\right]=0$.
} 
besitzt. Die Lösung von Gl. (2.2.8) ist somit auf die Lösung von Gl. (A.3.8) zurückgeführt [119], welche Ähnlichkeiten zur PRANDTL'schen Integralgleichung der Zirkulationsverteilung aufweist (vgl. [220, S.7 ff.]). Nach „notwendigen Integralumformungen und umfangreichen Rechnungen" führt dies auf einen direkten Zusammenhang zwischen der instationären Druckdifferenz $\Delta p(\Theta, t)$ und dem Abwind $w(\Theta, t)$ gemäß (Formulierung nach $[109$, S.258])

$$
\begin{aligned}
\Delta p(\Theta, t)= & \frac{2}{\pi} \rho_{\infty} u_{\infty} \int_{0}^{\pi} w(\vartheta, t)\left[\mathrm{i} \omega^{*} L(\Theta, \vartheta) \sin (\vartheta)+\frac{\sin (\Theta)}{\cos (\vartheta)-\cos (\Theta)}\right. \\
& \left.+\tan \left(\frac{\vartheta}{2}\right)\left(\frac{1+T\left(\omega^{*}\right)}{2}[1+\cos (\vartheta)]-\cos (\vartheta)\right)\right] \mathrm{d} \vartheta, \\
\text { mit } L(\Theta, \vartheta)= & \ln \left|\frac{\sin \left(\frac{\Theta+\vartheta}{2}\right)}{\sin \left(\frac{\Theta-\vartheta}{2}\right)}\right|=\frac{1}{2} \ln \left(\frac{1-\cos (\Theta+\vartheta)}{1-\cos (\Theta-\vartheta)}\right) .
\end{aligned}
$$

Mit $\Delta p(\Theta, t)$ folgt dann aus Gl. (2.2.9) und (2.2.10) wieder der Auftrieb Gl. (A.3.6) und das Moment Gl. (A.3.7).

\section{A.4. Berechnung der instationären Luftkräfte}
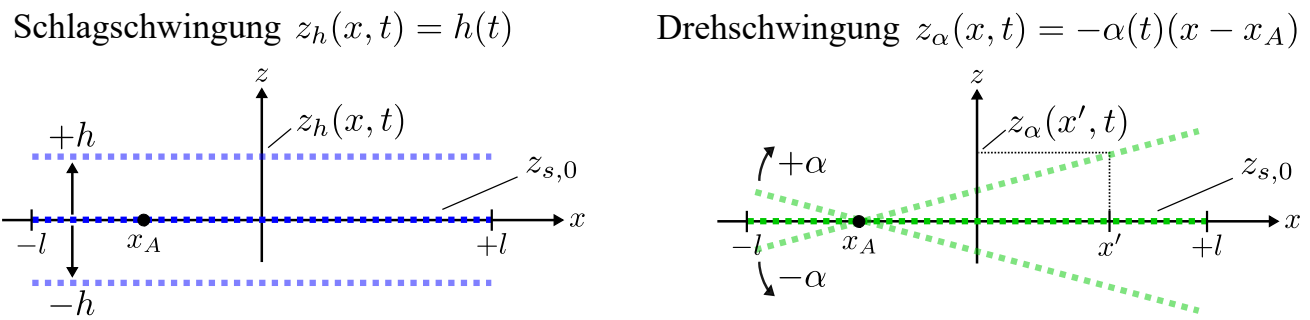

Abbildung A.2.: Bewegung der Skelettlinie am schwingenden Tragflügelprofil, unterteilt in eine Schlagschwingung $z_{h}$ (links) und eine Drehschwingung $z_{\alpha}$ (rechts). Die Vorzeichenkonvention orientiert sich an Abb. 2.9.

Für einen Tragflügel, der Schlag- und Drehschwingungen $h(t)$ und $\alpha(t)$ ausführt, beträgt die vertikale Auslenkung der Skelettlinie $z_{s}(x, t)$ für kleine Winkel $(\tan (\alpha) \approx \alpha)$

$$
z_{s}(x, t)=h-\alpha\left(x-x_{A}\right)
$$

Wie in Abb. A.2 dargestellt, setzt sich die Bewegung additiv aus der Schlagkomponente und der Drehkomponente zusammen. Für den Abwind folgt gemäß Gl. (2.2.7) dann

$$
w(x, t)=\dot{h}-\dot{\alpha}\left(x-x_{A}\right)-u_{\infty} \alpha .
$$

Dieser setzt sich ebenfalls additiv aus einem induzierten Abwind der Schlagbewegung $\dot{h}$, der Drehbewegung $-\dot{\alpha}\left(x-x_{a}\right)$ und des Anstellwinkels $-u_{\infty} \alpha$ zusammen, wie in Abb. A.3 dargestellt (vgl. auch [109, S.256 f.]). Eine positive, nach oben gerichtete 

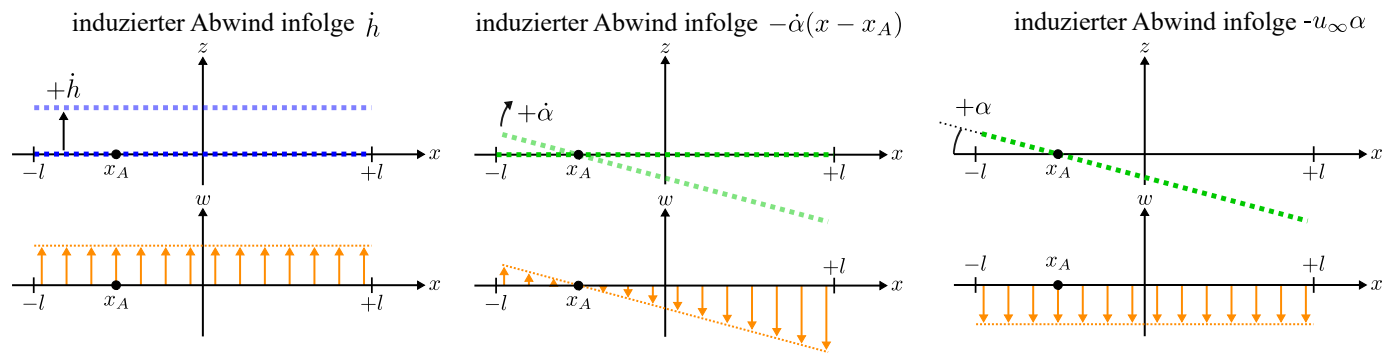

Abbildung A.3.: Schematische Darstellung des durch die jeweiligen Bewegungen der Skelettlinie induzierten Abwinde.

Schlagbewegung $\dot{h}$ induziert einen über die Flügeltiefe konstanten positiven Abwind (Abb. A.3 links). Eine positive, aufdrehende Drehbewegung $-\dot{\alpha}\left(x-x_{a}\right)$ induziert für $x<x_{A}$, also vor dem Drehpunkt, einen positiven und hinter dem Drehpunkt einen negativen Abwind (Abb. A.3 mittig). Dabei variiert die Stärke des Abwinds mit dem Abstand zum Drehpunkt. Abschließend induziert ein positiver Anstellwinkel $\alpha$ einen negativen Abwind, welcher wiederum konstant über die Flügeltiefe verteilt ist (Abb. A.3 rechts). Einsetzen von Gl. (A.4.2) in Gl. (A.3.2) liefert dann ${ }^{4}$

$$
\begin{aligned}
& P_{0}=-\frac{\dot{h}}{u_{\infty}}+\alpha-\frac{\dot{\alpha} x_{A}}{u_{\infty}}=-\mathrm{i} \omega^{*} \frac{h}{l}+\alpha\left(1-\mathrm{i} \omega^{*} \tilde{x}_{A}\right), \\
& P_{1}=\frac{l \dot{\alpha}}{2 u_{\infty}}=\mathrm{i} \omega^{*} \frac{\alpha}{2} \\
& P_{n}=0 \quad \forall n>1 .
\end{aligned}
$$

Für die Koeffizienten $a_{0}$ bis $a_{n}$ nach Gl. (A.3.4) folgt somit

$$
\begin{aligned}
a_{0} & =C\left(\omega^{*}\right)\left[-\frac{\dot{h}}{u_{\infty}}+\alpha-\frac{\dot{\alpha} x_{A}}{u_{\infty}}+\frac{l \dot{\alpha}}{2 u_{\infty}}\right]-\frac{l \dot{\alpha}}{2 u_{\infty}} \\
& =-\mathrm{i} \omega^{*} C\left(\omega^{*}\right) \frac{h}{l}+C\left(\omega^{*}\right) \alpha\left[1+\mathrm{i} \omega^{*}\left(\frac{1}{2}-\tilde{x}_{A}\right)\right]-\frac{\mathrm{i} \omega^{*}}{2} \alpha, \\
a_{1} & =\frac{\mathrm{i} \omega^{*}}{2}\left[-\frac{\dot{h}}{u_{\infty}}+\alpha-\frac{\dot{\alpha} x_{A}}{u_{\infty}}\right]+\frac{\dot{\alpha} l}{2 u_{\infty}} \\
& =\frac{\omega^{* 2}}{2} \frac{h}{l}+\left(\mathrm{i} \omega^{*}+\frac{\omega^{* 2}}{2} \tilde{x}_{A}\right) \alpha, \\
a_{2} & =\frac{\mathrm{i} \omega^{*}}{2} \frac{l \dot{\alpha}}{2 u_{\infty}}=-\frac{\omega^{* 2}}{4} \alpha, \\
a_{n} & =0 \quad \forall n>2 .
\end{aligned}
$$

\footnotetext{
${ }^{4}$ Bei der Berechnung von $P_{1}$ ist der Grenzwert $\lim _{n \rightarrow 1}\left[-\frac{n \sin (n \pi)}{n^{2}-1}\right]=\frac{\pi}{2}$ bei der Ausführung der Integration
} zu berücksichtigen. 
Dies eingesetzt in Gl. (A.3.6) und Gl. (A.3.7) liefert den instationäre Auftrieb (wiederum pro Flügelschnitt dy)

$$
\begin{aligned}
\frac{F_{L}(t)}{\mathrm{d} y}= & \pi \rho_{\infty} l^{2}\left[-\ddot{h}+u_{\infty} \dot{\alpha}-l \tilde{x}_{A} \ddot{\alpha}\right] \\
& +2 \pi \rho_{\infty} u_{\infty} l C\left(\omega^{*}\right)\left[-\dot{h}+u_{\infty} \alpha+l\left(\frac{1}{2}-\tilde{x}_{A}\right) \dot{\alpha}\right]
\end{aligned}
$$

und das instationäre Moment

$$
\begin{aligned}
\frac{M_{\alpha}(t)}{\mathrm{d} y}= & \pi \rho_{\infty} l^{2}\left[l \tilde{x}_{A} \ddot{h}-u_{\infty} l\left(\frac{1}{2}-\tilde{x}_{A}\right) \dot{\alpha}-l^{2}\left(\frac{1}{8}+\tilde{x}_{A}^{2}\right) \ddot{\alpha}\right] \\
& +2 \pi \rho_{\infty} u_{\infty} l^{2}\left(\tilde{x}_{A}+\frac{1}{2}\right) C\left(\omega^{*}\right)\left[-\dot{h}+u_{\infty} \alpha+l\left(\frac{1}{2}-\tilde{x}_{A}\right) \dot{\alpha}\right] .
\end{aligned}
$$

Dabei bezeichnet $\tilde{x}_{A}=\frac{x_{A}}{l}$ den mit der halben Flügeltiefe $l$ entdimensionalisierten Abstand des Flügeldrehpunktes zum Flügeltiefenmittelpunkt, bezogen auf das gewählte Koordinatensystem ${ }^{5}$ ( $c / 4$ bzw. $\tilde{x}_{A}=-\frac{1}{2}$ in der vorliegenden Arbeit).

Analoge Ergebnisse liefert eine Berechnung von Gl. (A.3.6) und Gl. (A.3.7) mit der direkten Berechnung der Druckdifferenz nach Gl. (A.3.10) und (A.4.2).

Führt man in Gl. (A.4.5) und Gl. (A.4.6) den Grenzübergang $\lim _{\omega^{*} \rightarrow 0} C\left(\omega^{*}\right)$ aus, was einer stationären Strömung entspricht, so verschwindet der Imaginärteil der THEODORSENFunktion $C\left(\omega^{*}\right)$ (vgl. 2.2.1) und der Realteil wird $\operatorname{Re}\left[C\left(\omega^{*}=0\right)\right]=1$. Dies bedeutet, dass der Einfluss der abgehenden Wirbel (siehe Abb. 2.9) vernachlässigt wird. Die instationären Kräfte gehen dabei über in quasi-stationäre Annahmen. Der verbleibende Term $-\dot{h}+u_{\infty} \alpha+l\left(\frac{1}{2}-\tilde{x}_{A}\right) \dot{\alpha}$ kann als ein totaler effektiver Anstellwinkel interpretiert werden, welcher von $\dot{h}$ und $\dot{\alpha}$ durch einen induzierten Anstellwinkel und eine effektive Wölbung beeinflusst wird [221]. Die Beschleunigungsterme $\ddot{h}$ und $\ddot{\alpha}$ stellen hingegen Trägheitsterme dar, beschreiben also die zusätzlichen Masseneffekte des durch die Profilbewegung beschleunigten Fluids. Wird des Weiteren ein starrer Tragflügel angenommen ( $\dot{h}=\dot{\alpha}=\ddot{h}=\ddot{\alpha}=0$ ), gehen Gl. (A.4.5) und Gl. (A.4.6) über in die stationären aerodynamischen Kräfte der angestellten ebenen Platte (vgl. [109, S.198 f.]).

\footnotetext{
${ }^{5}$ Bei der Definition der instationären Kräfte gibt es immer wieder eine Problematik bei der Vorzeichenfestlegung, wozu auf [109, S.262 f.] hingewiesen sei. Die hier verwendete Konvention bezieht sich auf das in Abb. 2.9 dargestellte Koordinatensystem.
} 



\section{B. Ergänzungen zu Kapitel 3}

\section{B.1. Berechnung von Strömungsparametern und Diskussion von Messfehlern}

Die Messung der Strömungsgrößen wird vom DNW-TWG durchgeführt und für jeden Messpunkt bereitgestellt. Die Ruhetemperatur $T_{0}$ wird in der Beruhigungskammer des TWG mittels Pt100-Temperatur-Sensoren mit einer Messgenauigkeit von $\pm 0,2 \mathrm{~K}$ gemessen. Der Plenumsdruck $p_{K}$, der Ruhedruck $p_{0}$ (Pitosonde in der Beruhigungskammer) und der statische Druck $p_{\infty}$ (Druckbohrungen am Eingang der adaptiven Messstrecke) werden mit einem Mensor CPG 2500 Druckmesssystem mit einer Genauigkeit von $\pm 15 \mathrm{~Pa}$ ermittelt. Ausgehend hiervon erfolgt seitens des DNW-TWG und im Preprocessing der AMIS (siehe 3.4) eine Umrechnung in die dimensionslosen Größen Sutherland-Konstante $\mathrm{Su}_{0}$, Mach-Zahl $\mathrm{Ma}_{\infty}$ und Reynolds-Zahl $\mathrm{Re}_{\infty}$, wobei zusätzliche, für den TWG kanalspezifische Korrekturen angewendet werden. Weitere Strömungsgrößen werden im Preprocessing aus diesen drei Größen mit den nachstehenden Gleichungen sowie den in Tab. B.1 aufgeführten Konstanten berechnet oder gegebenenfalls direkt aus den Messdaten übernommen.

Tabelle B.1.: Konstanten zur Berechnung von Strömungsparametern aus der dimensionslosen Sutherland-Konstante $\mathrm{Su}_{0}$, der Mach-Zahl $\mathrm{Ma}_{\infty}$ und der Reynolds-Zahl $\mathrm{Re}_{\infty}$.

\begin{tabular}{lll}
\hline Notation & Größe & Beschreibung \\
\hline$c$ & $0,3 \mathrm{~m}$ & Flügeltiefe des Windkanalmodells \\
$T_{r}$ & $273,15 \mathrm{~K}$ & Referenztemperatur $\left(0^{\circ} \mathrm{C}\right)$ \\
$\eta_{r}$ & $1,719 \cdot 10^{-5} \mathrm{~Pa} \cdot \mathrm{s}$ & Dynamische Viskosität $\left(\mathrm{Luft} ; 0^{\circ} \mathrm{C}\right)$ \\
$\mathrm{Su}$ & $110,4 \mathrm{~K}$ & Sutherlandkonstante $\left(\mathrm{Luft} ; 0^{\circ} \mathrm{C}\right)$ \\
$\kappa$ & 1,4 & Isentropenexponent $\left(\mathrm{Luft} ; 0^{\circ} \mathrm{C} ; 1013,25 \mathrm{hPa}\right)$ \\
$R_{s}$ & $287,041 \mathrm{~J} /(\mathrm{kg} \cdot \mathrm{K})$ & Spezifische Gaskonstante $($ Luft $)$ \\
\hline
\end{tabular}

Es sei angemerkt, dass zur Berechnung der strömungsmechanischen Größen isentrope Zustandsänderungen eines idealen Gases angenommen werden. Dem entsprechend sind die statischen Größen Temperatur, Druck und Dichte als Funktionen der Mach-Zahl und 
der jeweiligen Ruhegrößen gemäß

$$
T_{\infty}=f\left(T_{0}, \mathrm{Ma}_{\infty}\right), \quad p_{\infty}=g\left(p_{0}, \mathrm{Ma}_{\infty}\right), \quad \rho_{\infty}=h\left(\rho_{0}, \mathrm{Ma}_{\infty}\right)
$$

darstellbar (und umgekehrt). Die für die Berechnung und Verrechnung der Strömungsgrößen verwendeten Beziehungen sind:

Ruhetemperatur in $\mathrm{K}: \quad T_{0}=\frac{\mathrm{Su}}{\mathrm{Su}_{0}}$

Statische Temperatur in K: $\quad T_{\infty}=\frac{T_{0}}{1+\frac{\kappa-1}{2} \cdot \mathrm{Ma}_{\infty}^{2}}$

Dynamische Viskosität in Pa.s: $\eta_{\infty}=\eta_{r} \cdot\left(\frac{T_{\infty}}{T_{r}}\right)^{\frac{3}{2}} \cdot\left(\frac{T_{r}+\mathrm{Su}}{T_{\infty}+\mathrm{Su}}\right)$

Statische Dichte in $\mathrm{kg} / \mathrm{m}^{3}: \quad \rho_{\infty}=\frac{\operatorname{Re}_{\infty} \cdot \eta_{\infty}}{u_{\infty} \cdot c}$

Ruhedichte in $\mathrm{kg} / \mathrm{m}^{3}: \quad \rho_{0}=\rho_{\infty} \cdot\left(1+\frac{\kappa-1}{2} \cdot \mathrm{Ma}_{\infty}^{2}\right)^{\frac{1}{\kappa-1}}$

Anströmungsgeschwindigkeit in $\mathrm{m} / \mathrm{s}: \quad u_{\infty}=\mathrm{Ma}_{\infty} \cdot c_{\infty}=\mathrm{Ma}_{\infty} \cdot \sqrt{\kappa R_{s} T_{\infty}}$

Statischer Druck in Pa: $p_{\infty}=R_{s} \cdot \rho_{\infty} \cdot T_{\infty}$

Dynamischer Druck in Pa: $q_{\infty}=\frac{1}{2} \cdot \rho_{\infty} \cdot u_{\infty}^{2}$

Ruhedruck in Pa: $\quad p_{0}=p_{\infty} \cdot\left(1+\frac{\kappa-1}{2} \cdot \mathrm{Ma}_{\infty}^{2}\right)^{\frac{\kappa}{\kappa-1}}$

Die jeweiligen Messfehler $\sigma_{x}$ der Größen folgen direkt aus den oben angegebenen Sensorungenauigkeiten und der Gauß'schen Fehlerfortpflanzung. Für die Mach-Zahl folgt aus Gl. (B.1.9)

$$
\mathrm{Ma}_{\infty}=\sqrt{\frac{2}{\kappa-1}\left[\left(\frac{p_{0}}{p_{\infty}}\right)^{\frac{\kappa-1}{\kappa}}-1\right]}
$$

und mit den Sensorungenauigkeiten $\sigma_{p_{0}}=\sigma_{p_{\infty}}=\sigma_{p}$ ein Messfehler von

$$
\begin{aligned}
\sigma_{\mathrm{Ma}_{\infty}} & =\sigma_{p} \cdot \sqrt{\left(\frac{\partial \mathrm{Ma}_{\infty}}{\partial p_{0}}\right)^{2}+\left(\frac{\partial \mathrm{Ma}_{\infty}}{\partial p_{\infty}}\right)^{2}}, \\
\text { mit } \frac{\partial \mathrm{Ma}_{\infty}}{\partial p_{0}} & =\sqrt{\frac{\kappa-1}{2}} \cdot \frac{1}{p_{\infty} \kappa} \cdot\left(\frac{p_{0}}{p_{\infty}}\right)^{-\frac{1}{\kappa}} \cdot\left[\left(\frac{p_{0}}{p_{\infty}}\right)^{\frac{\kappa-1}{\kappa}}-1\right]^{-\frac{1}{2}} \\
\text { und } \frac{\partial \mathrm{Ma}_{\infty}}{\partial p_{\infty}} & =-\sqrt{\frac{\kappa-1}{2}} \cdot \frac{1}{p_{0} \kappa} \cdot\left(\frac{p_{0}}{p_{\infty}}\right)^{\frac{2 \kappa-1}{\kappa}} \cdot\left[\left(\frac{p_{0}}{p_{\infty}}\right)^{\frac{\kappa-1}{\kappa}}-1\right]^{-\frac{1}{2}} .
\end{aligned}
$$


Gl. (B.1.11) ist in Abb. B.1(a) unter Ausnutzung von Gl. (B.1.9) für einen Bereich von $0,5 \leq \mathrm{Ma}_{\infty} \leq 0,8$ und einem Ruhedruck $30 \mathrm{kPa} \leq p_{0} \leq 100 \mathrm{kPa}$ grafisch ausgewertet. Es zeigt sich, dass für den Messfehler der Mach-Zahl im für die durchgeführten Versuche

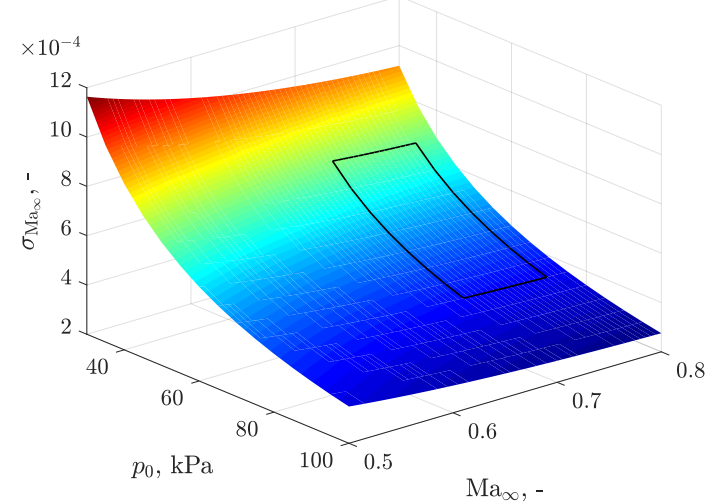

(a) Messfehler $\sigma_{\mathrm{Ma}_{\infty}}$ der Mach-Zahl

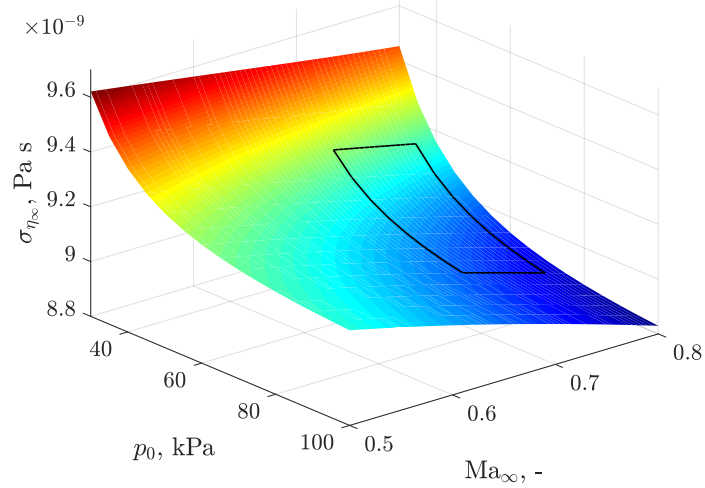

(c) Messfehler $\sigma_{\eta_{\infty}}$ der dynamischen Viskosität

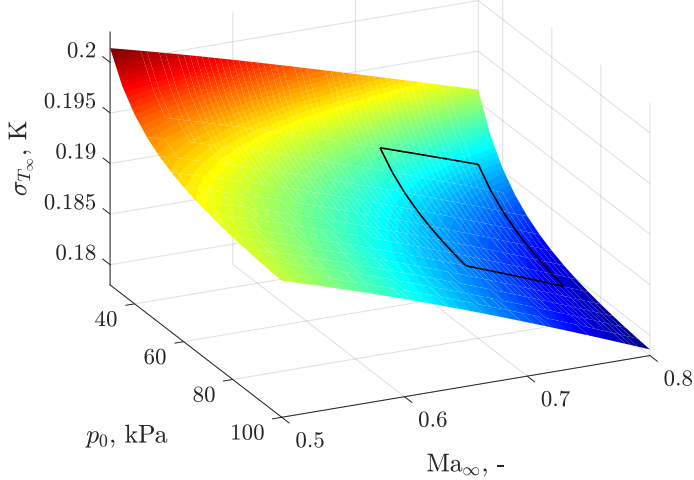

(b) Messfehler $\sigma_{T_{\infty}}$ der statischen Temperatur

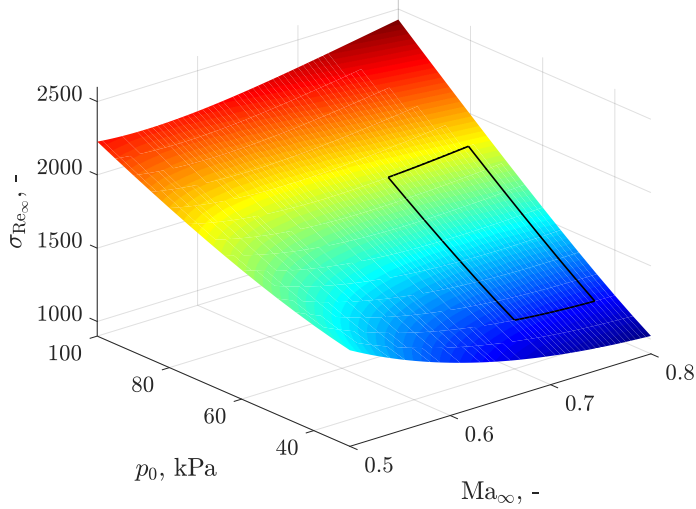

(d) Messfehler $\sigma_{\operatorname{Re}_{\infty}}$ der Reynolds-Zahl

Abbildung B.1.: Grafische Auswertung der Messfehler für die Mach-Zahl (a), der statischen Temperatur (b), der dynamischen Viskosität (c) und die Reynolds-Zahl (d) des TWG in Abhängigkeit von $\mathrm{Ma}_{\infty}$ und $p_{0}$. Die schwarze Umrandung $\left(0,7 \leq \mathrm{Ma}_{\infty} \leq 0,78\right.$ und $\left.40 \leq p_{0} \leq 75\right)$ markiert den ungefähren Strömungsbereich des gemessenen Transonic Dips des CAST 10-2 Profils.

relevanten Strömungsbereich (schwarz umrandeter Bereich in Abb. B.1) $\sigma_{\mathrm{Ma}_{\infty}}<\mathcal{O}\left(10^{-3}\right)$ gilt. Der Messfehler liegt somit noch unterhalb der Einstellgenauigkeit der Mach-Zahl (vgl. 3.1). Für die statische Temperatur folgt mit Gl. (B.1.2) ein Messfehler von

$$
\begin{gathered}
\sigma_{\mathrm{T}_{\infty}}=\sqrt{\sigma_{T_{0}}^{2} \cdot\left(\frac{\partial T_{\infty}}{\partial T_{0}}\right)^{2}+\sigma_{\mathrm{Ma}_{\infty}}^{2} \cdot\left(\frac{\partial T_{\infty}}{\partial \mathrm{Ma}_{\infty}}\right)^{2}} \\
\operatorname{mit} \frac{\partial T_{\infty}}{\partial T_{0}}=\left[1+\frac{\kappa-1}{2} \mathrm{Ma}_{\infty}^{2}\right]^{-1} \text { und } \frac{\partial T_{\infty}}{\partial \mathrm{Ma}_{\infty}}=\frac{-T_{0}(\kappa-1) \mathrm{Ma}_{\infty}}{\left[1+\frac{\kappa-1}{2} \mathrm{Ma}_{\infty}^{2}\right]^{2}}
\end{gathered}
$$

Die grafische Auswertung von $\sigma_{T_{\infty}}$ ist für eine gewählte Ruhetemperatur $T_{0}=310 \mathrm{~K}$ in Abb. B.1(b) dargestellt. Hier liegt der Messfehler im relevanten Strömungsbereich 
unterhalb von 0,2 K. Für den Messfehler der dynamischen Viskosität folgt dann direkt aus Gl. (B.1.3) zusammen mit Gl. (B.1.12)

$$
\sigma_{\eta_{\infty}}=\sigma_{T_{\infty}}\left|\frac{\partial \eta_{\infty}}{\partial T_{\infty}}\right|=\sigma_{T_{\infty}}\left|\frac{\eta_{r} \sqrt{T_{\infty}}\left(T_{r}+\mathrm{Su}\right)}{T_{r}^{3 / 2}\left(T_{\infty}+\mathrm{Su}\right)}\left[\frac{3}{2}-\frac{T_{\infty}}{T_{\infty}+\mathrm{Su}}\right]\right|
$$

Dieser Fehler ist in Abb. B.1(c) dargestellt, ebenfalls für eine Ruhetemperatur von $T_{0}=310 \mathrm{~K}$. Im gesamten dargestellten Strömungsbereich gilt für den Fehler $\sigma_{\eta_{\infty}}<$ $\mathcal{O}\left(10^{-8}\right) \mathrm{Pa}$ s. Abschließend lässt sich die Reynolds-Zahl gemäß Gl. (B.1.4) zusammen mit Gl. (B.1.2), (B.1.6), (B.1.7) und (B.1.9) schreiben als

$$
\operatorname{Re}_{\infty}=\frac{\mathrm{Ma}_{\infty} \sqrt{\kappa} c p_{0}}{\sqrt{R_{s} T_{0}} \eta_{\infty}}\left(1+\frac{\kappa-1}{2} \mathrm{Ma}_{\infty}^{2}\right)^{\frac{\kappa+1}{2(1-\kappa)}}
$$

Für den Fehler $\sigma_{\operatorname{Re}_{\infty}}$ der Reynolds-Zahl folgt mit den zuvor berechneten Messfehlern $\sigma_{\mathrm{Ma}_{\infty}}, \sigma_{\mathrm{T}_{0}}, \sigma_{p_{0}}$ und $\sigma_{\eta_{\infty}}$

$$
\begin{aligned}
\sigma_{\mathrm{Re}_{\infty}}= & {\left[\sigma_{\mathrm{Ma}_{\infty}}^{2}\left(\frac{\partial \mathrm{Re}_{\infty}}{\partial \mathrm{Ma}_{\infty}}\right)^{2}+\sigma_{T_{0}}^{2}\left(\frac{\partial \mathrm{Re}_{\infty}}{\partial T_{0}}\right)^{2}\right.} \\
& \left.+\sigma_{p_{0}}^{2}\left(\frac{\partial \mathrm{Re}_{\infty}}{\partial p_{0}}\right)^{2}+\sigma_{\eta_{\infty}}^{2}\left(\frac{\partial \mathrm{Re}_{\infty}}{\partial \eta_{\infty}}\right)^{2}\right]^{\frac{1}{2}}, \\
\operatorname{mit} \frac{\partial \mathrm{Re}_{\infty}}{\partial \mathrm{Ma}_{\infty}}= & \frac{\sqrt{\kappa} c p_{0}}{\sqrt{R_{s} T_{0}} \eta_{\infty}}\left[\left(1+\frac{\kappa-1}{2} \mathrm{Ma}_{\infty}^{2}\right)^{\frac{\kappa+1}{2(1-\kappa)}}\right. \\
& \left.-\mathrm{Ma}_{\infty}^{2} \frac{\kappa+1}{2}\left(1+\frac{\kappa-1}{2} \mathrm{Ma}_{\infty}^{2}\right)^{\frac{3 \kappa-1}{2(1-\kappa)}}\right] \\
\frac{\partial \mathrm{Re}_{\infty}}{\partial T_{0}}= & -\frac{\mathrm{Ma}_{\infty} \sqrt{\kappa} c p_{0}}{2 \sqrt{R_{s}} T_{0}^{3 / 2} \eta_{\infty}}\left(1+\frac{\kappa-1}{2} \mathrm{Ma}_{\infty}^{2}\right)^{\frac{\kappa+1}{2(1-\kappa)}}, \\
\frac{\partial \mathrm{Re}_{\infty}}{\partial p_{0}}= & \frac{\mathrm{Ma}_{\infty} \sqrt{\kappa} c}{\sqrt{R_{s} T_{0}} \eta_{\infty}}\left(1+\frac{\kappa-1}{2} \mathrm{Ma}_{\infty}^{2}\right)^{\frac{\kappa+1}{2(1-\kappa)}}, \\
\frac{\partial \mathrm{Re}_{\infty}}{\partial \eta_{\infty}}= & -\frac{\mathrm{Ma}_{\infty} \sqrt{\kappa} c p_{0}}{\sqrt{R_{s} T_{0}} \eta_{\infty}^{2}}\left(1+\frac{\kappa-1}{2} \mathrm{Ma}_{\infty}^{2}\right)^{\frac{\kappa+1}{2(1-\kappa)}}
\end{aligned}
$$

Der Fehler für die Reynolds-Zahl ist grafisch in Abb. B.1(d) dargestellt. Im relevanten Strömungsbereich $\left(\operatorname{Re}_{\infty} \sim 2 \cdot 10^{6}\right)$ ist $\sigma_{\operatorname{Re}_{\infty}}<2000$ und ist damit mit einem relativen Fehler $<0,1 \%$ vernachlässigbar.

Es sei hier angemerkt, dass die durchgeführten Fehlerbetrachtungen die zusätzlichen spezifischen Windkanalkorrekturen einzelner Größen nicht mit berücksichtigen. Diese stellen weitere Fehlerquellen dar, welche jedoch nicht genauer quantifiziert werden können. Im Allgemeinen liegen die Korrekturen an sich jedoch in einer ähnlichen Größenordnung wie die berechneten Messfehler selbst. 


\section{B.2. Federkonstanten und generalisierte Massen des FVS}

Die Federsteifigkeiten des FVS wurden mit einem statischen Auslenkversuch bestimmt. Hierfür wurden die Federn mit definierten Kräften $F_{i}$ und Momenten $M_{i}$ belastet und die Auslenkungen $x_{i}$ (bzw. $h$ und $\alpha$ ) gemessen. Durch die lineare Beziehung dieser Parameter (Hooke'sches Gesetz [172, S. 171 ff.]) wurden die Federkonstanten $k$ mithilfe einer linearen Regression [222, S. 153 ff.] zu

$$
k=\frac{\sum_{i=1}^{n}\left(x_{i}-\bar{x}\right)\left(F_{i}-\bar{F}\right)}{\sum_{i=1}^{n}\left(x_{i}-\bar{x}\right)^{2}}, \quad \text { mit } \quad \bar{x}=\frac{1}{n} \sum_{i=1}^{n} x_{i} \quad \text { und } \quad \bar{F}=\frac{1}{n} \sum_{i=1}^{n} F_{i}
$$

aus $n$ Einzelmessungen bestimmt. Die zugehörigen Fehler berechnen sich aus der Streuung der Messwerte um die Regressionsgerade gemäß

$$
\sigma_{k}=\sqrt{\frac{1}{n-2} \cdot \frac{\sum_{i=1}^{n}\left(F_{i}-F_{0}-k x_{i}\right)^{2}}{\sum_{i=1}^{n}\left(x_{i}-\bar{x}\right)^{2}}}, \quad \text { mit } \quad F_{0}=\bar{F}-k \bar{x} .
$$

Ferner wurden die generalisierten Massen und Trägheitsmomente der Federn samt mitschwingenden Bauteilen des FVSs bestimmt [155,223]. Die Federn wurden mit Zusatz-Massen $m_{Z}$ bzw. Trägheitsmomenten $I_{Z}$ belastet und die Eigenfrequenzen $f_{0}$ der Federsysteme verändert. Aus den verstimmten Systemfrequenzen $f_{Z}$ wurden die generalisierten Größen

$$
m^{*}=m_{Z} \cdot \frac{f_{Z}{ }^{2}}{f_{0}^{2}-f_{Z}{ }^{2}} \quad \text { und } \quad I^{*}=I_{Z} \cdot \frac{f_{Z}{ }^{2}}{f_{0}^{2}-f_{Z}{ }^{2}}
$$

berechnet. Die zugehörigen Fehler folgen hierbei aus den Fehlern der Frequenzbestimmung $\sigma_{f}=\sigma_{f_{0}}=\sigma_{f_{Z}}$ und der Massen $\sigma_{m_{Z}}$ und Trägheitsmomente $\sigma_{I_{Z}} \mathrm{zu}$

$$
\sigma_{m^{*}}=\sqrt{{\sigma_{m_{Z}}}^{2} \cdot\left[\frac{f_{Z}^{2}}{f_{0}^{2}-f_{Z}^{2}}\right]^{2}+\sigma_{f}^{2}\left(f_{0}^{2}+f_{Z}^{2}\right) \cdot\left[\frac{2 m_{Z} f_{Z} f_{0}}{\left(f_{0}^{2}-f_{Z}^{2}\right)^{2}}\right]^{2}},
$$

$\sigma_{I^{*}}$ berechnet sich analog. Die Werte der Einzelmessungen Gl. (B.2.3) wurden abschließend durch einen, durch den Fehler der Einzelmessung Gl. (B.2.4) gewichteten Mittelwert

$$
\bar{m}^{*}=\frac{\sum_{i=1}^{n} \frac{m_{i}^{*}}{\sigma_{m^{*}}{ }^{2}}}{\sum_{i=1}^{n} \frac{1}{\sigma_{m^{*}}{ }^{2}}} \quad \text { bzw. } \quad \bar{I}^{*}=\frac{\sum_{i=1}^{n} \frac{I_{i}^{*}}{\sigma_{I^{*}{ }^{2}}{ }^{2}}}{\sum_{i=1}^{n} \frac{1}{\sigma_{I^{*}{ }^{2}{ }^{2}}}}
$$


zusammengefasst. Die zugehörigen Fehler wurden durch die gewichtete Standardabweichung der einzelnen Messungen abgeschätzt.

\section{B.2.1. Federkonstanten der Rechteck-Parallelfedern}

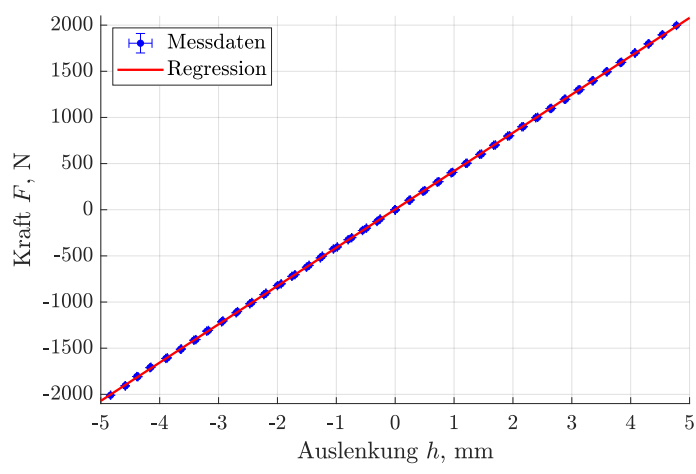

(a) Federkennlinie Ostseite

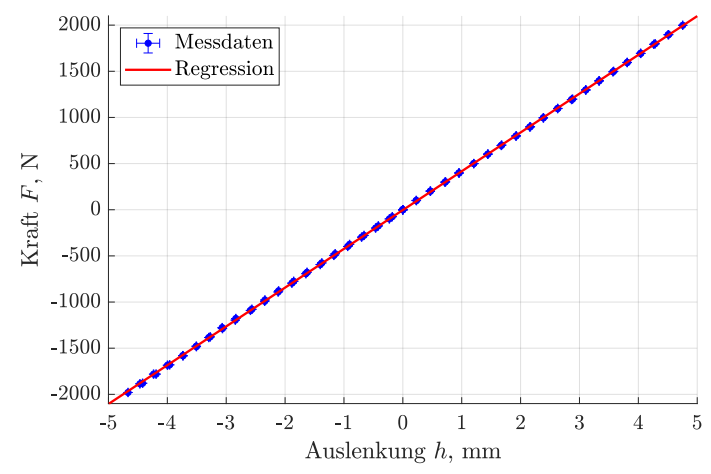

(b) Federkennlinie Westseite

Abbildung B.2.: Auslenkung der Rechteck-Parallelfedern unter Last und statische Abschätzung der Federkonstanten durch eine lineare Regression. Eine positive Auslenkung beschreibt eine Aufwärtsbewegung der Blattfedern, analog zu einer positiven Auftriebskraft.

Zur Auslenkung der Federn wurden mit einem mechanischen Spindeltrieb Kräfte zwischen $-2000 \mathrm{~N} \lesssim F_{i} \lesssim 2000 \mathrm{~N}$ auf die Blattfedern aufgebracht. Die Auslenkung wurde von einem Lasertriangulator gemessen, der sich an der Blattfederspitze befand. Die Kräfte wurden mit einem piezoelektrischen Kraftaufnehmer bestimmt, der zwischen den Blattfedern und dem Spindeltrieb eingebaut war. Die gemessenen Federkennlinien sind in Abb. B.2(a) (Ostseite) und Abb. B.2(b) (Westseite) dargestellt. Die Fehler jedes Datenpunkts wurden durch die Standardabweichung der Zeitreihen von $F_{i}$ und $h_{i}$ abgeschätzt. Die mit Gl. (B.2.1) und (B.2.2) berechneten Federkonstanten sind in Tab. B.2 aufgeführt, ebenso die kombinierte Federkonstante $k_{h}$ (Parallelschaltung, siehe 3.2.2). Die Streuung der Messdaten um die Regressionsgeraden ist gering, folglich ist der Fehler der Federkonstante klein. Weiterhin weisen beide Federn keine signifikante Hysterese auf. Das Schlagfedersystem ist somit statisch linear ${ }^{1}$.

Tabelle B.2.: Federkonstanten der Rechteck-Parallelfedern des FVS.

\begin{tabular}{cccc}
\hline Seite & Notation & $\mathbf{k}, \mathrm{kN} / \mathrm{m}$ & $\sigma_{\mathbf{k}}, \mathrm{kN} / \mathrm{m}$ \\
\hline ost & $k_{h, \text { ost }}$ & 414,96 & 0,21 \\
west & $k_{h, \text { west }}$ & 420,23 & 0,26 \\
kombiniert & $k_{h}$ & 835,19 & 0,33 \\
\hline
\end{tabular}

\footnotetext{
${ }^{1}$ Eine Prüfung der dynamischen Linearität (vgl. [141]) ist nicht direkt erfolgt. Diese kann indirekt aus exponentiell aufklingenden Flatterkurven gefolgert werden [26].
} 


\section{B.2.2. Generalisierte Massen der Rechteck-Parallelfedern}

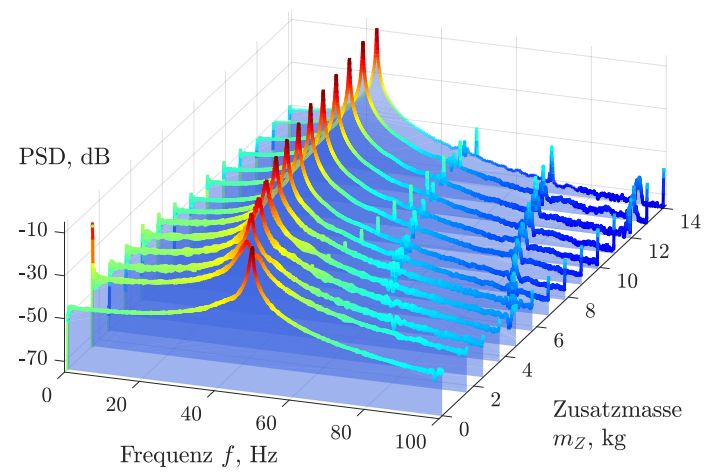

(a) Frequenzänderung Ostseite $f_{h, \text { ost }}\left(m_{Z}\right)$

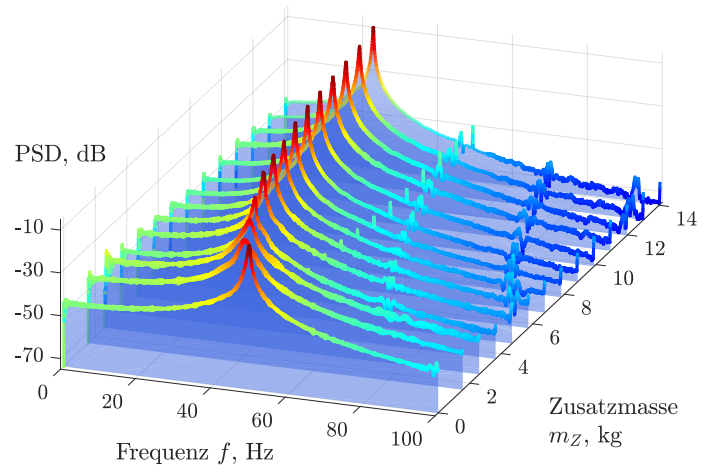

(c) Frequenzänderung Westseite $f_{h, \text { west }}\left(m_{Z}\right)$

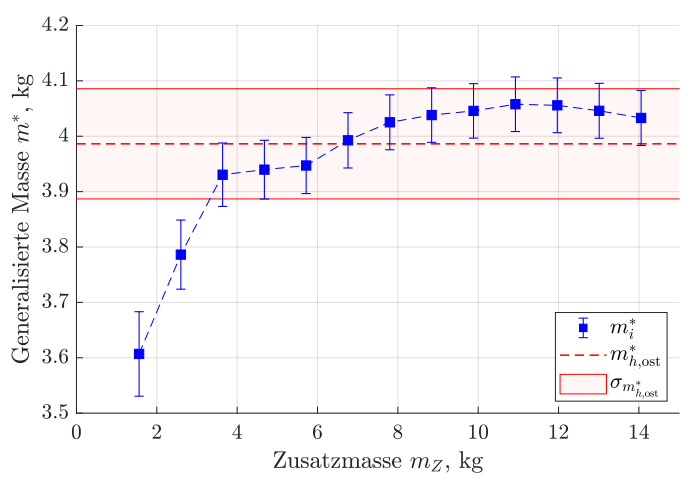

(b) Generalisierte Masse Ostseite $m_{h, \text { ost }}^{*}$

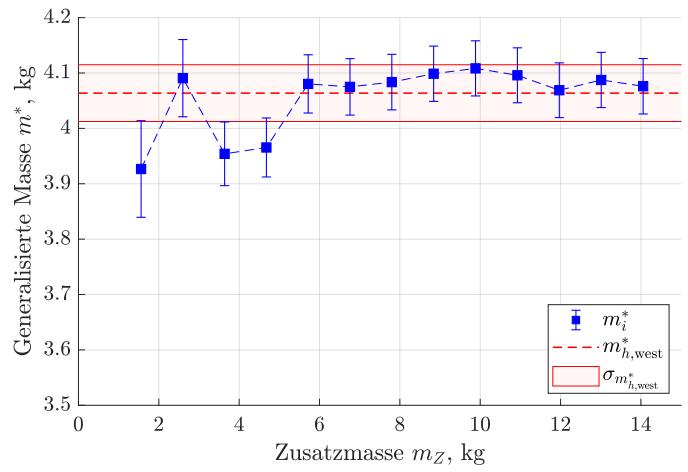

(d) Generalisierte Masse Westseite $m_{h \text {,west }}^{*}$

Abbildung B.3.: Veränderung der Frequenzen bzw. Autoleistungsspektren der Schlagfedern unter Zusatzmassen und berechnete generalisierte Massen.

Zur Verstimmung der Rechteck-Parallelfedern wurden definierte Massen an die Schlagfedersysteme angebracht. Die nicht verstimmten Eigenfrequenzen betrugen $f_{0 \text {,ost }}=$ $(49,38 \pm 0,10) \mathrm{Hz}$ und $f_{0, \text { west }}=(49,56 \pm 0,10) \mathrm{Hz}$, wobei der Fehler auf der Frequenzauflösung der berechneten Autoleistungsspektren und einer Dämpfungskorrektur ${ }^{2}$ basiert. Die Entwicklungen der Frequenzen für verschiedene Zusatzmassen sind in Abb. B.3(a)

Tabelle B.3.: Generalisierte Massen der Schlagfedersysteme und der zugehörigen Komponenten (Schlagkasten und Verbindungselemente) des FVS sowie die damit zu Vergleichszwecken berechneten Federkonstanten.

\begin{tabular}{ccccc}
\hline Seite & $\mathbf{m}^{*}, \mathrm{~kg}$ & $\sigma_{\mathbf{m}^{*}}, \mathrm{~kg}$ & $\mathbf{k}, \mathrm{kN} / \mathrm{m}$ & $\sigma_{\mathbf{k}}, \mathrm{kN} / \mathrm{m}$ \\
\hline ost & 3,99 & 0,10 & 383,71 & 9,66 \\
west & 4,06 & 0,06 & 394,07 & 5,29 \\
kombiniert & 8,05 & 0,12 & 777,78 & 11,01 \\
\hline
\end{tabular}

\footnotetext{
${ }^{2}$ Die Berechnung der generalisierten Massen und Momente basiert auf der Annahme einer ungedämpften harmonischen Schwingung, das reale System ist hingegen gedämpft. Die gemessenen, gedämpften Eigenfrequenzen $\omega_{d}$ sind daher reduziert. Die zugehörige, ungedämpfte Eigenfrequenz ist durch $\omega_{0}=\sqrt{\omega_{d}^{2}+\gamma^{2}}$ gegeben. Da die Abklingkonstante $\gamma \lesssim 2 \mathrm{~s}^{-1}$ ist, sind die gemessenen Frequenzen um den Faktor $\Delta \omega=\omega_{0}-\omega_{d} \lesssim 0.04 \mathrm{~Hz}$ zu klein. Der größer geschätzte Fehler $\sigma_{f}$ berücksichtigt dies.
} 
(Ostseite) und Abb. B.3(c) (Westseite) dargestellt. Die mit Gl. (B.2.3) und (B.2.4) berechneten generalisierten Massen sind in Abb. B.3(b) (Ostseite) und Abb. B.3(d) (Westseite) dargestellt. Darüber hinaus sind die gewichteten Mittelwerte nach Gl. (B.2.5) und die zugehörigen Fehlerintervalle in Abb. B.3(b) und Abb. B.3(d) eingezeichnet (rote Linie und rot hinterlegter Bereich). Ausgehend von den Eigenfrequenzen und den generalisierten Massen wurden die Federkonstanten mit $k=\left(2 \pi f_{0}\right)^{2} \cdot m^{*}$ auf eine weitere Art berechnet. Die Ergebnisse sind in Tab. B.3 zusammengefasst. Ein Vergleich mit der statisch bestimmten Steifigkeit $k_{h}$ (siehe B.2 und B.3) zeigt eine Abweichung von 6,9\%. Auch wenn diese Abweichung nicht von den Fehlern abgedeckt wird, liegt diese im erwarteten Bereich der Messmethode. Da der Fehler der statischen Methode deutlich geringer ausfällt, wird eine kombinierte Federsteifigkeit des Schlagfedersystems von $k_{h}=(835,19 \pm 0,33) \mathrm{kN} / \mathrm{m}$ angenommen (siehe B.2.1).

\section{B.2.3. Federkonstanten der Torsionsfedern}

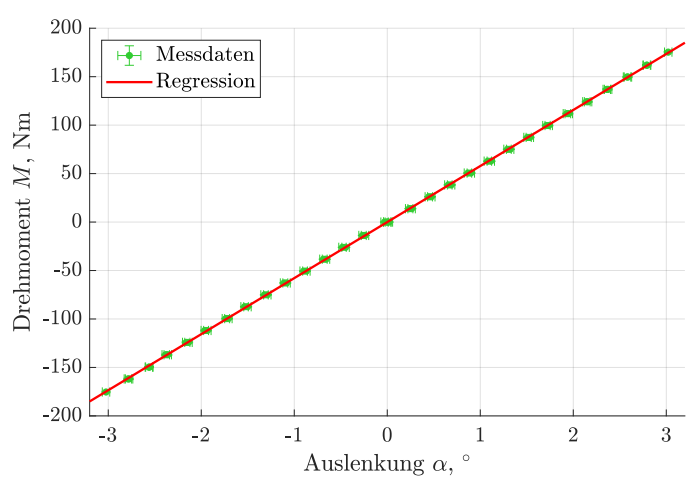

(a) Federkennlinie Ostseite

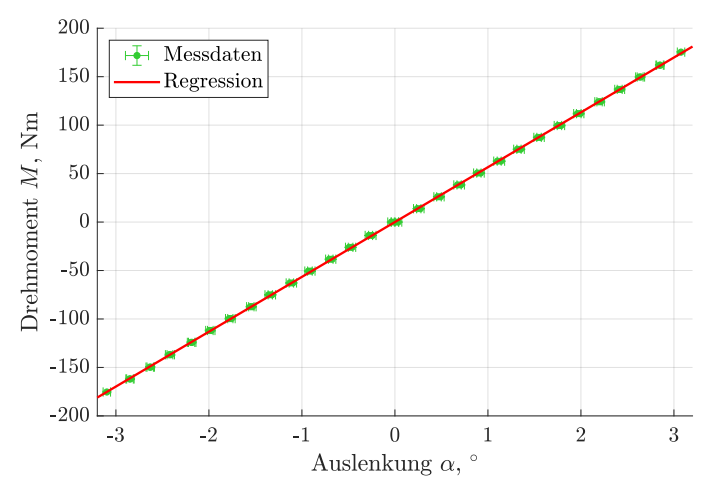

(b) Federkennlinie Westseite

Abbildung B.4.: Auslenkung der Torsionsfedern unter Last und statische Abschätzung der Federkonstanten durch eine lineare Regression. Ein positiver Auslenk-Winkel beschreibt ein Aufdrehen der Torsionsfedern, analog zu einem positiven aerodynamischen Nickmoment.

Die Steifigkeiten der Torsionsfedern wurden in analoger Vorgehensweise zu den Schlagfedern bestimmt. Hierzu wurde ein Balken mit einer Länge von $2 r=500 \mathrm{~mm}$ an den Torsionsfedern befestigt, an den Massen bis zu $m \approx 75 \mathrm{~kg}$ angebracht wurden, resultierend in Drehmomenten $M_{i}=g m_{i} r$ zwischen $-180 \mathrm{Nm} \lesssim M_{i} \lesssim 180 \mathrm{Nm}$. Die Auslenkung wurde mit zwei Lasertriangulatoren gemessen (vgl. 3.4 und B.4). Der Fehler für das aufgebrachte Moment wurde mit $\sigma_{M_{i}}=\sqrt{\sigma_{r}^{2}\left(g m_{i}\right)^{2}+\sigma_{m}^{2}(g r)^{2}}$ abgeschätzt. Die gemessenen Kennlinien der Torsionsfedern sind in Abb. B.4(a) (Ostseite) und B.4(b) (Westseite) dargestellt. Wie zuvor ist die Streuung der Messdaten vernachlässigbar klein. Die Regressionsgeraden verlaufen innerhalb der Fehlerintervalle der Messdaten, die resultierenden Fehler für die Federkonstanten sind wiederum klein. Des Weiteren tritt keine signifikante Hysterese auf, so dass auch das Torsionsfedersystem statisch linear ist (siehe Anm. B.2.1). Die Ergebnisse sind in Tab. B.4 eingetragen. 
Tabelle B.4.: Federkonstanten der Torsionsfedern des FVS.

\begin{tabular}{cccc}
\hline Seite & Notation & $\mathbf{k}, \mathrm{Nm} / \mathrm{rad}$ & $\sigma_{\mathbf{k}}, \mathrm{Nm} / \mathrm{rad}$ \\
\hline ost & $k_{\alpha, \text { east }}$ & 3313,25 & 4,14 \\
west & $k_{\alpha, \text { west }}$ & 3242,07 & 4,46 \\
kombiniert & $k_{\alpha}$ & 6555,32 & 6,09 \\
\hline
\end{tabular}

\section{B.2.4. Generalisierte Massen des Torsionsfedersystems}

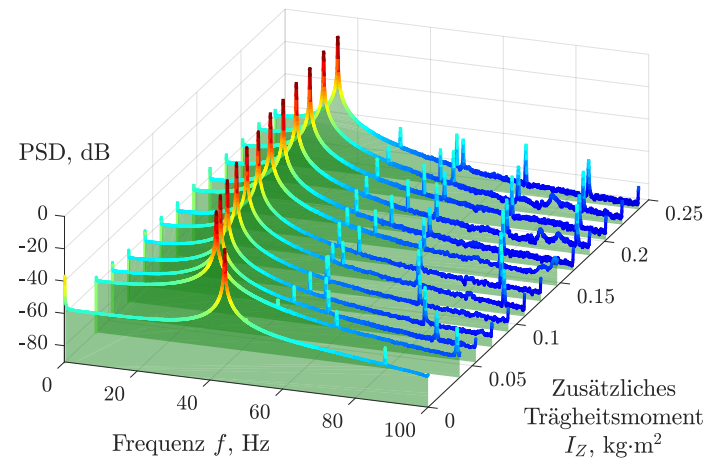

(a) Frequenzänderung Ostseite $f_{\alpha, \text { ost }}\left(I_{Z}\right)$

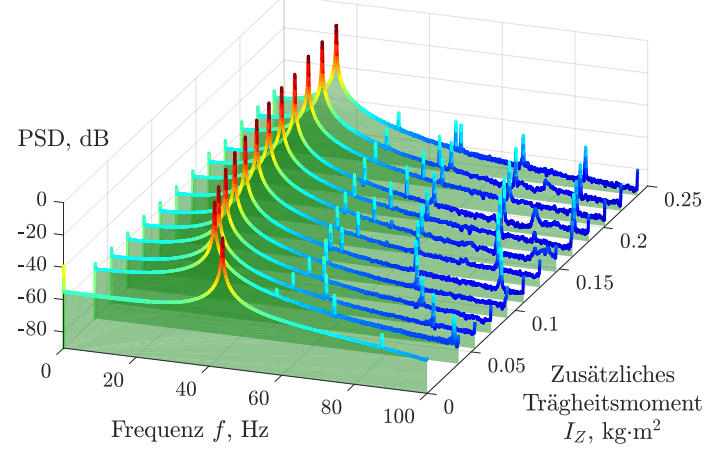

(c) Frequenzänderung Westseite $f_{\alpha, \text { west }}\left(I_{Z}\right)$

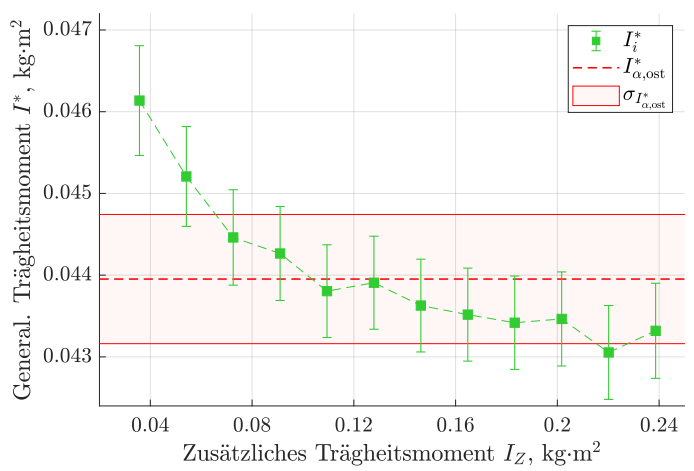

(b) Generalisierte Momente Ostseite $I_{\alpha, \text { ost }}^{*}$

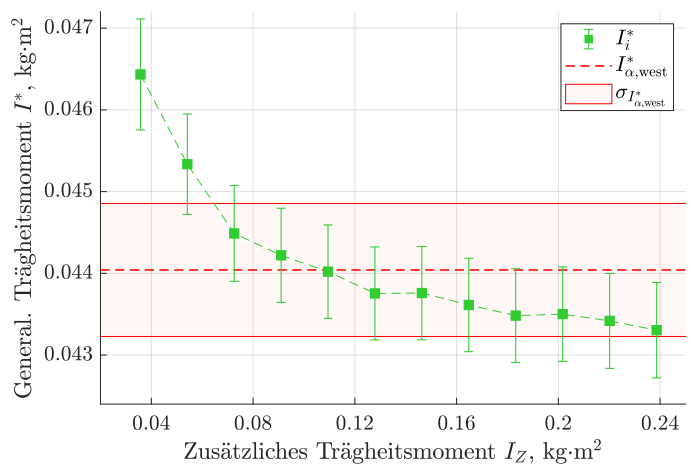

(d) Generalisierte Momente Westseite $I_{\alpha, \text { west }}^{*}$

Abbildung B.5.: Veränderung der Frequenzen bzw. Autoleistungsspektren der Torsionsfedern unter Zusatz-Trägheitsmomenten und berechnete generalisierte Trägheitsmomente (Torsionsfeder und Balken).

Analog zu B.2.2 wurde eine Frequenzverstimmung der Torsionsfedern durch das Anbringen von definierten Trägheitsmomenten an den Balken realisiert. Die Ergebnisse sind in Abb. B.5(a) bis Abb. B.5(d) dargestellt ${ }^{3}$. Ebenfalls wurden die Federkonstanten dynamisch aus den generalisierten Trägheitsmomenten berechnet. Sämtliche Ergebnisse sind in Tab. B.5 aufgelistet. Die Abweichung der durch das dynamische Verfahren bestimmten Federkonstante beträgt 2,2 \% im Vergleich zur statischen Bestimmung. Da der Fehler im letzteren Fall kleiner ist, wird für die kombinierte Federsteifigkeit des Torsionsfedersystems ein Wert von $k_{\alpha}=(6555,32 \pm 6,09) \mathrm{Nm} / \mathrm{rad}$ angenommen. Die generalisierten

\footnotetext{
${ }^{3}$ Die dargestellten Trägheitsmomente beziehen sich auf das System aus Torsionsfeder und den, für die Messung obligatorischen Balken.
} 
Trägheitsmomente der Torsionsfedern folgen aus den Ergebnissen (Tab. B.5) nach Abzug des Balkenmoments $I_{B}=(41,81 \pm 0,42) \cdot 10^{-3} \mathrm{~kg} \cdot \mathrm{m}^{2}$. Für die Ostseite ergibt sich somit $I_{\alpha, \text { ost }}^{*}=(2,14 \pm 0,89) \cdot 10^{-3} \mathrm{~kg} \cdot \mathrm{m}^{2}$, für die Westseite $I_{\alpha, \text { west }}^{*}=(2,23 \pm 0,91) \cdot 10^{-3} \mathrm{~kg} \cdot \mathrm{m}^{2}$ und kombiniert $I_{\alpha, s}^{*}=(4,37 \pm 1,27) \cdot 10^{-3} \mathrm{~kg} \cdot \mathrm{m}^{2}$. Die Resultate stellen den isolierten Teil des Trägheitsmoments dar, der ausschließlich dem Beitrag der Torsionfedern entspricht und somit relevant ist für das aeroelastische System.

Tabelle B.5.: Generalisierte Trägheitsmomente der Torsionsfedersysteme und des angeschlossenen Balkens sowie die damit berechneten Federkonstanten.

\begin{tabular}{cccccc}
\hline Seite & $\mathbf{I}^{*}, 10^{-3} \mathrm{~kg} \cdot \mathrm{m}^{2}$ & $\sigma_{\mathbf{I}^{*}}, 10^{-3} \mathrm{~kg} \cdot \mathrm{m}^{2}$ & $\mathbf{f}_{\mathbf{0}}, \mathrm{Hz}$ & $\mathbf{k}, \mathrm{Nm} / \mathrm{rad}$ & $\sigma_{\mathbf{k}}, \mathrm{Nm} / \mathrm{rad}$ \\
\hline ost & 43,95 & 0,79 & 44,07 & 3369,80 & 62,47 \\
west & 44,04 & 0,81 & 43,76 & 3329,37 & 63,10 \\
kombiniert & 87,99 & 1,13 & - & 6699,17 & 88,79 \\
\hline
\end{tabular}

\section{B.3. Vermessung der Profilgeometrie}

Zur Überprüfung der Modellgeometrie wurde eine Vermessung des Windkanalmodells in Auftrag gegeben. Insgesamt wurden sieben Profilschnitte in x-Richtung entlang der Flügelspannweite $s$ und sechs Konturen auf der Modelloberfläche in y-Richtung entlang der Flügeltiefe $c$ aufgezeichnet. Die Vermessung erfolgte mithilfe eines CNC gesteuerten Koordinatenmessgerätes entlang der jeweiligen Messschnitte. Der Modellbereich, welcher mit Heißfilmsensoren bestückt ist, wurde aufgrund deren Berührungsempfindlichkeit ausgespart. Die gemessenen Abweichungen $\Delta n / c$ in Normalenrichtung zur Oberfläche zwischen dem Windkanalmodell und dem CAD-Referenzmodell sind grafisch in Abb. B.6 und Abb. B.7 dargestellt. Die minimalen und maximalen Abweichungen samt deren Positionen auf der Modelloberfläche sowie die mittleren Abweichungen sind zusätzlich in Tab. B.6 und Tab. B.7 eingetragen.

Insgesamt zeigt sich eine geringfügige Aufdickung des gesamten Modells. Diese liegt im Durchschnitt jedoch unterhalb von 0,1\% der Flügeltiefe und beträgt auch maximal weniger als 0,13\% von c. Der aerodyanmische Einfluss dieser Aufdickung wird als vernachlässigbar klein angenommen. Des Weiteren sind entlang der Flügeltiefe (entlang der Anströmung) keine Stufen oder Unstetigkeiten in der Modellkontur zu erkennen, welche sich störend auf die Grenzschichtströmung auswirken könnten. Die kleine Eindellung an der Vorderkante, wie Abb. B.6 zeigt, ist auf die Klebenaht der Schalenbauweise des Modells zurückzuführen. Auch hier ist die aerodynamische Relevanz als vernachlässigbar einzustufen. 

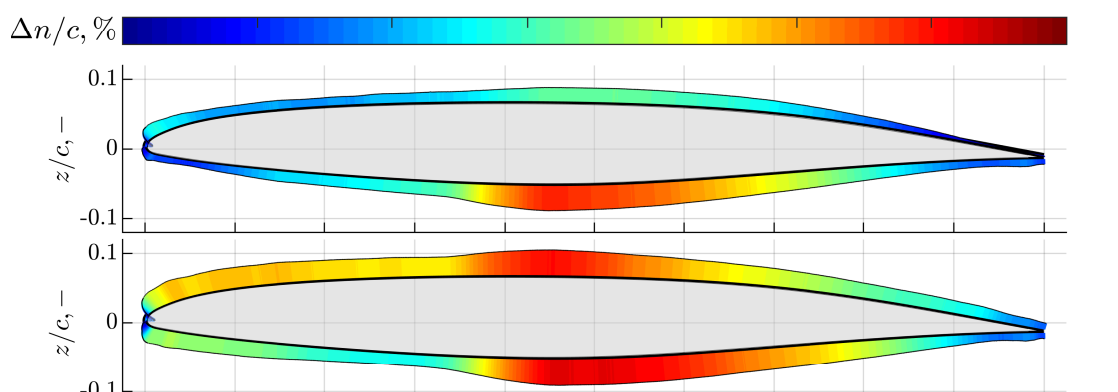

Schnitt bei $10 \%$ der Spannweite

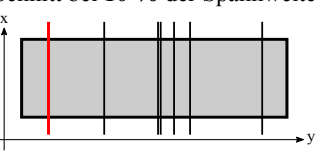

Schnitt bei $\mathbf{3 1} \%$ der Spannweite
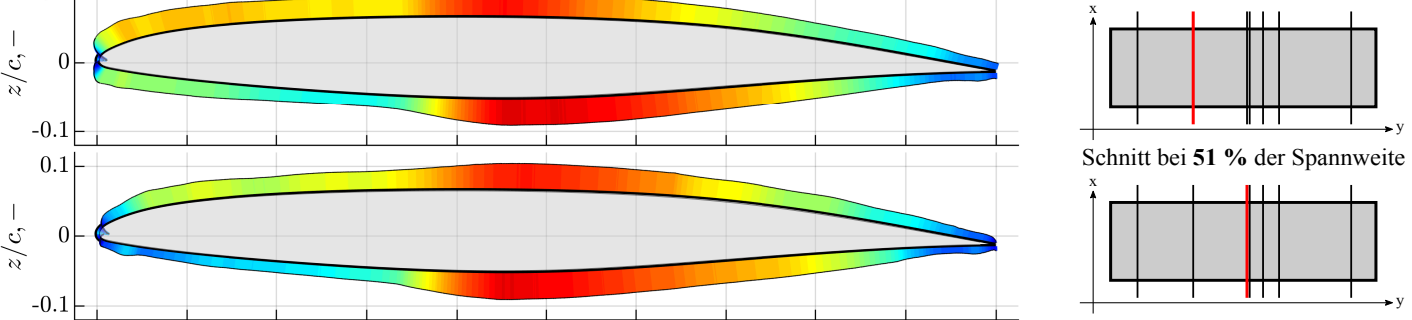

Schnitt bei $\mathbf{5 1} \%$ der Spannweite

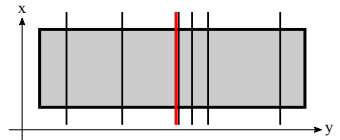

Schnitt bei $\mathbf{5 2} \%$ der Spannweite
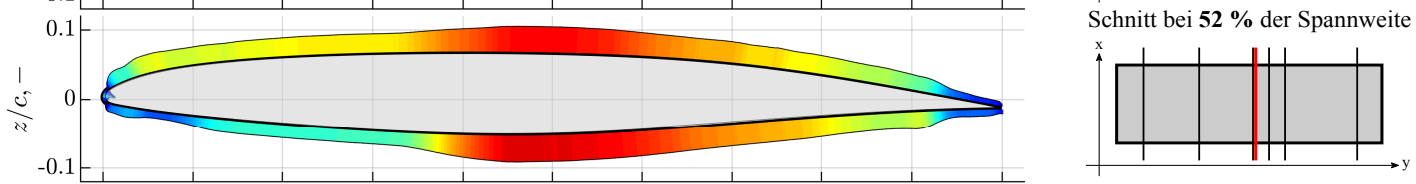

Schnitt bei $\mathbf{5 7} \%$ der Spannweite
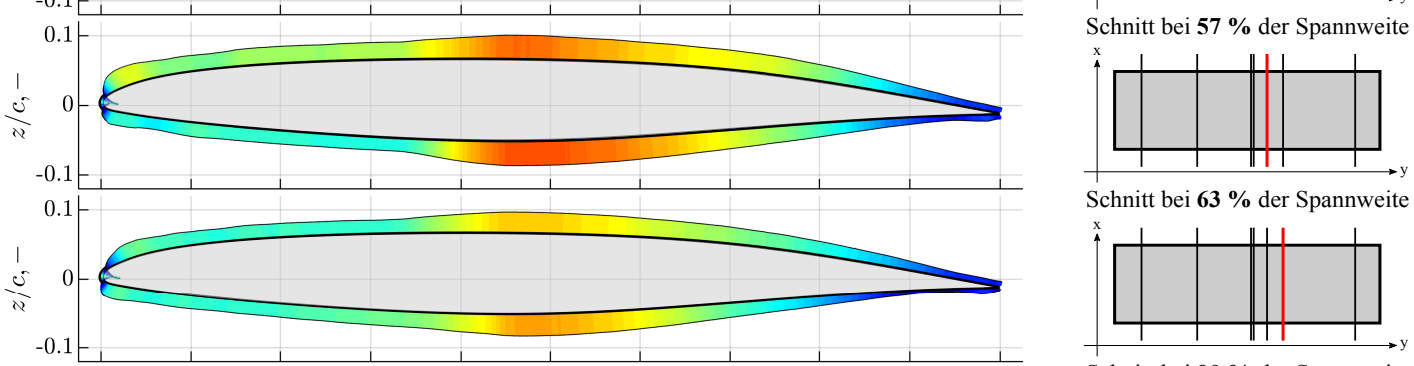

Schnitt bei $63 \%$ der Spannweite

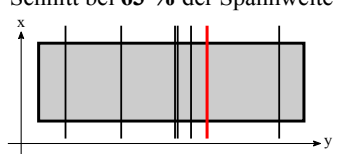

Schnitt bei $90 \%$ der Spannweite
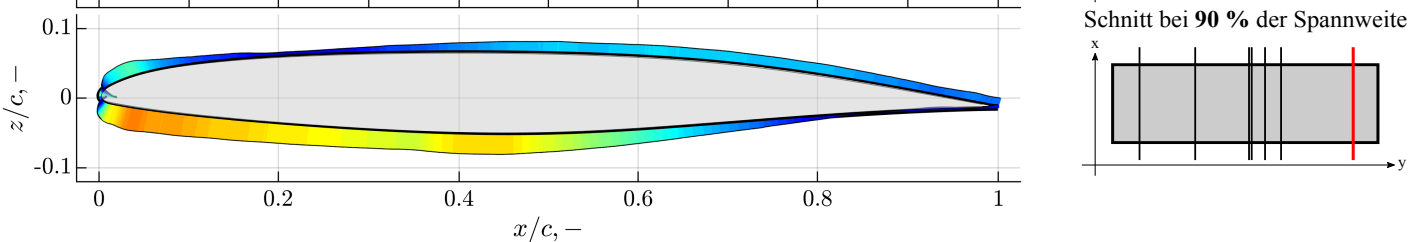

Abbildung B.6.: Abweichung $\Delta n / c$ der vermessenen Profilgeometrie des CAST 10-2 Windkanalmodells von der Sollkontur des CAD-Modells in Normalenrichtung zur Oberfläche entlang der Flügeltiefe $c$. Das Modell wurde an sieben Schnitten entlang der Spannweite $s$ vermessen. Die Abweichung ist in Prozent zur Flügeltiefe $c$ angegeben und farblich kodiert. Die Normalenvektoren sind zur Veranschaulichung 30 -fach überhöht dargestellt.

Tabelle B.6.: Minimale, maximale und mittlere Abweichung $\Delta n / c$ der CAST 10-2 Modellgeometrie entlang der Flügeltiefe. Die jeweiligen Positionen $x / c$ sind ebenfalls eingetragen, wobei sich $\uparrow$ auf die Profil-Oberseite und $\downarrow$ auf die Profil-Unterseite bezieht.

\begin{tabular}{cccccc}
\hline Messschnitt & $\min (\boldsymbol{\Delta} \mathbf{n} / \mathbf{c}), \boldsymbol{\%}$ & $\mathbf{x} / \mathbf{c}, \boldsymbol{\%}$ & $\max (\boldsymbol{\Delta} \mathbf{n} / \mathbf{c}), \boldsymbol{\%}$ & $\mathbf{x} / \mathbf{c}, \boldsymbol{\%}$ & $\overline{\mathbf{\Delta n} / \mathbf{c}, \%}$ \\
\hline $10 \%$ von s & $-0,029$ & $0,05 \uparrow$ & 0,119 & $44,95 \downarrow$ & 0,053 \\
$31 \%$ von s & $-0,037$ & $0,06 \uparrow$ & 0,127 & $46,25 \downarrow$ & 0,080 \\
$51 \%$ von s & $-0,046$ & $0,04 \uparrow$ & 0,126 & $46,15 \downarrow$ & 0,074 \\
$52 \%$ von s & $-0,045$ & $0,05 \uparrow$ & 0,128 & $46,15 \downarrow$ & 0,079 \\
$57 \%$ von s & $-0,063$ & $0,04 \uparrow$ & 0,112 & $46,25 \downarrow$ & 0,068 \\
$63 \%$ von s & $-0,071$ & $0,03 \uparrow$ & 0,100 & $47,35 \downarrow$ & 0,060 \\
$90 \%$ von s & $-0,067$ & $0,05 \uparrow$ & 0,105 & $4,05 \downarrow$ & 0,047 \\
\hline
\end{tabular}




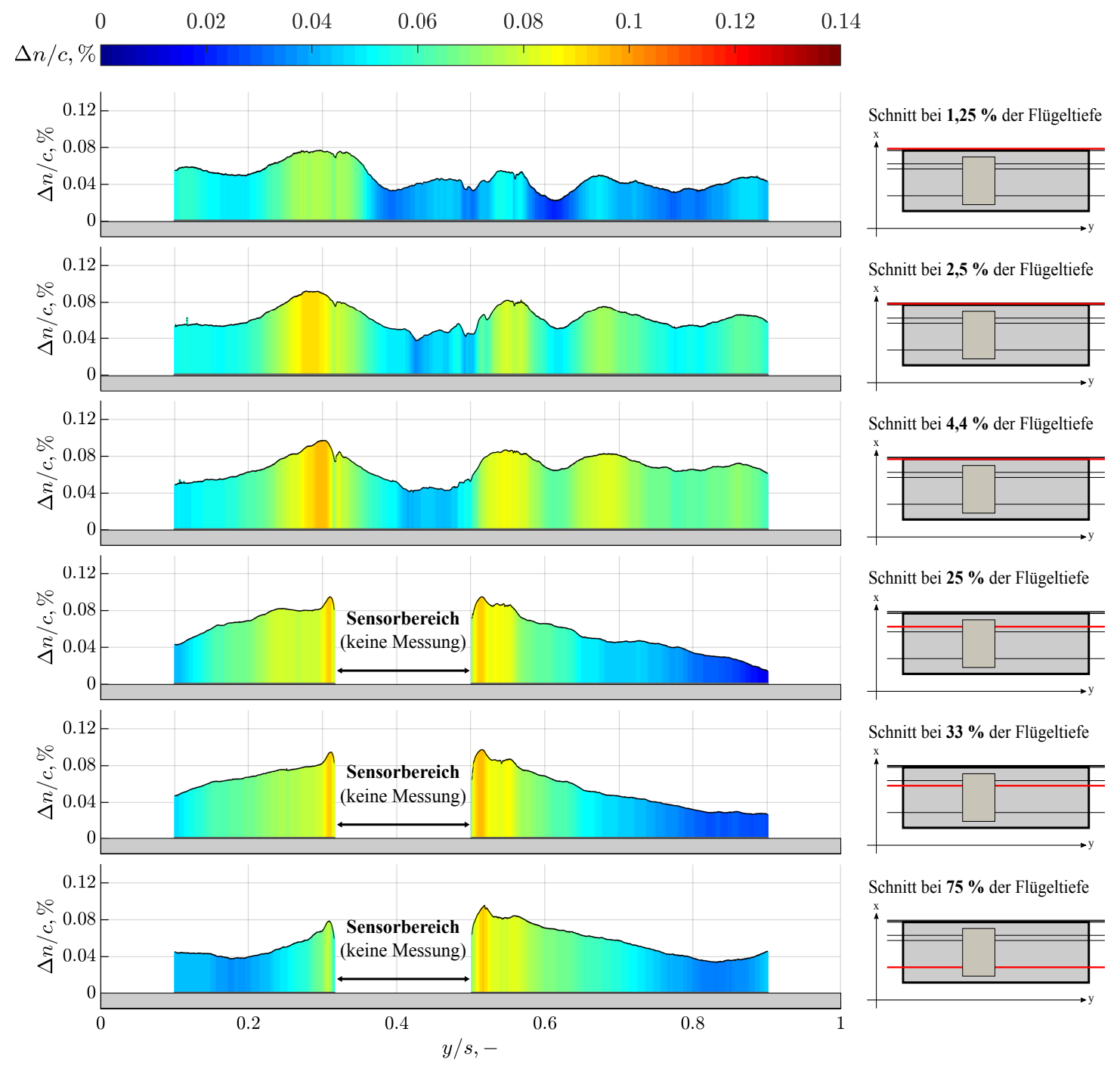

Abbildung B.7.: Abweichung $\Delta n / c$ der vermessenen Profilgeometrie des CAST 10-2 Windkanalmodells von der Sollkontur des CAD-Modells in Normalenrichtung zur Oberfläche entlang der Spannweite $s$. Das Modell wurde an sechs Schnitten auf der Profil-Oberseite entlang der Flügeltiefe $c$ vermessen. Die Abweichung ist in Prozent zur Flügeltiefe $c$ angegeben und farblich kodiert.

Tabelle B.7.: Minmiale, maximale und mittlere Abweichung $\Delta n / c$ der CAST 10-2 Modellgeometrie auf der Profil-Oberseite entlang der Spannweite. Die jeweiligen Positionen $y / s$ sind ebenfalls eingetragen.

\begin{tabular}{cccccc}
\hline Messschnitt & $\min (\boldsymbol{\Delta} \mathbf{n} / \mathbf{c}), \boldsymbol{\%}$ & $\mathbf{y} / \mathbf{s}, \boldsymbol{\%}$ & $\max (\boldsymbol{\Delta} \mathbf{n} / \mathbf{c}), \%$ & $\mathbf{y} / \mathbf{s}, \%$ & $\overline{\boldsymbol{\Delta} \mathbf{n} / \mathbf{c}}, \boldsymbol{\%}$ \\
\hline $1,25 \%$ von c & 0,022 & 61,42 & 0,077 & 29,62 & 0,048 \\
$2,5 \%$ von c & 0,038 & 42,71 & 0,092 & 27,81 & 0,063 \\
$4,4 \%$ von c & 0,041 & 42,25 & 0,097 & 30,30 & 0,068 \\
$25 \%$ von c & 0,014 & 90,02 & 0,095 & 31,05 & 0,058 \\
$33 \%$ von c & 0,027 & 88,16 & 0,097 & 51,65 & 0,058 \\
$75 \%$ von c & 0,034 & 83,24 & 0,096 & 51,89 & 0,054 \\
\hline
\end{tabular}




\section{B.4. Messungenauigkeiten der Sensorik}

Nachfolgend sind die Ungenauigkeiten der eingesetzten Sensorik diskutiert und die Messfehler quantifiziert, was sich jedoch im Wesentlichen auf die Messung der Modellbewegung mithilfe der Lasertriangulatoren und die Drucksensoren beschränkt. Die Beschleunigungssensoren messen nur Wechselanteile und sind ohnehin nicht für stationäre bzw. absolute Messungen geeignet. Im relevanten Frequenzbereich sprechen die Sensoren quasi instantan an. Ein zeitlicher Versatz, also ein möglicher Phasenfehler, ist hier zu vernachlässigen. Eine weiterführende Fehlerdiskussion für die Beschleunigungssensoren ist daher nicht erfolgt. Gleiches gilt für die Heißfilmsensoren, welche nur qualitative Werte messen.

\section{B.4.1. Messfehler der Drucksensoren}

Im Windkanalmodell sind Druckdifferenzsensoren vom Typ Kulite XCQ-093D (siehe 3.4) verbaut, welche mithilfe einer kubischen Funktion zu Beginn eines jeden Messtages kalibriert wurden. Zur Abschätzung der Messfehler der Sensoren sind in Abb. B.8 die Kalibrierungsdaten für acht exemplarisch ausgewählte Kulite-Drucksensoren dargestellt. Die Sensitivität $s$ der Sensoren wurde hier mit einer linearen Regression bestimmt,

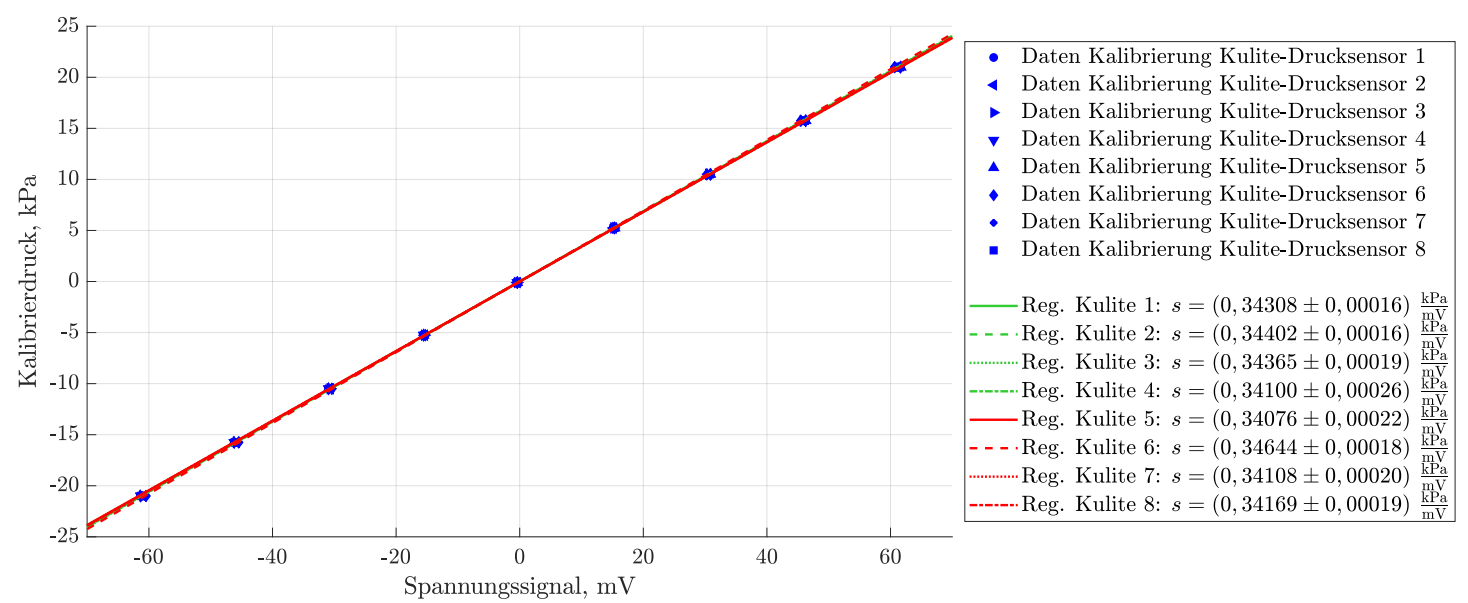

Abbildung B.8.: Beispiel- und stichprobenhafte Kalibrierung von acht Kulite-Drucksensoren (XCQ-093D) mit einer linearen Regression zur Bestimmung der Sensitivitäten samt Fehlern, abweichend zur kubischen Regression wie diese im Versuchsbetrieb verwendet wird.

wobei sich der Fehler $\sigma_{s}$ der Sensitivitäten analog zu Gl. (B.2.2) berechnet. Beide Werte sind Abb. B.8 zu entnehmen. Es zeigt sich, dass der Fehler bei den ausgewählten Sensoren maximal $\sigma_{s}=0,00026 \frac{\mathrm{kPa}}{\mathrm{mV}}$ beträgt und im Allgemeinen keine große Streuung, weder der Sensitivitäten noch der Spannungswerte (Kalibrierdaten und Regressionen der Kulites sind nahezu identisch) auftritt. Ausgehend hiervon wird angenommen, dass ein allgemeiner Fehler für die Sensorsensitivität von $\sigma_{s}=0,0003 \frac{\mathrm{kPa}}{\mathrm{mV}}$ eine großzügige Abschätzung darstellt. Zumal erfasst eine kubische Regression eventuelle Nichtlinearitäten und sollte somit zu einem geringeren Fehler führen. Da sich der lokale Druck bzw. 
Differenzdruck an der jeweiligen Stelle des Windkanalmodells nunmehr aus der Sensitivität und dem gemessenen Sensor-Spannungssignal $U_{S}$ ergibt, folgt für den Absolut-Fehler der Druckmessung $\sigma_{\Delta p}=\sigma_{s} \cdot\left|U_{S}\right|$, bzw. ausgedrückt als Funktion des Differenzdrucks $\sigma_{\Delta p}(\Delta p)=\sigma_{s} \cdot\left|\frac{\Delta p}{s}\right|$. Für den Fehler des $c_{p}$-Wertes folgt dann mit Gl. (3.4.1)

$$
\sigma_{c_{p}}=\sqrt{\sigma_{\Delta p}^{2}\left(\frac{\partial c_{p}}{\partial \Delta p}\right)^{2}+\sigma_{q_{\infty}}^{2}\left(\frac{\partial c_{p}}{\partial q_{\infty}}\right)^{2}}=\frac{\Delta p}{q_{\infty}} \sqrt{\frac{\sigma_{s}^{2}}{s^{2}}+\frac{\sigma_{q_{\infty}}^{2}}{q_{\infty}^{2}}} .
$$

Der dynamische Druck $q_{\infty}$ nach Gl. (B.1.8) kann mit Gl. (B.1.6) und (B.1.7) auch als Funktion von $\mathrm{Ma}_{\infty}$ und $p_{\infty}$ geschrieben werden als

$$
q_{\infty}=\frac{\kappa}{2} p_{\infty} \mathrm{Ma}_{\infty}^{2} \quad \text { mit } \quad \sigma_{q_{\infty}}=\mathrm{Ma}_{\infty} \kappa \sqrt{\frac{\sigma_{p_{\infty}}^{2} \mathrm{Ma}_{\infty}^{2}}{4}+\sigma_{\mathrm{Ma}_{\infty}}^{2} p_{\infty}^{2}}
$$

Somit ist Gl. (B.4.1) auf die in B.1 bereits behandelten Fehler zurückgeführt. Analog hierzu ist der Fehler $\sigma_{q_{\infty}}$ in Abb. B.9(a) unter Verwendung von Gl. (B.1.9) für $0,5 \leq$ $\mathrm{Ma} \leq 0,8$ und $30 \mathrm{kPa} \leq p_{0} \leq 100 \mathrm{kPa}$ grafisch dargestellt. Hierbei zeigt sich, dass $\sigma_{q_{\infty}}$

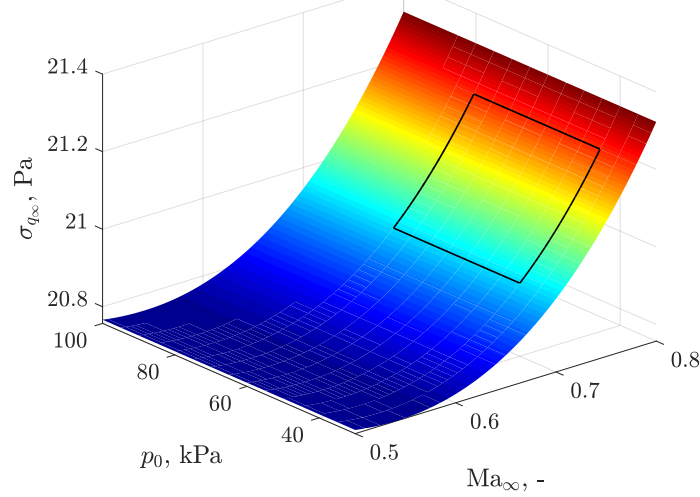

(a) Fehler des dynamischen Drucks $\sigma_{q_{\infty}}$

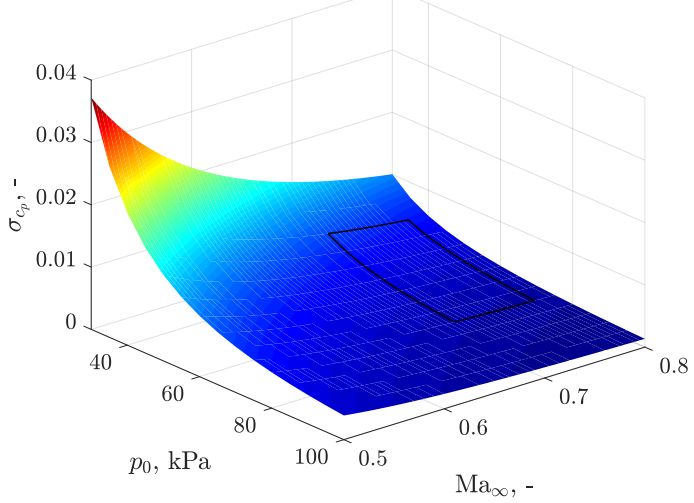

(b) Fehler des Druckbeiwertes $\sigma_{c_{p}}$

Abbildung B.9.: Messfehler $\sigma_{q_{\infty}}$ des dynamischen Drucks (a) und Fehler $\sigma_{c_{p}}$ des Druckbeiwertes (b) für eine Druckdifferenz von $\Delta p=34,5 \mathrm{kPa}$. Wie zuvor ist der ungefähre Strömungsbereich des Transonic Dips markiert.

unabhängig vom Druck $p_{\infty}$ bzw. $p_{0}$ ist, was auch nach einer Vereinfachung von Gl. B.1.11 ersichtlich wird. Der Fehler für die Mach-Zahl lässt sich mit etwas Rechenaufwand schreiben als

$$
\sigma_{\mathrm{Ma}_{\infty}}=\frac{\sigma_{p}}{\kappa \mathrm{Ma}_{\infty} p_{0}}\left(\frac{p_{0}}{p_{\infty}}\right)^{\frac{\kappa-1}{\kappa}} \sqrt{1+\left(\frac{p_{0}}{p_{\infty}}\right)^{2}} .
$$

Hier zeigt sich das $\sigma_{\mathrm{Ma}_{\infty}} \sim \frac{1}{p_{0}} \sim \frac{1}{p_{\infty}}$. Die Druckabhängigkeit von $\sigma_{q_{\infty}}$ bzw. Gl. (B.4.2) kürzt sich folglich heraus. Unter Verwendung von $\sigma_{q_{\infty}}$ ist in Abb. B.9(b) nunmehr auch der Fehler $\sigma_{c_{p}}$ des Druckbeiwerts grafisch dargestellt. Da Gl. (B.4.1) linear mit der gemessenen Druckdifferenz $\Delta p$ ansteigt, wurde $\sigma_{c_{p}}$ mit der größtmöglichen Druckdifferenz von $\Delta p=$ 
$34,5 \mathrm{kPa}$, entsprechend dem maximalen Messbereich der Kulite-Sensoren, ausgewertet. Für die Sensitivität wurde der Mittelwert der acht betrachteten Drucksensoren $s=$ $0,34271 \frac{\mathrm{kPa}}{\mathrm{mV}}$ verwendet. Daraus folgt im relevanten Strömungsbereich ein Fehler für den Druckbeiwert von $\sigma_{c_{p}} \leq 0,01$.

Es sei hier nochmals angemerkt, dass die vorausgehende Fehlerbetrachtung nur den Messfehler der absoluten Werte der Drucksensoren berücksichtigt. Weitere Ungenauigkeiten basieren auf einem veränderten Übertragungsverhalten durch die Zuleitungen zwischen Sensor und Druckbohrung. Hieraus resultieren mögliche Amplitudenänderungen und Phasenversätze, wie bereits in 3.4 diskutiert.

\section{B.4.2. Messfehler des Anstellwinkels bei Flatterversuchen}

Bei Flatterversuchen ist der Anstellwinkel des Windkanalmodells

$$
\alpha(t)=\bar{\alpha}+\alpha_{\text {inst }}(t)=\alpha_{2 D}+\alpha_{0}+\alpha_{\text {inst }}(t)
$$

eine Kombination des Winkels $\alpha_{\text {inst }}(t)$, gemessen mit den Lasertriangulatoren, dem Winkel $\alpha_{2 D}$ des 2D-Supports sowie dem Einstellwinkel des Modells $\alpha_{0}$. Der Fehler folgt aus der Gauß'schen Fehlerfortpflanzung zu

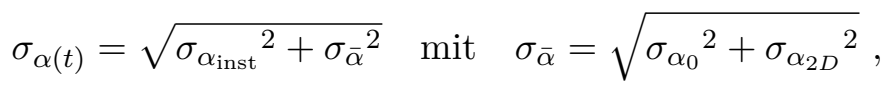

mit den Fehlern der jeweiligen Winkelkomponenten.

Die Messung des Einstellwinkels $\alpha_{0}$ erfolgt mit einer, parallel zur Bezugslinie (vgl. 3.3) verlaufenden Profilschablone, welche auf die Oberfläche des Windkanalmodells aufgebracht wird. Mit einem Clinotronic-Neigungsmessgerät wird der Winkel dieser Schablone gemessen, was dem Modell-Einstellwinkel entspricht. Der systematische Fehler des Inklinometers beträgt $\sigma_{\text {clin }}=(0,016+0,01)^{\circ} \approx 0,03^{\circ}$ im Messbereich von $\pm 10^{\circ}$ [224]. Der Fehler der sich aus der Anpassung und der Positionierung der Schablone auf der Modelloberfläche ergibt, wird mit $0,05^{\circ}$ abgeschätzt. Zusammengenommen folgt für den Einstellwinkel somit ein Fehler von $\sigma_{\alpha_{0}} \approx 0,06^{\circ}$.

Der 2D-Support des DNW-TWG (vgl. 3.2.2) weist eine Hysterese in der Winkelverstellung von etwa $0,06^{\circ}$ auf, welche bei der Änderung der Drehrichtung zu berücksichtigen ist. Da keine weiteren Informationen über die Messunsicherheit des 2D-Supports existieren, wird diese Hysterese als Unsicherheit für $\alpha_{2 D}$ angenommen und der Fehler mit $\sigma_{2 D}=0,06^{\circ}$ abgeschätzt. 
Zur Bestimmung von $\alpha_{\text {inst }}$ messen je zwei Lasertriangulatoren am FVS (analog zum NVS) den Winkel

$$
\alpha_{\text {ost } / \text { west }}=\tan ^{-1}\left(\frac{d_{L_{2 / 4}}-d_{L_{1 / 3}}}{\Delta x_{L}}\right)
$$

des Windkanalmodells auf der jeweiligen Seite (vgl. 3.4). Der Fehler $\sigma_{\alpha_{\text {ost }} \text { west }}$ basiert auf dem Fehler $\sigma_{\Delta x_{L}}$ des Abstands der Laser untereinander sowie dem Fehler des Abstandssignals $\sigma_{d_{L}}$. Gemäß Gauß'scher Fehlerfortpflanzung folgt

$$
\begin{aligned}
\sigma_{\alpha_{\text {ost } / \text { west }}} & =\sqrt{{\sigma_{d_{L}}}^{2} \cdot\left[\left(\frac{\partial \alpha_{\text {ost } / \text { west }}}{\partial d_{L 1 / 3}}\right)^{2}+\left(\frac{\partial \alpha_{\text {ost } / \text { west }}}{\partial d_{L 2 / 4}}\right)^{2}\right]+\sigma_{\Delta x_{L}}^{2} \cdot\left(\frac{\partial \alpha_{\text {ost } / \text { west }}}{\partial \Delta x_{L}}\right)^{2}} \\
& =\frac{\sqrt{2{\sigma_{d_{L}}}^{2} \Delta x_{L}^{2}+\sigma_{\Delta x_{L}}{ }^{2} \Delta d_{L}^{2}}}{\Delta x_{L}^{2}+\Delta d_{L}^{2}}
\end{aligned}
$$

mit $\Delta d_{L}=d_{L 1 / 3}-d_{L 2 / 4}$ und $\sigma_{d_{L 1 / 3}}=\sigma_{d_{L 2 / 4}}=\sigma_{d_{L}}=6 \cdot 10^{-5} \mathrm{~m}$ [175]. Der Abstand $\Delta x_{L}$ der Laser ist aufgrund der Laserhalterungen des FVS nahezu identisch mit $\Delta x_{L} \approx 218,5 \mathrm{~mm}\left(\Delta x_{L, \text { west }} \approx 219 \mathrm{~mm}\right.$ und $\left.\Delta x_{L, \text { ost }} \approx 218 \mathrm{~mm}\right)$. Hierfür wird ein Fehler von $\sigma_{\Delta x_{L}}=1 \mathrm{~mm}$ abgeschätzt. Des Weiteren wir angenommen, dass $\Delta d_{L, \text { ost }} \approx \Delta d_{L \text {, west }}$ und somit $\sigma_{\alpha_{\text {ost }}} \approx \sigma_{\alpha_{\text {west }}}$. Damit folgt für den Fehler des instationären Winkelanteils

$$
\sigma_{\alpha_{\mathrm{inst}}}=\frac{\sigma_{\alpha_{\mathrm{ost}} / \mathrm{west}}}{\sqrt{2}}
$$

infolge der Mittelwertbildung der Winkelanteile $\alpha_{\text {ost }}$ und $\alpha_{\text {west }}$ zur Berechnung von $\alpha_{\text {inst }}$. Der Fehler $\sigma_{\alpha_{\text {inst }}}$ hängt vom gemessenen Winkel selbst ab, da Gl. (B.4.7) und (B.4.6) Funktionen von $\Delta d_{L}$ sind. Unter Verwendung dieser Gleichungen und der oben bereits

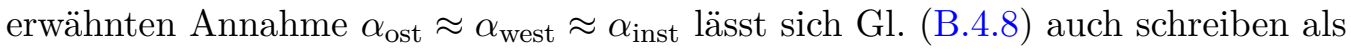

$$
\sigma_{\alpha_{\text {inst }}}\left(\alpha_{\text {inst }}\right)=\frac{\sqrt{2{\sigma_{d_{L}}}^{2}+\sigma_{\Delta x_{L}}^{2} \tan ^{2}\left(\alpha_{\text {inst }}\right)}}{\sqrt{2} \Delta x_{L}\left[1+\tan ^{2}\left(\alpha_{\text {inst }}\right)\right]} .
$$

Mit den jeweiligen Einzelfehlern folgt somit gemäß Gl. (B.4.5) für den mittleren Anstellwinkel ein Fehler von $\sigma_{\bar{\alpha}} \approx \mathbf{0}, \mathbf{0 8}^{\circ}$. Aus dem maximalen Messbereich der Lasertriangulatoren von $\pm 10 \mathrm{~mm}$ für $d_{L}$ folgt, dass $\Delta d_{L}=\left(d_{L 1}-d_{L 2}\right) \in[-20 \mathrm{~mm}: 20 \mathrm{~mm}]$ und somit $\alpha_{\text {inst }} \in\left[-5.23^{\circ}: 5.23^{\circ}\right]$. Der Messfehler $\sigma_{\alpha_{\text {inst }}}\left(\alpha_{\text {inst }}\right)$ gemäß Gl. (B.4.9) ist in Abb. B.10(a) grafisch dargestellt. Innerhalb des Messbereichs der Lasertriangulatoren beträgt der maximale Fehler $\sigma_{\alpha_{\text {inst }}} \approx \mathbf{0} \mathbf{, 0 2 3}^{\circ}$. Der Gesamtfehler für den Anstellwinkel nach Gl. (B.4.5) ist in Abb. B.10(b) grafisch ausgewertet. Hier beträgt der maximale Fehler innerhalb des Messbereiches $0,088^{\circ}$, so dass der Messfehler des Anstellwinkels bei Flatterversuchen mit $\sigma_{\alpha} \approx \mathbf{0}, \mathbf{0 9}^{\circ}$ abgeschätzt wird. 


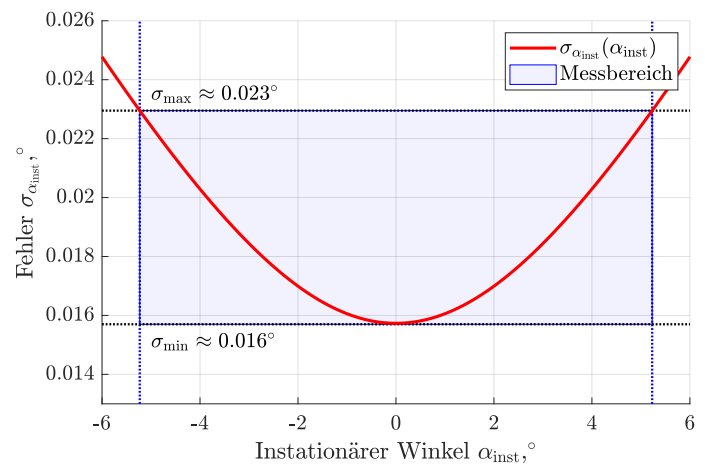

(a) Fehler des instationären Winkelanteils $\sigma_{\alpha_{\text {inst }}}$

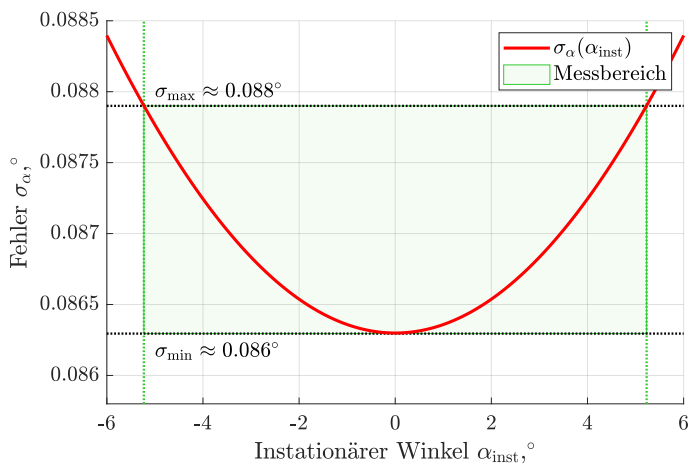

(b) Fehler des gesamten Anstellwinkels $\sigma_{\alpha}\left(\alpha_{\text {inst }}\right)$

Abbildung B.10.: Messfehler $\sigma_{\alpha_{\text {inst }}}$ des instationären Winkelanteils $\alpha_{\text {inst }}$, welcher mit den Lasertriangulatoren gemessen wird (a) und resultierender Gesamtfehler $\sigma_{\alpha}\left(\alpha_{\text {inst }}\right)$ des Anstellwinkels bei Flatterversuchen (b).

\section{B.4.3. Messfehler des Anstellwinkels bei Nickversuchen}

Der Abstand der Lasertriangulatoren am Nickversuchstand ist variabel und wird je nach erforderlichem Messbereich eingestellt. Zur Bestimmung des Abstands wurde im Vorfeld des Nickversuchs eine Winkelreihe aufgezeichnet, wobei die Spannungs- bzw. die Wegsignale $d_{\mathrm{L}_{i}}$ der vier eingesetzten Lasertriangulatoren und die eingestellten Winkel der Ost- und Westseite aufgezeichnet wurden. Letztere wurden mit zwei ClinotronicNeigungsmessgeräten mit einer Genauigkeit von $\sigma_{\text {clin }} \approx 0,03^{\circ}$ (vgl. B.4.2) bestimmt. Ausgehend von Gl. (B.4.6) folgt, dass zwischen der Differenz der Lasersignale und $\tan (\alpha)$ eine lineare Beziehung gemäß

$$
\Delta d_{L}=\Delta x_{L} \cdot \tan (\alpha)
$$

besteht. Mithilfe einer linearen Regression, analog zu Gl. (B.2.1), wurde somit der Laserabstand $\Delta x_{L}$ aus der gemittelten Lasersignaldifferenz $\Delta d_{L}$ und dem gemittelten Winkel $\bar{\alpha}$ bestimmt. Die Ergebnisse sind in Abb. B.11(a) zusammen mit der ermittelten Regressionsgeraden dargestellt. Es ergab sich somit ein mittlerer Abstand der Laser von $\Delta x_{L} \approx(262,26 \pm 0,08) \mathrm{mm}$. Der Fehler folgt bei dieser Vorgehensweise aus der Streuung der Messwerte um die Regressionsgerade, analog zu Gl. (B.2.2) und fällt bedeutend kleiner aus, als bei einer direkten Messung wie beim Flatterversuch (vgl. B.4.2). Für den Anstellwinkel ergibt sich dann analog zu Gl. (B.4.9) ein vom Winkel selbst abhängiger Fehler, welcher über den größtmöglichen Lasermessbereich $\alpha \in\left[-10,79^{\circ}: 10,79^{\circ}\right]$ in Abb. B.11(b) dargestellt ist ${ }^{4}$. Die Fehler der Lasersignale werden für den Nickversuch mit $\sigma_{d_{L}}=1 \cdot 10^{-4} \mathrm{~m}$ abgeschätzt [225]. Für den maximalen Fehler folgt dann $\sigma_{\alpha} \approx 0,022^{\circ}$, was dem eigentlichen Messfehler des Anstellwinkels entspricht. Für den realen Anstellwinkel

\footnotetext{
${ }^{4}$ Der hier deutlich vergrößerte Messbereich von $\alpha$ im Vergleich zum Flatterversuch begründet sich in dem Einsatz von Lasertriangulatoren mit einem größeren Abstands-Messbereich (vgl. 3.4).
} 


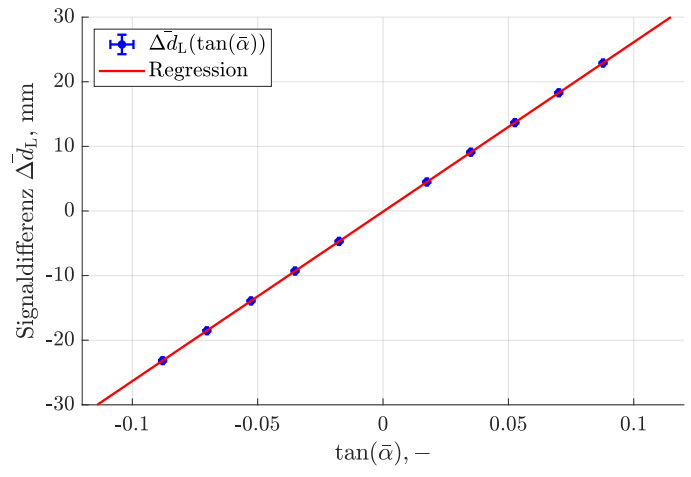

(a) Regression der Winkelkalibrierung

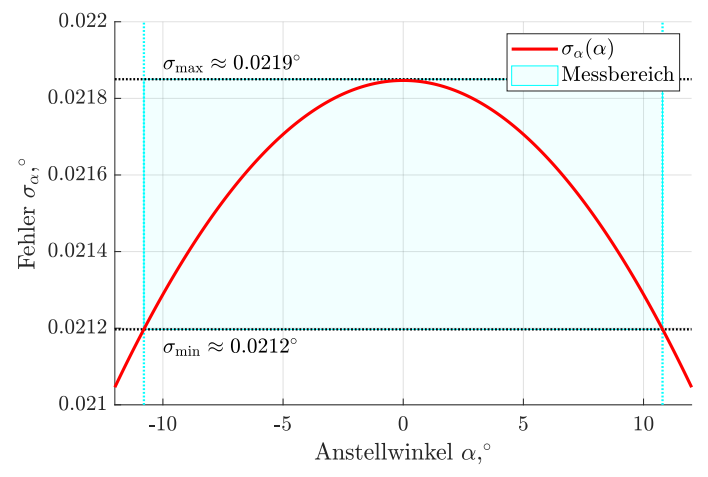

(b) Fehler des Anstellwinkels $\sigma_{\alpha}(\alpha)$

Abbildung B.11.: Lineare Regression der Winkelkalibrierung zur Bestimmung des Laserabstands $\Delta x_{L}$ (a) und Messfehler des Anstellwinkels (Anteil nur der Lasertriangulatoren) $\sigma_{\alpha}(\alpha)$ bei Nickversuchen (b).

des Modells gegenüber der Strömung muss jedoch zusätzlich der Fehler des Einbaubzw. Einstellwinkels berücksichtigt werden (vgl. B.4.2), entsprechend ergibt sich für den Anstellwinkel ein Gesamtfehler von $\sigma_{\alpha} \approx \mathbf{0 . 0 7}^{\circ}$.

Eine zusätzliche Fehlerquelle bei der Messung von Anstellwinkeln resultiert aus der strukturellen Verformung von Windkanalmodellen, wie z. B. einer Verdrehung des Mittelschnitts gegenüber den Fußpunkten der Modelle. Eine genauere Quantifizierung dieser Verdrehung ist nicht erfolgt. Hierin begründen sich jedoch geringfügige Abweichungen zwischen Messungen mit dem NVS und dem FVS infolge der unterschiedlichen Modellaufhängungen.

\section{B.4.4. Messfehler der Schlagbewegung}

Die Schlagbewegung berechnet sich aus dem Mittelwert der vier Signale der Lasertriangulatoren zu

$$
h(t)=\frac{1}{4} \cdot \sum_{i=1}^{4} d_{L_{i}} .
$$

Damit folgt auch der Messfehler $\sigma_{h}$ direkt aus den Messfehlern der einzelnen Lasersignale gemäß

$$
\sigma_{h}=\sqrt{\sum_{i=1}^{4} \sigma_{d_{L_{i}}}^{2} \cdot\left(\frac{\partial h}{\partial d_{L_{i}}}\right)^{2}}=\frac{\sigma_{d_{L}}}{\sqrt{4}}=\frac{\sigma_{d_{L}}}{2},
$$

mit $\sigma_{d_{L}}=6 \cdot 10^{-5} \mathrm{~m}$ (vgl. B.4.2). Somit folgt für den Messfehler des mit den Lasertriangulatoren gemessenen Schlagsignals $\sigma_{h}=3 \cdot 10^{-5} \mathrm{~m}$.

Infolge der Rotation des FVS kommt es darüber hinaus noch zu einer Verdrehung der Blattfedern aus ihrer horizontalen Ausgangsposition. Die gemessene Schlagbewegung verläuft nicht mehr vertikal, sondern um den Winkel $\alpha_{2 D}$ verdreht (vgl. 3.2.2). Die 
resultierende vertikale Komponente reduziert sich zu $h \cdot \cos \left(\alpha_{2 D}\right)$. Eine einfache Abschätzung mit Schlagamplituden von $\hat{h} \lesssim 0,01 \mathrm{~m}$ sowie Winkeln des 2 D-Support $\alpha_{2 D} \lesssim 4^{\circ}$ führen auf Reduzierungen der Vertikalkomponente von $\lesssim 2,5 \cdot 10^{-5} \mathrm{~m}$ im Vergleich zum gemessenen Schlagsignal. Auch wenn diese Abweichungen klein ausfallen, liegen sie doch in einer gleichen Größenordnung wie der Messfehler und werden somit, abweichend zu [28], auch berücksichtigt. Dies mit einbezogen wird für das Schlagsignal ein Fehler von $\sigma_{\mathbf{h}}=\mathbf{4} \cdot 10^{-5} \mathrm{~m}$ abgeschätzt.

Eine weitere Fehlerquelle bzgl. der Schlagbewegung bildet die endliche Dicke des Zielbalkens in Kombination mit einer möglichen, nicht mittigen Ausrichtung der Lasertriangulatoren zum Drehpunkt. Hierbei kommt es zu einer winkelabhängigen Verschiebung des Laserreflexpunktes, was einen zusätzlichen Anteil im Schlagsignal generiert. Dies wird hier jedoch nicht berücksichtigt. Eine ausführliche Diskussion dieses Zusammenhangs findet sich in [226]. 



\section{Ergänzungen zu Kapitel 4}

\section{C.1. Diskrete Fourier-Transformation}

Infolge der Abtastung eines zeitkontinuierlichen (analogen) Signals $x(t)$ mit der Abtastfrequenz $f_{s}=\frac{1}{T}$ geht dieses über in ein zeitdiskretes Signal $x[n]$, was als eine mit $x(t)$ gewichtete Diracstoßfolge

$$
x[n]=x(n T) \hat{=} x(t) \sum_{n=-\infty}^{\infty} \delta(t-n T)=\sum_{n=-\infty}^{\infty} x(n T) \delta(t-n T)
$$

aufgefasst werden kann [182, S.153]. Dabei beruht der letzte Schritt in Gl. (C.1.1) auf der Ausblendeigenschaft der DiRAC'schen $\delta$-Funktion. Die Abtastwerte $x[n]$ entsprechen den Werten von $x(t)$ zum Zeitpunkt $t=n T$. Wendet man die Fourier-Transformation gemäß Gl. (4.1.4) auf Gl. (C.1.1) an, so folgt (ohne Vorfaktor $\frac{1}{\sqrt{2 \pi}}$ )

$$
\begin{aligned}
X(\mathrm{i} \omega) & =\int_{-\infty}^{\infty} \sum_{n=-\infty}^{\infty} x(n T) \delta(t-n T) \exp (-\mathrm{i} \omega t) \mathrm{d} t \\
& =\sum_{n=-\infty}^{\infty} x(n T) \int_{-\infty}^{\infty} \delta(t-n T) \exp (-\mathrm{i} \omega t) \mathrm{d} t \\
& =\sum_{n=-\infty}^{\infty} x(n T) \exp (-\mathrm{i} \omega n T)=\sum_{n=-\infty}^{\infty} x[n] \exp (-\mathrm{i} \omega n T) .
\end{aligned}
$$

Dies ist die Fourier-Transformation für Abtastsignale (FTA) [182, S.154 ff.]. Es besteht jedoch das Problem, dass die FTA über unendlich viele Werte summiert. In der Praxis wird ein Signal mit $N$ Abtastwerten in einem endlichen Zeitfenster der Länge $N T$ abgetastet. Somit reduziert sich Gl. (C.1.2) zu

$$
\tilde{X}=\sum_{n=0}^{N-1} x[n] \exp (-\mathrm{i} \omega n T)=\sum_{n=0}^{N-1} x[n] \exp (-\mathrm{i} 2 \pi f n T),
$$

wobei das Spektrum periodisch (Periode $T=\frac{1}{f_{s}}$ ) und nunmehr beschränkt ist. Dies äußert sich in einer diskreten Frequenzachse $f[m]=\frac{m}{N T}$ mit N möglichen Frequenzen von 0 bis $f_{s}$ und einem Abstand von $\Delta f=f_{s} / N$. Setzt man die diskretisierte Frequenz 
in Gl. (C.1.3) ein, so folgt die diskrete Fourier-Transformation (DFT):

$$
\begin{aligned}
\text { DFT } & X[m]=\sum_{n=0}^{N-1} x[n] \exp \left(-\mathrm{i} 2 \pi \frac{m n}{N}\right) \\
\text { inverse DFT } & x[n]=\frac{1}{N} \sum_{n=0}^{N-1} X[m] \exp \left(\text { i } 2 \pi \frac{m n}{N}\right)
\end{aligned}
$$

Ein Vergleich von $X[m]$ mit den komplexen Fourier-Koeffizienten der Fourier-Reihe in Gl. (4.1.2) zeigt eine große Ähnlichkeit, welche im diskreten Fall mit $\hat{f}_{n}=\frac{X[m]}{N}$ gegeben ist $^{1}$. Dementsprechend enthalten das DFT-Spektrum und die Folge der komplexen Fourier-Koeffizienten gleichwertige Informationen [182, S.166 f.]. Die DFT kann durch die Diskretisierung in $n$ Abtastwerte und $m$ Spektrallinien $^{2}$ einfach numerisch implementiert werden. Jedoch steigt der Rechenaufwand recht schnell an. Für die Auswertung einer Frequenz (also ein $m$ ) sind $N$ komplexe Multiplikationen notwendig. Bei $N$ möglichen $m$ folgen somit $N^{2}$ komplexe Multiplikationen zur Berechnung des Spektrums $X[m]$.

Effizient berechnet wird die DFT durch die Fast Fourier Transformation (FFT) [182, S.174 ff.]. Dieser Algorithmus basiert auf Gl. (C.1.4), nutzt aber die Redundanz infolge der periodischen Winkelfunktionen aus. Der FFT-Algorithmus unterteilt die Blocklänge $N$ in mehrere ganzzahlige Teilsegmente und nutzt aus, dass die DFT von $\mathrm{X}[\mathrm{m}]$ mit $N$ Abtastwerten auch als Linearkombination von zwei DFTs mit $N / 2$ Abtastwerten geschrieben werden kann. Diese DFTs lassen sich weiter unterteilen, bis DFTs über 2 Abtastwerte gebildet werden. Am effizientesten funktioniert dies für eine Blocklänge $N=$ $2^{x}$, wobei die FFT die Anzahl an komplexen Multiplikationen auf $\frac{N}{2} \log _{2}(N)$ reduziert. Für die numerische Fourier-Transformationen wird in der vorliegenden Arbeit hauptsächlich der FFT-Algorithmus genutzt. Eine ausführlichere Darstellung des Algorithmus findet sich in [182, S.174 ff.] oder [227, S.36 ff.].

Abschließend sei noch erwähnt, dass es bei der Berechnung von Spektren mithilfe der DFT oftmals zu Fehlern in Folge der periodischen Fortsetzung eines endlichen, abgetasteten Signals kommt. Sofern eine periodische Fortsetzung des Signals ohne Sprungstellen nicht gegeben ist, kommt es zu Leckeffekten (engl.: Leakage) und das berechnete DFTSpektrum weicht vom realen Spektrum ab. Zur Reduzierung des Leakage-Effekts müssen die Transienten in der periodischen Fortsetzung weitestgehend reduziert werden. Hierzu wird das Signal bzw. ein Signalsegment vor Berechnung der DFT mit einer Fensterfunktion $w[n]$ gewichtet [186, S.187 ff.]. In der vorliegenden Arbeit wird hierfür das Hanning-Fenster

\footnotetext{
${ }^{1}$ Eine Division von Gl. C.1.4 mit $N$ liefert folglich physikalisch interpretierbare Resultate [186, S.180 f.], jedoch berechnet u.a. auch MATLAB ${ }^{\circledR}$ die DFT analog zu Gl. C.1.4.

${ }^{2}$ Bei der numerischen Implementierung von Gl. (C.1.4) läuft die Zahlvariable $n$ von 0 bis $N-1$. Die diskretisierte Frequenz $m$ wird infolge des Abtasttheorems auf die Werte $m<\frac{N}{2}$ begrenzt [182, S.167 ff.], $m=\frac{N}{2}$ entspricht der Nyquist Frequenz. Sofern für eine Rücktransformation nach Gl. (C.1.5) auch die Werte $X[m]$ für $m>\frac{N}{2}$ benötigt werden, folgen diese aus den Symmetrieeigenschaften [186, S.186].
} 


$$
w[n]=\sin ^{2}\left(\frac{\pi n}{N}\right)=\frac{1}{2}\left[1-\cos \left(\frac{2 \pi n}{N}\right)\right]
$$

verwendet. Gl. (C.1.6) führt jedoch zu einem Informationsverlust, was sich als Amplitudenfehler im Spektrum auswirkt. Dieser kann mit einem Skalierungs- bzw. AmplitudenKorrektur-Faktor korrigiert werden (siehe [182, S. 195]).

\section{C.2. Welch-Methode}

Wie in 4.1.2 erwähnt, sind die Leistungsdichtespektren PSD und CPSD von Interesse. Die numerische Berechnung erfolgt mit der WeLCH-Methode [186, S.211 ff.], welche im Wesentlichen wiederum auf die DFT/FFT zurückgreift. Mit der WELCH-Methode berechnet sich das diskrete Autoleistungsdichtespektrum (PSD) nach Gl. (4.1.7) gemäß

$$
S_{x x}[m]=S_{A} \cdot \frac{1}{k} \sum_{j=1}^{k} X_{w, j}^{*}[m] X_{w, j}[m]=S_{A} \cdot \frac{1}{k} \sum_{j=1}^{k}\left|X_{w, j}[m]\right|^{2}
$$

und das diskrete Kreuzleistungsdichtespektrum (CPSD) nach Gl. (4.1.8) zu

$$
S_{x y}[m]=S_{A} \cdot \frac{1}{k} \sum_{j=1}^{k} Y_{w, j}[m] X_{w, j}^{*}[m] .
$$

Dabei beschreibt $X_{w, j}$ die DFT des mit der Fensterfunktion $w[n]$ multiplizierten Signals $x_{j}[n]$, also gemäß Gl. (C.1.4)

$$
X_{w, j}[m]=\sum_{n=0}^{N-1} x_{j}[n] w[n] \exp \left(-\mathrm{i} 2 \pi \frac{m n}{N}\right) .
$$

Bei der Welch-Methode wird also das Signal $x[n]$ mit $k N$ Abtastwerten in $k$ Teilsegmente $x_{j}[n]$ mit jeweils $N$ Werten unterteilt. Jedes dieser Teilsegmente wird mit einer Fensterfunktion (hier: Hanning-Fenster, vgl. C.1) multipliziert und die DFT berechnet. Abschließend wird dann über die $k$ Teilsegmente gemittelt. Bei dem zusätzlichen Faktor $S_{A}$ in Gl. (C.2.2) bzw. (C.2.3) handelt es sich um eine Skalierungskonstante, die u. a. die Amplitudenkorrektur des verwendeten Fensters enthält (vgl. C.1).

In der vorliegenden Arbeit wird die Skalierungskonstante jedoch vernachlässigt $\left(S_{A} \rightarrow 1\right)$, da hier PSDs weitestgehend qualitativ, zur Bestimmung von Frequenzanteilen betrachtet, oder einzelne Amplituden untereinander ins Verhältnis gesetzt werden. Bei der Berechnung von Übertragungsfunktionen (vgl. 4.1.3 und C.3) kürzen sich die Skalierungsfaktoren ohnehin raus. Auch wird in der vorliegenden Arbeit eine Überlappung von 62,5\% der einzelnen Segmente bei der Bildung des Mittelwertes verwendet. Dies steigert die 
effektive Anzahl an Mittellungen und reduziert bestmöglich die Varianz bei Anwendung des Hanning-Fensters [186, S.217 ff.].

\section{C.3. Schätzung der Übertragungsfunktion}

In 4.1.3 ist mit Gl. (4.1.10) bereits eine einfache Möglichkeit zur Berechnung der Übertragungsfunktion gegeben, was durch eine Erweiterung mit $\frac{X^{*}(\mathrm{i} \omega)}{X^{*}(\mathrm{i} \omega)} \mathrm{zu}$ Gl. (4.1.11) bzw.

$$
H_{1}(\mathrm{i} \omega)=\frac{Y(\mathrm{i} \omega)}{X(\mathrm{i} \omega)} \cdot \frac{X^{*}(\mathrm{i} \omega)}{X^{*}(\mathrm{i} \omega)}=\frac{S_{y x}^{*}(\omega)}{S_{x x}(\omega)}=\frac{S_{x y}(\omega)}{S_{x x}(\omega)}
$$

führt. Eine Erweiterung von Gl. (4.1.10) mit $\frac{Y^{*}(\mathrm{i} \omega)}{Y^{*}(\mathrm{i} \omega)}$ liefert hingegen

$$
H_{2}(\mathrm{i} \omega)=\frac{Y(\mathrm{i} \omega)}{X(\mathrm{i} \omega)} \cdot \frac{Y^{*}(\mathrm{i} \omega)}{Y^{*}(\mathrm{i} \omega)}=\frac{S_{y y}(\omega)}{S_{x y}^{*}(\omega)}=\frac{S_{y y}(\omega)}{S_{y x}(\omega)} .
$$

Es ergeben sich somit zwei mögliche Varianten einer Übertragungsfunktion. Die Unterschiede von $H_{1}$ und $H_{2}$ werden jedoch erst für reale Signale deutlich, welche mit Störungen wie u.a. Rauschen überlagert sind [182, Anhang B S.56 ff.]. Durch die zur Berechnung der Übertragungsfunktionen durchgeführten Korrelationsanalysen (vgl. C.2) mitteln sich unkorrelierte Störungen weg. Damit ergibt sich ein Unterschied, so dass je nach Störung $H_{1}$ oder $H_{2}$ die bessere Schätzung der wahren Übertragungsfunktion liefert [182, Anhang S.62]). Insgesamt empfiehlt es sich $\mathbf{H}_{\mathbf{1}}$ bei ungestörtem Eingangssignal und $\mathbf{H}_{2}$ bei ungestörtem Ausgangssignal zu verwenden. Generell ist bei realen, mit Rauschen überlagerten Signalen Gl. (C.3.1) bzw. (C.3.2) einer Berechnung mit Gl. (4.1.10) vorzuziehen. Eine genaue Herleitung dieses Sachverhaltes findet sich in [182, Anhang B S.60 f.], weitere Informationen sind des Weiteren in [228] zu finden.

\section{C.4. Interpolation der Profilkontur und Druckverteilung}

Für die Berechnung der aerodynamischen Beiwerte wird eine Diskretisierung der CAST 10-2 Profilkontur vorgenommen. Ausgehend von einem bestehenden Satz an mit der Flügeltiefe $c$ entdimensionalisierten Profilkoordinaten $\vec{x}_{0}=\left(x_{0} z_{0}\right)^{\mathrm{T}}$, wird eine formerhaltende, stückweise kubische Interpolation ${ }^{3}$ durchgeführt. Für eine eindeutige Splinefunktion wird die Profilkoordinate $x_{i}$ durch $\psi_{i}=x_{i} \cdot \operatorname{sgn}($ Seite) substituiert, um der Koordinatenposition auf der Oberseite $(\psi>0)$ oder Unterseite $(\psi<0)$ des Profils Rechnung zu tragen (vgl. [173, S.62]), schematisch dargestellt in Abb. C.1. Durch die Interpolation wird eine äquidistante Verteilung $\mathrm{d} \psi_{i}=\psi_{i+1}-\psi_{i}$ der Stützstellen entlang der Profiltiefe erzeugt,

\footnotetext{
${ }^{3}$ Die verwendete Interpolation nutzt stückweise kubische, hermitesche Polynome $P(x)$, so dass die erste Ableitung $\mathrm{d} P / \mathrm{d} x$ stetig ist. $P(x)$ erhält sowohl die Monotonie als auch die Konvexität der Daten und unterbindet irrelevante „Beulen“ oder "Wackler" des interpolierten Splines [229].
} 


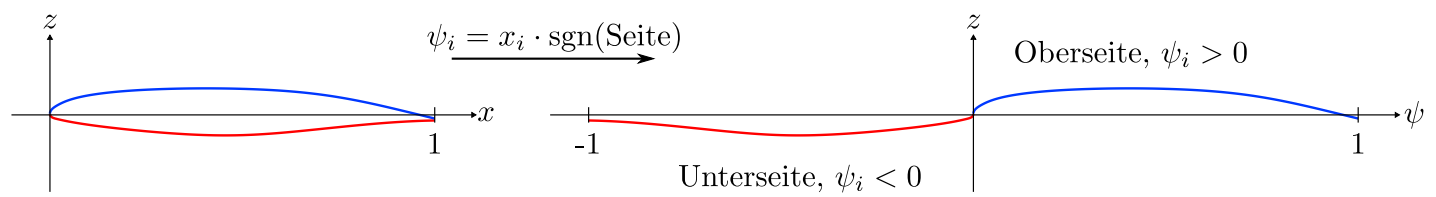

Abbildung C.1.: Schematische Darstellung zur Auswirkung der ProfilkoordinatenSubstitution $\psi=x_{i} \cdot \operatorname{sgn}($ Seite) für eine eindeutige Spline-Interpolation der Profilkontur.

womit sich die Profilkontur stückweise beschreiben lässt durch

$$
\mathrm{d} \vec{s}_{i}=\vec{x}_{i+1}-\vec{x}_{i}=\left(\begin{array}{c}
\mathrm{d} \psi_{i} \\
\mathrm{~d} z_{i}
\end{array}\right)=\left(\begin{array}{c}
\psi_{i+1}-\psi_{i} \\
z_{i+1}-z_{i}
\end{array}\right) .
$$

Für ein Bogenelement $\mathrm{d} s_{i}$ der Profilkontur gilt dementsprechend

$$
\left|\mathrm{d} \vec{s}_{i}\right|=\mathrm{d} s_{i}=\sqrt{\mathrm{d} \psi_{i}^{2}+\mathrm{d} z_{i}^{2}}=\sqrt{\left(\psi_{i+1}-\psi_{i}\right)^{2}+\left(z_{i+1}-z_{i}\right)^{2}} .
$$

Durch eine Multiplikation mit der Flügelspannweite $s$ folgt damit für ein Flächenelement des Profils $\mathrm{d} A_{i}=\mathrm{d} s_{i} \cdot s$. Die Orientierung des Flächenelementes ist gegeben durch den zugehörigen Normaleneinheitsvektor

$$
\hat{n}_{i}=\frac{1}{\left|\vec{n}_{i}\right|} \vec{n}_{i}=\frac{1}{\mathrm{~d} s_{i}}\left(\begin{array}{c}
-\mathrm{d} z_{i} \\
\operatorname{sgn}\left(\chi_{i}\right) \mathrm{d} \psi_{i}
\end{array}\right) .
$$

Jedem Flächenelement wird der Ortsvektor $\overrightarrow{\chi_{i}}=\left(\begin{array}{ll}\chi_{i} & \zeta_{i}\end{array}\right)^{T}$ zugeordnet, wobei die Stützstellen $\chi_{i}=\psi_{i}+\mathrm{d} \psi_{i} / 2$ und $\zeta_{i}=z_{i}+\mathrm{d} z_{i} / 2$ so gewählt sind, dass diese jeweils mittig auf dem zugehörigen Bogenelement d $s_{i}$ liegen. In Abb. C.2 (a) ist die Koordinaten- und Vektorbezeichnung bzgl. der Diskretisierung der Profilkontur sowie deren Berechnung schematisch dargestellt.

(a)

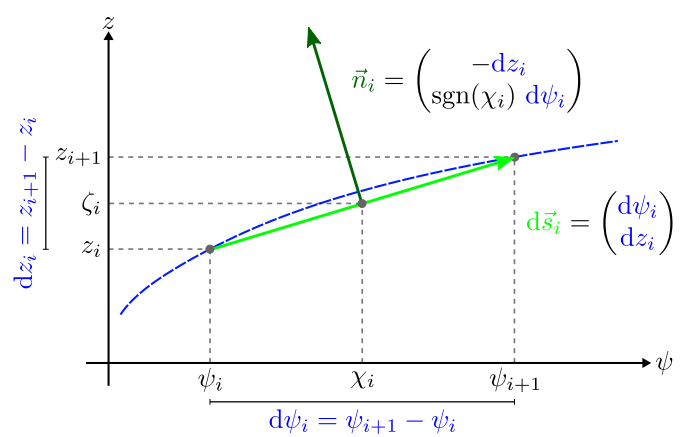

(b)

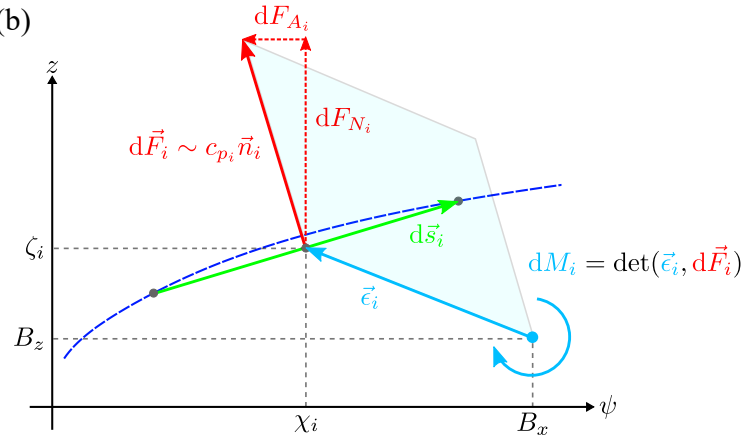

Abbildung C.2.: Ausschnitt der Profilkontur zur schematischen Darstellung der Diskretisierung der Profiloberfläche. In (a) sind die Koordinatenbezeichnungen und Vektorbeziehungen sowie deren Berechnung abgebildet. In (b) ist die Komponentenzerlegung der resultierenden Druckkraft $\mathrm{d} \vec{F}_{i}$ und die Berechnung des Drehmomentes grafisch veranschaulicht. Die Berücksichtigung des Vorzeichens von $\chi_{i}$ ist nicht ersichtlich. 
An den Stützstellen $\chi_{i}$ wird in gleicher Weise eine Interpolation der Druckbeiwerte $c_{p_{i}}(t)$ durchgeführt. An der Hinterkante wird als abschließende Stützstelle ein gewichteter Mittelwert der in der Nähe der Hinterkante gemessenen Druckbeiwerte $c_{p_{O}}$ und $c_{p_{U}}$ für die Ober- und Unterseite des Profils gebildet

$$
c_{p}(\chi=1, t)=\frac{c_{p_{O}} \cdot\left(1-\left.x\right|_{c_{p_{U}}}\right)+c_{p_{U}} \cdot\left(1-\left.x\right|_{c_{p_{O}}}\right)}{\left(1-\left.x\right|_{c_{p_{U}}}\right)+\left(1-\left.x\right|_{c_{p_{O}}}\right)} .
$$

Die Gewichtungsfaktoren ergeben sich aus dem Abstand der letzten Druckmessstellen zur Hinterkante $\left(1-\left.x\right|_{c_{p_{O}}}\right)$ bzw. $\left(1-\left.x\right|_{c_{p_{U}}}\right)$. Hiermit wird ein identischer Druck an der Hinterkante für beide Profilseiten erzeugt, womit dort für die Druckdifferenz $\left[c_{p_{U}}-c_{p_{O}}\right]=0$ folgt, entsprechend einer "Nulllastannahme“ der Hinterkante. Weiterhin wird somit die KuTTA-Bedingung erfüllt ${ }^{4}$. Es korrespondiert jeweils ein interpolierter Druckbeiwert $c_{p}\left(\chi_{i}, t\right)$ mit einem Flächenelement $\mathrm{d} A\left(\chi_{i}\right)$ und dessen Normalenvektor $\hat{n}\left(\chi_{i}\right)$.

\section{C.5. Diskretisierte Berechnung der aerodynamischen Kräfte}

Für die auf ein Flächenelement $\mathrm{d} A\left(\chi_{i}\right)$ entlang $\hat{n}\left(\chi_{i}\right)$ wirkende Druckkraft gilt nunmehr

$$
\mathrm{d} \vec{F}\left(\chi_{i}, t\right)=\left(\begin{array}{l}
\mathrm{d} F_{A}\left(\chi_{i}, t\right) \\
\mathrm{d} F_{N}\left(\chi_{i}, t\right)
\end{array}\right)=-q_{\infty} c c_{p}\left(\chi_{i}, t\right) \hat{n}\left(\chi_{i}\right) \mathrm{d} A\left(\chi_{i}\right)
$$

Die Kraft teilt sich in eine, zur Bezugslinie horizontale Axialkomponente $\mathrm{d} F_{A}\left(\chi_{i}, t\right)$ und eine, zur Bezugslinie vertikale Normalkomponente $\mathrm{d} F_{N}\left(\chi_{i}, t\right)$ auf. Dies ist in Abb. C.2(b) dargestellt. Eine zusätzliche Multiplikation mit der Flügeltiefe $c$ ist infolge der entdimensionalisierten Profilkoordinaten erforderlich. Nach Gl. (4.2.1) folgt die gesamte Druckkraft aus einer Integration über die Profilkontur. Im diskretisierten Fall folgt dies aus der Aufsummierung von Gl. (C.5.1), also der Summierung über alle Flächenelemente gemäß

$$
\vec{F}(t)=\sum_{i} \mathrm{~d} \vec{F}\left(\chi_{i}, t\right)=-q_{\infty} c \sum_{i} c_{p}\left(\chi_{i}, t\right) \hat{n}\left(\chi_{i}\right) \mathrm{d} A\left(\chi_{i}\right)
$$

Die Auftriebs- und Druckwiderstandskraft, $F_{L}(t)$ und $F_{D}(t)$, folgen dann durch die Transformation ins Laborkoordinatensystem zur Berücksichtigung des momentanen Anstellwinkels $\alpha(t)$ gemäß Gl. (4.2.2).

\footnotetext{
${ }^{4}$ Die Gültigkeit der KUTTA-Bedingung ist für stationäre, anliegende Strömungen gegeben. Im Falle von instationären Strömungen wird diese kontrovers diskutiert bzw. es existieren weitere Ansätze und Formulierungen für eine instationäre Kutta-Bedingung [230-232]. Für kleine, hier relevante reduzierte Frequenzen $\omega^{*} \leq 0,5$ behält die KuTTA-Bedingung jedoch ihre Gültigkeit [231,233]. Der zusätzliche Einfluss einer transsonischen Strömung mit einer Stoß-Grenzschicht-Interaktion und einer möglichen Hinterkantenablösung bzgl. der Gültigkeit der KUTTA-Bedingung ist jedoch kontrovers zu betrachten.
} 
Das aerodynamische Moment pro Flächenelement $\mathrm{d} M_{i}$ folgt aus $\mathrm{d} \vec{F}\left(\chi_{i}, t\right)$ nach Gl. C.5.1) und dem Vektor $\vec{\epsilon}\left(\chi_{i}\right)=\left(\begin{array}{lll}B_{x}-\operatorname{sgn}\left(\chi_{i}\right) \chi_{i} & B_{z}-\zeta_{i}\end{array}\right)^{T}$. Letzterer beschreibt die Verbindung zwischen dem Bezugspunkt ${ }^{5} \vec{B}=\left(\begin{array}{ll}B_{x} & B_{z}\end{array}\right)^{T}$ und dem Ortsvektor des Flächenelementes $\vec{\chi}_{i}$ unter Berücksichtigung des Vorzeichens von $\chi_{i}$. Das Moment entspricht dem Flächeninhalt des von den beiden Vektoren aufgespannten Parallelogramms, dargestellt in Abb. C.2(b) und berechnet sich aus der Determinante dieser beiden Vektoren zu

$$
\mathrm{d} M\left(\chi_{i}, t\right)=c \operatorname{det}\left(\vec{\epsilon}\left(\chi_{i}\right), \mathrm{d} \vec{F}\left(\chi_{i}, t\right)\right)=c\left[\epsilon_{x} \mathrm{~d} F_{N}\left(\chi_{i}, t\right)-\epsilon_{z} \mathrm{~d} F_{A}\left(\chi_{i}, t\right)\right]
$$

Das gesamte aerodynamische Nickmoment folgt wiederum aus einer Summierung von Gl. (C.5.3) über alle Flächenelemente $M(t)=\sum_{i} \mathrm{~d} M\left(\chi_{i}, t\right)$.

\section{C.6. Rekonstruktion der Modellbewegung}

Die Bewegung des Tragfügelmodells bzw. der Flächenelemente und Normalenvektoren (vgl. C.4) im Laborkoordinatensystem lässt sich als affine Transformation darstellen. Zur Beschreibung der Drehbewegung $\mathbf{A}(t)$ des Modells wird zunächst mithilfe der Translationsmatrix $\mathbf{T}\left(-E_{x},-E_{z}\right)$ das Labor-Koordinatensystem verschoben, so dass der Drehpunkt des Modells $\vec{E}=\left(\begin{array}{ll}E_{x} & E_{z}\end{array}\right)^{T}$ der neue Ursprung ist. Daraufhin wird eine Rotation $\mathbf{D}(\alpha)$ um den Anstellwinkel $\alpha(t)$ vorgenommen und die vorherige Translation mit $\mathbf{T}\left(E_{x}, E_{z}\right)$ invers durchgeführt

$$
\mathbf{A}(t)=\underbrace{\left(\begin{array}{ccc}
1 & 0 & E_{x} \\
0 & 1 & E_{z} \\
0 & 0 & 1
\end{array}\right)}_{\mathbf{T}\left(E_{x}, E_{z}\right)} \underbrace{\left(\begin{array}{ccc}
\cos (\alpha(t)) & \sin (\alpha(t)) & 0 \\
-\sin (\alpha(t)) & \cos (\alpha(t)) & 0 \\
0 & 0 & 1
\end{array}\right)}_{\mathbf{D}(\alpha(t))} \underbrace{\left(\begin{array}{ccc}
1 & 0 & -E_{x} \\
0 & 1 & -E_{z} \\
0 & 0 & 1
\end{array}\right)}_{\mathbf{T}\left(-E_{x},-E_{z}\right)} .
$$

Die Schlagbewegung $\mathbf{H}(t)$ des Modells wird beschrieben durch die Translation

$$
\mathbf{H}(t)=\mathbf{T}(0, h(t))=\left(\begin{array}{ccc}
1 & 0 & 0 \\
0 & 1 & \frac{h(t)}{c} \\
0 & 0 & 1
\end{array}\right)
$$

\footnotetext{
${ }^{5}$ Das aerodynamische Moment ist auf den $c / 4$-Punkt bei $25 \%$ der Profiltiefe bezogen, $\Rightarrow \vec{B}=\left(\begin{array}{ll}0,25 & 0\end{array}\right)^{T}$.
} 
Die zeitabhängigen Ortsvektoren $\overrightarrow{\chi_{i}}(t)$ und Normalenvektoren $\hat{n}\left(\chi_{i}, t\right)$ der Flächenelemente berechnen sich damit unter der Verknüpfung von Gl. (C.6.1) und (C.6.2) zu

$$
\begin{aligned}
\vec{\chi}_{i}(t) & =\mathbf{H}(t) \mathbf{A}(t) \overrightarrow{\chi_{i}}\left(t_{0}\right), \\
\hat{n}\left(\chi_{i}, t\right) & =\mathbf{A}(t) \hat{n}\left(\chi_{i}, t_{0}\right) .
\end{aligned}
$$

Die dritte Vektorkomponente ist lediglich zur Ausführung der Matrizenmultiplikation hinzugefügt und kann vernachlässigt werden. Die kinematische Geschwindigkeit der Modelloberfläche im Laborkoordinatensystem folgt aus Gl. (C.6.3) durch Differentiation nach der Zeit zu

$$
\vec{v}\left(\overrightarrow{\chi_{i}}, t\right)=\frac{\mathrm{d} \overrightarrow{\chi_{i}}}{\mathrm{~d} t}=\left(\begin{array}{c}
-\left(\chi_{0}-E_{x}\right) \sin (\alpha) \dot{\alpha}+\left(\zeta_{0}-E_{z}\right) \cos (\alpha) \dot{\alpha} \\
-\left(\chi_{0}-E_{x}\right) \cos (\alpha) \dot{\alpha}-\left(\zeta_{0}-E_{z}\right) \sin (\alpha) \dot{\alpha}+\dot{h}
\end{array}\right) .
$$

\section{C.7. Diskretisierte Berechnung des Energieeintrags}

Für die diskretisierte Berechnung bietet sich wiederum eine lokale Betrachtung des Energieeintrags an. So ist die durch die Druckkraft verrichtete Gesamtarbeit pro Flächenelement $\mathrm{d} W\left(\chi_{i}, t\right)$ gegeben durch Gl. (4.2.6) und (C.5.1) mit dem zeitabhängigen Normalenvektor Gl. (C.6.4) zu

$$
\mathrm{d} W\left(\chi_{i}, t\right)=c \mathrm{~d} \vec{F}\left(\chi_{i}, t\right) \cdot \mathrm{d} \overrightarrow{r_{i}}(t)=-q_{\infty} c^{2} c_{p}\left(\chi_{i}, t\right) \mathrm{d} A\left(\chi_{i}\right) \hat{n}\left(\chi_{i}, t\right) \cdot \mathrm{d} \overrightarrow{r_{i}}(t)
$$

Berücksichtigt man nur den instationären Anteil des Drucks $c_{p}\left(\chi_{i}, t\right)-\overline{c_{p}}\left(\chi_{i}\right)$ (vgl. 4.2.2) folgt aus Gl. (C.7.1) für die instationäre Arbeit pro Flächenelement $\mathrm{d} W_{\text {inst }}\left(\chi_{i}, t\right)$

$$
\mathrm{d} W_{\text {inst }}\left(\chi_{i}, t\right)=-q_{\infty} c^{2}\left[c_{p}\left(\chi_{i}, t\right)-\overline{c_{p}}\left(\chi_{i}\right)\right] \mathrm{d} A\left(\chi_{i}\right) \hat{n}\left(\chi_{i}, t\right) \cdot \mathrm{d} \overrightarrow{r_{i}}(t)
$$

Die Arbeit geleistet am gesamten Flügel ergibt sich wiederum durch eine Summierung über alle Flächenelemente

$$
W(t)=\sum_{i} \mathrm{~d} W\left(\chi_{i}, t\right) \quad \text { bzw. } \quad W_{\text {inst }}(t)=\sum_{i} \mathrm{~d} W_{\text {inst }}\left(\chi_{i}, t\right) .
$$

\section{C.8. Vorzeichenkonvention bei Phasenverschiebungen}

Im Folgenden ist die in der vorliegenden Arbeit verwendete Definition der Phasenverschiebung samt Vorzeichenkonvention bei der Betrachtung von Phasendifferenzen dargelegt, was insbesondere bei der Betrachtung von instationären Beiwerten relevant ist (vgl. 4.2.3). 
Gegeben seien zwei Schwingungen

$$
\Psi_{1}(t)=A \cdot \sin \left(\omega t+\varphi_{1}\right) \quad \text { und } \quad \Psi_{2}(t)=A \cdot \sin \left(\omega t+\varphi_{2}\right),
$$

mit identischer Amplitude $A$ und Kreisfrequenz $\omega$, jedoch unterschiedlichem Nullphasenwinkel $\varphi_{1}$ und $\varphi_{2} . \Psi_{1}$ wird als Bezugsschwingung und damit $\varphi_{1}$ als Bezugswinkel gewählt, womit für die Phasendifferenz bzw. den Phasenverschiebungswinkel

$$
\Delta \varphi_{\Psi_{2}, \Psi_{1}}=\varphi_{2}-\varphi_{1}
$$

folgt. In Abb. C.3 sind $\Psi_{1}$ und $\Psi_{2}$ grafisch über der Zeit $t$ dargestellt. Die Nullphasenwinkel im oberen Graphen sind so gewählt, dass $\varphi_{\mathbf{1}}<\varphi_{\mathbf{2}}$ und damit $\boldsymbol{\Delta} \varphi>\mathbf{0}$ ist. Das Zeigerdiagramm bildet diesen Zustand für $t=0 \mathrm{ab}$ (durchgezogene Linien). Betrachtet man das Zeigerdiagramm zum Zeitpunkt $t^{*}$ mit $\Psi_{1}\left(t^{*}\right)=0$ (gestrichelte Linien), ist die
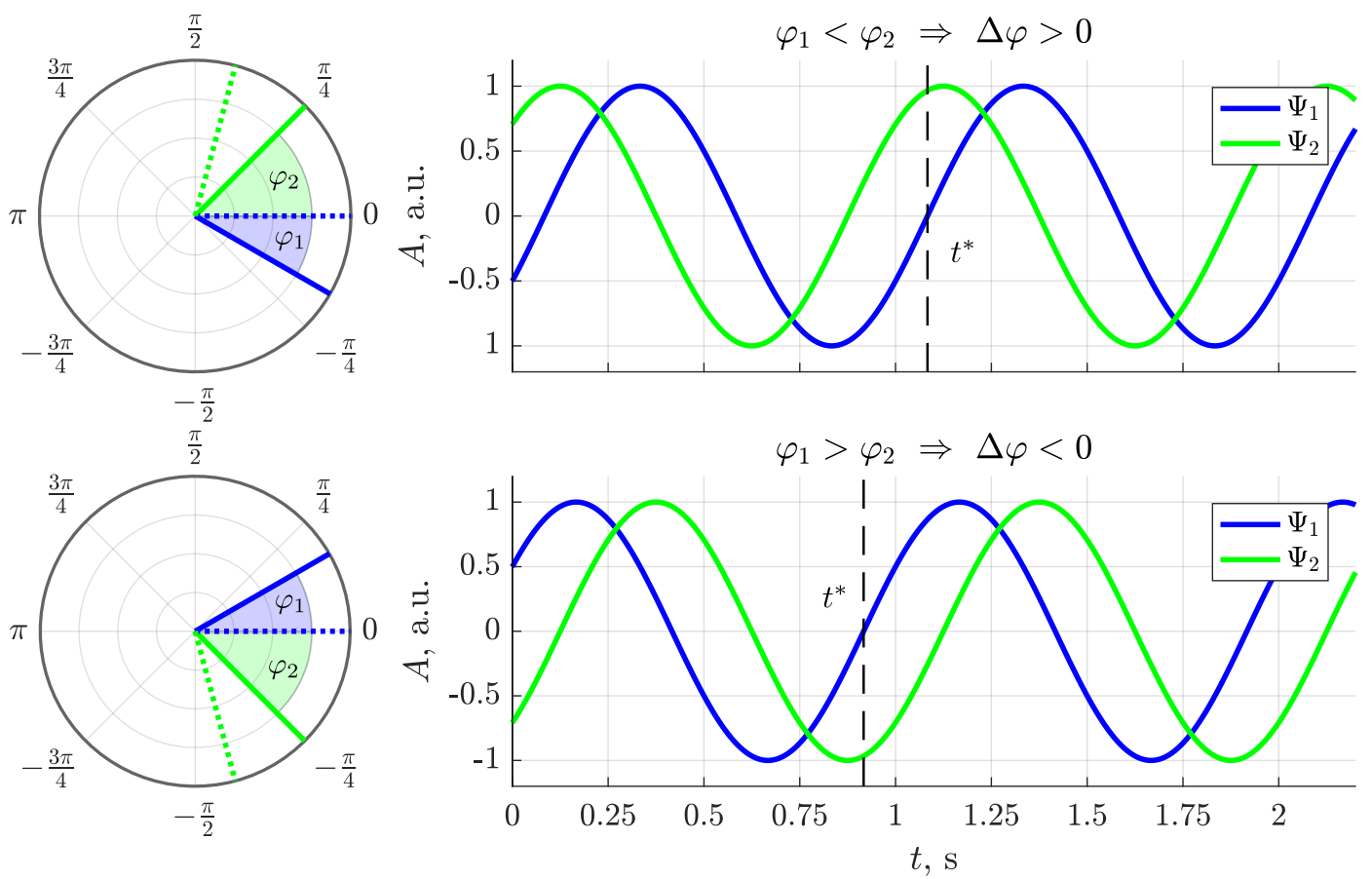

Abbildung C.3.: Grafische Veranschaulichung zur Definition der Phasenverschiebung. Oben: $\Psi_{1}(t)$ und $\Psi_{2}(t)$ für den Fall $\varphi_{1}<\varphi_{2}$ und damit $\Delta \varphi>0$. Unten: $\Psi_{1}(t)$ und $\Psi_{2}(t)$ für den Fall $\varphi_{1}>\varphi_{2}$ und damit $\Delta \varphi<0$. Die Zeigerdiagramme links veranschaulichen die gewählten Nullphasenwinkel $\varphi_{1}$ und $\varphi_{2}$ jeweils zum Zeitpunkt $t=0$ (durchgezogene Linien) und $t=t^{*}$, entsprechend einem Nulldurchgang der Bezugsschwingung $\Psi_{1}$ (gestrichelte Linien). Für $t^{*}$ ist die Phasendifferenz am Zeiger von $\Psi_{2}$ direkt abzulesen.

Phasendifferenz $\Delta \varphi$ im Zeigerdiagramm direkt abzulesen. Im Allgemeinen ist der Zeiger von $\Psi_{2}$ dem von $\Psi_{1}$ in mathematischen Drehsinn stehts voraus. Hier wird von einer Voreilung von $\Psi_{2}$ in Bezug zu $\Psi_{1}$ gesprochen. In Analogie zur Berechnung der instationären Beiwerte (vgl. 4.2.3) zeigt die mit $\Psi_{1}$ als Eingang und $\Psi_{2}$ als Ausgang berechnete 
Übertragungsfunktion $H(\mathrm{i} \omega)$ einen positiven Imaginärteil $\operatorname{Im}(\mathbf{H})>\mathbf{0}$, dargestellt im linken Argand-Diagramm in Abb. C.4.

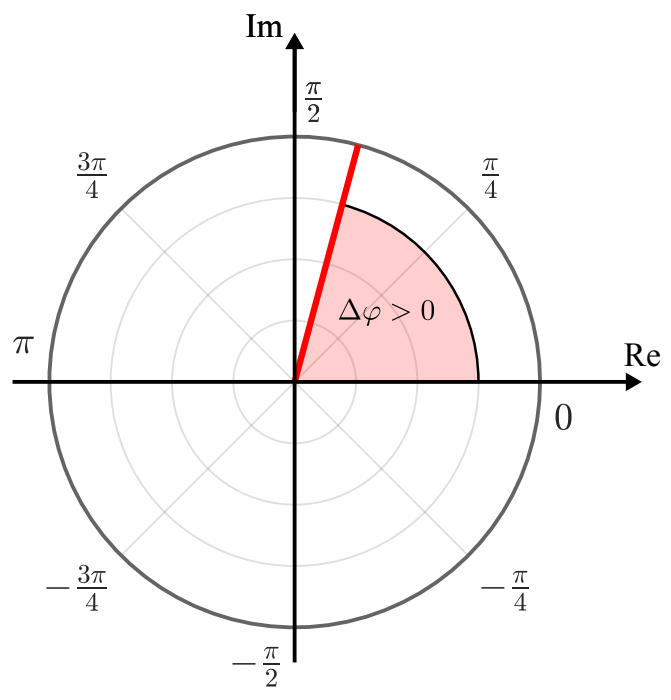

voreilende Schwingung

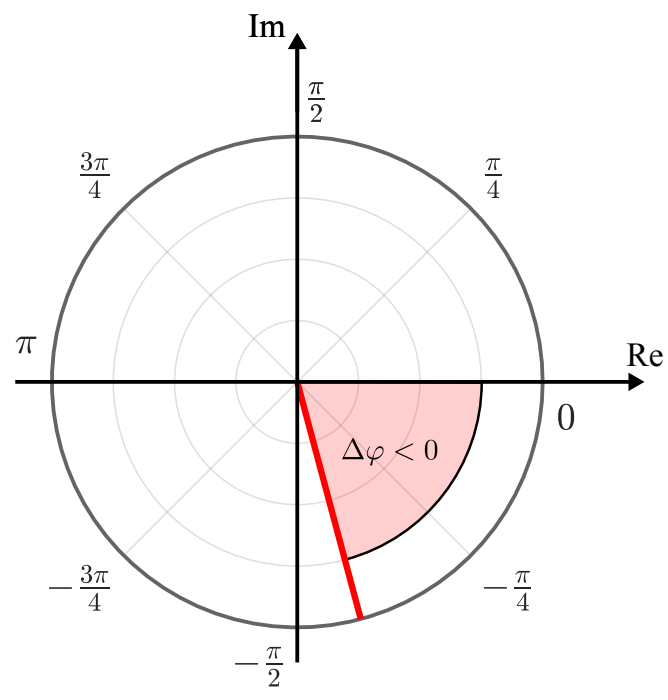

nacheilende Schwingung

Abbildung C.4.: Argand-Diagramm zur Darstellung der komplexen Übertragungsfunktion $H(\mathrm{i} \omega)$. Links: $H(\mathrm{i} \omega)$ für den in Abb. C.3 oben dargestellten Fall. Die Übertragungsfunktion hat einen positiven Imaginärteil $\operatorname{Im}(H)>0$, die Phasendifferenz $\Delta \varphi$ ist ebenfalls positiv und im Diagramm abzulesen. Rechts: $H(\mathrm{i} \omega)$ für den in Abb. C.3 unten dargestellten Fall mit einem negativen Imaginärteil $\operatorname{Im}(H)<0$, entsprechend einer negativen Phasendifferenz.

Der untere Graph in Abb. C.3 zeigt $\Psi_{1}$ und $\Psi_{2}$ für den Fall $\varphi_{\mathbf{1}}>\varphi_{\mathbf{2}}$ und damit $\boldsymbol{\Delta} \varphi<\mathbf{0}$. Das Zeigerdiagramm verdeutlicht hier, dass $\Psi_{2}$ der Schwingung $\Psi_{1}$ nacheilt. Für den Imaginärteil der Übertragungsfunktion folgt $\operatorname{Im}(\mathbf{H})<\mathbf{0}$, dargestellt im rechten ArgandDiagramm in Abb. C.4.

Wie in 4.2.3 erwähnt, wird bei der Berechnung der instationären Beiwerte das Signal des Anstellwinkels $\alpha(t)$ als Bezugsschwingung (Eingang) gewählt, womit die Terminologie »Vor- und Nacheilen« sich auf diesen bezieht, sofern nicht explizit anders angegeben. 


\section{Ergänzungen zu Kapitel 5}

\section{D.1. Zu 5.4: Instationäre Effekte bei statischen Anstellwinkeln}
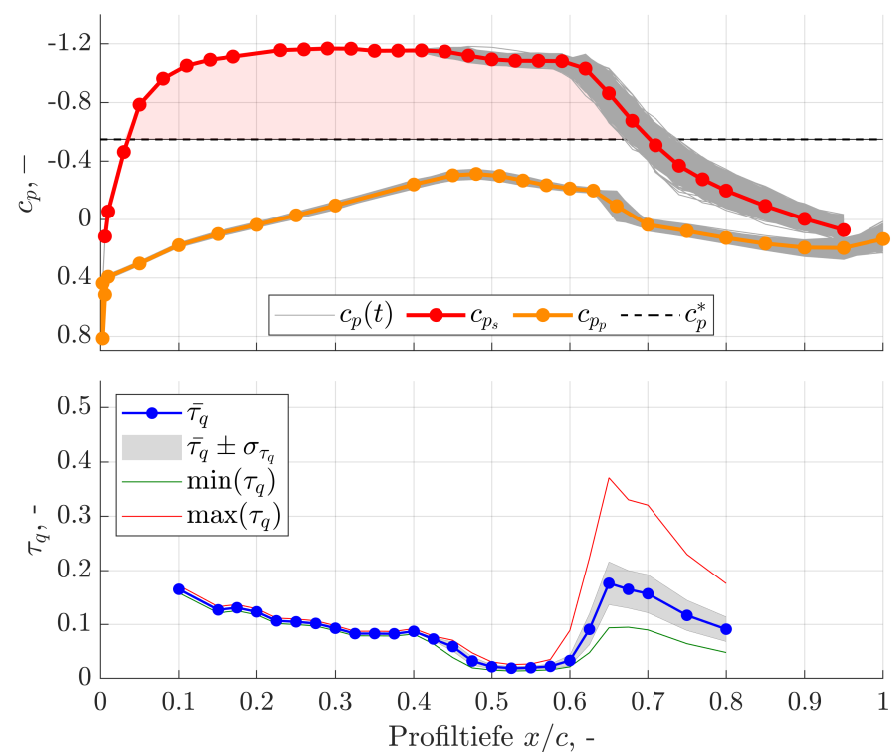

(a) $\alpha=1,54^{\circ}$
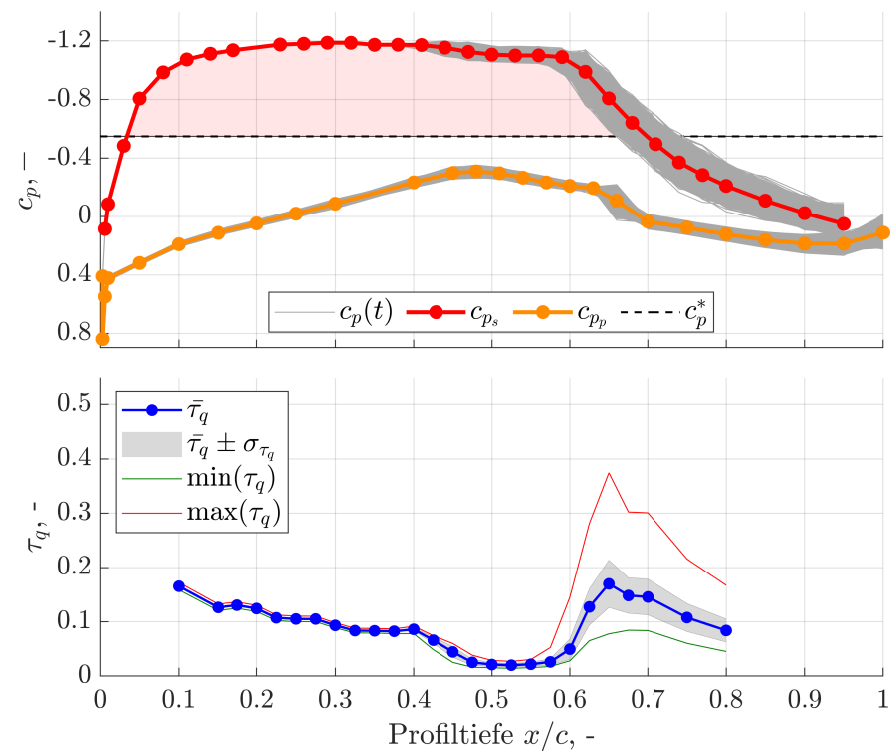

(b) $\alpha=1,75^{\circ}$

Abbildung D.1.: Druckverteilungen und Quasi-Wandschubspannungen für stationäre Messpunkte bei einem Anstellwinkel von $\alpha=1,54^{\circ}$ (a) und $\alpha=1,75^{\circ}$ (b) für $\mathrm{Ma}_{\infty}=0,76$. 

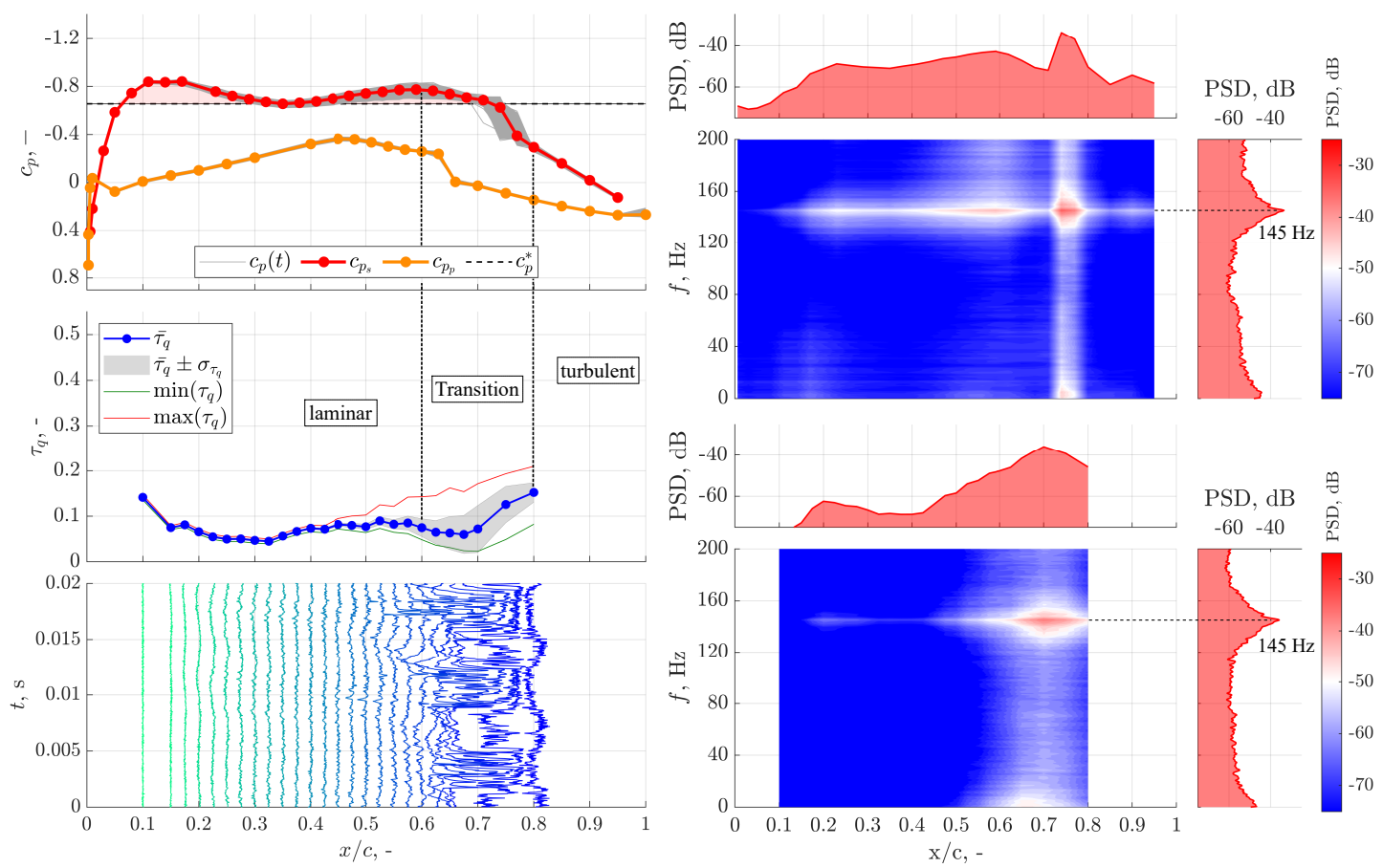

Abbildung D.2.: Druckverteilung, Quasi-Wandschubspannung, CTA-Zeitreihen und Leistungsdichtespektren für $\mathrm{Ma}_{\infty}=0,73$ und $\alpha=-0,37^{\circ}$.
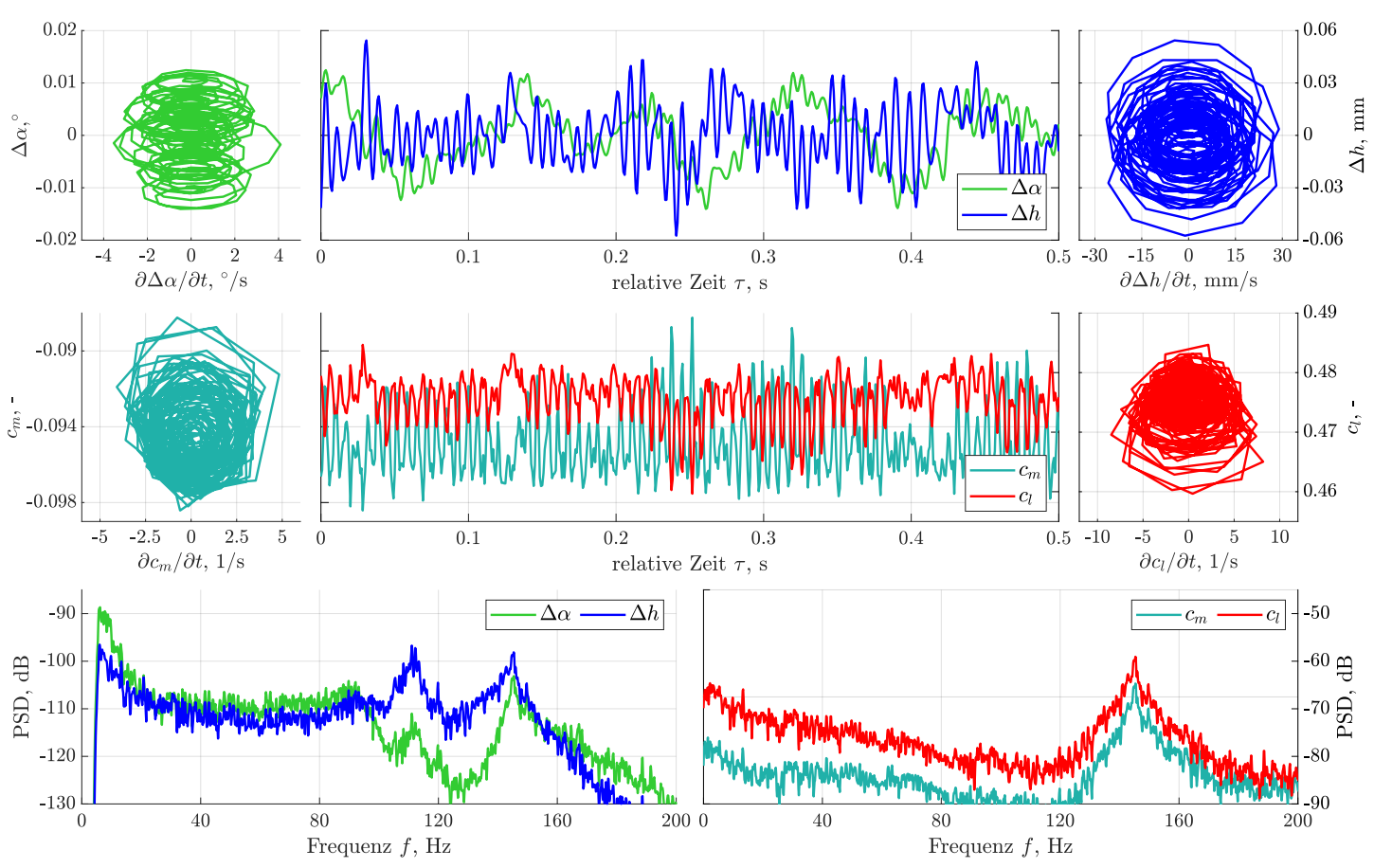

Abbildung D.3.: Bewegung des Modellmittelschnitts $\Delta \alpha$ und $\Delta h$ und Zeitreihenausschnitt der aerodynamischen Beiwerte $c_{m}$ und $c_{l}$ sowie Leistungsdichtespektren für $\mathrm{Ma}_{\infty}=0,73$ und $\alpha=-0,37^{\circ}$. 

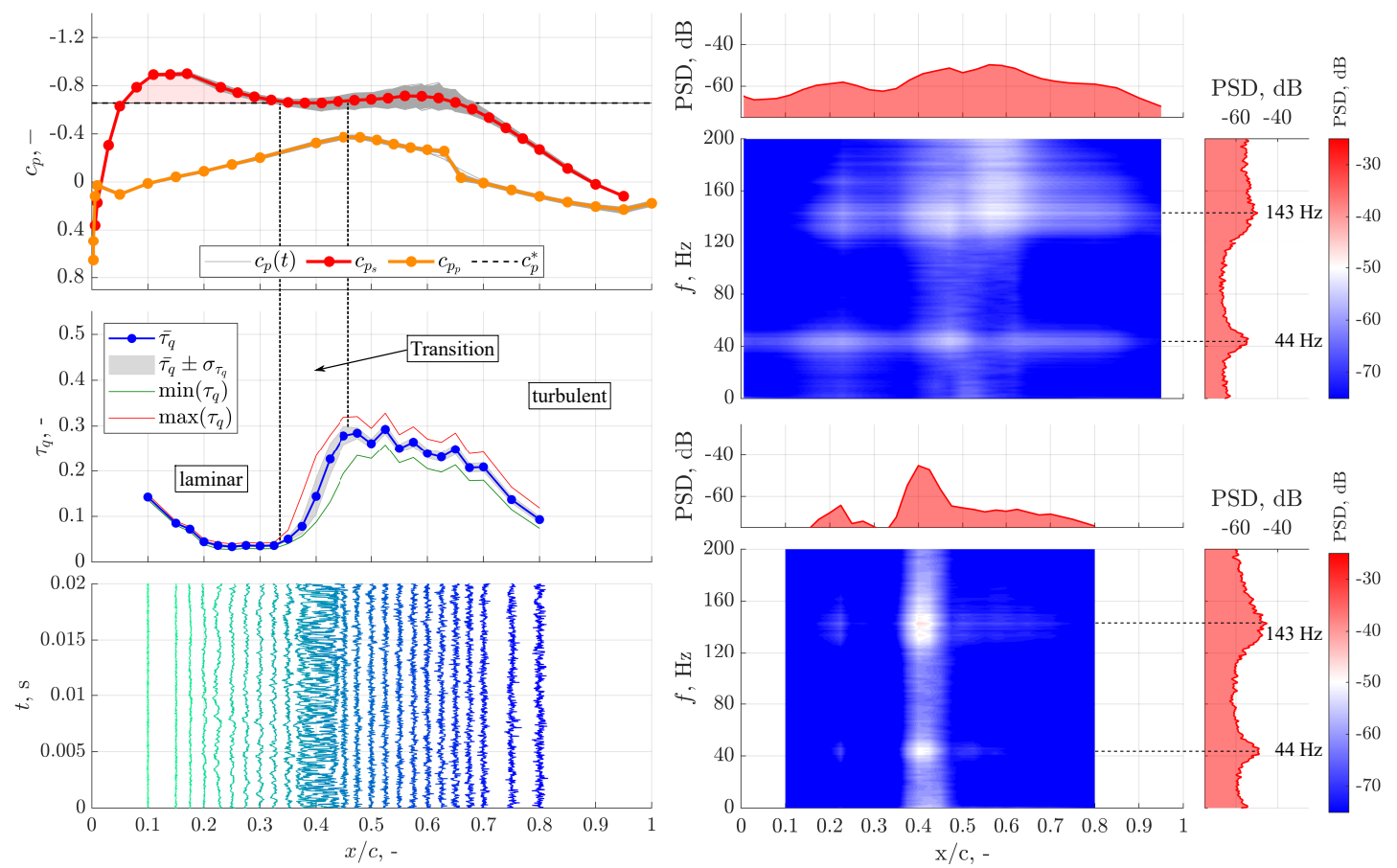

Abbildung D.4.: Druckverteilung, Quasi-Wandschubspannung, CTA-Zeitreihen und Leistungsdichtespektren für $\mathrm{Ma}_{\infty}=0,73$ und $\alpha=0,06^{\circ}$ (vgl. auch [62]).
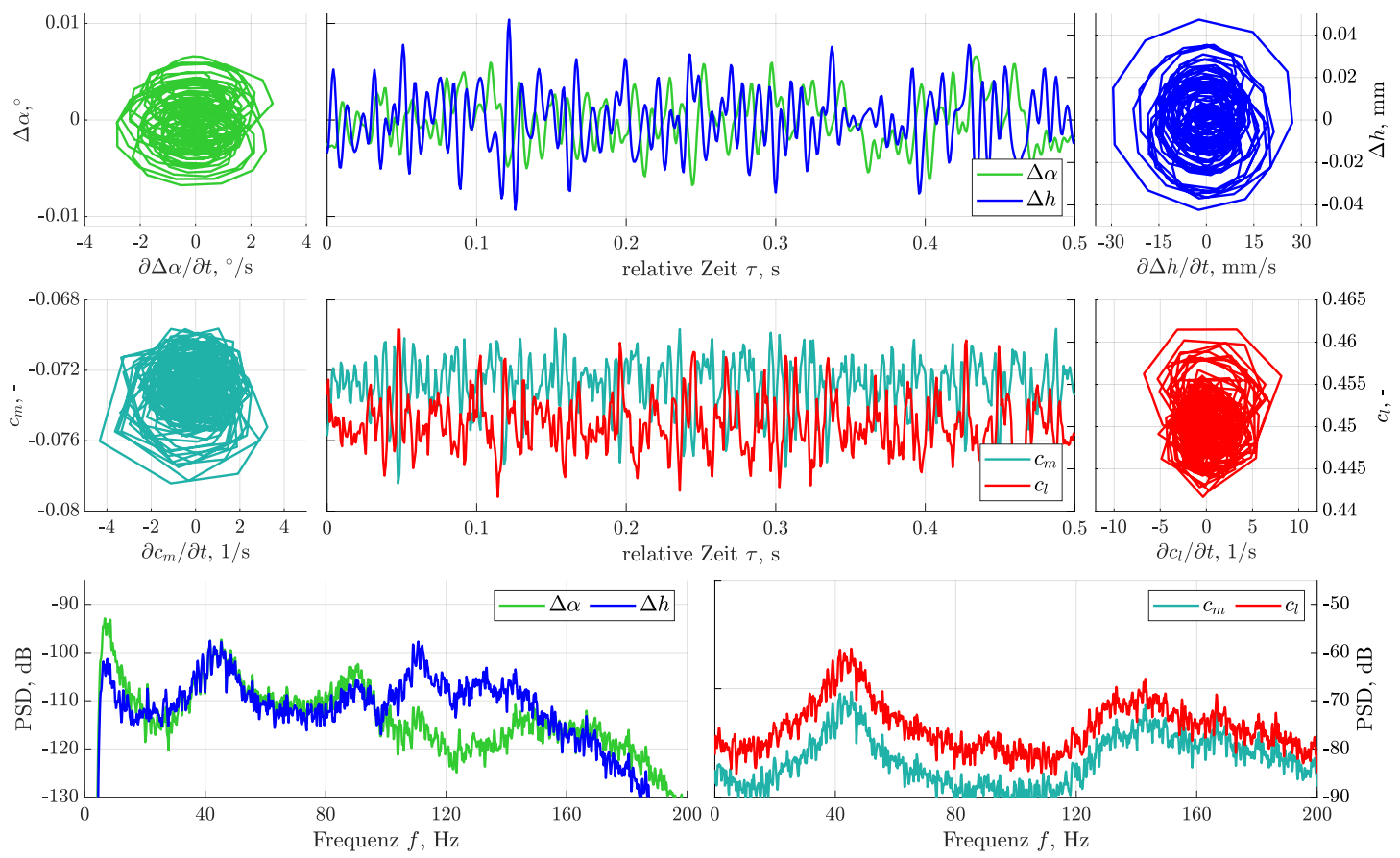

Abbildung D.5.: Bewegung des Modellmittelschnitts $\Delta \alpha$ und $\Delta h$ und Zeitreihenausschnitt der aerodynamischen Beiwerte $c_{m}$ und $c_{l}$ sowie Leistungsdichtespektren für $\mathrm{Ma}_{\infty}=0,73$ und $\alpha=0,06^{\circ}$. 

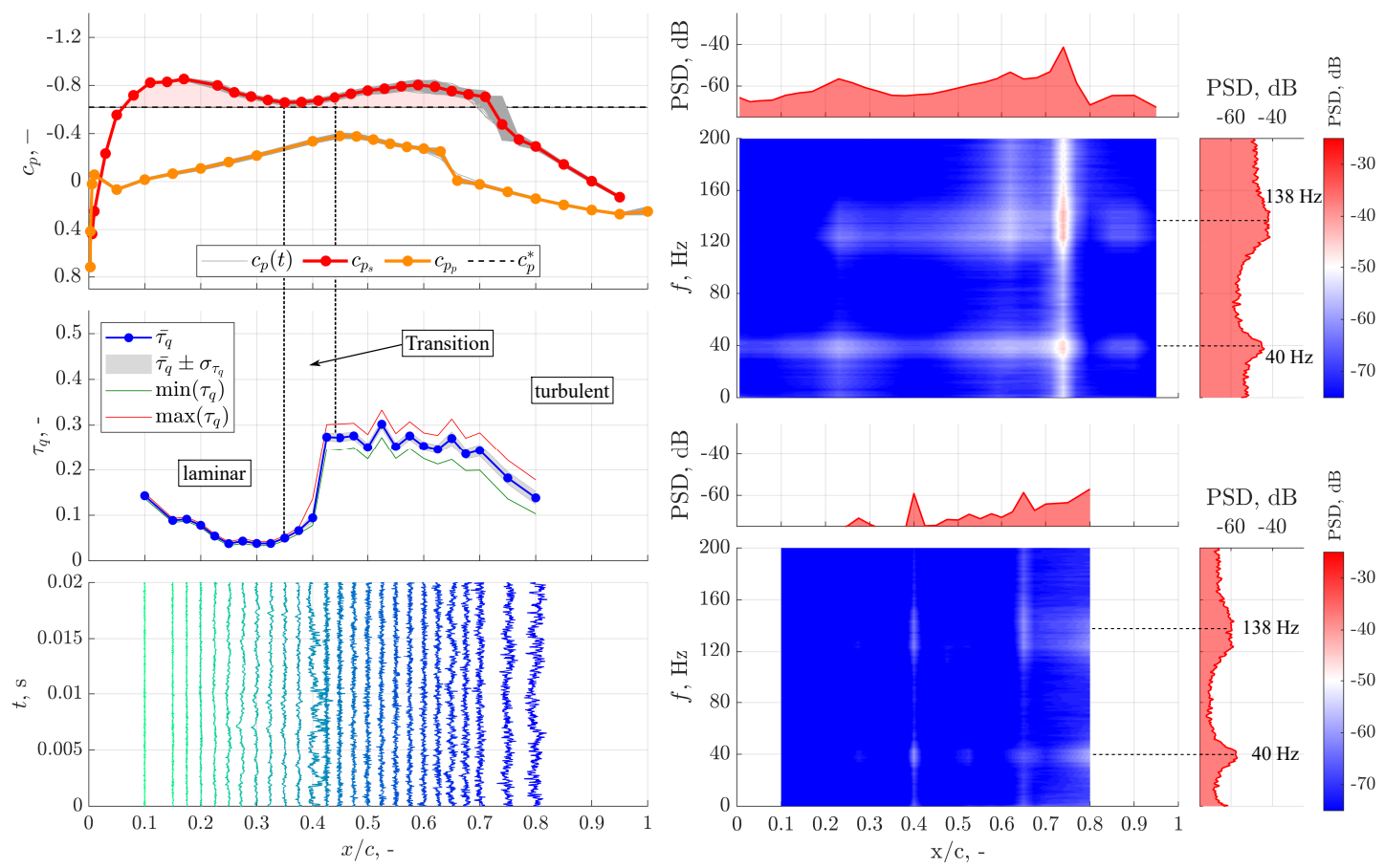

Abbildung D.6.: Druckverteilung, Quasi-Wandschubspannung, CTA-Zeitreihen und Leistungsdichtespektren für $\mathrm{Ma}_{\infty}=0,74$ und $\alpha=-0,38^{\circ}$.
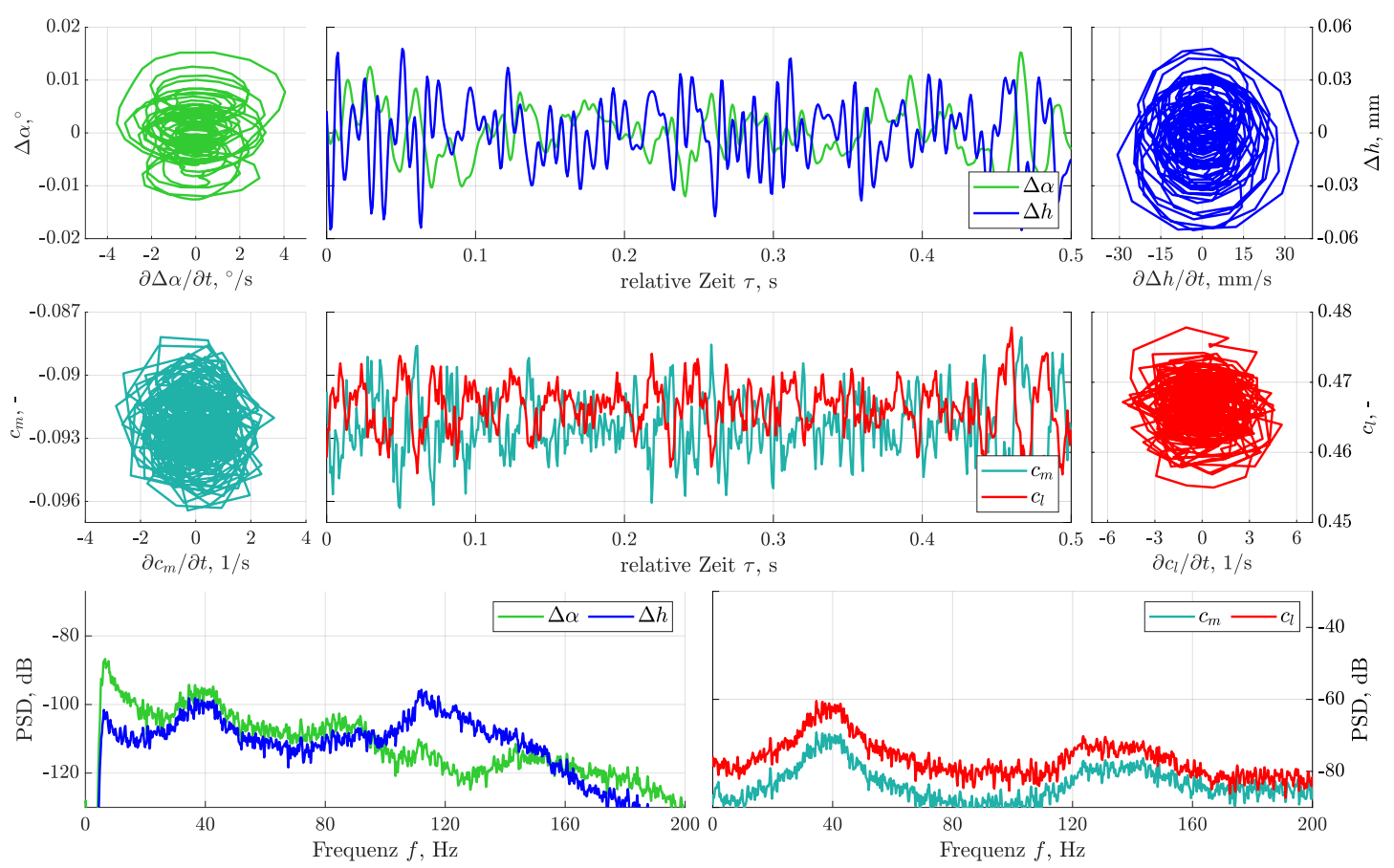

Abbildung D.7.: Bewegung des Modellmittelschnitts $\Delta \alpha$ und $\Delta h$ und Zeitreihenausschnitt der aerodynamischen Beiwerte $c_{m}$ und $c_{l}$ sowie Leistungsdichtespektren für $\mathrm{Ma}_{\infty}=0,74$ und $\alpha=-0,38^{\circ}$. 

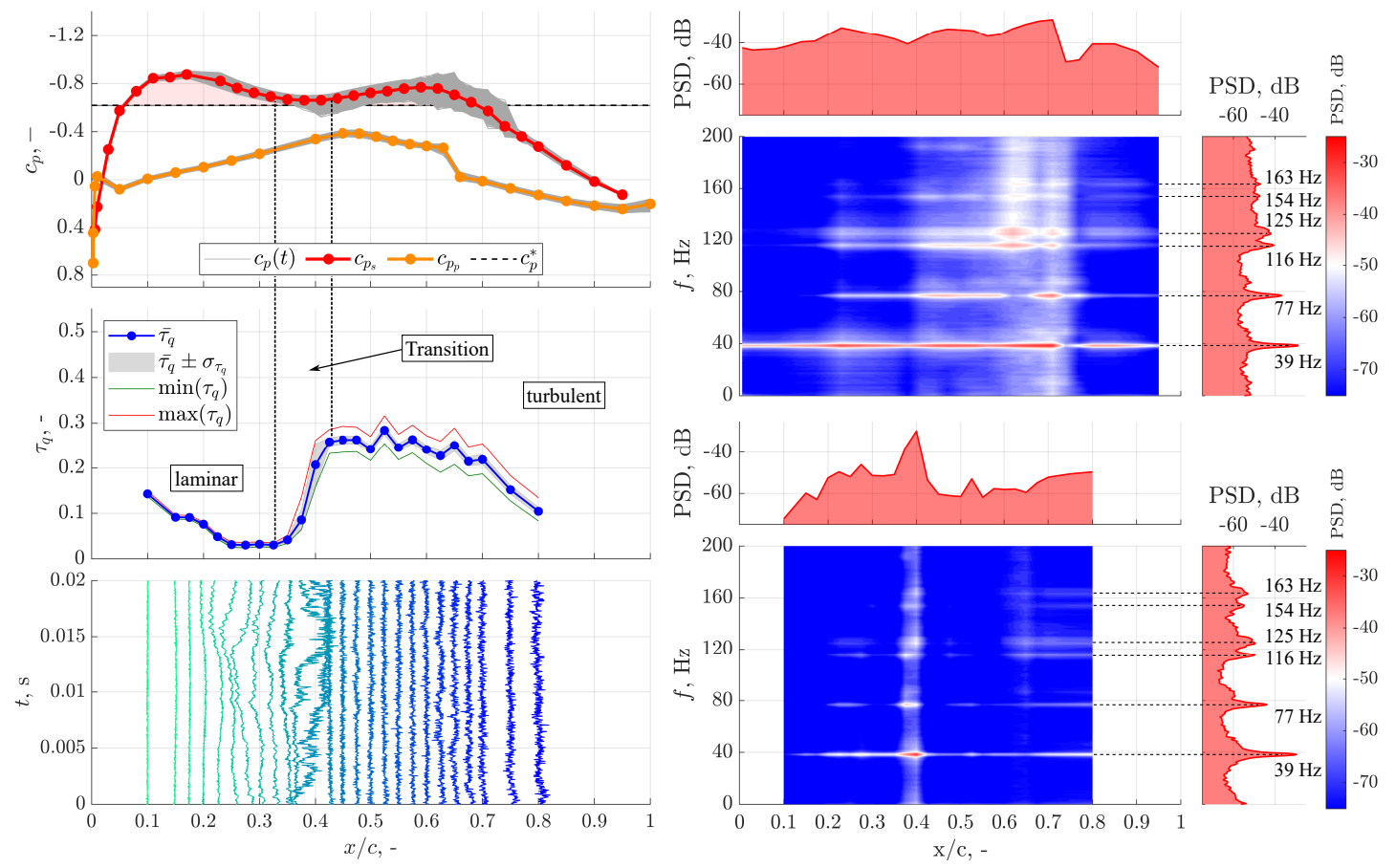

Abbildung D.8.: Druckverteilung, Quasi-Wandschubspannung, CTA-Zeitreihen und Leistungsdichtespektren für $\mathrm{Ma}_{\infty}=0,74$ und $\alpha=-0,16^{\circ}$.
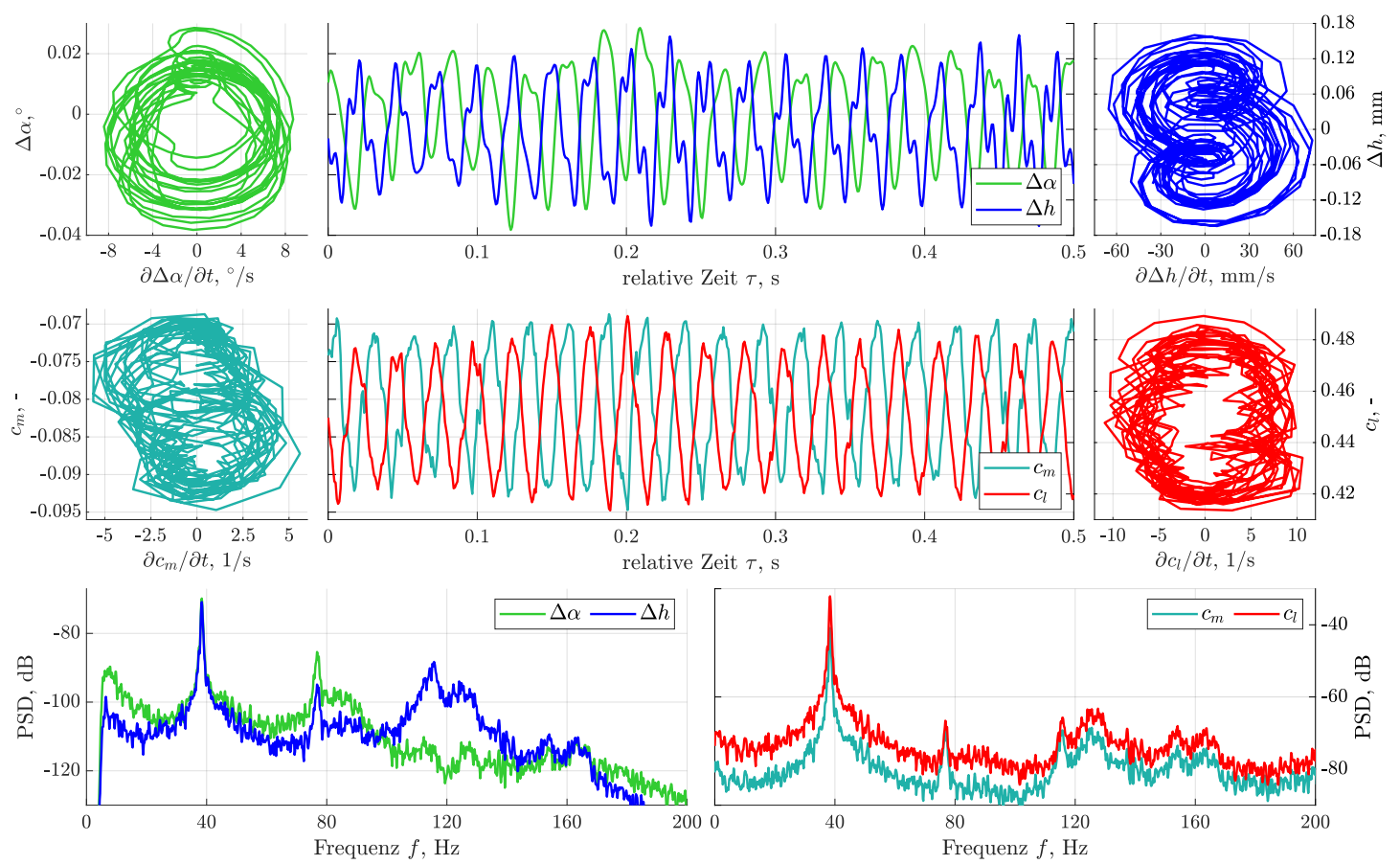

Abbildung D.9.: Bewegung des Modellmittelschnitts $\Delta \alpha$ und $\Delta h$ und Zeitreihenausschnitt der aerodynamischen Beiwerte $c_{m}$ und $c_{l}$ sowie Leistungsdichtespektren für $\mathrm{Ma}_{\infty}=0,74$ und $\alpha=-0,16^{\circ}$. 

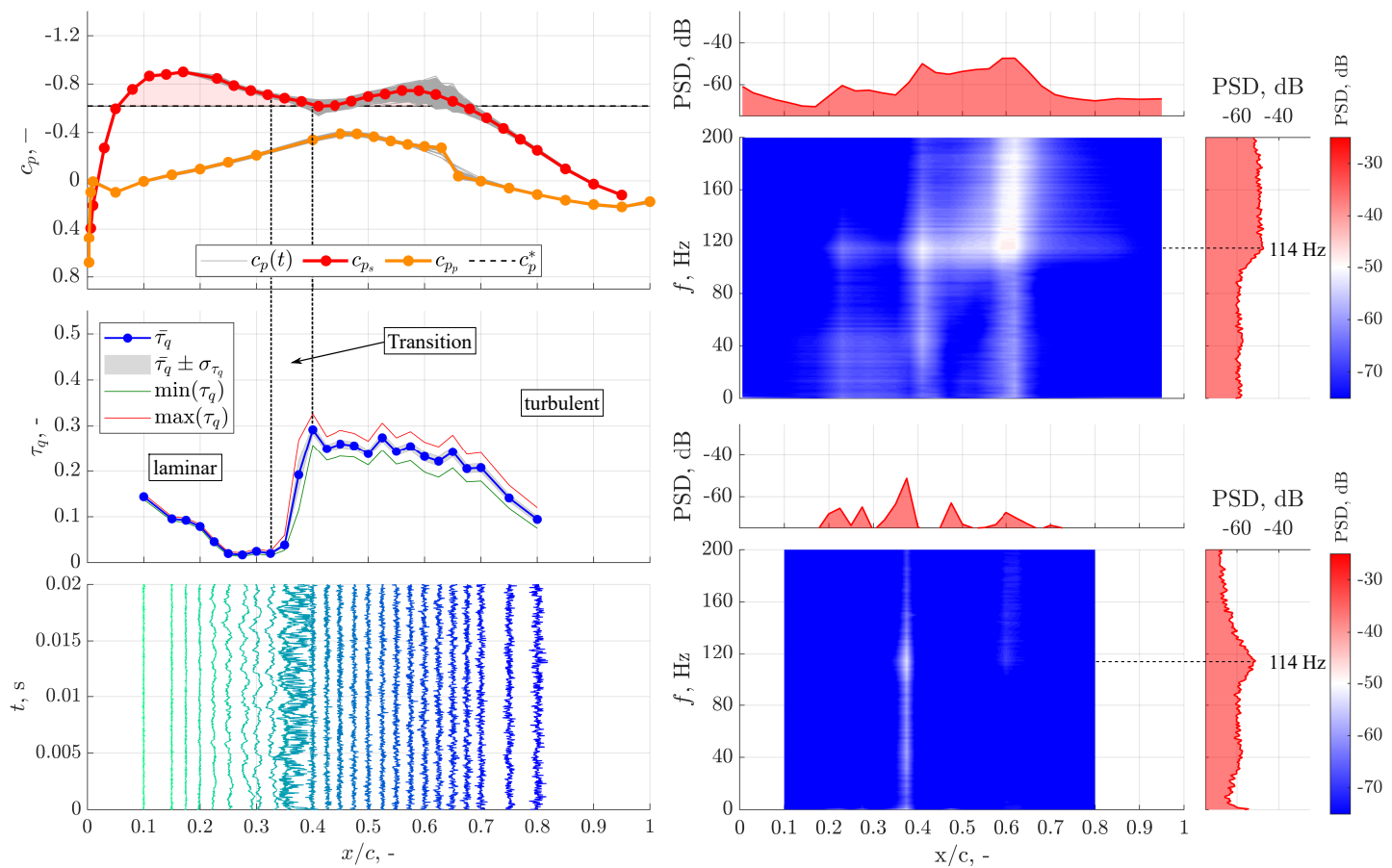

Abbildung D.10.: Druckverteilung, Quasi-Wandschubspannung, CTA-Zeitreihen und Leistungsdichtespektren für $\mathrm{Ma}_{\infty}=0,74$ und $\alpha=0,06^{\circ}$.
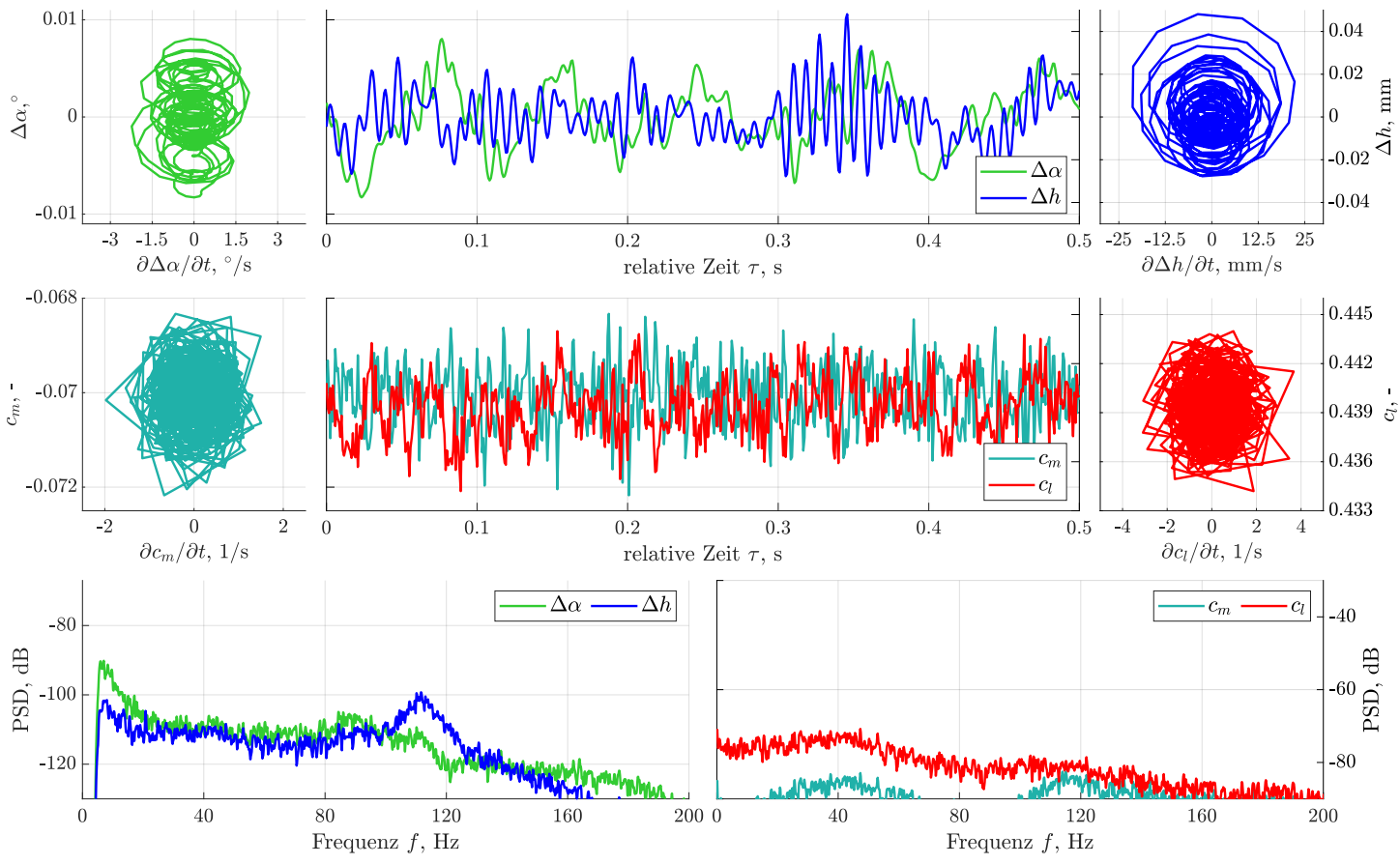

Abbildung D.11.: Bewegung des Modellmittelschnitts $\Delta \alpha$ und $\Delta h$ und Zeitreihenausschnitt der aerodynamischen Beiwerte $c_{m}$ und $c_{l}$ sowie Leistungsdichtespektren für $\mathrm{Ma}_{\infty}=0,74$ und $\alpha=0,06^{\circ}$. 

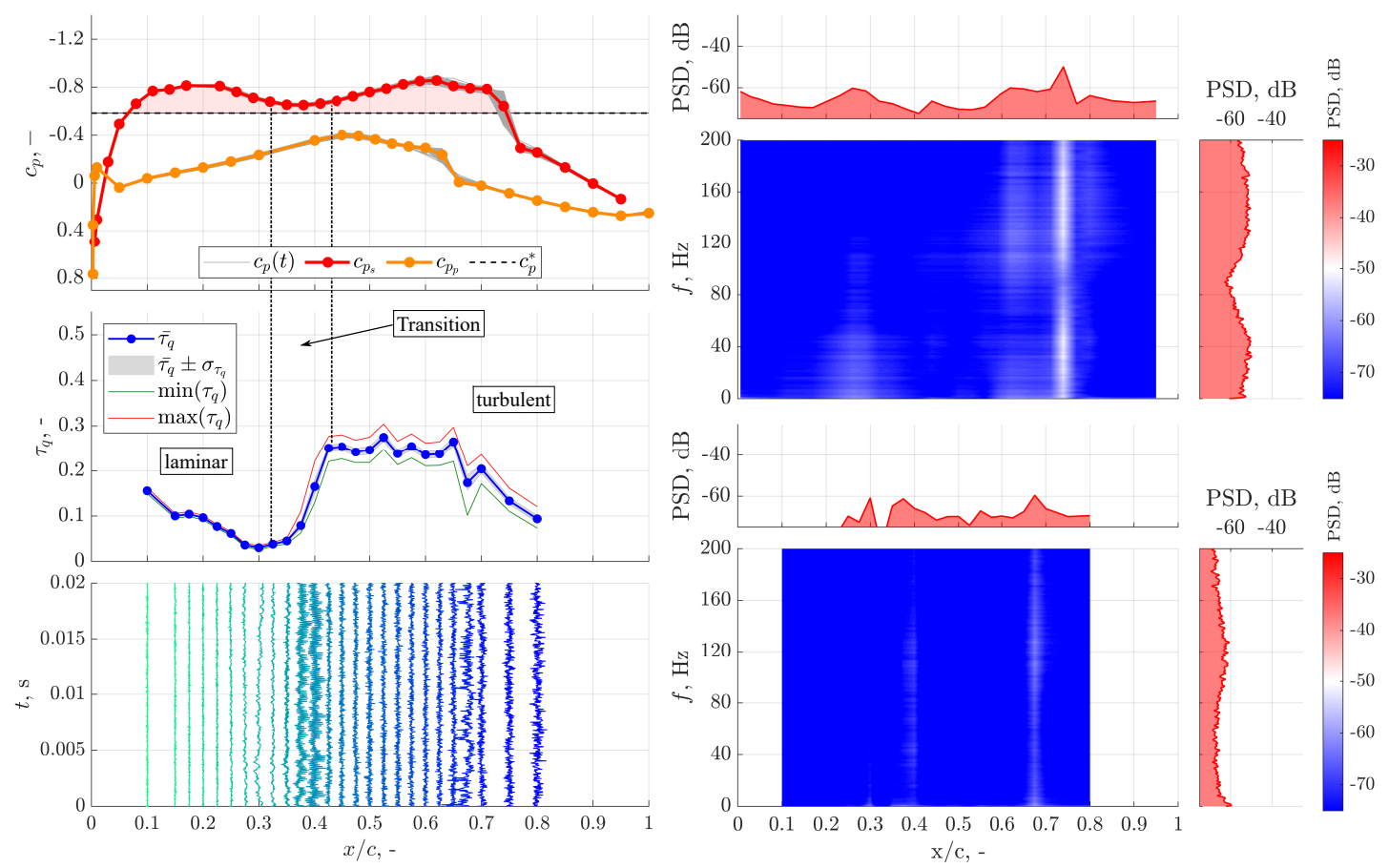

Abbildung D.12.: Druckverteilung, Quasi-Wandschubspannung, CTA-Zeitreihen und Leistungsdichtespektren für $\mathrm{Ma}_{\infty}=0,75$ und $\alpha=-0,58^{\circ}$.
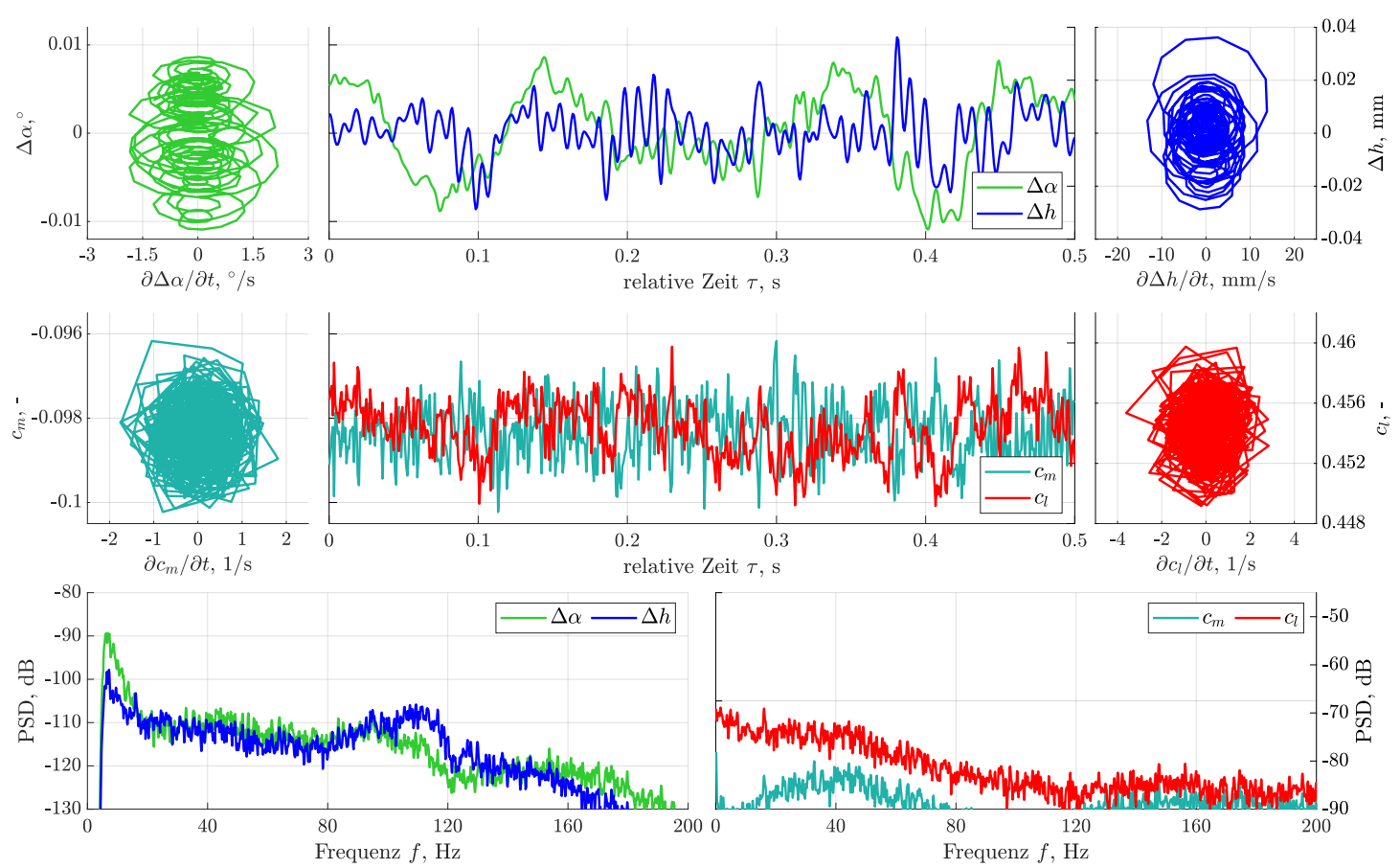

Abbildung D.13.: Bewegung des Modellmittelschnitts $\Delta \alpha$ und $\Delta h$ und Zeitreihenausschnitt der aerodynamischen Beiwerte $c_{m}$ und $c_{l}$ sowie Leistungsdichtespektren für $\mathrm{Ma}_{\infty}=0,75$ und $\alpha=-0,58^{\circ}$. 

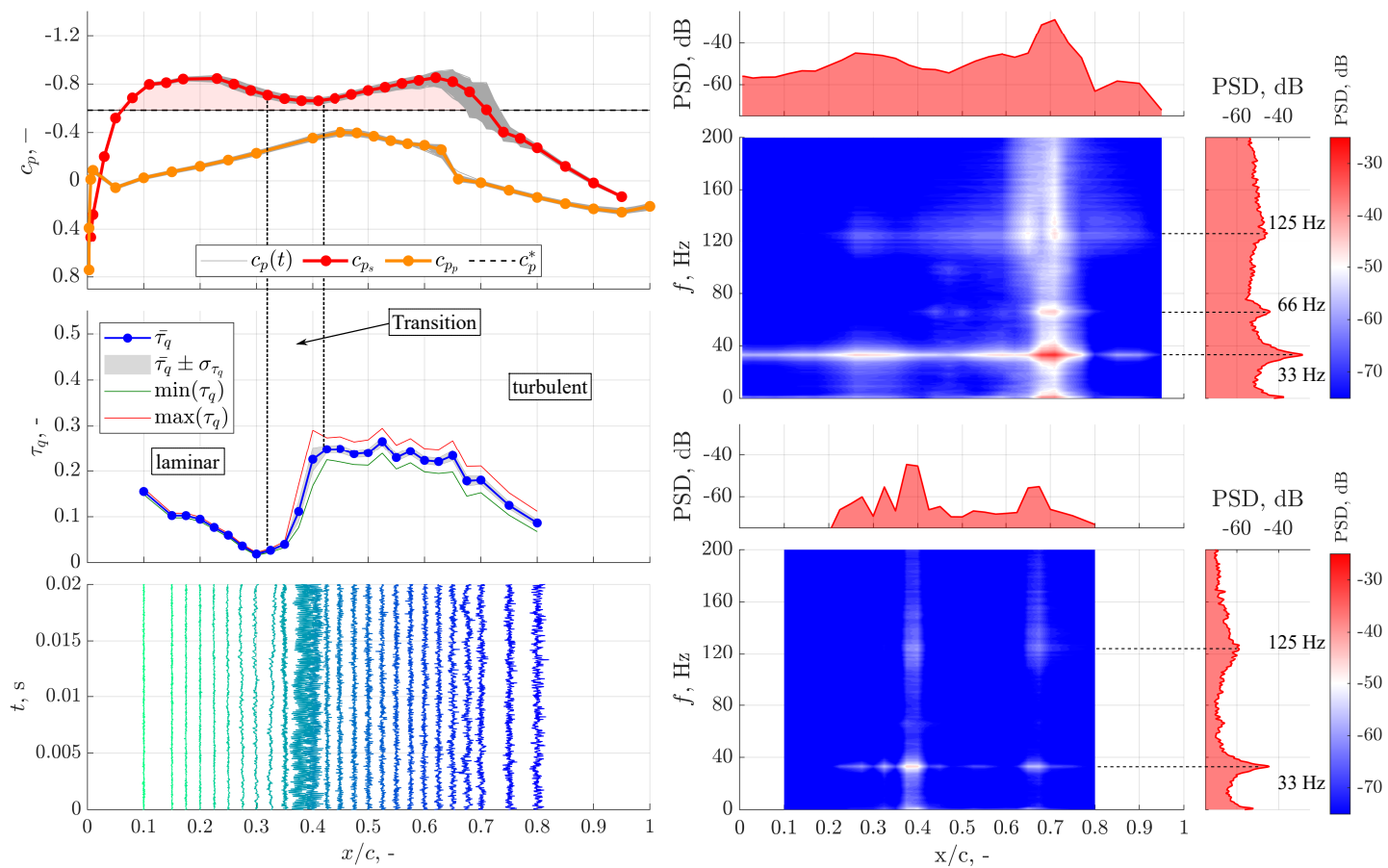

Abbildung D.14.: Druckverteilung, Quasi-Wandschubspannung, CTA-Zeitreihen und Leistungsdichtespektren für $\mathrm{Ma}_{\infty}=0,75$ und $\alpha=-0,37^{\circ}$.
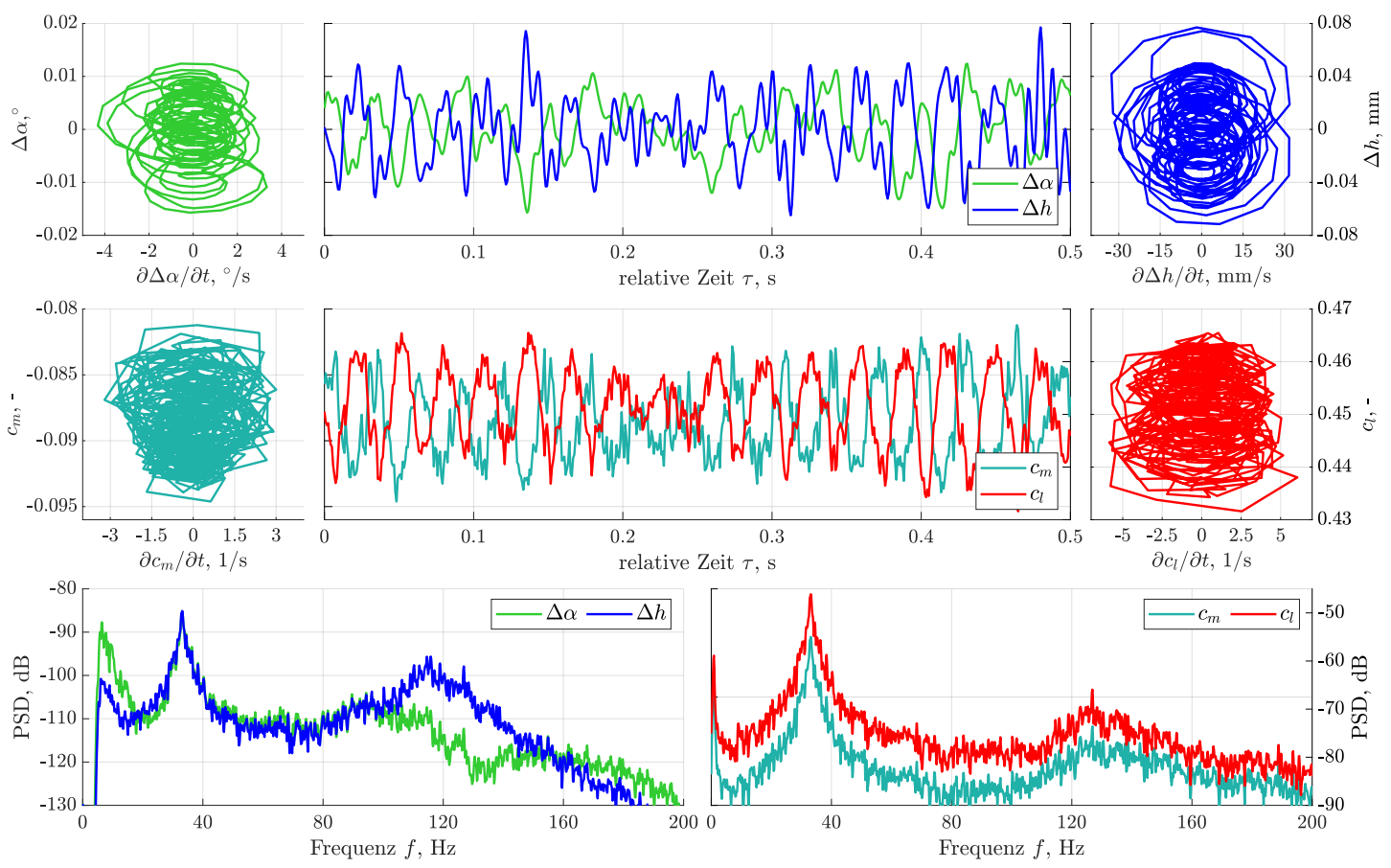

Abbildung D.15.: Bewegung des Modellmittelschnitts $\Delta \alpha$ und $\Delta h$ und Zeitreihenausschnitt der aerodynamischen Beiwerte $c_{m}$ und $c_{l}$ sowie Leistungsdichtespektren für $\mathrm{Ma}_{\infty}=0,75$ und $\alpha=-0,37^{\circ}$. 

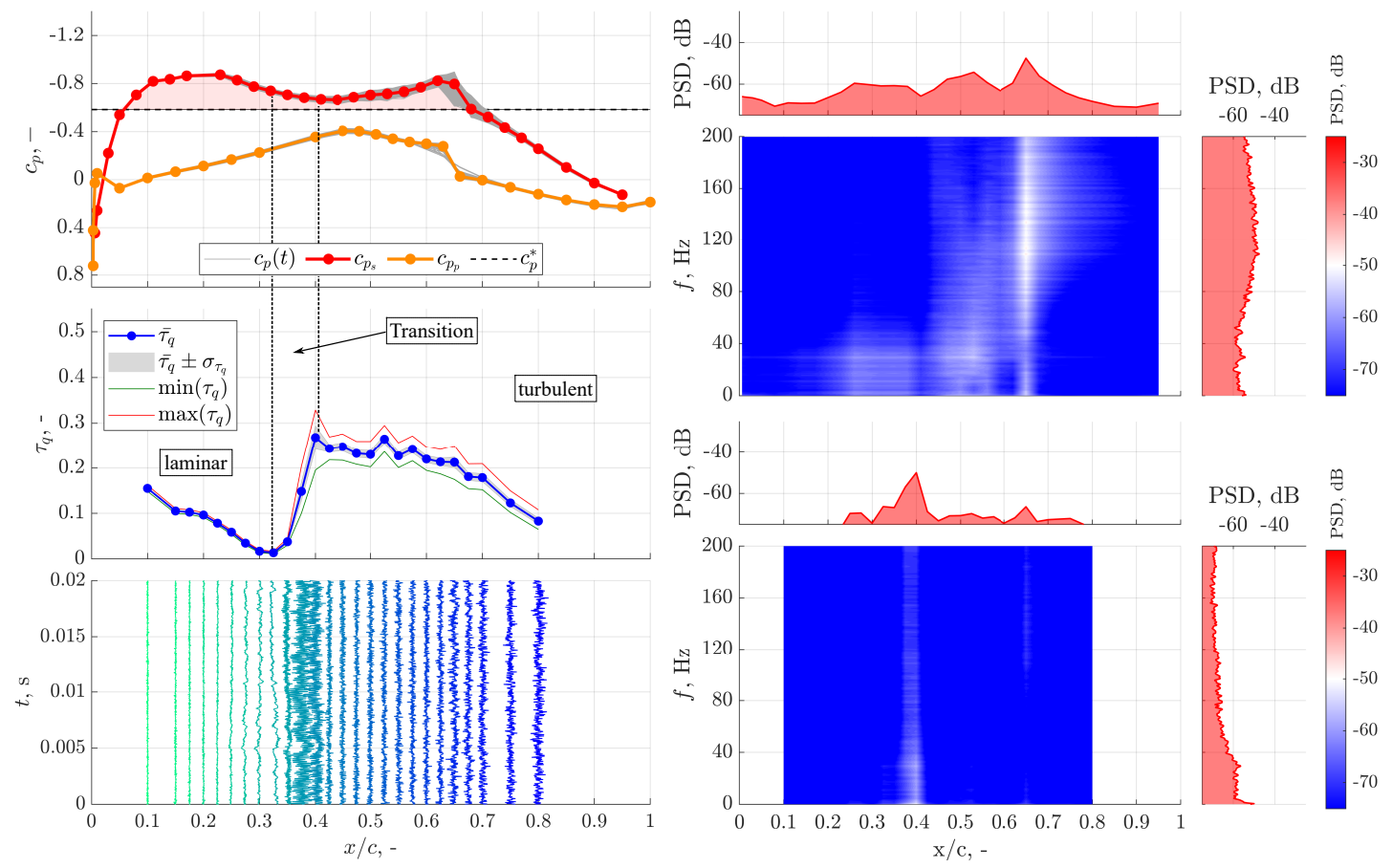

Abbildung D.16.: Druckverteilung, Quasi-Wandschubspannung, CTA-Zeitreihen und Leistungsdichtespektren für $\mathrm{Ma}_{\infty}=0,75$ und $\alpha=-0,15^{\circ}$.
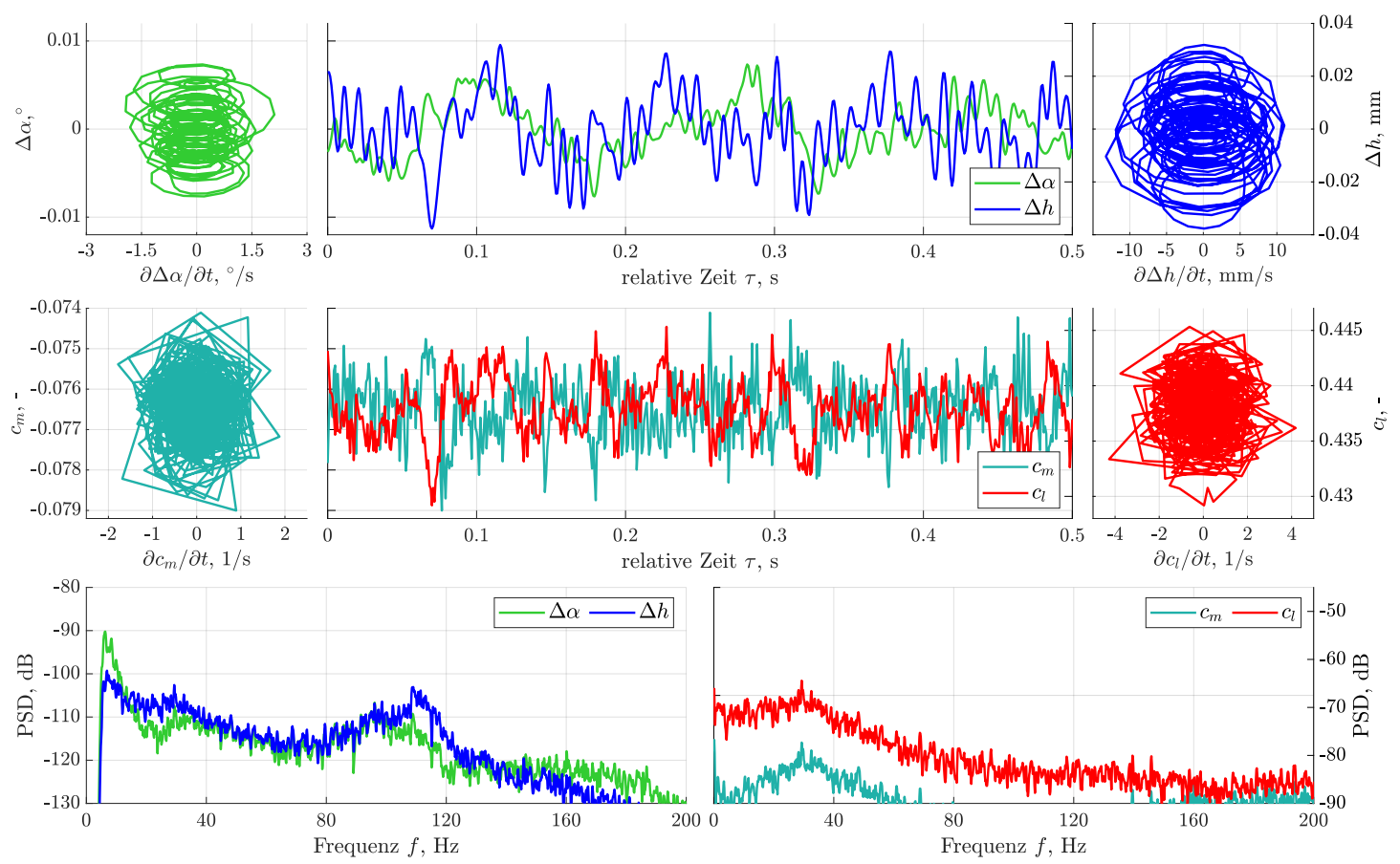

Abbildung D.17.: Bewegung des Modellmittelschnitts $\Delta \alpha$ und $\Delta h$ und Zeitreihenausschnitt der aerodynamischen Beiwerte $c_{m}$ und $c_{l}$ sowie Leistungsdichtespektren für $\mathrm{Ma}_{\infty}=0,75$ und $\alpha=-0,15^{\circ}$. 



\section{E. Ergänzungen zu Kapitel 6}

\section{E.1. Zu 6.1: Instationäre Derivativa der aerodynamischen Beiwerte}

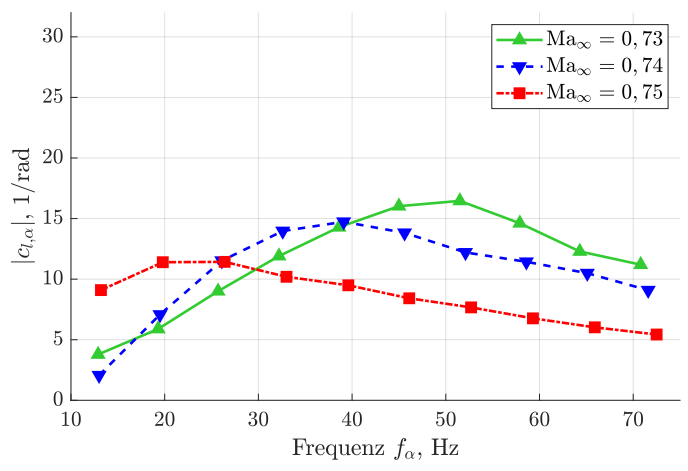

(a) Amplitude $\left|c_{l, \alpha}\right|$

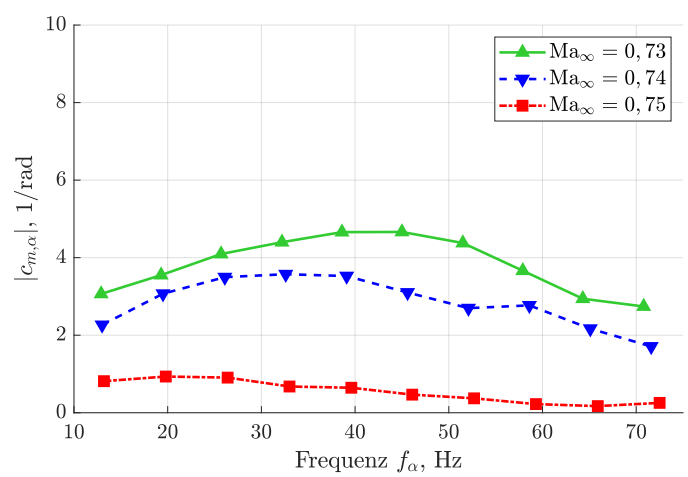

(c) Amplitude $\left|c_{m, \alpha}\right|$

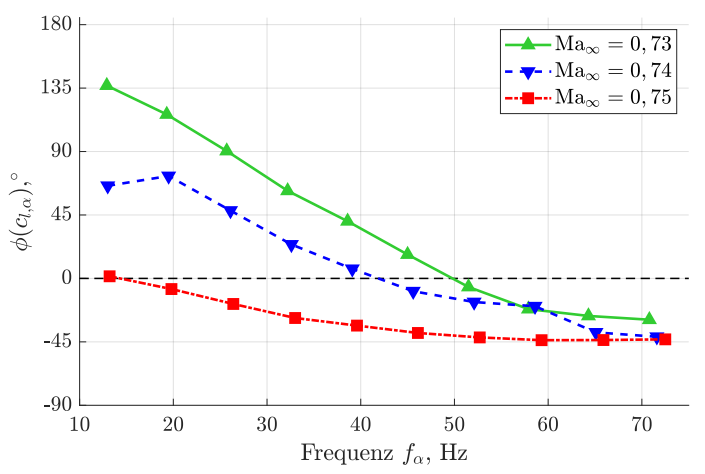

(b) Phase $\phi\left(c_{l, \alpha}\right)$

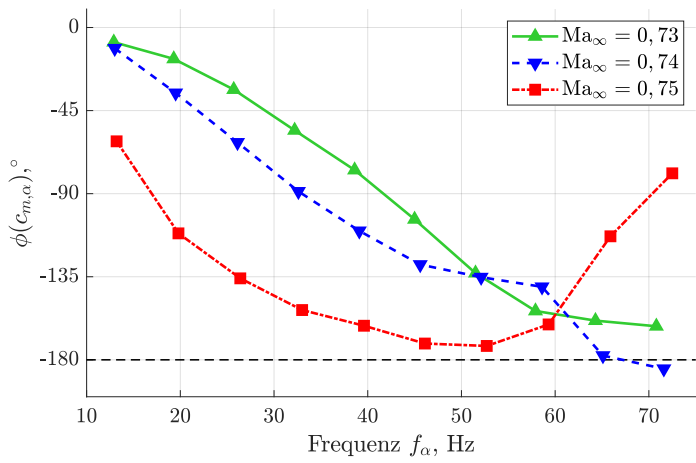

(d) Phase $\phi\left(c_{m, \alpha}\right)$

Abbildung E.1.: Instationäre Auftriebs- und Momentenderivativa in Betrag und Phase für eine Grenzschicht mit freier Transition, gemessen mit einer Frequenzvariation mit $\hat{\alpha}=0,25^{\circ}$ um $\bar{\alpha}=0,05^{\circ}$. Hierbei lag eine Grenzschichtströmung mit freier Transition vor. 


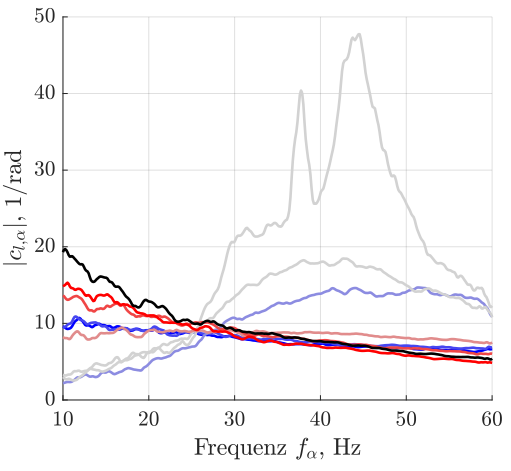

(a) Amplitude $\left|c_{l, \alpha}\right|$, freie Transition

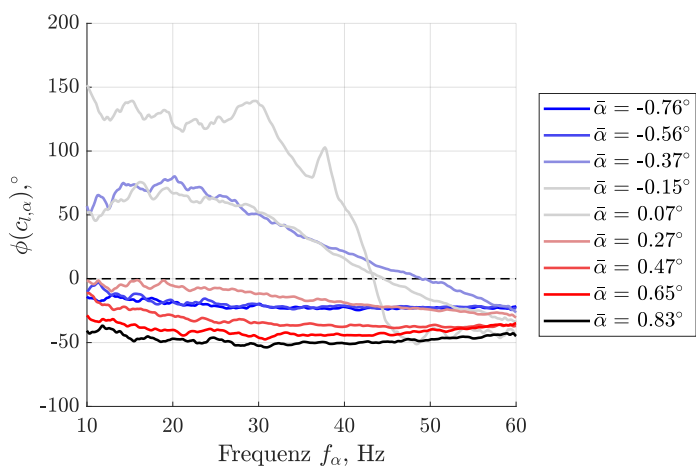

(c) Phase $\phi\left(c_{l, \alpha}\right)$, freie Transition

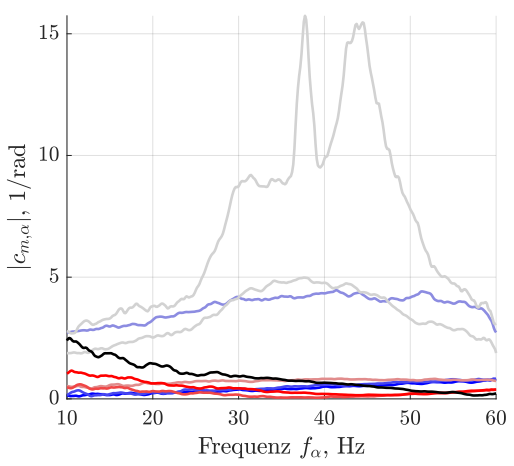

(e) Amplitude $\left|c_{m, \alpha}\right|$, freie Transition

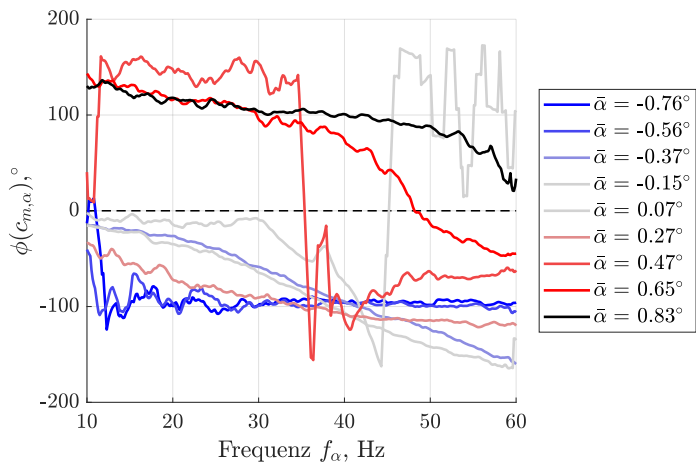

(g) Phase $\phi\left(c_{m, \alpha}\right)$, freie Transition

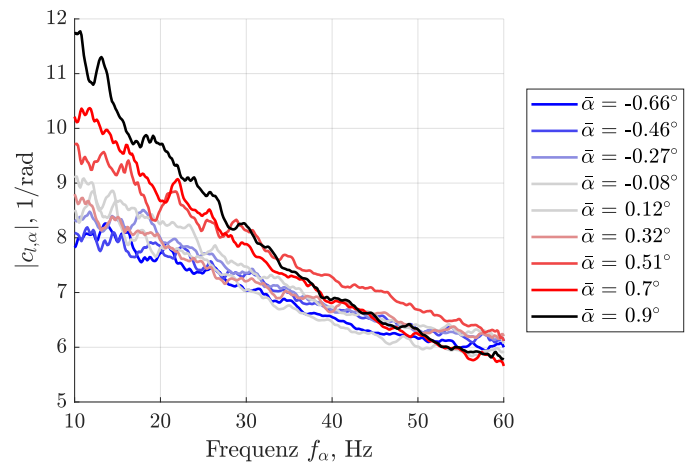

(b) Amplitude $\left|c_{l, \alpha}\right|$, fixierte Transition

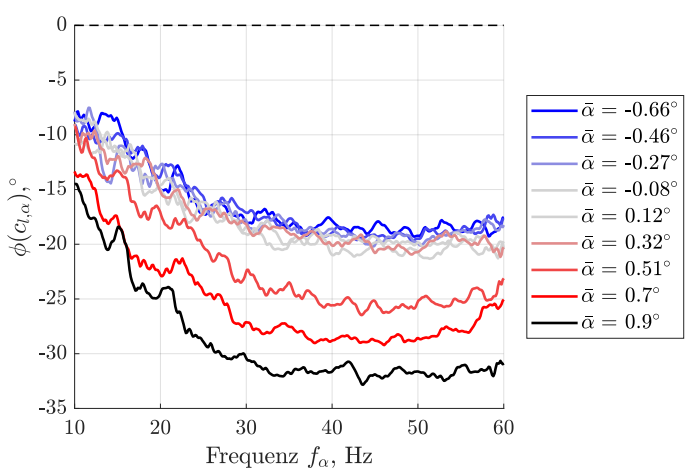

(d) Phase $\phi\left(c_{l, \alpha}\right)$, fixierte Transition

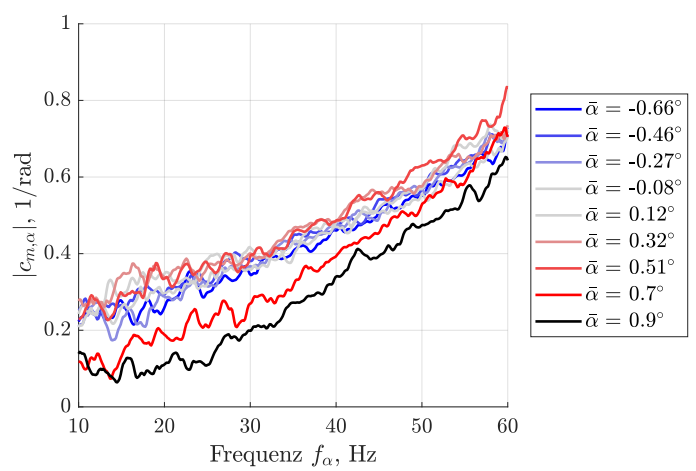

(f) Amplitude $\left|c_{m, \alpha}\right|$, fixierte Transition

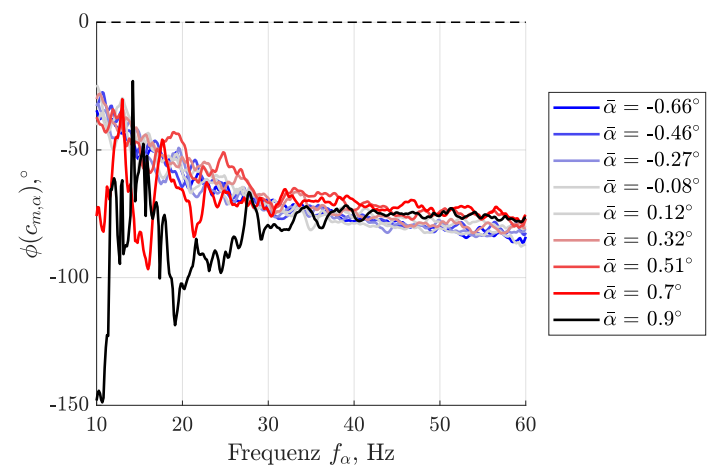

(h) Phase $\phi\left(c_{m, \alpha}\right)$, fixierte Transition

Abbildung E.2.: Instationäre Derivativa in Betrag und Phase für eine Grenzschicht mit freier (a, c, e und g) und fixierter Transition (b, d, f und h), gemessen mithilfe von Frequenzsweeps mit $\hat{\alpha}=0,08^{\circ}$ bei $\mathrm{Ma}_{\infty}=0,74$. 


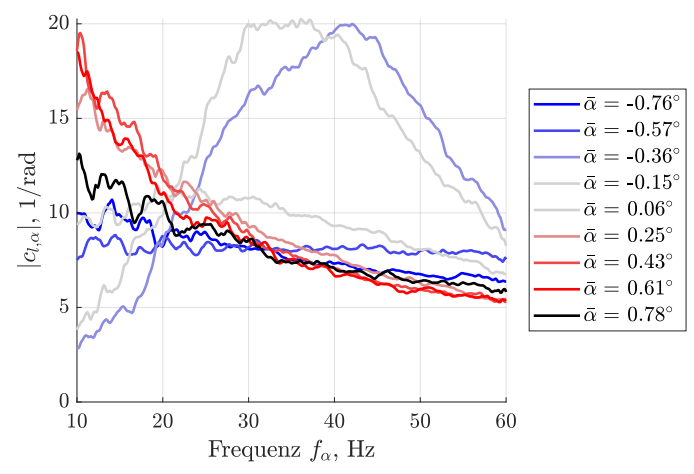

(a) Amplitude $\left|c_{l, \alpha}\right|$, freie Transition

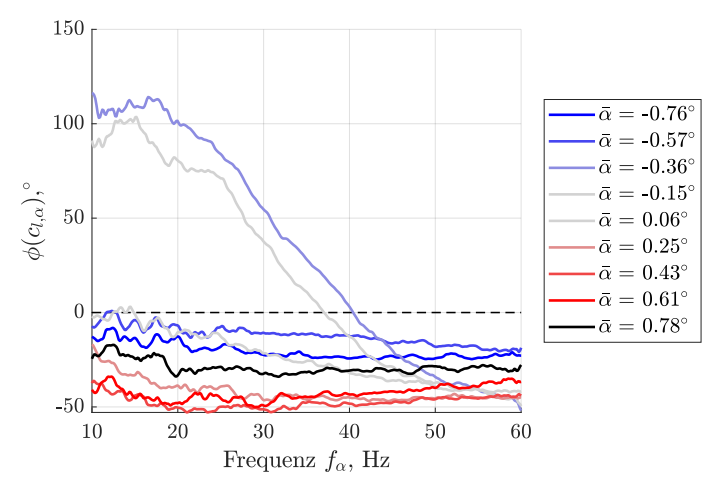

(c) Phase $\phi\left(c_{l, \alpha}\right)$, freie Transition

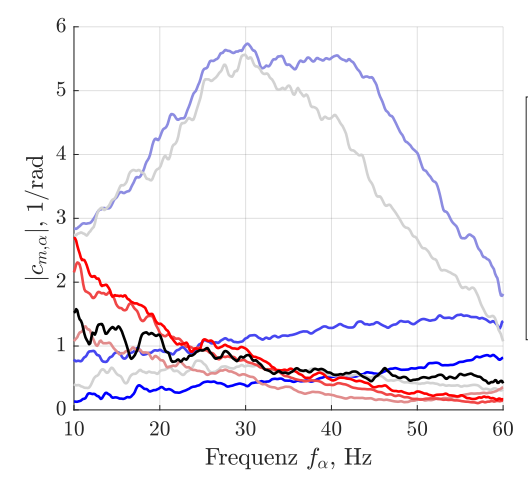

(e) Amplitude $\left|c_{m, \alpha}\right|$, freie Transition

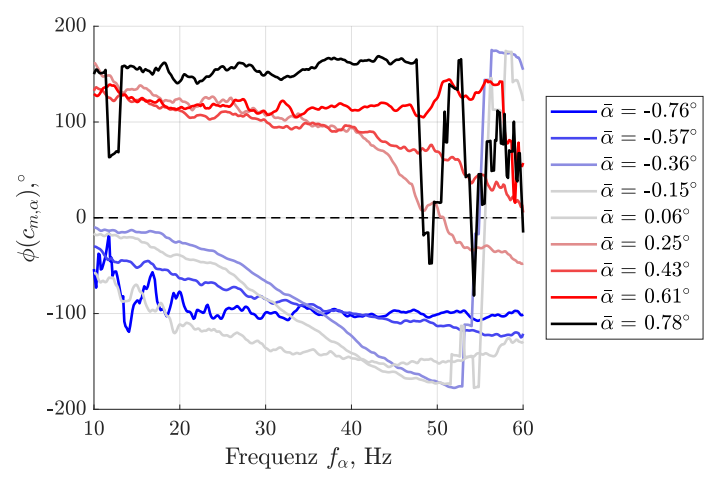

(g) Phase $\phi\left(c_{m, \alpha}\right)$, freie Transition

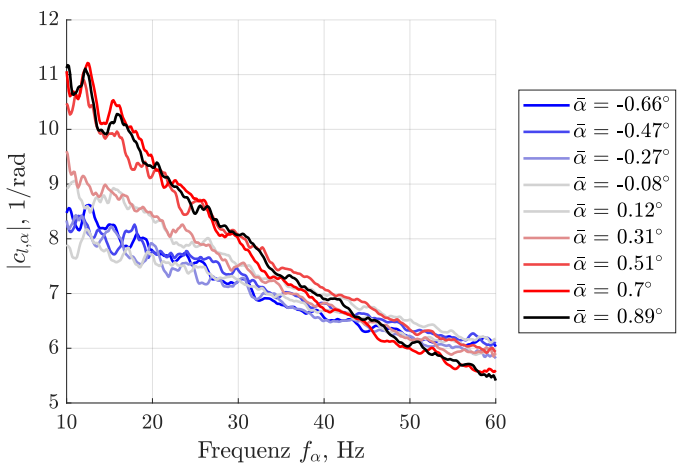

(b) Amplitude $\left|c_{l, \alpha}\right|$, fixierte Transition

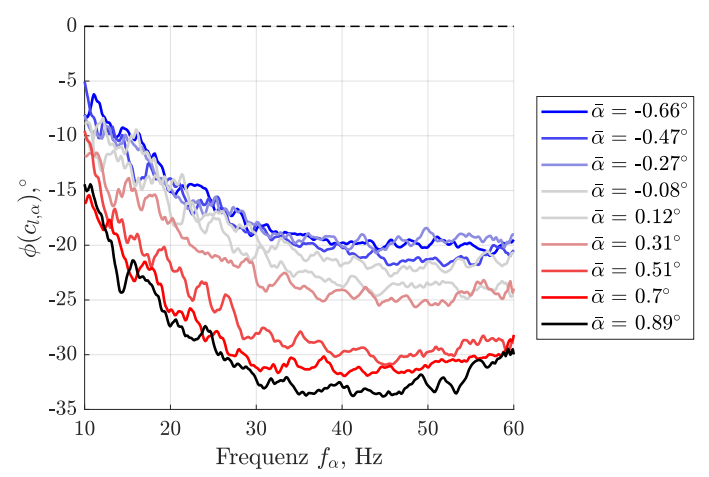

(d) Phase $\phi\left(c_{l, \alpha}\right)$, fixierte Transition

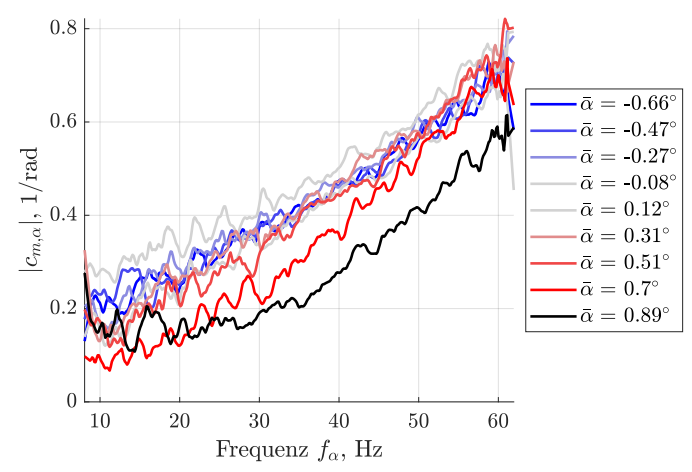

(f) Amplitude $\left|c_{m, \alpha}\right|$, fixierte Transition

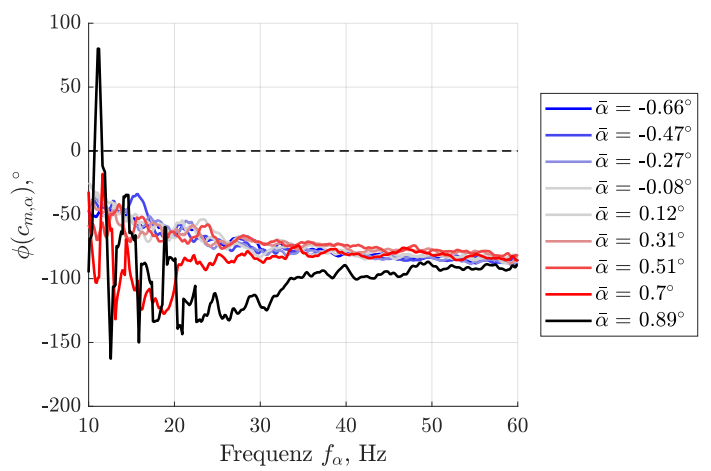

(h) Phase $\phi\left(c_{m, \alpha}\right)$, fixierte Transition

Abbildung E.3.: Instationäre Derivativa in Betrag und Phase für eine Grenzschicht mit freier (a, c, e und g) und fixierter Transition (b, d, f und h), gemessen mithilfe von Frequenzsweeps mit $\hat{\alpha}=0,08^{\circ}$ bei $\mathrm{Ma}_{\infty}=0,75$. 


\section{E.2. Zu 6.2: Energiebetrachtung}

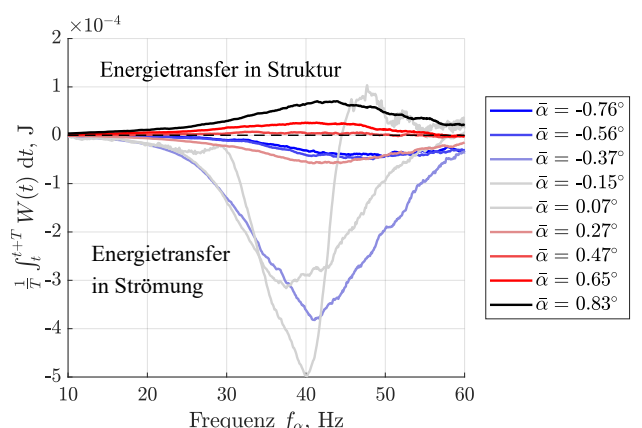

(a) Energie 2D, freie Transition, $\mathrm{Ma}_{\infty}=0,74$

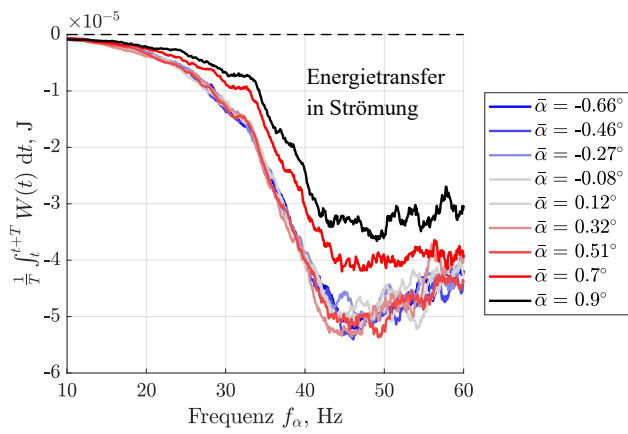

(c) Energie 2D, fix. Transition, $\mathrm{Ma}_{\infty}=0,74$

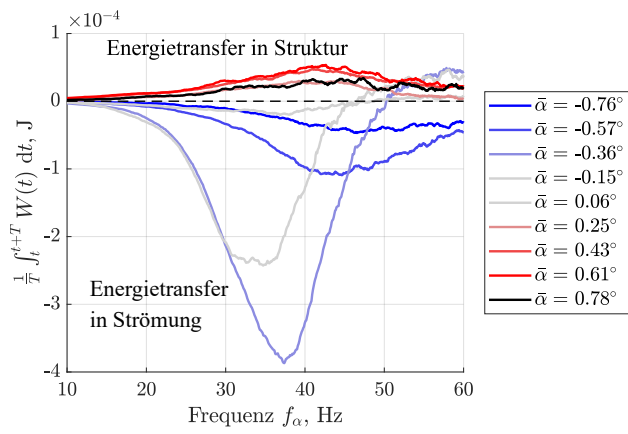

(e) Energie 2D, freie Transition, $\mathrm{Ma}_{\infty}=0,75$

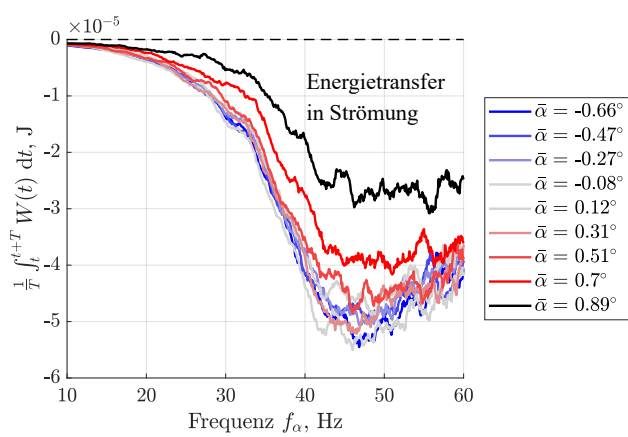

(g) Energie 2D, fix. Transition, $\mathrm{Ma}_{\infty}=0,75$

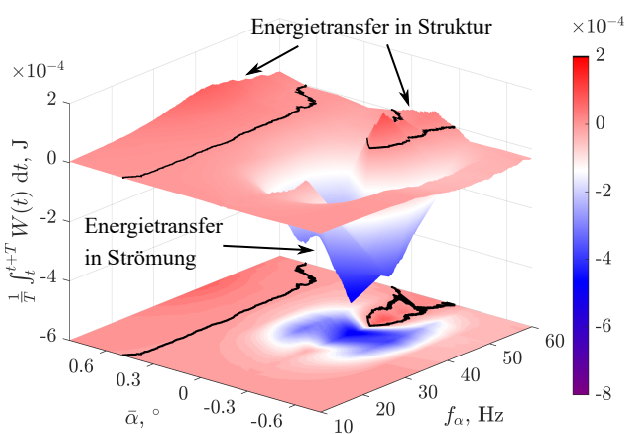

(b) Energie 3D, freie Transition, $\mathrm{Ma}_{\infty}=0,74$

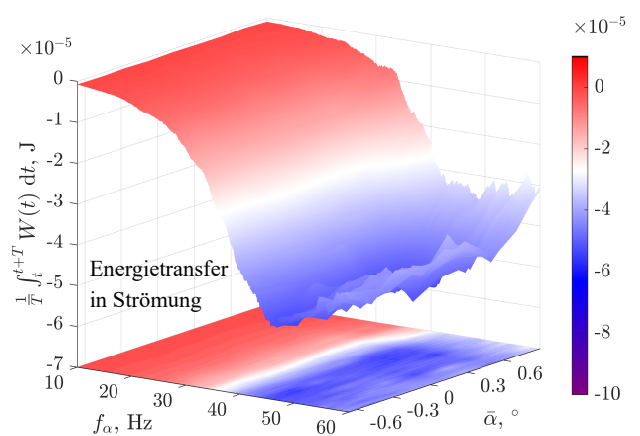

(d) Energie 3D, fix. Transition, $\mathrm{Ma}_{\infty}=0,74$

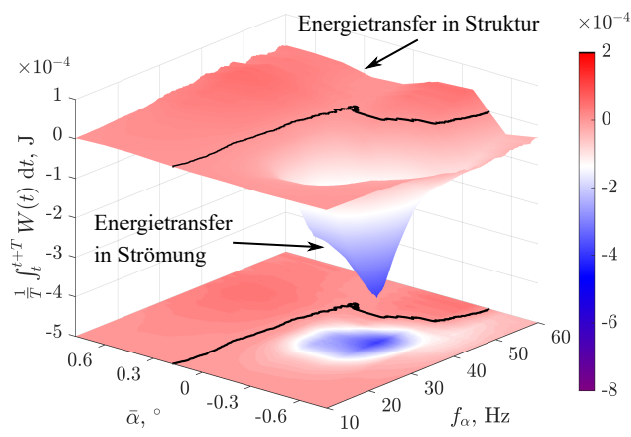

(f) Energie 3D, freie Transition, $\mathrm{Ma}_{\infty}=0,75$

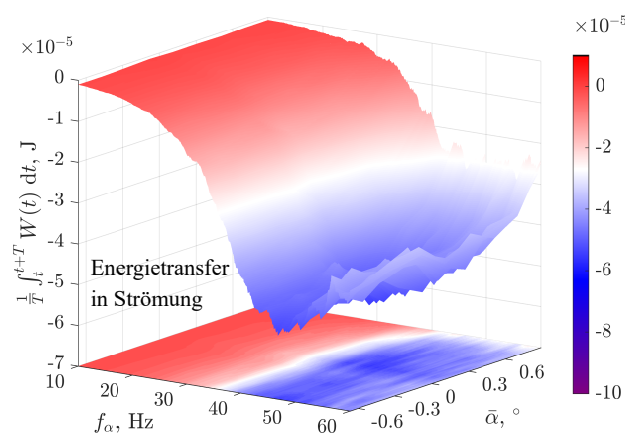

(h) Energie 3D, fix. Transition, $\mathrm{Ma}_{\infty}=0,75$

Abbildung E.4.: Energietransfer pro Schwingungsperiode zwischen Struktur und Strömung für eine freie ( $\mathrm{a}, \mathrm{b}$ und e,f) sowie eine fixierte Grenzschichttransition (c, d und g, h), gemessen mithilfe von Frequenzsweeps mit $\hat{\alpha}=0,08^{\circ}$ bei $\mathrm{Ma}_{\infty}=0,74$ und $\mathrm{Ma}_{\infty}=0,75$. Zum Zwecke der besseren Darstellung sind die 3D Grafiken für verschiedene Grenzschichtzustände gedreht. 


\section{E.3. Zu 6.3: Instationäres Druckverhalten}

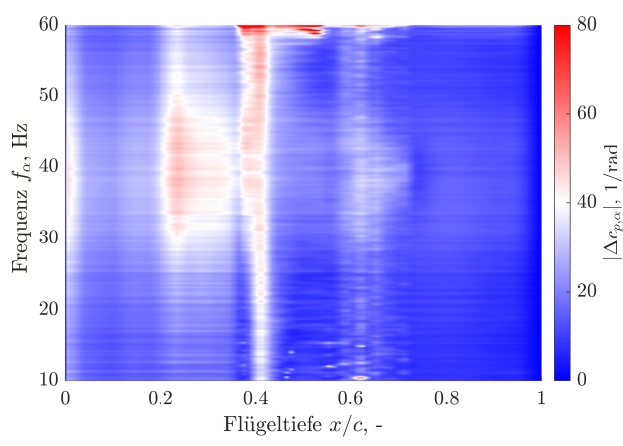

(a) Amplitude $\left|\Delta c_{p, \alpha}\right|$

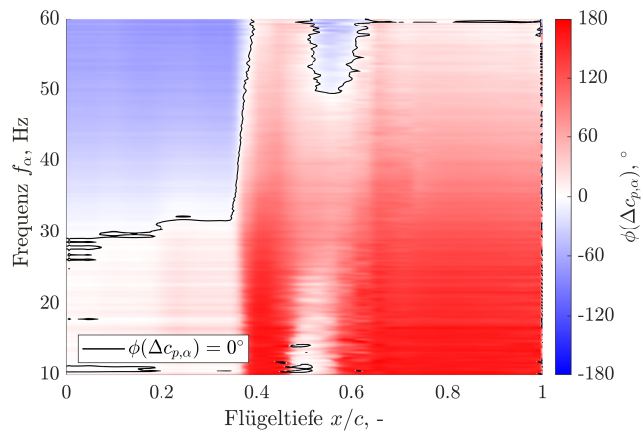

(b) Phase $\phi\left(\Delta c_{p, \alpha}\right)$
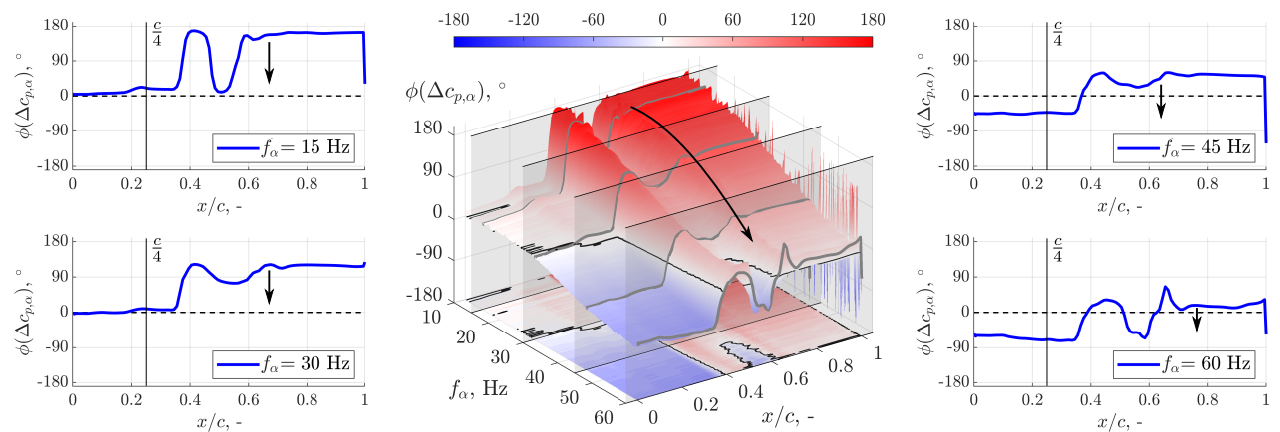

(c) Phase $\phi\left(\Delta c_{p, \alpha}\right)$ in 3D und Frequenzschnitte für $15 \mathrm{~Hz}, 30 \mathrm{~Hz}, 45 \mathrm{~Hz}$ und $60 \mathrm{~Hz}$

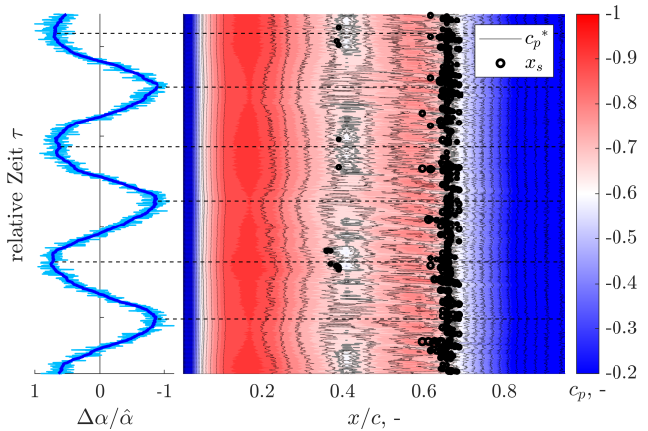

(d) $c_{p, s}$ für $f_{\alpha} \approx 15 \mathrm{~Hz}$

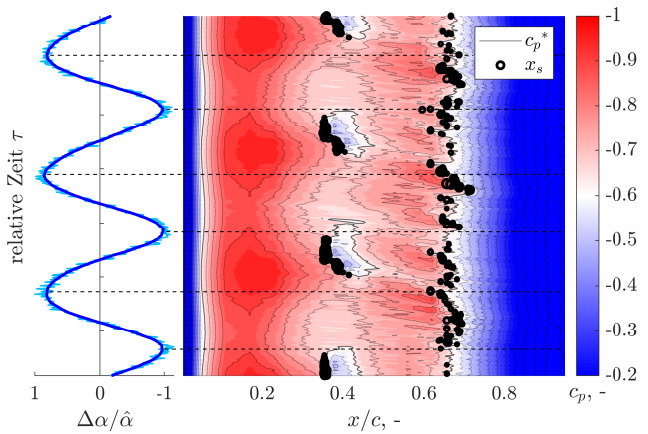

(f) $c_{p, s}$ für $f_{\alpha} \approx 45 \mathrm{~Hz}$

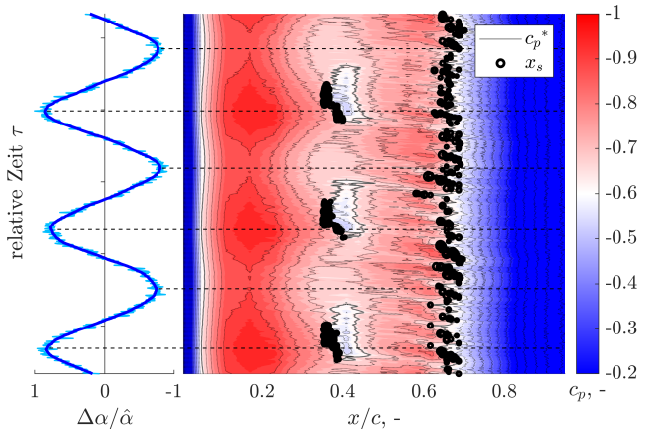

(e) $c_{p, s}$ für $f_{\alpha} \approx 30 \mathrm{~Hz}$

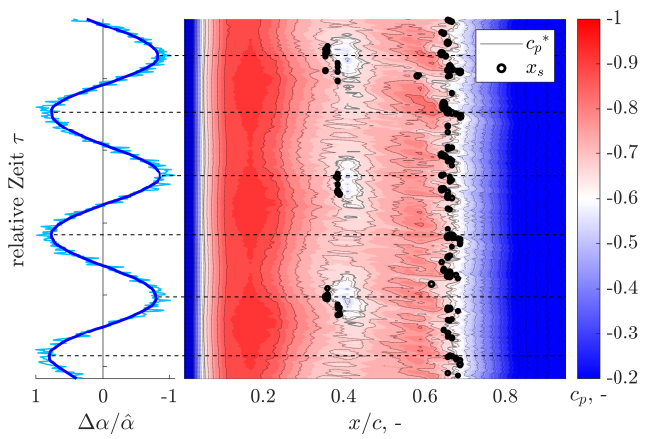

(g) $c_{p, s}$ für $f_{\alpha} \approx 60 \mathrm{~Hz}$

Abbildung E.5.: Instationäre Druckdifferenz $\Delta c_{p, \alpha}$ in Betrag (a) und Phase in 2D (b) sowie 3D (c) samt Frequenzschnitte für eine freie Grenzschichttransition, gemessen mithilfe eines Frequenzsweeps mit $\hat{\alpha}=0,08^{\circ}$ bei $\mathrm{Ma}_{\infty}=0,74$ und $\bar{\alpha}=0,07^{\circ}$. Zusätzlich sind die mit den Frequenzschnitten korrespondierenden Druckverteilungen $c_{p, s}$ in (d) bis (g) dargestellt. 


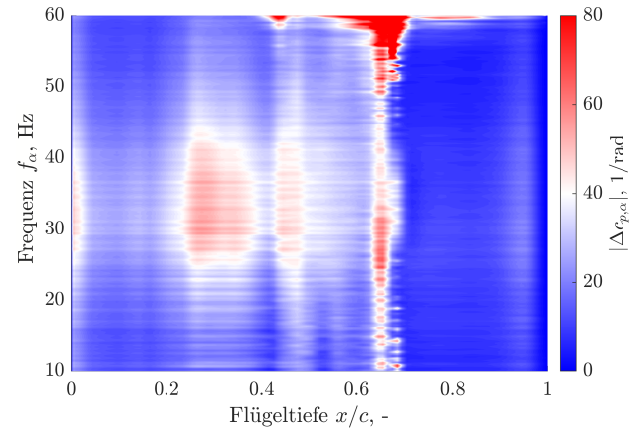

(a) Amplitude $\left|\Delta c_{p, \alpha}\right|$

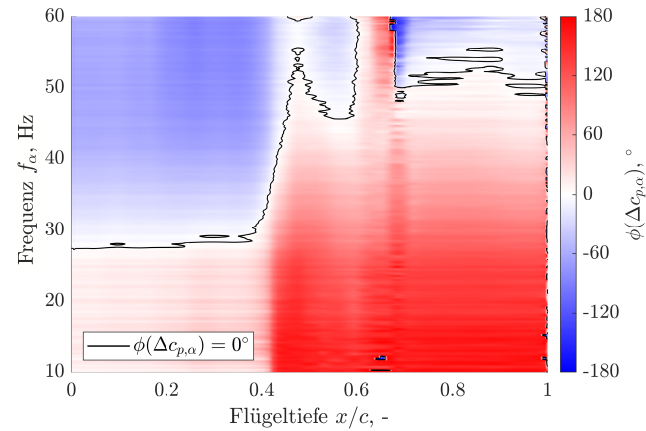

(b) Phase $\phi\left(\Delta c_{p, \alpha}\right)$
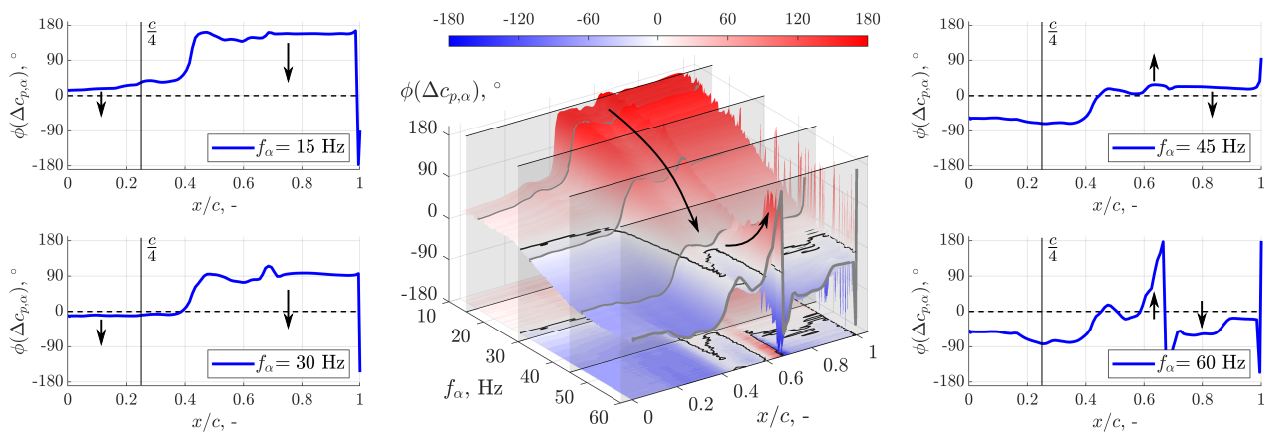

(c) Phase $\phi\left(\Delta c_{p, \alpha}\right)$ in 3D und Frequenzschnitte für $15 \mathrm{~Hz}, 30 \mathrm{~Hz}, 45 \mathrm{~Hz}$ und $60 \mathrm{~Hz}$

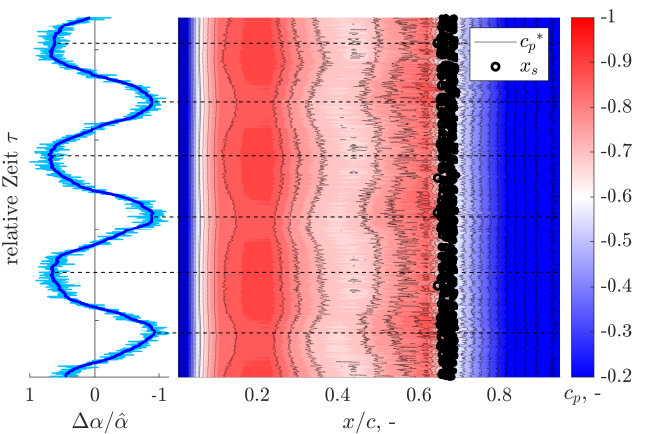

(d) $c_{p, s}$ für $f_{\alpha} \approx 15 \mathrm{~Hz}$

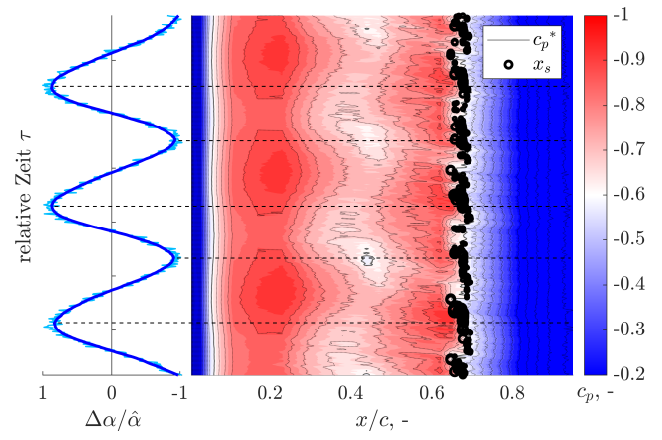

(f) $c_{p, s}$ für $f_{\alpha} \approx 45 \mathrm{~Hz}$

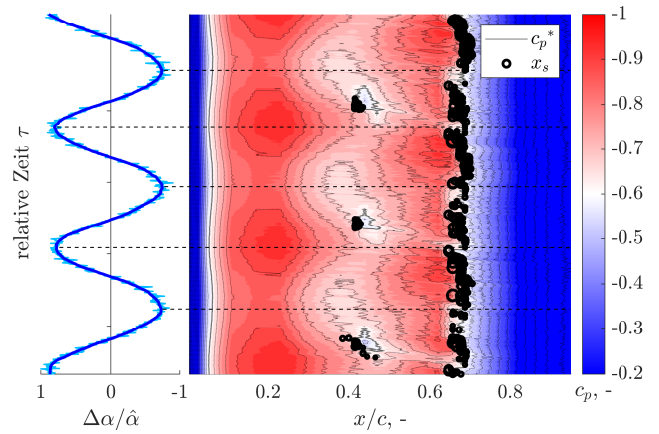

(e) $c_{p, s}$ für $f_{\alpha} \approx 30 \mathrm{~Hz}$

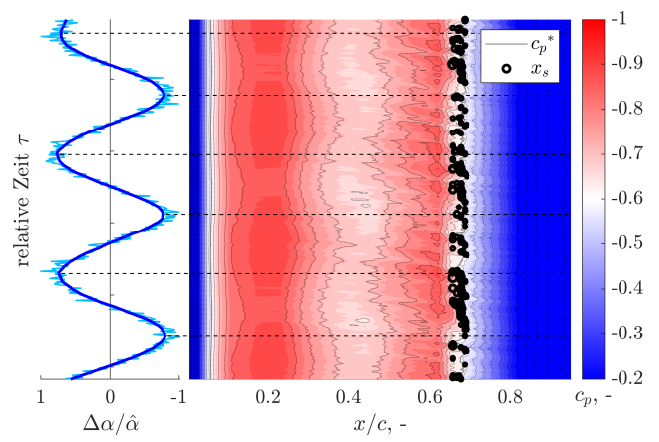

(g) $c_{p, s}$ für $f_{\alpha} \approx 60 \mathrm{~Hz}$

Abbildung E.6.: Instationäre Druckdifferenz $\Delta c_{p, \alpha}$ in Betrag (a) und Phase in 2D (b) sowie 3D (c) samt Frequenzschnitte für eine freie Grenzschichttransition, gemessen mithilfe eines Frequenzsweeps mit $\hat{\alpha}=0,08^{\circ}$ bei $\mathrm{Ma}_{\infty}=0,75$ und $\bar{\alpha}=-0,15^{\circ}$. Zusätzlich sind die mit den Frequenzschnitten korrespondierenden Druckverteilungen $c_{p, s}$ in (d) bis (g) dargestellt. 


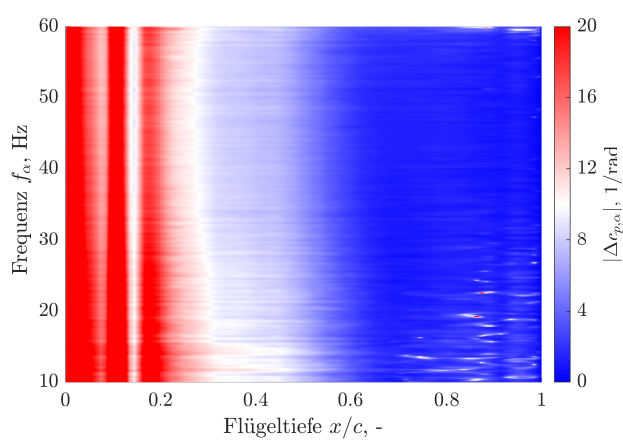

(a) Amplitude $\left|\Delta c_{p, \alpha}\right|, \mathrm{Ma}_{\infty}=0,74$

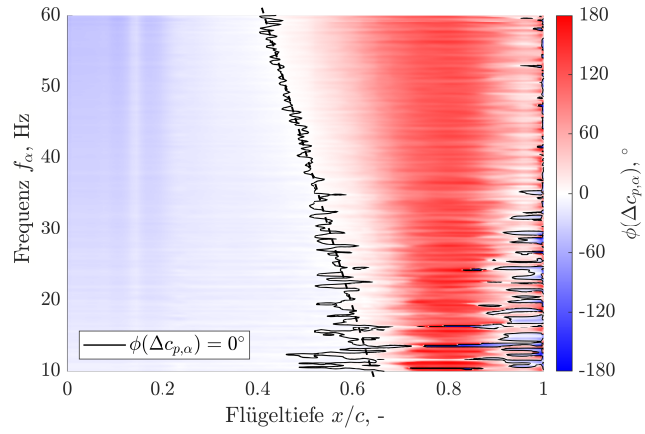

(b) Phase $\phi\left(\Delta c_{p, \alpha}\right), \mathrm{Ma}_{\infty}=0,74$
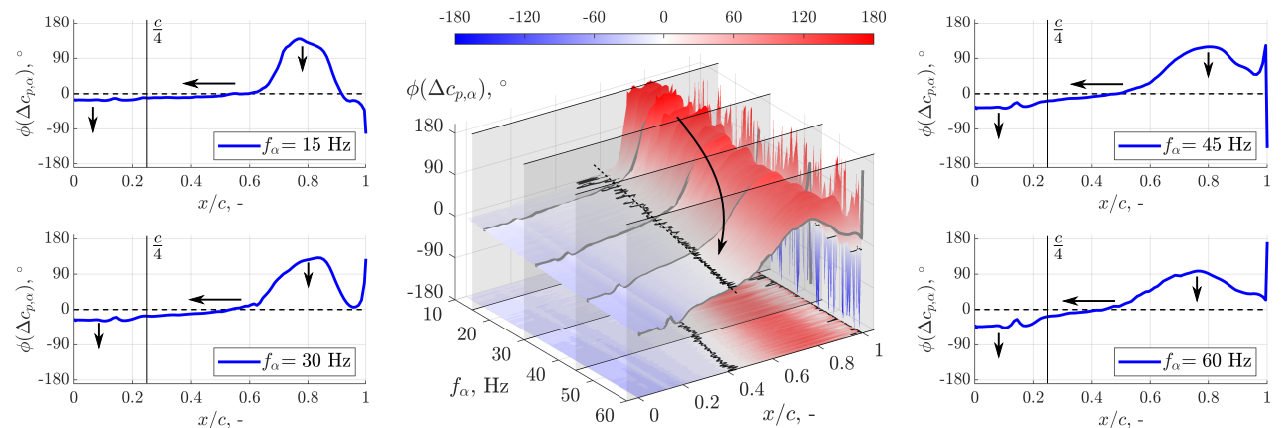

(c) Phase $\phi\left(\Delta c_{p, \alpha}\right)$ in 3D und Frequenzschnitte für $\mathrm{Ma}_{\infty}=0,74$

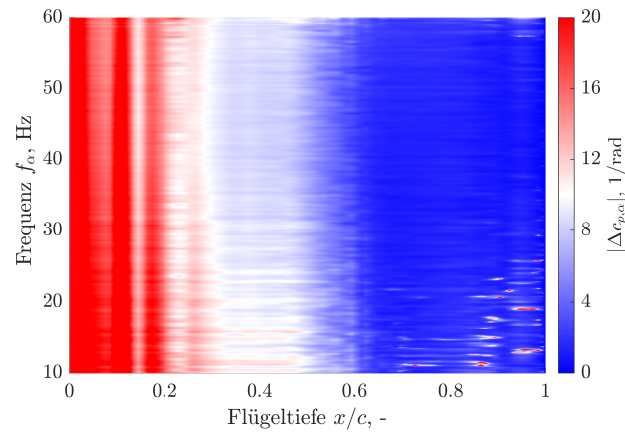

(d) Amplitude $\left|\Delta c_{p, \alpha}\right|, \mathrm{Ma}_{\infty}=0,75$

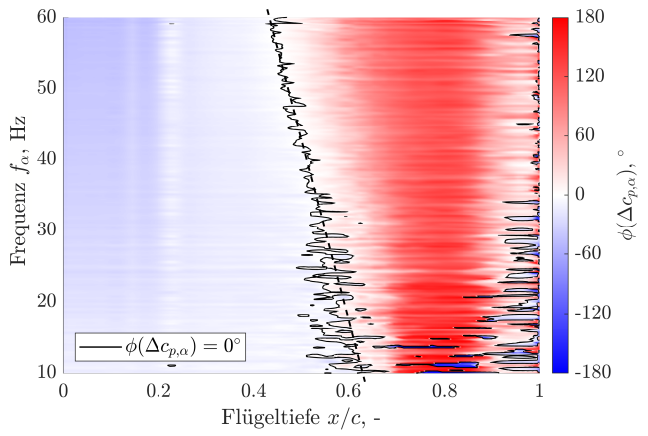

(e) Phase $\phi\left(\Delta c_{p, \alpha}\right), \mathrm{Ma}_{\infty}=0,75$
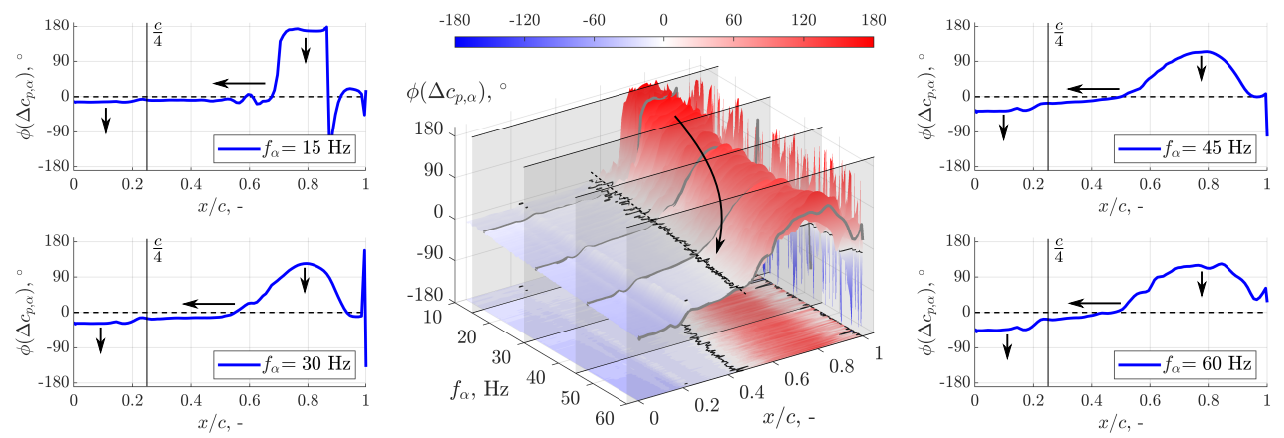

(f) Phase $\phi\left(\Delta c_{p, \alpha}\right)$ in 3D und Frequenzschnitte für $\mathrm{Ma}_{\infty}=0,75$

Abbildung E.7.: Instationäre Druckdifferenz $\Delta c_{p, \alpha}$ in Betrag und Phase sowie 3D-Darstellung der Phase samt Frequenzschnitte für eine fixierte Grenzschichttransition, gemessen mithilfe eines Frequenzsweeps mit $\hat{\alpha}=0,08^{\circ}$ bei $\mathrm{Ma}_{\infty}=0,74$ und $\bar{\alpha}=-0,08^{\circ}$ (a bis c) sowie bei $\mathrm{Ma}_{\infty}=0,75$ und $\bar{\alpha}=-0,27^{\circ}(\mathrm{d}$ bis f). 


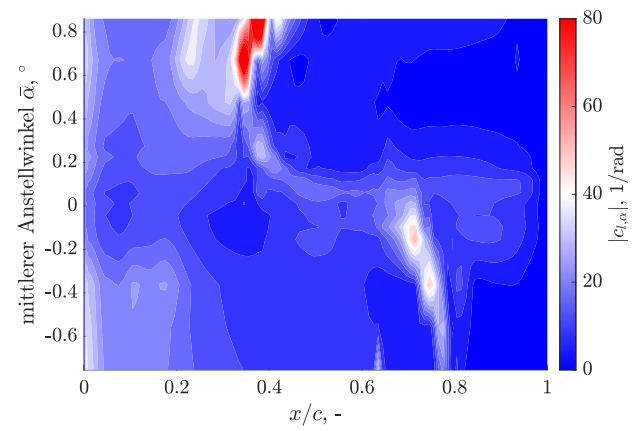

(a) Amplitude $\left|\Delta c_{p, \alpha}\right|$ für $f_{\alpha} \approx 15 \mathrm{~Hz}$

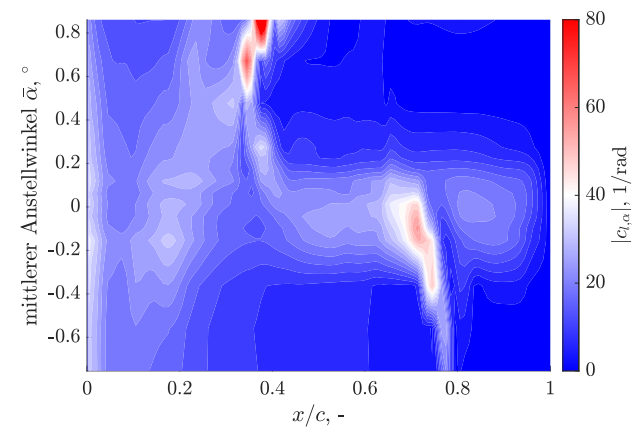

(c) Amplitude $\left|\Delta c_{p, \alpha}\right|$ für $f_{\alpha} \approx 30 \mathrm{~Hz}$

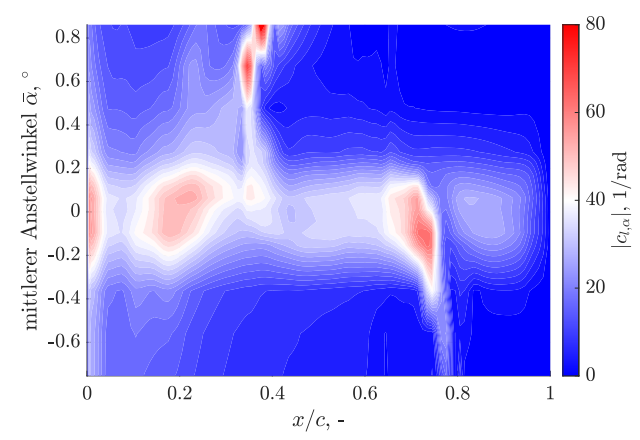

(e) Amplitude $\left|\Delta c_{p, \alpha}\right|$ für $f_{\alpha} \approx 45 \mathrm{~Hz}$

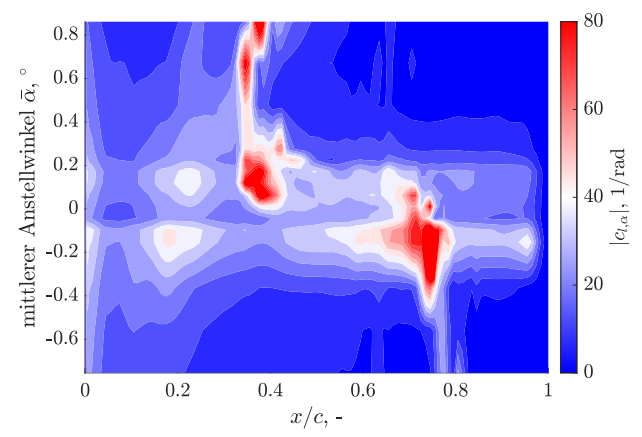

(g) Amplitude $\left|\Delta c_{p, \alpha}\right|$ für $f_{\alpha} \approx 60 \mathrm{~Hz}$

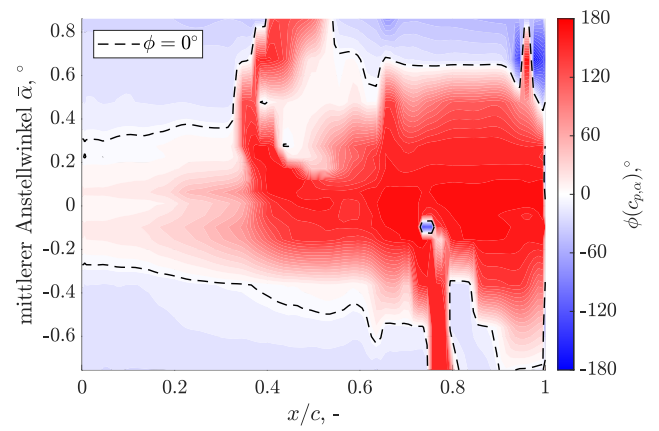

(b) Phase $\phi\left(\Delta c_{p, \alpha}\right)$ für $f_{\alpha} \approx 15 \mathrm{~Hz}$

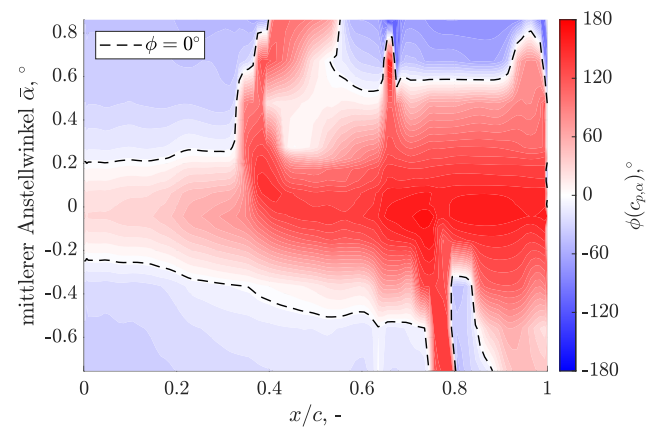

(d) Phase $\phi\left(\Delta c_{p, \alpha}\right)$ für $f_{\alpha} \approx 30 \mathrm{~Hz}$

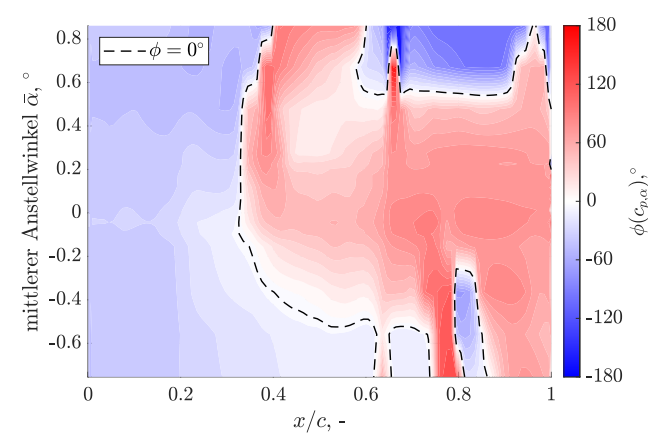

(f) Phase $\phi\left(\Delta c_{p, \alpha}\right)$ für $f_{\alpha} \approx 45 \mathrm{~Hz}$

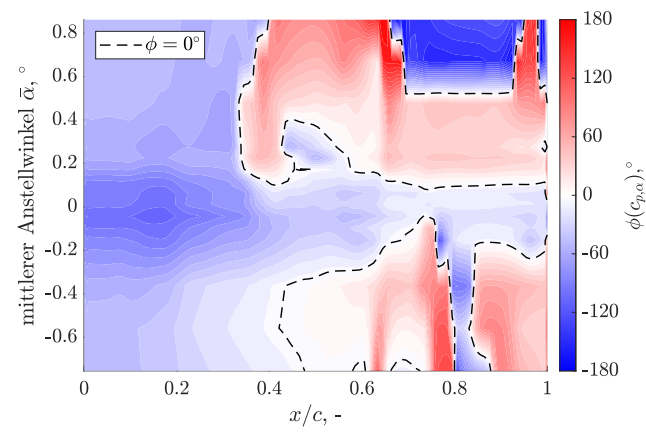

(h) Phase $\phi\left(\Delta c_{p, \alpha}\right)$ für $f_{\alpha} \approx 60 \mathrm{~Hz}$

Abbildung E.8.: Amplituden und Phasen der instationären Druckdifferenz $\Delta c_{p, \alpha}$ in Abhängigkeit des mittleren Anstellwinkels $\bar{\alpha}$, ausgewertet für vier Schwingungsfrequenzen $15 \mathrm{~Hz}$, $30 \mathrm{~Hz}, 45 \mathrm{~Hz}$ und $60 \mathrm{~Hz}$ für eine freie Grenzschichttransition. Die Ergebnisse basieren auf Frequenzsweeps mit $\hat{\alpha}=0,08^{\circ}$ bei $\mathrm{Ma}_{\infty}=0,73$. 


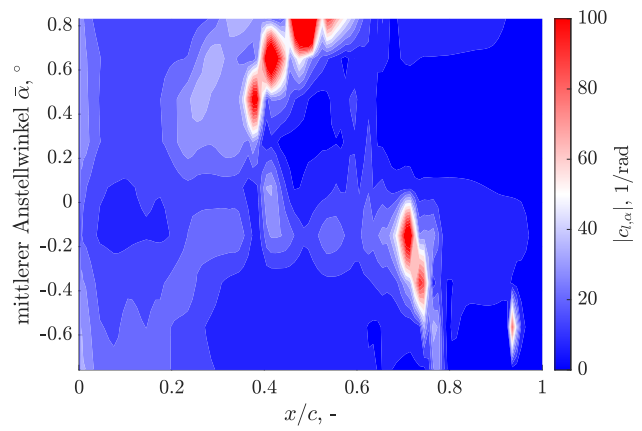

(a) Amplitude $\left|\Delta c_{p, \alpha}\right|$ für $f_{\alpha} \approx 15 \mathrm{~Hz}$

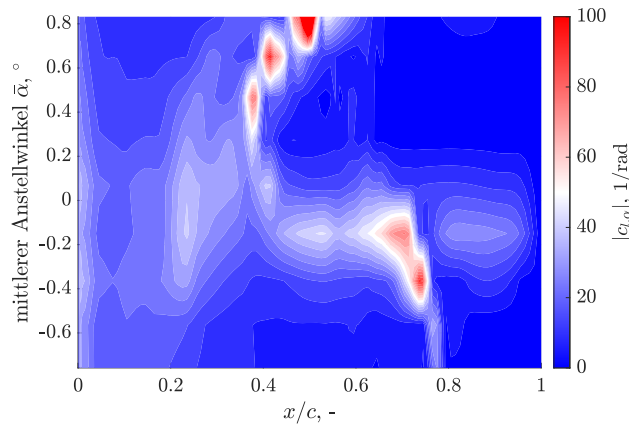

(c) Amplitude $\left|\Delta c_{p, \alpha}\right|$ für $f_{\alpha} \approx 30 \mathrm{~Hz}$

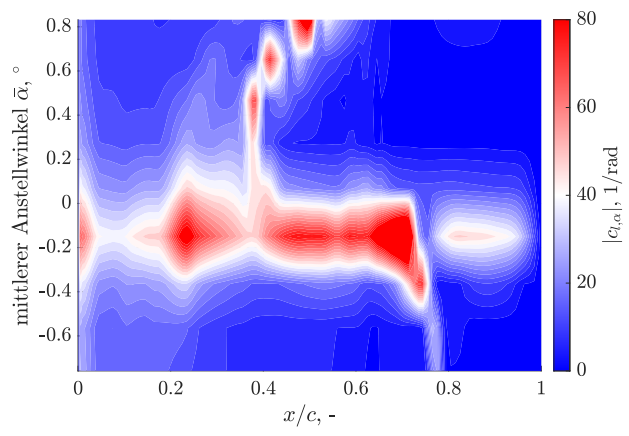

(e) Amplitude $\left|\Delta c_{p, \alpha}\right|$ für $f_{\alpha} \approx 45 \mathrm{~Hz}$

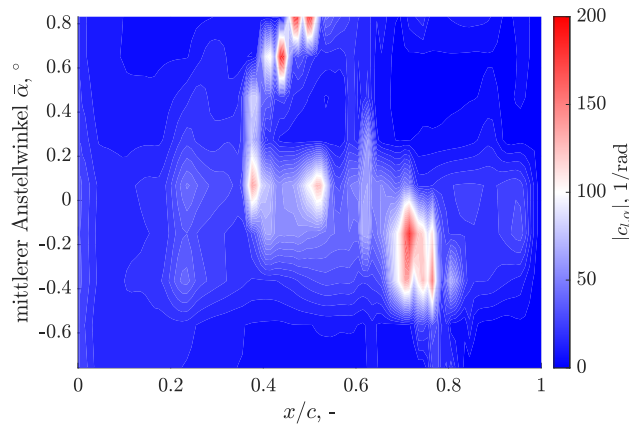

(g) Amplitude $\left|\Delta c_{p, \alpha}\right|$ für $f_{\alpha} \approx 60 \mathrm{~Hz}$

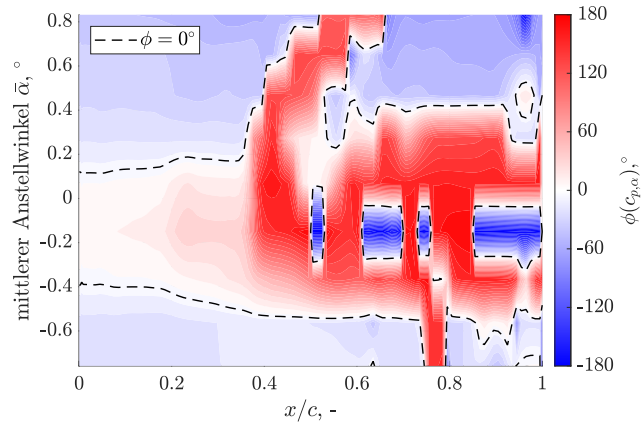

(b) Phase $\phi\left(\Delta c_{p, \alpha}\right)$ für $f_{\alpha} \approx 15 \mathrm{~Hz}$

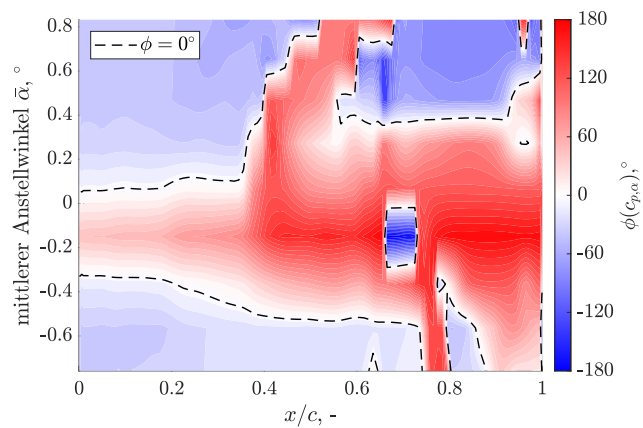

(d) Phase $\phi\left(\Delta c_{p, \alpha}\right)$ für $f_{\alpha} \approx 30 \mathrm{~Hz}$

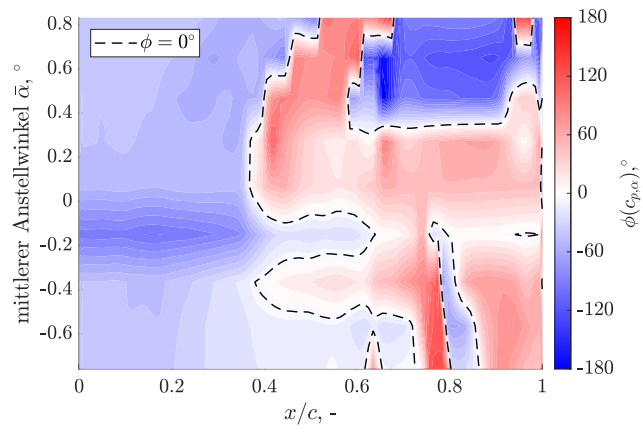

(f) Phase $\phi\left(\Delta c_{p, \alpha}\right)$ für $f_{\alpha} \approx 45 \mathrm{~Hz}$

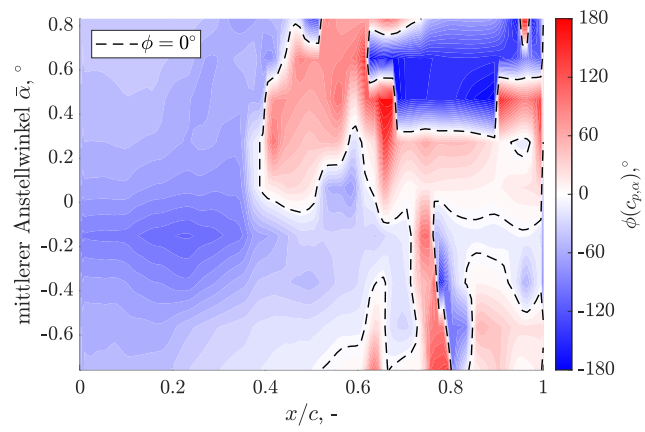

(h) Phase $\phi\left(\Delta c_{p, \alpha}\right)$ für $f_{\alpha} \approx 60 \mathrm{~Hz}$

Abbildung E.9.: Amplituden und Phasen der instationären Druckdifferenz $\Delta c_{p, \alpha}$ in Abhängigkeit des mittleren Anstellwinkels $\bar{\alpha}$, ausgewertet für vier Schwingungsfrequenzen $15 \mathrm{~Hz}$, $30 \mathrm{~Hz}, 45 \mathrm{~Hz}$ und $60 \mathrm{~Hz}$ für eine freie Grenzschichttransition. Die Ergebnisse basieren auf Frequenzsweeps mit $\hat{\alpha}=0,08^{\circ}$ bei $\mathrm{Ma}_{\infty}=0,74$. 


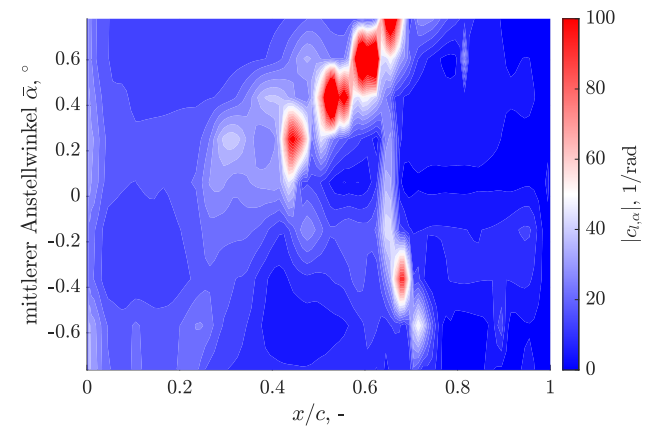

(a) Amplitude $\left|\Delta c_{p, \alpha}\right|$ für $f_{\alpha} \approx 15 \mathrm{~Hz}$

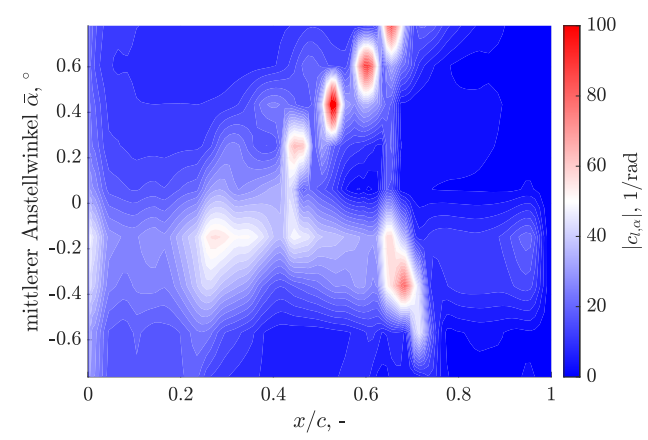

(c) Amplitude $\left|\Delta c_{p, \alpha}\right|$ für $f_{\alpha} \approx 30 \mathrm{~Hz}$

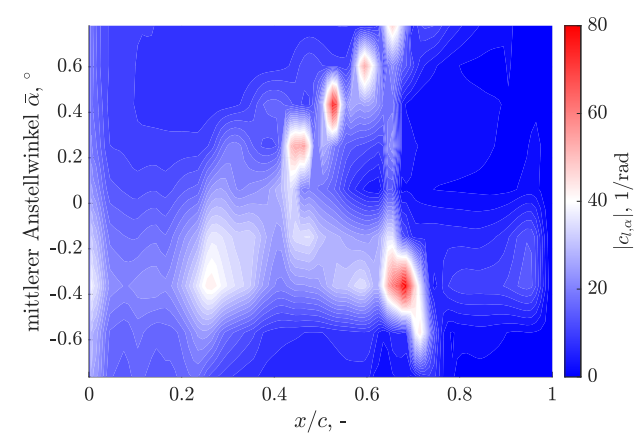

(e) Amplitude $\left|\Delta c_{p, \alpha}\right|$ für $f_{\alpha} \approx 45 \mathrm{~Hz}$

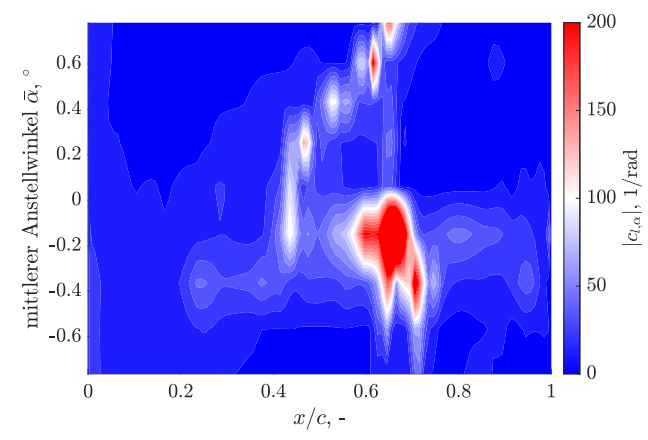

(g) Amplitude $\left|\Delta c_{p, \alpha}\right|$ für $f_{\alpha} \approx 60 \mathrm{~Hz}$

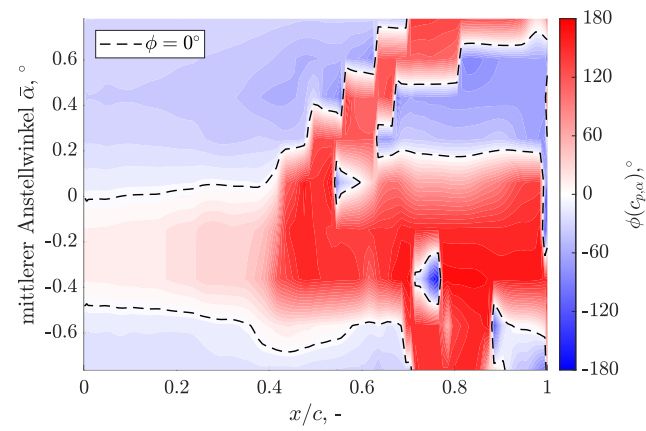

(b) Phase $\phi\left(\Delta c_{p, \alpha}\right)$ für $f_{\alpha} \approx 15 \mathrm{~Hz}$

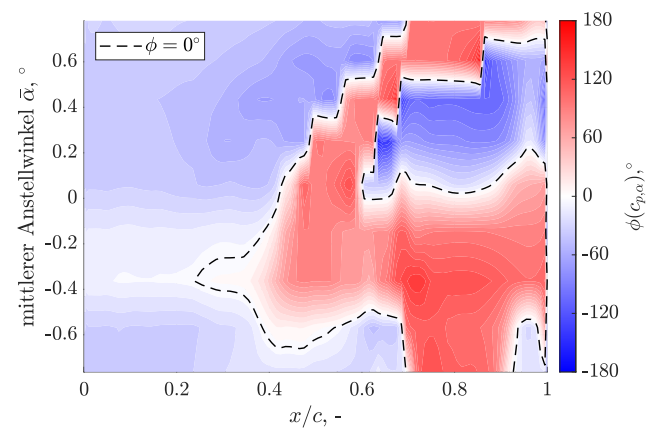

(d) Phase $\phi\left(\Delta c_{p, \alpha}\right)$ für $f_{\alpha} \approx 30 \mathrm{~Hz}$

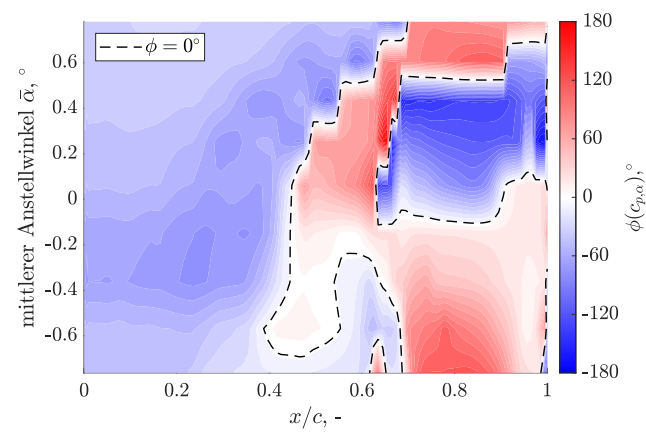

(f) Phase $\phi\left(\Delta c_{p, \alpha}\right)$ für $f_{\alpha} \approx 45 \mathrm{~Hz}$

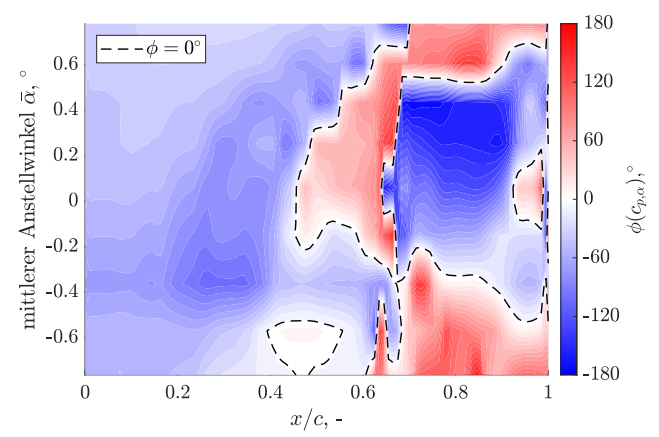

(h) Phase $\phi\left(\Delta c_{p, \alpha}\right)$ für $f_{\alpha} \approx 60 \mathrm{~Hz}$

Abbildung E.10.: Amplituden und Phasen der instationären Druckdifferenz $\Delta c_{p, \alpha}$ in Abhängigkeit des mittleren Anstellwinkels $\bar{\alpha}$, ausgewertet für vier Schwingungsfrequenzen $15 \mathrm{~Hz}$, $30 \mathrm{~Hz}, 45 \mathrm{~Hz}$ und $60 \mathrm{~Hz}$ für eine freie Grenzschichttransition. Die Ergebnisse basieren auf Frequenzsweeps mit $\hat{\alpha}=0,08^{\circ}$ bei $\mathrm{Ma}_{\infty}=0,75$. 


\section{E.4. Zu 6.4: Instationäres Transitionsverhalten}

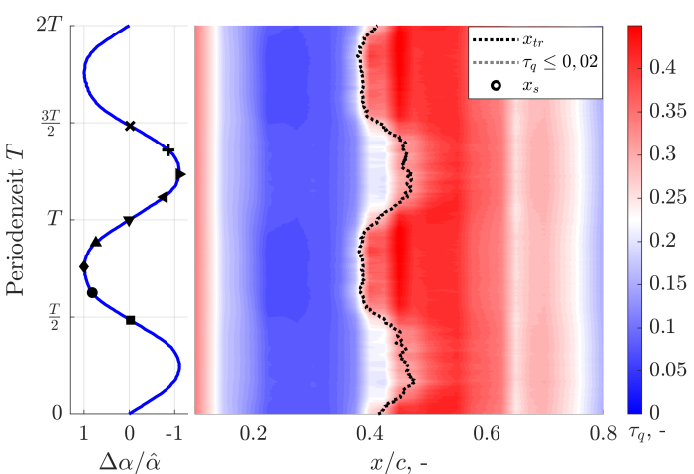

(a) $x_{t r}$ für $f_{\alpha}=12,9 \mathrm{~Hz}$

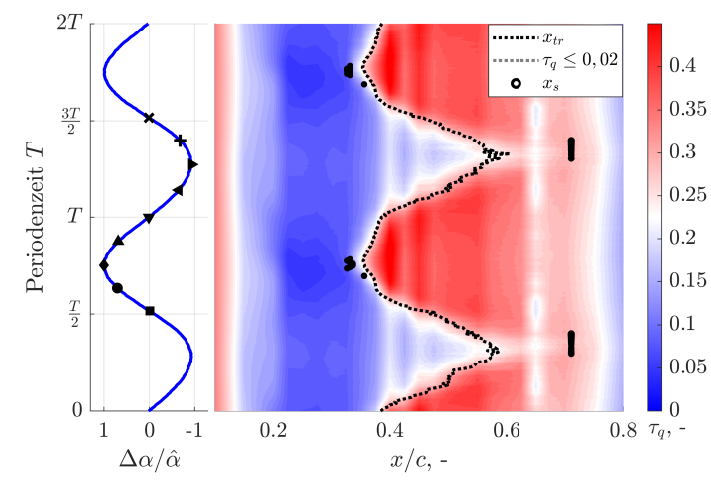

(c) $x_{t r}$ für $f_{\alpha}=32,2 \mathrm{~Hz}$

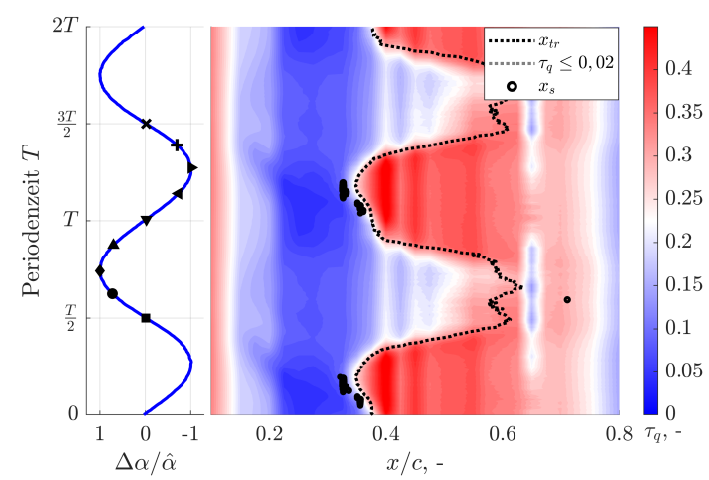

(e) $x_{t r}$ für $f_{\alpha}=51,5 \mathrm{~Hz}$

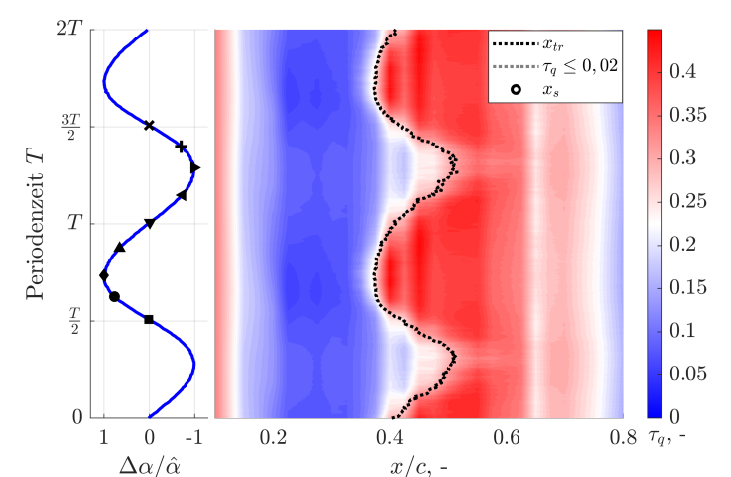

(b) $x_{t r}$ für $f_{\alpha}=25,7 \mathrm{~Hz}$

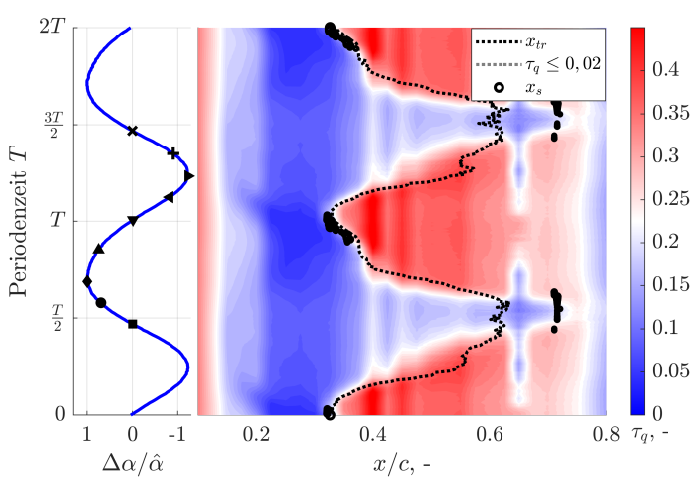

(d) $x_{t r}$ für $f_{\alpha}=45,0 \mathrm{~Hz}$

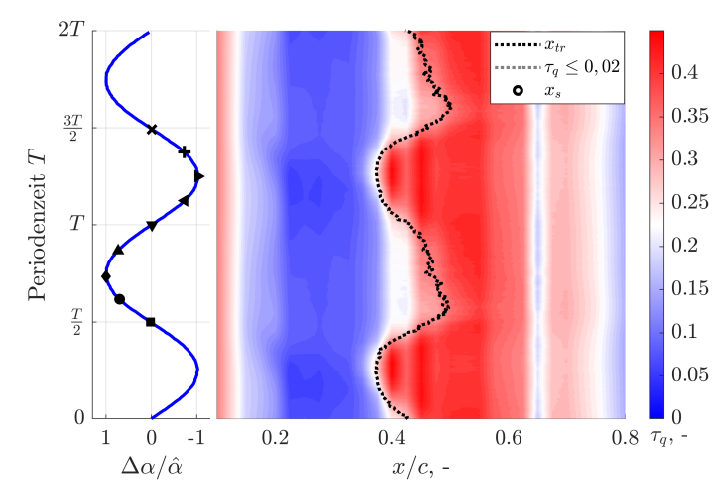

(f) $x_{t r}$ für $f_{\alpha}=64,3 \mathrm{~Hz}$

Abbildung E.11.: Phasengemittelte Quasi-Wandschubspannung $\tau_{q}$ für zwei Schwingungsperioden, gemessen und berechnet auf Basis einer diskreten Frequenzvariation um $\bar{\alpha}=0,05^{\circ}$ mit $\hat{\alpha}=0,1^{\circ}$ bei $\mathrm{Ma}_{\infty}=0,73$. 


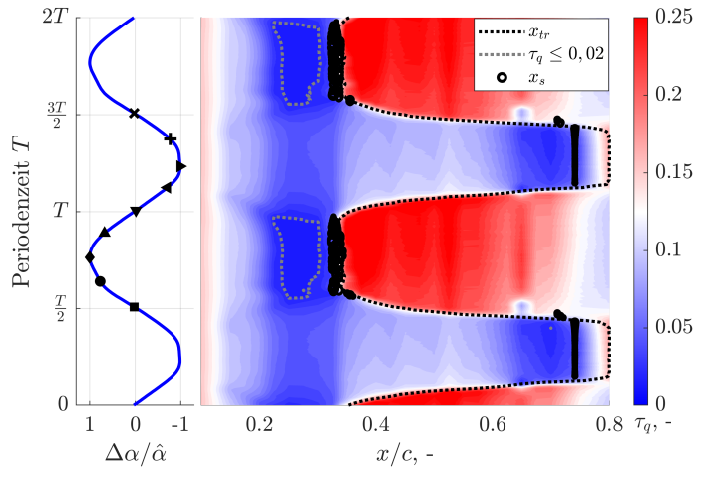

(a) $x_{t r}$ für $f_{\alpha}=12,9 \mathrm{~Hz}$

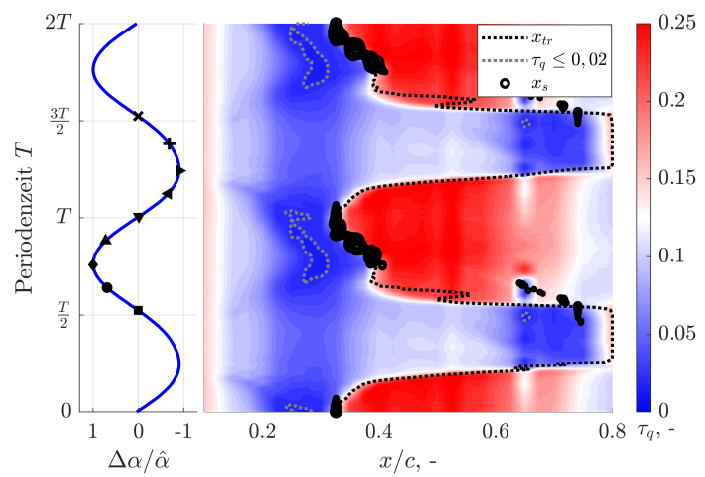

(c) $x_{t r}$ für $f_{\alpha}=32,2 \mathrm{~Hz}$

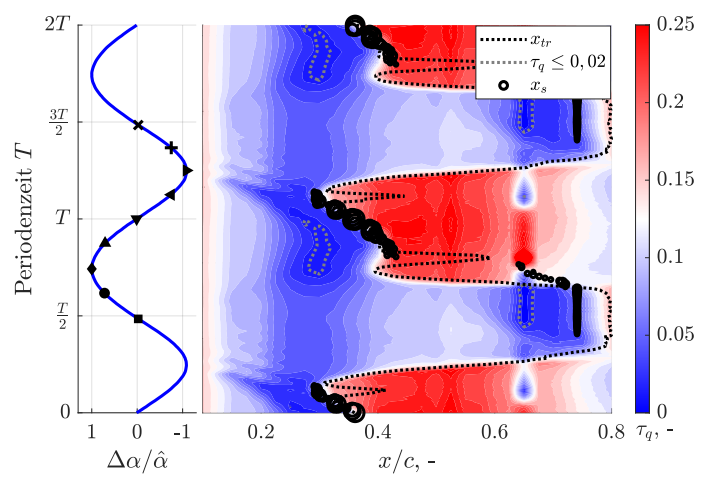

(e) $x_{t r}$ für $f_{\alpha}=45,0 \mathrm{~Hz}$

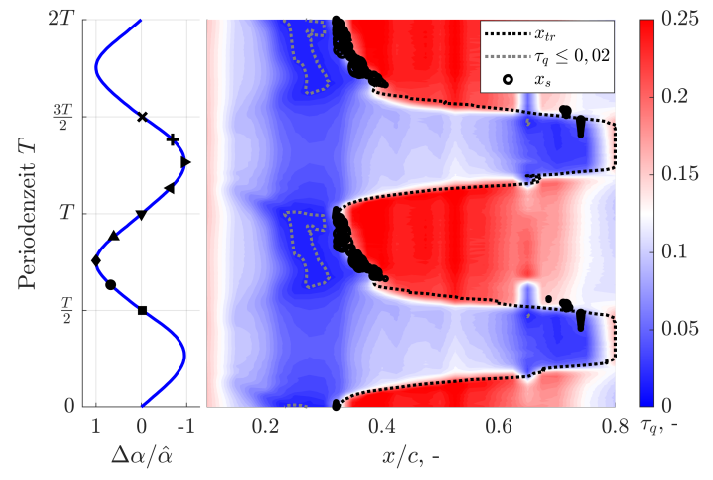

(b) $x_{t r}$ für $f_{\alpha}=25,7 \mathrm{~Hz}$

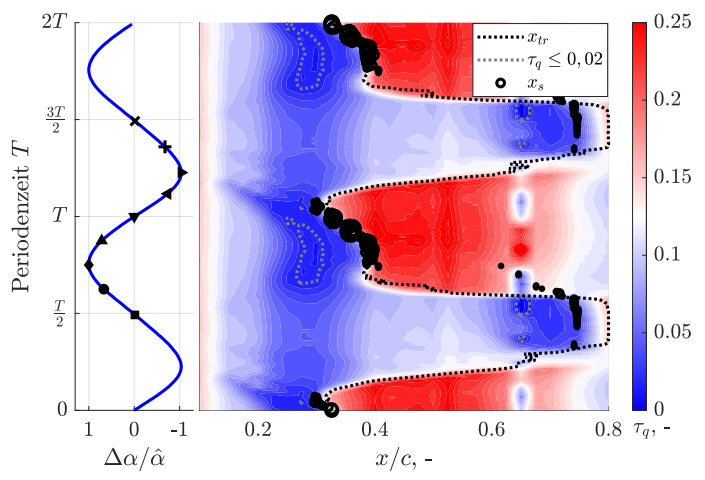

(d) $x_{t r}$ für $f_{\alpha}=38,6 \mathrm{~Hz}$

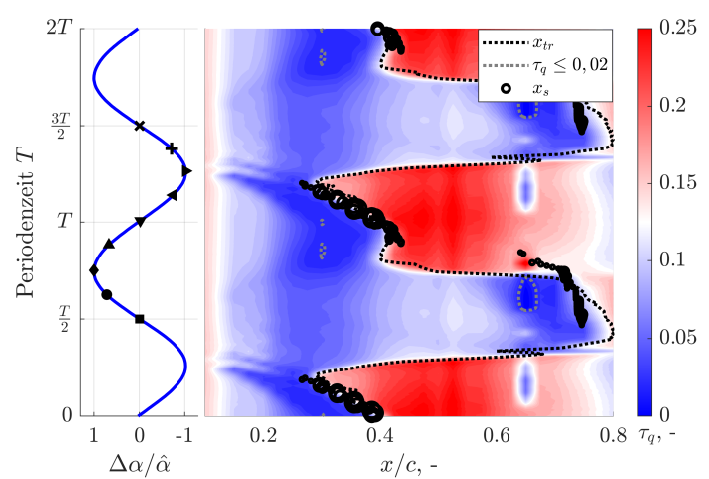

(f) $x_{t r}$ für $f_{\alpha}=51,5 \mathrm{~Hz}$

Abbildung E.12.: Phasengemittelte Quasi-Wandschubspannung $\tau_{q}$ für zwei Schwingungsperioden, gemessen und berechnet auf Basis einer diskreten Frequenzvariation um $\bar{\alpha}=0,05^{\circ}$ mit $\hat{\alpha}=0,5^{\circ}$ bei $\mathrm{Ma}_{\infty}=0,73$. 


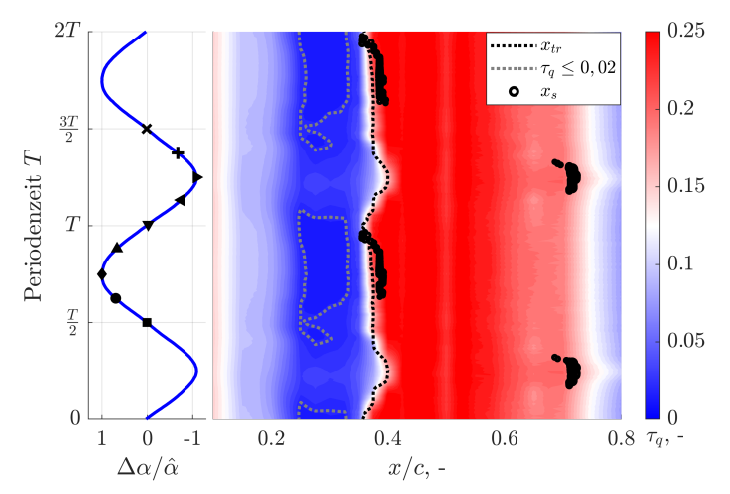

(a) $x_{t r}$ für $f_{\alpha}=13,0 \mathrm{~Hz}$

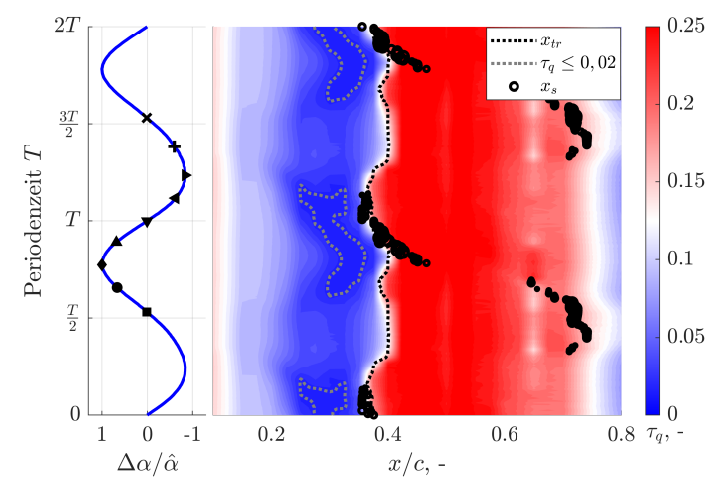

(c) $x_{t r}$ für $f_{\alpha}=32,6 \mathrm{~Hz}$

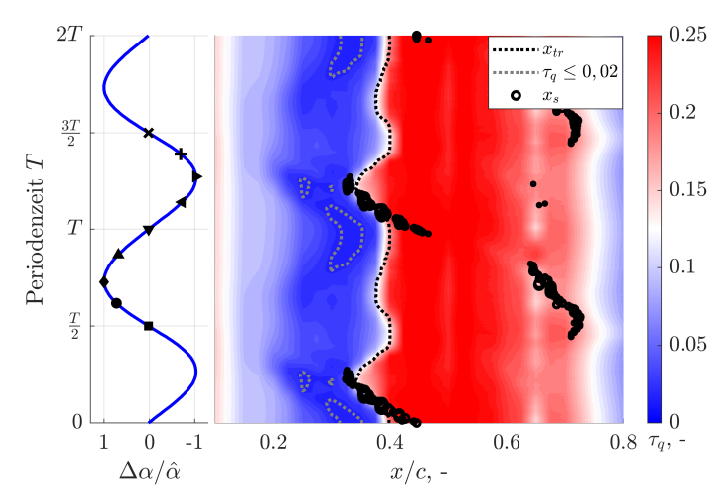

(e) $x_{t r}$ für $f_{\alpha}=52,1 \mathrm{~Hz}$

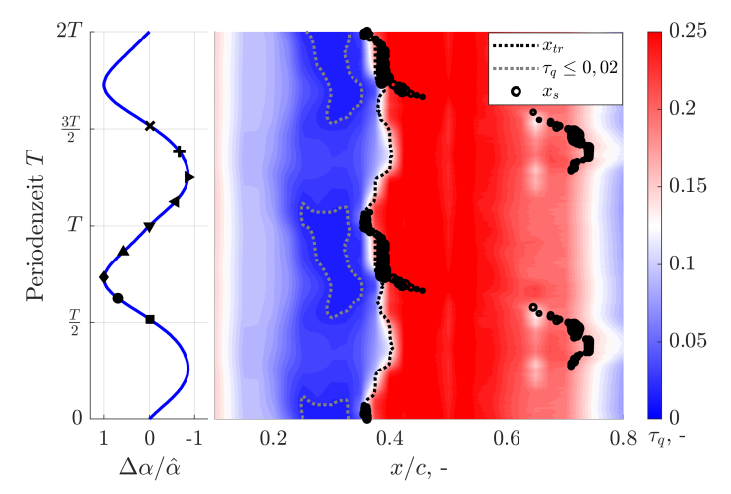

(b) $x_{t r}$ für $f_{\alpha}=26,1 \mathrm{~Hz}$

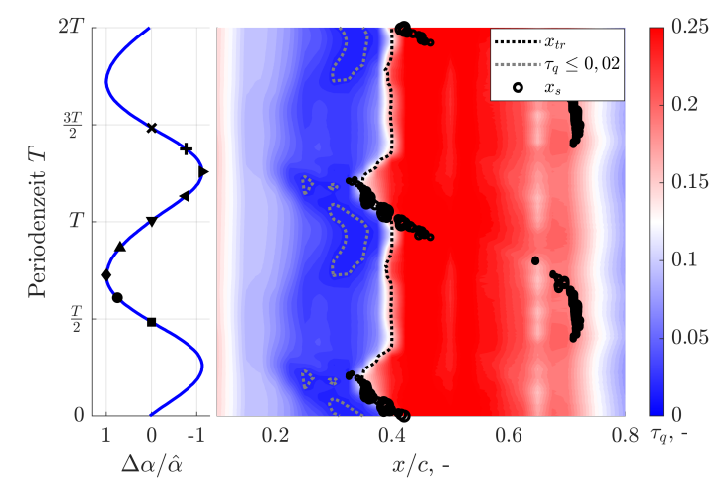

(d) $x_{t r}$ für $f_{\alpha}=45,6 \mathrm{~Hz}$

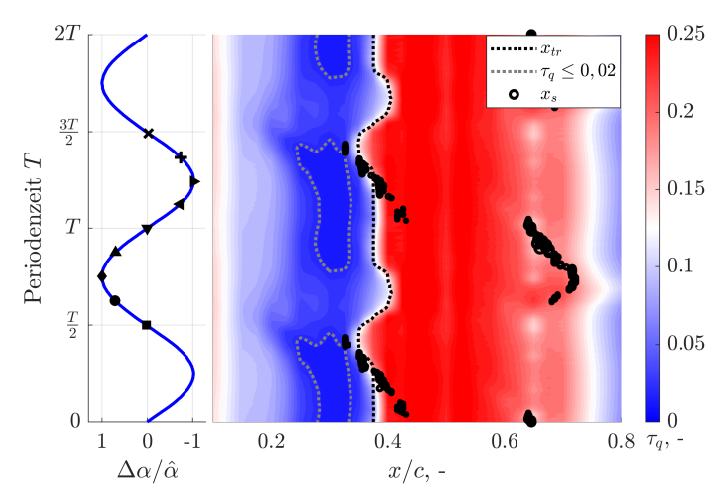

(f) $x_{t r}$ für $f_{\alpha}=65,1 \mathrm{~Hz}$

Abbildung E.13.: Phasengemittelte Quasi-Wandschubspannung $\tau_{q}$ für zwei Schwingungsperioden, gemessen und berechnet auf Basis einer diskreten Frequenzvariation um $\bar{\alpha}=0,05^{\circ}$ mit $\hat{\alpha}=0,25^{\circ}$ bei $\mathrm{Ma}_{\infty}=0,74$. 


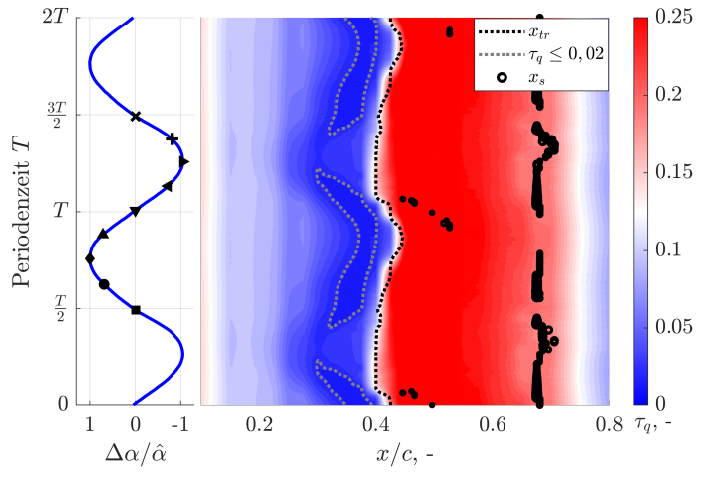

(a) $x_{t r}$ für $f_{\alpha}=13,2 \mathrm{~Hz}$

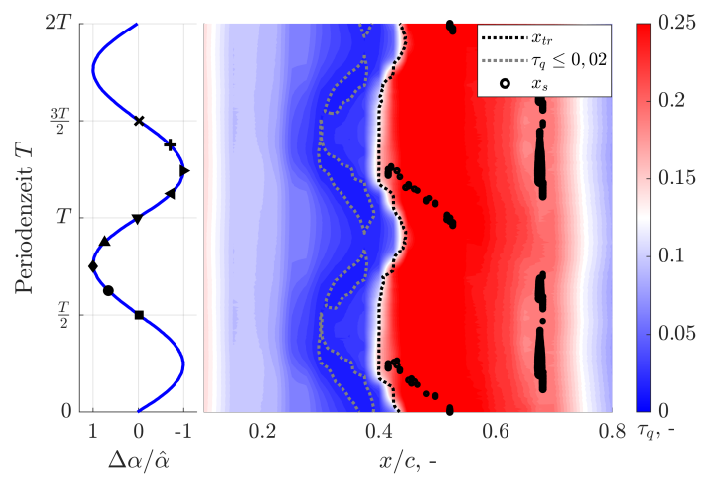

(c) $x_{t r}$ für $f_{\alpha}=33,0 \mathrm{~Hz}$

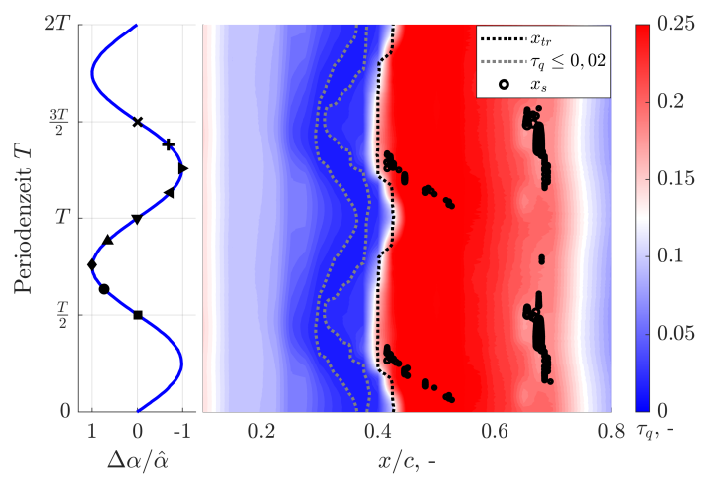

(e) $x_{t r}$ für $f_{\alpha}=52,7 \mathrm{~Hz}$

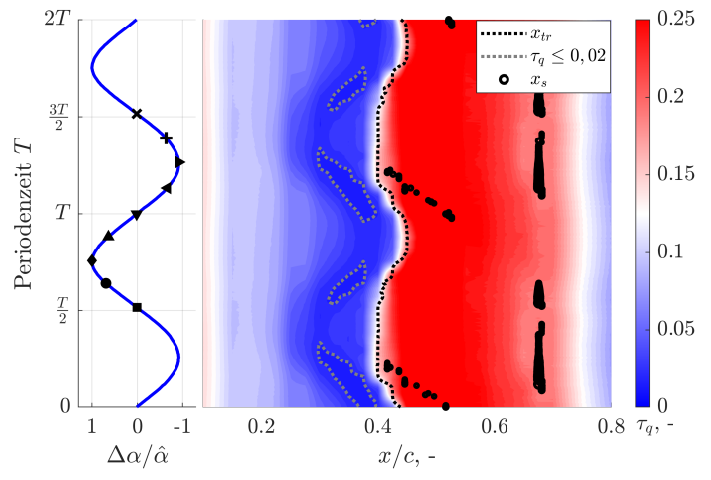

(b) $x_{t r}$ für $f_{\alpha}=26,4 \mathrm{~Hz}$

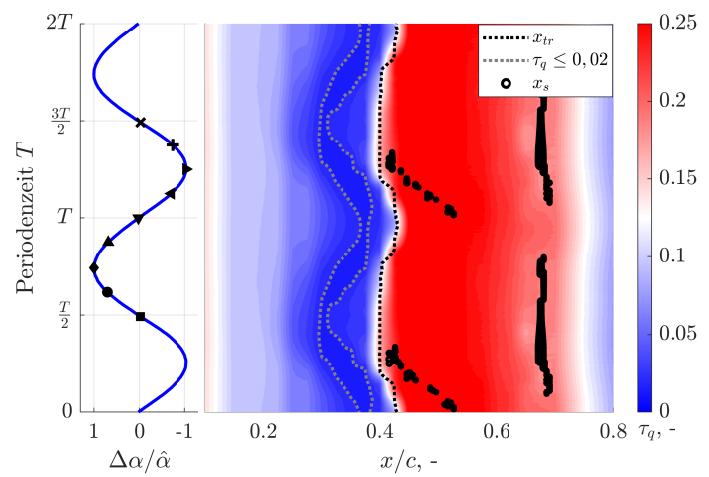

(d) $x_{t r}$ für $f_{\alpha}=46,1 \mathrm{~Hz}$

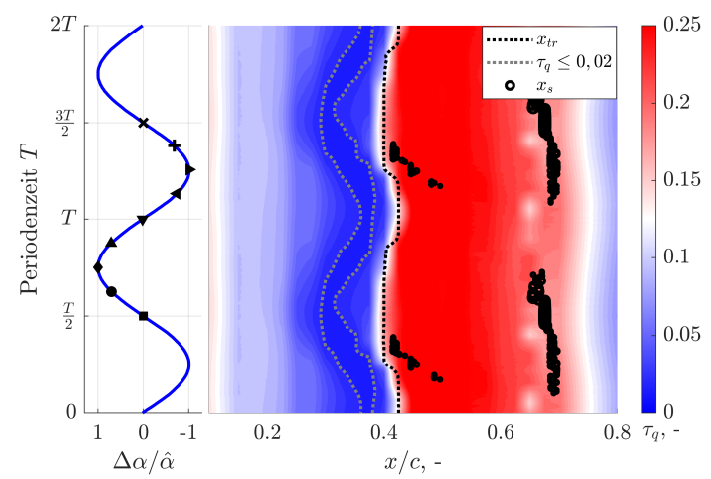

(f) $x_{t r}$ für $f_{\alpha}=65,9 \mathrm{~Hz}$

Abbildung E.14.: Phasengemittelte Quasi-Wandschubspannung $\tau_{q}$ für zwei Schwingungsperioden, gemessen und berechnet auf Basis einer diskreten Frequenzvariation um $\bar{\alpha}=0,05^{\circ}$ mit $\hat{\alpha}=0,25^{\circ}$ bei $\mathrm{Ma}_{\infty}=0,75$. 


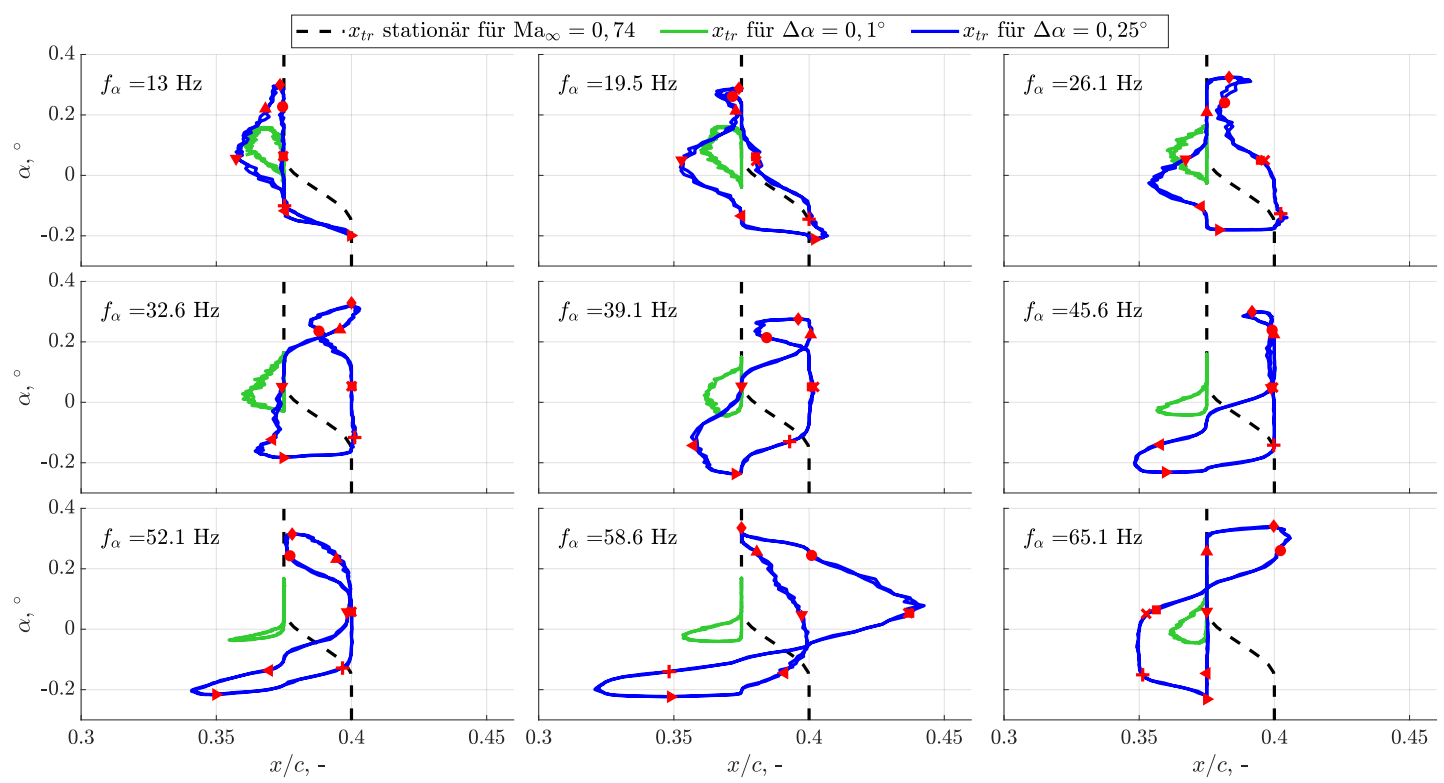

Abbildung E.15.: Ermittelte Transitionsbewegung für die, bei $\mathrm{Ma}_{\infty}=0,74$ um $\bar{\alpha}=0,05^{\circ}$ durchgeführten Frequenzvariationen mit $\hat{\alpha}=0,1^{\circ}$ und $\hat{\alpha}=0,25^{\circ}$.

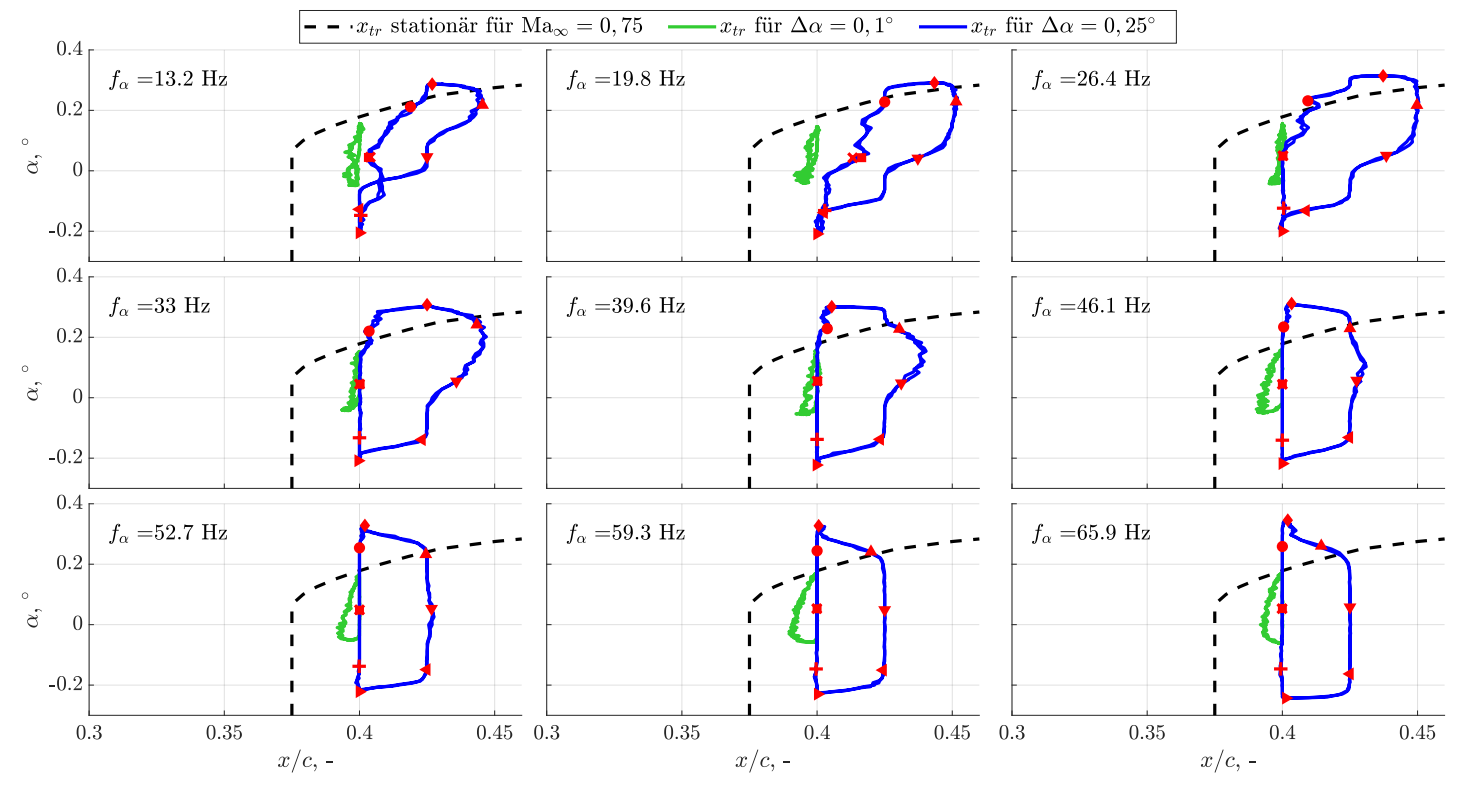

Abbildung E.16.: Ermittelte Transitionsbewegung für die, bei $\mathrm{Ma}_{\infty}=0,75$ um $\bar{\alpha}=0,05^{\circ}$ durchgeführten Frequenzvariationen mit $\hat{\alpha}=0,1^{\circ}$ und $\hat{\alpha}=0,25^{\circ}$. 



\section{F. Ergänzungen zu Kapitel 7}

\section{F.1. Dämpfungen der aeroelastischen Konfigurationen}

Zur Dämpfungsbestimmung der einzelnen Schwingungsmoden der aeroelastischen Konfigurationen wurden im Vorfeld der Windkanalversuche Vibrationstests durchgeführt. Hierzu wurde das Windkanalmodell sowie der FVS mit zusätzlichen Beschleunigungssensoren ausgestattet. Die Dämpfungen wurden dann anhand der Hüllkurven $f\left(\gamma_{j}\right)$ der abklingenden Beschleunigungssignale mehrerer Impulsantworten bestimmt. Das Verfahren ist in Abb. F.1 und Abb. F.2 für die zwei Schwingungsmoden Schlag und Drehung der 2DoF Konfiguration anhand eines repräsentativen Beschleunigungssignals veranschaulicht.
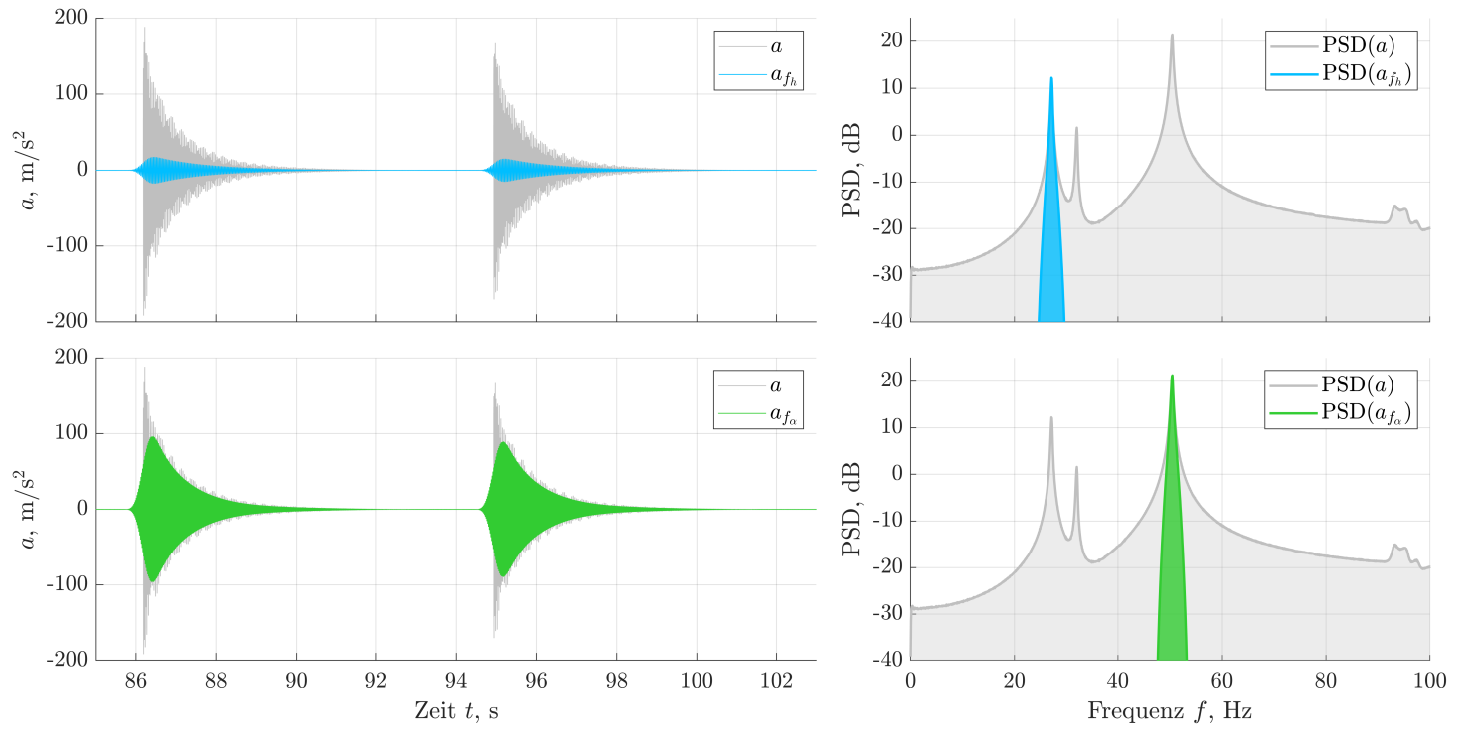

Abbildung F.1.: Zeitreihen und PSDs der Roh- (grau) und der gefilterten Signale, welche den Starrkörper-Schwingungsmoden Schlag $a_{f_{h}}$ (blau) und Drehung $a_{f_{\alpha}}$ (grün) der aeroelastischen 2-DoF Konfiguration zuzuordnen sind.

Zur Selektion der Schwingungsmode wurde das Rohsignal (grau in Abb. F.1) mit Hilfe eines digitalen Bandpassfilters gefiltert, der auf die jeweilige Frequenz der Schwingungsmode abgestimmt wurde. Die Filterbandweite wurde dahingehend optimiert, dass eine bestmögliche Approximation des jeweiligen Frequenzpeak, mindestens bis zu einem Abfall von $3 \mathrm{~dB}$ gewährleistet wurde. Für die ausgewählten Moden in Abb. F.1 betragen die 
Eigenfrequenzen $f_{h}=27,2 \mathrm{~Hz}$ und $f_{\alpha}=50,5 \mathrm{~Hz}$, die Filterbandweite betrug jeweils $\Delta f= \pm 0,2 \mathrm{~Hz}$. Die gefilterten Signale $a_{f_{h}}$ (blau) und $a_{f_{\alpha}}$ (grün) sind in Abb. F.1 sowohl als Zeitreihe sowie als PSD farblich dargestellt.
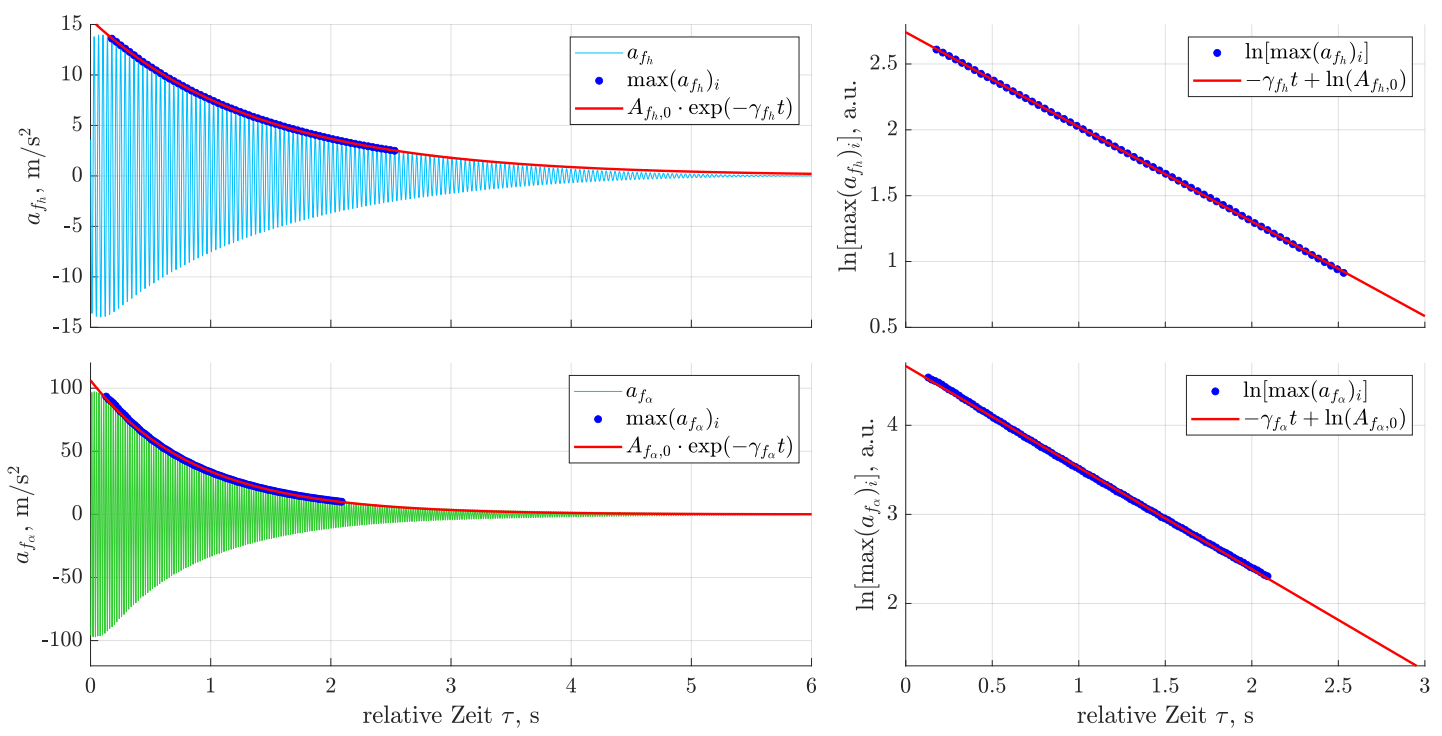

Abbildung F.2.: Darstellung der gefilterten Beschleunigungssignale der Schwingungsmoden $a_{f_{h}}$ (oben) und $a_{f_{\alpha}}$ (unten) sowie der ermittelten Abklingkurven, basierend auf der linearen Regression der ermittelten Maxima.

Für die gefilterten Kurven wurden jeweils die lokalen Amplitudenmaxima $\max \left(a_{f_{i}}\right)$ (blaue Punkte) bestimmt. Diese Maxima wurden unter Verwendung einer logarithmischen Skalierung durch eine lineare Regression mit

$$
\ln \left[f\left(\gamma_{f_{i}}\right)\right]=-\gamma_{f_{i}} t+\ln \left(A_{f_{i}, 0}\right)
$$

angenähert, was beispielhaft in Abb. F.2 für die zwei Schwingungsmoden dargestellt ist. Die Steigung der Regressionsgeraden repräsentiert den Abklingkoeffizienten $\gamma_{f_{i}}$ und der Schnittpunkt $f\left(\gamma_{f_{i}}\right)=0 \rightarrow \ln \left(A_{f_{i}, 0}\right)$ liefert die Amplitude $A_{f_{i}, 0}$ der zugehörigen Hüllkurve

$$
f\left(\gamma_{f_{i}}\right)=A_{f_{i}, 0} \cdot \exp \left(-\gamma_{f_{i}} t\right) .
$$

Diese sind ebenfalls in Abb. F.2 als rote Kurven dargestellt. Basierend auf dem Abklingkoeffizienten $\gamma_{f_{i}}$ kann der Dämpfungskoeffizient $d_{f_{i}}$ oder das LEHR'sche Dämpfungsmaß $D_{f_{i}}$ gemäß

$$
d_{f_{i}}=2 m \omega_{0, i} D_{f_{i}} \quad \text { bzw. } \quad D_{f_{i}}=\frac{\gamma_{f_{i}}}{\omega_{0, i}}
$$

berechnet werden. Dabei entspricht $m$ der schwingenden Masse bzw. dem Trägheitsmoment und $\omega_{0, i}$ der Kreisfrequenz der ungedämpften ${ }^{1}$ Schwingung [205, 50 ff.].

\footnotetext{
${ }^{1}$ Bedingt durch die geringe Dämpfung folgt $\omega_{0, i} \approx \omega_{d, i}$.
} 
Die Berechnung der finalen Dämpfungswerte der jeweiligen Schwingungsmoden basiert dann auf dem Mittelwert der ermittelten Abklingkoeffizienten aller aufgezeichneten Impulsantworten und Beschleunigungssensoren. Der Fehler $\sigma_{\gamma}$ entspricht der zugehörigen Standardabweichung. Das Lehr'sche Dämpfungsmaß folgt dann aus Gl. (F.1.3). Der Fehler $\sigma_{D}$ folgt aus dem Fehler $\sigma_{\gamma}$ und dem Fehler $\sigma_{f}$ der Eigenfrequenz der Schwingungsmode gemäß Gauß'scher Fehlerfortpflanzung zu

$$
\sigma_{D}=\sqrt{\sigma_{\gamma}^{2}\left(\frac{\partial D}{\partial \gamma}\right)^{2}+\sigma_{f}^{2}\left(\frac{\partial D}{\partial f}\right)^{2}}=\sqrt{\left(\frac{\sigma_{\gamma}}{2 \pi f}\right)^{2}+\left(\frac{\sigma_{f} \gamma}{2 \pi f^{2}}\right)^{2}} .
$$

Da die Bestimmung der Dämpfung von komplexen Strukturen im Allgemeinen mit größerer Unsicherheit behaftet ist, sollte der angegebene Dämpfungsfehler kritisch betrachtet werden. Vergleichbare Untersuchungen weisen auf einen deutlich höheren relativen Fehler von $\mathcal{O}(20 \%)$ [28] hin. Die Ergebnisse der Dämpfungsbestimmung für die zwei untersuchten aeroelastischen Konfigurationen sind in Tab. F.1 eingetragen.

Tabelle F.1.: Ermittelte Abklingkoeffizienten und LEHR'sche Dämpfungsmaße der zwei aeroelastischen Konfigurationen. Die Bezeichnung der Moden bezieht sich auf Abb. 7.2.

\begin{tabular}{cccccccc}
\hline System & Bez. & $\mathbf{f}, \mathrm{Hz}$ & $\sigma_{\mathbf{f}}, \mathrm{Hz}$ & $\gamma, \mathrm{s}^{-1}$ & $\sigma_{\gamma}, \mathrm{s}^{-1}$ & $\mathbf{D}, \%$ & $\sigma_{\mathbf{D}}, \%$ \\
\hline 2-DoF & $f_{h_{0}}$ & 27,2 & 0,1 & 0,80 & 0,05 & 0,47 & 0,03 \\
$"$ & $f_{r}$ & 32,1 & 0,2 & 0,74 & 0,07 & 0,36 & 0,03 \\
$"$ & $f_{\alpha_{0}}$ & 50,5 & 0,1 & 1,08 & 0,03 & 0,34 & 0,01 \\
$"$ & $f_{s_{1}}$ & 95,6 & 0,1 & 3,38 & 0,21 & 0,56 & 0,03 \\
$"$ & $f_{s_{2}}$ & 148,2 & 0,2 & 7,04 & 0,67 & 0,76 & 0,07 \\
" & $f_{s_{3}}$ & 179,3 & 0,3 & 7,17 & 1,18 & 0,64 & 0,10 \\
\hline 1-DoF & $f_{\alpha_{0}}$ & 47,7 & 0,1 & 1,18 & 0,03 & 0,39 & 0,01 \\
$"$ & $f_{s_{1}}$ & 83,5 & 0,1 & 4,84 & 0,56 & 0,92 & 0,11 \\
$"$ & $f_{s_{2}}$ & 124,6 & 0,2 & 3,80 & 0,07 & 0,48 & 0,01 \\
$"$ & $f_{s_{3}}$ & 167,6 & 0,2 & 9,39 & 1,94 & 0,89 & 0,18 \\
$"$ & $f_{s_{4}}$ & 186,5 & 0,2 & 9,63 & 1,24 & 0,82 & 0,11 \\
\hline
\end{tabular}




\section{F.2. Schwingungsmoden der aeroelastischen Konfigurationen}

Die Darstellung der Schwingungsmoden der aeroelastischen Konfigurationen basiert auf den Ergebnissen der Vibrationstests. Die in Abb. F.3 und F.4 dargestellten roten Punkte markieren jeweils einen Beschleunigungssensor. Die orange Linie markiert die Drehachse bei $e=c / 4$ und das graue Gitternetz zeigt die Nulllage der Struktur. Die dargestellten Moden sind nicht normiert und zur besseren Veranschaulichung unterschiedlich skaliert. Die Bezeichnung der Moden bezieht sich auf Abb. 7.2.

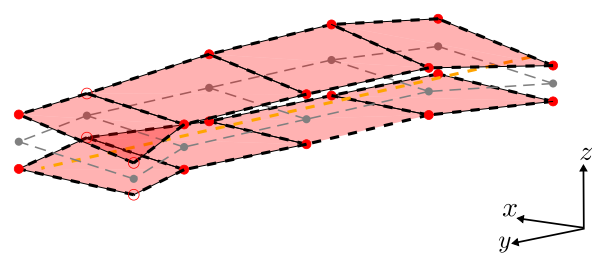

(a) Schlag bei $f_{h_{0}}=27,2 \mathrm{~Hz}$

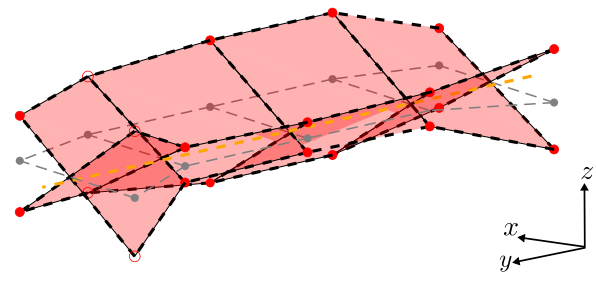

(c) Drehung bei $f_{\alpha_{0}}=50,5 \mathrm{~Hz}$

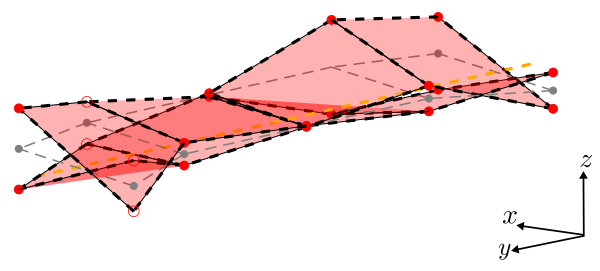

(e) 2. Biegung +1 . Torsion bei $f_{s_{2}}=148,2 \mathrm{~Hz}$

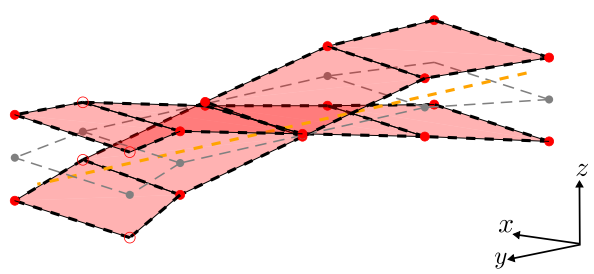

(b) Rollen bei $f_{r}=32,1 \mathrm{~Hz}$

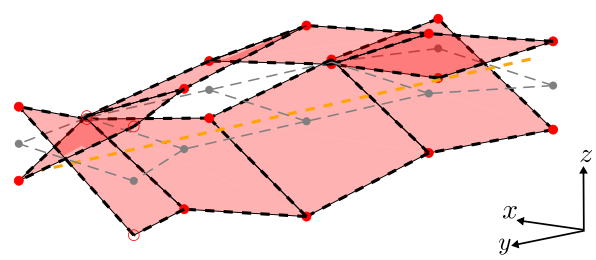

(d) 1. Biegung + Drehung bei $f_{s_{1}}=95,6 \mathrm{~Hz}$

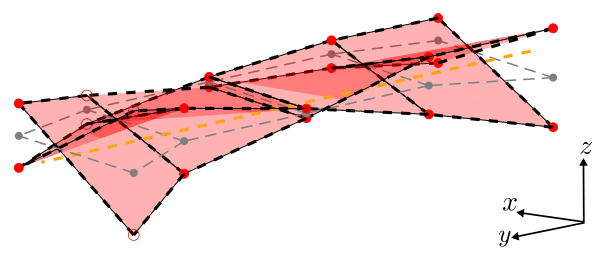

(f) 1. Torsion bei $f_{s_{3}}=179,3 \mathrm{~Hz}$

Abbildung F.3.: Identifizierte Schwingungsmoden der aeroelastischen 2-DoF Konfiguration. 


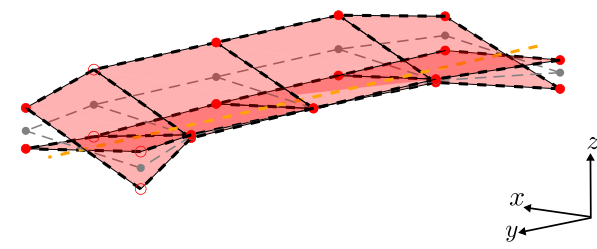

(a) Drehung bei $f_{\alpha_{0}}=47,7 \mathrm{~Hz}$

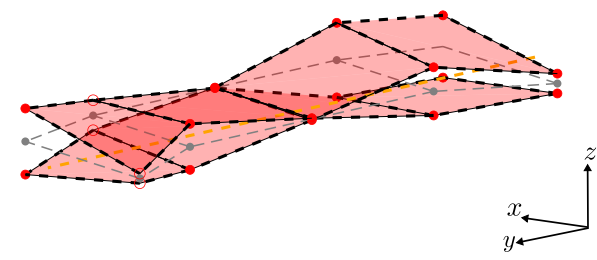

(c) 2. Biegung bei $f_{s_{2}}=124,6 \mathrm{~Hz}$

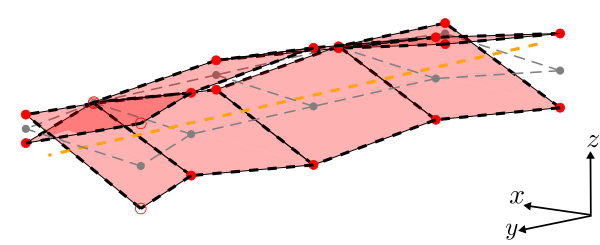

(b) 1 . Biegung + Drehung bei $f_{s_{1}}=83,5 \mathrm{~Hz}$

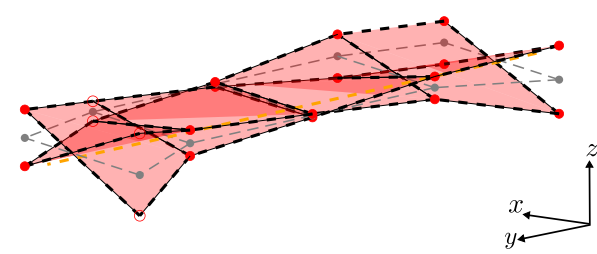

(d) 1 . Torsion bei $f_{s_{3}}=167,6 \mathrm{~Hz}$

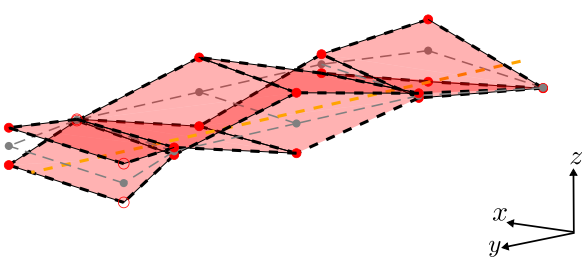

(e) 3. Biegung bei $f_{s_{4}}=186,5 \mathrm{~Hz}$

Abbildung F.4.: Identifizierte Schwingungsmoden der aeroelastischen 1-DoF Konfiguration. 


\section{F.3. Windkanalresonanzen}

In Windkanal-Messstrecken mit geschlossenen Wänden können Interferenzen auftreten, die sowohl das stationäre als auch das instationäre Strömungsfeld beeinflussen. Diese Störungen stellen eine zusätzliche instationäre Komponente der aerodynamischen Kräfte dar und können mit der Flügelstruktur interagieren [234]. Hierdurch kann es zu einer Beeinflussung der Dynamik der Versuchskonfiguration kommen. Zur Minimierung von Wandeffekten werden in der vorliegenden Arbeit adaptive Windkanalwände eingesetzt (vgl. 3.1), jedoch wird dabei keine vollständige Unterdrückung von Störungen erzielt.

So spielt das Auftreten von Kanalwandreflexionen in Form von Schallwellen eine wesentliche Rolle [235], insbesondere bei kompressiblen Strömungen und höheren Werten der reduzierten Frequenz [236]. Dabei bilden sich zwischen Kanalwand und Modell stehende Wellenmuster aus, wenn eine Resonanzbedingung erfüllt ist. Bei aeroelastischen Versuchen muss also sichergestellt werden, dass die Frequenzen von Windkanalresonanzen von den strukturellen Eigenfrequenzen bzw. von Flatterfrequenzen des aeroelastischen Systems getrennt sind.

Für eine Windkanal-Messstrecke mit geschlossenen, festen Wänden hängt die Resonanzkreisfrequenz $\omega_{n}$ von der Machzahl $\mathrm{Ma}_{\infty}$ und der Geschwindigkeit der freien Strömung $u_{\infty}$ ab. Eine einfache Abschätzung von $\omega_{n}[236]$ ist gegeben durch

$$
\omega_{n}=(2 n-1) \cdot \pi u_{\infty} \frac{\sqrt{1-\mathrm{Ma}_{\infty}^{2}}}{\mathrm{Ma}_{\infty}} \cdot \frac{1}{2 b} .
$$

Dabei bezeichnet $b$ die Hälfte der Höhe der Windkanal-Messstrecke und $n \in \mathbb{N}^{*}$ berücksichtigt höhere Harmonische. Durch Ersetzen der Strömungsgeschwindigkeit $u_{\infty}$ und der statischen Temperatur $T_{\infty}$ mit

$$
u_{\infty}=\mathrm{Ma}_{\infty} \cdot \sqrt{\kappa R_{s} T_{\infty}} \text { und } T_{\infty}=\frac{T_{0}}{1+\frac{\kappa-1}{2} \cdot \mathrm{Ma}_{\infty}^{2}}
$$

kann Gl. (F.3.1) in Abhängigkeit von $\mathrm{Ma}_{\infty}$ und $T_{0}$ ausgedrückt werden. Da die Ruhetemperatur $T_{0}$ im Verlauf einer Messung nahezu konstant ist, kann die Resonanzbedingung in Bezug auf die Mach-Zahl für gegebene $T_{0}$ ausgewertet werden.

In Abb. F.5 sind die Frequenzen $f_{1}=\frac{\omega_{1}}{2 \pi}$ für die erste Harmonische der Windkanalresonanzbedingung nach Gl. F.3.1 für die minimale und maximale Ruhetemperatur ausgewertet, die während der Messungen vorlagen ${ }^{2}$.

\footnotetext{
${ }^{2}$ Die Untersuchungen der stationären und instationären Aerodynamik des CAST 10-2 Windkanalmodells fanden unter vergleichbaren Strömungsbedingungen statt, so dass die Resonanzfrequenzen in einem ähnlichen Bereich liegen.
} 


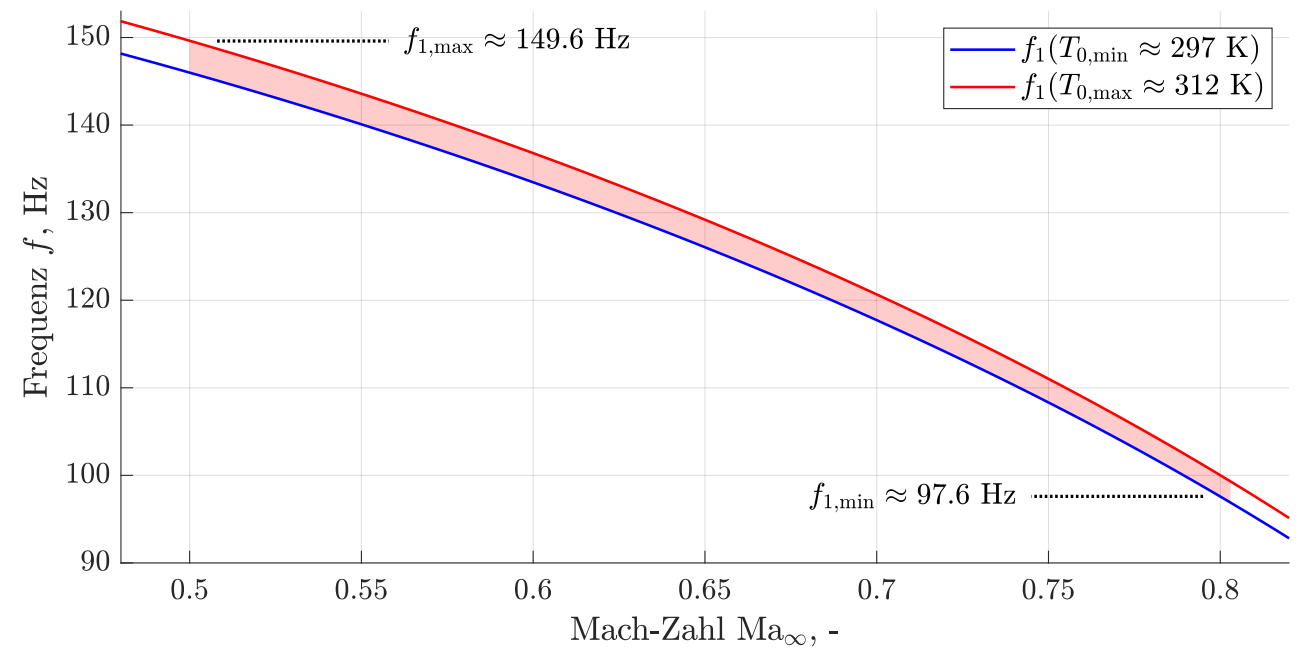

Abbildung F.5.: Windkanalresonanzfrequenzen $f_{1}$ für die geschlossene Teststrecke des DNWTWG mit einem Querschnitt von $1 \mathrm{~m} \times 1 \mathrm{~m}$, ausgewertet für zwei Ruhetemperaturen $T_{0, \text { min }}$ und $T_{0, \max }$, die den Messbereich der Flattermessungen abdecken.

Für den Mach-Zahl-Bereich, in dem Messungen durchgeführt wurden (rot hinterlegter Bereich in Abb. F.5), liegen die Resonanzfrequenzen bei $97.6 \mathrm{~Hz} \lesssim f_{1} \lesssim 149.6 \mathrm{~Hz}$. Ein Vergleich mit den Starrkörpereigenfrequenzen der aeroelastischen Konfigurationen (vgl. 7.1) sowie den auftretenden Flatterfrequenzen (vgl. 7.2 und 7.3) zeigt, dass diese deutlich unterhalb der Resonanzfrequenzen liegen. Es kann somit ausgeschlossen werden, dass Windkanalresonanzen mit aeroelastischen Starrkörper-Eigenfrequenzen des Windkanalmodells interagieren oder auch mit beobachteten Instationaritäten (vgl. 5.4 und 7.4) in Verbindung stehen.

\section{F.4. Zu 7.3.3: Einfluss des Anstellwinkels}

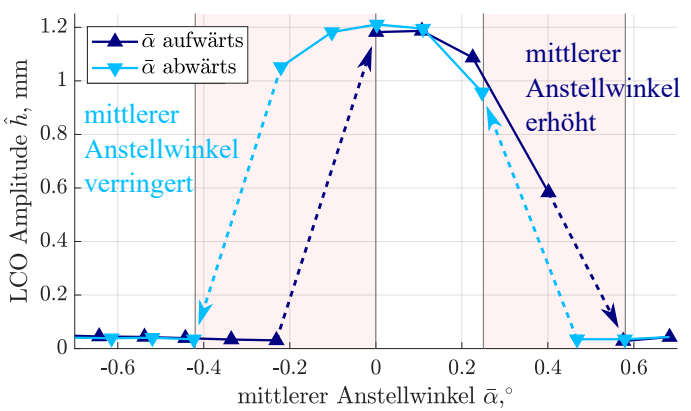

(a) LCO Amplituden der Dreh-Bewegung

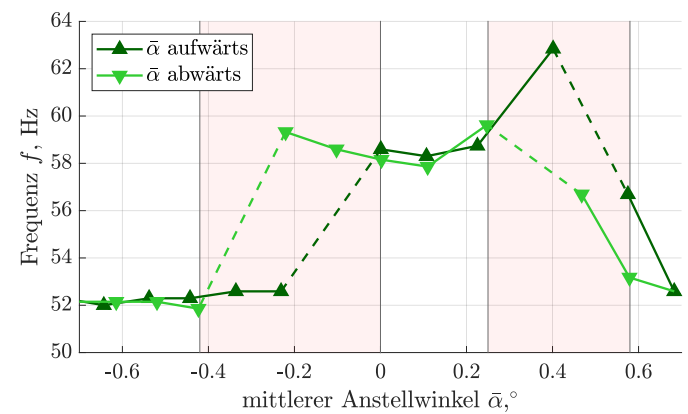

(b) Spektren der $\mathrm{Ma}_{\infty}$-Variation

Abbildung F.6.: Bifurkationsdiagramm des Schlags (a) und identifizierte Frequenzen (b) bei einer Variation des mittleren Anstellwinkels $\bar{\alpha}$ für die aeroelastische 1-DoF Konfiguration bei $\mathrm{Ma}_{\infty}=0,73$ und $p_{0}=55 \mathrm{kPa}$, verändert nach [63]. 


\section{F.5. Zu 7.4.1: Verhalten von Stoß und Transition}

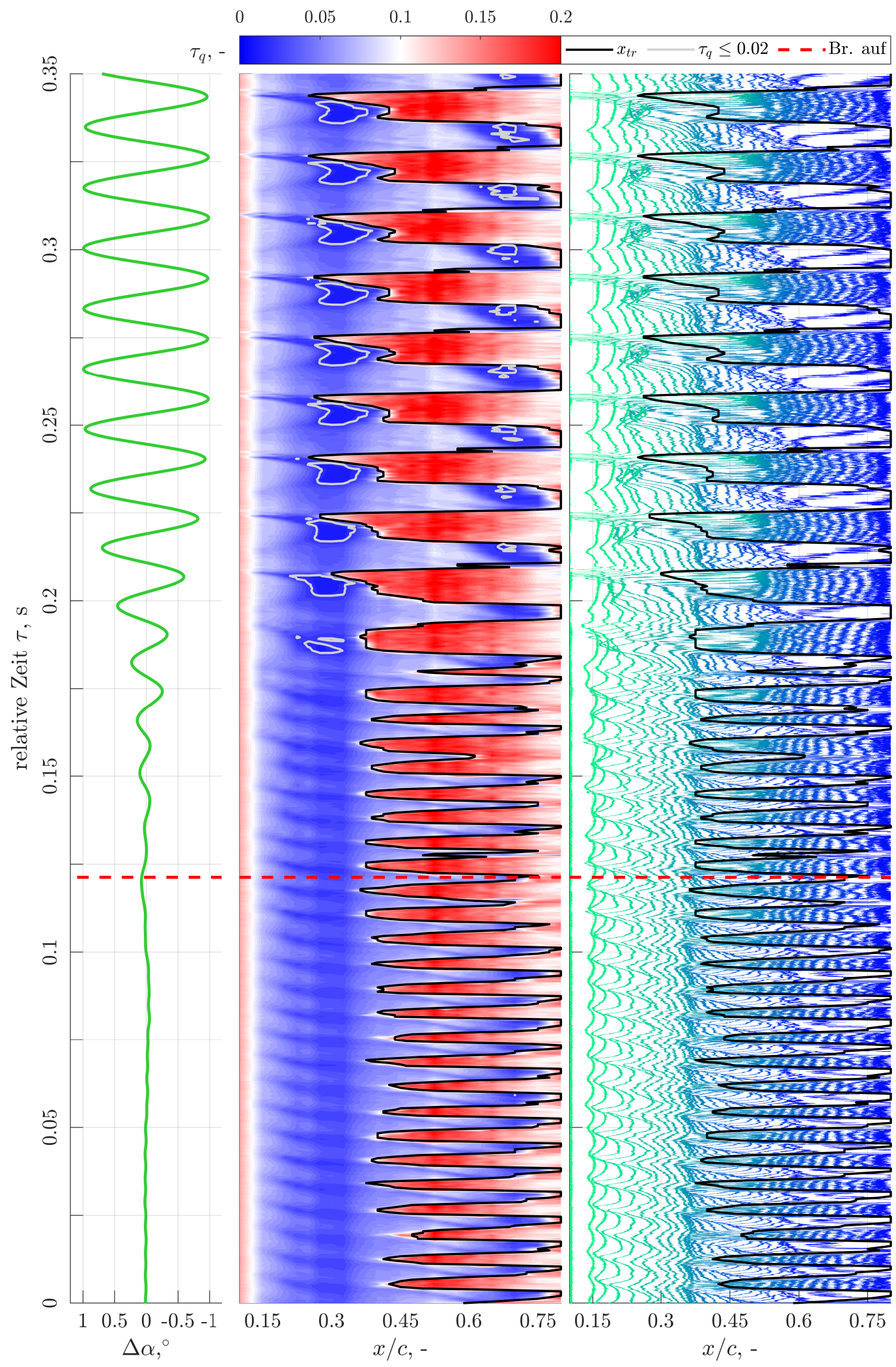

Abbildung F.7.: Verlauf der Quasi-Wandschubspannung $\tau_{q}$ (mitte) und der Transitionsposition $x_{t r}$ während des LCO (links) bei $\mathrm{Ma}_{\infty}=0,732, p_{0}=55 \mathrm{kPa}$ und $\bar{\alpha}=0,06^{\circ}$. Rechts sind die Spannungszeitschriebe der CTAs mit der ermittelten Transitionsposition dargestellt. 


\section{F.6. Zu 7.4.2: Energie- und Leistungsbetrachtung}

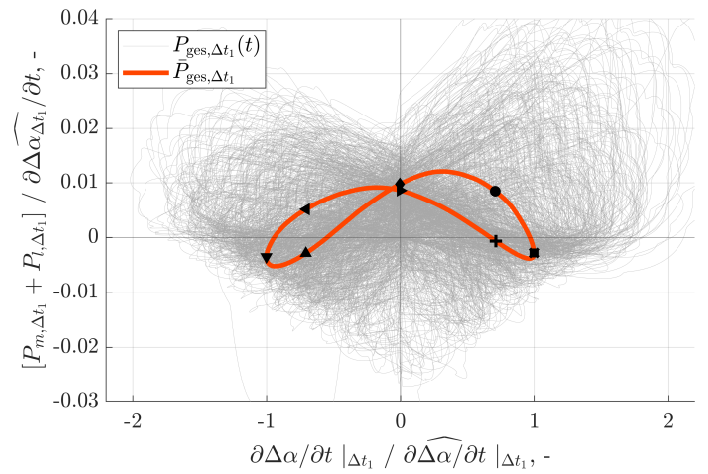

(a) Aerodynamische Gesamtleistung im Zeitausschnitt $\Delta t_{1}$, es gilt $\bar{T}_{\Delta t_{1}}^{-1} \int_{0}^{\bar{T}_{\Delta t_{1}}} \bar{P}_{\text {aero, } \Delta t_{1}} \mathrm{~d} t>0$

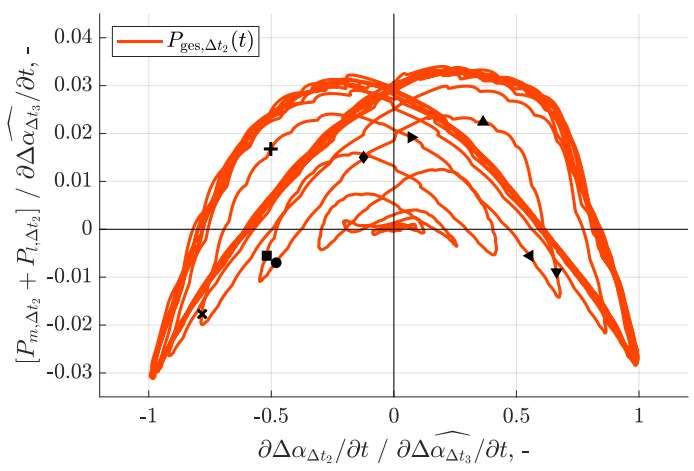

(b) Aerodynamische Gesamtleistung im Zeitausschnitt $\Delta t_{2}$, es gilt $\bar{T}_{\Delta t_{2}}^{-1} \int_{0}^{\bar{T}_{\Delta t_{2}}} \bar{P}_{\text {aero, } \Delta t_{2}} \mathrm{~d} t>0$

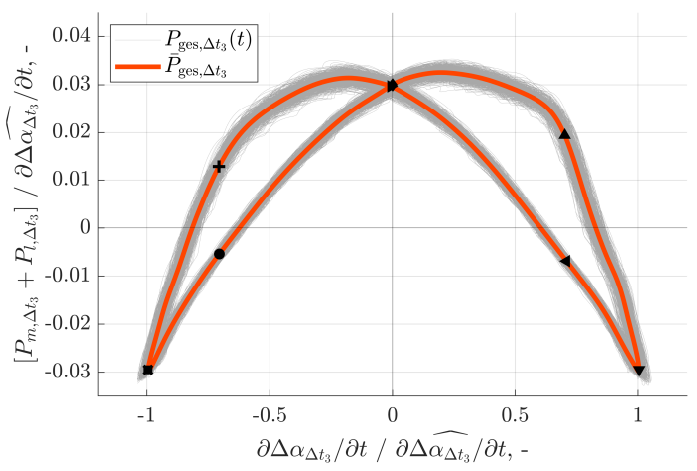

(c) Aerodynamische Gesamtleistung im Zeitausschnitt $\Delta t_{3}$, es gilt $\bar{T}_{\Delta t_{3}}^{-1} \int_{0}^{\bar{T}_{\Delta t_{3}}} \bar{P}_{\text {aero }, \Delta t_{3}} \mathrm{~d} t>0$

Abbildung F.8.: Aerodynamischer Leistungseintrag $P_{m}+P_{l}$ für drei Zeitausschnitte während des LCOs bei $\mathrm{Ma}_{\infty}=0,732, p_{0}=55 \mathrm{kPa}$ und $\bar{\alpha}=0,06^{\circ}$. Der erste Zeitausschnitt $\Delta t_{1}$ (a) betrifft den Zeitraum bei geschlossener Flatterbremse, also bei einem fixierten Modell. $\Delta t_{2}$ (b) umfasst den Zeitausschnitt des aufklingenden LCOs und der Zeitausschnitt $\Delta t_{3}$ (c) deckt den aufgezeichneten Zeitraum des ausgebildeten aeroelastischen Grenzzyklus ab. 
